\title{
Anti-fibrinolytic use for minimising perioperative allogeneic blood transfusion (Review)
}

Henry DA, Carless PA, Moxey AJ, O’Connell D, Stokes BJ, Fergusson DA, Ker K

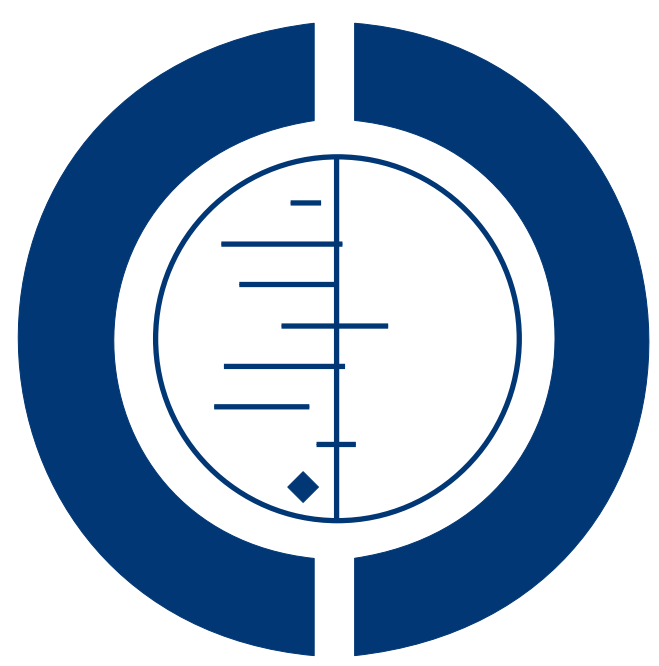

\section{THE COCHRANE COLLABORATION $^{\circledR}$}

This is a reprint of a Cochrane review, prepared and maintained by The Cochrane Collaboration and published in The Cochrane Library 2011, Issue 1

http://www.thecochranelibrary.com

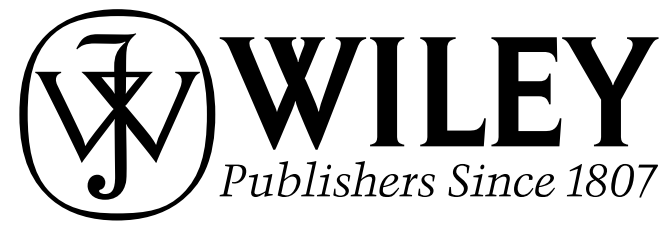

Anti-fibrinolytic use for minimising perioperative allogeneic blood transfusion (Review)

Copyright (C) 201 I The Cochrane Collaboration. Published by John Wiley \& Sons, Ltd. 
TABLE OF CONTENTS

HEADER . . . . . . . . . . . . . . . . . . . . . . . . . . . . . . . . . . . . . . . 1

ABSTRACT .. . . . . . . . . . . . . . . . . . . . . . . . . . . . . . . . . . . . . . . . . . . . . . . . .

PLAIN LANGUAGE SUMMARY . . . . . . . . . . . . . . . . . . . . . . . . . . . . . . . . . . . $\quad$. 2

BACKGROUND . . . . . . . . . . . . . . . . . . . . . . . . . . . . . . . . . . . . . 2

OBJECTIVES . . . . . . . . . . . . . . . . . . . . . . . . . . . . . . . . . . . . . . . . 3

METHODS . . . . . . . . . . . . . . . . . . . . . . . . . . . . . . . . . . . . . . 44

RESULTS . . . . . . . . . . . . . . . . . . . . . . . . . . . . . . . . . . . . . . . 6

Figure 1. . . . . . . . . . . . . . . . . . . . . . . . . . . . . . . . . . . . . . 8

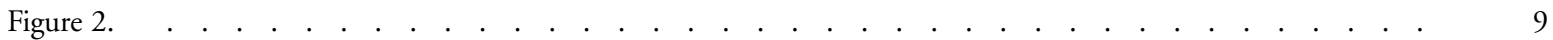

DISCUSSION . . . . . . . . . . . . . . . . . . . . . . . . . . . . . . . . . . . . . . . . .

Figure 3. . . . . . . . . . . . . . . . . . . . . . . . . . . . . . . . . . . . . . 25

Figure 4. . . . . . . . . . . . . . . . . . . . . . . . . . . . . . . . . . . . . . 26

Figure 5. . . . . . . . . . . . . . . . . . . . . . . . . . . . . . . . . . . . . . $\quad .27$

Figure 6. . . . . . . . . . . . . . . . . . . . . . . . . . . . . . . . 28

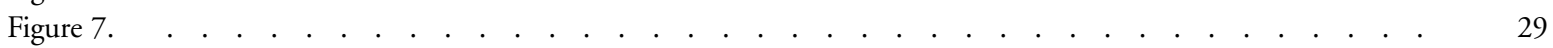

Figure 8. . . . . . . . . . . . . . . . . . . . . . . . . . . . . . . . . . . . . . 30

Figure 9. . . . . . . . . . . . . . . . . . . . . . . . . . . . . . . . . . . . . . 31

Figure 10. . . . . . . . . . . . . . . . . . . . . . . . . . . . . . . . . . . . . . $\quad 33$

AUTHORS' CONCLUSIONS . . . . . . . . . . . . . . . . . . . . . . . . . . . . . . . . . . . . . 35

ACKNOWLEDGEMENTS . . . . . . . . . . . . . . . . . . . . . . . . . . . . . . . . . . . . . . 36

REFERENCES . . . . . . . . . . . . . . . . . . . . . . . . . . . . . . . . . . . . . 36

CHARACTERISTICS OF STUDIES . . . . . . . . . . . . . . . . . . . . . . . . . . . . . . . 50

DATA AND ANALYSES . . . . . . . . . . . . . . . . . . . . . . . . . . . . . . . . . . . . . . . . . . . . . $\quad 238$

Analysis 1.1. Comparison 1 Aprotinin versus Control (Blood Transfusion \& Blood Loss), Outcome 1 No. Exposed to Allogeneic Blood. . . . . . . . . . . . . . . . . . . . . . . . . . . . . . . . .

Analysis 1.2. Comparison 1 Aprotinin versus Control (Blood Transfusion \& Blood Loss), Outcome 2 No. Exposed to Allogeneic Blood - Type of Surgery. . . . . . . . . . . . . . . . . . . . . . . . . . . . . . . . . . . . . . .

Analysis 1.3. Comparison 1 Aprotinin versus Control (Blood Transfusion \& Blood Loss), Outcome 3 No. Exposed to Allogeneic Blood - Transfusion Protocol. . . . . . . . . . . . . . . . . . . . . . . . .

Analysis 1.4. Comparison 1 Aprotinin versus Control (Blood Transfusion \& Blood Loss), Outcome 4 No. Exposed to Allogeneic Blood - Dose. . . . . . . . . . . . . . . . . . . . . . . . . . . . . . . 262

Analysis 1.5. Comparison 1 Aprotinin versus Control (Blood Transfusion \& Blood Loss), Outcome 5 No. Exposed to Allogeneic Blood - Dose (Cardiac Surgery). . . . . . . . . . . . . . . . . . . . . . . . . . . . . . . 267

Analysis 1.6. Comparison 1 Aprotinin versus Control (Blood Transfusion \& Blood Loss), Outcome 6 Trial Methodological Quality - Allocation Concealment. . . . . . . . . . . . . . . . . . . . . . . . . . . 271

Analysis 1.7. Comparison 1 Aprotinin versus Control (Blood Transfusion \& Blood Loss), Outcome 7 Units of Allogeneic Blood Transfused - Transfused Patients. . . . . . . . . . . . . . . . . . . . . . . . . . 275

Analysis 1.8. Comparison 1 Aprotinin versus Control (Blood Transfusion \& Blood Loss), Outcome 8 Units of Allogeneic Blood Transfused - All Patients. . . . . . . . . . . . . . . . . . . . . . . . . . . . . 277

Analysis 1.9. Comparison 1 Aprotinin versus Control (Blood Transfusion \& Blood Loss), Outcome 9 Blood loss - Intraoperative. . . . . . . . . . . . . . . . . . . . . . . . . . . . . . . . . . . . 280

Analysis 1.10. Comparison 1 Aprotinin versus Control (Blood Transfusion \& Blood Loss), Outcome 10 Blood loss - Postoperative. . . . . . . . . . . . . . . . . . . . . . . . . . . . . . . . . . . . 281

Analysis 1.11. Comparison 1 Aprotinin versus Control (Blood Transfusion \& Blood Loss), Outcome 11 Blood loss - Postoperative - Dose (Cardiac Surgery)...$\quad$. . . . . . . . . . . . . . . . . . . . . . . . . . . . . . . . 285

Analysis 1.12. Comparison 1 Aprotinin versus Control (Blood Transfusion \& Blood Loss), Outcome 12 Blood loss Total. . . . . . . . . . . . . . . . . . . . . . . . . . . . . . . . . . . . . 289

Analysis 2.1. Comparison 2 Tranexamic Acid versus Control (Blood Transfusion \& Blood Loss), Outcome 1 No. Exposed to Allogeneic Blood...$\quad$. . . . . . . . . . . . . . . . . . . . . . . . . . . . . . . . . . . . . . . . . 290

Analysis 2.2. Comparison 2 Tranexamic Acid versus Control (Blood Transfusion \& Blood Loss), Outcome 2 No. Exposed to Allogeneic Blood - Type of Surgery. 
Analysis 2.3. Comparison 2 Tranexamic Acid versus Control (Blood Transfusion \& Blood Loss), Outcome 3 No. Exposed to Allogeneic Blood - Transfusion Protocol. . . . . . . . . . . . . . . . . . . . . . . . .

Analysis 2.4. Comparison 2 Tranexamic Acid versus Control (Blood Transfusion \& Blood Loss), Outcome 4 No. Exposed to Allogeneic Blood - Dose (Cardiac Surgery). . . . . . . . . . . . . . . . . . . . . . . . . . . . .

Analysis 2.5. Comparison 2 Tranexamic Acid versus Control (Blood Transfusion \& Blood Loss), Outcome 5 Trial Methodological Quality - Allocation Concealment. . . . . . . . . . . . . . . . . . . . . . Analysis 2.6. Comparison 2 Tranexamic Acid versus Control (Blood Transfusion \& Blood Loss), Outcome 6 Units Allogeneic Blood Transfused - Transfused Patients. . . . . . . . . . . . . . . . . . . . . . Analysis 2.7. Comparison 2 Tranexamic Acid versus Control (Blood Transfusion \& Blood Loss), Outcome 7 Units of Allogeneic Blood Transfused - All Patients. . . . . . . . . . . . . . . . . . . . . . . . .

Analysis 2.8. Comparison 2 Tranexamic Acid versus Control (Blood Transfusion \& Blood Loss), Outcome 8 Blood loss Intra-operative.

Analysis 2.9. Comparison 2 Tranexamic Acid versus Control (Blood Transfusion \& Blood Loss), Outcome 9 Blood loss Post-operative. . . . . . . . . . . . . . . . . . . . . . . . . . . . . . . . . . . .

Analysis 2.10. Comparison 2 Tranexamic Acid versus Control (Blood Transfusion \& Blood Loss), Outcome 10 Blood loss Post-operative - Dose (Cardiac Surgery). . . . . . . . . . . . . . . . . . . . . . . . . . . . . . . . . .

Analysis 2.11. Comparison 2 Tranexamic Acid versus Control (Blood Transfusion \& Blood Loss), Outcome 11 Blood loss Total.

Analysis 3.1. Comparison 3 Epsilon Aminocaproic Acid versus Control (Blood Transfusion \& Blood Loss), Outcome 1 No. Exposed to Allogeneic Blood. . . . . . . . . . . . . . . . . . . . . . . . . . . . . . Analysis 3.2. Comparison 3 Epsilon Aminocaproic Acid versus Control (Blood Transfusion \& Blood Loss), Outcome 2 No. Exposed to Allogeneic Blood - Type of Surgery. . . . . . . . . . . . . . . . . . . . . . . . Analysis 3.3. Comparison 3 Epsilon Aminocaproic Acid versus Control (Blood Transfusion \& Blood Loss), Outcome 3 No. Exposed to Allogeneic Blood - Transfusion Protocol. . . . . . . . . . . . . . . . . . . . Analysis 3.4. Comparison 3 Epsilon Aminocaproic Acid versus Control (Blood Transfusion \& Blood Loss), Outcome 4 Trial Methodological Quality - Allocation Concealment. . . . . . . . . . . . . . . . . . . . Analysis 3.5. Comparison 3 Epsilon Aminocaproic Acid versus Control (Blood Transfusion \& Blood Loss), Outcome 5 Units of Allogeneic Blood Transfused - Transfused Patients. . . . . . . . . . . . . . . . . . . Analysis 3.6. Comparison 3 Epsilon Aminocaproic Acid versus Control (Blood Transfusion \& Blood Loss), Outcome 6 Units of Allogeneic Blood Transfused - All Patients. . . . . . . . . . . . . . . . . . . . . . Analysis 3.7. Comparison 3 Epsilon Aminocaproic Acid versus Control (Blood Transfusion \& Blood Loss), Outcome 7 Blood loss - Intra-operative.

Analysis 3.8. Comparison 3 Epsilon Aminocaproic Acid versus Control (Blood Transfusion \& Blood Loss), Outcome 8 Blood loss - Post-operative. . . . . . . . . . . . . . . . . . . . . . . . . . . . . . . . . . . . . .

Analysis 3.9. Comparison 3 Epsilon Aminocaproic Acid versus Control (Blood Transfusion \& Blood Loss), Outcome 9 Blood loss - Total.

Analysis 4.1. Comparison 4 Aprotinin versus Tranexamic Acid (Blood Transfusion \& Blood Loss), Outcome 1 No. Exposed to Allogeneic Blood. . . . . . . . . . . . . . . . . . . . . . . . . . . . . . . . . 32

Analysis 4.2. Comparison 4 Aprotinin versus Tranexamic Acid (Blood Transfusion \& Blood Loss), Outcome 2 No. Exposed to Allogeneic Blood - Type of Surgery. . . . . . . . . . . . . . . . . . . . . . . . . . . . . . . .

Analysis 4.3. Comparison 4 Aprotinin versus Tranexamic Acid (Blood Transfusion \& Blood Loss), Outcome 3 No. Exposed to Allogeneic Blood - Transfusion Protocol. . . . . . . . . . . . . . . . . . . . . . . . . . . Analysis 4.4. Comparison 4 Aprotinin versus Tranexamic Acid (Blood Transfusion \& Blood Loss), Outcome 4 Trial Methodological Quality - Allocation Concealment. . . . . . . . . . . . . . . . . . . . . .

Analysis 4.5. Comparison 4 Aprotinin versus Tranexamic Acid (Blood Transfusion \& Blood Loss), Outcome 5 Units Allogeneic Blood Transfused - Transfused Patients. . . . . . . . . . . . . . . . . . . . . .

Analysis 4.6. Comparison 4 Aprotinin versus Tranexamic Acid (Blood Transfusion \& Blood Loss), Outcome 6 Units Allogeneic Blood Transfused - All Patients. . . . . . . . . . . . . . . . . . . . . . . . .

Analysis 4.7. Comparison 4 Aprotinin versus Tranexamic Acid (Blood Transfusion \& Blood Loss), Outcome 7 Blood loss.

Analysis 5.1. Comparison 5 Aprotinin versus Epsilon Aminocaproic Acid (Blood Transfusion \& Blood Loss), Outcome 1 No. Exposed to Allogeneic Blood. 
Analysis 5.2. Comparison 5 Aprotinin versus Epsilon Aminocaproic Acid (Blood Transfusion \& Blood Loss), Outcome 2

No. Exposed to Allogeneic Blood - Type of Surgery. . . . . . . . . . . . . . . . . . . . . . . .

Analysis 5.3. Comparison 5 Aprotinin versus Epsilon Aminocaproic Acid (Blood Transfusion \& Blood Loss), Outcome 3

No. Exposed to Allogeneic Blood - Transfusion Protocol. . . . . . . . . . . . . . . . . . . . . .

Analysis 5.4. Comparison 5 Aprotinin versus Epsilon Aminocaproic Acid (Blood Transfusion \& Blood Loss), Outcome 4

Trial Methodological Quality - Allocation Concealment. . . . . . . . . . . . . . . . . . . .

Analysis 5.5. Comparison 5 Aprotinin versus Epsilon Aminocaproic Acid (Blood Transfusion \& Blood Loss), Outcome 5

Units of Allogeneic Blood Transfused - Transfused Patients. . . . . . . . . . . . . . . . . . .

Analysis 5.6. Comparison 5 Aprotinin versus Epsilon Aminocaproic Acid (Blood Transfusion \& Blood Loss), Outcome 6

Units of Allogeneic Blood Transfused - All Patients. . . . . . . . . . . . . . . . . . . . . . 335

Analysis 5.7. Comparison 5 Aprotinin versus Epsilon Aminocaproic Acid (Blood Transfusion \& Blood Loss), Outcome 7

Blood loss. . . . . . . . . . . . . . . . . . . . . . . . . . . . . . . . . . . .

Analysis 6.1. Comparison 6 Tranexamic Acid versus Epsilon Aminocaproic Acid (Blood Transfusion \& Blood Loss),

Outcome 1 No. Exposed to Allogeneic Blood. . . . . . . . . . . . . . . . . . . . . . . .

Analysis 6.2. Comparison 6 Tranexamic Acid versus Epsilon Aminocaproic Acid (Blood Transfusion \& Blood Loss),

Outcome 2 No. Exposed to Allogeneic Blood - Type of Surgery. . . . . . . . . . . . . . . . . . . . . .

Analysis 6.3. Comparison 6 Tranexamic Acid versus Epsilon Aminocaproic Acid (Blood Transfusion \& Blood Loss),

Outcome 3 No. Exposed to Allogeneic Blood - Transfusion Protocol. . . . . . . . . . . . . . . . .

Analysis 6.4. Comparison 6 Tranexamic Acid versus Epsilon Aminocaproic Acid (Blood Transfusion \& Blood Loss),

Outcome 4 Trial Methodological Quality - Allocation Concealment. . . . . . . . . . . . . . . .

Analysis 6.5. Comparison 6 Tranexamic Acid versus Epsilon Aminocaproic Acid (Blood Transfusion \& Blood Loss),

Outcome 5 Units of Allogeneic Blood Transfused - Transfused Patients. . . . . . . . . . . . . . . .

Analysis 6.6. Comparison 6 Tranexamic Acid versus Epsilon Aminocaproic Acid (Blood Transfusion \& Blood Loss),

Outcome 6 Units of Allogeneic Blood Transfused - All Patients.

Analysis 6.7. Comparison 6 Tranexamic Acid versus Epsilon Aminocaproic Acid (Blood Transfusion \& Blood Loss),

Outcome 7 Blood loss. . . . . . . . . . . . . . . . . . . . . . . . . . . . . . . .

Analysis 7.1. Comparison 7 Aprotinin versus Lysine Analogues (Blood Transfusion), Outcome 1 No. Exposed to Allogeneic

Blood. . . . . . . . . . . . . . . . . . . . . . . . . . . . . . . . . . . . .

Analysis 7.2. Comparison 7 Aprotinin versus Lysine Analogues (Blood Transfusion), Outcome 2 No. Exposed to Allogeneic

Blood - Type of Surgery. . . . . . . . . . . . . . . . . . . . . . . . . . . . . . . . . 344

Analysis 7.3. Comparison 7 Aprotinin versus Lysine Analogues (Blood Transfusion), Outcome 3 No. Exposed to Allogeneic

Blood - Transfusion Protocol. . . . . . . . . . . . . . . . . . . . . . . . . . . . . 346

Analysis 7.4. Comparison 7 Aprotinin versus Lysine Analogues (Blood Transfusion), Outcome 4 Units of Allogeneic Blood

Transfused - Transfused Patients. . . . . . . . . . . . . . . . . . . . . . . . . . . . 348

Analysis 7.5. Comparison 7 Aprotinin versus Lysine Analogues (Blood Transfusion), Outcome 5 Units of Allogeneic Blood

Transfused - All Patients. . . . . . . . . . . . . . . . . . . . . . . . . . . . . . .

Analysis 7.6. Comparison 7 Aprotinin versus Lysine Analogues (Blood Transfusion), Outcome 6 Trial Methodological

Quality - Allocation Concealment.

Analysis 8.1. Comparison 8 Adverse Events and Other Outcomes (Active versus Control), Outcome 1 Re-operation for bleeding.

Analysis 8.2. Comparison 8 Adverse Events and Other Outcomes (Active versus Control), Outcome 2 Mortality. . .

Analysis 8.3. Comparison 8 Adverse Events and Other Outcomes (Active versus Control), Outcome 3 Myocardial Infarction (MI). . . . . . . . . . . . . . . . . . . . . . . . . . . . . . . . . . . 360

Analysis 8.4. Comparison 8 Adverse Events and Other Outcomes (Active versus Control), Outcome 4 Stroke (CVA).

Analysis 8.5. Comparison 8 Adverse Events and Other Outcomes (Active versus Control), Outcome 5 Deep Vein Thrombosis (DVT).

Analysis 8.6. Comparison 8 Adverse Events and Other Outcomes (Active versus Control), Outcome 6 Pulmonary Embolism (PE).

Analysis 8.7. Comparison 8 Adverse Events and Other Outcomes (Active versus Control), Outcome 7 Other Thrombosis.

Analysis 8.8. Comparison 8 Adverse Events and Other Outcomes (Active versus Control), Outcome 8 Coronary artery graft occlusion. 
Analysis 8.9. Comparison 8 Adverse Events and Other Outcomes (Active versus Control), Outcome 9 Renal Failure / Dysfunction. . . . . . . . . . . . . . . . . . . . . . . . . . . . . . . . . . .

Analysis 8.10. Comparison 8 Adverse Events and Other Outcomes (Active versus Control), Outcome 10 Hospital Length of Stay. . . . . . . . . . . . . . . . . . . . . . . . . . . . . . . . . . . . .

Analysis 9.1. Comparison 9 Adverse Events and Other Outcomes (Active versus Active), Outcome 1 Re-operation for bleeding - Aprotinin versus Tranexamic Acid. . . . . . . . . . . . . . . . . . . . . . . .

Analysis 9.2. Comparison 9 Adverse Events and Other Outcomes (Active versus Active), Outcome 2 Re-operation for bleeding - Aprotinin versus Epsilon Aminocaproic Acid.

Analysis 9.3. Comparison 9 Adverse Events and Other Outcomes (Active versus Active), Outcome 3 Re-operation for bleeding - Tranexamic Acid versus Epsilon Aminocaproic Acid. . . . . . . . . . . . . . . . . . . .

Analysis 9.4. Comparison 9 Adverse Events and Other Outcomes (Active versus Active), Outcome 4 Mortality - Aprotinin versus Tranexamic Acid. . . . . . . . . . . . . . . . . . . . . . . . . . . . . . .

Analysis 9.5. Comparison 9 Adverse Events and Other Outcomes (Active versus Active), Outcome 5 Mortality - Aprotinin

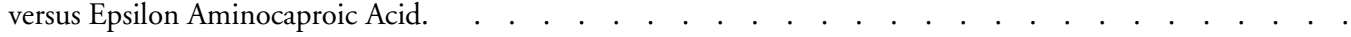

Analysis 9.6. Comparison 9 Adverse Events and Other Outcomes (Active versus Active), Outcome 6 Mortality - Tranexamic Acid versus Epsilon Aminocaproic Acid. . . . . . . . . . . . . . . . . . . . . . . . . . . . .

Analysis 9.7. Comparison 9 Adverse Events and Other Outcomes (Active versus Active), Outcome 7 Mortality - Aprotinin versus Lysine Analogues. . . . . . . . . . . . . . . . . . . . . . . . . . . . . .

Analysis 9.8. Comparison 9 Adverse Events and Other Outcomes (Active versus Active), Outcome 8 Myocardial Infarction - Aprotinin versus Tranexamic Acid. . . . . . . . . . . . . . . . . . . . . . . . . . .

Analysis 9.9. Comparison 9 Adverse Events and Other Outcomes (Active versus Active), Outcome 9 Myocardial Infarction - Aprotinin versus Epsilon Aminocaproic Acid. . . . . . . . . . . . . . . . . . . . . . . . . . . .

Analysis 9.10. Comparison 9 Adverse Events and Other Outcomes (Active versus Active), Outcome 10 Myocardial Infarction - Tranexamic Acid versus Epsilon Aminocaproic Acid. . . . . . . . . . . . . . . . . . . .

Analysis 9.11. Comparison 9 Adverse Events and Other Outcomes (Active versus Active), Outcome 11 Myocardial infarction - Aprotinin versus Lysine Analogues. . . . . . . . . . . . . . . . . . . . . . .

Analysis 9.12. Comparison 9 Adverse Events and Other Outcomes (Active versus Active), Outcome 12 Stroke (CVA) Aprotinin versus Tranexamic Acid.

Analysis 9.13. Comparison 9 Adverse Events and Other Outcomes (Active versus Active), Outcome 13 Stroke (CVA) Aprotinin versus Epsilon Aminocaproic Acid. . . . . . . . . . . . . . . . . . . . . . . . . . . . . . .

Analysis 9.14. Comparison 9 Adverse Events and Other Outcomes (Active versus Active), Outcome 14 Stroke (CVA) Tranexamic Acid versus Epsilon Aminocaproic Acid.

Analysis 9.15. Comparison 9 Adverse Events and Other Outcomes (Active versus Active), Outcome 15 Deep Vein Thrombosis (DVT) - Aprotinin versus Tranexamic Acid. . . . . . . . . . . . . . . . . . . .

Analysis 9.16. Comparison 9 Adverse Events and Other Outcomes (Active versus Active), Outcome 16 Deep Vein Thrombosis (DVT) - Aprotinin versus Epsilon Aminocaproic Acid. . . . . . . . . . . . . . . . . . Analysis 9.17. Comparison 9 Adverse Events and Other Outcomes (Active versus Active), Outcome 17 Deep Vein Thrombosis (DVT) - Tranexamic Acid versus Epsilon Aminocaproic Acid. . . . . . . . . . . . . . . . . Analysis 9.18. Comparison 9 Adverse Events and Other Outcomes (Active versus Active), Outcome 18 Pulmonary Embolism (PE) - Aprotinin versus Tranexamic Acid.

Analysis 9.19. Comparison 9 Adverse Events and Other Outcomes (Active versus Active), Outcome 19 Pulmonary Embolism (PE) - Aprotinin versus Epsilon Aminocaproic Acid. . . . . . . . . . . . . . . . . . . . . . Analysis 9.20. Comparison 9 Adverse Events and Other Outcomes (Active versus Active), Outcome 20 Pulmonary Embolism (PE) - Tranexamic Acid versus Epsilon Aminocaproic Acid.

Analysis 9.21. Comparison 9 Adverse Events and Other Outcomes (Active versus Active), Outcome 21 Other Thrombosis - Aprotinin versus Tranexamic Acid.

Analysis 9.22. Comparison 9 Adverse Events and Other Outcomes (Active versus Active), Outcome 22 Other Thrombosis - Aprotinin versus Epsilon Aminocaproic Acid. . . . . . . . . . . . . . . . . . . . . . . alysis 9.23. Comparison 9 Adverse Events and Other Outcomes (Active versus Active), Outcome 23 Other Thrombosis - Tranexamic Acid versus Epsilon Aminocaproic Acid. 
Analysis 9.25. Comparison 9 Adverse Events and Other Outcomes (Active versus Active), Outcome 25 Renal Failure /

Dysfunction - Aprotinin versus Epsilon Aminocaproic Acid. . . . . . . . . . . . . . . . . . .

Analysis 9.26. Comparison 9 Adverse Events and Other Outcomes (Active versus Active), Outcome 26 Renal Failure /

Dysfunction - Tranexamic Acid versus Epsilon Aminocaproic Acid. . . . . . . . . . . . . . . . . . . . .

Analysis 9.27. Comparison 9 Adverse Events and Other Outcomes (Active versus Active), Outcome 27 Hospital Length of

Stay - Aprotinin versus Tranexamic Acid. . . . . . . . . . . . . . . . . . . . . . . . . 39

Analysis 9.28. Comparison 9 Adverse Events and Other Outcomes (Active versus Active), Outcome 28 Hospital Length of

Stay - Aprotinin versus Epsilon Aminocaproic Acid. . . . . . . . . . . . . . . . . . . . . . . . . . . . . . .

Analysis 9.29. Comparison 9 Adverse Events and Other Outcomes (Active versus Active), Outcome 29 Hospital Length of

Stay - Tranexamic Acid versus Epsilon Aminocaproic Acid. . . . . . . . . . . . . . . . . . . . . . . . 395

Analysis 10.1. Comparison 10 Adverse Events and Other Outcomes (Active versus Control) - Cardiac Surgery, Outcome 1

Re-operation for bleeding. . . . . . . . . . . . . . . . . . . . . . . . . . . . . . . . . . .

Analysis 10.2. Comparison 10 Adverse Events and Other Outcomes (Active versus Control) - Cardiac Surgery, Outcome 2

Mortality. . . . . . . . . . . . . . . . . . . . . . . . . . . . . . . . . . . . 400

Analysis 10.3. Comparison 10 Adverse Events and Other Outcomes (Active versus Control) - Cardiac Surgery, Outcome 3

Myocardial Infarction. . . . . . . . . . . . . . . . . . . . . . . . . . . . . . . . 404

Analysis 10.4. Comparison 10 Adverse Events and Other Outcomes (Active versus Control) - Cardiac Surgery, Outcome 4

Stroke. . . . . . . . . . . . . . . . . . . . . . . . . . . . . . . . . . . . . 407

Analysis 10.5. Comparison 10 Adverse Events and Other Outcomes (Active versus Control) - Cardiac Surgery, Outcome 5

Deep Vein Thrombosis (DVT). . . . . . . . . . . . . . . . . . . . . . . . . . . . . . . . . .

Analysis 10.6. Comparison 10 Adverse Events and Other Outcomes (Active versus Control) - Cardiac Surgery, Outcome 6

Pulmonary Embolism. . . . . . . . . . . . . . . . . . . . . . . . . . . . . . . . 410

Analysis 10.7. Comparison 10 Adverse Events and Other Outcomes (Active versus Control) - Cardiac Surgery, Outcome 7

Other Thrombosis. . . . . . . . . . . . . . . . . . . . . . . . . . . . . . . . . . . . . . .

Analysis 10.8. Comparison 10 Adverse Events and Other Outcomes (Active versus Control) - Cardiac Surgery, Outcome 8

Renal Failure / Dysfunction. . . . . . . . . . . . . . . . . . . . . . . . . . . . . .

Analysis 10.9. Comparison 10 Adverse Events and Other Outcomes (Active versus Control) - Cardiac Surgery, Outcome 9

Hospital Length of Stay. . . . . . . . . . . . . . . . . . . . . . . . . . . . . . . 414

APPENDICES . . . . . . . . . . . . . . . . . . . . . . . . . . . . . . . . . . . . . . . 415

WHAT'S NEW . . . . . . . . . . . . . . . . . . . . . . . . . . . . . . . . . . . . . 418

HISTORY . . . . . . . . . . . . . . . . . . . . . . . . . . . . . . . . . . . . . . . 418

CONTRIBUTIONS OF AUTHORS . . . . . . . . . . . . . . . . . . . . . . . . . . . . . . . . . . . . . . . . 418

DECLARATIONS OF INTEREST . . . . . . . . . . . . . . . . . . . . . . . . . . . . . . . . . . . . . . 418

SOURCES OF SUPPORT . . . . . . . . . . . . . . . . . . . . . . . . . . . . . . . . . . . . . . . . . . . . 419

INDEX TERMS . . . . . . . . . . . . . . . . . . . . . . . . . . . . . . . . . . . . . . . . . . . . . . . . 419

Anti-fibrinolytic use for minimising perioperative allogeneic blood transfusion (Review)

Copyright @ 201 I The Cochrane Collaboration. Published by John Wiley \& Sons, Ltd. 


\title{
Anti-fibrinolytic use for minimising perioperative allogeneic blood transfusion
}

\author{
David A Henry ${ }^{1}$, Paul A Carless ${ }^{2}$, Annette J Moxey ${ }^{3}$, Dianne O’Connell ${ }^{4}$, Barrie J Stokes ${ }^{2}$, Dean A Fergusson ${ }^{5}$, Katharine Ker ${ }^{6}$ \\ ${ }^{1}$ Institute of Clinical Evaluative Sciences, Toronto, Canada. ${ }^{2}$ Discipline of Clinical Pharmacology, Faculty of Health, University of \\ Newcastle, Newcastle, Australia. ${ }^{3}$ Research Centre for Gender, Health \& Ageing, Faculty of Health, University of Newcastle, Newcastle, \\ Australia. ${ }^{4}$ Cancer Epidemiology Research Unit, Cancer Council, Sydney, Australia. ${ }^{5}$ Ottawa Health Research Institute, University of \\ Ottawa Centre for Transfusion Research, Ottawa, Canada. ${ }^{6}$ Cochrane Injuries Group, London School of Hygiene \& Tropical Medicine, \\ London, UK
}

Contact address: David A Henry, Institute of Clinical Evaluative Sciences, 2075 Bayview Avenue, G1 06, Toronto, Ontario, M4N 3M5, Canada. david.henry@ices.on.ca.

Editorial group: Cochrane Injuries Group.

Publication status and date: Edited (conclusions changed), published in Issue 1, 2011.

Review content assessed as up-to-date: 31 January 2010.

Citation: Henry DA, Carless PA, Moxey AJ, O’Connell D, Stokes BJ, Fergusson DA, Ker K. Anti-fibrinolytic use for minimising perioperative allogeneic blood transfusion. Cochrane Database of Systematic Reviews 2011, Issue 1. Art. No.: CD001886. DOI: 10.1002/14651858.CD001886.pub3.

Copyright (C) 2011 The Cochrane Collaboration. Published by John Wiley \& Sons, Ltd.

\begin{abstract}
A B S T R A C T
Background

Concerns regarding the safety of transfused blood have led to the development of a range of interventions to minimise blood loss during major surgery. Anti-fibrinolytic drugs are widely used, particularly in cardiac surgery, and previous reviews have found them to be effective in reducing blood loss, the need for transfusion, and the need for re-operation due to continued or recurrent bleeding. In the last few years questions have been raised regarding the comparative performance of the drugs. The safety of the most popular agent, aprotinin, has been challenged, and it was withdrawn from world markets in May 2008 because of concerns that it increased the risk of cardiovascular complications and death.
\end{abstract}

\section{Objectives}

To assess the comparative effects of the anti-fibrinolytic drugs aprotinin, tranexamic acid (TXA), and epsilon aminocaproic acid (EACA) on blood loss during surgery, the need for red blood cell (RBC) transfusion, and adverse events, particularly vascular occlusion, renal dysfunction, and death.

\section{Search strategy}

We searched: the Cochrane Injuries Group's Specialised Register (July 2010), Cochrane Central Register of Controlled Trials (The Cochrane Library 2010, Issue 3), MEDLINE (Ovid SP) 1950 to July 2010, EMBASE (Ovid SP) 1980 to July 2010. References in identified trials and review articles were checked and trial authors were contacted to identify any additional studies. The searches were last updated in July 2010.

\section{Selection criteria}

Randomised controlled trials (RCTs) of anti-fibrinolytic drugs in adults scheduled for non-urgent surgery. Eligible trials compared antifibrinolytic drugs with placebo (or no treatment), or with each other. 


\section{Data collection and analysis}

Two authors independently assessed trial quality and extracted data.

\section{Main results}

This review summarises data from 252 RCTs that recruited over 25,000 participants. Data from the head-to-head trials suggest an advantage of aprotinin over the lysine analogues TXA and EACA in terms of reducing perioperative blood loss, but the differences were small. Compared to control, aprotinin reduced the probability of requiring RBC transfusion by a relative $34 \%$ (relative risk [RR] $0.66,95 \%$ confidence interval [CI] 0.60 to 0.72 ). The RR for RBC transfusion with TXA was 0.61 (95\% CI 0.53 to 0.70 ) and was 0.81 (95\% CI 0.67 to 0.99 ) with EACA. When the pooled estimates from the head-to-head trials of the two lysine analogues were combined and compared to aprotinin alone, aprotinin appeared more effective in reducing the need for RBC transfusion (RR 0.90; $95 \%$ CI 0.81 to 0.99$)$.

Aprotinin reduced the need for re-operation due to bleeding by a relative $54 \%$ (RR $0.46,95 \%$ CI 0.34 to 0.62 ). This translates into an absolute risk reduction of $2 \%$ and a number needed-to-treat (NNT) of 50 (95\% CI 33 to 100). A similar trend was seen with EACA (RR $0.32,95 \%$ CI 0.11 to 0.99 ) but not TXA (RR $0.80,95 \%$ CI 0.55 to 1.17 ). The blood transfusion data were heterogeneous and funnel plots indicate that trials of aprotinin and the lysine analogues may be subject to publication bias.

When compared with no treatment aprotinin did not increase the risk of myocardial infarction (RR 0.87, 95\% CI 0.69 to 1.11 ), stroke (RR $0.82,95 \%$ CI 0.44 to 1.52 ), renal dysfunction (RR $1.10,95 \%$ CI 0.79 to 1.54 ) or overall mortality (RR $0.81,95 \%$ CI 0.63 to 1.06). Similar trends were seen with the lysine analogues, but data were sparse. These data conflict with the results of recently published non-randomised studies, which found increased risk of cardiovascular complications and death with aprotinin. There are concerns about the adequacy of reporting of uncommon events in the small clinical trials included in this review.

When aprotinin was compared directly with either, or both, of the two lysine analogues it resulted in a significant increase in the risk of death (RR 1.39, 95\% CI 1.02, 1.89), and a non-significant increase in the risk of myocardial infarction (RR $1.1195 \%$ CI 0.82 , 1.50). Most of the data contributing to this added risk came from a single study - the BART trial (2008).

\section{Authors' conclusions}

Anti-fibrinolytic drugs provide worthwhile reductions in blood loss and the receipt of allogeneic red cell transfusion. Aprotinin appears to be slightly more effective than the lysine analogues in reducing blood loss and the receipt of blood transfusion. However, head to head comparisons show a lower risk of death with lysine analogues when compared with aprotinin. The lysine analogues are effective in reducing blood loss during and after surgery, and appear to be free of serious adverse effects.

\section{PLAIN LANGUAGE SUMMARY}

\section{Anti-fibrinolytic drugs reduce blood loss and the need for red blood cell transfusions during and after surgery.}

Aprotinin, although effective in reducing bleeding, had a higher rate of death than tranexamic acid and aminocaproic acid, which appeared free of serious side-effects. Aprotinin has been withdrawn from world markets because of safety concerns. This review of over 250 clinical trials found that anti-fibrinolytic drugs used at the time of major surgery reduce bleeding, the need for transfusions of red blood cells and the need for repeat surgery because of bleeding. With the exception of aprotinin the drugs appear safe.

\section{B A C K G R O U N D}

Public concern regarding the safety of transfused blood has prompted a reconsideration of the role of allogeneic blood transfusion (whole blood or packed red cells from an unrelated donor). The risks associated with receiving transfusion of allogeneic blood that has been screened by a competent blood transfusion program are considered minimal, with very low risks of transmission of HIV, and hepatitis C (Whyte 1997). However, this only applies where there is a safe, plentiful, well-regulated supply. The majority of the 
world's population does not have access to such a system, and the risks of transfusion in developing countries may be much higher (McFarland 1997). Concerns of patients and clinicians regarding blood safety have generated enthusiasm for the use of technologies intended to reduce the use of allogeneic blood (Bryson 1998; Forgie 1998; Huet 1999; Laupacis 1997). Although allogeneic blood transfusion has had a unique place in medical practice, we are obliged to examine the evidence on the benefits, harms and costs of a range of techniques designed to minimise the use of this resource. Some of the alternatives to allogeneic blood have their own risks, and are expensive (Coyle 1999; Fergusson 1999).

Perioperative bleeding is one of the major indications for allogeneic blood transfusions worldwide (Levy 2006). However, massive surgical blood loss is a serious problem that affects many cardiac surgery patients in particular and has been shown to have a strong, independent association with in-hospital mortality (Karkouti 2004). There is also considerable evidence that blood loss that leads to the transfusion of blood products is harmful, and that the degree of harm is directly related to the amount of blood loss (Karkouti 2006). To reduce perioperative blood loss a number of pharmacological agents have been used, these include the antifibrinolytic drugs aprotinin, tranexamic acid (TXA), and epsilon aminocaproic acid (EACA).

Aprotinin is a non-specific, serine protease inhibitor, derived from bovine lung, with anti-fibrinolytic properties. It acts as an inhibitor of several serine proteases, including trypsin, plasmin, plasmakallikrein and tissue kallikrein. Aprotinin also inhibits the contact phase activation of coagulation that both initiates coagulation and promotes fibrinolysis (Fritz 1983; Royston 1998). During cardiopulmonary bypass (CPB) the negatively charged surface of the $\mathrm{CPB}$ circuit activates factor XII, converting prekallikrein to kallikrein which further activates factor XII. This positive feedback loop acts to intensify the intrinsic coagulation cascade. By inhibiting plasma kallikrein, aprotinin minimises derangements in coagulation and fibrinolysis (Smith 1998). There is also evidence that aprotinin exerts an indirect preservative effect on platelet function during extracorporeal circulation (ECC) (Mohr 1992). In many countries aprotinin is specifically indicated for the reduction of blood loss during cardiopulmonary bypass.

TXA and EACA are synthetic lysine analogues (synthetic derivatives of the amino acid lysine) that act as effective inhibitors of fibrinolysis. TXA and EACA act principally by blocking the lysine binding sites on plasminogen molecules, inhibiting the formation of plasmin and therefore inhibiting fibrinolysis (Faught 1998). Tranexamic acid is about ten times more potent than aminocaproic acid and binds much more strongly to both the strong and weak sites of the plasminogen molecule than EACA (Faught 1998; Mannucci 1998).

\section{Why it is important to do this review}

The efficacy of these three anti-fibrinolytic drugs to reduce perioperative blood loss and allogeneic blood transfusion has been studied extensively. Systematic reviews of these drugs (Henry 1999; Laupacis 1997; Levi 1999; Munoz 1999; Sedrakyan 2004) have shown that the use of aprotinin is associated with statistically significant reductions in allogeneic blood transfusion requirements and re-operation due to bleeding. Systematic reviews have also shown TXA to be effective in reducing exposure to allogeneic blood transfusion without significant increases in adverse effects (Henry 1999; Laupacis 1997). In the case of EACA, the evidence of effect is equivocal with most systematic reviews severely hampered by the small number of trials of this agent.

Based on the evidence of efficacy anti-fibrinolytic drugs have become widely used, particularly in cardiac surgery. Because of their mode of actions there have been longstanding concerns about the possibility of adverse effects, with most attention directed at the risk of thrombosis and renal failure. However meta-analyses of randomised trials, including previous versions of this Cochrane review (Henry 1999; Henry 2007), have been reassuring in providing no convincing evidence of an increased risk of these events in treated subjects. However, in the case of aprotinin, this view of an attractive benefit to harm ratio was thrown into doubt by the publication of several large non-randomised studies (Mangano 2006; Mangano 2007; Schneeweiss 2008). The serious safety concerns raised by these and other studies prompted the United States Food and Drug Administration (FDA) to re-evaluate its position regarding the use of aprotinin in cardiac surgery, some thirteen years after it was initially approved for prophylactic treatment to reduce perioperative blood loss and blood transfusion (Ferguson 2007). Aprotinin was finally removed from world markets in May 2008. The other drugs reviewed here are still in use.

In the light of these developments and in order to inform decisions about the use of the two lysine analogues as an alternative to aprotinin in cardiac surgery we have updated the Cochrane systematic review of the three anti-fibrinolytic drugs used as blood-sparing agents in surgery. This review updates previous estimates of the efficacy of aprotinin, tranexamic acid, and epsilon aminocaproic acid in reducing perioperative allogeneic blood transfusion in elective surgery. In light of the adverse findings from pharmaco-epidemiological studies we also provide updated estimates of the effects of these drugs on clinical outcomes such as all-cause mortality, thrombosis and renal failure.

\section{O B J E C T I VES}

To examine the evidence for the efficacy of aprotinin, tranexamic acid, and epsilon aminocaproic acid in reducing allogeneic blood transfusion, and the evidence for any effect on clinical outcomes 
such as mortality and re-operation rates and complications such as thrombosis and renal failure.

\section{METHODS}

\section{Criteria for considering studies for this review}

\section{Types of studies}

Randomised controlled trials (RCTs) with a concurrent control group, or randomised head-to-head comparative trials.

\section{Types of participants}

The study participants were adults (over 18 years). Trials were included if participants aged less than 18 years were enrolled, but the type of surgery was predominantly carried out in adult patients. The surgery performed was primarily elective but trials were included if urgent cases were proportionately similar across trial arms.

\section{Types of interventions}

The interventions considered are the anti-fibrinolytic agents: aprotinin, tranexamic acid (TXA), and epsilon-aminocaproic acid (EACA).

\section{Types of outcome measures}

\section{Primary outcomes}

- the proportion of patients who were transfused with allogeneic blood, autologous blood, or with both;

- the amounts of allogeneic and autologous blood transfused.

\section{Secondary outcomes}

- perioperative blood loss,

- re-operation due to bleeding,

- mortality,

- post-operative complications (myocardial infarction, stroke, deep vein thrombosis, pulmonary embolism, any thrombosis, renal failure),

- length of hospital stay.

\section{Search methods for identification of studies}

We did not limit the searches by date language or publication status

\section{Electronic searches}

The original review drew on the literature search that was constructed as part of the International Study on Perioperative Transfusion (ISPOT) (Laupacis 1997). The original search is listed in full in Appendix 1.

\section{July 2006 update}

To maximise the sensitivity for the retrieval of all potentially relevant studies, the electronic searches of MEDLINE were initially unrestricted and updated to July 2006. In MEDLINE, two search filters were then used to restrict the electronic searches and improve the specificity of the updated searches. Firstly, the ISPOT filter, which identifies blood transfusion trials, and secondly, a modified version of the Cochrane Collaboration filter, which primarily identifies randomised controlled trials. These search filters were coupled with the specified MeSH headings and the relevant text-word terms. These restricted searches were updated in MEDLINE to July 2006. Electronic database searches of Excerpta Medica (EMBASE) were updated to July 2006 using similar search strategies to those used in MEDLINE.

\section{July 2010 update}

Searches were carried out in July 2010 as part of a larger project to identify trials in the use of antifibrinolytics.

We searched the following databases;

- the Cochrane Injuries Group's Specialised Register (searched July 2010),

- Cochrane Central Register of Controlled Trials (The Cochrane Library 2010, Issue 3),

- MEDLINE (Ovid SP) 1950 to July 2010,

- EMBASE (Ovid SP) 1980 to July 2010.

Full details of the search strategies are presented in Appendix 2.

\section{Searching other resources}

The web sites of International Health Technology Assessment Agencies were also searched through the International Network of Agencies of Health Technology Assessment (INAHTA), and the International Society of Technology Assessment in Health Care (ISTAHC). The Internet was widely searched using Google ${ }^{\mathrm{TM}}$ and Google ${ }^{\mathrm{TM}}$ Scholar. Contact was also made with experts in the field to identify reports or projects in progress relevant to the review. The reference lists of related reviews and identified articles were checked for relevant trials. In addition references in the identified trials were checked and authors contacted, where possible, to identify any additional published or unpublished data. 


\section{Data collection and analysis}

Electronic database searches were carried out by the Cochrane Injuries Group Trials Search Co-ordinator, who then collated the results and passed them on to the author (KK).

\section{Selection of studies}

The titles and abstracts identified in the electronic searches were independently screened by two authors to identify trials in which adult patients, scheduled for elective or urgent surgery, were randomised to either/or aprotinin, TXA, EACA or to a control group, who did not receive the intervention. From the results of the screened electronic searches, bibliographic searches, and contacts with experts, two of the authors independently selected trials that met previously defined inclusion criteria.

\section{Data extraction and management}

At least two authors independently extracted study characteristics and outcomes using an article extraction form. The extraction form was used to record information regarding randomisation criteria, methodology descriptions, the presence of a transfusion protocol, the type of surgery involved, treatment outcomes, and general comments.

Data on the following outcomes were recorded on the data extraction form: the number of patients exposed to allogeneic blood, the amount of allogeneic blood transfused, the number of patients receiving any transfusion (allogeneic blood, autologous blood, or both), the number of patients experiencing post-operative complications (thrombosis, myocardial infarction, renal failure), and mortality. Data were also recorded on blood loss, and the proportion of patients requiring re-operation for bleeding. Information regarding demographics (age, sex), type of surgery, and the presence or absence of a transfusion protocol was also recorded. Data were extracted for allogeneic blood transfusion if they were expressed as whole blood or packed red cells. Data were extracted regarding dose size for each drug regimen.

\section{Assessment of risk of bias in included studies}

Articles that met the inclusion criteria were independently assessed for methodological quality by two authors using criteria proposed by Schulz 1995. Disagreements were resolved by consensus. Methodological quality scores obtained for each trial using the criteria proposed by Schulz 1995 were then entered into Review Manager using the Cochrane Collaboration's tool for assessing risk of bias presented in Higgins 2009.

The following domains were assessed for each study:

- sequence generation,

- allocation concealment,

- blinding.
We completed a risk of bias table for each study, incorporating a description of the study's performance against each of the above domains and our overall judgement of the risk of bias for each entry is as follows; 'Yes' indicates low risk of bias, 'Unclear' indicates unknown risk of bias (not enough information was reported to assess methodological quality); and 'No' indicates a high risk of bias.

\section{Assessment of reporting biases}

Funnel plots were inspected for evidence of publication bias.

\section{Data synthesis}

Extraction of trial data was performed by one author and checked by the review team's statistician if necessary. Data were checked and entered into Review Manager by one author. Articles identified as duplicate publications were combined to obtain one set of data. The study report with the greatest number of patients was then represented in the analysis. Studies that did not report data for the number or proportion of patients transfused with allogeneic blood, or the amounts of allogeneic blood transfused, were not included for review. However, trials not reporting blood transfusion data that could be used in the meta-analysis were still included if they reported adverse event data. For dichotomous outcome data to be included in the analysis, trial reports had to provide either numeric data, that is the numbers of events that occurred in the treatment and control groups, or where there were no events recorded numerically, the trial report had to provide a clear statement qualifying and/or quantifying specific events had or had not occurred.

All analyses were performed using Review Manager software. Data on the numbers of patients exposed to allogeneic blood, and the numbers of patients in each treatment arm, were entered into Review Manager. The relative risks (RR) for allogeneic blood transfusion in the intervention group as compared with the control group, and the corresponding 95\% confidence intervals (CI), were calculated for each trial using the random effects model. The presence of heterogeneity of treatment effect was assessed using the $\mathrm{Q}$ statistic, which has an approximate chi-square distribution with degrees of freedom equal to the number of studies minus one (Der Simonian 1986). A P-value less than or equal to 0.1 was used to define statistically significant heterogeneity. Statistical heterogeneity was also assessed using the $\mathrm{I}^{2}$ test. The $\mathrm{I}^{2}$ test describes the percentage of total variation across studies due to heterogeneity rather than chance. A value of $0 \%$ indicates no observed heterogeneity and larger values show increasing heterogeneity. Substantial heterogeneity is considered to exist when $\mathrm{I}^{2}>50 \%$ (Higgins 2002).

The mean number of units of allogeneic blood transfused to each group, and the corresponding standard deviations, were also entered. As the majority of trials reported the means and standard 
deviations for the amount of blood transfused in all patients in each comparison group, the data included a number of zero values for those patients not receiving transfusion. The data are therefore likely to be highly skewed. Wherever possible, the mean and standard deviation for the numbers of units of blood transfused in those receiving transfusion were recalculated. The new mean was calculated by dividing the total number of units transfused in the group by the number of patients transfused. The reported standard deviation and mean were used to calculate the sum of squares of the numbers of units transfused for the group. As this is equal to the sum of squares of the numbers of units transfused in those receiving transfusion, the new standard deviation was calculated using this, the new mean and the number of patients transfused. Thus the new values estimate the average amount of blood received by those transfused in each group. The new values were then entered into Review Manager to obtain the mean difference (MD) and 95\% CIs to express the average reduction in the number of units of allogeneic blood given to those patients transfused. Data in millilitres ( $\mathrm{mls}$ ) were converted to units by dividing by 300 .

\section{Subgroup analysis and investigation of heterogeneity}

Analysis of a-priori subgroups was performed to determine whether effect sizes varied according to factors such as;

- the type of surgery,

- the use of transfusion protocols,

- dose regimen, and

- trial methodological quality.

\section{R E S U L T S}

\section{Description of studies}

See: Characteristics of included studies; Characteristics of excluded studies; Characteristics of ongoing studies.

Two hundred and fifty-two trials met the inclusion criteria. Four trials were excluded (refer to 'Characteristics of excluded studies' section of this review). Of the 252 included trials, 131 evaluated aprotinin, 60 evaluated tranexamic acid (TXA), and 12 evaluated epsilon aminocaproic acid (EACA) versus control. Forty-nine trials studied head-to-head comparisons of aprotinin, TXA, and EACA with or without an untreated control. Of these 49 trials, 25 compared aprotinin with TXA, 12 compared aprotinin with EACA, seven compared TXA with EACA, and five compared aprotinin with TXA and EACA. Trials were conducted in many countries including:

United States $(\mathrm{n}=45)$, Germany $(\mathrm{n}=24)$, UK $(\mathrm{n}=17)$, Canada $(\mathrm{n}=17)$, Italy $(\mathrm{n}=16)$, Spain $(\mathrm{n}=14)$, Belgium $(\mathrm{n}=12)$, France $(\mathrm{n}=10)$, Turkey $(\mathrm{n}=9)$, Australia $(\mathrm{n}=8)$, Sweden $(\mathrm{n}=8)$, The
Netherlands $(\mathrm{n}=8)$, Japan $(\mathrm{n}=7)$, China $(\mathrm{n}=6)$, Austria $(\mathrm{n}=$ 5), Israel $(n=5)$, Switzerland $(n=5)$, Finland $(n=5)$, Czech Republic $(\mathrm{n}=3)$, Denmark $(\mathrm{n}=3)$, Taiwan $(\mathrm{n}=2)$, Ireland $(\mathrm{n}$ $=2)$, Greece $(n=2)$, Poland $(n=2)$, Brazil $(n=1)$, Chile $(n$ $=1)$, Dubai $(n=1)$, Egypt $(n=1)$, India $(n=1)$, Norway $(n=$ 1), Oman ( $n=1)$, Saudi Arabia $(n=1)$ and South Africa $(n=1)$. Three studies were multicentre trials, one conducted across sites in the UK and the United States, one in sites in Australia, New Zealand, Asia and Europe and one in sites in the United States and Canada. The majority of included trials were published in English. Thirteen trials required translation (Carrera 1994; Corbeau 1995; Cvachovec 2001; Deleuze 1991; Gherli 1992; Hei 2005; Kahveci 1996; Katzel 1998; Kratzer 1997; Locatelli 1990; Maccario 1994; Trinh-Duc 1992; Utada 1997). The data from these trials were included in the analysis.

Of the 252 included trials, 173 were conducted in cardiac surgery, 53 trials were in orthopaedic surgery, 14 involved liver surgery, five were conducted in vascular surgery, four involved thoracic surgery, one involved gynaecological surgery, one involved neurosurgery, and one trial was in orthognathic surgery.

The trial conducted by Lemmer 1994 stratified patients according to the type of procedure being performed, that is, either primary CABG or redo CABG surgery. Patients from each group were then randomised to either aprotinin or placebo. The data obtained from each of these two groups (primary CABG and redo CABG) have been analysed separately by the authors. Therefore from this single trial (Lemmer 1994), two comparisons of aprotinin versus control have been obtained. This review presents the data from this trial as follows:

(1) Lemmer_1 1994: represents those patients who underwent primary CABG and were randomised to either aprotinin or placebo. (2) Lemmer_2 1994: represents those patients who underwent redo $\mathrm{CABG}$ and were randomised to either aprotinin or placebo.

\section{Description of Dose Regimens}

\section{Aprotinin dose range}

Three dose stratifications were used: (1) high-dose aprotinin, (2) low-dose aprotinin, and, (3) cardiopulmonary bypass (CPB) pump prime aprotinin. For the purposes of this review, any aprotinin regimen that did not follow the 'full Hammersmith' regimen, including those studies that described their regimens as 'half Hammersmith', were classified as low-dose aprotinin. For those trials that did not involve cardiac surgery, classification of the dose-regimen was based on the total quantity of aprotinin administered. Trials were classified as 'high-dose' where participants received a total dose equal to or exceeding five million kallikrein inactivator units (KIU) or $700 \mathrm{mg}$ of aprotinin.

High-dose aprotinin, described as the 'full Hammersmith' regimen, entails an initial loading dose of two million kallikrein inactivator units (KIU) of aprotinin given intravenously (IV) (280mg) 
over a 20 to 30 minute period commencing at the induction of anaesthesia, followed by a continuous infusion of 500,000 KIU per hour $(70 \mathrm{mg} / \mathrm{hr})$ until the end of the operation. In addition, two million KIU of aprotinin $(280 \mathrm{mg})$ is added to the oxygenator prime or pump prime of the $\mathrm{CPB}$. A 'half Hammersmith' regimen is described as follows: a loading dose of one million KIU (140mg) of aprotinin infused over a 20 to 30 minute period followed by a continuous IV infusion of 250,000 KIU of aprotinin per hour, until the end of the operation. An additional dose of one million $\mathrm{KIU}$ is added to the pump prime.

'Prime' dose aprotinin, for the purposes of this review, included those regimens that added aprotinin to the pump prime solution of the CPB exclusively. The dose of aprotinin used in the 'prime' regimen varied between trials. Sixteen trials studied the efficacy of 'prime' dose aprotinin and reported data on the proportion of participants exposed to allogeneic blood transfusion. Of these trials 12 studied a 'prime' dose of two million KIU of aprotinin, two studied a 'prime' dose of one million KIU of aprotinin, one studied a 'prime' dose of 500,000 KIU of aprotinin, and one trial studied a 'prime' dose of $25,000 \mathrm{KIU} / \mathrm{kg}$ (range 1.375 to 2.3 million KIU in total) of aprotinin.

\section{Tranexamic acid (TXA) dose range}

Of the 65 trials that studied the efficacy of TXA versus placebo or control (current standard practice) and were included in the metaanalysis of allogeneic blood transfusion exposure; 34 involved cardiac surgery, 27 involved orthopaedic surgery, two involved liver surgery, one trial involved gynaecological surgery and one trial involved vascular surgery. Dose regimens for TXA varied significantly between trials with varying dose sizes and time frames for drug delivery. Of the 34 trials involving cardiac surgery, the TXA loading or bolus dose ranged from $2.5 \mathrm{mg} / \mathrm{kg}$ to $100 \mathrm{mg} / \mathrm{kg}$. The maintenance dose of TXA for the cardiac trials, ranged from $0.25 \mathrm{mg} / \mathrm{kg} / \mathrm{hr}$ to $4.0 \mathrm{mg} / \mathrm{kg} / \mathrm{hr}$ delivered over 1 to 12 hours. Similar variation was observed in trials not involving cardiac surgery. More detailed information regarding dose regimens is provided in the 'Characteristics of included studies' section of this review.

\section{Epsilon aminocaproic acid (EACA) dose range}

Of the 16 trials that studied the efficacy of EACA versus placebo or control (current standard practice) and were included in the metaanalysis of allogeneic blood transfusion exposure; 11 involved cardiac surgery, four involved orthopaedic surgery, and one involved liver surgery. Dose regimens for EACA also varied significantly between trials. Generally trials used different dose sizes and time frames for drug delivery. The EACA loading or bolus dose ranged from $80 \mathrm{mg}$ to $15 \mathrm{~g}$ or 75 to $150 \mathrm{mg} / \mathrm{kg}$. The maintenance dose of EACA ranged from $1 \mathrm{~g} / \mathrm{hr}$ to $2 \mathrm{~g} / \mathrm{hr}$ or $12.5 \mathrm{mg} / \mathrm{kg} / \mathrm{hr}$ to $30 \mathrm{mg} / \mathrm{kg} /$ $\mathrm{hr}$ infused over varying time periods. More detailed information regarding dose regimens is provided in the 'Characteristics of included studies' section of this review.

\section{Transfusion 'triggers' / thresholds}

Of the 189 trials of aprotinin, TXA, and EACA versus control included in the analysis of allogeneic blood transfusion exposure, 158 trials (84\%) reported the use of a transfusion protocol, the remainder did not report the use of a transfusion protocol. Of those trials that reported the use of a transfusion protocol, all included a transfusion "trigger" value, that being the haemoglobin or haematocrit value, at which point a transfusion of allogeneic and/or autologous blood, was considered necessary. There was significant variation between trials in the type and value of transfusion threshold used. The lowest transfusion threshold level for haemoglobin was $5.0 \mathrm{~g} / \mathrm{dL}$ with blood being transfused if the haemoglobin level during CPB fell below 5.0g/dL (Green 1995). The transfusion protocol used by Brown 1997 advocated a haemoglobin threshold level of $6.0 \mathrm{~g} / \mathrm{dL}$ during $\mathrm{CPB}$, whereas other trials involving $\mathrm{CPB}$ advocated a haemoglobin threshold level of $7.0 \mathrm{~g} / \mathrm{dL}$, or haematocrit levels (Hct) between $18 \%$ to $20 \%$ during CPB. In general, post-operative transfusion threshold levels ranged from $\mathrm{Hb} 7.0 \mathrm{~g} /$ dL to $10.0 \mathrm{~g} / \mathrm{dL}$, or Hct $20 \%$ to $30 \%$.

\section{Risk of bias in included studies}

For further details regarding the performance of the studies against each domain, please see the 'Risk of bias' tables (Figure 1; Figure 2). A summary of the information in the tables is given below. Additionally, a visual summary of judgements about each methodological quality item for each included trial is shown in Figure 1. 
Figure I. Risk of bias graph: review authors' judgements about each risk of bias item presented as percentages across all included studies.

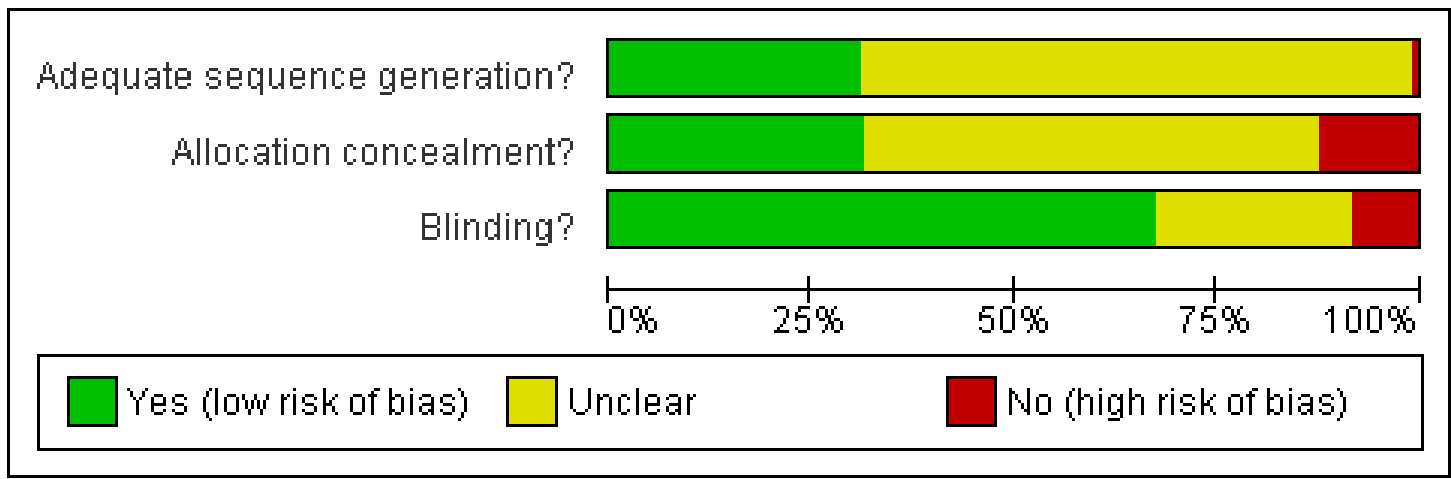


Figure 2. Risk of bias summary: review authors' judgements about each risk of bias item for each included study.

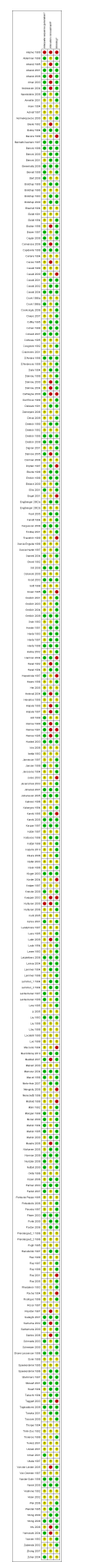

Anti-fibrinolytic use for minimising perioperative allogeneic blood transfusion (Review)

Copyright (C) 201 I The Cochrane Collaboration. Published by John Wiley \& Sons, Ltd. 


\section{Generation of allocation sequences}

The method used to generate allocation sequences was judged to be adequate in only 78 trials. For all but two of the remaining trials the method used to generate allocation sequences was judged to be unclear. For two trials the method of randomisation that was judged to be inadequate. Refer to results presented in the 'Risk of bias' tables.

\section{Allocation concealment}

Only 79 trials were judged to have adequately concealed treatment allocation. For 31 trials the method used to conceal treatment allocation was judged to be inadequate. For the remaining trials allocation concealment was judged to be unclear. Refer to results presented in the 'Risk of bias' tables.

\section{Blinding}

For 170 trials blinding was judged to be adequate (double blinded), and unclear for 61 trials. Refer to results presented in the 'Risk of bias' tables.

\section{Inclusion of all randomised participants}

Of those trials able to be assessed for methodological quality, 124 trials either reported there were no exclusions, or used intentionto-treat analysis. In 80 trials, where exclusions were reported, these exclusions were judged unlikely to cause bias. For 37 trials exclusions were either judged to be excessive and likely to cause bias, or were not reported.

\section{Effects of interventions}

\section{Aprotinin}

There were 108 trials of aprotinin versus control that reported data on the proportion of patients exposed to allogeneic blood transfusion. These trials included a total of 11,172 patients, of whom 6259 were randomised to receive aprotinin and 4913 patients were randomised to a control group who did not receive aprotinin. The apparent imbalance between the aprotinin and control groups resulted from pooling data across different aprotinin dose groups within trials. Overall, the use of aprotinin significantly reduced the rate of allogeneic blood transfusion by a relative $34 \%$ (RR $0.66,95 \%$ CI 0.60 to 0.72 ) compared to control. Heterogeneity between these trials was statistically significant $\left(\mathrm{Chi}^{2}=961.52, \mathrm{df}\right.$ $\left.=107, \mathrm{P}<0.00001 ; \mathrm{I}^{2}=89 \%\right)$. The absolute risk reduction (ARR) was $20 \%$ (RD $-0.20,95 \% \mathrm{CI}-0.24$ to -0.17 ).

\section{Type of surgery}

There were 84 trials of aprotinin versus control that involved cardiac surgery and provided data on the number of patients exposed to allogeneic blood transfusion. These trials included a total of 9497 patients, of whom 5329 were randomised to receive aprotinin and 4168 patients were randomised to a control group who did not receive aprotinin. Overall, the use of aprotinin in cardiac surgery significantly reduced the need for allogeneic blood transfusion by a relative $32 \%$ (RR $0.68,95 \%$ CI 0.63 to 0.73 ) compared to control. Heterogeneity between these trials was statistically significant $\left(\mathrm{Chi}^{2}=329.48, \mathrm{df}=83, \mathrm{P}<0.00001 ; \mathrm{I}^{2}=75 \%\right)$. The ARR was $21 \%$ (RD $-0.21,95 \%$ CI -0.24 to -0.18 ).

There were 15 trials of aprotinin versus control that involved orthopaedic surgery and provided data on the number of patients exposed to allogeneic blood transfusion. These trials included a total of 1146 patients, of whom 655 were randomised to receive aprotinin and 491 patients were randomised to a control group who did not receive aprotinin. Overall, the use of aprotinin in orthopaedic surgery significantly reduced the need for allogeneic blood transfusion by a relative $32 \%$ (RR $0.68,95 \%$ CI 0.52 to $0.89)$ compared to control. Heterogeneity between these trials was statistically significant $\left(\mathrm{Chi}^{2}=45.47, \mathrm{df}=14, \mathrm{P}<0.0001 ; \mathrm{I}^{2}=\right.$ $69 \%$ ). The ARR was $13 \%$ (RD $-0.13,95 \%$ CI -0.20 to -0.05 ).

There were three trials of aprotinin versus control that involved thoracic surgery and provided data on the number of patients exposed to allogeneic blood transfusion. These trials included a total of 78 patients, of whom 38 were randomised to receive aprotinin and 40 patients were randomised to a control group who did not receive aprotinin. The use of aprotinin in thoracic surgery significantly reduced the need for allogeneic blood transfusion by a relative $71 \%$ (RR $0.29,95 \%$ CI 0.14 to 0.59 ). Heterogeneity between these trials was not statistically significant $\left(\mathrm{Chi}^{2}=0.37\right.$, $\left.\mathrm{df}=2, \mathrm{P}=0.83 ; \mathrm{I}^{2}=0 \%\right)$.

There were two trials of aprotinin versus control that involved vascular surgery and provided data on the number of patients exposed to allogeneic blood transfusion. These trials included a total of 188 patients, of whom 105 were randomised to aprotinin and 83 patients were randomised to a control group who did not receive aprotinin. The use of aprotinin in vascular surgery had no effect on the need for allogeneic blood transfusion (RR 1.00, 95\% CI 0.97 to 1.03 ). Heterogeneity between these trials was not statistically significant $\left(\mathrm{Chi}^{2}=0.01, \mathrm{df}=1, \mathrm{P}=0.84 ; \mathrm{I}^{2}=0 \%\right)$.

There were two trials of aprotinin versus control that involved liver surgery and provided data on the number of patients exposed to allogeneic blood transfusion. These trials included a total of 177 patients, of whom 87 were randomised to aprotinin and 90 patients were randomised to a control group who did not receive aprotinin. The use of aprotinin in liver surgery reduced the need for allogeneic blood transfusion by a relative $42 \%$ (RR $0.58,95 \%$ 
CI 0.37 to 0.90$)$. Heterogeneity between these trials was not statistically significant $\left(\mathrm{Chi}^{2}=1.03, \mathrm{df}=1, \mathrm{P}=0.31 ; \mathrm{I}^{2}=3 \%\right)$.

Data from the trials involving neurosurgery, and orthognathic surgery could not be analysed due to the small number of trials in each of these surgical subgroups.

\section{Effect of transfusion protocols}

There were 87 trials that compared aprotinin with control and reported the use of transfusion protocols. These trials included a total of 9974 patients, of whom 5599 were randomised to aprotinin and 4375 were randomised to a control group who did not receive aprotinin. In those trials where a transfusion protocol was used, aprotinin significantly reduced the need for allogeneic blood transfusion by a relative $35 \%$ (RR $0.65,95 \%$ CI 0.59 to 0.71 ). Heterogeneity between these trials was statistical significant $\left(\mathrm{Chi}^{2}\right.$ $\left.=924.12, \mathrm{df}=86, \mathrm{P}<0.00001 ; \mathrm{I}^{2}=91 \%\right)$.

There were 21 trials of aprotinin versus control that reported data on the number of patients exposed to allogeneic blood transfusion but did not report the use of a transfusion protocol. These trials included a total of 1198 patients, of whom 660 were randomised to aprotinin and 538 were randomised to a control group who did not receive aprotinin. The use of aprotinin statistically significantly reduced the need for allogeneic blood transfusion by a relative $29 \%$ (RR $0.71,95 \%$ CI 0.61 to 0.84 ) compared to control. Heterogeneity between these trials was statistically significant $\left(\mathrm{Chi}^{2}=49.74, \mathrm{df}=20, \mathrm{P}=0.0002 ; \mathrm{I}^{2}=60 \%\right)$.

\section{Effect of dose}

In those trials that used a low-dose aprotinin regimen the RR of requiring an allogeneic blood transfusion was 0.65 (95\% CI 0.55 to $0.77)$ compared to control. Whereas in those trials that used a highdose aprotinin regimen the RR of receiving an allogeneic blood transfusion was 0.66 (95\% CI 0.61 to 0.71 ) compared to control. Therefore there was little difference in effect between high-dose and low-dose aprotinin. In cardiac surgery when aprotinin was given as a prime-dose only, the RR of requiring allogeneic blood transfusion was 0.83 ( $95 \%$ CI 0.71 to 0.96 ). There was statistically significant heterogeneity present in all three subgroups (P $>0.0001 ; \mathrm{I}^{2}>74 \%$ ).

The study conducted by Green 1995 was not included in this analysis as it only provided aggregate data for the number of patients exposed to allogeneic blood transfusion, without stratifying allogeneic blood transfusion exposure by dose.

\section{Volume of blood transfused}

Seventy-four trials of aprotinin versus control provided data on the volume of allogeneic blood transfused in all patients. These trials included a total of 7820 patients, of whom 4198 were randomised to aprotinin and 3622 were randomised to a control group who did not receive aprotinin. The use of aprotinin resulted in a significant saving of 1.02 units of allogeneic blood (MD -1.02 units, 95\% CI -1.26 to -0.79 units). Heterogeneity between these trials was statistically significant $\left(\mathrm{Chi}^{2}=1627.35, \mathrm{df}=69, \mathrm{P}<0.00001 ; \mathrm{I}^{2}\right.$ $=96 \%)$.

Forty trials of aprotinin versus control provided data on the volume of allogeneic blood transfused in those patients transfused. These trials provided data for a total of 3563 patients, of whom 1680 were treated with aprotinin and 1883 did not receive aprotinin treatment. In those patients transfused the use of aprotinin resulted in a significant saving of 0.98 units of allogeneic blood per patient (MD -0.98 units, 95\% CI -1.29 to -0.66 units). Heterogeneity between these trials was statistically significant $\left(\mathrm{Chi}^{2}=197.82, \mathrm{df}\right.$ $\left.=36, \mathrm{P}<0.00001 ; \mathrm{I}^{2}=82 \%\right)$.

\section{Blood loss - all surgery combined}

A total of 16 trials of aprotinin versus control reported intra-operative blood loss data. These trials included a total of 883 patients, of whom 449 were randomised to aprotinin and 434 were randomised to a control group. These trials involved cardiac surgery $(n=7)$, orthopaedic surgery $(n=5)$, thoracic surgery $(n=2)$, liver surgery $(\mathrm{n}=2)$ and vascular surgery $(\mathrm{n}=1)$. In aggregate, aprotinin treatment reduced intra-operative blood loss on average by around $192 \mathrm{mls}$ per patient (MD $-191.87 \mathrm{mls}, 95 \% \mathrm{CI}-280.45$ to $-103.28 \mathrm{mls}$ ). Heterogeneity between these trials was statistically significant $\left(\mathrm{Chi}^{2}=40.04, \mathrm{df}=16, \mathrm{P}=0.0008 ; \mathrm{I}^{2}=60 \%\right)$.

A total of 87 trials of aprotinin versus control reported post-operative blood loss data. These trials included a total of 7896 patients, of whom 4394 were randomised to aprotinin and 3502 were randomised to a control group. These trials involved cardiac surgery $(n=75)$, orthopaedic surgery $(n=7)$, thoracic surgery $(n=2)$, orthognathic surgery $(\mathrm{n}=1)$, liver surgery $(\mathrm{n}=1)$, and vascular surgery $(n=1)$. In aggregate, aprotinin treatment significantly reduced post-operative blood loss on average by around $346 \mathrm{mls}$ per patient (MD -345.88 mls, 95\% CI -383.47 to $-308.29 \mathrm{mls}$ ). Heterogeneity between these trials was statistically significant $\left(\mathrm{Chi}^{2}=\right.$ $\left.620.49, \mathrm{df}=86, \mathrm{P}<0.00001 ; \mathrm{I}^{2}=86 \%\right)$.

A total of 17 trials of aprotinin versus control reported total blood loss data (intra-operative and post-operative blood loss combined). These trials included a total of 1789 patients, of whom 932 patients were randomised to aprotinin and 857 were randomised to a control group. These trials involved cardiac surgery $(n=7)$ and orthopaedic surgery $(n=10)$. In aggregate, the use of aprotinin significantly reduced perioperative blood loss by around $416 \mathrm{mls}$ per patient (MD $-415.95 \mathrm{mls}$, 95\% CI -520.38 to $-311.51 \mathrm{mls}$ ). Heterogeneity between these trials was statistically significant $\left(\mathrm{Chi}^{2}=\right.$ 66.96, $\left.\mathrm{df}=16, \mathrm{P}<0.0001 ; \mathrm{I}^{2}=76 \%\right)$.

\section{Blood loss - cardiac surgery}

Seven trials of aprotinin versus control involving cardiac surgery reported intra-operative blood loss data. These trials included a 
total of 470 patients, of whom 242 were randomised to aprotinin and 228 were randomised to a control group. Aprotinin treatment in cardiac surgery appeared to be only marginally effective in reducing intra-operative blood loss (MD - $148.18 \mathrm{mls}$, 95\% CI 240.21 to $-56.14 \mathrm{mls}$ ). Heterogeneity between these trials was statistically significant $\left(\mathrm{Chi}^{2}=13.63, \mathrm{df}=6, \mathrm{P}=0.03 ; \mathrm{I}^{2}=56 \%\right)$. Seventy-five trials of aprotinin versus control involving cardiac surgery reported post-operative blood loss data. These trials included a total of 7371 patients, of whom 4132 were randomised to aprotinin and 3239 were randomised to a control group. The use aprotinin in cardiac surgery reduced post-operative blood loss on average by $370 \mathrm{mls}$ per patient (MD $-369.62 \mathrm{mls}$, 95\% CI 408.95 to $-330.29 \mathrm{mls})$. Heterogeneity between these trials was statistically significant $\left(\mathrm{Chi}^{2}=513.91, \mathrm{df}=74, \mathrm{P}<0.00001 ; \mathrm{I}^{2}=\right.$ $86 \%)$.

Seven trials of aprotinin versus control involving cardiac surgery reported total blood loss data (intra-operative and post-operative blood loss combined). These trials included a total of 1359 patients, of whom 716 were randomised to aprotinin and 643 were randomised to a control group. The use of aprotinin in cardiac surgery significantly reduced the total volume of blood lost during the perioperative period (MD $-448.86 \mathrm{mls}, 95 \% \mathrm{CI}-612.82$ to $-284.91 \mathrm{mls}$ ). Heterogeneity between these trials was statistically significant $\left(\mathrm{Chi}^{2}=42.60, \mathrm{df}=6, \mathrm{P}<0.00001 ; \mathrm{I}^{2}=86 \%\right)$.

\section{Blood loss - orthopaedic surgery}

Five trials of aprotinin versus control involving orthopaedic surgery reported intra-operative blood loss data. These trials included a total of 201 patients, of whom 103 were randomised to aprotinin and 98 were randomised to a control group. The use of aprotinin in orthopaedic surgery did not reduce the volume of blood lost during the intra-operative period (MD -151.05 mls, $95 \%$ CI -317.63 to $15.52 \mathrm{mls}$ ). Heterogeneity between these trials was not statistically significant $\left(\mathrm{Chi}^{2}=6.62, \mathrm{df}=4, \mathrm{P}=0.16 ; \mathrm{I}^{2}=\right.$ $40 \%)$.

Seven trials of aprotinin versus control involving orthopaedic surgery reported post-operative blood loss data. These trials included a total of 318 patients, of whom 160 were randomised to aprotinin and 158 were randomised to a control group. The use of aprotinin in orthopaedic surgery was only marginally effective in reducing post-operative blood loss (MD $-113.58 \mathrm{mls}, 95 \% \mathrm{CI}$ -223.69 to $-3.46 \mathrm{mls}$ ). Heterogeneity between these trials was statistically significant $\left(\mathrm{Chi}^{2}=18.56, \mathrm{df}=6, \mathrm{P}=0.005 ; \mathrm{I}^{2}=68 \%\right)$. Ten trials of aprotinin versus control involving orthopaedic surgery reported total blood loss data (intra-operative and post-operative blood loss combined). These trials included a total of $430 \mathrm{pa}-$ tients, of whom 216 were randomised to aprotinin and 214 were randomised to a control group. Aprotinin reduced the total volume of blood lost during the perioperative period on average by around $399 \mathrm{mls}$ per patient (MD -399.09 $\mathrm{mls}, 95 \% \mathrm{CI}-562.81$ to $-235.37 \mathrm{mls}$ ). Heterogeneity between these trials was statistically significant $\left(\mathrm{Chi}^{2}=22.67, \mathrm{df}=9, \mathrm{P}=0.007 ; \mathrm{I}^{2}=60 \%\right)$.

\section{Re-operation for bleeding}

Sixty-one trials of aprotinin versus control reported data on re-operation for bleeding. These trials included a total of 6117 patients, of whom 3392 were randomised to aprotinin and 2725 were randomised to a control group who did not receive aprotinin. The use of aprotinin significantly reduced the need for re-operation for bleeding by a relative $54 \%$ (RR $0.46,95 \%$ CI 0.34 to 0.62 ). Heterogeneity between these trials was not significant $\left(\mathrm{Chi}^{2}=35.44\right.$, $\mathrm{df}=42, \mathrm{P}=0.75 ; \mathrm{I}^{2}=0 \%$ ). When aprotinin was used in cardiac surgery, the RR of requiring re-operation due to bleeding was 0.46 (95\% CI 0.34 to 0.63 ). Again heterogeneity between these trials was not significant $\left(\mathrm{Chi}^{2}=34.56, \mathrm{df}=39, \mathrm{P}=0.67 ; \mathrm{I}^{2}=0 \%\right)$.

\section{Mortality}

Sixty-three trials of aprotinin versus control reported data on mortality. These trials included a total of 8876 patients, of whom 4889 were randomised to aprotinin and 3987 were randomised to a control group who did not receive aprotinin. The use of aprotinin was not associated with an increased risk of death (RR 0.81, 95\% CI 0.63 to 1.06). Heterogeneity between these trials was not significant $\left(\mathrm{Chi}^{2}=29.54, \mathrm{df}=43, \mathrm{P}=0.94 ; \mathrm{I}^{2}=0 \%\right)$. In the case of cardiac surgery, the use of aprotinin was not associated with an increased risk of death (RR $0.84,95 \%$ CI 0.64 to 1.10 ).

\section{Myocardial infarction}

Forty-nine trials of aprotinin versus control reported data for myocardial infarction. These trials included a total of 7137 patients, of whom 4032 were randomised to aprotinin and 3105 were randomised to a control group who did not receive aprotinin. The use aprotinin did not increase the risk of myocardial infarction (RR $0.87,95 \%$ CI 0.69 to 1.11 ). Heterogeneity between these trials was not statistically significant $\left(\mathrm{Chi}^{2}=27.71, \mathrm{df}=38, \mathrm{P}=0.89\right.$; $\left.\mathrm{I}^{2}=0 \%\right)$. When aprotinin was used in cardiac surgery, the relative risk of myocardial infarction was not statistically significant (RR $0.90,95 \%$ CI 0.71 to 1.14 ).

\section{Stroke}

Twenty-three trials of aprotinin versus control reported data for stroke. These trials included a total of 3122 patients, of whom 1862 were randomised to aprotinin and 1260 were randomised to a control group who did not receive aprotinin. The use aprotinin did not increase the risk of stroke (RR 0.82, 95\% CI 0.44 to 1.52 ). Heterogeneity between these trials was not statistically significant $\left(\mathrm{Chi}^{2}=11.97, \mathrm{df}=19, \mathrm{P}=0.89 ; \mathrm{I}^{2}=0 \%\right)$. The use of aprotinin in cardiac surgery was not associated with an increased risk of stroke (RR $0.81,95 \%$ CI 0.40 to 1.67 ). 


\section{Deep vein thrombosis}

Sixteen trials of aprotinin versus control reported data for deep vein thrombosis (DVT). These trials included a total of 1456 patients, of whom 854 were randomised to aprotinin and 602 were randomised to a control group who did not receive aprotinin. The use aprotinin did not increase the risk of deep vein thrombosis (RR $0.78,95 \%$ CI 0.47 to 1.29 ). Heterogeneity between these trials was not statistically significant $\left(\mathrm{Chi}^{2}=6.22, \mathrm{df}=11, \mathrm{P}=\right.$ $\left.0.86 ; \mathrm{I}^{2}=0 \%\right)$. Three cardiac trials reported data for DVT. The use of aprotinin was not associated with a statistically significant increased risk of DVT (RR 1.29, 95\% CI 0.36 to 4.58).

\section{Pulmonary embolus}

Four trials of aprotinin versus control reported data for pulmonary embolus (PE). These trials included a total of 585 patients, of whom 304 were randomised to aprotinin and 281 were randomised to a control group who did not receive aprotinin. The use of aprotinin did not statistically significantly increase the risk of PE (RR 1.49, 95\% CI 0.42 to 5.29).

\section{Renal failure / dysfunction}

Twenty-seven trials of aprotinin versus control reported data for renal failure / dysfunction. These trials included a total of 5185 patients, of whom 2904 were randomised to aprotinin and 2281 were randomised to a control group who did not receive aprotinin. The use aprotinin did not statistically significantly increase the risk of renal failure / dysfunction (RR 1.10, 95\% CI 0.79 to 1.54 ). Heterogeneity between these trials was not statistically significant $\left(\mathrm{Chi}^{2}=7.64, \mathrm{df}=16, \mathrm{P}=0.96 ; \mathrm{I}^{2}=0 \%\right)$. Although there appeared to be a trend toward an increased risk of renal failure/dysfunction when aprotinin was used in cardiac surgery, the result was not statistically significant (RR $1.07,95 \%$ CI 0.76 to 1.51 ).

\section{Length of hospital stay}

Twenty-three trials of aprotinin versus control reported data for hospital length of stay. These trials included a total of $2017 \mathrm{pa}-$ tients, of whom 1011 were randomised to aprotinin and 1006 were randomised to a control group who did not receive aprotinin. Aprotinin treatment did not reduce the length of hospital stay (MD -0.25 days, $95 \%$ CI -0.71 to 0.20 days). Heterogeneity between these trials was statistically significant $\left(\mathrm{Chi}^{2}=50.13 \mathrm{df}\right.$ $\left.=22, \mathrm{P}=0.0006 ; \mathrm{I}^{2}=56 \%\right)$.

\section{Tranexamic acid}

Sixty-five trials compared TXA with control, and reported data on the number of patients exposed to allogeneic blood transfusion. These trials included a total of 4842 patients, of whom 2528 were randomised to TXA and 2314 were randomised to a control group who did not receive TXA. The use of TXA significantly reduced the need for allogeneic blood transfusion by a relative 39\% (RR $0.61,95 \%$ CI 0.53 to 0.70 ). Heterogeneity between these trials was statistically significant $\left(\mathrm{Chi}^{2}=249.33, \mathrm{df}=63, \mathrm{P}<0.0001 ; \mathrm{I}^{2}\right.$ $=75 \%)$. This represents an absolute risk reduction of $18 \%(\mathrm{RD}$ $0.18,95 \%$ CI -0.22 to -0.14$)$.

\section{Type of surgery}

Thirty-four trials of TXA versus control involved cardiac surgery. These trials included a total of 3006 patients, of whom 1578 were randomised to TXA, and 1428 were randomised to a control group who did not receive TXA. There was a significant $32 \%$ relative reduction in the rate of exposure to allogeneic blood transfusion in those patients treated with TXA (RR 0.68, 95\% CI 0.57 to $0.81)$. Heterogeneity between these trials was statistically significant $\left(\mathrm{Chi}^{2}=137.35, \mathrm{df}=33, \mathrm{P}<0.00001 ; \mathrm{I}^{2}=76 \%\right)$.

Twenty-seven trials of TXA versus control involved orthopaedic surgery. These trials included a total of 1381 patients of whom of whom 722 were randomised to TXA and 659 were randomised to a control group who did not receive TXA. Again there was a significant $R R$ reduction of $51 \%$ in those participants treated with TXA (RR $0.49,95 \%$ CI 0.39 to 0.62 ). Heterogeneity between these trials was statistically significant $\left(\mathrm{Chi}^{2}=53.86, \mathrm{df}=25, \mathrm{P}=\right.$ $\left.0.0007 ; \mathrm{I}^{2}=54 \%\right)$.

Two trials of TXA versus control involved liver surgery. These trials included a total of 296 patients of whom 148 were randomised to TXA and 148 were randomised to a control group who did not receive TXA. In liver surgery treatment with TXA did not reduce the risk of receiving an allogeneic blood transfusion (RR $0.16,95 \%$ CI 0.00 to 32.47$)$. Heterogeneity between these trials was statistically significant $\left(\mathrm{Chi}^{2}=14.23, \mathrm{df}=1, \mathrm{P}=0.0002 ; \mathrm{I}^{2}=\right.$ 93\%).

One trial of TXA versus control involved vascular surgery. This trial included 59 patients of whom 30 were randomised to TXA and 29 were randomised to a control group who did not receive TXA. In vascular surgery treatment with TXA reduced the risk of receiving an allogeneic blood transfusion (RR $0.56,95 \%$ CI 0.33 to 0.96 ).

One trial of TXA versus control involved gynaecological surgery. This trial included 100 patients of whom 50 were randomised to TXA and 50 were randomised to a control group who did not receive TXA. In gynaecological surgery treatment with TXA did not reduce the risk of receiving an allogeneic blood transfusion (RR $1.50,95 \%$ CI 0.75 to 3.01 ).

\section{Effect of transfusion protocols}

Fifty-six trials of TXA versus control reported the use of transfusion protocols and provided data on the number of patients exposed to allogeneic blood transfusion. These trials included a total of 4125 patients, of whom 2156 were randomised to TXA and 1969 were 
randomised to a control group who did not receive TXA. The use of TXA reduced the need for allogeneic blood transfusion by a relative $43 \%$ (RR $0.57,95 \%$ CI 0.48 to 0.67 ). Heterogeneity between these trials was statistically significant $\left(\mathrm{Chi}^{2}=248.97\right.$, $\left.\mathrm{df}=55, \mathrm{P}<0.00001 ; \mathrm{I}^{2}=78 \%\right)$. There were nine trials that did not report the use of transfusion protocols. These trials included a total of 717 patients of whom 372 were randomised to TXA and 345 were randomised to a control group. The use of TXA reduced the need for allogeneic blood transfusion compared to control (RR $0.76,95 \%$ CI 0.61 to 0.96$)$. Heterogeneity between these trials was statistically significant $\left(\mathrm{Chi}^{2}=15.48, \mathrm{df}=7, \mathrm{P}=0.03\right.$; $\mathrm{I}^{2}=$ $55 \%)$.

Although the baseline rate of transfusion remained relatively constant across both subgroups (transfusion protocol $44 \%$ versus no transfusion protocol $45 \%$ ) transfusion rates in the intervention arms was collectively greater in those trials that did not report the use of a transfusion protocol compared to those trials that did use a transfusion protocol to guide transfusion practice $(37 \%$ versus $26 \%$, respectively).

\section{Volume of blood transfused}

Twenty-three trials of TXA versus control reported data on the volume of blood transfused in all patients. These trials included a total of 1814 patients, of whom 943 were randomised to TXA and 871 were randomised to a control group. The use of TXA resulted in a saving of 0.87 units of allogeneic blood per patient (MD -0.87 units, $95 \%$ CI -1.20 to -0.53 units). Heterogeneity between these trials was statistically significant $\left(\mathrm{Chi}^{2}=154.24, \mathrm{df}\right.$ $\left.=20, \mathrm{P}<0.00001 ; \mathrm{I}^{2}=87 \%\right)$.

Thirteen trials of TXA versus control provided data on the volume of blood transfused in those patients transfused. All 481 patients received allogeneic blood transfusion. The use of TXA did not statistically significantly reduce the volume of blood transfused compared to control (MD - 0.34 units, $95 \%$ CI -0.80 to 0.11 units). Heterogeneity between these trials was statistically significant $\left(\mathrm{Chi}^{2}=45.89, \mathrm{df}=12, \mathrm{P}<0.0001 ; \mathrm{I}^{2}=74 \%\right)$.

\section{Blood loss - all surgery combined}

A total of 17 trials of TXA versus control reported intra-operative blood loss data. These trials included a total of 1173 patients, of whom 599 were randomised to TXA and 574 were randomised to a control group. These trials involved cardiac surgery $(n=4)$, orthopaedic surgery $(n=12)$ and gynaecological surgery $(n=1)$. In aggregate, TXA treatment reduced intra-operative blood loss (MD -121.41 mls, 95\% CI -180.19 to $-62.63 \mathrm{mls}$ ). Heterogeneity between these trials was statistically significant $\left(\mathrm{Chi}^{2}=49.05, \mathrm{df}\right.$ $\left.=16, \mathrm{P}<0.0001 ; \mathrm{I}^{2}=67 \%\right)$.

A total of 35 trials of TXA versus control reported post-operative blood loss data. These trials included a total of 2501 patients, of whom 1285 were randomised to TXA and 1216 were randomised to a control group. These trials involved cardiac surgery $(n=22)$ orthopaedic surgery $(n=12)$ and gynaecological surgery $(n=1)$. In aggregate, TXA treatment significantly reduced post-operative blood loss on average by around $247 \mathrm{mls}$ per patient (MD -247.17 $\mathrm{mls}, 95 \%$ CI -294.76 to $-199.58 \mathrm{mls}$ ). Heterogeneity between these trials was statistically significant $\left(\mathrm{Chi}^{2}=248.36, \mathrm{df}=34, \mathrm{P}\right.$ $<0.00001 ; \mathrm{I}^{2}=86 \%$ ).

A total of 28 trials of TXA versus control reported total blood loss data (intra-operative and post-operative blood loss combined). These trials included a total of 1712 patients, of whom 875 patients were randomised to TXA and 837 were randomised to a control group. These trials involved cardiac surgery $(n=6)$, orthopaedic surgery $(n=20)$, gynaecological surgery $(n=1)$ and liver surgery $(n=1)$. In aggregate, the use of TXA significantly reduced perioperative blood loss by around $414 \mathrm{mls}$ per patient (MD - $414.06 \mathrm{mls}$, 95\% CI -525.19 to $-302.92 \mathrm{mls}$ ). Heterogeneity between these trials was statistically significant $\left(\mathrm{Chi}^{2}=249.58\right.$, $\left.\mathrm{df}=27, \mathrm{P}<0.00001 ; \mathrm{I}^{2}=89 \%\right)$.

\section{Blood loss - cardiac surgery}

Four trials of TXA versus control involving cardiac surgery reported intra-operative blood loss data. These trials included a total of 244 patients, of whom 138 were randomised to TXA and 106 randomised to a control group. The use of TXA in cardiac surgery reduced intra-operative blood loss on average by around $167 \mathrm{mls}$ per patient (MD - $166.76 \mathrm{mls}$, $95 \% \mathrm{CI}-331.24$ to -2.27 $\mathrm{mls})$. There is some evidence of statistical heterogeneity between these trials $\left(\mathrm{Chi}^{2}=5.36, \mathrm{df}=3, \mathrm{P}=0.15 ; \mathrm{I}^{2}=44 \%\right)$.

Twenty-two trials of TXA versus control involving cardiac surgery reported post-operative blood loss data. These trials included a total of 1597 patients, of whom 827 were randomised to TXA and 770 were randomised to a control group. On average, TXA treatment reduced post-operative blood loss by around $273 \mathrm{mls}$ per patient compared to control (MD -272.87 mls, 95\% CI 328.85 to $-216.89 \mathrm{mls}$ ). Heterogeneity between these trials was statistically significant $\left(\mathrm{Chi}^{2}=83.41, \mathrm{df}=21, \mathrm{P}<0.00001\right.$ I $^{2}=$ $75 \%)$.

Six trials of TXA versus control involving cardiac surgery reported total blood loss data (intra-operative and post-operative blood loss combined). These trials included a total of 391 patients, of whom 210 were randomised to TXA and 181 were randomised to a control group. TXA treatment reduced the total amount of blood lost during the perioperative period by around $300 \mathrm{mls}$ per patient (MD -300.47 mls, 95\% CI -470.74 to $-130.21 \mathrm{mls}$ ). Heterogeneity between these trials was statistically significant $\left(\mathrm{Chi}^{2}=12.19\right.$, $\left.\mathrm{df}=5, \mathrm{P}=0.03 ; \mathrm{I}^{2}=59 \%\right)$.

\section{Blood loss - orthopaedic surgery}

Twelve trials of TXA versus control involving orthopaedic surgery reported intra-operative blood loss data. These trials included a 
total of 829 patients, of whom 411 were randomised to TXA and 418 were randomised to a control group. The use of TXA in orthopaedic surgery reduced intra-operative blood loss by around $116 \mathrm{mls}$ per patient (MD $-115.52 \mathrm{mls}, 95 \%$ CI -187.88 to -43.16 $\mathrm{mls})$. Heterogeneity between these trials was statistically significant $\left(\mathrm{Chi}^{2}=42.52, \mathrm{df}=11, \mathrm{P}<0.0001 ; \mathrm{I}^{2}=74 \%\right)$.

Twelve trials of TXA versus control involving orthopaedic surgery reported post-operative blood loss data. These trials included a total of 804 patients, of whom 408 were randomised to TXA and 396 were randomised to a control group. On average, TXA treatment in orthopaedic surgery reduced post-operative blood loss by around $229 \mathrm{mls}$ per patient (MD $-228.52 \mathrm{mls}$, 95\% CI -321.76 to $-135.27 \mathrm{mls}$ ). Heterogeneity between these trials was statistically significant $\left(\mathrm{Chi}^{2}=125.01, \mathrm{df}=11, \mathrm{P}<0.00001 ; \mathrm{I}^{2}=\right.$ $91 \%)$.

Twenty trials of TXA versus control involving orthopaedic surgery reported total blood loss data (intra-operative and post-operative blood loss combined). These trials included a total of $1201 \mathrm{pa}-$ tients, of whom 605 were randomised to TXA and 596 were randomised to a control group. The use of TXA in orthopaedic surgery significantly reduced the total amount of blood lost during the perioperative period (MD $-446.19 \mathrm{mls}, 95 \% \mathrm{CI}-554.61$ to $-337.78 \mathrm{mls}$ ). Heterogeneity between these trials was statistically significant $\left(\mathrm{Chi}^{2}=85.30, \mathrm{df}=19, \mathrm{P}<0.00001 ; \mathrm{I}^{2}=78 \%\right)$.

\section{Re-operation for bleeding}

Twenty-seven trials of TXA versus control reported data on reoperation for bleeding. These trials included a total of $2386 \mathrm{pa}-$ tients, of whom 1224 were randomised to TXA and 1162 were randomised to a control group. The use of TXA did not statistically significantly decrease the risk of re-operation for bleeding (RR $0.80,95 \%$ CI 0.55 to 1.17 ). Heterogeneity between these trials was not statistically significant $\left(\mathrm{Chi}^{2}=12.66, \mathrm{df}=23, \mathrm{P}=\right.$ $0.96 ; \mathrm{I}^{2}=0 \%$ ). Of the 27 trials of TXA that reported data for this outcome 26 involved cardiac surgery. Therefore in the context of cardiac surgery the use of TXA did not statistically significantly reduce the risk of re-operation for bleeding (RR $0.79,95 \% \mathrm{CI}$ 0.54 to 1.17$)$.

\section{Mortality}

Thirty trials of TXA versus control reported mortality data. These trials included a total of 2917 patients, of whom 1478 were randomised to TXA and 1439 were randomised to a control group. The use of TXA was not associated with an increased risk of death (RR $0.60,95 \%$ CI 0.33 to 1.10 ). Heterogeneity between these trials was not statistically significant $\left(\mathrm{Chi}^{2}=10.00, \mathrm{df}=17, \mathrm{P}=\right.$ $\left.0.90 ; \mathrm{I}^{2}=0 \%\right)$. Of the 30 trials of TXA that reported data for mortality 23 involved cardiac surgery. The use of TXA in cardiac surgery was not associated with an increased risk of death (RR $0.58,95 \%$ CI 0.26 to 1.28 ).

\section{Myocardial infarction}

Twenty-one trials of TXA versus control reported data for myocardial infarction. These trials included a total of 2186 patients, of whom 1117 were randomised to TXA and 1069 were randomised to a control group who did not receive TXA. The use of TXA was not associated with an increased risk of myocardial infarction (RR $0.79,95 \%$ CI 0.41 to 1.52 ). Heterogeneity between these trials was not statistically significant $\left(\mathrm{Chi}^{2}=7.84, \mathrm{df}=12, \mathrm{P}=0.80\right.$; $\mathrm{I}^{2}$ $=0 \%)$. Of the 21 trials of TXA that reported data for myocardial infarction 19 involved cardiac surgery. The use of TXA in cardiac surgery did not increase the risk of myocardial infarction (RR $0.74,95 \%$ CI 0.37 to 1.47 ).

\section{Stroke}

Eighteen trials of TXA versus control reported data for stroke. These trials included a total of 2027 patients, of whom 1050 were randomised to TXA and 977 were randomised to a control group. The use of TXA was not associated with a statistically significant increase in the risk of stroke (RR 1.23, 95\% CI 0.49 to 3.07). Heterogeneity between these trials was not statistically significant $\left(\mathrm{Chi}^{2}=3.18, \mathrm{df}=7, \mathrm{P}=0.87 ; \mathrm{I}^{2}=0 \%\right)$. Of the 18 trials of TXA that reported data for this outcome 17 involved cardiac surgery. In this surgical setting the risk of stroke was not statistically significantly increased with the use of TXA (RR 1.44, 95\% CI 0.53 to 3.91 ).

\section{Deep vein thrombosis}

Twenty-three trials of TXA versus control reported data for deep vein thrombosis. These trials included a total of 1472 , of whom 746 were randomised to TXA and 726 were randomised to a control group. TXA treatment did not appear to be associated with an increase in the risk of developing a DVT (RR 0.71, 95\% CI 0.35 to 1.43$)$. Heterogeneity between these trials was not statistically significant $\left(\mathrm{Chi}^{2}=5.71, \mathrm{df}=11, \mathrm{P}=0.89 ; \mathrm{I}^{2}=0 \%\right)$. Of the 23 trials of TXA that reported data for DVT four involved cardiac surgery. Of the 422 patients that underwent cardiac surgical procedures two patients developed a DVT. These were single events occurring in the control arms of two separate trials.

\section{Pulmonary embolism}

Fourteen trials of TXA versus control reported data for pulmonary embolism. These trials included a total of 1006 patients, of whom 527 were randomised to TXA and 479 were randomised to a control group who did not receive TXA. The use of TXA did not increase the risk of developing a pulmonary embolus (RR 0.67, $95 \%$ CI 0.23 to 1.99$)$. Heterogeneity between these trials was not statistically significant $\left(\mathrm{Chi}^{2}=2.81, \mathrm{df}=7, \mathrm{P}=0.90 ; \mathrm{I}^{2}=0 \%\right)$. Of the 16 trials that reported data for pulmonary embolism six involved cardiac surgery. Of the 569 patients that underwent cardiac surgical procedures only two patients developed a pulmonary 
embolus. As was the case with deep vein thrombosis these were single events occurring in the control arms of two separate trials.

\section{Renal failure / dysfunction}

Nine trials of TXA versus control provided data for renal failure / dysfunction. These nine cardiac surgery trials included a total of 912 patients, of whom 454 were randomised to TXA and 458 were randomised to a control group. Treatment with TXA did not appear to increase the risk of developing renal failure or renal dysfunction (RR 0.89, 95\% CI 0.33 to 2.37). Heterogeneity between these trials was not statistically significant $\left(\mathrm{Chi}^{2}=2.52, \mathrm{df}=6, \mathrm{P}\right.$ $\left.=0.87 ; \mathrm{I}^{2}=0 \%\right)$.

\section{Hospital length of stay}

Ten trials of TXA versus control provided data for hospital length of stay. These trials included a total of 772 patients, of whom 379 were randomised to TXA and 393 were randomised to a control group. The use of TXA did not significantly impact on the length of hospital stay (MD - 0.34 days, $95 \%$ CI -0.82 to 0.13 days). Heterogeneity between these trials was statistically significant $\left(\mathrm{Chi}^{2}=\right.$ $\left.18.42, \mathrm{df}=9, \mathrm{P}=0.03 ; \mathrm{I}^{2}=51 \%\right)$. For the five trials that involved cardiac surgery the use of TXA did not significantly reduce the length of hospital stay (MD -0.08 days, $95 \%$ CI -0.34 to 0.18 days).

\section{Epsilon aminocaproic acid}

Sixteen trials of EACA versus control provided data on the number of patients exposed to allogeneic blood transfusion. These trials included a total of 1035 patients, of whom 530 were randomised to EACA and 505 were randomised to a control who did not receive EACA. The use of EACA significantly reduced the need for allogeneic blood transfusion by a relative 19\% (RR 0.81, 95\% CI 0.67 to 0.99$)$. Heterogeneity between these trials was statistically significant $\left(\mathrm{Chi}^{2}=41.12, \mathrm{df}=15, \mathrm{P}=0.0003 ; \mathrm{I}^{2}=64 \%\right)$. This represents an absolute risk reduction of $10 \%$ (RD $-0.10,95 \% \mathrm{CI}$ -0.18 to -0.03$)$.

\section{Type of surgery}

Eleven trials of EACA versus control involved cardiac surgery. These trials included a total of 649 patients, of whom 338 were randomised to EACA and 311 were randomised to a control group. When used in cardiac surgery EACA reduced the need for allogeneic blood transfusion by a relative $30 \%$ (RR $0.70,95 \%$ CI 0.52 to 0.93$)$. There is some evidence of statistical heterogeneity between these trials $\left(\mathrm{Chi}^{2}=16.38, \mathrm{df}=10, \mathrm{P}=0.09 ; \mathrm{I}^{2}=\right.$ $39 \%)$. Four trials of EACA versus control involved orthopaedic surgery. These trials included a total of 304 patients, of whom 150 were randomised to EACA and 154 patients were randomised to a control group. The use of EACA in orthopaedic surgery did not reduce the need for allogeneic blood transfusion compared to control (RR 1.00, 95\% CI 0.93 to 1.08). Heterogeneity between these trials was not statistically significant $\left(\mathrm{Chi}^{2}=1.01, \mathrm{df}=3, \mathrm{P}\right.$ $\left.=0.80 ; \mathrm{I}^{2}=0 \%\right)$. One trial of EACA involved liver surgery. For this single trial the relative risk of requiring an allogeneic blood transfusion was 0.93 (95\% CI 0.80 to 1.08 ).

\section{Effect of transfusion protocols}

Of the 16 trials of EACA versus control that provided data for the number of patients exposed to allogeneic blood transfusion, 15 reported the use of a transfusion protocol to guide transfusion practice. Therefore stratification of the data by the presence or absence of a transfusion protocol was uninformative.

\section{Volume of blood transfused}

Six trials of EACA versus control provided data for the volume of blood transfused in all patients. These trials included a total of 432 patients, of whom 215 were randomised to EACA and 217 were randomised to a control group who did not receive EACA. On average, the use of EACA reduced the volume of allogeneic blood transfused by 1.3 units per patient (MD -1.30 units, $95 \%$ CI -2.14 to -0.45 units). Heterogeneity between these trials was statistically significant $\left(\mathrm{Chi}^{2}=23.45, \mathrm{df}=5, \mathrm{P}=0.0003 ; \mathrm{I}^{2}=\right.$ $79 \%)$. Three trials of EACA versus control provided data for the volume of blood transfused in those patients transfused. When the volume of allogeneic blood transfused was assessed in only those patients that actually received a blood transfusion the use of EACA did not reduce the amount of blood transfused (MD 0.22 units, $95 \%$ CI -0.34 to 0.79 units). Heterogeneity between these trials was not statistically significant $\left(\mathrm{Chi}^{2}=0.56, \mathrm{df}=2, \mathrm{P}=0.76 ; \mathrm{I}^{2}=\right.$ $0 \%)$.

\section{Blood loss - all surgery combined}

Five trials of EACA versus control reported intra-operative blood loss data. These trials included a total of 353 patients, of whom 175 were randomised to EACA and 178 were randomised to a control group. These trials involved cardiac surgery $(n=2)$ and orthopaedic surgery $(n=3)$. In aggregate, EACA treatment reduced the amount of blood lost during the intra-operative period by around $157 \mathrm{mls}$ per patient (MD - $156.63 \mathrm{mls}$, 95\% CI -276.92 to $-36.33 \mathrm{mls})$. Heterogeneity between these trials was not statistically significant $\left(\mathrm{Chi}^{2}=5.01, \mathrm{df}=4, \mathrm{P}=0.29 ; \mathrm{I}^{2}=20 \%\right)$.

Fourteen trials of EACA versus control reported post-operative blood loss data. These trials included a total of 1174 patients, of whom 580 were randomised to EACA and 594 were randomised to a control group. These trials involved cardiac surgery $(n=12)$ and orthopaedic surgery $(\mathrm{n}=2)$. In aggregate, EACA treatment reduced post-operative blood loss on average by $207 \mathrm{mls}$ per patient (MD $-207.49 \mathrm{mls}$, 95\% CI -276.43 to $-138.54 \mathrm{mls}$ ). Het- 
erogeneity between these trials was statistically significant $\left(\mathrm{Chi}^{2}=\right.$ 97.46, $\left.\mathrm{df}=13, \mathrm{P}<0.00001 ; \mathrm{I}^{2}=87 \%\right)$.

Two trials of EACA versus control reported total blood loss data (intra-operative and post-operative blood loss combined). These orthopaedic trials included a total of 92 patients, of whom 44 were randomised to EACA and 48 were randomised to a control group. The use of EACA in orthopaedic surgery was only marginally effective in reducing blood loss during the perioperative period (MD -299.69 mls, 95\% CI -522.54 to $-76.84 \mathrm{mls}$ ). Heterogeneity between these trials was not statistically significant $\left(\mathrm{Chi}^{2}=0.73\right.$, $\left.\mathrm{df}=1, \mathrm{P}=0.39 ; \mathrm{I}^{2}=0 \%\right)$.

\section{Blood loss - cardiac surgery}

Two trials of EACA versus control involving cardiac surgery reported intra-operative blood loss data. These trials included a total of 79 patients, of whom 40 patients were randomised to EACA and 39 were randomised to a control group. On average, the use of EACA in cardiac surgery reduced the amount of blood lost during the intra-operative period by around $214 \mathrm{mls}$ per patient (MD $213.58,95 \%$ CI -310.03 to $-117.13 \mathrm{mls}$ ). Heterogeneity between these trials was not statistically significant $\left(\mathrm{Chi}^{2}=0.12, \mathrm{df}=1, \mathrm{P}\right.$ $=0.73 ; \mathrm{I}^{2}=0 \%$ ).

Twelve trials of EACA versus control involving cardiac surgery reported post-operative blood loss data. These trials included a total of 946 patients, of whom 467 were randomised to EACA and 479 were randomised to control group who did not receive EACA treatment. The use of EACA in cardiac surgery reduced the amount of blood lost during the post-operative period on average by around $200 \mathrm{mls}$ per patient (MD $-200.27 \mathrm{mls}, 95 \% \mathrm{CI}$ -273.44 to $-127.09 \mathrm{mls}$ ). Heterogeneity between these trials was statistically significant $\left(\mathrm{Chi}^{2}=97.18, \mathrm{df}=11, \mathrm{P}<0.00001, \mathrm{I}^{2}=\right.$ $89 \%)$.

\section{Blood loss - orthopaedic surgery}

Three trials of EACA versus control involving orthopaedic surgery provided intra-operative blood loss data. These trials included a total of 274 patients, of whom 135 were randomised to EACA and 139 were randomised to a control group. EACA treatment in orthopaedic surgery did not reduce the amount of blood lost during the intra-operative period (MD $-40.66 \mathrm{mls}$, 95\% CI 236.71 to $155.38 \mathrm{mls}$ ). Heterogeneity between these trials was not statistically significant $\left(\mathrm{Chi}^{2}=2.10, \mathrm{df}=2, \mathrm{P}=0.35 ; \mathrm{I}^{2}=5 \%\right)$. Two trials of EACA versus control involving orthopaedic surgery reported post-operative blood loss. These trials included a total of 228 patients, of whom 113 were randomised to EACA and 115 were randomised to a control group. The use of EACA in orthopaedic surgery reduced blood loss during the post-operative period by around $285 \mathrm{mls}$ per patient (MD -285.06 mls, 95\% CI -452.73 to $-117.39 \mathrm{mls})$. Heterogeneity between these trials was not statistically significant $\left(\mathrm{Chi}^{2}=0.18, \mathrm{df}=1, \mathrm{P}=0.67 ; \mathrm{I}^{2}=0 \%\right)$.
Two trials of EACA versus control involving orthopaedic surgery reported total blood loss data (intra-operative and post-operative blood loss combined). These trials included a total of 92 patients, of whom 44 were randomised to EACA and 48 were randomised to a control group. The use of EACA in orthopaedic surgery reduced blood loss during the perioperative period by around $300 \mathrm{mls}$ per patient (MD -299.69 mls, 95\% CI -522.54 to $-76.84 \mathrm{mls}$ ). Heterogeneity between these trials was not statistically significant $\left(\mathrm{Chi}^{2}=0.73, \mathrm{df}=1, \mathrm{P}=0.39 ; \mathrm{I}^{2}=0 \%\right)$.

\section{Re-operation for bleeding}

Eight trials of EACA versus control reported data on the number of patients requiring re-operation for bleeding. These trials included a total of 922 patients, of whom 470 were randomised to EACA and 452 were randomised to a control group. The use of EACA was not associated with an increased risk of re-operation compared to control (RR $0.32,95 \%$ CI 0.11 to 0.99 ). Heterogeneity between these trials was not statistically significant $\left(\mathrm{Chi}^{2}=1.84, \mathrm{df}=5, \mathrm{P}\right.$ $\left.=0.87, \mathrm{I}^{2}=0 \%\right)$. Of the eight trials of EACA that reported data on re-operations, seven involved cardiac surgery. In this surgical setting the use of EACA did not increase the risk of re-operation (RR 0.35 , 95\% CI 0.11 to 1.17 ).

\section{Mortality}

Eight trials of EACA versus control reported data on mortality. These trials included a total of 988 patients, of whom 504 were randomised to EACA and 484 were randomised to a control group. The use of EACA was not associated with a statistically significant increased risk of death compared to control (RR 1.07, 95\% CI 0.44 to 2.57). Heterogeneity between these trials was not statistically significant $\left(\mathrm{Chi}^{2}=2.30, \mathrm{df}=5, \mathrm{P}=0.81 ; \mathrm{I}^{2}=0 \%\right)$. Of the eight trials of EACA that reported data on mortality six involved cardiac surgery. In this surgical setting the use of EACA did not statistically significantly increase the risk of death (RR 1.65, 95\% CI 0.50 to 5.43).

\section{Myocardial infarction}

Seven trials of EACA versus control reported data for myocardial infarction. These trials included a total of 896 patients, of whom 456 were randomised to EACA and 440 were randomised to a control group. The use of EACA was not associated with an increased risk of myocardial infarction compared to control (RR $0.88,95 \%$ CI 0.48 to 1.63$)$. Heterogeneity between these trials was not statistically significant $\left(\mathrm{Chi}^{2}=3.44, \mathrm{df}=4, \mathrm{P}=0.49 ; \mathrm{I}^{2}=\right.$ $0 \%)$. Of the seven trials of EACA that reported data on myocardial infarction six involved cardiac surgery. In this surgical setting the use of EACA did not increase the risk of myocardial infarction (RR $0.88,95 \%$ CI 0.48 to 1.63 ). 


\section{Stroke}

Eight trials of EACA versus control reported data for stroke. These trials included a total of 936 patients, of whom 477 were randomised to EACA and 459 were randomised to a control group. The use of EACA was not associated with an increased risk of stroke compared to control (RR $0.6295 \%$ CI 0.16 to 2.36). Heterogeneity between these trials was not statistically significant $\left(\mathrm{Chi}^{2}\right.$ $\left.=1.84, \mathrm{df}=4, \mathrm{P}=0.77 ; \mathrm{I}^{2}=0 \%\right)$. Of the eight trials of EACA that reported data on stroke, seven involved cardiac surgery. In this surgical setting the use of EACA did not increase the risk of stroke (RR 0.70, 95\% CI 0.16 to 3.10 ).

\section{Deep vein thrombosis}

Four trials of EACA versus control reported data for DVT. These trials included a total of 304 patients, of whom 150 were randomised to EACA and 154 were randomised to a control group. The use of EACA was not associated with an increased risk of DVT compared to control (RR $0.78,95 \%$ CI 0.20 to 3.03). Heterogeneity between these trials was not statistically significant $\left(\mathrm{Chi}^{2}\right.$ $\left.=1.02, \mathrm{df}=1, \mathrm{P}=0.31 ; \mathrm{I}^{2}=2 \%\right)$.

\section{Pulmonary embolism}

Three trials of EACA versus control provided data for pulmonary embolism. These trials included a total of 274 patients, of whom 135 were randomised to EACA and 139 were randomised to a control group. The use of EACA was not associated with an increased risk of pulmonary embolism compared to control (RR $0.34,95 \%$ CI 0.06 to 2.13 ). Heterogeneity between these trials was not statistically significant $\left(\mathrm{Chi}^{2}=0.00, \mathrm{df}=1, \mathrm{P}=0.97 ; \mathrm{I}^{2}=\right.$ $0 \%)$.

\section{Renal failure / dysfunction}

Two trials of EACA versus control reported data for renal failure / dysfunction. These trials included a total of 235 patients, of whom 117 were randomised to EACA and 118 were randomised to a control group. The use of EACA was not associated with an increased risk of renal failure / dysfunction (RR $0.41,95 \%$ CI 0.14 to 1.22 ). Heterogeneity between these trials was not statistically significant $\left(\mathrm{Chi}^{2}=0.48, \mathrm{df}=1, \mathrm{P}=0.49 ; \mathrm{I}^{2}=0 \%\right)$.

\section{Hospital length of stay}

Two trials of EACA versus control reported data for hospital length of stay. These trial included a total of 228 patients, of whom 113 were randomised to EACA and 115 were randomised to a control group. The use of EACA did not impact of the length of hospital stay (MD 0.58 days, $95 \%$ CI -3.17 to 4.33 days). Heterogeneity between these trials was statistically significant $\left(\mathrm{Chi}^{2}=3.13, \mathrm{df}=\right.$ $\left.1, \mathrm{P}=0.08 ; \mathrm{I}^{2}=68 \%\right)$.

\section{Aprotinin versus tranexamic acid}

Twenty-one trials of aprotinin versus TXA reported data on the number of patients exposed to allogeneic blood transfusion. These trials included a total of 4185 patients, of whom 2124 were randomised to aprotinin and 2061 were randomised to TXA. There was no statistically significant difference in the rates of allogeneic blood transfusion between those patients treated with aprotinin compared to those treated with TXA (RR $0.90,95 \%$ CI 0.81 to $1.01)$. Heterogeneity between these trials was statistically significant $\left(\mathrm{Chi}^{2}=60.78, \mathrm{df}=20, \mathrm{P}<0.0001 ; \mathrm{I}^{2}=67 \%\right)$.

\section{Type of surgery}

Eighteen of the 21 trials of aprotinin versus TXA that reported data on the number patients exposed to allogeneic blood transfusion involved cardiac surgery. These trials included a total of 3983 patients, of whom 2025 were randomised to aprotinin and 1958 were randomised to TXA. Compared to TXA, aprotinin reduced the rate of allogeneic blood transfusion (RR $0.87,95 \%$ CI 0.76 to 0.99$)$. Heterogeneity between these trials was statistically significant $\left(\mathrm{Chi}^{2}=45.01, \mathrm{df}=17, \mathrm{P}=0.0002 ; \mathrm{I}^{2}=62 \%\right)$.

\section{Effect of transfusion protocols}

Of the 21 trials of aprotinin versus TXA that reported data on the number patients exposed to allogeneic blood transfusion, all but one reported the use of a transfusion protocol to guide transfusion practice. Therefore stratification of the data by the presence or absence of a transfusion protocol proved uninformative.

\section{Volume of blood transfused}

Ten trials of aprotinin versus TXA provided data on the volume of allogeneic blood transfused in all patients. These trials included a total of 992 patients, of whom 496 were randomised to aprotinin and 496 were randomised to TXA. There was a small but statistically significant difference between aprotinin and TXA in the volume of allogeneic blood transfused (MD - 0.24 units, 95\% CI -0.45 to -0.04 units). Heterogeneity between these trials was not statistically significant $\left(\mathrm{Chi}^{2}=10.87, \mathrm{df}=9 ; \mathrm{P}=0.28 ; \mathrm{I}^{2}=\right.$ $17 \%)$. Six trials of aprotinin versus TXA provided data on the volume of allogeneic blood transfused in those patients transfused. These trials provided data for 207 transfused patients, of whom 97 were treated with aprotinin and 110 were treated with TXA. There was no statistically significant difference between aprotinin and TXA in the volume of allogeneic blood transfused in those patients transfused (MD - 0.07 units, $95 \% \mathrm{CI}-0.44$ to 0.30 units). Heterogeneity between these trials was not statistically significant $\left(\mathrm{Chi}^{2}=0.97, \mathrm{df}=5, \mathrm{P}=0.97 ; \mathrm{I}^{2}=0 \%\right)$. 


\section{Blood loss}

Thirteen trials of aprotinin versus TXA involving cardiac surgery provided data for post-operative blood loss. These trials included a total of 831 patients, of whom 412 were randomised to aprotinin and 419 were randomised to TXA. On average, aprotinin appeared to be more effective in reducing post-operative blood loss than TXA (MD - $145.81 \mathrm{mls}$, 95\% CI -209.99 to $-81.62 \mathrm{mls}$ ). Heterogeneity between these trials was statistically significant $\left(\mathrm{Chi}^{2}=\right.$ $\left.33.86, \mathrm{df}=12, \mathrm{P}=0.0007 ; \mathrm{I}^{2}=65 \%\right)$.

\section{Re-operation for bleeding}

Seventeen trials of aprotinin versus TXA provided data on the number of patients requiring re-operation for bleeding. These trials included a total of 4010 patients, of whom 2005 were randomised to aprotinin and 2005 were randomised to TXA. Aprotinin appeared to reduce the need for re-operation compared to TXA (RR 0.69 , 95\% CI 0.51 to 0.93 ). Heterogeneity between these trials was not statistically significant $\left(\mathrm{Chi}^{2}=8.90, \mathrm{df}=13\right.$, $\mathrm{P}=0.78 ; \mathrm{I}^{2}=0 \%$ ). The BART study (Fergusson 2008) provided $61.4 \%$ (weight) of the information for this outcome.

\section{Mortality}

Seventeen trials of aprotinin versus TXA reported mortality data. These trials included a total of 4130 patients, of whom 2060 were randomised to aprotinin and 2070 were randomised to TXA. There was no statistically significant difference between aprotinin and TXA (RR 1.35, 95\% CI 0.94 to 1.93). Heterogeneity between these trials was not statistically significant $\left(\mathrm{Chi}^{2}=6.78, \mathrm{df}=9, \mathrm{P}\right.$ $\left.=0.66, \mathrm{I}^{2}=0 \%\right)$. BART study data (Fergusson 2008) dominated the analysis of this outcome ( $65.5 \%$ weight).

\section{Myocardial infarction}

Thirteen trials of aprotinin versus TXA reported data for myocardial infarction. These trials included a total of 3574 patients, of whom 1778 were randomised to aprotinin and 1796 were randomised to TXA. There was statistically significant difference between aprotinin and TXA (RR 1.00, 95\% CI 0.71 to 1.42 ). Heterogeneity between these trials was not statistically significant $\left(\mathrm{Chi}^{2}=6.18, \mathrm{df}=10, \mathrm{P}=0.80 ; \mathrm{I}^{2}=0 \%\right)$. The BART study (Fergusson 2008) provided $49.6 \%$ (weight) of the information for this outcome.

\section{Stroke}

Six trials of aprotinin versus TXA reported data for stroke. These trials include a total of 2030 patients of whom 1017 were randomised to aprotinin and 1013 were randomised to TXA. There was no statistically significant difference between aprotinin and TXA (RR $0.88,95 \%$ CI 0.52 to 1.47 ). Heterogeneity between these trials was not statistically significant $\left(\mathrm{Chi}^{2}=1.91, \mathrm{df}=4, \mathrm{P}\right.$ $\left.=0.75 ; \mathrm{I}^{2}=0 \%\right)$. BART study data (Fergusson 2008) dominated the analysis of this outcome ( $88.5 \%$ weight).

\section{Renal failure / dysfunction}

Six trials of aprotinin versus TXA reported data for renal failure / dysfunction. These trials included a total of 2238 patients, of whom 1119 were randomised to aprotinin and 1119 were randomised to TXA. There was no statistically significant difference between aprotinin and TXA (RR 1.02, 95\% CI 0.79 to 1.31). Heterogeneity between these trials was not statistically significant $\left(\mathrm{Chi}^{2}=1.20, \mathrm{df}=3, \mathrm{P}=0.75 ; \mathrm{I}^{2}=0 \%\right)$. BART study data (Fergusson 2008) dominated the analysis of this outcome $(94.5 \%$ weight).

\section{Hospital length of stay}

Six trials of aprotinin versus TXA reported data for hospital length of stay. These trials include a total of 2174 patients, of whom 1090 were randomised to aprotinin and 1084 were randomised to TXA. There was no statistically significant difference between aprotinin and TXA (MD - $0.05,95 \%$ CI -0.92 to 0.83 days). There was some evidence of statistical heterogeneity between these trials $\left(\mathrm{Chi}^{2}=\right.$ 9.14, $\left.\mathrm{df}=5, \mathrm{P}=0.10 ; \mathrm{I}^{2}=45 \%\right)$.

\section{Aprotinin versus epsilon aminocaproic acid}

Twelve trials of aprotinin versus EACA reported data on the number of patients exposed to allogeneic blood transfusion. These trials included a total of 2200 patients, of whom 1102 were randomised to aprotinin and 1098 were randomised to EACA. The use of aprotinin significantly reduced the rate of allogeneic blood transfusion compared to EACA (RR 0.82, 95\% CI 0.76 to 0.89 ). Heterogeneity between these trials was not statistically significant $\left(\mathrm{Chi}^{2}=9.33, \mathrm{df}=11, \mathrm{P}=0.59 ; \mathrm{I}^{2}=0 \%\right)$.

\section{Type of surgery}

Of the 12 trials of aprotinin versus EACA that reported data on the number of patients exposed to allogeneic blood transfusion, 10 involved cardiac surgery and two involved orthopaedic surgery. Compared to EACA, aprotinin reduced the rate of allogenic blood transfusion in cardiac surgery (RR $0.82,95 \%$ CI 0.76 to 0.89 ) but not in orthopaedic surgery (RR $0.82,95 \%$ CI 0.48 to 1.40 ).

\section{Effect of transfusion protocols}

Of the 12 trials of aprotinin versus EACA that reported data on the number of patients exposed to allogeneic blood transfusion, nine reported the use of a transfusion protocol to guide transfusion practice and three did not. For the nine trials that reported the use of a transfusion protocol, aprotinin reduced the rate of allogeneic 
blood transfusions compared to EACA by a relative $18 \%$ (RR 0.82 , $95 \%$ CI 0.76 to 0.89$)$. Heterogeneity between these trials was not statistically significant $\left(\mathrm{Chi}^{2}=6.45, \mathrm{df}=8, \mathrm{P}=0.60 ; \mathrm{I}^{2}=0 \%\right)$. For those trials that did not report the use of a transfusion protocol there was no statistically significant difference aprotinin and EACA (RR $0.78,95 \%$ CI 0.47 to 1.31 ). Heterogeneity between these trials was not statistically significant $\left(\mathrm{Chi}^{2}=2.86, \mathrm{df}=2, \mathrm{P}=0.24\right.$; $\left.\mathrm{I}^{2}=30 \%\right)$

\section{Volume of blood transfused}

Five trials of aprotinin versus EACA reported data for the volume of allogeneic blood transfused in all patients. These trials included a total of 329 patients, of whom 166 were randomised to aprotinin and 163 were randomised to EACA. There was no statistically significant difference between aprotinin and EACA (MD -0.21 units, $95 \%$ CI -0.55 to 0.14 units). Heterogeneity between these trials was not statistically significant $\left(\mathrm{Chi}^{2}=5.14, \mathrm{df}=4, \mathrm{P}=0.27\right.$; $\left.\mathrm{I}^{2}=22 \%\right)$. Two trials of aprotinin versus EACA provided data for the volume of allogeneic blood transfused in those patients transfused. These trials included a total of 63 transfused patients, of whom 28 were treated with aprotinin and 35 were treated with EACA. There was no statistically significant difference between aprotinin and EACA treatment (MD - 0.18 units, $95 \%$ CI -0.63 to 0.28 units). Heterogeneity between these trials was not statistically significant $\left(\mathrm{Chi}^{2}=0.66, \mathrm{df}=1, \mathrm{P}=0.41 ; \mathrm{I}^{2}=0 \%\right)$.

\section{Blood loss}

There were seven trials of aprotinin versus EACA involving cardiac surgery that reported post-operative blood loss data. These trials included a total of 454 patients, of whom 230 were randomised to aprotinin and 224 were randomised to EACA. Aprotinin appeared to be marginally more effective in reducing post-operative blood loss than EACA (MD - $111.43 \mathrm{mls}$, 95\% CI -220.64 to -2.21 $\mathrm{mls})$. Heterogeneity between these trials was statistically significant $\left(\mathrm{Chi}^{2}=25.74, \mathrm{df}=6, \mathrm{P}=0.0002 ; \mathrm{I}^{2}=77 \%\right)$.

\section{Re-operation for bleeding}

Six trials of aprotinin versus EACA reported data on the number of patients requiring re-operation for bleeding. These trials included a total of 2075 patients, of whom 1034 were randomised to aprotinin and 1041 were randomised to EACA. Although aprotinin appeared to be more effective than EACA in reducing the number patients requiring re-operation due to bleeding the difference did not reach statistical significance (RR $0.70,95 \%$ CI 0.49 to 1.00 ). Heterogeneity between these trials was not statistically significant $\left(\mathrm{Chi}^{2}=0.93, \mathrm{df}=2, \mathrm{P}=0.63 ; \mathrm{I}^{2}=0 \%\right)$. However, the data from the BART study (Fergusson 2008) provided $90.1 \%$ of the information (weight) for this outcome. The results of this one trial showed that aprotinin was statistically significantly more effective than EACA in reducing the risk of re-operation for bleeding (RR $0.67,95 \%$ CI 0.46 to 0.98 ).

\section{Mortality}

There were five trials of aprotinin versus EACA that reported mortality data. These trials included a total of 1891 patients, of whom 949 were randomised to aprotinin and 942 were randomised to EACA. Although the result failed to reach statistical significance, there appeared to be a trend toward an increased risk of death in the aprotinin group compared to EACA (RR 1.51, 95\% CI 0.99 to 2.30). Again, the results of the BART study (Fergusson 2008) provided most of the information for this outcome $(89.9 \%$ weight). Heterogeneity between these trials was not statistically significant $\left(\mathrm{Chi}^{2}=0.26, \mathrm{df}=3, \mathrm{P}=0.97 ; \mathrm{I}^{2}=0 \%\right)$.

\section{Myocardial infarction}

Four trials of aprotinin versus EACA reported data for myocardial infarction. These trials included a total of 1676 patients, of whom 830 were randomised to aprotinin and 846 were randomised to EACA. There was no statistically significant difference in the risk of myocardial infarction between aprotinin and EACA (RR 1.42, 95\% CI 0.90 to 2.22). Heterogeneity between these trials was not statistically significant $\left(\mathrm{Chi}^{2}=1.27, \mathrm{df}=3, \mathrm{P}=0.74 ; \mathrm{I}^{2}=\right.$ $0 \%)$. Data from the BART study (Fergusson 2008) dominated this outcome (68.2\% weight).

\section{Stroke}

Two trials of aprotinin versus EACA reported data for stroke (cerebrovascular accident). These trials included a total of 1578 patients, of whom 785 were randomised to aprotinin and 793 were randomised to EACA. There was no difference in the risk of stroke between aprotinin and EACA (RR 1.05, 95\% CI 0.60 to 1.85). Heterogeneity between these trials was not statistically significant $\left(\mathrm{Chi}^{2}=0.27, \mathrm{df}=1, \mathrm{P}=0.60 ; \mathrm{I}^{2}=0 \%\right)$. The BART study (Fergusson 2008) results provided $94.2 \%$ of the information for this outcome.

\section{Deep vein thrombosis}

Four trials of aprotinin versus EACA reported data for deep vein thrombosis. These trials included a total of 300 patients, of whom 153 were randomised to aprotinin and 147 were randomised to EACA. One trial reported three cases of DVT all of which occurred in EACA treated patients (RR 0.14, 95\% CI 0.01 to 2.51). There were no reported cases of DVT in the three remaining trials. 


\section{Pulmonary embolism}

Three trials of aprotinin versus EACA reported data for pulmonary embolism. These trials included a total of 270 patients, of whom 138 were randomised to aprotinin and 132 were randomised to EACA. Three events of pulmonary embolism were reported; two in aprotinin treated patients and one in EACA treated patients. There was no statistically significant difference between aprotinin and EACA treatment (RR $1.33,95 \%$ CI 0.10 to 18.42). Heterogeneity between these trials was not statistically significant $\left(\mathrm{Chi}^{2}\right.$ $\left.=1.45, \mathrm{df}=1, \mathrm{P}=0.23 ; \mathrm{I}^{2}=31 \%\right)$.

\section{Renal failure / dysfunction}

Two trials of aprotinin versus EACA reported data for renal failure / dysfunction. These trials included a total of 1595 patients, of whom 796 were randomised to aprotinin and 799 were randomised to EACA. Although the analysis was dominated by the data from the BART study (71.6\% weight) there was no statistically significant difference between aprotinin and EACA in the number patients experiencing renal failure / dysfunction (RR 1.33, 95\% CI 0.59 to 2.99 ). Heterogeneity between these trials was moderate $\left(\mathrm{Chi}^{2}=2.12, \mathrm{df}=1, \mathrm{P}=0.15 ; \mathrm{I}^{2}=53 \%\right)$.

\section{Hospital length of stay}

Two trials of aprotinin versus EACA reported data for hospital length of stay. These trials included a total of 1605 patients, of whom 803 were randomised to aprotinin and 802 patients were randomised to EACA. There was no statistically significant difference between aprotinin and EACA (MD -0.49 days, 95\% CI 1.74 to 0.77 days).

\section{Tranexamic acid versus epsilon aminocaproic acid}

Eight trials provided direct 'head-to-head' comparisons of TXA and EACA and reported data on the number of patients exposed to allogeneic blood transfusion. These trials included a total of 2003 patients, of whom 1000 were randomised to TXA and 1003 were randomised to EACA. There was no statistically significant difference between TXA and EACA in the rates of allogeneic blood transfusion (RR $0.97,95 \%$ CI 0.77 to 1.21 ). Heterogeneity between these trials was statistically significant $\left(\mathrm{Chi}^{2}=14.01, \mathrm{df}=\right.$ $\left.7, \mathrm{P}=0.05 ; \mathrm{I}^{2}=50 \%\right)$. All eight trials included in this analysis reported the use of a transfusion protocol to guide transfusion practice. Six of the eight trials included in this analysis involved cardiac surgery. A subgroup analysis of the data from these cardiac trials showed that the relative risk of receiving an allogeneic blood transfusion in patients treated with TXA compared to patients treated with EACA was 1.07 (95\% CI 0.79 to 1.46 ).

\section{Volume of blood transfused}

Three trials of TXA versus EACA provided data for the volume of allogeneic blood transfused in all patients. These trials included a total of 268 patients, of whom 136 were randomised to TXA and 132 were randomised to EACA. There was no statistically significant difference between TXA and EACA (MD -0.28 units, $95 \%$ CI -0.59 to 0.03 units). Heterogeneity between these trials was not statistically significant $\left(\mathrm{Chi}^{2}=0.96, \mathrm{df}=2, \mathrm{P}=0.62 ; \mathrm{I}^{2}\right.$ $=0 \%)$. Four trials of TXA versus EACA provided data for the volume of allogeneic blood transfused to those patients transfused. These trials included a total of 133 patients, of whom 59 were randomised to TXA and 74 were randomised to EACA. Again there was no statistically significant difference between TXA and EACA treatment (MD - 0.34 units, $95 \%$ CI -0.74 to 0.07 units). Heterogeneity between these trials was not statistically significant $\left(\mathrm{Chi}^{2}=0.12, \mathrm{df}=2, \mathrm{P}=0.94 ; \mathrm{I}^{2}=0 \%\right)$.

\section{Blood loss}

Six trials of TXA versus EACA involving cardiac surgery reported post-operative blood loss data. These trials included a total of 402 patients, of whom 209 were randomised to TXA and 193 were randomised to EACA. There was no difference between TXA and EACA in the volume of blood lost during the post-operative period (MD $-4.36 \mathrm{mls}$, 95\% CI -163.35 to $154.63 \mathrm{mls}$ ). Heterogeneity between these trials was statistically significant $\left(\mathrm{Chi}^{2}=33.81, \mathrm{df}\right.$ $\left.=5, \mathrm{P}<0.00001 ; \mathrm{I}^{2}=85 \%\right)$.

\section{Re-operation for bleeding}

Five trials of TXA versus EACA provided data on re-operation for bleeding. These trials included a total of 1853 patients, of whom 922 were randomised to TXA and 931 were randomised to EACA. There was no statistically significant difference between TXA and EACA (RR 1.00, 95\% CI 0.73 to 1.39). Heterogeneity between these trials was not statistically significant $\left(\mathrm{Chi}^{2}=1.81, \mathrm{df}=3, \mathrm{P}\right.$ $=0.61 ; \mathrm{I}^{2}=0 \%$ ). The data of the BART study (Fergusson 2008) dominated the results of this analysis ( $93.4 \%$ weight $)$.

\section{Mortality}

Five trials of TXA versus EACA provided mortality data. These trials included a total of 1958 patients, of whom 980 were randomised to TXA and 978 were randomised to EACA. There was no statistically significant difference between TXA and EACA (RR $0.93,95 \%$ CI 0.59 to 1.47 ). Heterogeneity between these trials was not statistically significant $\left(\mathrm{Chi}^{2}=1.43, \mathrm{df}=3, \mathrm{P}=0.70 ; \mathrm{I}^{2}\right.$ $=0 \%$ ). The data of the BART study (Fergusson 2008) dominated the results of this analysis $(86.8 \%$ weight $)$. 


\section{Myocardial infarction}

Three trials of TXA versus EACA reported data for myocardial infarction. These trials included a total of 1687 patients, of whom 840 were randomised to TXA and 847 were randomised to EACA. There was no statistically significant difference between TXA and EACA (RR $1.33,95 \%$ CI 0.80 to 2.23). Heterogeneity between these trials was not statistically significant $\left(\mathrm{Chi}^{2}=0.62, \mathrm{df}=2, \mathrm{P}\right.$ $=0.73 ; \mathrm{I}^{2}=0 \%$ ). The data of the BART study (Fergusson 2008) dominated the results of this analysis $(82.9 \%$ weight $)$.

\section{Stroke}

Three trials of TXA versus EACA reported data for stroke (cerebrovascular accident). These trials included a total of $1658 \mathrm{pa}-$ tients, of whom 820 were randomised to TXA and 838 were randomised to EACA. There was no statistically significant difference between TXA and EACA (RR 1.33, 95\% CI 0.78 to 2.29). Heterogeneity between these trials was not statistically significant $\left(\mathrm{Chi}^{2}=0.30, \mathrm{df}=1, \mathrm{P}=0.58 ; \mathrm{I}^{2}=0 \%\right)$. The data of the BART study (Fergusson 2008) provided $97.1 \%$ (weight) of the information for this analysis.

\section{Pulmonary embolism}

Three trials of TXA versus EACA reported data for pulmonary embolism. These trials included a total of 284 patients, of whom 150 were randomised to TXA and 134 were randomised to EACA. There was only one reported case of pulmonary embolism, this occurred in EACA treated patients.

\section{Renal failure / dysfunction}

Only the BART study (Fergusson 2008) provided data on renal failure / dysfunction in patients treated with either TXA or EACA. The results of the BART study showed that there was no statistically significant difference between TXA and EACA in the rates of patients experiencing renal failure / dysfunction (RR 0.98, 95\% CI 0.76 to 1.27 ).

\section{Hospital length of stay}

Only the BART study (Fergusson 2008) hospital length of stay data in patients treated with either TXA or EACA. The results of the BART study showed that there was no statistically significant difference between TXA and EACA in the length of hospital stay (MD - 0.64 days, $95 \%$ CI -1.82 to 0.54 days).

\section{Aprotinin versus either lysine analogue}

Thirty trials of aprotinin versus either TXA or EACA provided data on the number of patients exposed to allogeneic blood transfusion. These trials included a total of 5566 patients, of whom
2407 were randomised to aprotinin and 3159 were randomised to a lysine analogue. The use of aprotinin reduced the need for allogeneic blood transfusion by a relative 10\% (RR 0.90, 95\% CI 0.81 to 0.99$)$. Heterogeneity between these trials was statistically significant $\left(\mathrm{Chi}^{2}=70.06, \mathrm{df}=29\left(\mathrm{P}<0.0001 ; \mathrm{I}^{2}=59 \%\right)\right.$.

In view of the importance of the data on death and myocardial infarction we compared aprotinin with either tranexamic acid or aminocaproic acid. There were nineteen trials that reported on mortality. Of 2115 subjects randomised to aprotinin 71 died, compared with 85 of 3012 randomised to either lysine analogue. The increase in mortality with aprotinin was statistically significant (RR 1.39 , 95\% CI 1.02 to 1.89 ). Seventy percent of the statistical weight came from the Bart trial (Fergusson 2008). In contrast, there was no significant increase in the risk of myocardial infarction with aprotinin compared with either lysine analogue (RR 1.11, 95\% CI 0.82 to 1.50 ).

\section{Impact of trial quality}

\section{Aprotinin}

Of the 108 trials of aprotinin that provided data on the number of patients exposed to allogeneic blood transfusion, 33 trials were assessed as having adequate allocation concealment of treatment schedule. For these 33 trials the use of aprotinin reduced the rate of allogeneic blood transfusion by a relative 36\% (RR 0.64, 95\% CI 0.53 to 0.79$)$. Heterogeneity between these trials was statistically significant $\left(\mathrm{Chi}^{2}=665.70, \mathrm{df}=32, \mathrm{P}<0.00001 ; \mathrm{I}^{2}=95 \%\right)$. In the 63 trials where there was uncertainty regarding the method of allocation concealment (Unclear), the use of aprotinin reduced the rate of allogeneic blood transfusion by a relative $31 \%$ (RR $0.69,95 \%$ CI 0.64 to 0.75 ). Heterogeneity between these trials was statistically significant $\left(\mathrm{Chi}^{2}=179.31, \mathrm{df}=62, \mathrm{P}<0.00001\right.$; $\left.\mathrm{I}^{2}=65 \%\right)$. In the remaining 12 trials where the method of allocation concealment was assessed as being inadequate $(\mathrm{No})$, the use of aprotinin reduced the rate of allogeneic blood transfusion by a relative $37 \%$ (RR $0.63,95 \%$ CI 0.54 to 0.75 ). Heterogeneity between these trials was not statistically significant $\left(\mathrm{Chi}^{2}=15.50, \mathrm{df}\right.$ $\left.=11, \mathrm{P}=0.16 ; \mathrm{I}^{2}=29 \%\right)$. These data indicate the effects of aprotinin were not significantly greater in those studies that reported inferior techniques for concealing the randomisation sequence.

\section{Tranexamic acid}

Of the 65 trials of TXA that provided data on the number of patients exposed to allogeneic blood transfusion, 28 were assessed as having adequate allocation concealment of treatment schedule. For these 28 trials the use of TXA reduced the rate of allogeneic blood transfusion by a relative $41 \%$ (RR $0.59,95 \%$ CI 0.51 to 0.69). Heterogeneity between these trials was statistically significant $\left(\mathrm{Chi}^{2}=41.35, \mathrm{df}=27, \mathrm{P}=0.04 ; \mathrm{I}^{2}=35 \%\right)$. In the 24 trials where there was uncertainty regarding the method of allocation 
concealment (Unclear), the use of TXA reduced the rate of allogeneic blood transfusion by a relative $47 \%$ (RR $0.53,95 \%$ CI 0.37 to 0.76 ). Heterogeneity between these trials was statistically significant $\left(\mathrm{Chi}^{2}=209.62, \mathrm{df}=23, \mathrm{P}<0.00001 ; \mathrm{I}^{2}=89 \%\right)$. In the remaining 13 trials where the method of allocation concealment was assessed as being inadequate $(\mathrm{No})$, the use of TXA reduced the rate of allogeneic blood transfusion by a relative $27 \%$ (RR $0.73,95 \%$ CI 0.62 to 0.86 ). Heterogeneity between these trials was not statistically significant $\left(\mathrm{Chi}^{2}=16.38, \mathrm{df}=11(\mathrm{P}=0.13)\right.$, $\left.\mathrm{I}^{2}=33 \%\right)$

\section{Epsilon aminocaproic acid}

Of the 16 trials that provided data on the number of patients exposed to allogeneic blood transfusion, five were assessed as having adequate allocation concealment of treatment schedule. For these trials the use of EACA did not statistically significantly reduce the rate of allogeneic blood transfusion (RR $0.82,95 \%$ CI 0.58 to 1.16). Heterogeneity between trials was statistically significant $\left(\mathrm{Chi}^{2}=14.35, \mathrm{df}=4, \mathrm{P}=0.006 ; \mathrm{I}^{2}=72 \%\right)$. In the nine trials where there was uncertainty regarding the method of allocation concealment (Unclear), the use of EACA did not statistically significantly reduce the rate of allogeneic blood transfusion (RR $0.68,95 \% \mathrm{CI}$ 0.46 to 1.03$)$. Heterogeneity between trials was not statistically significant $\left(\mathrm{Chi}^{2}=12.54, \mathrm{df}=8, \mathrm{P}=0.13 ; \mathrm{I}^{2}=36 \%\right)$. In the remaining two trials where the method of allocation concealment was assessed as being inadequate (No), the use of EACA did not statistically significantly reduce the rate of allogeneic blood transfusion (RR $0.93,95 \%$ CI 0.81 to 1.08 ). Heterogeneity between these trials was not statistically significant $\left(\mathrm{Chi}^{2}=0.13, \mathrm{df}=1, \mathrm{P}\right.$ $\left.=0.72 ; \mathrm{I}^{2}=0 \%\right)$.

\section{Aprotinin versus tranexamic acid}

Of the 21 trials that compared aprotinin to TXA, four were assessed as having adequate allocation concealment of treatment schedule. For these trials the RR of receiving an allogeneic blood transfusion in those patients treated with aprotinin compared to those patients treated with TXA was 0.80 (95\% CI 0.69 to 0.92 ). Heterogeneity between these trials was not statistically significant $\left(\mathrm{Chi}^{2}=3.60, \mathrm{df}=3, \mathrm{P}=0.31 ; \mathrm{I}^{2}=17 \%\right)$. In the 13 trials where there was uncertainty regarding the method of allocation concealment (Unclear), the RR of receiving an allogeneic blood transfusion was statistically significantly different between aprotinin and TXA (RR $0.97,95 \%$ CI 0.88 to 1.07). Heterogeneity between these trials was not statistically significant $\left(\mathrm{Chi}^{2}=19.25, \mathrm{df}=12\right.$, $\left.\mathrm{P}=0.08 ; \mathrm{I}^{2}=38 \%\right)$. In the remaining four trials where the method of allocation concealment was assessed as being inadequate ( $\mathrm{No})$, the RR of receiving an allogeneic blood transfusion was not statistically significantly different between aprotinin treated patients and TXA treated patients (RR $0.93,95 \%$ CI 0.62 to 1.39 ). Heterogeneity between these trials was statistically significant $\left(\mathrm{Chi}^{2}=\right.$ $\left.10.29, \mathrm{df}=3, \mathrm{P}=0.02 ; \mathrm{I}^{2}=71 \%\right)$.

\section{Aprotinin versus epsilon aminocaproic acid}

Of the 12 trials of aprotinin versus EACA that were assessed for methodological quality, three were assessed as having adequate allocation concealment. For these trials the RR of receiving an allogeneic blood transfusion in those patients treated with aprotinin compared to those patients treated with EACA was 0.86 (95\%CI 0.71 to 1.05$)$. Heterogeneity between these trials was not statistically significant $\left(\mathrm{Chi}^{2}=2.75, \mathrm{df}=2, \mathrm{P}=0.25 ; \mathrm{I}^{2}=27 \%\right)$. For eight trials there was uncertainty regarding the method of allocation concealment (Unclear), the RR of receiving an allogeneic blood transfusion was not statistically significantly different between aprotinin and EACA (RR $0.76,95 \%$ CI 0.58 to 0.99 ). Heterogeneity between these trials was not statistically significant $\left(\mathrm{Chi}^{2}=6.19, \mathrm{df}=7, \mathrm{P}=0.52 ; \mathrm{I}^{2}=0 \%\right)$. For one trial the method of allocation concealment was assessed as being inadequate $(\mathrm{No})$.

\section{Tranexamic acid versus epsilon aminocaproic acid}

Of the eight trials of TXA versus EACA that were assessed for methodological quality, one trial was assessed as having adequate allocation concealment (Yes). For five trials there was uncertainty regarding the method of allocation concealment (Unclear), and for two trials the method of allocation concealment was assessed as being inadequate (No). There were too few trials to formally assess the impact that methodological quality had on treatment effect.

\section{Aprotinin versus lysine analogues (TXA and EACA combined)}

Of the 29 trials that compared aprotinin to the lysine analogues, six were assessed as having adequate allocation concealment of treatment schedule. For these trials the RR of receiving an allogeneic blood transfusion in those patients treated with aprotinin compared to those patients treated with a lysine analogue was 0.82 (95\% CI 0.71 to 0.95 ). Heterogeneity between these trials was not statistically significant $\left(\mathrm{Chi}^{2}=6.44, \mathrm{df}=3, \mathrm{P}=0.27 ; \mathrm{I}^{2}=\right.$ $22 \%)$. In the 18 trials where there was uncertainty regarding the method of allocation concealment (Unclear), the RR of receiving an allogeneic blood transfusion was statistically significantly different between aprotinin and the lysine analogues (RR 0.95, 95\% CI 0.86 to 1.04$)$. Heterogeneity between these trials was not statistically significant $\left(\mathrm{Chi}^{2}=26.77, \mathrm{df}=18, \mathrm{P}=0.08 ; \mathrm{I}^{2}=33 \%\right)$. In the remaining five trials where the method of allocation concealment was assessed as being inadequate $(\mathrm{No})$, the $\mathrm{RR}$ of receiving an allogeneic blood transfusion was not statistically significantly different between aprotinin treated patients and lysine analogue treated patients (RR $0.92,95 \%$ CI 0.67 to 1.28 ). Heterogeneity between these trials was statistically significant $\left(\mathrm{Chi}^{2}=10.34, \mathrm{df}\right.$ $\left.=4, \mathrm{P}=0.04 ; \mathrm{I}^{2}=61 \%\right)$. 


\section{DISCUSSION}

This systematic review of the three anti-fibrinolytic drugs, aprotinin, tranexamic acid (TXA), and epsilon aminocaproic acid (EACA), includes a total of 252 randomised controlled trials (RCTs), which recruited over 25,000 participants. The previous versions of this Cochrane review (Henry 1999; Henry 2007), included a total of 89 trials with 9876 participants and 211 trials with 20,781 participants, respectively. Although the three drugs differ somewhat in their modes of action, the results of this review confirm and strengthen previous findings that they reduce surgical blood loss and exposure to allogeneic red blood cell transfusion to a degree that is both statistically and clinically significant. Importantly, the risk of re-operation necessitated by recurrent or continued bleeding after cardiac surgery was lowered by treatment with aprotinin and a clear trend was also seen with TXA for that outcome. These findings are not new, but this updated review provides additional information regarding two significant questions: how do the drugs compare with each other and to what extent are the clinical benefits offset by adverse effects, in particular vascular occlusion? In addressing these questions the updated review includes data from 49 active comparisons between aprotinin and the lysine analogues, compared with 29 in the previous review (Henry 2007). This updated review also adds to the information about vascular events - capturing 54 more episodes of myocardial infarction than the earlier version.

The analyses of active comparator trials (direct head-to-head comparisons) indicate that aprotinin was slightly more effective than TXA in reducing the need for red cell transfusion in patients undergoing cardiac surgery (RR $0.87,95 \%$ CI 0.76 to 0.99 ). However, the results of the head-to-head comparison showed that aprotinin was marginally more effective than TXA in reducing postoperative blood loss. In the context of cardiac surgery, aprotinin appeared to be more effective than EACA in reducing the need for red cell transfusion and post-operative blood loss. Our confidence in ascribing an advantage to aprotinin needs to be moderated by evidence of possible publication bias and uncertainty over the comparative dose response relationships.

Mortality appeared to be unaffected by treatment with any of the drugs and there was no evidence that aprotinin, or the lysine analogues, increased the risks of myocardial infarction or other serious thrombosis. These latter results conflict with the findings of recently published observational studies by Mangano et al ( Mangano 2006; Mangano 2007) and Karkouti et al (Karkouti 2006), which showed that the use of aprotinin in cardiac surgery was associated with an increase in the incidence of renal failure, myocardial infarction, and all-cause mortality (over five years).

\section{Measures of efficacy: blood loss and need for transfusion}

Aprotinin appeared to be the most efficacious of the three drugs in reducing perioperative blood loss, the confidence interval (CI) for the average reduction in blood loss with aprotinin seen in placebo/ inactive controlled trials does not overlap with those of either TXA or EACA. This conclusion was supported by the sparser literature from active comparator trials, which found that aprotinin reduced post-operative blood loss to a greater extent than TXA; a similar result was seen in the comparison of aprotinin and EACA. It was notable that the apparent differences between the drugs were only seen in the context of cardiac surgery. There was no advantage of aprotinin over TXA when the drugs were used as an adjunct to orthopaedic procedures.

The three drugs were effective in reducing the proportions of patients who required transfusion with red blood cells. The pooled relative risk (RR) values from placebo/inactive controlled trials were similar. When considering these results it may be relevant that the baseline rates of transfusion differed considerably between the trials of aprotinin and the trials of TXA and EACA. The control-arms of the aprotinin trials had an average transfusion rate of $62 \%$, compared with $44 \%$ for the control-arm of the TXA trials and $54 \%$ for the control-arms of the EACA trials. A possible explanation for this difference is that aprotinin has been studied more extensively and for a longer period of time than TXA and EACA. It is generally accepted that improvements in surgical technique, advancements in cardiopulmonary bypass technology, the introduction of auto-transfusion procedures and acceptance of lower transfusion thresholds have been responsible for a reduction in the rates of perioperative blood transfusion over time. This time dependant trend was observed in the trials of aprotinin in cardiac surgery. It is also possible that trials of aprotinin included more high-risk patients than trials of the lysine analogues. Such highrisk patients tend to have a greater propensity for blood loss and hence transfusion. Thus, comparisons between drugs based on the placebo/inactive controlled trials of anti-fibrinolytic drugs may be confounded at trial level by differences in patient populations. Publication bias is a further consideration when considering the placebo/inactive controlled studies of these drugs. As in the previous versions of this review, an examination of the generated funnel plots suggested a degree of publication bias (favouring active treatment) in the aprotinin trials (Figure 3), and a similar pattern was also seen with the trials of TXA (Figure 4) and EACA (Figure 5). 
Figure 3. Funnel plot of comparison: I Aprotinin versus Control (Blood Transfusion \& Blood Loss), outcome: I.I No. Exposed to Allogeneic Blood.

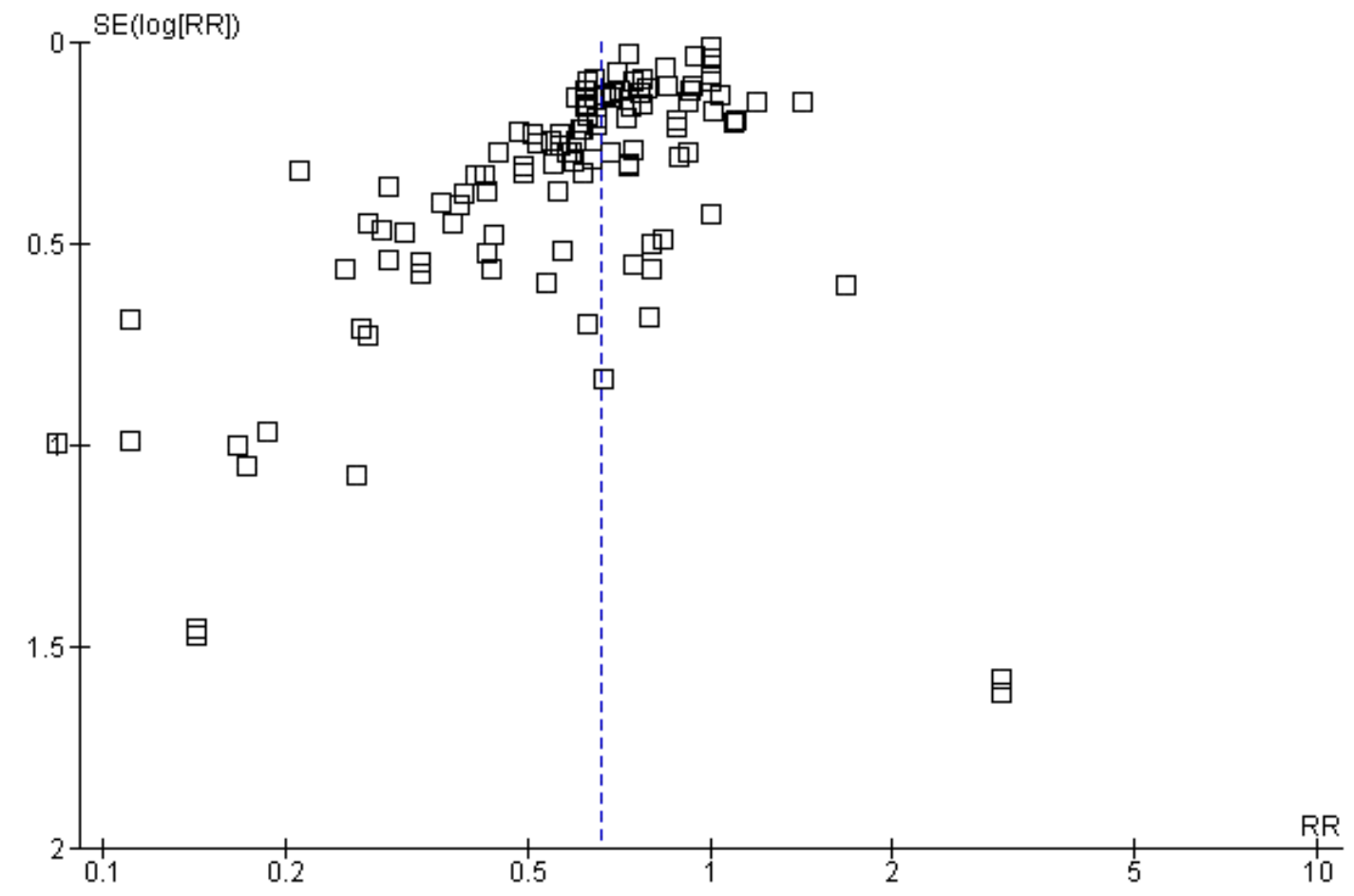


Figure 4. Funnel plot of comparison: 2 Tranexamic Acid versus Control (Blood Transfusion \& Blood Loss), outcome: 2.I No. Exposed to Allogeneic Blood.

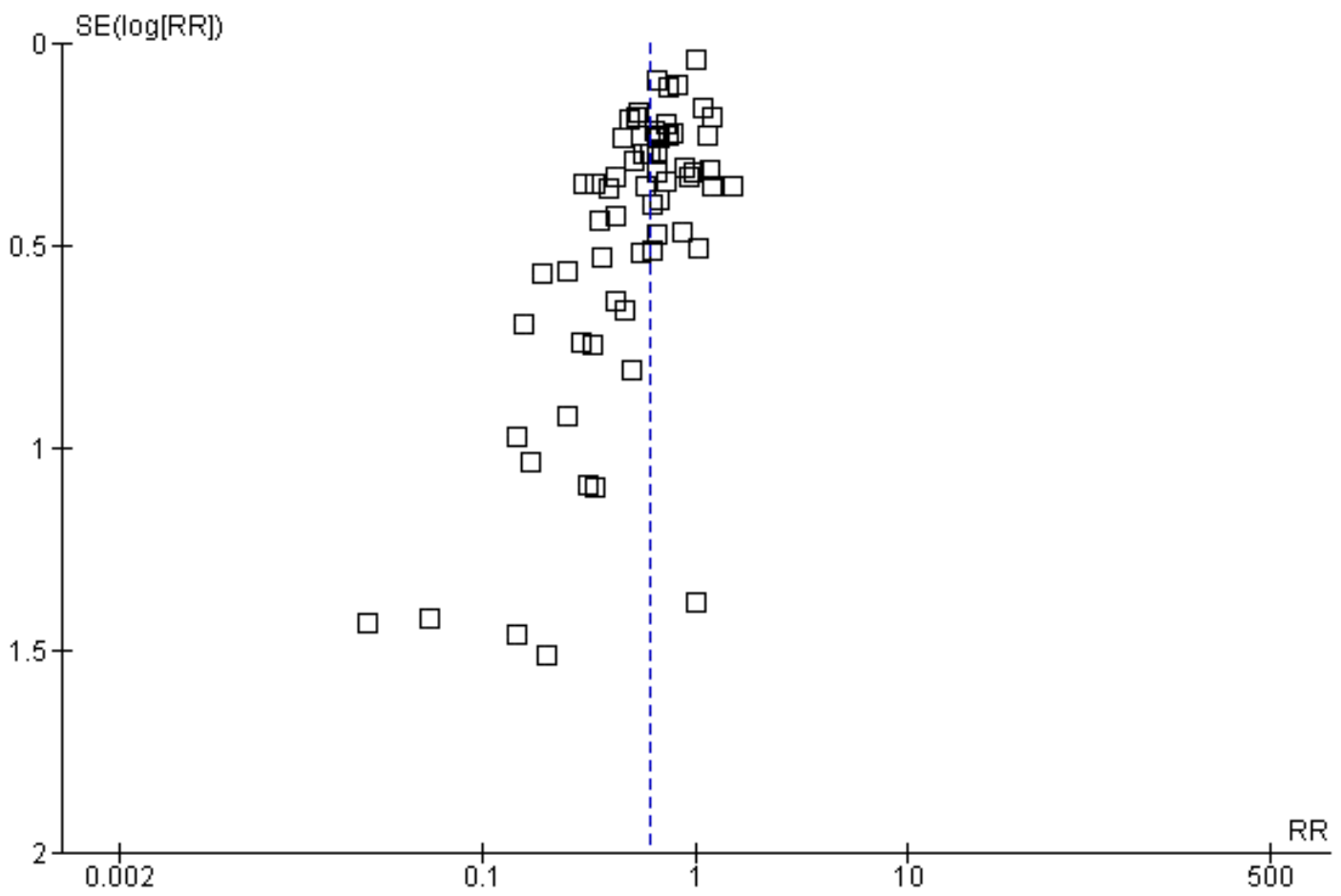


Figure 5. Funnel plot of comparison: 3 Epsilon Aminocaproic Acid versus Control (Blood Transfusion \& Blood Loss), outcome: 3.I No. Exposed to Allogeneic Blood.

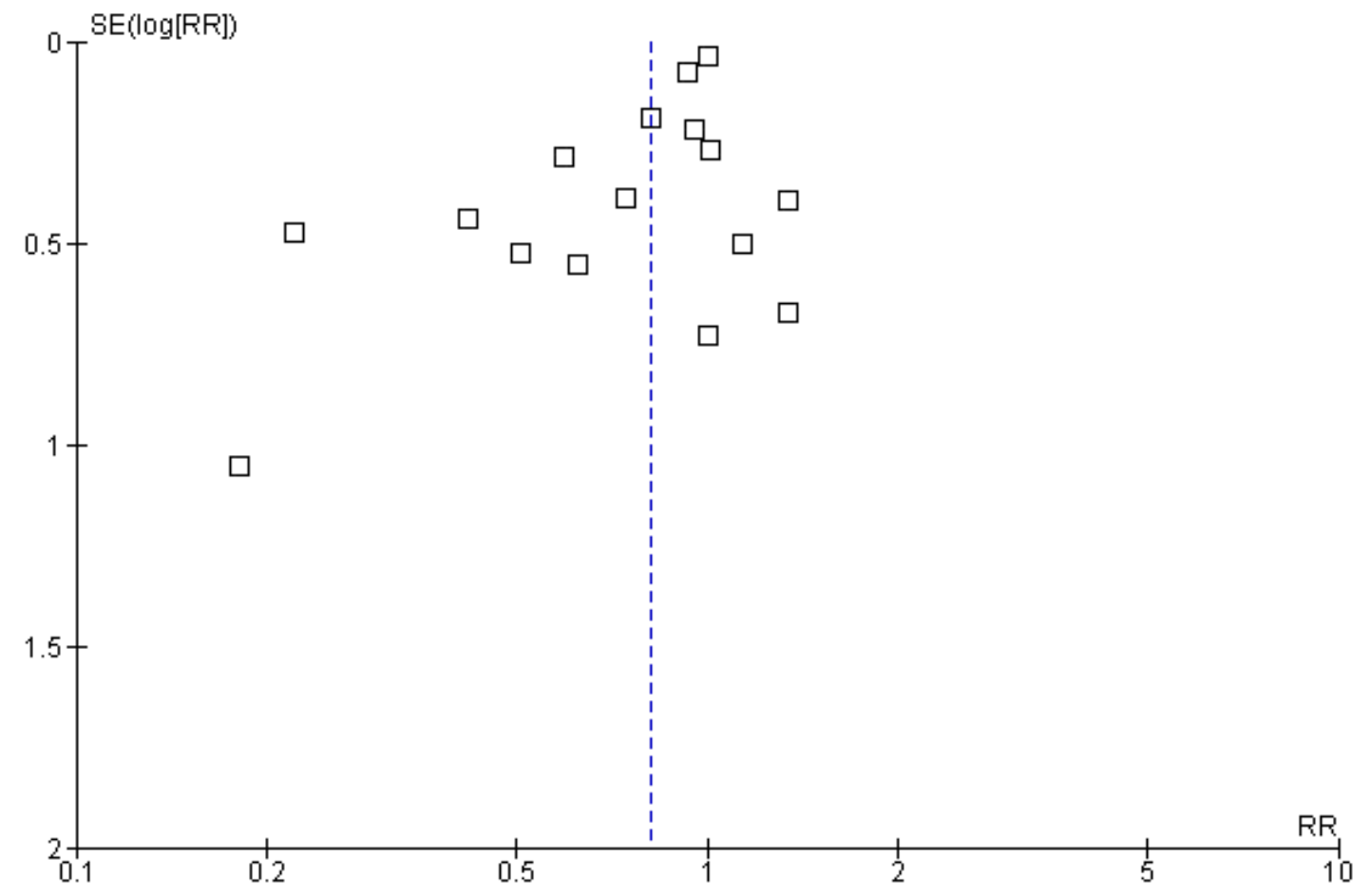

In the case of cardiac surgery, when aprotinin was included in pairwise comparisons of blood transfusion requirements with TXA and EACA a small trend in favour of aprotinin was seen in each comparison. When we pooled data on blood transfusions for head-to-head comparisons of aprotinin with either of the lysine analogues the advantage of aprotinin was borderline significant - pooled RR 0.90 (95\% CI 0.81 to 0.99 ). We have previously published a meta-analysis of the comparative trials of aprotinin and lysine analogues in cardiac surgery (Carless 2005). In that study we used a Bayesian meta-analytic approach to determine if TXA and EACA could be considered equivalent (non-inferior) to aprotinin in reducing the rate of allogeneic blood transfusion. Although hampered by the small number and size of the trials, our results showed that for blood transfusion, using a non-inferiority boundary of $20 \%$, the posterior probability that TXA is equivalent to aprotinin was 0.82 .

In other words, the updated analyses make us less sure about the equivalence of the lysine analogues and aprotinin when used to re- duce the need for red cell transfusion in cardiac surgery. But these conclusions do not take account of two additional factors, dose effects and the possibility of publication bias. As the funnel plots generated from the head-to-head trials of aprotinin and the lysine analogues show there appears to be a gap that should be occupied by small trials favouring the latter drugs (Figure 6; Figure 7; Figure 8; Figure 9). The data are sparse but if this represents non-publication of such trials it could explain some of the apparent advantages of aprotinin seen in the overall analyses. This suggestion was made originally by Beattie 2006 and our updated analysis supports their conclusions. To find evidence of publication bias in the placebocontrolled trials of these drugs is perhaps not surprising, but we thought it less likely to affect the active comparison studies. The commercial interests in the role of aprotinin (an expensive and popular drug) as an adjunct to cardiac surgery may lie behind this. However, it should be noted that none of the reports of the comparative trials mentions commercial sponsorship. 
Figure 6. Funnel plot of comparison: 4 Aprotinin versus Tranexamic Acid (Blood Transfusion \& Blood Loss), outcome: 4.I No. Exposed to Allogeneic Blood.

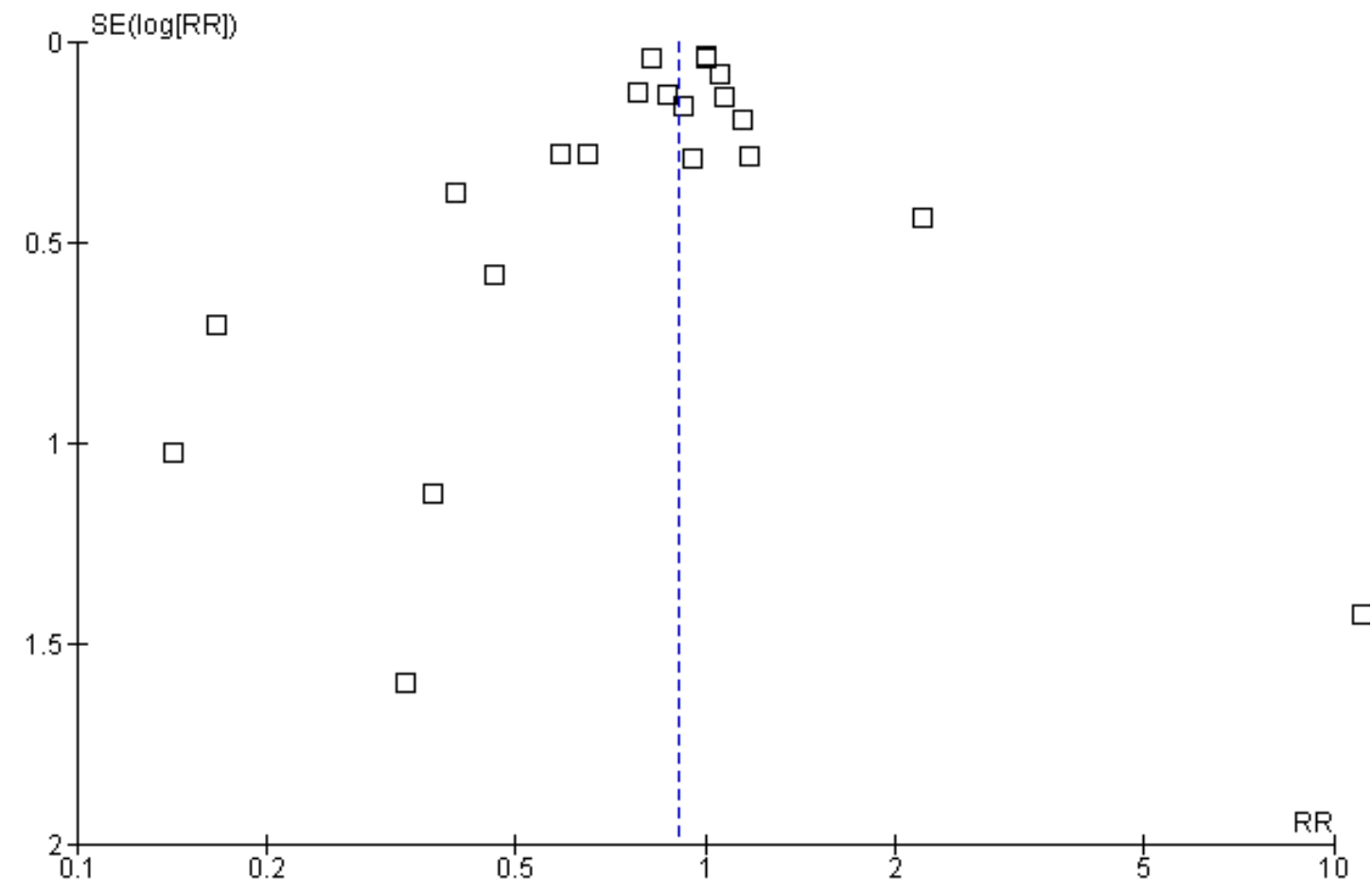


Figure 7. Funnel plot of comparison: 5 Aprotinin versus Epsilon Aminocaproic Acid (Blood Transfusion \& Blood Loss), outcome: 5.I No. Exposed to Allogeneic Blood.

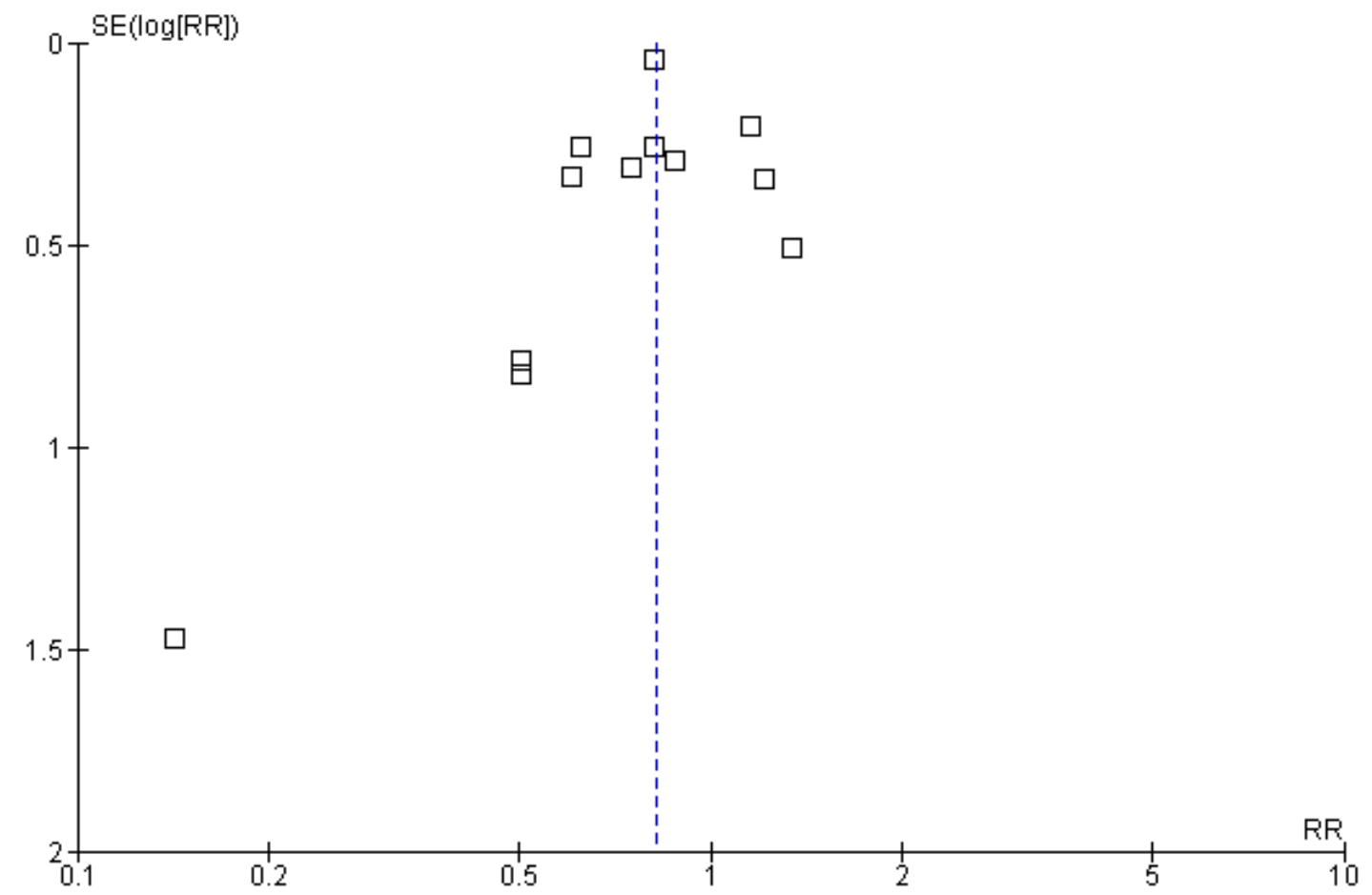


Figure 8. Funnel plot of comparison: 6 Tranexamic Acid versus Epsilon Aminocaproic Acid (Blood Transfusion \& Blood Loss), outcome: 6.I No. Exposed to Allogeneic Blood.

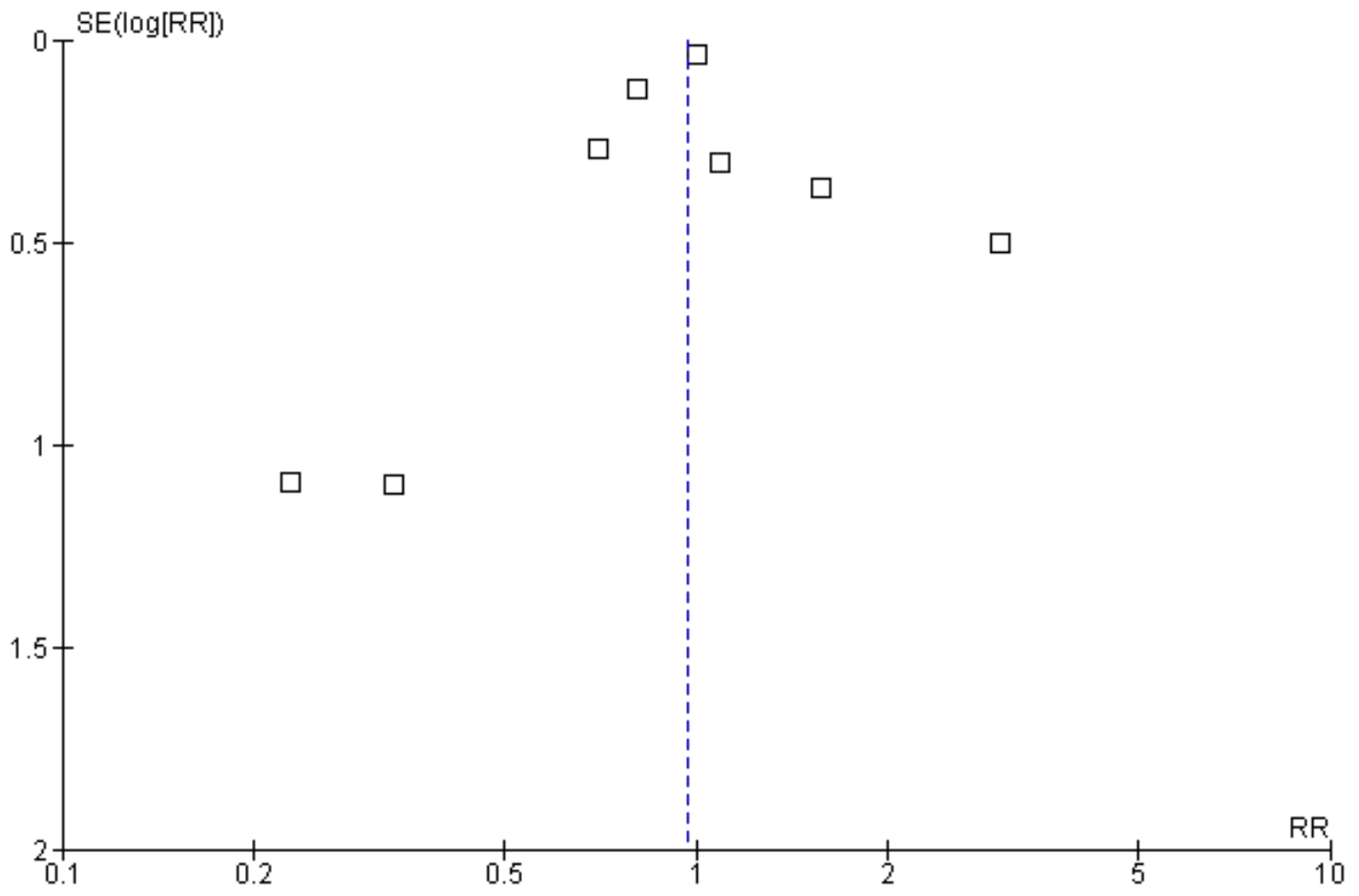


Figure 9. Funnel plot of comparison: 7 Aprotinin versus Lysine Analogues (Blood Transfusion), outcome: 7.I No. Exposed to Allogeneic Blood.

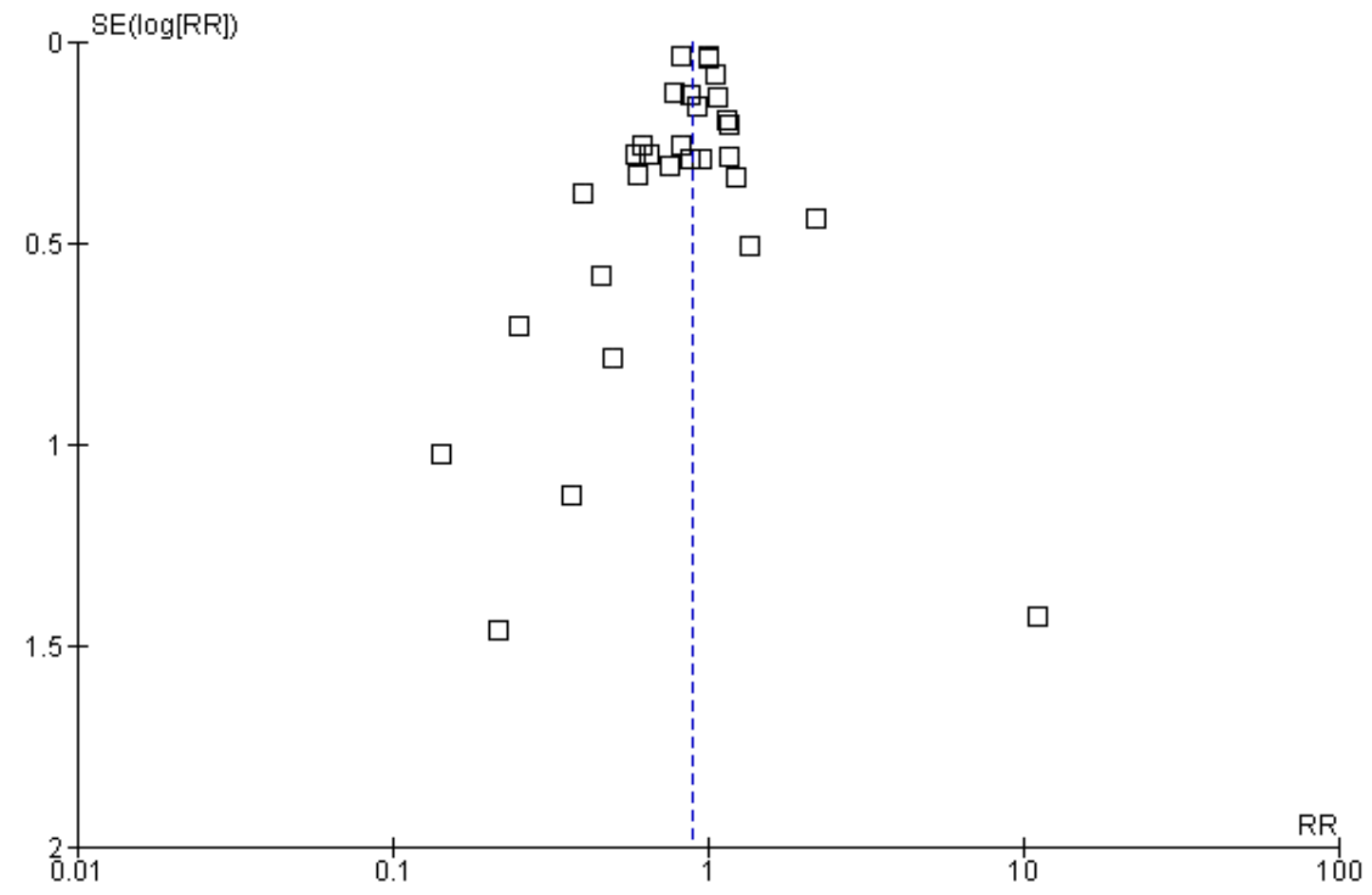

In making comparisons between the average efficacy of the drugs it is important to consider the possible role of dose as a treatment effect modifier. When the pooled RR values for aprotinin were stratified, both low and high doses reduced the incidence of allogeneic red cell transfusion by around 35\%. This was greater than the effect of aprotinin when given only as a priming dose - a RR reduction of $17 \%$. So extending duration of treatment beyond the priming dose may be important. TXA in doses of 2 to $10 \mathrm{~g}$ and in doses below $2 \mathrm{~g}$ had a similar effect, reducing allogeneic red cell transfusion by around $30 \%$. There were insufficient data to explore dose effects in the head-to-head trials of aprotinin and TXA. Analyses of the comparative trials of aprotinin and the lysine analogues in orthopaedic surgery were hampered by sparse data. When the results of placebo/inactive controlled trials were combined TXA appeared to be as effective as aprotinin in reducing the number of patients exposed to allogeneic blood transfusion. Conclusions about the relative efficacy of EACA and aprotinin in orthopaedic surgery were hampered by the small number of trials. Of the fourteen trials of aprotinin eight $(57.1 \%)$ were published between 2000 and 2006. In comparison, of the 21 trials that compared TXA to placebo-control 16 (76.2\%) were published in this time period. As with cardiac surgery, conclusions about the relative efficacy of the drugs may be confounded by changes in surgical technique and transfusion practices that have occurred over time. However, as with the data on blood loss, the apparent advantage of aprotinin over the lysine analogues on the need for blood transfusion observed in cardiac surgery was not seen in orthopaedic surgery.

The analyses of the volumes of red cells transfused were difficult to interpret because of incomplete data in many trials. When all randomised subjects were included in the analyses (which included some who did not receive a transfusion) the average volumes of blood transfused were reduced modestly by all three drugs. When the analysis was confined to individuals who received red cell transfusions the reductions in volume were less marked and a statistically significant treatment effect was observed only for aprotinin. There were insufficient data from head-to-head trials to assess the comparative effectiveness of the three drugs in reducing the volumes of blood transfused.

\section{Clinical significance of avoiding red cell transfusion}

The true value of avoiding allogeneic red cell transfusion remains unclear (Vamvakas 2001). Patients who are concerned about the risks of contracting illness as a result of blood transfusion (or ob- 
ject to transfusion on religious grounds) will be more interested in avoiding it completely, rather than just reducing the volume of transfused blood. The importance of avoiding the need for transfusion depends on the probability of avoiding disease transmission or other adverse effects, in particular immunomodulation thought to be due to transfused white blood cells (Blumberg 1997; Vamvakas 2001). The significance of the latter remains although a number of countries now perform leukocyte depletion, either selectively, or universally, before administering red cell transfusions, despite a lack of convincing evidence that this provides clinical benefits (Vamvakas 2004). The rate of transmission of HIV or viral hepatitis in most developed countries is very low, because of the quality of screening of donated blood (Coyle 1999; Whyte 1997). These broad assumptions do not apply equally in developing countries. Allogeneic red cell transfusion is administered frequently and blood products may be inadequately screened; the prevalence of viral pathogens amongst donors is high (Kimball 1995; McFarland 1997). In these settings there may be much greater clinical value in a range of interventions that diminish or avoid the need for allogeneic blood. However, the costs of the drugs reviewed here are likely to be prohibitive in developing countries.

Most of the red cell transfusion data reviewed here have been collected in the context of major cardiac surgery, where blood loss may be substantial. The applicability of the efficacy data to clinical settings where blood loss is minor is questionable. Anti-fibrinolytic drugs may be used alongside other interventions designed to minimise the need for allogeneic red cell transfusion. A variety of techniques have been employed; most involve the re-infusion of autologous blood either from pre-operative deposit, acute normovolemic haemodilution, or cell salvage. The latter, in most instances involves the re-infusion of red blood cells that have been shed into the operative field. The evidence on the efficacy and safety of these techniques was reviewed extensively by the Interna- tional Study on Perioperative Transfusion (ISPOT) (Bryson 1998; Forgie 1998; Huet 1999). The literature on re-infusion techniques is generally viewed as being of indifferent quality, because of inadequate randomisation and lack of blinding of outcomes assessment. However, these techniques probably have a modest blood sparing effect. Significantly, the efficacy of autologous re-infusion techniques appears lower when they are used in the context of a rigorous transfusion protocol. This and the growing evidence on the efficacy of transfusion triggers indicates that a more conservative approach to blood transfusion decisions is desirable (Carson 1998; Hebert 1999). This conservative approach, combined with the selective use of anti-fibrinolytic drugs, may offer the best approach for managing the transfusion requirements of participants in high-risk settings such as cardiac surgery.

\section{Other measures of efficacy: need for re- operation due to bleeding}

If the significance of avoiding red cell transfusion is unclear the importance of avoiding re-operation is not. Going back to theatre because of continued or recurrent bleeding is a serious development after cardiac surgery and any reduction in the incidence of this event is clinically significant (O'Brien 2002). The updated metaanalysis confirmed that aprotinin reduces the rate of re-operation due to bleeding by about half. This translates into an absolute risk reduction of $2 \%$ and a number needed-to-treat of 50 (95\% CI 33 to 100). Similar trends were seen with TXA and EACA, but the data were sparse and the differences failed to reach statistical significance. We did not see evidence of publication bias in the data relating to re-operation rates (Figure 10). When aprotinin was compared directly with TXA in head-to-head comparative trials the analysis suggested that aprotinin reduced re-operations by $31 \%$. 
Figure 10. Funnel plot of comparison: 7 Adverse Events and Other Outcomes (Active versus Control), outcome: 7.I Re-operation for bleeding.

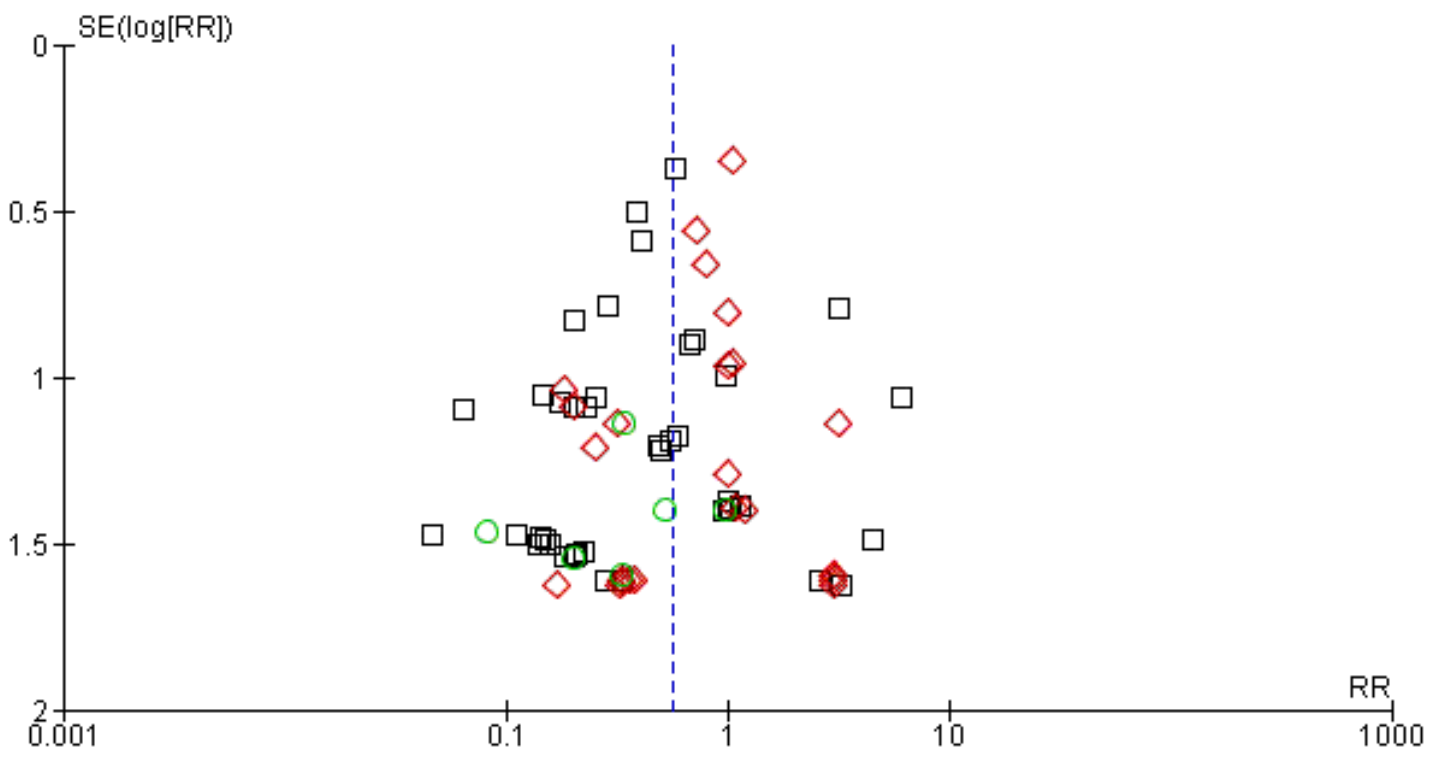

Subgroups

Aprotinin versus Control

Tranexamic Acid versus Control

Epsilon Aminocaproic Acid versus Control

\section{Effects of treatment on all cause mortality}

Regardless of the type of surgery, when aprotinin was compared with no treatment there was no apparent effect on all-cause mortality (RR $0.81,95 \%$ CI 0.63 to 1.06). In the subset of cardiac surgery trials the result was similar: RR 0.84 (95\% CI 0.64 to 1.10). Likewise, when TXA was compared to no treatment the effect on mortality rate in cardiac surgery was not statistically significant and the CI was fairly wide (RR $0.60,95 \%$ CI 0.33 to 1.10 ). In head to head comparisons there was a trend to higher mortality with aprotinin than either tranexamic acid or aminocaproic acid but the analyses were constrained by the relatively small numbers of outcomes. As there were no qualitative differences between tranexamic acid and aminocaproic acid, and any quantitative differences between these drugs were small, we compared aprotinin with either lysine analogue for the outcomes of mortality and myocardial infarction. The risk of death was higher with aprotinin than with either lysine analogue, although this result was very dependent on the results of the BART trial (Fergusson 2008). There was no significant increase in the risk of myocardial infarction that could explain the higher mortality and indeed there were no other outcome analyses from the head to head trials that could provide an explanation. It is also possible that the difference was due to a benefit of the lysine analogues rather than an adverse effect of aprotinin. In any event this distinction is academic as aprotinin has been withdrawn from world markets and the lysine analogues appear almost equally effective in reducing the need for transfusion with allogeneic blood.

\section{Adverse events and other outcomes}

Neither aprotinin nor the lysine analogues appeared to increase the risk of myocardial infarction. In each case the pooled relative risk was close to one. Most data have been collected for aprotinin, which is more often used in cardiac surgery that the lysine analogues. This probably explains the higher rates of myocardial infarction in the placebo-treated subjects in the aprotinin trials (4.5\%) than the TXA trials $(2.3 \%)$. Similarly, the risk of stroke was not increased by any of these drugs; nor was there any apparent increase in the risk of developing other thrombotic events (deep vein thrombosis, pulmonary embolism, 'other thrombosis').

Data aggregated from 28 randomised trials of aprotinin and nine trials of TXA showed that neither drug increased the risk of renal dysfunction compared to control. Although the event rate was slightly higher in aprotinin-treated patients compared to the con- 
trol group (2.4\% versus $1.5 \%)$ the difference was not statistically significant.

\section{Potential sources of bias in this review}

In our review we found a large number of small trials. These continue to be published in the literature, even though individually they contribute very little additional information. In the case of aprotinin, redundancy in terms of new information has long since been reached and there is no justification for continuing to perform placebo-controlled trials. Future investigation should involve large trials of the relative efficacy and safety of the different drugs ( Hebert 2005). The small size of most of the existing trials raises concerns about the effects of publication bias. The funnel plot of the aprotinin trials reveals possible evidence of this - in the form of a 'missing' population of small negative trials (Figure 3).

The main study outcome used in these trials was a practice variable - the decision to transfuse a patient with allogeneic red cells. Although this requires a degree of subjectivity on the part of clinicians it is probably not a major source of bias in this meta-analysis as around $70 \%$ of the trials were assessed as being double-blind, involving the use of an identical placebo.

\section{Sources of heterogeneity}

Substantial heterogeneity in trial outcomes was seen. This was seen in the case of aprotinin for blood loss and blood transfusion outcomes. However, it was not apparent in the analyses of more significant clinical outcomes, such as re-operation, myocardial infarction and death. It is therefore possible that the subjective nature of the intermediate outcomes, which require judgement about the degree of blood loss, and the need for transfusion, contributed to the between study heterogeneity. Despite this heterogeneity we have little doubt about the existence of a treatment benefit with these drugs. The variation for blood transfusion variables was in terms of the size, not the direction, of effect.

We considered a number of other factors that might explain variation in the size of the treatment effect for blood loss and rates of transfusion. In the case of transfusion, we stratified the data by the clinical setting, operation type, the concomitant use of clinical transfusion thresholds (transfusion triggers), and trial methodological quality. In the case of blood loss, we stratified the data by the type surgery performed and the period in which blood loss was assessed (that is, intra-operative and/or post-operative blood loss). Basically, none of these provided an adequate explanation for the degree of heterogeneity seen in these studies. Although effect size varied somewhat with dose, considerable heterogeneity was seen within dose strata. Likewise, there was substantial heterogeneity within the trials of aprotinin in cardiac surgery (that is, for intraand-post-operative blood loss, and the rates of transfusion). For the rates of exposure to allogeneic blood transfusion the adequacy of concealment of treatment allocation was associated with a small variation in treatment effect size, but once again there was hetero- geneity within the different strata of methodological quality.

\section{How do the results compare with the observational studies?}

The most controversial aspect of this review is the lack of evidence of an increase in the risks of myocardial infarction, stroke, renal dysfunction and death with aprotinin when compared with no treatment. This is in keeping with previous published meta-analyses of the randomised controlled trials of anti-fibrinolytic drugs. In the case of aprotinin this review includes $77 \%$ more myocardial infarctions, but only $7 \%$ more deaths, than the previous version of this review. The updated data-sets comparing aprotinin with no treatment conflict with those from four recent observational studies (Karkouti 2006; Mangano 2006; Mangano 2007; Schneeweiss 2008). Mangano and colleagues (2007) showed that during five years of follow-up aprotinin-treated patients had a death rate around 1.6 times higher than that of the untreated control group. The adjusted hazard ratio (HR) for all-cause mortality was 1.48 (95\% confidence interval 1.19 to 1.85 ). This study generated considerable scientific debate with calls for the use of aprotinin in cardiac surgery to be abandoned. In 2008 a large pharmaco-epidemiological study by Schneeweiss 2008 confirmed the increased risk of death with aprotinin. These investigators studied the use of aprotinin (33,517 patients) or aminocaproic acid (44,682 patients) on the day coronary bypass surgery was performed. In this non-randomised study they found that 1512 of the 33,517 aprotinin recipients (4.5\%) and 1101 of the 44,682 aminocaproic acid recipients (2.5\%) died. After adjustment, the estimated risk of death was $64 \%$ higher in the aprotinin group than in the aminocaproic acid group (relative risk, 1.64; $95 \%$ confidence interval [CI], 1.50 to 1.78). This difference remained statistically significant after a range of analytical procedures including a propensity score matched analysis and an instrumental variable analysis.

The first large observational study to find and adverse effect of aprotinin (Mangano 2006, Mangano 2007) was criticized on several grounds, including the fact that it was based on a multi-centre patient registry, not a true population based cohort, that there were important differences between centres and that a range of selection biases may have influenced the between-drug comparisons. These arguments will not be repeated here as full details are available in the relevant publications (Bidstrup 2006; Body 2006; Ferguson 2007; Levy 2006). Our view is not that the studies of Karkouti 2006; Mangano 2006 and Mangano 2007 were badly done, but that they have inherent limitations, mainly due to their observational nature and selection biases that probably cannot be completely overcome through statistical adjustments by propensity scores and co-variates. These weaknesses were addressed in the larger study performed by Schneeweiss and colleagues (2008), described above. The agreement between these studies adds weight to the claim that aprotinin does indeed increase the chances of 
death.

In considering the apparently conflicting results of the different study types it is also important to acknowledge weaknesses in the database of randomised trials, in particular under-recording of infrequent events that were not the primary outcomes of the trials. It is important to note that for dichotomous data to be included in our analyses, trial reports had to provide either numeric data, that is the numbers of events that occurred in the treatment and control groups, or where there were no events recorded, the trial report had to clearly state this. So, we have some confidence in the data included in the meta-analyses. However, we acknowledge that under-reporting of uncommon events that were not the primary outcomes of these generally small trials is a potential problem with this literature. In the case of aprotinin our analyses of myocardial infarction were based on data from 37 (49\%) out of a total of 76 trials included in the analyses of blood transfusions. These trials were larger than average and included $64 \%$ of all participants. Nevertheless, the incomplete data are a potential source of bias in this and the analyses of other vascular outcomes. We are more confident of our analyses of mortality in cardiac surgery where, in the case of aprotinin, data were reported for $60 \%$ of all trials and $80 \%$ of participants. The most likely effect of under-reporting is to make estimates imprecise, meaning that fairly small changes in mortality or occurrence of thrombotic events might have been missed.

There was a disappointing lack of information in the randomised trials regarding this putative adverse effect of the drug. Only 18 out of 76 trials of aprotinin documented this outcome, so there is a potential for bias due to under-reporting. Based on analysis of 107 events in 4174 individuals the point estimate of the pooled RR with aprotinin (compared with placebo or no treatment) was 1.16 (95\% CI 0.79 to 1.70 ), so we are not confident that we have ruled out a modest increase in risk. On the other hand the suggestion of an increase in risk from Mangano 2006 was based on a total of only 18 events, of which eight occurred in patients treated with aprotinin. Karkouti 2006 carried out a closely matched analysis of 898 individuals who received either aprotinin or TXA. Using a very sensitive measure of renal dysfunction they documented 182 instances, with a higher incidence in aprotinin treated subjects (RR 1.43 , 95\% CI 1.10 to 1.86 ).

There was greater agreement when we consider the results of the summary analyses of the head to head trials of aprotinin and lysine analogues and the observational studies described above. The comparison of aprotinin with the combined results of the lysine analogues found a significantly increased risk of death; similar in magnitude to what was found in the observational studies, but no apparent increase in the risk of major thrombotic events. The absence of a no treatment control group from these analyses means that we are unable to say whether the differences in mortality were due to an adverse effect of aprotinin or a protective effect of the lysine analogues. In addition, the meta-analyses for death and myocardial infarction were heavily weighted by the results of the BART trial (Fergusson 2008). These factors limit our ability to draw firm conclusions about the true effects of the drugs. But the summary data now available, and the regulatory action taken against aprotinin, enable us to make some pragmatic recommendations. Despite the possibility that they are inferior to aprotinin in minimising perioperative blood loss and the need for allogeneic red cell transfusion both tranexamic acid and aminocaproic acid appear effective and safe. The experience is greatest with tranexamic acid and confidence in the use of this drug has been strengthened by the recent publication of the CRASH-2 trial (CRASH-2 2010), which found that two doses of tranexamic acid reduced overall mortality when administered soon after major trauma.

\section{Conclusions}

Antifibrinolytic drugs are effective in reducing blood loss, the need for allogeneic red cell transfusion, and the need for re-operation due to continued post-operative bleeding (in cardiac surgery). Aprotinin appears more effective than the lysine analogues in minimising peri and post operative blood loss when used as adjunctive therapy in cardiac surgery. Strictly speaking, based on their average effects on the need for red cell transfusion, the lysine analogues do not meet the criteria for being considered equivalent to aprotinin. However, comparisons between the drugs need to take account of the clinical significance of any small advantage of aprotinin, the dose response relationships for each of the drugs, and the possible effects of publication bias, which appears to favour aprotinin. Taking these factors into consideration it may reasonably be concluded that tranexamic acid is as effective as aprotinin, particularly when it is used as an adjunct to non-cardiac surgical procedures. The data for epsilon aminocaproic acid are sparser and as a consequence not so convincing.

The updated meta-analyses of the randomised trials comparing aprotinin with no treatment do not confirm the evidence from observational studies that aprotinin increases the risks of vascular occlusive events and mortality. However, there has been a degree of under-reporting of these adverse events in trials of anti-fibrinolytic drugs. The head to head comparisons of aprotinin and the lysine analogues have yielded results that are closer to those seen in the observational studies and indicate that aprotinin carries an increased risk of death. Consequently, the balance of benefit and harm favours the use of the lysine analogues over aprotinin, and justifies the regulatory action that resulted in the withdrawal of aprotinin from international markets in 2008.

\section{AUTHORS' CONCLUSIONS}

\section{Implications for practice}

Tranexamic acid and epsilon aminocaproic acid provide worthwhile reductions in blood loss and the need for allogeneic red cell 
transfusion. Based on the results of randomised trials their efficacy does not appear to be offset by serious adverse effects. The evidence is stronger for tranexamic acid than for epsilon aminocaproic acid.

\section{Implications for research}

There is no need for further placebo-controlled trials of anti-fibrinolytic drugs in cardiac surgery. The principal need is for large comparative trials to assess the relative efficacy, safety and cost-ef- fectiveness of the lysine analogues in different surgical procedures.

\section{ACKNOWLEDGEMENTS}

The authors wish to thank Sonya Murray, Kim Henderson, Ann Wilkinson, Ketrina Sly, June McLeod, Jayne Fryer, Rosemary Aldrich, and Patricia McGettigan for their assistance at various stages during the development of this review.

\section{REFER E N C E S}

\section{References to studies included in this review}

Alajmo 1989 \{published data only\}

Alajmo F, Calamai G, Perna AM, Melissano G, Pretelli P, Palmarini MF, et al.High-dose aprotinin: hemostatic effects in open heart operations. Annals of Thoracic Surgery 1989;48(4):536-9.

Alderman 1998 \{published data only\} Alderman EL, Levy JH, Rich JB, Nili M, Vidne B, Schaff H, et al.Analyses of coronary graft patency after aprotinin use: results from the International Multicenter Aprotinin Graft Patency Experience (IMAGE) trial. Journal of Thoracic \& Cardiovascular Surgery 1998;116(5):716-30.

Alvarez 1995 \{published data only\}

Alvarez JM, Quiney NF, McMillan D, Joscelyne K, Connelly T, Brady $\mathrm{P}$, et al.The use of ultra-low-dose aprotinin to reduce blood loss in cardiac surgery. Journal of Cardiothoracic \& Vascular Anesthesia 1995;9(1):29-33.

Alvarez 2001 \{published data only\}

Alvarez JM, Jackson LR, Chatwin C, Smolich JJ. Low-dose postoperative aprotinin reduces mediastinal drainage and blood product use in patients undergoing primary coronary artery bypass grafting who are taking aspirin: a prospective, randomized, doubleblind, placebo-controlled trial. Journal of Thoracic \& Cardiovascular Surgery 2001;122(3):457-63.

Alvarez 2008 \{published data only\}

Álvarez JC, Santiveri FX, Ramos I, Vela E, Puig L, Escolano F. Tranexamic acid reduce blood transfusion in total knee arthroplasty event when a blood conservation program is applied. Blood Conservation \& Transfusion Alternatives 2008;48(3):519-25.

Amar 2003 \{published data only\} Amar D, Grant FM, Zhang H, Boland PJ, Leung DH, Healey JA. Antifibrinolytic therapy and perioperative blood loss in cancer patients undergoing major orthopedic surgery. Anesthesiology 2003; 98(2):337-42.

Andreasen 2004 \{published data only\}

Andreasen JJ, Nielsen C. Prophylactic tranexamic acid in elective, primary coronary artery bypass surgery using cardiopulmonary bypass. European Journal of Cardio-Thoracic Surgery 2004;26(2): $311-7$.
Apostolakis 2008 \{published data only\} Apostolakis E, Panagopoulos N, Koletsis E N, Crockett J, StamouKouki H, Sourgiadaki E, et al.Influence of ultra-low dose Aprotinin on thoracic surgical operations: a prospective randomized trial. Journal of cardiothoracic surgery 2008;3:14.

Armellin 2001 \{published data only\} Armellin G, Casella S, Guzzinati S, Pasini L, Marcassa A, Giron G. Tranexamic acid in aortic valve replacement. Journal of Cardiothoracic \& Vascular Anesthesia 2001;15(3):331-5.

Arom 1994 \{published data only\} Arom KV, Emery RW. Decreased postoperative drainage with addition of epsilon-aminocaproic acid before cardiopulmonary bypass. Annals of Thoracic Surgery 1994;57(5):1108-12.

Ashraf 1997 \{published data only\}

Ashraf S, Tian Y, Cowan D, Nair U, Chatrath R, Saunders NR, et al. "Low-dose" aprotinin modifies hemostasis but not proinflammatory cytokine release. Annals of Thoracic Surgery 1997; 63(1):68-73.

Asimakopoulos 2000 \{published data only\} Asimakopoulos G, Kohn A, Stefanou DC, Haskard DO, Landis RC, Talyor KM. Leukocyte integrin expression in patients undergoing cardiopulmonary bypass. Annals of Thoracic Surgery 2000;69:1192-7.

Baele 1992 \{published data only\} Baele PL, Ruiz-Gomez J, Londot C, Sauvage M, Van Dyck MJ, Robert A. Systematic use of aprotinin in cardiac surgery: influence on total homologous exposure and hospital cost. Acta Anaesthesiologica Belgica 1992;43(2):103-12.

Bailey 1994 \{published data only\} Bailey CR, Kelleher A, Wielogorski AK. Randomised placebocontrolled double blind study of three aprotinin regimes in primary cardiac surgery. British Journal of Surgery 1994;81:969-73.

Basora 1999 \{published data only\}

Basora M, Gomar C, Escolar G, Pacheco M, Fita G, Rodriguez E, Ordinas A. Platelet function during cardiac surgery and cardiopulmonary bypass with low-dose aprotinin. Journal of Cardiothoracic \& Vascular Anesthesia 1999;13(4):382-7.

Bennett-Guerrero 1997 \{published data only\} Bennett-Guerrero E, Sorohan JG, Gurevich ML, Kazanjian PE, Levy RR, Barbera AV, et al.Cost-benefit and efficacy of aprotinin 
compared with epsilon-aminocaproic acid in patients having repeated cardiac operations: a randomized, blinded clinical trial. Anesthesiology 1997;87(6):1373-80.

Benoni 1996 \{published data only\}

Benoni G, Fredin H. Fibrinolytic inhibition with tranexamic acid reduces blood loss and blood transfusion after knee arthroplasty: a prospective, randomised, double-blind study of 86 patients. Journal of Bone \& Joint Surgery - British Volume 1996;78(3):434-40.

Benoni 2000 \{published data only\}

Benoni G, Lethagen S, Nilsson P, Fredin H. Tranexamic acid, given at the end of the operation, does not reduce postoperative blood loss in hip arthroplasty. Acta Orthopaedica Scandinavica 2000;71 (3):250-4.

Benoni 2001 \{published data only\}

Benoni G, Fredin H, Knebel R, Nilsson P. Blood conservation with tranexamic acid in total hip arthroplasty: a randomized, doubleblind study in 40 primary operations. Acta Orthopaedica Scandinavica 2001;72(5):442-8.

Berenholtz 2009 \{published data only\}

Berenholtz SM, Cuong Pham J, Garrett-Mayer E, Atchison CW, Kostuik JP, Cohen DB, et al.Effect of Epsilon Aminocaproic Acid on red-cell transfusion requirements in major spinal surgery. Spine 2009;34(19):2096-103.

Bernet 1999 \{published data only\}

Bernet F, Carrel T, Marbet G, Skarvan K, Stulz P. Reduction of blood loss and transfusion requirements after coronary artery bypass grafting: similar efficacy of tranexamic acid and aprotinin in aspirin-treated patients. Journal of Cardiac Surgery 1999;14(2): $92-7$.

Bert 2008 \{published data only\}

Bert C, De Buck F, Sergeant P, Van Hemelrijck J, Kasran A, Van Duppen $\mathrm{V}$, et al.Aprotinin reduces cardiac troponin I release and inhibits apoptosis of polymorphonuclear cells during off-pump coronary artery bypass surgery. Journal of cardiothoracic and vascular anesthesia 2008;22(1):16-22.

Bidstrup 1989 \{published data only\}

Bidstrup BP, Royston D, Sapsford RN, Taylor KM. Reduction in blood loss and blood use after cardiopulmonary bypass with high dose aprotinin (Trasylol). Journal of Thoracic \& Cardiovascular Surgery 1989;97(3):364-72.

Bidstrup 1990 \{published data only\} Bidstrup BP, Royston D, McGuinness C, Sapsford RN. Aprotinin in aspirin-pretreated patients. Perfusion 1990;5:77-81.

Bidstrup 1993 \{published data only\} Bidstrup BP, Underwood SR, Sapsford RN, Streets EM. Effect of aprotinin (Trasylol) on aorta-coronary bypass graft patency. Journal of Thoracic \& Cardiovascular Surgery 1993;105(1):147-52.

Bidstrup 2000 \{published data only\}

Bidstrup BP, Hunt BJ, Sheikh S, Parratt RN, Bidstrup JM, Sapsford $\mathrm{RN}$. Amelioration of the bleeding tendency of preoperative aspirin after aortocoronary bypass grafting. Annals of Thoracic Surgery 2000;69(2):541-7.

Blauhut 1994 \{published data only\} Blauhut B, Harringer W, Bettelheim P, Doran JE, Spath P, Lundsgaard-Hansen P. Comparison of the effects of aprotinin and tranexamic acid on blood loss and related variables after cardiopulmonary bypass. Journal of Thoracic \& Cardiovascular Surgery 1994;108(6):1083-91.

\section{Boldt 1991 \{published data only\}}

Boldt J, Zickmann B, Czeke A, Herold C, Dapper F, Hempelmann $\mathrm{G}$. Blood conservation techniques and platelet function in cardiac surgery. Anesthesiology 1991;75:426-32.

Boldt 1994 \{published data only\}

Boldt J, Schindler E, Knothe C, Hammermann H, Stertmann WA, Hempelmann G. Does aprotinin influence endothelial-associated coagulation in cardiac surgery?. Journal of Cardiothoracic \& Vascular Anesthesia 1994;8(5):527-31.

Boylan 1996 \{published data only\}

Boylan JF, Klinck JR, Sandler AN, Arellano R, Greig PD,

Nierenberg $\mathrm{H}$, et al.Tranexamic acid reduces blood loss, transfusion requirements, and coagulation factor use in primary orthotopic liver transplantation. Anesthesiology 1996;85(5):1043-8.

\section{Brown 1997 \{published data only\}}

Brown RS, Thwaites BK, Mongan PD. Tranexamic acid is effective in decreasing postoperative bleeding and transfusions in primary coronary artery bypass operations: A double-blind, randomized, placebo-controlled trial. Anesthesia \& Analgesia 1997;85(5): 963-970.

Caglar 2008 \{published data only\}

Caglar G S, Tasci Y, Kayikcioglu F, Haberal A. Intravenous tranexamic acid use in myomectomy: a prospective randomized double-blind placebo controlled study. European journal of obstetrics, gynecology, and reproductive biology 2008;137(2):227-31.

\section{Camarasa 2006 \{published data only\}}

Camarasa MA, Olle G, Serra-Prat M, Martin A, Sanchez M, Ricos $P$, et al.Efficacy of aminocaproic, tranexamic acids in the control of bleeding during total knee replacement: a randomized clinical trial. British Journal of Anaesthesia 2006;96(5):576-82.

Capdevila 1998 \{published data only\}

Capdevila X, Calvet Y, Biboulet P, Biron C, Rubenovitch J, d'Athis F. Aprotinin decreases blood loss and homologous transfusions in patients undergoing major orthopedic surgery. Anesthesiology 1998; 88(1):50-7.

\section{Carrera 1994 \{published data only\}}

Carrera A, Martinez MV, Garcia Guiral M, Herrero E, Peral A, Planas A. Utilizacion de altas dosis de aprotinina en cirugia cardiaca - High doses of aprotinin in cardiac surgery. Revista Espanola de Anestesiologia y Reanimacion 1994;41:13-9.

\section{Casas 1995 \{published data only\}}

Casas JI, Zuazu-Jausoro I, Mateo J, Oliver A, Litvan H, MunizDiaz E, et al.Aprotinin versus desmopressin for patients undergoing operations with cardiopulmonary bypass. A double-blind placebocontrolled study. Journal of Thoracic \& Cardiovascular Surgery 1995;110(4 Pt 1):1107-17.

Casati 1999 \{published data only\}

Casati V, Guzzon D, Oppizzi M, Cossolini M, Torri G, Calori G, Alfieri O. Hemostatic effects of aprotinin, tranexamic acid and epsilon-aminocaproic acid in primary cardiac surgery. Annals of Thoracic Surgery 1999;68(6):2252-6. 
Casati 2000 \{published data only\}

Casati V, Guzzon D, Oppizzi M, Bellotti F, Franco A, Gerli C, et al.Tranexamic acid compared with high-dose aprotinin in primary elective heart operations: effects on perioperative bleeding and allogeneic transfusions. Journal of Thoracic \& Cardiovascular Surgery 2000;120(3):520-7.

Casati 2001 \{published data only\}

Casati V, Gerli C, Franco A, Torri G, D’Angelo A, Benussi S, Alfieri O. Tranexamic acid in off-pump coronary surgery: a preliminary, randomized, double-blind, placebo-controlled study. Annals of Thoracic Surgery 2001;72(2):470-5.

Casati 2002 \{published data only\}

Casati V, Sandrelli L, Speziali G, Calori G, Grasso MA, Spagnolo S. Hemostatic effects of tranexamic acid in elective thoracic aortic surgery: a prospective, randomized, double-blind, placebocontrolled study. Journal of Thoracic \& Cardiovascular Surgery 2002;123(6):1084-91.

\section{Casati 2004 \{published data only\}}

Casati V, Della VP, Benussi S, Franco A, Gerli C, Baili P, et al.Effects of tranexamic acid on postoperative bleeding and related hematochemical variables in coronary surgery: Comparison between on-pump and off-pump techniques. Journal of Thoracic \& Cardiovascular Surgery 2004;128(1):83-91.

Cicek 1996a \{published data only\}

Cicek S, Demirkilic U, Kuralay E, Ozal E, Tatar H. Postoperative Aprotinin: Effect on blood loss and transfusion requirements in cardiac operations. Annals of Thoracic Surgery 1996;61:1372-6.

Cicek 1996b \{published data only\}

Cicek S, Demirkilic U, Ozal E, Kuralay E, Bingol H, Tatar H, Ozturk OY. Postoperative use of aprotinin in cardiac operations: An alternative to its prophylactic use. Journal of Thoracic \& Cardiovascular Surgery 1996;112(6):1462-7.

Cicekcioglu 2006 \{published data only\} Cicekcioglu F, Cagli K, Emir M, Topbas M, Catav Z, Sener E, et al.Effects if minimal dose aprotinin on blood loss and fibrinolytic system-complement activation in coronary artery bypass grafting. Journal of Cardiac Surgery 2006;21:336-41.

Claeys 2007 \{published data only\}

Claeys M A, Vermeersch N, Haentjens P. Reduction of blood loss with tranexamic acid in primary total hip replacement surgery. Acta chirurgica Belgica 2007;107(4):397-401.

Coffey 1995 \{published data only\}

Coffey A, Pittmam J, Halbrook H, Fehrenbacher J, Beckman D, Hormuth D, et al.The use of tranexamic acid to reduce postoperative bleeding following cardiac surgery: A double-blind randomized trial. American Surgeon 1995;61(7):566-8.

Cohen 1998 \{published data only\}

Cohen G, Ivanov J, Weisel RD, Rao V, Mohabeer MK, Mickle DA. Aprotinin and dipyridamole for the safe reduction of postoperative blood loss. Annals of Thoracic Surgery 1998;65(3):674-83.

Colwell 2007 \{published data only\}

Colwell CW, Chelly JE, Murkin JM, Stevens D, O’Keefe TJ, Hall $\mathrm{R}$, et al.Randomized study of Aprotinin effect in transfusions and blood loss in primary THA. Clinical Orthopaedics \& Relation Research 2007;465:189-95.

\section{Corbeau 1995 \{published data only\}}

Corbeau JJ, Monrigal JP, Jacob JP, Cottineau C, Moreau X, Bukowski JG, et al.Comparaison des effets de l'aprotinine et de l'acide tranexamique sur le saignment en chirurgie cardiaque Comparative effects of aprotinin and tranexamic acid on blood loss in cardiac surgery. Annales Francaises d'Anesthesie et de Reanimation 1995;14:154-61

Cosgrove 1992 \{published data only\}

Cosgrove DM, 3d, Heric B, Lytle BW, Taylor PC, Novoa R, Golding LA, et al.Aprotinin therapy for reoperative myocardial revascularization: a placebo-controlled study. Annals of Thoracic Surgery 1992;54(6):1031-6.

\section{Cvachovec 2001 \{published data only\}} Cvachovec K, Sechovska H, Berousek J, Popelka S, Hladikova M. Aprotinin (Antilysin Spofa)--its effect on decreasing hemorrhage in the postoperative period in hip arthroplasty [Czech]. Acta Chirurgiae Orthopaedicae et Traumatologiae Cechoslovaca 2000;68 (5):311-4.

\section{D'Ambra 1996 \{published data only\}}

* D'Ambra MN, Akins CW, Blackstone EH, Bonney SL, Cohn $\mathrm{LH}$, Cosgrove DM, et al.Aprotinin in primary valve replacement and reconstruction: a multicenter, double-blind, placebocontrolled trial. Journal of Thoracic \& Cardiovascular Surgery 1996; 112(4):1081-9.

D’Ambra MN, Lynch KE, Boccagno J, et al.The effect of perioperative administration of recombinant human erythropoietin (R-HuEPO) in CABG patients: A double blind, placebo-controlled trial. Anesthesiology: ASA Abstracts 1992;77(3A):A159.

D'Ambrosio 1999 \{published data only\}

D’Ambrosio A, Borghi B, Damato A, D'Amato G, Antonacci D, Valeri F. Reducing perioperative blood loss in patients undergoing total hip arthroplasty. International Journal of Artificial Organs 1999;22(1):47-51.

\section{Daily 1994 \{published data only\}}

Daily PO, Lamphere JA, Dembitsky WP, Adamson RM, Dans NF. Effect of prophylactic epsilon-aminocaproic acid on blood loss and transfusion requirements in patients undergoing first-time coronary artery bypass grafting. A randomized, prospective, double-blind study. Journal of Thoracic \& Cardiovascular Surgery 1994;108(1): 99-106.

\section{Dalmau 1999 \{published data only\}}

Dalmau A, Sabate A, Acosta F, Garcia-Huete L, Koo M, Reche M, et al.Comparative study of antifibrinolytic drugs in orthotopic liver transplantation. Transplantation Proceedings 1999;31(6):2361-2.

Dalmau 2000 \{published data only\}

Dalmau A, Sabate A, Acosta F, Garcia-Huete L, Koo M, Sansano T, et al.Tranexamic acid reduces red cell transfusion better than epsilon-aminocaproic acid or placebo in liver transplantation. Anesthesia \& Analgesia 2000;91(1):29-34.

Dalmau 2004 \{published data only\}

Dalmau A, Sabate A, Koo M, Bartolome C, Rafecas A, Figueras J, et al.The prophylactic use of tranexamic acid and aprotinin in orthotopic liver transplantation: a comparative study. Liver Transplantation 2004;10(2):279-84. 
Defraigne 2000 \{published data only\}

Defraigne JO, Pincemail J, Larbuisson R, Blaffart F, Limet R. Cytokine release and neutrophil activation are not prevented by heparin- coated circuits and aprotinin administration. Annals of Thoracic Surgery 2000;69(4):1084-91.

Del Rossi 1989 \{published data only\} DelRossi AJ, Cernaianu AC, Botros S, Lemole GM, Moore R. Prophylactic treatment of postperfusion bleeding using EACA. Chest 1989;96(1):27-30.

\section{Deleuze 1991 \{published data only\}}

Deleuze P, Loisance DY, Feliz A, Hillion ML, Castanie JB, Richemond J, et al.Reduction of per- and postoperative blood loss with aprotinin (Trasylol) during extracorporeal circulation [French]. Archives des Maladies du Coeur et des Vaisseaux 1991;84 (12):1797-802.

\section{Demeyere 2006 \{published data only\}}

Demeyere R, Bosteels A, Arnout J, Gasthuisberg UV. Comparison of the effects of tranexamic acid, aprotinin and placebo on blood conservation, fibrinolysis and platelet function with extensive heart surgery. Critical Care 2006;10(Suppl 1):S67.

\section{Desai 2009 \{published data only\}}

Desai P H, Kurian D, Thirumavalavan N, Desai S P, Ziu P, Grant $\mathrm{M}$, et al.A randomized clinical trial investigating the relationship between aprotinin and hypercoagulability in off-pump coronary surgery. Anesthesia and analgesia 2009;109(5):1387-94.

Dietrich 1990 \{published data only\}

Dietrich W, Spannagl M, Jochum M, Wendt P, Schramm W, Barankay A, Sebening F, Richter JA. Influence of high-dose aprotinin treatment on blood loss and coagulation patterns in patients undergoing myocardial revascularization. Anesthesiology 1990;73(6):1119-26.

Dietrich 1992 \{published data only\}

Dietrich W, Barankay A, Hahnel C, Richter JA. High-dose aprotinin in cardiac surgery: three years' experience in 1,784 patients. Journal of Cardiothoracic \& Vascular Anesthesia 1992;6(3): 324-7.

\section{Dietrich 1995 \{published data only\}}

Dietrich W, Dilthey G, Spannagl M, Jochum M, Braun SL, Richter JA. Influence of high-dose aprotinin on anticoagulation, heparin requirement, and celite- and kaolin-activated clotting time in heparin-pretreated patients undergoing open-heart surgery. A double-blind, placebo-controlled study. Anesthesiology 1995;83(4): $679-89$.

\section{Dietrich 2008 \{published data only\}}

Dietrich W, Spannagl M, Boehm J, Hauner K, Braun S, Schuster T, et al.Tranexamic acid and aprotinin in primary cardiac operations: an analysis of 220 cardiac surgical patients treated with tranexamic acid or aprotinin. Anesthesia and analgesia 2008;107(5):1469-78.

Dignan 2001 \{published data only\}

Dignan RJ, Law DW, Seah PW, Manganas CW, Newman DC, Grant PW, et al.Ultra-low dose aprotinin decreases transfusion requirements and is cost effective in coronary operations. Annals of Thoracic Surgery 2001;71(1):158-63.

Diprose 2005 \{published data only\}

Diprose P, Herbertson MJ, O'Shaughnessy D, Deakin CD, Gill RS. Reducing allogeneic transfusion in cardiac surgery: a randomized double-blind placebo-controlled trial of antifibrinolytic therapies used in addition to intra-operative cell salvage. British Journal of Anaesthesia 2005;94(3):271-8.

Dorman 2008 \{published data only\} Dorman B H, Stroud R E, Wyckoff M M, Zellner J L, Botta D, Leonardi A H, et al.Differential effects of epsilon-aminocaproic acid and aprotinin on matrix metalloproteinase release in patients following cardiopulmonary bypass. Journal of cardiovascular pharmacology 2008;51(4):418-23.

Dryden 1997 \{published data only\}

Dryden PJ, O'Connor JP, Jamieson WR, Reid I, Ansley D, Sadeghi $\mathrm{H}$, et al.Tranexamic acid reduces blood loss and transfusion in reoperative cardiac surgery. Canadian Journal of Anaesthesia 1997; 44(9):934-41.

\section{Eberle 1998 \{published data only\}} Eberle B, Mayer E, Hafner G, Heinermann J, Dahm M, Prellwitz $\mathrm{W}$, et al.High-dose epsilon-aminocaproic acid versus aprotinin: antifibrinolytic efficacy in first-time coronary operations. Annals of Thoracic Surgery 1998;65(3):667-73.

Ehrlich 1998 \{published data only\} Ehrlich M, Grabenwoger M, Cartes-Zumelzu F, Luckner D, Kovarik J, Laufer G, et al.Operations on the thoracic aorta and hypothermic circulatory arrest: is aprotinin safe?. Journal of Thoracic \& Cardiovascular Surgery 1998;115(1):220-5.

Ekback 2000 \{published data only\} Ekback G, Axelsson K, Ryttberg L, Edlund B, Kjellberg J, Weckstrom J, et al.Tranexamic acid reduces blood loss in total hip replacement surgery. Anesthesia \& Analgesia 2000;91(5):1124-30.

\section{Ellis 2001 \{published data only\}}

Ellis MH, Fredman B, Zohar E, Ifrach N, Jedeikin R. The effect of tourniquet application, tranexamic acid, and desmopressin on the procoagulant and fibrinolytic systems during total knee replacement. Journal of Clinical Anesthesia 2001;13(7):509-13.

Engel 2001 \{published data only\}

Engel JM, Hohaus T, Ruwoldt R, Menges T, Jurgensen I, Hempelmann G. Regional hemostatic status and blood requirements after total knee arthroplasty with and without tranexamic acid or aprotinin. Anesthesia \& Analgesia 2001;92(3): $775-80$.

Englberger 2002a \{published data only\} Englberger L, Markart P, Eckstein FS, Immer FF, Berdat PA, Carrel TP. Aprotinin reduces blood loss in off-pump coronary artery bypass (OPCAB) surgery. European Journal of Cardio-Thoracic Surgery 2002;22(4):545-51.

Englberger 2002b \{published data only\} Englberger L, Kipfer B, Berdat PA, Nydegger UE, Carrel TP. Aprotinin in coronary operation with cardiopulmonary bypass: does "low-dose" aprotinin inhibit the inflammatory response? Annals of Thoracic Surgery 2002;73(6):1897-904.

Fauli 2005 \{published data only\}

Fauli A, Gomar C, Campistol JM, Alvarez L, Manig AM, Matute P. Kidney-specific proteins in patients receiving aprotinin at high- and low-dose regimens during coronary artery bypass graft with cardiopulmonary bypass. European Journal of Anaesthesiology 2005; 22(9):666-71. 
Feindt 1994 \{published data only\}

Feindt P, Seyfert U, Volkmer I, Huwer H, Kalweit G, Gams E. Is there a phase of hypercoagulability when aprotinin is used in cardiac surgery?. European Journal of Cardio-thoracic Surgery 1994; 8:308-14.

\section{Fergusson 2008 \{published data only\}}

Fergusson DA, Hébert PC, Mazer CD, Fremes S, MacAdams C, Murkin JM, et al.A comparison of aprotinin and lysine analogues in high-risk cardiac surgery. The New England Journal of Medicine 2008;358(22):2319-31.

\section{Findlay 2001 \{published data only\}}

Findlay JY, Rettke SR, Ereth MH, Plevak DJ, Krom RA, Kufner RP. Aprotinin reduces red blood cell transfusion in orthotopic liver transplantation: a prospective, randomized, double-blind study. Liver Transplantation 2001;7(9):802-7.

\section{Fraedrich 1989 \{published data only\}}

Fraedrich G, Weber C, Bernard C, Hettwer A, Schlosser V. Reduction of blood transfusion requirement in open heart surgery by administration of high doses of aprotinin - preliminary results. Thoracic \& Cardiovascular Surgeon 1989;37(2):89-91.

Garcia-Enguita 1998 \{published data only\} Garcia-Enguita MA, Ortega JP, Gomez C, Rasal S, Pascual A, Urieta SA. High dose aprotinin in complex total hip arthroplasty [abstract]. British Journal of Anaesthesia 1998;80(Suppl 1):80.

Garcia-Huete 1997 \{published data only\} Garcia-Huete L, Domenech P, Sabate A, Martinez-Brotons F, Jaurrieta E, Figueras J. The prophylactic effect of aprotinin on intraoperative bleeding in liver transplantation: A randomized clinical study. Hepatology 1997;26(5):1143-8.

\section{Garneti 2004 \{published data only\}} Garneti N, Field J. Bone bleeding during total hip arthroplasty after administration of tranexamic acid. Journal of Arthroplasty 2004;19 (4):488-92.

Gherli 1992 \{published data only\}

Gherli T, Porcu A, Padua G, Marongiu GM, Antona C, Alamanni F, Bacciu PP. Reducing bleeding during extracorporeal circulation interventions by high doses of aprotinin [Italian]. Minerva Cardioangiologica 1992;40(4):121-6.

\section{Gill 2009 \{published data only\}}

Gill JB, Chase E, Rosenstein AD. The use of tranexamic acid in revision total hip arthroplasty: a pilot study. Current Orthopaedic Practice 2009;20(2):152-6.

Golanski 2000 \{published data only\} Golanski R, Golanski J, Chizynski K, Iwaszkiewicz A, Zaslonka J, Pietrucha T, et al.Low doses of aprotinin in aortocoronary bypass surgery - advantages and disadvantages. Medical Science Monitor 2000;6(4):722-8.

\section{Good 2003 \{published data only\}}

Good L, Peterson E, Lisander B. Tranexamic acid decreases external blood loss but not hidden blood loss in total knee replacement. British Journal of Anaesthesia 2003;90(5):596-9.

\section{Gott 1998 \{published data only\}}

Gott JP, Cooper WA, Schmidt FE, Brown WM, Wright CE, Merlino JD, et al.Modifying risk for extracorporeal circulation: trial of four antiinflammatory strategies. Annals of Thoracic Surgery 1998;66:747-54

\section{Green 1995 \{published data only\}}

Green D, Sanders J, Eiken M, Wong CA, Frederiksen J, Joob A, et al.Recombinant aprotinin in coronary artery bypass graft operations. Journal of Thoracic \& Cardiovascular Surgery 1995;110 (4 Pt 1):963-70.

\section{Greilich 2001 \{published data only\}}

Greilich PE, Okada K, Latham P, Kumar RR, Jessen ME. Aprotinin but not epsilon-aminocaproic acid decreases interleukin-10 after cardiac surgery with extracorporeal circulation: randomized, double-blind, placebo-controlled study in patients receiving aprotinin and epsilon-aminocaproic acid. Circulation 2001;104(12 Suppl 1):265-9.

\section{Greilich 2003 \{published data only\}} Greilich PE, Brouse CF, Whitten CW, Chi L, Dimaio JM, Jessen ME. Antifibrinolytic therapy during cardiopulmonary bypass reduces proinflammatory cytokine levels: a randomized, doubleblind, placebo-controlled study of epsilon-aminocaproic acid and aprotinin. Journal of Thoracic \& Cardiovascular Surgery 2003;126 (5):1498-503

Greilich 2004 \{published data only\} Greilich PE, Brouse CF, Rinder CS, Smith BR, Sandoval BA, Rinder HM, et al.Effects of epsilon-aminocaproic acid and aprotinin on leukocyte-platelet adhesion in patients undergoing cardiac surgery. Anesthesiology 2004;100(2):225-33.

\section{Greilich 2009 \{published data only\}}

Greilich PE, Jessen ME, Satyanarayana N, Whitten CW, Nuttall GA, Beckham GA, et al.The effects of Epsilon-Aminocaproic Acid and Aprotinin on fibrinolysis and blood loss in patients undergoing primary, isolated coronary artery bypass surgery: a randomized double-blind, placebo-controlled, noninferiority trial. Anesthesia \& Analgesia 2009;109(1):15-24.

Groh 1993 \{published data only\}

Groh J, Welte M, Azad SC, Anthuber M, Haller M, Kratzer MA. Does aprotinin really reduce blood loss in orthotopic liver transplantation?. Seminars in Thrombosis \& Hemostasis 1993;19(3): 306-8.

\section{Harder 1991 \{published data only\}}

Harder MP, Eijsman L, Roozendaal KJ, van Oeveren W, Wildevuur CR. Aprotinin reduces intraoperative and postoperative blood loss in membrane oxygenator cardiopulmonary bypass. Annals of Thoracic Surgery 1991;51(6):936-41.

Hardy 1993 \{published data only\}

Hardy JF, Desroches J, Belisle S, Perrault J, Carrier M, Robitaille D. Low-dose aprotinin infusion is not clinically useful to reduce bleeding and transfusion of homologous blood products in highrisk cardiac surgical patients. Canadian Journal of Anaesthesia 1993; 40(7):625-31.

Hardy 1997 \{published data only\}

Hardy JF, Belisle S, Couturier A, Robitaille D. Randomized, placebo-controlled, double-blind study of an ultra-low-dose aprotinin regimen in reoperative and/or complex cardiac operations. Journal of Cardiac Surgery 1997;12(1):15-22. 
Hardy 1998 \{published data only\}

Hardy JF, Belisle S, Dupont C, Harel F, Robitaille D, Roy M, et al.Prophylactic tranexamic acid and epsilon-aminocaproic acid for primary myocardial revascularization. Annals of Thoracic Surgery 1998;65(2):371-6.

Harley 2002 \{published data only\}

Harley BJ, Beaupre LA, Jones CA, Cinats JG, Guenther CR. The effect of epsilon aminocaproic acid on blood loss in patients who undergo primary total hip replacement: a pilot study. Canadian Journal of Surgery 2002;45(3):185-90.

Harmon 2004 \{published data only\}

Harmon DC, Ghori KG, Eustace NP, O'Callaghan SJ, O'Donnell AP, Shorten GD. Aprotinin decreases the incidence of cognitive deficit following CABG and cardiopulmonary bypass: a pilot randomized controlled study. Canadian Journal of Anaesthesia 2004;51(10):1002-9.

Havel 1992 \{published data only\}

Havel M, Owen AN, Simon P, Teufelsbauer H, Zwoelfer W, Laczkovics A, et al.Decreasing use of donated blood and reduction of bleeding after orthotopic heart transplantation by use of aprotinin. Journal of Heart \& Lung Transplantation 1992;11(2 Pt 1):348-9.

\section{Havel 1994 \{published data only\}}

Havel M, Grabenwoger F, Schneider J, Laufer G, Wollenek, G, Owen A, et al.Aprotinin does not decrease early graft patency after coronary artery bypass grafting despite reducing postoperative bleeding and use of donated blood. Journal of Thoracic \& Cardiovascular Surgery 1994;107(3):807-10.

\section{Hayashida 1997 \{published data only\}}

Hayashida N, Isomura T, Sato T, Maruyama H, Kosuga K, Aoyagi $S$. Effects of minimal-dose aprotinin on coronary artery bypass grafting. Journal of Thoracic \& Cardiovascular Surgery 1997;114(2): 261-9.

Hayes 1996 \{published data only\}

Hayes A, Murphy DB, McCarroll M. The efficacy of single-dose aprotinin 2 million KIU in reducing blood loss and its impact on the incidence of deep venous thrombosis in patients undergoing total hip replacement surgery. Journal of Clinical Anesthesia 1996;8 (5):357-60.

Hei 2005 \{published data only\} Hei ZQ, Luo CF, Li SR, Ma WH, Luo GJ, Chi XJ. Effect of aprotinin on perioperative requirements of blood and liquid in severe hepatisis patients undergoing liver transplantation. Chinese Pharmaceutical Journal 2005;40(18):1432-5.

Hekmat 2004 \{published data only\}

Hekmat K, Zimmermann T, Kampe S, Kasper SM, Weber HJ, Geissler HJ, et al.Impact of tranexamic acid vs. aprotinin on blood loss and transfusion requirements after cardiopulmonary bypass: a prospective, randomised, double-blind trial. Current Medical Research \& Opinion 2004;20(1):121-6.

Hendrice 1995 \{published data only\}

Hendrice C, Schmartz D, Pradier O, Coron T, Kucharcezwski C, d'Hollander A, et al.Effects of aprotinin on blood loss, heparin monitoring tests, and heparin doses in patients undergoing coronary artery bypass surgery. Journal of Cardiothoracic \& Vascular Anesthesia 1995;9(3):245-9.

\section{Hiipala 1995 \{published data only\}}

Hiippala S, Strid L, Wennerstrand M, Arvela V, Mantyla S, Ylinen $\mathrm{J}$, et al.Tranexamic acid (Cyklokapron) reduces perioperative blood loss associated with total knee arthroplasty. British Journal of Anaesthesia 1995;74(5):534-7.

Hiipala 1997 \{published data only\} Hiippala ST, Strid LJ, Wennerstrand MI, Arvela JVV, Niemela HM, Mantyla SK, et al.Tranexamic acid radically decreases blood loss and transfusions associated with total knee arthroplasty. Anesthesia \& Analgesia 1997;84(4):839-44.

Hill 1998 \{published data only\} Hill GE, Diego RP, Stammers AH, Huffman SM, Pohorecki R. Aprotinin enhances the endogenous release of interleukin-10 after cardiac operations. Annals of Thoracic Surgery 1998;65(1):66-9.

Horrow 1990 \{published data only\} Horrow JC, Hlavacek J, Strong MD, Collier W, Brodsky I, Goldman SM, Goel IP. Prophylactic tranexamic acid decreases bleeding after cardiac operations. Journal of Thoracic \& Cardiovascular Surgery 1990;99(1):70-4.

Horrow 1991 \{published data only\} Horrow JC, Van Riper DF, Strong MD, Brodsky I, Parmet JL. Hemostatic effects of tranexamic acid and desmopressin during cardiac surgery. Circulation 1991;84(5):2063-70.

Horrow 1995 \{published data only\} Horrow JC, Van Riper DF, Strong MD, Grunewald KE, Parmet JL. The dose-response relationship of tranexamic acid. Anesthesiology 1995;82(2):383-92.

Husted 2003 \{published data only\} Husted H, Blond L, Sonne-Holm S, Holm G, Jacobsen TW, Gebuhr P. Tranexamic acid reduces blood loss and blood transfusions in primary total hip arthroplasty: a prospective randomized double-blind study in 40 patients. Acta Orthopaedica Scandinavica 2003;74(6):665-9.

Ickx 2006 \{published data only\} Ickx BE, Van Der Linden PJ, Melot C, Wijns W, De Pauw L, Vandestadt J, et al.Comparison of the effects of aprotinin and tranexamic acid on blood loss and red blood cell transfusion requirements during the late stages of liver transplantation. Transfusion 2006;46(4):595-605.

Isetta 1993 \{published data only\} Isetta C, Gunness TK, Samat C, Paolini G, Lugrin D, Sanchez B, et al.Antifibrinolytic Treatment and Homologeous Transfusion in Cardiac Surgery. European Heart Journal 1993;14(Suppl):424.

Jamieson 1997 \{published data only\} Jamieson WRE, Dryden PJ, O'Connor JP, Sadeghi H, Ansley DM, Merrick PM, et al.Beneficial effect of both tranexamic acid and aprotinin on blood loss reduction in reoperative valve replacement surgery. Circulation 1997;96(9 SUPPL):II96-101.

Jansen 1999 \{published data only\} Jansen AJ, Andreica S, Claeys M, D'Haese J, Camu F, Jochmans K. Use of tranexamic acid for an effective blood conservation strategy after total knee arthroplasty. British Journal of Anaesthesia 1999;83 (4):596-601. 
Janssens 1994 \{published data only\}

Janssens M, Joris J, David JL, Lemaire R, Lamy M. High-dose aprotinin reduces blood loss in patients undergoing total hip replacement surgery. [see comments]. Anesthesiology 1994;80(1): 23-9.

\section{Jares 2003 \{published data only\}}

Jares M, Vanek T, Straka Z, Brucek P. Tranexamic acid reduces bleeding after off-pump coronary artery bypass grafting. Journal of Cardiovascular Surgery 2003;44(2):205-8.

Jeserschek 2003 \{published data only\} Jeserschek R, Clar H, Aigner C, Rehak P, Primus B, Windhager R Reduction of blood loss using high-dose aprotinin in major orthopaedic surgery: a prospective, double-blind, randomised and placebo-controlled study. Journal of Bone \& Joint Surgery - British Volume 2003;85(2):174-7.

\section{Jimenez 2007 \{published data only\}}

* Jimenez J J, Iribarren J L, Lorente L, Rodriguez J M, Hernandez D, Nassar I, et al.Tranexamic acid attenuates inflammatory response in cardiopulmonary bypass surgery through blockade of fibrinolysis: a case control study followed by a randomized double-blind controlled trial. Critical care (London, England) 2007;11(6):R117. Jimenez Rivera J, Iribarren Sarrias J, Nassar I, Rodriguez J, Raya J, Garrido P, et al.Tranexamic acid decreased postoperative bleeding and systemic inflammatory response syndrome associated with cardiopulmonary bypass: a prospective, randomized, double-blind controlled study. Critical Care 2006;10(Suppl 1):S95.

Johansson 2005 \{published data only\}

Johansson T, Pettersson LG, Lisander B. Tranexamic acid in total hip arthroplasty saves blood and money: a randomized, doubleblind study in 100 patients. Acta Orthopaedica 2005;76(3):314-9.

\section{Kahveci 1996 \{published data only\}}

Kahveci SF, Ozkan U, Korfali G, Sahin S. Effects of intraoperative aprotinin administration on hemostatic parameters in patients undergoing cardiac surgery [Turkish]. Turk Anesteziyoloji Ve Reanimasyon 1996;24(9):404-7.

Kalangos 1994 \{published data only\}

Kalangos A, Tayyareci G, Pretre R, Di Dio P, Sezerman O. Influence of aprotinin on early graft thrombosis in patients undergoing myocardial revascularization. European Journal of Cardiothoracic Surgery 1994;8(12):651-6.

Karski 1995 \{published data only\}

Karski JM, Teasdale SJ, Norman P, Carroll J, VanKessel K, Wong P, et al.Prevention of bleeding after cardiopulmonary bypass with high-dose tranexamic acid. Double-blind, randomized clinical trial. Journal of Thoracic \& Cardiovascular Surgery 1995;110(3):835-42.

Karski 2005 \{published data only\}

Karski J, Djaiani G, Carroll J, Iwanochko M, Seneviratne P, Liu P, et al.Tranexamic acid and early saphenous vein graft patency in conventional coronary artery bypass graft surgery: a prospective randomized controlled clinical trial. Journal of Thoracic \& Cardiovascular Surgery 2005;130(2):309-14.

Kaspar 1997 \{published data only\} Kaspar M, Ramsay MAE, Nguyen AT, Cogswell M, Hurst G, Ramsay KJ. Continuous small-dose tranexamic acid reduces fibrinolysis but not transfusion requirements during orthotopic liver transplantation. Anesthesia \& Analgesia 1997;85(2):281-5.

Katoh 1997 \{published data only\}

Katoh J, Tsuchiya K, Sato W, Nakajima M, Iida Y. Additional postbypass administration of tranexamic acid reduces blood loss after cardiac operations. Journal of Thoracic \& Cardiovascular Surgery 1997;113(4):802-4.

\section{Katsaros 1996 \{published data only\}}

Katsaros D, Petricevic M, Snow NJ, Woodhall DD, Van Bergen R. Tranexamic acid reduces postbypass blood use: a double-blinded, prospective, randomized study of 210 patients. Annals of Thoracic Surgery 1996;61(4):1131-5.

Katzel 1998 \{published data only\}

Katzel R, Keuper H, Wiedemann B, Brethner L, Voigt B. Effects of aprotinin application on perioperative changes in hemostasis and fibrinolysis and its consequence for blood loss and transfusion requirement in lung surgery. [German]. Infusionstherapie und Transfusionsmedizin 1998;25(4):236-245.

\section{Kazemi 2010 \{published data only\}}

Kazemi SM, Mosaffa F, Eajazi A, Kaffashi M, Daftari Besheli L, Reza Bigdeli M, et al.The effects of tranexamic acid on reducing blood loss in cementless total hip arthroplasty under epidural anesthesia. Orthopedics 2010;33(1):17.

Kikura 2006 \{published data only\}

Kikura M, Levy JH, Tanaka KA, Ramsay JG. A double-blind, placebo-controlled trial of epsilon-aminocaproic acid for reducing blood loss in coronary artery bypass grafting surgery. Journal of the American College of Surgeons 2006;202(2):216-222.

Kipfer 2003 \{published data only\}

Kipfer B, Englberger L, Gygax E, Nydegger U, Carrel T. Is reduced systemic heparinization justified with heparin-bonded bypass circuits in cardiac surgery? - Experience with and without aprotinin. Transfusion \& Apheresis Science 2003;29(1):17-24.

\section{Klein 1998 \{published data only\}}

Klein M, Keith PR, Dauben HP, Schulte HD, Beckmann H, Mayer G, Elert O, Gams E. Aprotinin counterbalances an increased risk of peri-operative hemorrhage in $C A B G$ patients pre-treated with Aspirin. European Journal of Cardio-Thoracic Surgery 1998;14(4): 360-6.

\section{Kluger 2003 \{published data only\}}

Kluger R, Olive DJ, Stewart AB, Blyth CM. Epsilon-aminocaproic acid in coronary artery bypass graft surgery: preincision or postheparin?. Anesthesiology 2003;99(6):1263-9.

\section{Koster 2004 \{published data only\}}

Koster A, Huebler S, Merkle F, Hentschel T, Grundel M, Krabatsch T, Tambeur L, Praus M, Habazettl H, Kuebler WM, Kuppe H. Heparin-level-based anticoagulation management during cardiopulmonary bypass: a pilot investigation on the effects of a half-dose aprotinin protocol on postoperative blood loss and hemostatic activation and inflammatory response. Anesthesia \& Analgesia 2004;98(2):285-90.

Kratzer 1997 \{published data only\} Kratzer MAA, Azad SC, Groh J, Welte M, Haller M, Pratschke E. Effect of aprotinin on blood loss and coagulation parameters in 
orthotopic liver transplantation. [German]. Anaesthesist 1997;46 (4):294-302.

\section{Kreisler 2005 \{published data only\}}

Kreisler KR, Vance RA, Cruzzavala J, Mahnken JD. Heparinbonded cardiopulmonary bypass circuits reduce the rate of red blood cell transfusion during elective coronary artery bypass surgery. Journal of Cardiothoracic \& Vascular Anesthesia 2005;19(5):608-11.

\section{Kuepper 2003 \{published data only\}}

Kuepper F, Dangas G, Mueller-Chorus A, Kulka PM, Zenz M, Wiebalck A. Fibrinolytic activity and bleeding after cardiac surgery with cardiopulmonary bypass and low-dose aprotinin therapy. Blood Coagulation \& Fibrinolysis 2003;14(2):147-53.

\section{Kuitunen 2005 \{published data only\}}

Kuitunen A, Hiippala S, Vahtera E, Rasi V, Salmenpera M. The effects of aprotinin and tranexamic acid on thrombin generation and fibrinolytic response after cardiac surgery. Acta Anaesthesiologica Scandinavica 2005;49(9):1272-9.

Kuitunen 2006 \{published data only\} Kuitunen AH, Suojaranta-Ylinen RT, Kukkonen SI, Niemi TT. Tranexamic acid does not correct the haemostatic impairment caused by hydroxyethyl starch $(200 \mathrm{kDa} / 0.5)$ after cardiac surgery. Blood Coagulation and Fibrinolysis 2006;17:639-45.

\section{Kunt 2005 \{published data only\}}

Kunt AS, Darcin OT, Aydin S, Demir D, Selli C, Andac MH Mini-dose pump-prime aprotinin inhibited enhanced fibrinolytic activity and reduced blood loss and transfusion requirements after coronary artery bypass surgery. Journal of Thrombosis \& Thrombolysis 2005;19(3):197-200.

\section{Kyriss 2001 \{published data only\}}

Kyriss T, Wurst H, Friedel G, Jaki R, Toomes H. Reduced blood loss by aprotinin in thoracic surgical operations associated with high risk of bleeding. A placebo-controlled, randomized phase IV study. European Journal of Cardio-Thoracic Surgery 2001;20(1):38-41.

\section{Landymore 1997 \{published data only}

Landymore RW, Murphy JT, Lummis H, Carter C. The use of lowdose aprotinin, epsilon-aminocaproic acid or tranexamic acid for prevention of mediastinal bleeding in patients receiving aspirin before coronary artery bypass operations.. European Journal of Cardio-Thoracic Surgery 1997;11:798-800.

\section{Lass 1995 \{published data only\}}

Lass M, Simic O, Ostermeyer J. Re-Graft patency and clinical efficacy of aprotinin in elective bypass surgery. Cardiovascular Surgery 1997;5(6):604-7.

* Lass M, Welz A, Kochs M, Mayer G, Schwandt M, Hannekum A. Aprotinin in elective primary bypass surgery. European Journal of Cardio-thoracic Surgery 1995;9:206-10.

\section{Later 2009 \{published data only}

Later A F, Maas J J, Engbers F H, Versteegh M I, Bruggemans E F, Dion R A, et al.Tranexamic acid and aprotinin in low- and intermediate-risk cardiac surgery: a non-sponsored, double-blind, randomised, placebo-controlled trial. European journal of cardiothoracic surgery : official journal of the European Association for Cardio-thoracic Surgery 2009;36(2):322-9.
Laub 1994 \{published data only\}

Laub GW, Riebman JB, Chen C, Adkins MS, Anderson WA, Fernandez J, McGrath LB. The impact of aprotinin on coronary artery bypass graft patency. Chest 1994;106(5):1370-5.

\section{Lavee 1993 \{published data only\}}

Lavee J, Raviv Z, Smolinsky A, Savion N, Varon D, Goor DA,

Mohr R. Platelet protection by low-dose aprotinin in cardiopulmonary bypass: electron microscopic study. Annals of Thoracic Surgery 1993;55(1):114-9.

Leijdekkers 2006 \{published data only\}

Leijdekkers VJ, Vahl AC, Mackaay AJC, Huijgens PC, Rauwerda JA. Aprotinin does not diminish blood loss in elective operations for infrarenal abdominal aneurysms: a randomized double-blind controlled trial. Annals of Vascular Surgery 2006;20:322-9.

Lemay 2004 \{published data only\}

Lemay E, Guay J, Cote C, Roy A. Tranexamic acid reduces the need for allogenic red blood cell transfusions in patients undergoing total hip replacement. Canadian Journal of Anaesthesia 2004;51(1):31-7.

Lemmer 1994 \{published data only\}

Lemmer JH, Jr, Stanford W, Bonney SL, Breen JF, Chomka EV, Eldredge WJ, Holt WW, Karp RB, Laub GW, Lipton MJ, et al.Aprotinin for coronary bypass operations: efficacy, safety, and influence on early saphenous vein graft patency. A multicenter, randomized, double-blind, placebo-controlled study. Journal of Thoracic \& Cardiovascular Surgery 1994;107(2):543-51.

Lemmer 1996 \{published data only\}

Lemmer JH, Jr, Dilling EW, Morton JR, Rich JB, Robicsek F, Bricker DL, Hantler CB, Copeland JG, 3rd, Ochsner JL, Daily $\mathrm{PO}$, et al.Aprotinin for primary coronary artery bypass grafting: a multicenter trial of three dose regimens. Annals of Thoracic Surgery 1996;62(6):1659-67.

Lemmer'1 1994 \{published data only\}

* Lemmer JH, Jr, Stanford W, Bonney SL, Breen JF, Chomka EV, Eldredge WJ, Holt WW, Karp RB, Laub GW, Lipton MJ, et al.Aprotinin for coronary bypass operations: efficacy, safety, and influence on early saphenous vein graft patency. A multicenter, randomized, double-blind, placebo-controlled study. Journal of Thoracic \& Cardiovascular Surgery 1994;107(2):543-51.

Lemmer JH, Jr, Stanford W, Bonney SL, Chomka EV, Karp RB, Laub GW, Rumberger JA, Schaff HV. Aprotinin for coronary artery bypass grafting: effect on postoperative renal function. Annals of Thoracic Surgery 1995;59(1):132-6.

\section{Lemmer'2 1994 \{published data only\}}

* Lemmer JH, Jr, Stanford W, Bonney SL, Breen JF, Chomka EV, Eldredge WJ, Holt WW, Karp RB, Laub GW, Lipton MJ, et al.Aprotinin for coronary bypass operations: efficacy, safety, and influence on early saphenous vein graft patency. A multicenter, randomized, double-blind, placebo-controlled study. Journal of Thoracic \& Cardiovascular Surgery 1994;107(2):543-51. Lemmer JH, Jr, Stanford W, Bonney SL, Chomka EV, Karp RB Laub GW, Rumberger JA, Schaff HV. Aprotinin for coronary artery bypass grafting: effect on postoperative renal function. Annals of Thoracic Surgery 1995;59(1):132-6.

Lentschener 1997 \{published data only\}

Lentschener C, Benhamou D, Mercier FJ, Boyer-Neumann C, Naveau S, Smadja C, Wolf M, Franco D. Aprotinin reduces blood 
loss in patients undergoing elective liver resection. Anesthesia \& Analgesia 1997;84(4):875-81.

Lentschener 1999 \{published data only\}

Lentschener C, Cottin P, Bouaziz H, Mercier FJ, Wolf M, Aljabi Y, et al.Reduction of blood loss and transfusion requirement by aprotinin in posterior lumbar spine fusion. Anesthesia \& Analgesia 1999;89(3):590-7.

Levy 1995 \{published data only\}

Levy JH, Pifarre R, Schaff HV, Horrow JC, Albus R, Spiess B, Rosengart TK, Murray J, Clark RE, Smith P. A multicenter, double-blind, placebo-controlled trial of aprotinin for reducing blood loss and the requirement for donor-blood transfusion in patients undergoing repeat coronary artery bypass grafting. Circulation 1995;92(8):2236-44.

Li 2005 \{published data only\}

Li S, Ji H, Lin J, Lenehan E, Ji B, Liu J, et al.Combination of acute preoperative plateletpheresis, cell salvage, and aprotinin minimizes blood loss and requirement during cardiac surgery. Journal of ExtraCorporeal Technology 2005;37(1):9-14.

Liu 1993 \{published data only\}

* Liu B, Belboul A, Radberg G, Tengborn L, Dernevik L, Roberts

D, William-Olsson G. Effect of reduced aprotinin dosage on blood loss and use of blood products in patients undergoing cardiopulmonary bypass. Scandinavian Journal of Thoracic \& Cardiovascular Surgery 1993;27(3-4):149-55.

Liu B, Tengborn L, Larson G, Radberg LO, Belboul A, Dernevik L, Roberts D. Half-dose aprotinin preserves hemostatic function in patients undergoing bypass operations. Annals of Thoracic Surgery 1995;59(6): 1534-40.

Liu 1998 \{published data only\}

Liu YC, Tsai TP. The effect of coagulation protection with combination of epsilon aminocaproic acid and plasma saver in open-heart surgery. Acta Anaesthesiologica Sinica 1998;36(3): 149-154.

Llau 1998 \{published data only\}

Llau JV, Aguilar G, Soliveres J, Minguez MF, Saz T, Belda FJ. Aprotinin administration reduces blood loss and transfusion requirements in patients undergoing total hip arthroplasty. [abstract]. British Journal of Anaesthesia 1998;80(Suppl 1):80.

Locatelli 1990 \{published data only\}

Locatelli A, Bertollo D, Bianchi T, et al.Aprotinin in cardiosurgery: a randomized prospective study with different protocols for use. [Italian]. Minerva Anestesiologica 1990;56:973-5.

Luo 1998 \{published data only\}

Luo J, Huang Y, Lan H. Effect of Aprotinin on the red cell immunity in cardiopulmonary bypass. Journal of Tongi Medical University 1998;18(2):97-100.

Maccario 1994 \{published data only\}

Maccario M, Fumagalli C, Deangelis R, Delfino R, Pergolo A, Dottori V, Barberis L. Comparison between low and high doses of aprotinin in heart surgery. [Italian]. Minerva Anestesiologica 1994; 60(6):315-20

MacGillivray 2010 \{published data only\}

MacGillivray RG, Tarabichi SB, Hawari MF, Raoof NT.

Tranexamic acid to reduce blood loss after bilateral total knee arthroplasty. The Journal of Arthroplasty 2010; [In press].
Maddali 2007 \{published data only\}

Maddali M M, Rajakumar M C. Tranexamic acid and primary coronary artery bypass surgery: a prospective study. Asian cardiovascular \& thoracic annals 2007;15(4):313-9.

Maineri 2000 \{published data only\}

Maineri P, Covaia G, Realini M, Caccia G, Ucussich E, Luraschi M, Crosta A, Foresti B, Chiaranda M. Postoperative bleeding after coronary revascularization. Comparison between tranexamic acid and epsilon-aminocaproic acid. Minerva Cardioangiologica 2000;48 (6):155-60.

Mansour 2004 \{published data only\}

Mansour EE, Mustafa B. Aprotinin versus tranexamic acid in patients receiving aspirin and undergoing off-pump coronary artery bypass. Egyptian Journal of Anaesthesia 2004;20(3):229-36.

Marcel 1996 \{published data only\}

Marcel RJ, Stegall WC, Suit CT, Arnold JC, Vera RL, Ramsay MA, O'Donnell MB, Swygert TH, Hein HA, Whitten CW. Continuous small-dose aprotinin controls fibrinolysis during orthotopic liver transplantation. Anesthesia \& Analgesia 1996;82(6):1122-5.

\section{Mehr-Aein 2007 \{published data only\}}

Mehr-Aein A, Sadeghi M, Madani-civi M. Does tranexamic acid reduce blood loss in off-pump coronary artery bypass?. Asian cardiovascular \& thoracic annals 2007;15(4):285-9.

\section{Mengistu 2008 \{published data only\}}

Mengistu A M, Röhm K D, Boldt J, Mayer J, Suttner S W, Piper S $\mathrm{N}$. The influence of aprotinin and tranexamic acid on platelet function and postoperative blood loss in cardiac surgery. Anesthesia and analgesia 2008;107(2):391-7.

\section{Menichetti 1996 \{published data only\}}

Menichetti A, Tritapepe L, Ruvolo G, Speziale G, Cogliati A, Di Giovann C, Pacilli M, Criniti A. Changes in coagulation patterns, blood loss and blood use after cardiopulmonary bypass: aprotinin vs tranexamic acid vs epsilon aminocaproic acid. Journal of Cardiovascular Surgery 1996;37(4):401-7.

Misfeld 1998 \{published data only\}

Misfeld M, Dubbert S, Eleftheriadis S, Siemens HJ, Wagner T, Sievers HH. Fibrinolysis-adjusted perioperative low-dose aprotinin reduces blood loss in bypass operations. Annals of Thoracic Surgery 1998;66(3):792-9

\section{Mohr 1992 \{published data only\}}

Mohr R, Goor DA, Lusky A, Lavee J. Aprotinin prevents cardiopulmonary bypass-induced platelet dysfunction. A scanning electron microscope study. Circulation 1992;86(5:Suppl):II405-9.

Mongan 1998 \{published data only\}

Mongan PD, Brown RS, Thwaites BK. Tranexamic acid and aprotinin reduce postoperative bleeding and transfusions during primary coronary revascularization. Anesthesia \& Analgesia 1998;87 (2):258-65.

\section{Moran 2000 \{published data only\}}

Moran SV, Lema G, Medel J, Irarrazaval MJ, Zalaquett R, Garayar B, Flaskamp R. Comparison of two doses of aprotinin in patients receiving aspirin before coronary bypass surgery. Perfusion 2000;15 (2):105-10. 
Murkin 1994 \{published data only\}

Murkin JM, Lux J, Shannon NA, Guiraudon GM, Menkis AH, McKenzie FN, Novick RJ. Aprotinin significantly decreases bleeding and transfusion requirements in patients receiving aspirin and undergoing cardiac operations. Journal of Thoracic \& Cardiovascular Surgery 1994;107(2):554-61.

\section{Murkin 1995 \{published data only\}}

Murkin JM, Shannon NA, Bourne RB, Rorabeck CH, Cruickshank M, Wyile G. Aprotinin decreases blood loss in patients undergoing revision or bilateral total hip arthroplasty. Anesthesia \& Analgesia 1995;80(2):343-8.

\section{Murkin 2000 \{published data only\}}

Murkin JM, Haig GM, Beer KJ, Cicutti N, McCutchen J, Comunale ME, Hall R, Ruzicka BB. Aprotinin decreases exposure to allogeneic blood during primary unilateral total hip replacement. Journal of Bone \& Joint Surgery 2000;82(5):675-84.

Murphy 2006 \{published data only\}

Murphy GJ, Mango E, Lucchetti V, Battaglia F, Catapano D, Rogers $\mathrm{CA}$, et al.A randomized trial of tranexamic acid in combination with cell salvage plus a meta-analysis of randomized trials evaluating tranexamic acid in off-pump coronary artery bypass grafting. Cardiopulmonary \& Physiology 2006;132(3):475-80.

Niskanen 2005 \{published data only\}

Niskanen RO, Korkala OL. Tranexamic acid reduces blood loss in cemented hip arthroplasty: a randomized, double-blind study of 39 patients with osteoarthritis. Acta Orthopaedica 2005;76(6):829-32.

Norman 2009 \{published data only\}

Norman P H, Thall P F, Purugganan R V, Riedel B J, Thakar D R, Rice D C, et al.A possible association between aprotinin and improved survival after radical surgery for mesothelioma. Cancer 2009;115(4):833-41

\section{Nurözler 2008 \{published data only\}}

Nurözler F, Kutlu T, Küçük G. Aprotinin for patients exposed to clopidogrel before off-pump coronary bypass. Asian cardiovascular \& thoracic annals 2008;16(6):483-7.

Nuttall 2000 \{published data only\}

Nuttall GA, Oliver WC, Ereth MH, Santrach PJ, Bryant SC, Orszulak TA, Schaff HV. Comparison of blood-conservation strategies in cardiac surgery patients at high risk for bleeding. Anesthesiology 2000;92(3):674-82.

\section{Okita 1996 \{published data only\}}

Okita Y, Takamoto S, Ando M, Morota T, Yamaki F, Kawashima Y. Is use of aprotinin safe with deep hypothermic circulatory arrest in aortic surgery? Investigations on blood coagulation. Circulation 1996;94(Suppl):II177-81.

\section{Orpen 2006 \{published data only\}}

Orpen NM, Little C, Walker G, Crawfurd EJP. Tranexamic acid reduces early post-operative blood loss after total knee arthroplasty: A prospective randomised controlled trial of 29 patients. Knee 2006;13(2):106-10.

Palmer 2003 \{published data only\} Palmer JD, Francis JL, Pickard JD, Iannotti F. The efficacy and safety of aprotinin for hemostasis during intracranial surgery. Journal of Neurosurgery 2003;98(6):1208-16.

\section{Parvizi 2007 \{published data only\}}

Parvizi R, Azarfarin R, Hassanzadeh S. Ultra-low dose aprotinin effects on reducing the need for blood transfusion in cardiac surgery. Saudi Medical Journal 2007;28(1):49-53.

\section{Penta de Peppo 1995 \{published data only\}}

Penta de Peppo A, Pierri MD, Scafuri A, De Paulis R, Colantuono G, Caprara E, Tomai F, Chiariello L. Intraoperative antifibrinolysis and blood-saving techniques in cardiac surgery. Prospective trial of 3 antifibrinolytic drugs. Texas Heart Institute Journal 1995;22(3): 231-6.

Petsatodis 2006 \{published data only\}

Petsatodis G, Samoladas E, Christodoulou A, Hatzokos I, Pournaras I. Does aprotinin reduce blood loss in total hip arthroplasty?. Orthopedics 2006;29(1):75-7.

Pinosky 1997 \{published data only\}

Pinosky ML, Kennedy DJ, Fishman RL, Reeves ST, Alpert CC, Ecklund J, Kribbs S, Spinale FG, Kratz JM, Crawford R, Gravlee GP, Dorman BH. Tranexamic acid reduces bleeding after cardiopulmonary bypass when compared to epsilon aminocaproic acid and placebo. Journal of Cardiac Surgery 1997;12(5):330-8.

Pleym 2003 \{published data only\}

Pleym H, Stenseth R, Wahba A, Bjella L, Karevold A, Dale O. Single-dose tranexamic acid reduces postoperative bleeding after coronary surgery in patients treated with aspirin until surgery. Anesthesia \& Analgesia 2003;96(4):923-8.

Porte 2000 \{published data only\}

Porte RJ, Molenaar IQ, Begliomini B, Groenland THN, Januszkiewicz A, Lindgren L, Palareti G, Hermans J, Terpstra OT. Aprotinin and transfusion requirements in orthotopic liver transplantation: a multicentre randomised double-blind study. Lancet 2000;355(9212):1303-9.

Poston 2006 \{published data only\}

Poston RS, White C, Gu J, Brown J, Gammie J, Pierson RN, et al.Aprotinin shows both hemostatic and antithrombotic effects during off-pump coronary artery bypass grafting. Annals of Thoracic Surgery 2006;81(1):104-11.

\section{Prendergast 11996 \{published data only\}} Prendergast TW, Furukawa S, Beyer AJ, 3rd, Eisen HJ, McClurken JB, Jeevanandam V. Defining the role of aprotinin in heart transplantation. Annals of Thoracic Surgery 1996;62(3):670-4.

Prendergast 21996 \{published data only\} Prendergast TW, Furukawa S, Beyer AJ, 3rd, Eisen HJ, McClurken $\mathrm{JB}$, Jeevanandam V. Defining the role of aprotinin in heart transplantation. Annals of Thoracic Surgery 1996;62(3):670-4.

Pugh 1995 \{published data only\}

Pugh SC, Wielogorski AK. A comparison of the effects of tranexamic acid and low-dose aprotinin on blood loss and homologous blood usage in patients undergoing cardiac surgery. Journal of Cardiothoracic \& Vascular Anesthesia 1995;9:240-4.

Ranaboldo 1997 \{published data only\} Ranaboldo CJ, Thompson JF, Davies JN, Shutt AM, Francis JN, Roath OS, Webster JHH, Chant ADB. Prospective randomized placebo-controlled trial of aprotinin for elective aortic reconstruction. British Journal of Surgery 1997;84(8):1110-3. 
Rao 1999 \{published data only\}

Rao BH, Saxena N, Chauhan S, Sashikanth M. Use of EAminocaproic acid in the management of aspirin related postoperative bleeding in patients undergoing coronary revascularization. Journal of Anaesthesiology Clinical Pharmacology 1999;15(3):261-4.

\section{Ray 1997 \{published data only\}}

Ray MJ, Marsh NA, Just SJ, Perrin EJ, O’Brien MF, Hawson GA. Preoperative platelet dysfunction increases the benefit of aprotinin in cardiopulmonary bypass. Annals of Thoracic Surgery 1997;63(1): 57-63.

\section{Ray 1999 \{published data only\}}

Ray MJ, Brown KF, Burrows CA, O’Brien MF. Economic evaluation of high-dose and low-dose aprotinin therapy during cardiopulmonary bypass. Annals of Thoracic Surgery 1999;68(3): $940-5$.

\section{Ray 2001 \{published data only\}}

Ray MJ, O’Brien MF. Comparison of epsilon aminocaproic acid and low-dose aprotinin in cardiopulmonary bypass: efficiency, safety and cost. Annals of Thoracic Surgery 2001;71(3):838-43.

\section{Ray 2005 \{published data only\}}

Ray M, Hatcher S, Whitehouse SL, Crawford S, Crawford R. Aprotinin and epsilon aminocaproic acid are effective in reducing blood loss after primary total hip arthroplasty--a prospective randomized double-blind placebo-controlled study. Journal of Thrombosis \& Haemostasis 2005;3(7):1421-7.

Rhydderch 1993 \{published data only\}

Rhydderch RD, Khan B, Saleh A. Single dose Aprotinin in routine cardiac surgery. Middle East Journal of Anesthesiology 1993;12(3): 287-97.

Rocha 1994 \{published data only\}

Rocha E, Hidalgo F, Llorens R, Melero JM, Arroyo JL, Paramo JA. Randomized study of aprotinin and DDAVP to reduce postoperative bleeding after cardiopulmonary bypass surgery. Circulation 1994;90(2):921-7.

Rodrigus 1996 \{published data only\}

Rodrigus IE, Vermeyen KM, De Hert SG, Amsel BJ, Walter PJ. Efficacy and safety of aprotinin in aortocoronary bypass and valve replacement operations: a placebo-controlled randomized doubleblind study. Perfusion 1996;11(4):313-8.

Rossi 1997 \{published data only\}

Rossi M, Storti S, Martinelli L, Varano C, Marra R, Zamparelli R, Possati G, Schiavello R. A pump-prime aprotinin dose in cardiac surgery: appraisal of its effects on the hemostatic system. Journal of Cardiothoracic \& Vascular Anesthesia 1997;11(7):835-9.

Royston 1987 \{published data only\}

Royston D, Bidstrup BP, Taylor KM, Sapsford RN. Effect of aprotinin on need for blood transfusion after repeat open-heart surgery. Lancet 1987;2(8571):1289-91.

\section{Sadeghi 2007 \{published data only\}}

Sadeghi M, Mehr-Aein A. Does a single bolus dose of tranexamic acid reduce blood loss and transfusion requirements during hip fracture surgery? A prospective randomized double blind study in 67 patients. Acta Medica Iranica 2007;45(6):437-42.
Samama 2002 \{published data only\}

Samama CM, Langeron O, Rosencher N, Capdevila X, Rouche P, Pegoix M, Berniere J, Coriat P. Aprotinin versus placebo in major orthopedic surgery: a randomized, double-blinded, dose-ranging study. Anesthesia \& Analgesia 2002;95(2):287-93.

Santamaria 2000 \{published data only\} Santamaria A, Mateo J, Oliver A, Litvan H, Murillo J, Souto JC, Fontcuberta J. The effect of two different doses of aprotinin on hemostasis in cardiopulmonary bypass surgery: similar transfusion requirements and blood loss. Haematologica 2000;85(12):1277-84.

Santos 2006 \{published data only\}

Santos ATL, Kalil RAK, Bauemann C, Pereira JB, Nesralla IA. A randomized, double-blind, and placebo-controlled study with tranexamic acid of bleeding and fibrinolytic activity after primary coronary artery bypass grafting. Brazilian Journal of Medical హ Biological Research 2006;39(1):63-9.

Schmartz 2003 \{published data only\}

Schmartz D, Tabardel Y, Preiser JC, Barvais L, d'Hollander A, Duchateau J, Vincent JL. Does aprotinin influence the inflammatory response to cardiopulmonary bypass in patients?. Journal of Thoracic \& Cardiovascular Surgery 2003;125(1):184-90.

\section{Schweizer 2000 \{published data only\}} Schweizer A, Hohn L, Morel DR, Kalangos A, Licker M. Aprotinin does not impair renal haemodynamics and function after cardiac surgery. British Journal of Anaesthesia 2000;84(1):16-22.

Shore-Lesserson 1996 \{published data only\}

Shore-Lesserson L, Reich DL, Vela-Cantos F, Ammar T, Ergin MA. Tranexamic acid reduces transfusions and mediastinal drainage in repeat cardiac surgery. Anesthesia \& Analgesia 1996;83(1):18-26.

Sorin 1999 \{published data only\}

Sorin A, Claeys MA, Jansen A, D'Haese J, Camu F. Reduction of blood loss by tranexamic acid in total knee replacement. Journal of Bone \& Joint Surgery - British Volume. 81-B 1999; Vol. 81-B, issue Suppl.II:234.

Speekenbrink 1995 \{published data only\}

Speekenbrink RG, Vonk AB, Wildevuur CR, Eijsman L. Hemostatic efficacy of dipyridamole, tranexamic acid, and aprotinin in coronary bypass grafting. Annals of Thoracic Surgery 1995;59(2):438-42.

Speekenbrink 1996 \{published data only\} Speekenbrink RGH, Wildevuur CRH, Sturk A, Eijsman L. Lowdose and high-dose aprotinin improve hemostasis in coronary operations. Journal of Thoracic \& Cardiovascular Surgery 1996;112 (2):523-30.

\section{Stammers 1997 \{published data only\}}

Stammers AH, Huffman S, Alonso A, Fristoe LW, Hill G, Casebeer D, Diego RP, Song Z. The antiinflammatory effects of aprotinin in patients undergoing cardiac surgery with cardiopulmonary bypass. Journal of Extra-Corporeal Technology 1997;29(3):114-22.

Stewart 2001 \{published data only\}

Stewart A, Newman L, Sneddon K, Harris M. Aprotinin reduces blood loss and the need for transfusion in orthognathic surgery. British Journal of Oral \& Maxillofacial Surgery 2001;39(5):365-70. 
Swart 1994 \{published data only\}

Swart MJ, Gordon PC, Hayse-Gregson PB, Dyer RA, Swanepoel AL, Buckels NJ, Schall R, Odell JA. High-dose aprotinin in cardiac surgery--a prospective, randomized study. Anaesthesia \& Intensive Care 1994;22(5):529-33.

Tabuchi 1994 \{published data only\}

Tabuchi N, Huet RC, Sturk A, Eijsman L, Wildevuur CR. Aprotinin preserves hemostasis in aspirin-treated patients undergoing cardiopulmonary bypass. Annals of Thoracic Surgery 1994;58(4):1036-9.

Taggart 2003 \{published data only\}

Taggart DP, Djapardy V, Naik M, Davies A. A randomized trial of aprotinin (Trasylol) on blood loss, blood product requirement, and myocardial injury in total arterial grafting. Journal of Thoracic \& Cardiovascular Surgery 2003;126(4):1087-94.

Taghaddomi 2009 \{published data only\}

Taghaddomi RJ Mirzaee A Attar AS Shirdel A. Tranexamic Acid Reduces Blood Loss in Off-Pump Coronary Artery Bypass Surgery. Journal of Cardiothoracic and Vascular Anesthesia 2009;23(3):312-5.

Tanaka 2001 \{published data only\}

Tanaka N, Sakahashi H, Sato E, Hirose K, Ishima T, Ishii S. Timing of the administration of tranexamic acid for maximum reduction in blood loss in arthroplasty of the knee. Journal of Bone \& Joint Surgery - British Volume 2001;83(5):702-5.

Tassani 2000 \{published data only\}

Tassani P, Augustin N, Barankay A, Braun SL, Zaccaria F, Richter JA. High-dose aprotinin modulates the balance between proinflammatory and anti-inflammatory responses during coronary artery bypass graft surgery. Journal of Cardiothoracic \& Vascular Anesthesia 2000;14(6):682-6.

Thorpe 1994 \{published data only\}

Thorpe CM, Murphy WG, Logan M. Use of aprotinin in knee replacement surgery. British Journal of Anaesthesia 1994;73(3): $408-10$.

Trinh-Duc 1992 \{published data only\}

Trinh-Duc P, Wintrebert P, Boulfroy D, Albat B, Thevenet A, Roquefeuil B. Comparison of the effects of epsilon-aminocaproic acid and aprotinin on intra- and postoperative bleeding in heart surgery. [French]. Annales de Chirurgie 1992;46(8):677-683.

Troianos 1999 \{published data only\}

Troianos CA, Sypula RW, Lucas DM, D’Amico F, Mathie TB, Desai M, Pasqual RT, Pellegrini RV, Newfeld ML. The effect of prophylactic epsilon-aminocaproic acid on bleeding, transfusions, platelet function and fibrinolysis during coronary artery bypass grafting. Anesthesiology 1999;91(2):430-5.

Turkoz 2001 \{published data only\}

Turkoz A, Cigli A, But K, Sezgin N, Turkoz R, Gulcan O, Ersoy $\mathrm{MO}$. The effects of aprotinin and steroids on generation of cytokines during coronary artery surgery. Journal of Cardiothoracic \& Vascular Anesthesia 2001;15(5):603-10.

Uozaki 2001 \{published data only\}

Uozaki Y, Watanabe G, Kotou K, Ueyama K, Doi Y, Misaki T.

Effect of tranexamic acid on blood loss reduction after cardiopulmonary bypass. Japanese Journal of Thoracic \& Cardiovascular Surgery 2001;49(5):273-8.
Urban 2001 \{published data only\}

Urban MK, Beckman J, Gordon M, Urquhart B, Boachie-Adjei O. The efficacy of antifibrinolytics in the reduction of blood loss during complex adult reconstructive spine surgery. Spine 2001;26 (10):1152-6.

Utada 1997 \{published data only\}

Utada K, Matayoshi Y, Sumi C, Itaya M, Miyawaki H, Ito M, Tamura H, Mitsukuji T. Aprotinin 2 million KIU reduces perioperative blood loss in patients undergoing primary total hip replacement. [Japanese]. Masui - Japanese Journal of Anesthesiology 1997;46(1):77-82.

Van der Linden 2005 \{published data only\} Van der Linden J, Lindvall G, Sartipy U. Aprotinin decreases postoperative bleeding and number of transfusions in patients on clopidogrel undergoing coronary artery bypass graft surgery: A double-blind, placebo-controlled, randomized clinical trial. Circulation 2005;112(Suppl I):I-276-80.

Van Oeveren 1987 \{published data only\} Van Oeveren W, Jansen NJ, Bidstrup BP, Royston D, Westaby S, Neuhof H, Wildevuur CR. Effects of aprotinin on hemostatic mechanisms during cardiopulmonary bypass. Annals of Thoracic Surgery 1987;44(6):640-5.

Vander-Salm 1996 \{published data only\} Vander Salm TJ, Kaur S, Lancey RA, Okike ON, Pezzella AT, Stahl RF, Leone L, Li J-M, Valeri CR, Michelson AD. Reduction of bleeding after heart operations through the prophylactic use of epsilon-aminocaproic acid. Journal of Thoracic \& Cardiovascular Surgery 1996;112(4):1098-107.

Vanek 2005 \{published data only\}

Vanek T, Jares M, Fajt R, Straka Z, Jirasek K, Kolesar M, Brucek P, Maly M. Fibrinolytic inhibitors in off-pump coronary surgery: A prospective, randomized, double-blind TAP study (tranexamic acid, aprotinin, placebo). European Journal of Cardio-Thoracic Surgery 2005;28(4):563-8.

Vedrinne 1992 \{published data only\}

Vedrinne C, Girard C, Jegaden O, Blanc P, Bouvier H, Ffrench P, Mikaeloff P, Estanove S. Reduction in blood loss and blood use after cardiopulmonary bypass with high-dose aprotinin versus autologous fresh whole blood transfusion. Journal of Cardiothoracic \& Vascular Anesthesia 1992;6(3):319-23.

Veien 2002 \{published data only\}

Veien M, Sorensen JV, Madsen F, Juelsgaard P. Tranexamic acid given intraoperatively reduces blood loss after total knee replacement: a randomized, controlled study. Acta Anaesthesiologica Scandinavica 2002;46(10):1206-11.

Wei 2006 \{published data only\}

Wei M, Jian K, Guo Z, Wang L, Jiang D, Zhang L, Tarkka M. Tranexamic acid reduces postoperative bleeding in off-pump coronary artery bypass grafting. Scandinavian Cardiovascular Journal 2006; 40 (2):105-9.

Wendel 1995 \{published data only\} Wendel HP, Heller W, Michel J, Mayer G, Ochsenfahrt C, Graeter U, Schulze J, Hoffmeister HM, Hoffmeister HE. Lower cardiac troponin $\mathrm{T}$ levels in patients undergoing cardiopulmonary bypass and receiving high-dose aprotinin therapy indicate reduction of 
perioperative myocardial damage. Journal of Thoracic \& Cardiovascular Surgery 1995;109(6):1164-72.

Wong 2000 \{published data only\}

Wong BI, McLean RF, Fremes SE, Deemar KA, Harrington EM, Christakis GT, Goldman BS. Aprotinin and tranexamic acid for high transfusion risk cardiac surgery. Annals of Thoracic Surgery 2000;69(3):808-16.

Wong 2008 \{published data only\}

Wong J, El-Beheiry H, Rampersaud R, Lewis S, Fehlings M, Chung F. Tranexamic acid reduces blood loss and transfusion in adult patients having spinal fusion surgery. Canadian Journal of Surgery 2007;50(3 Suppl):S12-3.

* Wong J, El Beheiry H, Rampersaud Y R, Lewis S, Ahn H, De Silva Y, et al.Tranexamic Acid reduces perioperative blood loss in adult patients having spinal fusion surgery. Anesthesia and analgesia 2008;107(5):1479-86.

Wu 2006 \{published data only\}

Wu CC, Ho WM, Cheng SB, Yeh DC, Wen MC, Liu TJ, et al.Perioperative parenteral tranexamic acid in liver tumor resection: a prospective randomized trial toward a "blood transfusion"-free hepatectomy. Annals of Surgery 2006;243(2):173-80.

Yamasaki 2004 \{published data only\} Yamasaki S, Masuhara K, Fuji T. Tranexamic acid reduces blood loss after cementless total hip arthroplasty-prospective randomized study in 40 cases. International Orthopaedics 2004;28(2):69-73.

Yassen 1993 \{published data only\}

Yassen K, Bellamy MC, Sadek SA, Webster NR. Tranexamic acid reduces blood loss during orthotopic liver transplantation. Clinical Transplantation 1993;7(5):453-8.

Zabeeda 2002 \{published data only\}

Zabeeda D, Medalion B, Sverdlov M, Ezra S, Schachner A, Ezri T, Cohen AJ. Tranexamic acid reduces bleeding and the need for blood transfusion in primary myocardial revascularization. Annals of Thoracic Surgery 2002;74(3):733-8.

Zhang 2007 \{published data only\}

Zhang F, Gao Z, Yu J. [Clinical comparative studies on effect of tranexamic acid on blood loss associated with total knee arthroplasty]. [Chinese]. Chinese Journal of Reparative \& Reconstructive Surgery 2007;21(12):1302-4.

Zohar 2004 \{published data only\}

Zohar E, Ellis M, Ifrach N, Stern A, Sapir O, Fredman B. The postoperative blood-sparing efficacy of oral versus intravenous tranexamic acid after total knee replacement. Anesthesia \& Analgesia 2004;99(6):1679-83.

\section{References to studies excluded from this review}

Fejer 1998 \{published data only\}

Fejer C, Mezofi M, Varga PP. Use of high-dose aprotinin during spinal surgery results in a reduction in both blood loss and the amount of blood transfused. [abstract]. British Journal of Anaesthesia 1998;80(Suppl 1):80.

Langdown 2000 \{published data only\} Langdown AJ, Field J, Grote J, Himayat H. Aprotinin (Trasylol) does not reduce bleeding in primary total hip arthroplasty. Journal of Arthroplasty 2000;15(8):1009-12.
Montesano 1996 \{published data only\}

Montesano RM, Gustafson PA, Palanzo DA, Manley NJ, Sadr FS. The effect of low-dose epsilon-aminocaproic acid on patients following coronary artery bypass surgery. Perfusion 1996;11(1): 53-6.

Zufferey 2010 \{published data only\}

Zufferey P J, Miquet M, Quenet S, Laporte S, Martin P, Chambefort V, et al.Does tranexamic acid decrease erythrocyte transfusion in patients undergoing hip fracture surgery with fondaaparinux for prevention of venous thromboembolism?. http:/ /www isth2007 com/ 2007.

Zufferey PJ Miquet M Quenet S Martin P Adam P Albaladejo P Mismetti P Molliex S. Tranexamic acid in hip fracture surgery: A randomized controlled trial. British Journal of Anaesthesia 2010;104 (1):23-30.

\section{References to ongoing studies}

Myles 2008 \{published data only\}

Myles P S, Smith J, Knight J, Cooper D J, Silbert B, McNeil J, et al.Aspirin and Tranexamic Acid for Coronary Artery Surgery (ATACAS) Trial: rationale and design. American heart journal 2008;155(2):224-30.

Verma 2010 \{published data only\}

Verma K, Errico TJ, Vaz KM, Lonner BS. A prospective, randomized, double-blinded single-site control study comparing blood loss prevention of tranexamic acid (TXA) to epsilon aminocaproic acid (EACA) for corrective spinal surgery. BMC Surgery 2010;10(13).

\section{Additional references}

\section{Beattie 2006}

Beattie WS, Karkouti K. Con: Aprotinin has a good efficacy and safety profile relative to other alternatives for prevention of bleeding in cardiac surgery. [comment]. Anesthesia \& Analgesia 2006;103(6): $1360-4$.

\section{Bidstrup 2006}

Bidstrup BP. Aprotinin and tranexamic acid in high-transfusionrisk cardiac surgery. Transfusion 2006;46(12):2208-10.

\section{Blumberg 1997}

Blumberg N. Allogeneic transfusion and infection: economic and clinical implications. Seminars in Hematology 1997;34(3 Suppl 2): 34-40.

Body 2006

Body SC, Mazer CD. Pro: Aprotinin has a good efficacy and safety profile relative to other alternatives for prevention of bleeding in cardiac surgery. Anesthesia \& Analgesia 2006;103(6):1354-9.

Bryson 1998

Bryson GL, Laupacis A, Wells GA. Does acute normovolemic hemodilution reduce perioperative allogeneic transfusion? A metaanalysis. The International Study of Perioperative Transfusion. Anesthesia \& Analgesia 1998;86(1):9-15.

Carless 2005

Carless PA, Moxey AJ, Stokes BJ, Henry DA. Are antifibrinolytic drugs equivalent in reducing blood loss and transfusion in cardiac 
surgery? A meta-analysis of randomized head-to-head trials. BMC Cardiovascular Disorders 2005;5:19.

\section{Carson 1998}

Carson JL, Terrin ML, Barton FB, Aaron R, Greenburg AG, Heck DA, Magaziner J, Merlino FE, Bunce G, McClelland B, et al.A pilot randomized trial comparing symptomatic vs. hemoglobinlevel-driven red blood cell transfusions following hip fracture. Transfusion 1998;38(6):522-9.

\section{Coyle 1999}

Coyle D, Lee KM, Fergusson DA, Laupacis A. Economic analysis of erythropoietin use in orthopaedic surgery. Transfusion Medicine 1999;9(1):21-30.

\section{CRASH-2 2010}

Shakur H, Roberts I, Bautista R, Caballero J, Coats T, Dewan Y, et al.Effects of tranexamic acid on death, vascular occlusive events, and blood transfusion in trauma patients with significant haemorrhage (CRASH-2): a randomised, placebo-controlled trial. Lancet 2010;376(9734):23-32. [PUBMED: 20554319]

\section{Der Simonian 1986}

Der Simonian R, Laird N. Meta-analysis in clinical trials. Controlled Clinical Trials 1986;7:177-88.

\section{Faught 1998}

Faught C, Wells P, Fergusson D, Laupacis A. Adverse effects of methods for minimizing perioperative allogeneic transfusion: a critical review of the literature. Transfusion Medicine Reviews 1998; 12(3):206-25.

Ferguson 2007

Ferguson TB, Jr. Aprotinin--are there lessons learned?. JAMA 2007;297(5):527-9.

\section{Fergusson 1999}

Fergusson D, van Walraven C, Coyle D, Laupacis A. Economic evaluations of technologies to minimize perioperative transfusion: a systematic review of published studies. International Study of Perioperative Transfusion (ISPOT) investigators. Transfusion Medicine Reviews 1999;13(2):106-17.

\section{Forgie 1998}

Forgie MA, Wells PS, Laupacis A, Fergusson D. Pre-operative Autologous Donation deceases allogeneic transfusion but increases exposure to all red cell transfusion. Results of a Meta-Analysis. Archives of Internal Medicine 1998;158(6):610-16.

\section{Fritz 1983}

Fritz H, Wunderer G. Biochemistry and applications of aprotinin, the kallikrein inhibitor from bovine organs. Arzneimittel-Forschung 1983;33(4):479-94.

\section{Hebert 1999}

Hebert PC, Wells G, Blajchman MA, Marshall J, Martin C, Pagliarello G, et al.A multicenter, randomized, controlled clinical trial of transfusion requirements in critical care. Transfusion Requirements in Critical Care Investigators, Canadian Critical Care Trials Group. New England Journal of Medicine 1999;340(6): 409-17.

\section{Hebert 2005}

Hebert P, Fergusson DA, BART Investigators. Blood conservation using antifibrinolytics: A randomized trial in a cardiac surgery population - the BART study. (http://www.controlled-trials.com/ ISRCTN15166455/BART) 2005.

\section{Henry 1999}

Henry DA, Moxey AJ, Carless PA, O'Connell D, McClelland B, Henderson KM, et al.Anti-fibrinolytic use for minimising perioperative allogeneic blood transfusion. Cochrane Database of Systematic Reviews 1999, Issue 4. [DOI: 10.1002/ 14651858.CD001886.pub2]

\section{Henry 2007}

Henry DA, Carless PA, Moxey AJ, O’Connell D, Stokes BJ, McClelland B, et al.Anti-fibrinolytic use for minimising perioperative allogeneic blood transfusion. Cochrane Database of Systematic Reviews 2007, Issue 4. Art. No.: CD001886. DOI: 10.1002/14651858.CD001886.pub2.. [PUBMED: 17943760][Art. No.: CD001886. DOI: 10.1002/ 14651858.CD001886.pub3]

\section{Higgins 2002}

Higgins JP, Thompson SG. Quantifying heterogeneity in a metaanalysis. Statistics in Medicine 2002;21 (11):1539-58.

\section{Higgins 2009}

Higgins JPT, Green S. Cochrane Handbook for Systematic Reviews of Interventions. The Cochrane Collaboration. Chichester, UK: Wiley \& Sons, 2009.

Huet 1999

Huet C, Salmi LR, Fergusson D, Koopman-van Gemert AW, Rubens F, Laupacis A. A meta-analysis of the effectiveness of cell salvage to minimize perioperative allogeneic blood transfusion in cardiac and orthopedic surgery. International Study of Perioperative Transfusion (ISPOT) Investigators. Anesthesia \& Analgesia 1999;89(4):861-9.

Karkouti 2004

Karkouti K, Wijeysundera DN, Yau TM, Beattie WS, Abdelnaem E, McCluskey SA, et al.The independent association of massive blood loss with mortality in cardiac surgery. Transfusion 2004;44 (10):1453-62.

\section{Karkouti 2006}

Karkouti K, Beattie WS, Dattilo KM, McCluskey SA, Ghannam M, Hamdy A, et al.A propensity score case-control comparison of aprotinin and tranexamic acid in high-transfusion-risk cardiac surgery. Transfusion 2006;46(3):327-38.

\section{Kimball 1995}

Kimball AM, Berkley S, Ngugi E, Gayle H. International aspects of the AIDS/HIV epidemic. Annual Review of Public Health 1995;16: 253-82.

\section{Laupacis 1997}

Laupacis A, Fergusson D. Drugs to minimize perioperative blood loss in cardiac surgery: meta-analyses using perioperative blood transfusion as the outcome. The International Study of Perioperative Transfusion (ISPOT) Investigators. Anesthesia \& Analgesia 1997;85(6):1258-67.

\section{Levi 1999}

Levi M, Cromheecke ME, de Jonge E, Prins MH, de Mol BJM, Briet E, et al.Pharmacological strategies to decrease excessive blood loss in cardiac surgery: a meta-analysis of clinically relevant endpoints. Lancet 1999;354(9194):1940-7. 
Levy 2006

Levy JH, Ramsay JG, Guyton RA. Aprotinin in cardiac surgery. New England Journal of Medicine 2006; Vol. 354, issue 18 1953-7.

\section{Mangano 2006}

Mangano DT, Tudor IC, Dietzel C, Multicenter Study of Perioperative Ischemia Research Group, Ischemia Research and Education Foundation. The risk associated with aprotinin in cardiac surgery. New England Journal of Medicine 2006;354(4): 353-65.

\section{Mangano 2007}

Mangano DT, Miao Y, Vuylsteke A, Tudor IC, Juneja R, Filipescu $\mathrm{D}$, et al. Investigators of The Multicenter Study of Perioperative Ischemia Research Group, Ischemia Research and Education Foundation. Mortality associated with aprotinin during 5 years following coronary artery bypass graft surgery. JAMA 2007;297(5): 471-9.

Mannucci 1998

Mannucci PM. Hemostatic drugs. New England Journal of Medicine 1998;339(4):245-53.

\section{McFarland 1997}

McFarland W, Mvere D, Shandera W, Reingold A. Epidemiology and prevention of transfusion-associated human immunodeficiency virus transmission in sub-Saharan Africa. Vox Sanguinis 1997;72 (2):85-92.

\section{Munoz 1999}

Munoz JJ, Birkmeyer NJ, Birkmeyer JD, O’Connor GT, Dacey LJ. Is epsilon-aminocaproic acid as effective as aprotinin in reducing bleeding with cardiac surgery?: a meta-analysis. Circulation 1999; 99(1):81-9.

\section{O'Brien 2002}

O’Brien MF, Harrocks S, Clarke A, Garlick B, Barnett AG. How to do safe sternal reentry and the risk factors of redo cardiac surgery: a 21-year review with zero major cardiac injury. Journal of Cardiac Surgery 2002;17(1):4-13.

\section{Review Manager}

The Nordic Cochrane Centre. Review Manager. 4.2.8.

Copenhagen: The Cochrane Collaboration, 2003.

\section{Royston 1998}

Royston D. Aprotinin versus lysine analogues: the debate continues. Annals of Thoracic Surgery 1998;65(4):S9-19.

\section{Schneeweiss 2008}

Schneeweiss S, Seeger JD, Landon J, Walker AM. Aprotinin during coronary-artery bypass grafting and risk of death. The New England journal of medicine 2008;358(8):771-83. [PUBMED: 18287600]

\section{Schulz 1995}

Schulz KF, Chalmers I, Hayes RJ, Altman DJ. Empirical evidence of bias. Dimensions of methodological quality associated with estimates of treatment effects in controlled trials. JAMA 1995;273 (5):408-12.

\section{Sedrakyan 2004}

Sedrakyan A, Treasure T, Elefteriades JA. Effect of aprotinin on clinical outcomes in coronary artery bypass graft surgery: a systematic review and meta-analysis of randomized clinical trials. Journal of Thoracic \& Cardiovascular Surgery 2004;128(3):442-8.

\section{Smith 1998}

Smith CR. Management of bleeding complications in redo cardiac operations. Annals of Thoracic Surgery 1998;65(4):S2-8; discussion S27-8

\section{Vamvakas 2001}

Vamvakas EC, Blajchman MA. Deleterious clinical effects of transfusion-associated immunomodulation: fact or fiction?. Blood 2001;97(5):1180-95.

\section{Vamvakas 2004}

Vamvakas EC. White-blood-cell-containing allogeneic blood transfusion, postoperative infection and mortality: a meta-analysis of observational 'before-and-after' studies. Vox Sanguinis 2004;86 (2):111-9.

\section{Whyte 1997}

Whyte GS, Savoia HF. The risk of transmitting HCV, HBV or HIV by blood transfusion in Victoria. Medical Journal of Australia 1997;166(11):584-6.

* Indicates the major publication for the study 


\section{CHARACTERISTICS OF STUDIES}

\section{Characteristics of included studies [ordered by study ID]}

\section{Alajmo 1989}

\begin{tabular}{|c|c|c|}
\hline Methods & \multicolumn{2}{|c|}{$\begin{array}{l}\text { Patients were randomly divided into two groups according to birth date until an appropriate number } \\
\text { of treated patients was reached. Method of blinding and generation of allocation sequences were } \\
\text { not described. }\end{array}$} \\
\hline Participants & \multicolumn{2}{|c|}{$\begin{array}{l}34 \text { consecutive patients undergoing cardiac operations were randomly divided into two groups: } \\
\text { - Aprotinin group: } \mathrm{n}=22, \mathrm{M} / \mathrm{F}=12 / 8 \text {, mean }(\mathrm{sd}) \text { age }=62(6.6) \text { years } \\
\text { - Control group: } \mathrm{n}=12, \mathrm{M} / \mathrm{F}=7 / 5 \text {, mean }(\mathrm{sd}) \text { age }=57.8(16.3) \text { years } \\
\text { NB: Possible error in the gender data provided for the aprotinin group. }\end{array}$} \\
\hline Interventions & \multicolumn{2}{|c|}{$\begin{array}{l}\text { - Aprotinin group received } 2 \text { million kallikrein inactivation units (KIU) of aprotinin ( } 280 \mathrm{mg} \text { ) } \\
\text { at the start of anaesthesia (Trasylol, Bayer Leverkusen, FRG; } 10,000 \mathrm{KIU} / \mathrm{ml} \text { pure aprotinin with } \\
\text { no additives) infused over } 20 \text { to } 30 \text { minutes. Subsequently, 500,000 KIU/hr }(70 \mathrm{mg} / \mathrm{hr} \text { ) of } \\
\text { aprotinin was given until the end of the operation. Additionally, } 1 \mathrm{million} \mathrm{KIU} \text { of aprotinin ( } 140 \\
\mathrm{mg} \text { ) was given via the priming solution of the extracorporeal circuit. } \\
\text { - Control group did not receive aprotinin. }\end{array}$} \\
\hline Outcomes & \multicolumn{2}{|c|}{$\begin{array}{l}\text { Outcomes reported: Number of patients exposed to allogeneic blood, blood loss, haemoglobin } \\
\text { levels, platelet counts }\end{array}$} \\
\hline Notes & \multicolumn{2}{|c|}{$\begin{array}{l}\text { Quality assessment score (Schulz criteria): 2/7 } \\
\text { Transfusion protocol used }\end{array}$} \\
\hline \multicolumn{3}{|l|}{ Risk of bias } \\
\hline Item & Authors' judgement & Description \\
\hline Adequate sequence generation? & No & $\begin{array}{l}\text { Patients were randomly allocated into two } \\
\text { groups according to birth date until an appro- } \\
\text { priate number of treated patients was reached }\end{array}$ \\
\hline Allocation concealment? & No & Inadequate \\
\hline $\begin{array}{l}\text { Blinding? } \\
\text { All outcomes }\end{array}$ & No & \\
\hline
\end{tabular}

\section{Alderman 1998}

\section{Methods}

Participants
Patients were randomly divided into two groups by random code, generated in blocks with clinical center and stratum. Allocation concealment was not described.

870 patients were randomised into two groups:

- Aprotonin group $\mathrm{n}=436, \mathrm{M}=87.4 \%$, mean $(\mathrm{sd})$ age $=61.8(9.1)$ years

- Control group (Placebo) $\mathrm{n}=434, \mathrm{M}=86.9 \%$, mean (sd) age $=62.3$ (9.1) years 
Alderman 1998 (Continued)

\begin{tabular}{|c|c|c|}
\hline Interventions & \multicolumn{2}{|c|}{$\begin{array}{l}\text { - Aprotonin group received a loading dose of } 2 \text { million KIU }(280 \mathrm{mg}) \text {, a maintenance dose of } \\
\text { 500,000 KIU and a prime dose of } 2 \text { million KIU. } \\
\text { - No details were described on the placebo used. }\end{array}$} \\
\hline Outcomes & \multicolumn{2}{|c|}{$\begin{array}{l}\text { Outcomes reported: Number of patients exposed to allogeneic blood, blood loss, deaths, myocardial } \\
\text { infarction, CABG thrombosis, re-operation for bleeding. }\end{array}$} \\
\hline Notes & \multicolumn{2}{|c|}{$\begin{array}{l}\text { Quality assessment score (Schulz criteria): 3/7 } \\
\text { Transfusion protocol used }\end{array}$} \\
\hline \multicolumn{3}{|l|}{ Risk of bias } \\
\hline Item & Authors' judgement & Description \\
\hline Adequate sequence generation? & Yes & Random code generated in blocks \\
\hline Allocation concealment? & Unclear & Unclear \\
\hline $\begin{array}{l}\text { Blinding? } \\
\text { All outcomes }\end{array}$ & Yes & Double blind \\
\hline
\end{tabular}

Alvarez 1995

\begin{tabular}{l|l} 
Methods & $\begin{array}{l}\text { The hospital pharmacy made up identical infusions of the study drugs identifiable only by random } \\
\text { number. Patients were prospectively randomised into two groups by sealed envelopes. The method } \\
\text { used to generate allocation sequences was not described. }\end{array}$ \\
\hline Participants & $\begin{array}{l}100 \text { patients undergoing primary elective cardiac surgery were randomised into one of two groups: } \\
\text { - Aprotinin group: } \mathrm{n}=49, \mathrm{M} / \mathrm{F}=38 / 11, \text { mean }(\mathrm{sd}) \text { age }=63.3 \text { (11.0) years } \\
\text { - Control group (Placebo): } \mathrm{n}=51, \mathrm{M} / \mathrm{F}=34 / 17, \text { mean }(\mathrm{sd}) \text { age }=62.7 \text { (8.2) years }\end{array}$ \\
\hline Interventions & $\begin{array}{l}\text { - Aprotinin group received } 250,000 \text { kallikrein inactivation units (KIU) of aprotinin added to } \\
\text { the prime solution of the cardiopulmonary bypass (CPB) system. Before the start of CPB a } \\
\text { further } 250,000 \text { KIU of aprotinin, made up to } 100 \text { ml with } 0.9 \% \text { saline, was infused } \\
\text { intravenously over } 30 \text { minutes. } \\
\text { - Control group received a placebo of equal volumes of } 0.9 \% \text { saline administered at identical } \\
\text { times. } \\
\text { NB: Both the intervention and control group were combined with cell salvage. }\end{array}$ \\
\hline Outcomes & $\begin{array}{l}\text { Outcomes reported: } \text { Number of patients exposed to allogeneic blood, patients receiving autotrans- } \\
\text { fusion, blood loss, mortality, myocardial infarctions, re-operation, patients receiving cell salvage. }\end{array}$ \\
\hline Notes & $\begin{array}{l}\text { Quality assessment score (Schulz criteria): } 5 / 7 \\
\text { Transfusion protocol used }\end{array}$ \\
\hline
\end{tabular}

Risk of bias 
Alvarez 1995 (Continued)

\begin{tabular}{l|ll}
\hline Item & Authors' judgement & Description \\
\hline Adequate sequence generation? & Unclear & Unclear \\
\hline Allocation concealment? & No & $\begin{array}{l}\text { Inadequate - sealed envelopes were used to con- } \\
\text { ceal treatment allocation }\end{array}$ \\
\hline $\begin{array}{l}\text { Blinding? } \\
\text { All outcomes }\end{array}$ & Yes & Double blind \\
\hline
\end{tabular}

\begin{tabular}{|c|c|c|}
\hline Methods & \multicolumn{2}{|c|}{$\begin{array}{l}\text { Patients were randomised by a computer-generated random number sequence into either treatment } \\
\text { group. All clinical participants were double blinded until the completion of the trial. Placebo and } \\
\text { treatment solutions were identical in their appearance and packaging. }\end{array}$} \\
\hline Participants & \multicolumn{2}{|c|}{$\begin{array}{l}55 \text { patients undergoing either elective or urgent cardiac surgery were randomised into one of two } \\
\text { groups: } \\
\text { - Aprotonin group: } \mathrm{n}=26, \mathrm{M} / \mathrm{F}=23 / 3 \text {, mean }(\mathrm{sd}) \text { age }=63(8) \text { years } \\
\text { - Control group (Placebo): } \mathrm{n}=29, \mathrm{M} / \mathrm{F}=22 / 7 \text {, mean (sd) age }=64(8) \text { years }\end{array}$} \\
\hline Interventions & \multicolumn{2}{|c|}{$\begin{array}{l}\text { - Aprotinin group received } 250,000 \text { kallikrein inactivation units (KIU) of aprotinin } 280 \mathrm{mg} \\
\text { IV at the time of sternal skin closure. } \\
\text { - Control group received a placebo of an equal volume of normal saline solution infused over } \\
20 \text { mins. }\end{array}$} \\
\hline Outcomes & \multicolumn{2}{|c|}{$\begin{array}{l}\text { Outcomes reported: Number of patients exposed to allogeneic blood, patients receiving autotrans- } \\
\text { fusion, blood loss, mortality, myocardial infarctions, re-operation. }\end{array}$} \\
\hline Notes & \multicolumn{2}{|c|}{$\begin{array}{l}\text { Quality assessment score (Schulz criteria): 7/7 } \\
\text { Transfusion protocol used }\end{array}$} \\
\hline \multicolumn{3}{|l|}{ Risk of bias } \\
\hline Item & Authors' judgement & Description \\
\hline Adequate sequence generation? & Yes & Computer-generated random number sequence \\
\hline Allocation concealment? & Yes & Adequate \\
\hline $\begin{array}{l}\text { Blinding? } \\
\text { All outcomes }\end{array}$ & Yes & Double blind \\
\hline
\end{tabular}




\section{Alvarez 2008}

\begin{tabular}{|c|c|c|}
\hline Methods & \multicolumn{2}{|c|}{$\begin{array}{l}\text { Patients were allocated according to a computer-generated randomisation sequence. Allocation was } \\
\text { concealed using sealed, numbered envelopes. }\end{array}$} \\
\hline Participants & \multicolumn{2}{|c|}{$\begin{array}{l}95 \text { patients undergoing orthopaedic (knee arthroplasty) surgery. Patients were randomly allocated } \\
\text { to one of two groups: } \\
\text { - Tranexamic acid group: } \mathrm{n}=46, \mathrm{M} / \mathrm{F}=7 / 39 \text {, mean (sd) age }=71 \text { (9) years } \\
\text { - Control group (Placebo): } \mathrm{n}=49, \mathrm{M} / \mathrm{F}=10 / 39 \text {, mean (sd) age }=72(7) \text { years }\end{array}$} \\
\hline Interventions & \multicolumn{2}{|c|}{$\begin{array}{l}\text { - Tranexamic acid group received bolus of } 10 \mathrm{mg} / \mathrm{kg} \text { before deflation of tourniquet then } \\
\text { infusion of } 1 \mathrm{mg} / \mathrm{kg} / \mathrm{hr} \text { starting at the end of operation for six hours post-operation. } \\
\text { - Control group received saline. }\end{array}$} \\
\hline Outcomes & \multicolumn{2}{|c|}{$\begin{array}{l}\text { Outcomes reported: Number of patients requiring blood transfusion, blood loss, volume of blood } \\
\text { transfused, thrombosis. }\end{array}$} \\
\hline Notes & \multicolumn{2}{|c|}{$\begin{array}{l}\text { Quality assessment score (Schulz criteria): 5/7 } \\
\text { Transfusion protocol used }\end{array}$} \\
\hline \multicolumn{3}{|l|}{ Risk of bias } \\
\hline Item & Authors' judgement & Description \\
\hline Adequate sequence generation? & Yes & Computer-generated random number sequence \\
\hline Allocation concealment? & No & $\begin{array}{l}\text { Inadequate - randomised assignment was sealed } \\
\text { in a numbered envelope }\end{array}$ \\
\hline $\begin{array}{l}\text { Blinding? } \\
\text { All outcomes }\end{array}$ & Yes & Double blind \\
\hline
\end{tabular}

Amar 2003

Methods

Randomisation of patients in blocks of 20 were done by the Biostatistics Department and the hospital pharmacy using sealed, opaque treatment code envelopes.

\begin{tabular}{l|l}
\hline Participants & $\begin{array}{l}69 \text { patients undergoing elective orthopaedic surgery were randomised into one of three groups: } \\
\text { - Epsilon aminocaproic acid group: } \mathrm{n}=22, \mathrm{M} / \mathrm{F}=11 / 11, \text { mean }(\mathrm{sd}) \text { age }=53(18) \text { years } \\
\text { - Aprotinin group: } \mathrm{n}=23, \mathrm{M} / \mathrm{F}=13 / 10, \text { mean }(\mathrm{sd}) \text { age }=48(17) \text { years } \\
\text { - Control group (Placebo): } \mathrm{n}=24, \mathrm{M} / \mathrm{F}=13 / 11, \text { mean }(\mathrm{sd}) \text { age }=55(16) \text { years }\end{array}$ \\
\hline Interventions & $\begin{array}{l}\text { - Epsilon aminocaproic acid (EACA) group received } 150 \mathrm{mg} / \mathrm{kg} \text { EACA bolus in an equal } \\
\text { volume given over } 30 \text { minutes followed by an infusion of } 15 \mathrm{mg} / \mathrm{kg} / \mathrm{hr} \text { until the end of surgery. } \\
\text { - Aprotinin group received a bolus of } 2 \mathrm{million} \mathrm{KIU}(280 \mathrm{mg}) \text { given over } 30 \text { minutes followed } \\
\text { by an infusion of } 500,000 \mathrm{KIU} / \text { hour }(70 \mathrm{mg} / \mathrm{hr}) \text { until the end of surgery. } \\
\text { - Control group received a placebo of an equal volume of normal saline bolus and infusion. }\end{array}$
\end{tabular}




\begin{tabular}{|c|c|c|}
\hline Outcomes & \multicolumn{2}{|c|}{$\begin{array}{l}\text { Outcomes reported: } \text { Number of patients exposed to allogeneic blood, allogeneic blood units - tota } \\
\text { includes intra-operative \& } 48 \text { hours post-operative, blood loss - total blood loss = intra-operative } \\
\& 48 \text { hours post-operative, deep venous thrombosis, pulmonary embolus, hospital length of stay } \\
\text { (days), wound infection, thrombocytopenia, Haemoglobin levels (pre-operative \& post-operative) }\end{array}$} \\
\hline Notes & \multicolumn{2}{|c|}{$\begin{array}{l}\text { Quality assessment score (Schulz criteria): 6/7 } \\
\text { Transfusion protocol used }\end{array}$} \\
\hline \multicolumn{3}{|l|}{ Risk of bias } \\
\hline Item & Authors' judgement & Description \\
\hline Adequate sequence generation? & Yes & Computer-generated random numbers \\
\hline Allocation concealment? & No & $\begin{array}{l}\text { Inadequate - sealed opaque treatment coded en- } \\
\text { velopes were used to conceal treatment alloca- } \\
\text { tion }\end{array}$ \\
\hline $\begin{array}{l}\text { Blinding? } \\
\text { All outcomes }\end{array}$ & Yes & Double blind \\
\hline
\end{tabular}

\section{Andreasen 2004}

\begin{tabular}{l|l} 
Methods & $\begin{array}{l}\text { Patients were randomised by a random number sequence. The randomisation schedule was provided } \\
\text { in sealed envelopes and preparation of the drug or placebo was carried out just prior to anaesthesia } \\
\text { by a staff member not involved in the treatment of the patient. }\end{array}$ \\
\hline Participants & $\begin{array}{l}46 \text { patients undergoing elective coronary surgery. Patients were randomly allocated to one of two } \\
\text { groups: } \\
\text { - Tranexamic acid group }(\mathrm{n}=21), \mathrm{M} / \mathrm{F}=18 / 3, \text { mean age }(+/-\mathrm{SD})=62.3(9.5) \text { years } \\
\text { - Control group (Placebo) }(\mathrm{n}=23), \mathrm{M} / \mathrm{F}=19 / 4, \text { mean age }(+/-\mathrm{SD})=63.8(7.6) \text { years }\end{array}$ \\
\hline Interventions & $\begin{array}{l}\text { - Tranexamic acid group }(\mathrm{TXA}) \text { group }(\text { Cyklokapron, Phizer Consumer Healthcare) received } \\
\text { infusion of } 200 \mathrm{mg} / \mathrm{hr} \text { until additional } 1.5 \mathrm{~g} \text { was given. } \\
\text { - Control group received a placebo of } 0.9 \% \text { normal saline solution. }\end{array}$ \\
\hline $\begin{array}{l}\text { NB: Cell salvage - postoperatively shed mediastinal blood was returned in all patients using a closed } \\
\text { autotransfusion system. }\end{array}$ \\
\hline
\end{tabular}

Outcomes

Outcomes reported: Number of patients exposed to allogeneic blood, blood loss, deaths, myocardial Infarctions, CABG thrombosis, renal insufficiency, re-operation for bleeding, cell salvage autotransfusion 6 hrs, transient ischemic attack (30 day), stroke 30 day. 


\begin{tabular}{lll} 
Risk of bias & & \\
\hline Item & Authors' judgement & Description \\
\hline Adequate sequence generation? & Yes & Random number sequence \\
\hline Allocation concealment? & No & $\begin{array}{l}\text { Inadequate - used sealed envelopes to conceal } \\
\text { treatment allocation }\end{array}$ \\
\hline $\begin{array}{l}\text { Blinding? } \\
\text { All outcomes }\end{array}$ & Yes & Double blind \\
\hline
\end{tabular}

\section{Apostolakis 2008}

\begin{tabular}{|c|c|c|}
\hline Methods & \multicolumn{2}{|c|}{$\begin{array}{l}\text { A randomisation table was used to generate the allocation sequence. No information was provided } \\
\text { regarding allocation concealment. }\end{array}$} \\
\hline Participants & \multicolumn{2}{|c|}{$\begin{array}{l}59 \text { patients undergoing elective thoracic surgery. Patients were randomly allocated to one of two } \\
\text { groups: } \\
\text { - Aprotinin group: } \mathrm{n}=29, \mathrm{M} / \mathrm{F}=26 / 3 \text {, mean }(\mathrm{sd}) \text { age }=57.5(16.3) \text { years } \\
\text { - Control group (Placebo): } \mathrm{n}=30, \mathrm{M} / \mathrm{F}=27 / 3 \text {, mean }(\mathrm{sd}) \text { age }=58.5(9.8) \text { years }\end{array}$} \\
\hline Interventions & \multicolumn{2}{|c|}{$\begin{array}{l}\text { - Aprotinin group, administered immediately after intubation, received a test dose of } 1 \mathrm{ml} \text { then } \\
500,000 \mathrm{KIU} \text { intravenously in } 50 \mathrm{ml} \text { of solution over } 15 \text { minutes, received the same dose again } \\
\text { after thoracotomy closure. } \\
\text { - Control group received a placebo of an equal volume of normal saline. }\end{array}$} \\
\hline Outcomes & \multicolumn{2}{|c|}{$\begin{array}{l}\text { Outcomes reported: Blood loss, volume of transfused blood (units), mortality, re-operation for } \\
\text { bleeding, length of hospital stay (days). }\end{array}$} \\
\hline Notes & \multicolumn{2}{|c|}{$\begin{array}{l}\text { Quality assessment score (Schulz criteria): 5/7 } \\
\text { Transfusion protocol used }\end{array}$} \\
\hline \multicolumn{3}{|l|}{ Risk of bias } \\
\hline Item & Authors' judgement & Description \\
\hline Adequate sequence generation? & Yes & Random number table \\
\hline Allocation concealment? & Unclear & Unclear \\
\hline $\begin{array}{l}\text { Blinding? } \\
\text { All outcomes }\end{array}$ & Yes & Double blind \\
\hline
\end{tabular}


Armellin 2001

\begin{tabular}{|c|c|c|}
\hline Methods & \multicolumn{2}{|c|}{ Method of randomisation and allocation concealment were not described. } \\
\hline Participants & \multicolumn{2}{|c|}{$\begin{array}{l}300 \text { patients undergoing elective cardiac surgery were randomised to one of two groups: } \\
\text { - Tranexamic acid group: } \mathrm{n}=150, \mathrm{M} / \mathrm{F}=71 / 72, \text { mean }(\mathrm{sd}) \text { age }=65.7(11.7) \text { years } \\
\text { - Control group (Placebo): } \mathrm{n}=150, \mathrm{M} / \mathrm{F}=90 / 50 \text {, mean (sd) age }=65.9(12.8) \text { years }\end{array}$} \\
\hline Interventions & \multicolumn{2}{|c|}{$\begin{array}{l}\text { - Tranexamic acid group (TXA) received } 2.5 \mathrm{~g} \text { of TXA before the skin incision with a further } \\
\text { 2.5g of TXA added to the CPB prime solution. } \\
\text { - Control group received a placebo of an equal dose of saline at the same times as TXA. }\end{array}$} \\
\hline Outcomes & \multicolumn{2}{|c|}{$\begin{array}{l}\text { Outcomes reported: Number of patients exposed to allogeneic blood, allogeneic blood usage (units) } \\
\text {, blood loss, mortality, myocardial infarction, re-operation for bleeding, hospital length of stay } \\
\text { (days), fresh frozen plasma (FFP), platelets (units). }\end{array}$} \\
\hline Notes & \multicolumn{2}{|c|}{$\begin{array}{l}\text { Quality assessment score (Schulz criteria): 3/7 } \\
\text { Transfusion protocol used }\end{array}$} \\
\hline \multicolumn{3}{|l|}{ Risk of bias } \\
\hline Item & Authors' judgement & Description \\
\hline Adequate sequence generation? & Unclear & Not reported \\
\hline Allocation concealment? & Unclear & Unclear \\
\hline $\begin{array}{l}\text { Blinding? } \\
\text { All outcomes }\end{array}$ & Yes & Double blind \\
\hline
\end{tabular}

\section{Arom 1994}

\begin{tabular}{l|l}
\hline Methods & Method of randomisation and allocation concealment were not described. \\
\hline Participants & $\begin{array}{l}\text { 200 patients undergoing cardiac surgery were randomised to one of two groups: } \\
\text { - Epsilon aminocaproic acid group: } \mathrm{n}=100, \mathrm{M} / \mathrm{F}=70 / 30, \text { mean age }=60 \text { years } \\
\text { - Control group: } \mathrm{n}=100, \mathrm{M} / \mathrm{F}=71 / 29, \text { mean age }=55 \text { years }\end{array}$ \\
\hline Interventions & $\begin{array}{l}\text { CPB. Epsilon aminocaproic acid group received } 5 \mathrm{~g} \text { of intravenous EACA just before going on } \\
\text { - Control group did not receive EACA treatment. }\end{array}$ \\
\hline NB: Both groups received 0.03ug/kg of intravenous desmopressin (DDAVP) after CPB.
\end{tabular}

\section{Risk of bias}


Arom 1994 (Continued)

\begin{tabular}{lll}
\hline Item & Authors' judgement & Description \\
\hline Adequate sequence generation? & Unclear & Unclear \\
\hline Allocation concealment? & Unclear & Unclear \\
\hline $\begin{array}{l}\text { Blinding? } \\
\text { All outcomes }\end{array}$ & Unclear & Unclear \\
\hline
\end{tabular}

\section{Ashraf 1997}

\begin{tabular}{|c|c|c|}
\hline Methods & \multicolumn{2}{|c|}{ Method of randomisation and allocation concealment were not described. } \\
\hline Participants & \multicolumn{2}{|c|}{$\begin{array}{l}38 \text { patients undergoing coronary artery bypass graft surgery were randomised to one of two groups: } \\
\text { - Aprotinin group: } \mathrm{n}=19, \mathrm{M} / \mathrm{F}=16 / 3 \text {, median (range) age }=61(49-72) \text { years } \\
\text { - Control group: } \mathrm{n}=19, \mathrm{M} / \mathrm{F}=15 / 4 \text {, median (range) age }=65(50-79) \text { years }\end{array}$} \\
\hline Interventions & \multicolumn{2}{|c|}{$\begin{array}{l}\text { - Aprotinin group received } 2 \text { million KIU }(280 \mathrm{mg}) \text { of aprotinin added to the pump prime } \\
\text { solution of the extracorporeal circuit. } \\
\text { - Control group did not receive aprotinin. }\end{array}$} \\
\hline Outcomes & \multicolumn{2}{|c|}{$\begin{array}{l}\text { Outcomes reported: Number of patients exposed to allogeneic blood, allogeneic blood usage (units) } \\
\text {, blood loss } 24 \mathrm{hrs,} \mathrm{mortality,} \mathrm{re-exploration} \mathrm{for} \mathrm{bleeding,} \mathrm{pro-inflammatory} \mathrm{cytokine} \mathrm{levels.}\end{array}$} \\
\hline Notes & \multicolumn{2}{|c|}{$\begin{array}{l}\text { Quality assessment score (Schulz criteria): } 2 / 7 \\
\text { Transfusion protocol used }\end{array}$} \\
\hline \multicolumn{3}{|l|}{ Risk of bias } \\
\hline Item & Authors' judgement & Description \\
\hline Adequate sequence generation? & Unclear & Unclear \\
\hline Allocation concealment? & Unclear & Unclear \\
\hline $\begin{array}{l}\text { Blinding? } \\
\text { All outcomes }\end{array}$ & Unclear & Unclear \\
\hline
\end{tabular}

\section{Asimakopoulos 2000}

\begin{tabular}{l|l}
\hline Methods & Method of randomisation and allocation concealment not specified. \\
\hline Participants & $\begin{array}{l}18 \text { adults undergoing elective coronary artery bypass grafting were randomly allocated to one of } \\
\text { two groups: } \\
\text { - Aprotinin group: } \mathrm{n}=8, \mathrm{M} / \mathrm{F}=7 / 1, \text { mean }(\mathrm{sd}) \text { age }=59(3.9) \text { years } \\
\text { - Control group: } \mathrm{n}=10, \mathrm{M} / \mathrm{F}=10 / 0, \text { mean }(\mathrm{sd}) \text { age }=65(1.9) \text { years }\end{array}$ \\
\hline
\end{tabular}


Asimakopoulos 2000 (Continued)

\begin{tabular}{|c|c|c|}
\hline Interventions & \multicolumn{2}{|c|}{$\begin{array}{l}\text { - Aprotinin group received full-dose aprotinin. } \\
\text { - Control group did not receive aprotinin. }\end{array}$} \\
\hline Outcomes & \multicolumn{2}{|c|}{$\begin{array}{l}\text { Outcomes reported: Allogeneic blood (units), blood loss, myocardial infarction, renal failure, re- } \\
\text { operation for bleeding, cerebrovascular accident (stroke), hospital length of stay (days). }\end{array}$} \\
\hline Notes & \multicolumn{2}{|c|}{ Quality assessment score (Schulz criteria): 4/7 } \\
\hline \multicolumn{3}{|l|}{ Risk of bias } \\
\hline Item & Authors' judgement & Description \\
\hline Adequate sequence generation? & Unclear & Unclear \\
\hline Allocation concealment? & Unclear & Unclear \\
\hline $\begin{array}{l}\text { Blinding? } \\
\text { All outcomes }\end{array}$ & Yes & Double blind \\
\hline
\end{tabular}

Baele 1992

Methods

Method of randomisation was not described. Allocation concealment was inadequately concealed (sealed envelopes).

\begin{tabular}{l|l}
\hline Participants & $\begin{array}{l}115 \text { consecutive adults undergoing cardiac surgery were randomly allocated to one of two groups: } \\
\text { - Aprotinin group: } \mathrm{n}=58, \mathrm{M} / \mathrm{F}=45 / 13, \text { mean }(\mathrm{sd}) \text { age }=61.6(9.6) \text { years } \\
\text { - Control group: } \mathrm{n}=57, \mathrm{M} / \mathrm{F}=41 / 16, \text { mean }(\mathrm{sd}) \text { age }=62.9(10.5) \text { years }\end{array}$ \\
\hline Interventions & $\begin{array}{l}\text { - Aprotinin group received } 2 \text { million kallikrein inactivator units (KIU) before incision, } 2 \\
\text { million (KIU) prior to bypass and a continuous infusion of } 500,000 \mathrm{KIU} / \mathrm{hr} \text { for } 5 \text { hours. } \\
\text { - Control group did not receive aprotinin. }\end{array}$ \\
NB: Both the intervention and control groups were exposed to pre-operative autologous donation \\
(7 control and 4 intervention patients), acute normovolemic haemodilution $(13$ patients in each \\
group), and/or cell salvage (data not presented).
\end{tabular}

Outcomes $\quad$ Outcomes reported: Number of patients exposed to allogeneic blood, allogeneic blood (units) blood loss, mortality, myocardial infarction, myocardial ischemia, pericarditis, cardiac failure, pneumonia, renal insufficiency, hemiplegia, re-operation, allogeneic + autologous blood usage (units), intensive care unit (ICU) length of stay (hrs), hospital length of stay (days).

Notes Quality assessment score (Schulz criteria): 3/7

Transfusion protocol used

\section{Risk of bias}

Item

Authors' judgement

\section{Description}


Baele 1992 (Continued)

\begin{tabular}{lll}
\hline Adequate sequence generation? & Unclear & Not reported \\
\hline $\begin{array}{l}\text { Allocation concealment? } \\
\text { No }\end{array}$ & Unclear & $\begin{array}{l}\text { Inadequate - sealed envelopes were used to con- } \\
\text { ceal treatment allocation }\end{array}$ \\
\hline $\begin{array}{l}\text { Alinding? } \\
\text { outcomes }\end{array}$ & Unclear \\
\hline
\end{tabular}

\section{Bailey 1994}

Methods $\begin{aligned} & \text { Generation of allocation sequences was by a computer generated random number table. One } \\ & \text { investigator made up all the test solutions; a known volume of sterile } 0.9 \% \text { saline was discarded from } \\ & 500 \mathrm{ml} \text { bags and replaced with the same volume of test solution so that all bags contained the same } \\ & \text { equal volume }(500 \mathrm{ml}) \text {. Each set of bags was given a consecutive number. A separate investigator } \\ & \text { performed all the patient measurements. }\end{aligned}$

Participants
$\begin{aligned} & 100 \text { patients scheduled to undergo primary elective cardiac surgery employing cardiopulmonary } \\ & \text { bypass were consecutively allocated to one of four groups. } \\ & \text { - Control group (Placebo): } \mathrm{n}=25, \mathrm{M} / \mathrm{F}=17 / 8, \text { mean }(\mathrm{sd}) \text { age }=63(10) \text { years } \\ & \text { - Aprotinin group (High dose): } \mathrm{n}=25, \mathrm{M} / \mathrm{F}=18 / 7, \text { mean }(\mathrm{sd}) \text { age }=64 \text { (13) years } \\ & \text { - Aprotinin group (Prime dose): } \mathrm{n}=24, \mathrm{M} / \mathrm{F}=17 / 7, \text { mean }(\mathrm{sd}) \text { age }=59(11) \text { years } \\ & \text { - Aprotinin group (Low dose) } \mathrm{n}=26, \mathrm{M} / \mathrm{F}=20 / 6, \text { mean }(\mathrm{sd}) \text { age }=63(10) \text { years }\end{aligned}$

\begin{tabular}{l} 
Interventions \\
$\begin{array}{l}\text { - Control group received a placebo of an intravenous bolus of } 500 \mathrm{ml} \text { of } 0.9 \% \text { saline at } \\
\text { induction of anaesthesia, followed by } 500 \mathrm{ml} \text { of } 0.9 \% \text { saline every hour; a further } 500 \mathrm{ml} \text { of } 0.9 \% \\
\text { saline was added to the pump prime. } \\
\text { - Aprotinin (High dose) group received an intravenous bolus of } 300 \mathrm{ml} \text { of } 0.9 \% \text { saline with } \\
200 \mathrm{ml} \text { of aprotinin ( } 2 \text { million kallikrein inactivator units) at induction of anaesthesia, followed by } \\
450 \mathrm{ml} \text { of } 0.9 \% \text { saline with } 50 \mathrm{ml} \text { aprotinin }(500,000 \mathrm{KIU}) \text { every hour; a further } 300 \mathrm{ml} \text { of } 0.9 \% \\
\text { saline with } 200 \mathrm{ml} \text { aprotinin }(2 \mathrm{million} \mathrm{KIU}) \text { was added to the pump prime. } \\
\text { - Aprotinin (Prime dose) group received an intravenous bolus of } 500 \mathrm{ml} \text { of } 0.9 \% \text { saline at } \\
\text { induction of anaesthesia, followed by } 500 \mathrm{ml} \text { of } 0.9 \% \text { saline every hour; a further } 300 \mathrm{ml} \text { of } 0.9 \% \\
\text { saline with } 200 \mathrm{ml} \text { of aprotinin }(2 \text { million KIU) was added to the prime pump. } \\
\text { - Aprotinin (Low dose) group received an intravenous bolus of } 400 \mathrm{ml} \text { of } 0.9 \% \text { saline with } \\
100 \mathrm{ml} \text { of aprotinin (1 million KIU) at induction of anaesthesia, followed by } 500 \mathrm{ml} \text { of } 0.9 \% \\
\text { saline every hour; a further } 400 \mathrm{ml} \text { of } 0.9 \% \text { saline with } 100 \mathrm{ml} \text { of aprotinin } 1 \mathrm{million} \mathrm{KIU}) \text { was } \\
\text { added to the pump prime. }\end{array}$ \\
\hline
\end{tabular}

Outcomes

Outcomes reported: Number of patients exposed to allogeneic blood, allogeneic blood (units), fresh frozen plasma usage (units), blood loss.

$\begin{array}{ll}\text { Notes } & \begin{array}{l}\text { Quality assessment score (Schulz criteria): } 7 / 7 \\ \text { Transfusion protocol used }\end{array}\end{array}$

\section{Risk of bias}

Item

Authors' judgement

\section{Description}


Bailey 1994 (Continued)

\begin{tabular}{l|ll}
\hline Adequate sequence generation? & Yes & $\begin{array}{l}\text { Generation of allocation sequences was by a } \\
\text { computer generated random number table }\end{array}$ \\
\hline $\begin{array}{l}\text { Allocation concealment? } \\
\begin{array}{l}\text { Blinding? } \\
\text { All outcomes }\end{array}\end{array}$ & Yes & Adequate \\
\hline
\end{tabular}

\section{Basora 1999}

\begin{tabular}{|c|c|c|}
\hline Methods & \multicolumn{2}{|c|}{ Method of randomisation and allocation concealment were not described. } \\
\hline Participants & \multicolumn{2}{|c|}{$\begin{array}{l}59 \text { patients undergoing elective cardiac surgery were randomised to one of three groups: } \\
\text { - Control group: } \mathrm{n}=21, \mathrm{M} / \mathrm{F}=16 / 5, \text { mean }(\mathrm{sd}) \text { age }=59.8(10.3) \text { years } \\
\text { - Aprotinin group (Low dose }-\mathrm{A} 2): \mathrm{n}=17, \mathrm{M} / \mathrm{F}=12 / 5 \text {, mean }(\mathrm{sd}) \text { age }=61.2(13.1) \text { years } \\
\text { - Aprotinin group (Low dose }-\mathrm{A} 4): \mathrm{n}=19, \mathrm{M} / \mathrm{F}=14 / 5 \text {, mean }(\mathrm{sd}) \text { age }=60.9(7.6) \text { years }\end{array}$} \\
\hline Interventions & \multicolumn{2}{|c|}{$\begin{array}{l}\text { - Control group did not receive aprotinin. } \\
\text { - Aprotinin group (Low dose - A2) received 14,286 KIU } / \mathrm{kg}(2 \mathrm{mg} / \mathrm{kg}) 15 \text { mins before surgery, } \\
\text { then a continuous dose of } 7,143 \mathrm{KIU} / \mathrm{kg} / \mathrm{hr}(1 \mathrm{mg} / \mathrm{kg} / \mathrm{hr}) \text { until the end of surgery. } \\
\text { - Aprotinin group (Low dose - A4) received } 28,572 \mathrm{KIU} / \mathrm{kg}(4 \mathrm{mg} / \mathrm{kg}) 15 \text { mins before surgery, } \\
\text { then a continuous dose of } 7,143 \mathrm{KIU} / \mathrm{kg} / \mathrm{hr}(1 \mathrm{mg} / \mathrm{kg} / \mathrm{hr}) \text { until the end of surgery. }\end{array}$} \\
\hline Outcomes & \multicolumn{2}{|c|}{ Outcomes reported: Number of patients exposed to allogeneic blood, blood loss, platelet function. } \\
\hline Notes & \multicolumn{2}{|c|}{$\begin{array}{l}\text { Quality assessment score (Schulz criteria): 2/7 } \\
\text { Transfusion protocol used }\end{array}$} \\
\hline \multicolumn{3}{|l|}{ Risk of bias } \\
\hline Item & Authors' judgement & Description \\
\hline Adequate sequence generation? & Unclear & Not reported \\
\hline Allocation concealment? & Unclear & Unclear \\
\hline $\begin{array}{l}\text { Blinding? } \\
\text { All outcomes }\end{array}$ & No & Single blind \\
\hline
\end{tabular}

\section{Bennett-Guerrero 1997}

Methods Patients were randomised by means of a computer-generated schedule. Study drug was prepared according to a protocol by hospital pharmacies.

Participants

204 patients undergoing repeat cardiac surgery were randomised to one of two groups:

- Aprotinin group (High dose): $\mathrm{n}=99, \mathrm{M} / \mathrm{F}=66 / 33$, mean (sd) age = 62 (14) years

- Epsilon aminocaproic acid group: $\mathrm{n}=105, \mathrm{M} / \mathrm{F}=68 / 37$, mean (sd) age $=63$ (12) years 
Bennett-Guerrero 1997 (Continued)

- Aprotinin group (High dose) received 2 million KIU (280mg) of aprotinin on skin incision,
500,000 KIU/hr as a continuous infusion for 4 hours on initiation of CPB. An additional 2
million KIU $(280 \mathrm{mg})$ was added to the CPB prime solution. Patients received $1 \mathrm{ml}$ of the study
drug in a blinded manner before the loading dose to test for possible allergy.
- Epsilon aminocaproic acid group received $150 \mathrm{mg} / \mathrm{kg}$ on skin incision, $30 \mathrm{mg} / \mathrm{kg}$ over $4 \mathrm{hours}$
as a continuous infusion on initiation of CPB. In addition, normal saline solution was added to
the CPB prime solution. Patients received $1 \mathrm{ml}$ of the study drug in a blinded manner before the
loading dose to test for possible allergy.
NB: Both groups were exposed to cell salvage.

Outcomes

Outcomes reported: Number of participants exposed to allogeneic blood, allogeneic blood usage (units), fresh frozen plasma usage (units), platelet usage (units), blood loss, re-operation for bleeding.

$\begin{array}{ll}\text { Notes } & \text { Quality assessment score (Schulz criteria): 7/7 } \\ \text { Transfusion protocol used }\end{array}$

\section{Risk of bias}

\begin{tabular}{l|ll}
\hline Item & Authors' judgement & Description \\
\hline Adequate sequence generation? & Yes & $\begin{array}{l}\text { Patients were randomised by means of a com- } \\
\text { puter-generated schedule }\end{array}$ \\
\hline $\begin{array}{l}\text { Allocation concealment? } \\
\begin{array}{l}\text { Blinding? } \\
\text { All outcomes }\end{array}\end{array}$ & Yes & Adequate \\
\hline
\end{tabular}

\section{Benoni 1996}

Methods
Randomisation into blocks of 12 was done by an independent pharmacologist who was not other-
or the placebo were numbered and packed into envelopes which were opened by the anaesthetist
before administration. These ampoules could be identified only by their numbers, and the ran-
domisation code was known only to the independent pharmacologist. The code was not broken
until the end of the study and until all data had been corrected and included in the database. Ten
patients were excluded from the study after randomisation.

\begin{tabular}{l|l} 
Participants & patients undergoing total knee arthroplasty were randomly allocated to one of two groups: \\
& - Tranexamic acid group: $\mathrm{n}=43, \mathrm{M} / \mathrm{F}=13 / 30$, mean $(\mathrm{sd})$ age $=76(7)$ years \\
- Control group (placebo): $\mathrm{n}=43, \mathrm{M} / \mathrm{F}=10 / 33$, mean $(\mathrm{sd})$ age $=74(7)$ years
\end{tabular}

Interventions

- TXA group received $10 \mathrm{mg} / \mathrm{kg}$ of TXA as a slow intravenous injection towards the end of the operation (median time 12 minutes - range 1-40 minutes) before deflation of the limb tourniquet. This dose was repeated after 3 hours from the other ampoule of the pair provided in the envelope.

- Control group received a placebo of equal volumes of normal saline solution $(0.9 \%)$.

NB: 15 patients from the placebo group received an extra dose of TXA for severe post-operative 
bleeding, these patients represented the 'placebo-extra' group.

\begin{tabular}{|c|c|c|}
\hline Outcomes & \multicolumn{2}{|c|}{$\begin{array}{l}\text { Outcomes reported: Number of participants exposed to allogeneic blood, allogeneic blood usage } \\
\text { (units), blood loss, deep venous thrombosis, pulmonary embolus, wound haematomas, chest pain, } \\
\text { haemoglobin concentrations. }\end{array}$} \\
\hline Notes & \multicolumn{2}{|c|}{$\begin{array}{l}\text { Quality assessment score (Schulz criteria): 4/7 } \\
\text { Transfusion protocol used }\end{array}$} \\
\hline \multicolumn{3}{|l|}{ Risk of bias } \\
\hline Item & Authors' judgement & Description \\
\hline Adequate sequence generation? & Yes & Adequate \\
\hline Allocation concealment? & Yes & Adequate \\
\hline $\begin{array}{l}\text { Blinding? } \\
\text { All outcomes }\end{array}$ & Yes & Double blind \\
\hline
\end{tabular}

Benoni 2000

\begin{tabular}{|c|c|c|}
\hline Methods & \multicolumn{2}{|c|}{$\begin{array}{l}\text { Medication was administered using numbered ampoules and the randomisation was performed by } \\
\text { a pharmacist not otherwise engaged in the study. }\end{array}$} \\
\hline Participants & \multicolumn{2}{|c|}{$\begin{array}{l}40 \text { patients undergoing total hip arthroplasty were randomly assigned to one of two groups: } \\
\text { - Tranexamic acid group: } n=20, M / F=6 / 14 \text {, mean (sd) age }=69.5(10) \text { years } \\
\text { - Control group (Placebo): } n=20, M / F=11 / 8 \text {, mean (sd) age }=68(10) \text { years }\end{array}$} \\
\hline Interventions & \multicolumn{2}{|c|}{$\begin{array}{l}\text { - Tranexamic acid group received } 10 \mathrm{mg} / \mathrm{kg} \text { IV of TXA (Cyklokapron) at the end of the } \\
\text { operation and received another } 10 \mathrm{mg} / \mathrm{kg} \text { IV } 3 \text { hours later. } \\
\text { - Control group received corresponding volumes of normal saline (placebo). }\end{array}$} \\
\hline Outcomes & \multicolumn{2}{|c|}{$\begin{array}{l}\text { Outcomes reported: Number of participants exposed to allogeneic blood, allogeneic blood usage } \\
\text { (units), blood loss, amount of pre-operative autologous donated blood ( } 2 \text { units), infection. }\end{array}$} \\
\hline Notes & \multicolumn{2}{|c|}{$\begin{array}{l}\text { Quality assessment score (Schulz criteria): 5/7 } \\
\text { Transfusion protocol used }\end{array}$} \\
\hline \multicolumn{3}{|l|}{ Risk of bias } \\
\hline Item & Authors' judgement & Description \\
\hline Adequate sequence generation? & Unclear & Unclear \\
\hline Allocation concealment? & Yes & Adequate \\
\hline
\end{tabular}


Benoni 2000 (Continued)

\begin{tabular}{|c|c|c|}
\hline $\begin{array}{l}\text { Blinding? } \\
\text { All outcomes }\end{array}$ & Yes & Double blind \\
\hline
\end{tabular}

Benoni 2001

\begin{tabular}{|c|c|c|}
\hline Methods & \multicolumn{2}{|c|}{$\begin{array}{l}\text { Method of randomisation was not described. Medication was concealed by a code only known by } \\
\text { the hospitals chief pharmacist who was not involved in the study. }\end{array}$} \\
\hline Participants & \multicolumn{2}{|c|}{$\begin{array}{l}40 \text { patients undergoing total hip arthroplasty were randomly assigned to one of two treatments } \\
\text { groups: } \\
\text { - Tranexamic acid group: } \mathrm{n}=20, \mathrm{M} / \mathrm{F}=9 / 9 \text {, mean }(\mathrm{sd}) \text { age }=66(9.5) \text { years } \\
\text { - Control group (Placebo): } \mathrm{n}=20, \mathrm{M} / \mathrm{F}=10 / 10 \text {, mean }(\mathrm{sd}) \text { age }=68(9.4) \text { years }\end{array}$} \\
\hline Interventions & \multicolumn{2}{|c|}{$\begin{array}{l}\text { - Tranexamic acid group received } 100 \mathrm{mg} / \mathrm{ml} \text { of TXA (Cyklokapron), } 10 \mathrm{mg} / \mathrm{kg} \text { (maximum } 1 \mathrm{~g} \text { ) } \\
\text { in a slow (5-10 minutes) IV injection immediately before the operation. } \\
\text { - Control group received a similar volume of saline as the same times as TXA. }\end{array}$} \\
\hline Outcomes & \multicolumn{2}{|c|}{$\begin{array}{l}\text { Outcomes reported: Number of participants exposed to allogeneic blood, allogeneic blood usage } \\
\text { (units), pulmonary embolus. }\end{array}$} \\
\hline Notes & \multicolumn{2}{|c|}{$\begin{array}{l}\text { Quality assessment score (Schulz criteria): } 5 / 7 \\
\text { Transfusion protocol used }\end{array}$} \\
\hline \multicolumn{3}{|l|}{ Risk of bias } \\
\hline Item & Authors' judgement & Description \\
\hline Adequate sequence generation? & Unclear & Unclear \\
\hline Allocation concealment? & Yes & $\begin{array}{l}\text { Adequate - medication was concealed by a code } \\
\text { only known by the hospitals chief pharmacist } \\
\text { who was not involved in the study }\end{array}$ \\
\hline $\begin{array}{l}\text { Blinding? } \\
\text { All outcomes }\end{array}$ & Yes & Double blind \\
\hline
\end{tabular}

\section{Berenholtz 2009}

Methods

Patients were randomised according to a computer-generated randomisation schedule. Allocation was concealed through central (pharmacy) allocation.

Participants

182 patients undergoing orthopaedic surgery were randomly allocated to one of two groups:

- EACA group: $\mathrm{n}=91, \mathrm{M} / \mathrm{F}=26 / 65$, mean (sd) age $=55.5$ (14.0) years

- Control group: $\mathrm{n}=91, \mathrm{M} / \mathrm{F}=29 / 62$, mean $(\mathrm{sd})$ age $=55.4(15.5)$ years 
Berenholtz 2009 (Continued)

\begin{tabular}{|c|c|c|}
\hline Interventions & \multicolumn{2}{|c|}{$\begin{array}{l}\text { - EACA group, received } 100 \mathrm{mg} / \mathrm{kg} \text { administered immediately after anaesthesia followed by } \\
\text { infusion of } 10 \mathrm{mg} / \mathrm{kg} / \mathrm{hr} \text { continued for } 8 \text { hours after surgery. } \\
\text { - Control group received saline. }\end{array}$} \\
\hline Outcomes & \multicolumn{2}{|c|}{$\begin{array}{l}\text { Outcomes reported: Number of patients receiving blood transfusion, volume of blood transfused } \\
\text { (units), blood loss, mortality, pulmonary embolism, myocardial infarction, renal failure, stroke, } \\
\text { thrombosis, deep vein thrombosis, length of hospital stay (days). }\end{array}$} \\
\hline Notes & \multicolumn{2}{|c|}{$\begin{array}{l}\text { Quality assessment score (Schulz criteria): 5/7 } \\
\text { Transfusion protocol used }\end{array}$} \\
\hline \multicolumn{3}{|l|}{ Risk of bias } \\
\hline Item & Authors' judgement & Description \\
\hline Adequate sequence generation? & Yes & $\begin{array}{l}\text { Patients were randomised according to a com- } \\
\text { puter-generated randomisation schedule }\end{array}$ \\
\hline Allocation concealment? & Yes & $\begin{array}{l}\text { Adequate - allocation was concealed through } \\
\text { central (pharmacy) allocation }\end{array}$ \\
\hline $\begin{array}{l}\text { Blinding? } \\
\text { All outcomes }\end{array}$ & Yes & Double blind \\
\hline
\end{tabular}

\section{Bernet 1999}

Methods

Participants

Participants

Interventions

Outcomes

Notes
Random code used for randomisation. Drug solutions were prepared by the hospital pharmacy.

70 patients were randomised to one of two groups:

- Tranexamic acid group: $\mathrm{n}=28, \mathrm{M} / \mathrm{F}=25 / 3$, mean (sd) age $=61.3(2.86)$ years

- Aprotinin group: $\mathrm{n}=28, \mathrm{M} / \mathrm{F}=24 / 4$, mean $(\mathrm{sd})$ age $=58.4$ (3.76) years

- Tranexamic group received $200 \mathrm{~mL}(10 \mathrm{~g})$ of TXA administered 20 minutes before sternotomy. Normal saline placebo was given at the same time as aprotinin doses for the purpose of blinding.

- Aprotinin group received $200 \mathrm{ml}(2$ million $\mathrm{KIU}=280 \mathrm{mg}$ ) of aprotinin administered 20 minutes before sternotomy and $200 \mathrm{~mL}(2$ million $\mathrm{KIU}=280 \mathrm{mg})$ administered as a continuous infusion of $50 \mathrm{ml} / \mathrm{hr}(500,000 \mathrm{KIU})$ until closure of the chest.

Outcomes reported: Number of patients exposed to allogeneic blood, allogeneic blood usage (units) , fresh frozen plasma (units), platelets (units), blood loss, mortality, myocardial Infarctions, haematocrit levels, stroke, thrombotic complications, re-exploration for bleeding.

Quality assessment score (Schulz criteria): 4/7

Transfusion protocol used. All patients received ASA until the day of the operation (100mg/day). All patients received cell salvage (Imed 960) - 8 hours post-operatively. 
Bernet 1999 (Continued)

\begin{tabular}{l|ll} 
Risk of bias & & \\
\hline Item & Authors' judgement & Description \\
\hline Adequate sequence generation? & Yes & Random code used for randomisation \\
\hline Allocation concealment? & Unclear & $\begin{array}{l}\text { Adequate - drug solutions were prepared by the } \\
\text { hospital pharmacy }\end{array}$ \\
\hline $\begin{array}{l}\text { Blinding? } \\
\text { All outcomes }\end{array}$ & Yes & Double blind \\
\hline
\end{tabular}

\section{Bert 2008}

\begin{tabular}{|c|c|c|}
\hline Methods & \multicolumn{2}{|c|}{$\begin{array}{l}\text { A computer-generated randomisation list was used to generate the allocation sequence. No infor- } \\
\text { mation was provided regarding allocation concealment. }\end{array}$} \\
\hline Participants & \multicolumn{2}{|c|}{$\begin{array}{l}50 \text { patients undergoing elective cardiac surgery were randomised to one of two groups: } \\
\text { - Aprotinin group: } \mathrm{n}=25, \mathrm{M} / \mathrm{F}=20 / 5 \text {, mean }(\mathrm{sd}) \text { age }=65.7(10.2) \text { years } \\
\text { - Control group: } \mathrm{n}=25, \mathrm{M} / \mathrm{F}=20 / 5 \text {, mean (sd) age }=67.8(8.3) \text { years }\end{array}$} \\
\hline Interventions & \multicolumn{2}{|c|}{$\begin{array}{l}\text { - Aprotinin group received loading dose of 2,000,000 KIU before sternotomy, then } \\
\text { continuous infusion of 500,000 KIU until wound closure. } \\
\text { - Control group did not receive aprotinin. }\end{array}$} \\
\hline Outcomes & \multicolumn{2}{|c|}{$\begin{array}{l}\text { Outcomes reported: Blood loss, volume of blood transfused (units), re-operation for bleeding, } \\
\text { inflammatory cytokines. }\end{array}$} \\
\hline Notes & \multicolumn{2}{|c|}{$\begin{array}{l}\text { Quality assessment score (Schulz criteria): } 4 / 7 \\
\text { Transfusion protocol was not reported }\end{array}$} \\
\hline \multicolumn{3}{|l|}{ Risk of bias } \\
\hline Item & Authors' judgement & Description \\
\hline Adequate sequence generation? & Yes & $\begin{array}{l}\text { A computer-generated randomisation list was } \\
\text { used to generate the allocation sequence }\end{array}$ \\
\hline Allocation concealment? & Unclear & Unclear \\
\hline $\begin{array}{l}\text { Blinding? } \\
\text { All outcomes }\end{array}$ & Unclear & Unclear \\
\hline
\end{tabular}


Bidstrup 1989

\begin{tabular}{|c|c|c|}
\hline Methods & \multicolumn{2}{|c|}{$\begin{array}{l}\text { The trial drug was provided by the manufacturer (Bayer AG, Leverkusen) in identical case packs, } \\
\text { each of } 12 \text { bottles identifiable only by the random number. Method of generating allocation se- } \\
\text { quences was not described. }\end{array}$} \\
\hline Participants & \multicolumn{2}{|c|}{$\begin{array}{l}80 \text { patients undergoing primary aorto-coronary bypass grafting were randomised to either one of } \\
\text { two groups: } \\
\text { - Aprotinin group: } \mathrm{n}=40, \mathrm{M} / \mathrm{F}=37 / 3 \text {, mean }(\mathrm{sd}) \text { age }=58.1(8.6) \text { years } \\
\text { - Control group (Placebo): } \mathrm{n}=37, \mathrm{M} / \mathrm{F}=32 / 5 \text {; mean (sd) age }=57.7(8.3) \text { years }\end{array}$} \\
\hline Interventions & \multicolumn{2}{|c|}{$\begin{array}{l}\text { - Aprotinin group received after induction of anaesthesia, a loading dose of } 280 \mathrm{mg} \text { of } \\
\text { aprotinin given intravenously through a central venous cannula over } 20 \text { mins, then a continuous } \\
\text { infusion of } 70 \mathrm{mg} / \mathrm{hr} \text { was begun and maintained until the patient left the operating theatre. In } \\
\text { addition to the intravenous infusion, another } 280 \mathrm{mg} \text { of aprotinin was added to the priming } \\
\text { volume of the heart lung machine by replacement of an aliquot of the priming volume. } \\
\text { - Control group received an equal volume of saline. } \\
\text { NB: Both intervention and control received preoperative autologous donation (PAD) }\end{array}$} \\
\hline Outcomes & \multicolumn{2}{|c|}{$\begin{array}{l}\text { Outcomes reported: Number of participants exposed to allogeneic blood, allogeneic blood usage } \\
\text { (units), blood loss ( } 18-24 \mathrm{hrs}) \text {, mortality. }\end{array}$} \\
\hline Notes & \multicolumn{2}{|c|}{$\begin{array}{l}\text { Quality assessment score (Schulz criteria): 5/7 } \\
\text { Transfusion protocol used }\end{array}$} \\
\hline \multicolumn{3}{|l|}{ Risk of bias } \\
\hline Item & Authors' judgement & Description \\
\hline Adequate sequence generation? & Unclear & Not reported \\
\hline Allocation concealment? & Yes & Adequate \\
\hline $\begin{array}{l}\text { Blinding? } \\
\text { All outcomes }\end{array}$ & Yes & Double blind \\
\hline
\end{tabular}

\section{Bidstrup 1990}

\begin{tabular}{l|l}
\hline Methods & Method of randomisation and allocation concealment were not described. \\
\hline Participants & $\begin{array}{l}44 \text { patients undergoing aortocoronary artery bypass graft surgery were randomly allocated to one } \\
\text { of two groups: } \\
\text { - Aprotinin group: } \mathrm{n}=26, \mathrm{M} / \mathrm{F}=21 / 5, \text { mean }(\mathrm{sd}) \text { age }=59(8) \text { years } \\
\text { - Control group: } \mathrm{n}=18, \mathrm{M} / \mathrm{F}=15 / 3, \text { mean }(\mathrm{sd}) \text { age }=58(8) \text { years }\end{array}$ \\
\hline Interventions & $\begin{array}{l}\text { - Aprotinin group received a loading dose of } 280 \mathrm{mg}(2 \mathrm{million} \mathrm{KIU}) \text { of aprotinin after } \\
\text { induction of anaesthesia and a constant infusion of } 70 \mathrm{mg} / \mathrm{hr} \text { during the operation. A further } \\
\text { 280mg was added to the pump prime. } \\
\text { - Control group did not receive aprotinin. }\end{array}$ \\
\hline
\end{tabular}


Bidstrup 1990 (Continued)

\begin{tabular}{|c|c|c|}
\hline Outcomes & \multicolumn{2}{|c|}{$\begin{array}{l}\text { Outcomes reported: Number of participants exposed to allogeneic blood, allogeneic blood usage } \\
\text { (units), fresh frozen plasma usage (units), platelets (units), blood loss (18-24hrs), re-operation for } \\
\text { bleeding. }\end{array}$} \\
\hline Notes & \multicolumn{2}{|c|}{$\begin{array}{l}\text { Quality assessment score (Schulz criteria): 2/7 } \\
\text { Transfusion protocol used }\end{array}$} \\
\hline \multicolumn{3}{|l|}{ Risk of bias } \\
\hline Item & Authors' judgement & Description \\
\hline Adequate sequence generation? & Unclear & Not reported \\
\hline Allocation concealment? & Unclear & Unclear \\
\hline $\begin{array}{l}\text { Blinding? } \\
\text { All outcomes }\end{array}$ & Unclear & Unclear \\
\hline
\end{tabular}

\section{Bidstrup 1993}

Methods Patients received aprotinin or placebo (normal saline) from identical bottles supplied by the manufacturer, identifiable only by their random number. Method of randomisation was not described.

Participants

96 adult male patients undergoing first-time isolated coronary bypass grafting were randomised to either one of two groups:

- Aprotinin group: $\mathrm{n}=47$, mean $(\mathrm{sd})$ age $=59.1(7.4)$ years

- Control group (Placebo): $\mathrm{n}=49$, mean $(\mathrm{sd})$ age $=58.8$ (8.5) years

NB: Six patients withdrew from the study, four in the aprotinin group and two in the placebo group.

- Aprotinin group received $280 \mathrm{mg}$ of aprotinin (contained in $200 \mathrm{ml}$ ) as a loading dose before
the commencement of bypass. An additional $280 \mathrm{mg}$ of aprotinin was added to the prime of the
heart-lung machine. A constant infusion of $70 \mathrm{mg} / \mathrm{hr}$ was maintained during the procedure until
skin closure.
- Control group (placebo) received identical volumes of normal saline.

Outcomes

Outcomes reported: Allogeneic blood usage (units), blood loss, haemoglobin levels, platelet counts, haemoglobin loss, activated clotting times, adverse events, graft patency, re-operation for bleeding, wound infection.

Notes

Quality assessment score (Schulz criteria): 5/7

Transfusion protocol used

\section{Risk of bias}

Item

Authors' judgement

Description 
Bidstrup 1993 (Continued)

\begin{tabular}{lll}
\hline Adequate sequence generation? & Unclear & Not reported \\
\hline Allocation concealment? & Yes & Adequate \\
\hline $\begin{array}{l}\text { Blinding? } \\
\text { All outcomes }\end{array}$ & Yes & Double blind \\
\hline
\end{tabular}

Bidstrup 2000

\begin{tabular}{|c|c|c|}
\hline Methods & \multicolumn{2}{|c|}{$\begin{array}{l}\text { Patients were allocated to receive either placebo or active treatment in accordance with a previously } \\
\text { determined randomization schedule in a double blind fashion. Allocation concealment was ade- } \\
\text { quate, active drug and placebo were contained in identical bottles, identifiable only by a random } \\
\text { number. }\end{array}$} \\
\hline Participants & \multicolumn{2}{|c|}{$\begin{array}{l}60 \text { patients undergoing aortocoronary bypass were randomised to one of two groups: } \\
\text { - Aprotinin group (High dose): } \mathrm{n}=30, \mathrm{M} / \mathrm{F}=24 / 6 \text {, mean }(\mathrm{sd}) \text { age }=63.0(7.8) \text { years } \\
\text { - Control group (Placebo): } \mathrm{n}=30, \mathrm{M} / \mathrm{F}=27 / 3 \text {, mean }(\mathrm{sd}) \text { age }=61.7(6.8) \text { years }\end{array}$} \\
\hline Interventions & \multicolumn{2}{|c|}{$\begin{array}{l}\text { - Aprotinin group (High dose) received a loading dose } 280 \mathrm{mg} \text { ( } 2 \text { million KIU) of aprotinin } \\
\text { over } 20 \text { minutes after anaesthesia, } 280 \mathrm{mg} \text { of aprotinin added to the pump prime and a } \\
\text { continuous infusion of } 70 \mathrm{mg} / \mathrm{hr} \text { until the end of the procedure. } \\
\text { - Control group received a placebo of } 0.9 \% \text { normal saline. }\end{array}$} \\
\hline Outcomes & \multicolumn{2}{|c|}{$\begin{array}{l}\text { Outcomes reported: Number of participants exposed to allogeneic blood, mortality, myocardial } \\
\text { infarction, re-operation for bleeding, wound infection, neurologic disturbance, } \\
\text { atrial fibrillation/flutter. }\end{array}$} \\
\hline Notes & \multicolumn{2}{|c|}{$\begin{array}{l}\text { Quality assessment score (Schulz criteria): 6/7 } \\
\text { Transfusion protocol used }\end{array}$} \\
\hline \multicolumn{3}{|l|}{ Risk of bias } \\
\hline Item & Authors' judgement & Description \\
\hline Adequate sequence generation? & Unclear & Not reported \\
\hline Allocation concealment? & Yes & Adequate \\
\hline $\begin{array}{l}\text { Blinding? } \\
\text { All outcomes }\end{array}$ & Yes & Double blind \\
\hline
\end{tabular}


Blauhut 1994

\begin{tabular}{l|l}
\hline Methods & $\begin{array}{l}\text { Method of randomisation and allocation concealment were not described. No exclusions or loss to } \\
\text { follow-up reported. }\end{array}$ \\
\hline Participants & $\begin{array}{l}45 \text { patients undergoing cardiopulmonary bypass for coronary surgery were allocated at random to } \\
\text { one of three groups: } \\
\text { - Aprotinin group: } \mathrm{n}=15, \mathrm{M} / \mathrm{F}=13 / 2, \text { mean }(\mathrm{sd}) \text { age }=64.1(2.2) \text { years } \\
\text { - Tranexamic group: } \mathrm{n}=16, \mathrm{M} / \mathrm{F}=13 / 3, \text { mean }(\mathrm{sd}) \text { age }=62.5(2.2) \text { years } \\
\text { - Control group: } \mathrm{n}=14, \mathrm{M} / \mathrm{F}=11 / 3, \text { mean }(\mathrm{sd}) \text { age }=62.7(2.6) \text { years }\end{array}$ \\
\hline Interventions & $\begin{array}{l}\text { - Aprotinin group received a loading dose of } 2 \text { million kallikrein inactivator units (KIU) plus a } \\
\text { maintenance dose of } 500,000 \mathrm{KIU} / \mathrm{hr} \text { until the patient was transferred to the recovery area of the } \\
\text { intensive care unit. In addition, } 1 \mathrm{million} \mathrm{KIU} \text { was added to the oxygenator priming fluid, giving } \\
\text { an average total dose of } 4.2 \mathrm{million} \mathrm{KIU} \text { of aprotinin. } \\
\text { - Tranexamic (TXA) group received } 10 \mathrm{mg} / \mathrm{kg} \text { of TXA beginning } 30 \text { minutes before incision } \\
\text { of the skin and followed by } 1 \mathrm{mg} / \mathrm{kg} / \mathrm{hr} \text { for } 10 \text { hours after the beginning of the surgical procedures. } \\
\text { - Control group did not receive aprotinin or TXA treatment. }\end{array}$ \\
\hline
\end{tabular}

Outcomes Outcomes reported: Number of participants exposed to allogeneic blood, allogeneic blood usage (units), blood loss (24 hrs), mortality, platelet function, coagulation, haematocrit levels.

\begin{tabular}{l|ll}
\hline Notes & $\begin{array}{l}\text { Quality assessment score (Schulz criteria): 1/7 } \\
\text { Transfusion protocol used }\end{array}$ & \\
\hline Risk of bias & Authors' judgement & Description \\
\hline Item & Unclear & Not reported \\
\hline Adequate sequence generation? & Unclear \\
\hline Allocation concealment? & Unclear & Unclear \\
\hline $\begin{array}{l}\text { Blinding? } \\
\text { All outcomes }\end{array}$ & Unclear & \\
\hline
\end{tabular}

\section{Boldt 1991}

Methods

Participants
Method of randomisation and allocation concealment were not described. No exclusions or loss to follow-up reported.

30 male patients undergoing elective aortocoronary bypass grafting were randomised to one of three groups:

- Cell Salvage group: $\mathrm{n}=10$, mean $(\mathrm{sd})$ age $=60.4(7.1)$ years

- Hemofiltration group: $\mathrm{n}=10$, mean $(\mathrm{sd})$ age $=62.4$ (8.6) years

- Aprotinin group: $\mathrm{n}=10$, mean $(\mathrm{sd})$ age $=62.7(7.8)$ years

- Control group: $\mathrm{n}=10$, mean (sd) age $=46.6$ (16.2) years

NB: Control group did not appear to be part of the randomised schedule. Possibly a non-concurrent or historical control group. 
Boldt 1991 (Continued)

Interventions
CPB. Cell Salvage group - a cell separator (Cell Saver IV, Hemonetics) was used during and after
- Haemofiltration group had blood concentrated during and after CPB by means of a
hemofiltration device (HF-80, Fresenius, Bad Homburg, FRG).
- Aprotinin group received an infusion of 2 million kallikrein inactivator units (KIU) before
the operation (loading dose) and then as a continuous infusion of 500,000 units/hr until the end
of the operation. In addition, 2 million KIU of aprotinin was added to the priming of the heart-
lung machine. In addition blood concentration during and after CPB was performed with a
hemofiltration device (HF-80, Fresenius, Bad Homburg, FRG) the same as for Group 2.
- Control group underwent neurosurgery operations.
NB: Only Group 2 and Group 3 were compared.

Outcomes

Outcomes reported: Number of participants exposed to allogeneic blood, allogeneic blood usage (units), blood loss (24hrs).

Notes Quality assessment score (Schulz criteria): 2/7

Transfusion protocol used. Study used neurosurgical patients as a control group.

Risk of bias

\begin{tabular}{lll}
\hline Item & Authors' judgement & Description \\
\hline Adequate sequence generation? & Unclear & Unclear \\
\hline Allocation concealment? & Unclear & Unclear \\
\hline $\begin{array}{l}\text { Blinding? } \\
\text { All outcomes }\end{array}$ & Unclear & Unclear \\
\hline
\end{tabular}

Boldt 1994

\begin{tabular}{l|l}
\hline Methods & Method of randomisation and allocation concealment were not described. \\
\hline Participants & 40 patients undergoing cardiac surgery were randomised to one of two groups: \\
& $\begin{array}{l}\text { - Aprotinin group (High dose): } \mathrm{n}=20, \text { mean }(\mathrm{sd}) \text { age }=64(4) \text { years } \\
\text { - Control group: } \mathrm{n}=20, \text { mean }(\mathrm{sd}) \text { age }=63(5) \text { years }\end{array}$ \\
NB: Gender data were not reported
\end{tabular}

Interventions

- Aprotinin group (High dose) received 2 million KIU of aprotinin after the induction of anaesthesia, 500,000 KIU/hr of aprotinin as a continuous infusion until the end of the operation, and 2 million KIU was added to the $\mathrm{CPB}$ pump prime.

- Control group received the same amount of saline solution as aprotinin was administered.

Outcomes

Outcomes reported: Allogeneic blood usage (units), fresh frozen plasma usage (units), blood loss (24hrs), re-operation for bleeding, haemoglobin levels.

Notes

Quality assessment score (Schulz criteria): 4/7

Transfusion protocol used 
Boldt 1994 (Continued)

\begin{tabular}{l|ll} 
Risk of bias & & \\
\hline Item & Authors' judgement & Description \\
\hline Adequate sequence generation? & Unclear & Unclear \\
\hline Allocation concealment? & Unclear & Unclear \\
\hline $\begin{array}{l}\text { Blinding? } \\
\text { All outcomes }\end{array}$ & Yes & Double blind \\
\hline
\end{tabular}

\section{Boylan 1996}

\begin{tabular}{|c|c|c|}
\hline Methods & \multicolumn{2}{|c|}{$\begin{array}{l}\text { Study agents were prepared by the hospital pharmacy using a randomisation schedule provided in } \\
\text { sealed envelopes. The method used to generate allocation sequences was not described. }\end{array}$} \\
\hline Participants & \multicolumn{2}{|c|}{$\begin{array}{l}45 \text { patients undergoing primary, isolated orthotopic liver transplantation were randomised to one } \\
\text { of two groups: } \\
\text { - Tranexamic acid group: } \mathrm{n}=25 \text {, mean }(\mathrm{sd}) \text { age }=49.5(9.1) \text { years } \\
\text { - Control group (Placebo): } \mathrm{n}=20 \text {, mean (sd) age }=48.8 \text { (9.6) years }\end{array}$} \\
\hline Interventions & \multicolumn{2}{|c|}{$\begin{array}{l}\text { - Tranexamic acid (TXA) group received a continuous infusion of TXA in normal saline } \\
\text { ( } 40 \mathrm{mg} / \mathrm{kg} / \mathrm{hr} \text { to a maximum dose of } 20 \mathrm{~g} \text { ). } \\
\text { - Control group (placebo) received an equivalent volume of } 0.9 \% \text { normal saline alone. }\end{array}$} \\
\hline Outcomes & \multicolumn{2}{|c|}{$\begin{array}{l}\text { Outcomes reported: Allogeneic blood usage (units), fresh frozen plasma usage (units), platelet usage } \\
\text { (units), blood loss, mortality, portal vein thrombosis, hepatic thrombosis, hospital length of stay, } \\
\text { intensive care unit (ICU) length of stay, overall donor exposure. }\end{array}$} \\
\hline Notes & \multicolumn{2}{|c|}{$\begin{array}{l}\text { Quality assessment score (Schulz criteria):5/7 } \\
\text { Transfusion protocol used }\end{array}$} \\
\hline \multicolumn{3}{|l|}{ Risk of bias } \\
\hline Item & Authors' judgement & Description \\
\hline Adequate sequence generation? & Unclear & Unclear \\
\hline Allocation concealment? & No & $\begin{array}{l}\text { Inadequate - randomisation schedule was pro- } \\
\text { vided in sealed envelopes }\end{array}$ \\
\hline $\begin{array}{l}\text { Blinding? } \\
\text { All outcomes }\end{array}$ & Yes & Double blind \\
\hline
\end{tabular}


Brown 1997

\begin{tabular}{|c|c|c|}
\hline Methods & \multicolumn{2}{|c|}{$\begin{array}{l}\text { Method of allocation concealment was not described. Patients were randomised using a computer- } \\
\text { generated random number sequence. }\end{array}$} \\
\hline Participants & \multicolumn{2}{|c|}{$\begin{array}{l}91 \text { patients scheduled for elective coronary revascularisation were randomly allocated to one of } \\
\text { three groups: } \\
\text { - Control group (Placebo): } \mathrm{n}=30, \mathrm{M} / \mathrm{F}=24 / 6 \text {, mean }(\mathrm{sd}) \text { age }=59(7) \text { years } \\
\text { - Tranexamic acid group (TXA before } \mathrm{CPB}): \mathrm{n}=30, \mathrm{M} / \mathrm{F}=25 / 5 \text {, mean }(\mathrm{sd}) \text { age }=61 \text { (9) years } \\
\text { - Tranexamic acid group (TXA after } \mathrm{CPB}): \mathrm{n}=30, \mathrm{M} / \mathrm{F}=24 / 6 \text {, mean }(\mathrm{sd}) \text { age }=62(10) \text { years }\end{array}$} \\
\hline Interventions & \multicolumn{2}{|c|}{$\begin{array}{l}\text { - Control group received equivalent volumes of normal saline solution. } \\
\text { - Tranexamic acid group received } 15 \mathrm{mg} / \mathrm{kg} \text { of TXA before CPB, followed by a TXA infusion } \\
\text { of } 1 \mathrm{mg} / \mathrm{kg} / \mathrm{hr} \text { for } 5 \mathrm{hrs} \text {. } \\
\text { - Tranexamic acid group received } 15 \mathrm{mg} / \mathrm{kg} \text { of TXA after CPB, followed by a TXA infusion of } \\
1 \mathrm{mg} / \mathrm{kg} / \mathrm{hr} \text { for } 5 \mathrm{hrs} \text {. }\end{array}$} \\
\hline Outcomes & \multicolumn{2}{|c|}{$\begin{array}{l}\text { Outcomes reported: Number of participants exposed to allogeneic blood, haematologic/throm- } \\
\text { boelastographic/coagulation characteristics, mortality, myocardial infarction, stroke, re-exploration } \\
\text { for bleeding, infection. }\end{array}$} \\
\hline Notes & \multicolumn{2}{|c|}{$\begin{array}{l}\text { Quality assessment score (Schulz criteria): } 4 / 7 \\
\text { Transfusion protocol used }\end{array}$} \\
\hline \multicolumn{3}{|l|}{ Risk of bias } \\
\hline Item & Authors' judgement & Description \\
\hline Adequate sequence generation? & Yes & $\begin{array}{l}\text { Adequate - computer-generated random num- } \\
\text { ber sequence }\end{array}$ \\
\hline Allocation concealment? & Unclear & Unclear \\
\hline $\begin{array}{l}\text { Blinding? } \\
\text { All outcomes }\end{array}$ & Yes & Double blind \\
\hline
\end{tabular}

Caglar 2008

Methods A computer-generated randomisation list was used to generate the allocation sequence. No information was provided regarding allocation concealment.

Participants 100 female patients undergoing myomectomy were randomised to one of two groups:

- Tranexamic acid group: $\mathrm{n}=50$, mean $(\mathrm{sd})$ age $=34.2(5.5)$ years

- Control group: $\mathrm{n}=50$, mean $(\mathrm{sd})$ age $=36.5$ (4.5) years

Interventions

- Tranexamic acid group received bolus of $10 \mathrm{mg} / \mathrm{kg}$ over 10 minutes 15 minutes before incision, then continuous infusion of $1 \mathrm{mg} / \mathrm{kg} / \mathrm{hr}$ for 10 hours.

- Control group received saline. 


\begin{tabular}{|c|c|c|}
\hline Outcomes & \multicolumn{2}{|c|}{$\begin{array}{l}\text { Outcomes reported: number of patients exposed to allogeneic blood, blood loss, volume of blood } \\
\text { transfused. }\end{array}$} \\
\hline Notes & \multicolumn{2}{|l|}{$\begin{array}{l}\text { Quality assessment score (Schulz criteria): 5/7 } \\
\text { Transfusion protocol used }\end{array}$} \\
\hline \multicolumn{3}{|l|}{ Risk of bias } \\
\hline Item & Authors' judgement & Description \\
\hline Adequate sequence generation? & Yes & Computer-generated randomisation \\
\hline Allocation concealment? & Unclear & Unclear \\
\hline $\begin{array}{l}\text { Blinding? } \\
\text { All outcomes }\end{array}$ & Yes & Double blind \\
\hline
\end{tabular}

\section{Camarasa 2006}

Methods

ment was sealed in an opaque, numbered envelope which was opened only by the nurse who pre-
pared the solutions. This nurse was the only person who knew the patients study groups and did
not participate in any other phase of the trial.

- Tranexamic acid group received $10 \mathrm{mg} / \mathrm{kg}$ of TXA administered over 30 minutes immediately before releasing the tourniquet followed by a continuous intravenous infusion of $10 \mathrm{mg} / \mathrm{kg}$ for 3 hours.

- Epsilon aminocaproic acid group received $100 \mathrm{mg} / \mathrm{kg}$ of EACA administered over 30 minutes immediately before releasing the tourniquet followed by a continuous intravenous infusion of $1 \mathrm{~g} / \mathrm{hr}$ for 3 hours.

NB: Both groups were exposed to cell salvage and pre-operative autologous blood donation (PAD)

Outcomes

Outcomes reported: Number of participants exposed to allogeneic blood, allogeneic blood usage (units), blood loss, deep vein thrombosis.

Notes

Quality assessment score (Schulz criteria): 5/7

Transfusion protocol used

\section{Risk of bias}

Item
Authors' judgement

\section{Description}




\section{Camarasa 2006 (Continued)}

\begin{tabular}{l|l|l}
\hline Adequate sequence generation? & Yes & Computer-generated random numbers \\
\hline Allocation concealment? & No & $\begin{array}{l}\text { Inadequate - randomised assignment was sealed } \\
\text { in an opaque, numbered envelope }\end{array}$ \\
\hline $\begin{array}{l}\text { Blinding? } \\
\text { All outcomes }\end{array}$ & Yes & Double blind \\
\hline
\end{tabular}

\section{Capdevila 1998}

\begin{tabular}{|c|c|c|}
\hline Methods & \multicolumn{2}{|c|}{$\begin{array}{l}\text { Randomisation was performed using a random number list generated by computer programme. } \\
\text { Allocation was adequately concealed (administered fluids were prepared by the hospitals central } \\
\text { pharmacy in identical } 100 \text {-ml bottles). }\end{array}$} \\
\hline Participants & \multicolumn{2}{|c|}{$\begin{array}{l}23 \text { patients scheduled for orthopaedic surgery of the hip, femur or pelvis for sepsis or malignant } \\
\text { tumours were randomly allocated to one of two groups: } \\
\text { - Aprotinin group: } \mathrm{n}=12, \mathrm{M} / \mathrm{F}=7 / 5, \text { mean }(\mathrm{sd}) \text { age }=48.6(17.3) \text { years } \\
\text { - Control group (Placebo): } \mathrm{n}=11, \mathrm{M} / \mathrm{F}=6 / 5 \text {, mean }(\mathrm{sd}) \text { age }=48.5(16.3) \text { years }\end{array}$} \\
\hline Interventions & \multicolumn{2}{|c|}{$\begin{array}{l}\text { - Aprotinin group was administered a bolus of } 1 \text { million kallikrein inactivation units (KIU) } \\
\text { during a } 30 \text { minute injection period, followed by a continuous infusion of 500,000 KIU/hr } \\
\text { throughout the duration of surgery. } \\
\text { - Control group received identical volumes of saline over the same time periods. }\end{array}$} \\
\hline Outcomes & \multicolumn{2}{|c|}{$\begin{array}{l}\text { Outcomes reported: Number of patients exposed to allogeneic blood, allogeneic blood usage (units) } \\
\text {, fresh frozen plasma usage (units), platelet usage (units), blood loss, haemoglobin and haematocrit } \\
\text { levels, coagulation and fibrinolytic pathway explorations, allergic reactions. }\end{array}$} \\
\hline Notes & \multicolumn{2}{|c|}{$\begin{array}{l}\text { Quality assessment score (Schulz criteria): 7/7 } \\
\text { Transfusion protocol used }\end{array}$} \\
\hline \multicolumn{3}{|l|}{ Risk of bias } \\
\hline Item & Authors' judgement & Description \\
\hline Adequate sequence generation? & Yes & $\begin{array}{l}\text { Randomisation was performed using a random } \\
\text { number list generated by computer programme }\end{array}$ \\
\hline Allocation concealment? & Yes & Adequate \\
\hline $\begin{array}{l}\text { Blinding? } \\
\text { All outcomes }\end{array}$ & Yes & Double blind \\
\hline
\end{tabular}




\begin{tabular}{|c|c|c|}
\hline Methods & \multicolumn{2}{|c|}{$\begin{array}{l}\text { Randomisation and allocation concealment not specified. No exclusions or loss to follow-up re- } \\
\text { ported. [Spanish language] }\end{array}$} \\
\hline Participants & \multicolumn{2}{|c|}{$\begin{array}{l}102 \text { patients undergoing cardiac surgery were randomly assigned to one of two groups: } \\
\text { - Aprotinin group: } \mathrm{n}=51, \mathrm{M} / \mathrm{F}=20 / 31 \text {, mean }(\mathrm{sd}) \text { age }=54(13.1) \text { years } \\
\text { - Control group: } \mathrm{n}=51, \mathrm{M} / \mathrm{F}=21 / 30 \text {, mean }(\mathrm{sd}) \text { age }=55(13.0) \text { years }\end{array}$} \\
\hline Interventions & \multicolumn{2}{|c|}{$\begin{array}{l}\text { - Aprotinin group received } 2 \text { million kallikrein inhibiting units (KIU) of aprotinin upon } \\
\text { anaesthesia induction, a similar dose in the extracorporeal circulation priming pump, and a } \\
\text { maintenance dose of } 500,000 \mathrm{KIU} / \mathrm{hr} \text { until the removal from the operating theatre. } \\
\text { - Control group did not receive aprotinin. }\end{array}$} \\
\hline Outcomes & \multicolumn{2}{|c|}{$\begin{array}{l}\text { Outcomes reported: Number of participants exposed to allogeneic blood, allogeneic blood usage, } \\
\text { fresh frozen plasma usage, platelet usage, blood loss, myocardial infarction. }\end{array}$} \\
\hline Notes & \multicolumn{2}{|c|}{$\begin{array}{l}\text { Quality assessment score (Schulz criteria): } 2 / 7 \\
\text { Transfusion protocol used }\end{array}$} \\
\hline \multicolumn{3}{|l|}{ Risk of bias } \\
\hline Item & Authors' judgement & Description \\
\hline Adequate sequence generation? & Unclear & Unclear \\
\hline Allocation concealment? & Unclear & Unclear \\
\hline $\begin{array}{l}\text { Blinding? } \\
\text { All outcomes }\end{array}$ & Unclear & Unclear \\
\hline
\end{tabular}

\section{Casas 1995}

Methods Generation of allocation sequences was not specified. Allocation concealment was by sealed envelopes. The pharmacy prepared the encoded infusions.

Participants

149 patients scheduled to undergo either coronary artery bypass grafting (CABG), heart valve replacement or annuloplasty, combined valve replacement and CABG, or closure of atrial septal defects, were randomised to one of three groups:

- Aprotinin group: $\mathrm{n}=48, \mathrm{M} / \mathrm{F}=31 / 17$, mean (sd) age $=57$ (10) years

- Desmopressin group: $\mathrm{n}=50, \mathrm{M} / \mathrm{F}=33 / 17$, mean (sd) age $=58$ (12) years

- Control group (Placebo): $\mathrm{n}=51, \mathrm{M} / \mathrm{F}=31 / 20$, mean $(\mathrm{sd})$ age $=54$ (12) years

Interventions

- Aprotinin group received 2 million kallikrein inactivator units (KIU) of aprotinin before anaesthesia (time stage 1) given over 20 to 30 minutes. A dose of 2 million KIU was added to the prime solution of the heart-lung machine (time stage 2). Aprotinin was administered continuously (time stage 3 ) at 500,000 KIU/hr $(50 \mathrm{ml} / \mathrm{hr}$ ) until the end of the operation (from skin incision to skin closure), then patients received $50 \mathrm{ml}$ of saline (time stage 4 ).

- Desmopressin group received desmopressin infusions corresponding to 0.3 to $0.4 \mathrm{ug} / \mathrm{kg}$ body weight. Desmopressin was infused in $50 \mathrm{ml}$ of physiologic saline solution for 20 to 30 
minutes, 15 minutes after protamine administration (time stage 4). In other time phases (1-3) patients received saline solution only.

- Control group received a placebo of saline solution during all four stages.

\begin{tabular}{|c|c|c|}
\hline Outcomes & \multicolumn{2}{|c|}{$\begin{array}{l}\text { Outcomes reported: Number of patients exposed to allogeneic blood, allogeneic blood usage (units) } \\
\text {, number of patients exposed to fresh frozen plasma, number of patients exposed to platelets, blood } \\
\text { loss ( } 24 \mathrm{hrs} \text { ), re-operation for bleeding, femoral embolism, stroke. }\end{array}$} \\
\hline Notes & \multicolumn{2}{|c|}{$\begin{array}{l}\text { Quality assessment score (Schulz criteria): 4/7 } \\
\text { Transfusion protocol used }\end{array}$} \\
\hline \multicolumn{3}{|l|}{ Risk of bias } \\
\hline Item & Authors' judgement & Description \\
\hline Adequate sequence generation? & Unclear & Unclear \\
\hline Allocation concealment? & No & $\begin{array}{l}\text { Inadequate - allocation concealment was by } \\
\text { sealed envelopes }\end{array}$ \\
\hline $\begin{array}{l}\text { Blinding? } \\
\text { All outcomes }\end{array}$ & Unclear & Double blind \\
\hline
\end{tabular}

\section{Casati 1999}

\begin{tabular}{l|l}
\hline Methods & Method ofrandomisation and allocation concealment were not described. \\
\hline Participants & $\begin{array}{l}210 \text { patients undergoing cardiac surgery were randomly assigned to one of three groups: } \\
\text { - Epsilon aminocaproic group: } \mathrm{n}=68, \mathrm{M} / \mathrm{F}=54 / 12, \text { mean }(\mathrm{sd}) \text { age }=58.7(10) \text { years } \\
\text { - Tranexamic acid group: } \mathrm{n}=72, \mathrm{M} / \mathrm{F}=57 / 13, \mathrm{mean}(\mathrm{sd}) \text { age }=61.9(9.6) \text { years } \\
\text { - Aprotinin group: } \mathrm{n}=70, \mathrm{M} / \mathrm{F}=54 / 13, \text { mean }(\mathrm{sd}) \text { age }=63.6(9.6) \text { years }\end{array}$ \\
\hline Interventions & $\begin{array}{l}\text { - EACA group received } 5 \mathrm{~g} \text { during } 20 \text { minutes after induction of anaesthesia before } \\
\text { sternotomy followed by a continuous infusion of } 2 \mathrm{~g} / \mathrm{hr} \text { until the end of the operation }+2.5 \mathrm{~g} \\
\text { added to the pump prime. } \\
\text { - TXA group received } 1 \mathrm{~g} \text { over } 20 \text { minutes before sternotomy, followed by a continuous } \\
\text { infusion of } 400 \mathrm{mg} / \text { hour during operative period and } 500 \mathrm{mg} \text { added to the pump prime. } \\
\text { - Aprotinin group received } 280 \mathrm{mg} \text { throughout } 20 \text { minutes before sternotomy, followed by a } \\
\text { constant infusion of } 70 \mathrm{mg} \text { throughout the operation and } 280 \mathrm{mg} \text { added to the pump prime. } \\
\begin{array}{l}\text { NB: All groups were exposed to cell salvage. Pre-operative autologous blood donation use was evenly } \\
\text { distributed between groups. }\end{array}\end{array}$ \\
\hline
\end{tabular}

Outcomes

Outcomes reported: Number of patients exposed to allogeneic blood, allogeneic blood usage (units) blood loss, mortality, myocardial infarction, deep venous thrombosis, pulmonary embolus, pre-operative autologous donation of blood, neurological complications, re-operation for surgical bleeding. 


\begin{tabular}{|c|c|c|}
\hline Notes & \multicolumn{2}{|c|}{$\begin{array}{l}\text { Quality assessment score (Schulz criteria): 1/7 } \\
\text { Transfusion protocol used }\end{array}$} \\
\hline \multicolumn{3}{|l|}{ Risk of bias } \\
\hline Item & Authors' judgement & Description \\
\hline Adequate sequence generation? & Unclear & Unclear \\
\hline Allocation concealment? & Unclear & Unclear \\
\hline $\begin{array}{l}\text { Blinding? } \\
\text { All outcomes }\end{array}$ & Unclear & Unclear \\
\hline
\end{tabular}

\section{Casati 2000}

\begin{tabular}{l|l}
\hline Methods & $\begin{array}{l}\text { Patients were randomised into treatment groups by means of a computer generated random number } \\
\text { sequence. Allocation concealment was not described. Trial was unblinded. }\end{array}$ \\
\hline Participants & $\begin{array}{l}1040 \text { patients undergoing primary elective cardiac operations were randomly assigned to one of } \\
\text { two groups: } \\
\text { - Tranexamic acid group: } \mathrm{n}=522, \mathrm{M} / \mathrm{F}=415 / 107, \text { mean }(\mathrm{sd}) \text { age }=61(10) \text { years } \\
\text { - Aprotinin group: } \mathrm{n}=518, \mathrm{M} / \mathrm{F}=412 / 106, \text { mean }(\mathrm{sd}) \text { age }=62(10) \text { years }\end{array}$ \\
\hline Interventions & $\begin{array}{l}\text { - Tranexamic acid group received } 1 \mathrm{~g} \text { over } 20 \text { minutes before surgical incision followed by a } \\
\text { constant infusion of } 400 \mathrm{mg} / \mathrm{hr} \text { during the entire operative period and } 500 \mathrm{mg} \text { was added to the } \\
\text { pump prime. } \\
\text { - Aprotinin group received } 280 \mathrm{mg} \text { for } 20 \text { minutes before surgical incision followed by a } \\
\text { constant infusion of } 70 \mathrm{mg} / \mathrm{hr} \text { until the end of the operation and } 280 \mathrm{mg} \text { was added to the pump } \\
\text { prime. } \\
\text { NB: Both groups were exposed to cell salvage. }\end{array}$ \\
\hline
\end{tabular}

Outcomes

Outcomes reported: Number of patients exposed to allogeneic blood, allogeneic blood usage (units) , blood loss, mortality, myocardial infarction, fresh frozen plasma usage, (units), platelet usage (units), pulmonary embolus.

$\begin{array}{ll}\text { Notes } & \begin{array}{l}\text { Quality assessment score (Schulz criteria): 3/7 } \\ \text { Transfusion protocol used }\end{array}\end{array}$

\section{Risk of bias}

\begin{tabular}{l|ll}
\hline Item & Authors' judgement & Description \\
\hline Adequate sequence generation? & Yes & Computer generated random number sequence \\
\hline Allocation concealment? & Unclear & Unclear
\end{tabular}




\begin{tabular}{l|l}
\hline $\begin{array}{l}\text { Blinding? } \\
\text { All outcomes }\end{array}$ & No \\
\hline
\end{tabular}

\section{Casati 2001}

Methods

Method of randomisation was not described. Coded infusion syringes were used and prepared by a staff member not directly involved with perioperative clinical treatment.

\begin{tabular}{|c|c|c|}
\hline Participants & \multicolumn{2}{|c|}{$\begin{array}{l}40 \text { patients undergoing elective cardiac surgery were randomised to one of two groups: } \\
\text { - Tranexamic acid group: } \mathrm{n}=20, \mathrm{M} / \mathrm{F}=15 / 5 \text {, mean (sd) age }=64(13) \text { years } \\
\text { - Control group (Placebo): } \mathrm{n}=20, \mathrm{M} / \mathrm{F}=17 / 3 \text {, mean (sd) age }=62(11) \text { years }\end{array}$} \\
\hline Interventions & \multicolumn{2}{|c|}{$\begin{array}{l}\text { - TXA group (off-pump surgery) received a bolus of } 1 \mathrm{~g} \text { of TXA over } 20 \text { minutes after the } \\
\text { induction of anaesthesia but before skin incision and a continuous infusion of } 400 \mathrm{mg} / \mathrm{hr} \text { during } \\
\text { the whole surgical period. } \\
\text { - Control group received an infusion of saline. }\end{array}$} \\
\hline Outcomes & \multicolumn{2}{|c|}{$\begin{array}{l}\text { Outcomes reported: Number of patients exposed to allogeneic blood, allogeneic blood usage (units } \\
\text {, blood loss. }\end{array}$} \\
\hline Notes & \multicolumn{2}{|c|}{$\begin{array}{l}\text { Quality assessment score (Schulz criteria): 6/7 } \\
\text { Transfusion protocol used }\end{array}$} \\
\hline \multicolumn{3}{|l|}{ Risk of bias } \\
\hline Item & Authors' judgement & Description \\
\hline Adequate sequence generation? & Unclear & Unclear \\
\hline Allocation concealment? & Yes & Adequate \\
\hline $\begin{array}{l}\text { Blinding? } \\
\text { All outcomes }\end{array}$ & Yes & Double blind \\
\hline
\end{tabular}

\section{Casati 2002}

\begin{tabular}{l|l} 
Methods & $\begin{array}{l}\text { Coded infusion syringes were used to conceal which medication was placebo and which was TXA. } \\
\text { Method of randomisation was not described. }\end{array}$ \\
\hline Participants & $\begin{array}{l}60 \text { patients undergoing elective thoracic-aorto surgery were randomised to one of two groups: } \\
\text { - Tranexamic acid group: } \mathrm{n}=30, \mathrm{M} / \mathrm{F}=23 / 6, \text { mean }(\mathrm{sd}) \text { age }=59 \text { (13) years } \\
\text { - Control group (Placebo): } \mathrm{n}=30, \mathrm{M} / \mathrm{F}=19 / 10, \text { mean }(\mathrm{sd}) \text { age }=63(11) \text { years }\end{array}$ \\
\hline Interventions & $\begin{array}{l}\text { - Tranexamic acid group received a bolus of TXA } 1 \mathrm{~g} \text { in } 20 \text { minutes after the induction of } \\
\text { anaesthesia but before skin incision and a continuous infusion of } 400 \mathrm{mg} / \mathrm{hr} \text { during the whole } \\
\text { surgical period and an additional } 500 \mathrm{mg} \text { of TXA was added to the pump prime of CPB. } \\
\text { - Control group received an infusion of saline solution. }\end{array}$
\end{tabular}




\begin{tabular}{|c|c|c|}
\hline Outcomes & \multicolumn{2}{|c|}{$\begin{array}{l}\text { Outcomes reported: Number of patients exposed to allogeneic blood, allogeneic blood usage (units) } \\
\text {, number of patients exposed to fresh frozen plasma, number of patients exposed to platelets, blood } \\
\text { loss ( } 24 \mathrm{hrs} \text { ), re-operation for bleeding, mortality, myocardial infarction, pulmonary embolus, pre- } \\
\text { operative aspirin, pre-operative anticoagulant, stroke, hospital length of stay (days). }\end{array}$} \\
\hline Notes & \multicolumn{2}{|c|}{$\begin{array}{l}\text { Quality assessment score (Schulz criteria): 5/7 } \\
\text { Transfusion protocol used }\end{array}$} \\
\hline \multicolumn{3}{|l|}{ Risk of bias } \\
\hline Item & Authors' judgement & Description \\
\hline Adequate sequence generation? & Unclear & Unclear \\
\hline Allocation concealment? & Yes & Adequate \\
\hline $\begin{array}{l}\text { Blinding? } \\
\text { All outcomes }\end{array}$ & Yes & Double blind \\
\hline
\end{tabular}

\section{Casati 2004}

Methods
Randomisation was by means of two seperate computer-generated random number sequences. Coded syringes were used to administer medication.

102 patients scheduled for cardiac surgery with either 'on-pump' or 'off-pump' procedures were randomised to one of four groups:

- Control group (Placebo) ('Off-pump' surgery): $\mathrm{n}=25, \mathrm{M} / \mathrm{F}=21 / 4$, mean (sd) age =61 (11) years

- Tranexamic acid group ('Off-pump' surgery): $\mathrm{n}=26, \mathrm{M} / \mathrm{F}=20 / 6$, mean (sd) age $=64$ (12) years

- Control group (placebo) ('On-pump' surgery): $\mathrm{n}=25, \mathrm{M} / \mathrm{F}=21 / 4$, mean (sd) age = 60 (9) years

- Tranexamic acid group ('On-pump' surgery): $\mathrm{n}=26, \mathrm{M} / \mathrm{F}=24 / 2$, mean (sd) age $=64(9)$ years

Interventions

- Control group ('Off-pump' surgery) received an equivalent volume of saline solution administered as a bolus injection in 20 minutes before skin incision, followed by a continuous infusion of saline until the completion of surgery.

- Tranexamic acid group ('Off-pump' surgery) received a bolus injection of $1 \mathrm{~g}$ of TXA in 20 minutes before skin incision followed by a continuous infusion of $400 \mathrm{mg} / \mathrm{hr}$ until completion of surgery.

- Control group ('On-pump' surgery) received the same treatment as Group 1 plus received an equivalent volume of saline solution added to the $\mathrm{CPB}$ pump.

- Tranexamic acid ('On-pump' surgery) received the same treatment as Group 2 plus received $500 \mathrm{mg}$ of TXA added to the pump prime.

NB: 'On-pump' surgery patients (Groups $3 \& 4$ ) the remaining blood in the cardiopulmonary bypass $(\mathrm{CPB})$ circuit and that blood aspirated from the surgical field was concentrated with a cell 


\begin{tabular}{|c|c|c|}
\hline & \multicolumn{2}{|c|}{$\begin{array}{l}\text { separator and reinfused. For 'Off-pump' surgery patients only in cases of significant intra-operative } \\
\text { bleeding was the shed blood concentrated in a cell separator and reinfused. No autotransfusion of } \\
\text { shed mediastinal blood was performed during the post-operative period for any group. } \\
\text { ONCAB-Cell Salvage. Only in cases of significant intra-operative bleeding was the shed blood } \\
\text { concentrated in a cell separator and reinfused. No autotransfusion of shed mediastinal blood was } \\
\text { performed during the post-operative period. }\end{array}$} \\
\hline Outcomes & \multicolumn{2}{|c|}{$\begin{array}{l}\text { Outcomes reported: Number of patients exposed to allogeneic blood, allogeneic blood usage (units) } \\
\text {, number of patients exposed to fresh frozen plasma, number of participants exposed to platelets, } \\
\text { blood loss ( } 24 \mathrm{hrs} \text { ), re-exploration for bleeding, stroke, intra-operative resternotomy, fresh frozen } \\
\text { plasma usage (units), platelet usage (units). }\end{array}$} \\
\hline Notes & \multicolumn{2}{|c|}{$\begin{array}{l}\text { Quality assessment score (Schulz criteria): 7/7 } \\
\text { Transfusion protocol used }\end{array}$} \\
\hline \multicolumn{3}{|l|}{ Risk of bias } \\
\hline Item & Authors' judgement & Description \\
\hline Adequate sequence generation? & Yes & Computer-generated random number sequences \\
\hline Allocation concealment? & Yes & Adequate \\
\hline $\begin{array}{l}\text { Blinding? } \\
\text { All outcomes }\end{array}$ & Yes & Double blind \\
\hline
\end{tabular}

\section{Cicek 1996a}

\begin{tabular}{l|l}
\hline Methods & Method of randomisation and allocation concealment were not described. \\
\hline Participants & $\begin{array}{l}75 \text { patients scheduled for elective cardiac operations with cardiopulmonary bypass were randomly } \\
\text { assigned to one of three groups: } \\
\text { - Aprotinin group (High dose): } \mathrm{n}=25, \mathrm{M} / \mathrm{F}=22 / 3, \mathrm{mean}(\mathrm{sd}) \text { age }=44.9(18.6) \text { years } \\
\text { - Aprotinin group (Post-operative low dose): } \mathrm{n}=25, \mathrm{M} / \mathrm{F}=19 / 6, \mathrm{mean} \text { (sd) age }=52.9(12.4) \\
\text { years } \\
\text { - Control group: } \mathrm{n}=25, \mathrm{M} / \mathrm{F}=21 / 4, \text { mean }(\mathrm{sd}) \text { age }=46.7 \text { (15) years }\end{array}$ \\
\hline Interventions & $\begin{array}{l}\text { - Aprotinin group (High dose) received a bolus of } 2 \text { million kallikrein inhibiting units (KIU) } \\
\text { of aprotinin (280mg), plus a maintenance dose of } 500,000 \mathrm{KIU} / \mathrm{hr}(70 \mathrm{mg} / \mathrm{hr}) \text { until the end of } \\
\text { the operation. In addition } 2 \text { million KIU (280mg) was added to the oxygenator priming fluid. } \\
\text { - Aprotinin group (Post-operative low dose) received a bolus of } 2 \text { million KIU (280mg) at the } \\
\text { end of the procedure before transfer to the intensive care unit. } \\
\text { - Control group did not receive aprotinin. }\end{array}$ \\
\hline Outcomes & $\begin{array}{l}\text { Outcomes reported: } \text { Number of patients exposed to allogeneic blood, allogeneic blood usage (units) } \\
\text {, blood loss, myocardial infarction. }\end{array}$
\end{tabular}


Cicek 1996a (Continued)

\begin{tabular}{|c|c|c|}
\hline Notes & \multicolumn{2}{|c|}{$\begin{array}{l}\text { Quality assessment score (Schulz criteria): } 2 / 7 \\
\text { Transfusion protocol used }\end{array}$} \\
\hline \multicolumn{3}{|l|}{ Risk of bias } \\
\hline Item & Authors' judgement & Description \\
\hline Adequate sequence generation? & Unclear & Unclear \\
\hline Allocation concealment? & Unclear & Unclear \\
\hline $\begin{array}{l}\text { Blinding? } \\
\text { All outcomes }\end{array}$ & Unclear & Unclear \\
\hline
\end{tabular}

Cicek 1996b

\begin{tabular}{|c|c|c|}
\hline Methods & \multicolumn{2}{|c|}{$\begin{array}{l}\text { Patients were randomised to receive aprotinin or placebo by means of a random numbers table. } \\
\text { Method of allocation concealment was not described. }\end{array}$} \\
\hline Participants & \multicolumn{2}{|c|}{$\begin{array}{l}57 \text { patients undergoing cardiac operations with cardiopulmonary bypass }(\mathrm{CPB}) \text { were randomly } \\
\text { assigned to one of two groups: } \\
\text { - Aprotinin group: } \mathrm{n}=29, \mathrm{M} / \mathrm{F}=21 / 8 \text {, mean }(\mathrm{sd}) \text { age }=51.6(15.4) \text { years } \\
\text { - Control group (Placebo): } \mathrm{n}=28, \mathrm{M} / \mathrm{F}=19 / 9 \text {, mean }(\mathrm{sd}) \text { age }=48.2(14.2) \text { years }\end{array}$} \\
\hline Interventions & \multicolumn{2}{|c|}{$\begin{array}{l}\text { - Aprotinin group received a bolus of } 2 \text { million kallikrein inhibiting units (KIU) of aprotinin } \\
(280 \mathrm{mg}) \text { infused over } 15 \text { minutes when they arrived in intensive care. } \\
\text { - Control group received an equal volume of normal saline solution at corresponding times to } \\
\text { the aprotinin treated group. }\end{array}$} \\
\hline Outcomes & \multicolumn{2}{|c|}{$\begin{array}{l}\text { Outcomes reported: Number of patients exposed to allogeneic blood, allogeneic blood usage (units) } \\
\text {, blood loss. }\end{array}$} \\
\hline Notes & \multicolumn{2}{|c|}{$\begin{array}{l}\text { Quality assessment score (Schulz criteria): 5/7 } \\
\text { Transfusion protocol used }\end{array}$} \\
\hline \multicolumn{3}{|l|}{ Risk of bias } \\
\hline Item & Authors' judgement & Description \\
\hline Adequate sequence generation? & Yes & Random numbers table \\
\hline Allocation concealment? & Unclear & Unclear \\
\hline $\begin{array}{l}\text { Blinding? } \\
\text { All outcomes }\end{array}$ & Yes & Double blind \\
\hline
\end{tabular}




\section{Cicekcioglu 2006}

\begin{tabular}{|c|c|c|}
\hline Methods & \multicolumn{2}{|c|}{$\begin{array}{l}\text { Method of randomisation and allocation concealment were not described. Data were collected in } \\
\text { a blinded fashion. }\end{array}$} \\
\hline Participants & \multicolumn{2}{|c|}{$\begin{array}{l}44 \text { patients undergoing coronary artery bypass grafting were randomly assigned to one of two } \\
\text { groups: } \\
\text { - Aprotinin group: } \mathrm{n}=24, \mathrm{M} / \mathrm{F}=19 / 5, \text { mean }(\mathrm{sd}) \text { age }=48.6(12.1) \text { years } \\
\text { - Control group (Placebo): } \mathrm{n}=20, \mathrm{M} / \mathrm{F}=18 / 2 \text {, mean }(\mathrm{sd}) \text { age }=48.3(9.0) \text { years }\end{array}$} \\
\hline Interventions & \multicolumn{2}{|c|}{$\begin{array}{l}\text { - Aprotinin group (low-dose) administered in two equal doses - bolus of } 250,000 \mathrm{KIU} 5 \\
\text { minutes before skin incision just after induction of anaesthesia, second dose of 250,000 KIU was } \\
\text { added to the prime pump. } \\
\text { - Control group received saline. }\end{array}$} \\
\hline Outcomes & \multicolumn{2}{|c|}{$\begin{array}{l}\text { Outcomes reported: Number of patients exposed to allogeneic blood, amount of allogeneic blood } \\
\text { transfused (units), blood loss, mortality, length of hospital stay, post-operative morbidity. }\end{array}$} \\
\hline Notes & \multicolumn{2}{|c|}{$\begin{array}{l}\text { Quality assessment score (Schulz criteria): } 4 / 7 \\
\text { Transfusion protocol used }\end{array}$} \\
\hline \multicolumn{3}{|l|}{ Risk of bias } \\
\hline Item & Authors' judgement & Description \\
\hline Adequate sequence generation? & Unclear & Unclear \\
\hline Allocation concealment? & Unclear & Unclear \\
\hline $\begin{array}{l}\text { Blinding? } \\
\text { All outcomes }\end{array}$ & Yes & Double blind \\
\hline
\end{tabular}

\section{Claeys 2007}

\begin{tabular}{l|l}
\hline Methods & Methods of sequence generation and allocation concealment were not described. \\
\hline Participants & $\begin{array}{l}40 \text { patients undergoing orthopaedic (hip) surgery were randomised to one of two groups: } \\
\text { - Tranexamic acid group: } \mathrm{n}=20, \mathrm{M} / \mathrm{F}=5 / 15, \text { mean }(\mathrm{sd}) \text { age }=73 \text { (8) years } \\
\text { - Control group (Placebo): } \mathrm{n}=20, \text { all females, } \mathrm{M} / \mathrm{F}=7 / 13, \text { mean }(\mathrm{sd}) \text { age }=68(11) \text { years }\end{array}$ \\
\hline Interventions & $\begin{array}{l}\text { - Tranexamic acid group received single pre-operative dose of } 15 \mathrm{mg} / \mathrm{kg} . \\
\text { - Control group received a placebo of saline. }\end{array}$ \\
\hline Outcomes & $\begin{array}{l}\text { Outcomes reported: } \text { Number of patients receiving blood transfusions, blood loss, deep vein throm- } \\
\text { bosis. }\end{array}$ \\
\hline Notes & $\begin{array}{l}\text { Quality assessment score (Schulz criteria): } 4 / 7 \\
\text { Transfusion protocol used }\end{array}$ \\
\hline
\end{tabular}

\section{Risk of bias}


Claeys 2007 (Continued)

\begin{tabular}{|c|c|c|}
\hline Item & Authors' judgement & Description \\
\hline Adequate sequence generation? & Unclear & Unclear \\
\hline Allocation concealment? & Unclear & Unclear \\
\hline $\begin{array}{l}\text { Blinding? } \\
\text { All outcomes }\end{array}$ & Yes & Double blind \\
\hline
\end{tabular}

\section{Coffey 1995}

\begin{tabular}{|c|c|c|}
\hline Methods & \multicolumn{2}{|c|}{$\begin{array}{l}\text { Method of randomisation was not described. Pharmacy controlled the randomisation process. } \\
\text { Method of allocation concealment was not clear. }\end{array}$} \\
\hline Participants & \multicolumn{2}{|c|}{$\begin{array}{l}30 \text { patients undergoing cardiac surgery were randomised to either one of two groups: } \\
\text { - Tranexamic acid group: } n=16, M / F=5 / 11 \text {, mean age }=63.94 \text { years } \\
\text { - Control group (Placebo): } n=14, M / F=5 / 9 \text {, mean age }=64.75 \text { years }\end{array}$} \\
\hline Interventions & \multicolumn{2}{|c|}{$\begin{array}{l}\text { - Tranexamic acid group received a loading dose of } 10 \mathrm{mg} / \mathrm{kg} \text { of TXA over a period of } 30 \\
\text { minutes at the time of skin incision followed by a } 1 \mathrm{mg} / \mathrm{kg} / \mathrm{hr} \text { infusion over } 12 \text { hours. } \\
\text { - Control group received an equal volume of saline. }\end{array}$} \\
\hline Outcomes & \multicolumn{2}{|c|}{ Outcomes reported: Number of patients exposed to allogeneic blood, blood loss, mortality. } \\
\hline Notes & \multicolumn{2}{|c|}{$\begin{array}{l}\text { Quality assessment score (Schulz criteria): 6/7 } \\
\text { Transfusion protocol not specified }\end{array}$} \\
\hline \multicolumn{3}{|l|}{ Risk of bias } \\
\hline Item & Authors' judgement & Description \\
\hline Adequate sequence generation? & Unclear & Unclear \\
\hline Allocation concealment? & Yes & Adequate \\
\hline $\begin{array}{l}\text { Blinding? } \\
\text { All outcomes }\end{array}$ & Yes & Double blind \\
\hline
\end{tabular}

\section{Cohen 1998}

\begin{tabular}{l|l} 
Methods & $\begin{array}{l}\text { Patients were randomised by the hospital pharmacy after stratification and blocking in groups of } \\
\text { six. The pharmacy supplied bags that contained dipyridamole (DIP), aprotinin (APR) or a saline } \\
\text { placebo. }\end{array}$ \\
\hline Participants & $\begin{array}{l}115 \text { patients undergoing cardiac operations for valve replacement or myocardial revascularization, } \\
\text { or a combined procedure were randomised to one of two groups: } \\
\text { - Aprotinin group: } \mathrm{n}=56, \mathrm{M} / \mathrm{F}=44 / 12, \text { mean }(\mathrm{sd}) \text { age }=63(9) \text { years }\end{array}$
\end{tabular}


Cohen 1998 (Continued)

- Control group (Placebo): $\mathrm{n}=59, \mathrm{M} / \mathrm{F}=47 / 12$, mean $(\mathrm{sd})$ age $=61(8)$ years

- Aprotinin group received a high dose of aprotinin (Full Hammersmith) with a loading dose
of $280 \mathrm{mg}(2$ million KIU) plus a pump prime dose of $280 \mathrm{mg}$ and a maintenance dose $70 \mathrm{mg} / \mathrm{hr}$
intra-operatively and continued for 1 hour post-operatively.
- Control group received a saline placebo.
NB: All patients were administered dipyridamole (DIP) orally (100mg four times daily for three or
more doses pre-operatively) and intravenously (at a rate of $0.24 \mathrm{mg} / \mathrm{kghr}$ beginning before anaes-
thesia induction and continuing for 1 hour post-operatively). Autologous blood shed into sterile
cardiotomy reservoirs from chest drains was autotransfused to the patient when drainage exceeded
$150 \mathrm{ml}$ during the first 4 hours post-operatively.

Outcomes

Outcomes reported: Number of patients exposed to allogeneic blood, blood loss, mortality, myocardial infarction, autologous shed blood transfused, blood loss ( $24 \mathrm{hrs}$ ),

renal failure, stroke, intensive care unit length of stay (days), hospital length of stay (days).

$\begin{array}{ll}\text { Notes } & \begin{array}{l}\text { Quality assessment score (Schulz criteria): 5/7 } \\ \text { Transfusion protocol used }\end{array}\end{array}$

Transfusion protocol used

\section{Risk of bias}

\begin{tabular}{lll}
\hline Item & Authors' judgement & Description \\
\hline Adequate sequence generation? & Unclear & Unclear \\
\hline Allocation concealment? & Yes & Adequate \\
\hline $\begin{array}{l}\text { Blinding? } \\
\text { All outcomes }\end{array}$ & Yes & Double blind \\
\hline
\end{tabular}

Colwell 2007

\begin{tabular}{l|l}
\hline Methods & $\begin{array}{l}\text { Patients were randomly assigned on the day of surgery to a treatment group in a } 1: 1 \text { ration from a } \\
\text { computer-generated list managed by an interactive voice response system. Aprotinin and placebo } \\
\text { were provided to the pharmacy in the same packaging and were dispensed by the randomisation } \\
\text { assignment, blinding the patient and staff to the actual treatment group. The primary efficacy } \\
\text { analysis was performed on the intention-to-treat population. }\end{array}$ \\
\hline Participants & $\begin{array}{l}359 \text { patients undergoing orthopaedic surgery (hip arthroplasty) were randomised to one of two } \\
\text { groups: } \\
\text { - Aprotinin group: } \mathrm{n}=180, \mathrm{M} / \mathrm{F}=61 / 84, \text { mean }(\mathrm{sd}) \text { age }=63.4(12.1) \text { years } \\
\text { - Control group: } \mathrm{n}=179, \mathrm{M} / \mathrm{F}=81 / 96, \text { mean }(\mathrm{sd}) \text { age }=64.4(12.7) \text { years }\end{array}$ \\
\hline Interventions & $\begin{array}{l}\text { - Aprotinin group - received a test dose of } 10,000 \mathrm{KIU}, \text { loading dose of } 2 \text { million KIU, then } \\
0.5 \text { million KIU per hour until end of surgery. } \\
\text { - Control group received saline. }\end{array}$
\end{tabular}


Colwell 2007 (Continued)

\begin{tabular}{|c|c|c|}
\hline Outcomes & \multicolumn{2}{|c|}{$\begin{array}{l}\text { Outcomes reported: Number of patients receiving blood transfusions, volume of blood transfused } \\
\text { (units), blood loss, deep vein thrombosis, renal failure, myocardial infarction, stroke, mortality, } \\
\text { pulmonary embolism. }\end{array}$} \\
\hline Notes & \multicolumn{2}{|l|}{$\begin{array}{l}\text { Quality assessment score (Schulz criteria): 6/7 } \\
\text { Transfusion protocol used }\end{array}$} \\
\hline \multicolumn{3}{|l|}{ Risk of bias } \\
\hline Item & Authors' judgement & Description \\
\hline Adequate sequence generation? & Yes & Computer-generated list \\
\hline Allocation concealment? & Yes & Adequate \\
\hline $\begin{array}{l}\text { Blinding? } \\
\text { All outcomes }\end{array}$ & Yes & Double blind \\
\hline
\end{tabular}

\section{Corbeau 1995}

\begin{tabular}{|c|c|}
\hline Methods & $\begin{array}{l}\text { Method of randomisation and allocation concealment were not described. Two patients were ex- } \\
\text { cluded after randomisation. [French language] }\end{array}$ \\
\hline Participants & $\begin{array}{l}104 \text { adults undergoing either coronary artery bypass grafting }(\mathrm{CABG}) \text { or aortic valve replacement } \\
\text { (AVR) were randomised to one of three groups: } \\
\text { - Aprotinin group: } \mathrm{n}=43 \text { [AVR: } \mathrm{n}=20, \mathrm{M} / \mathrm{F}=13 / 7 \text {, mean }(\mathrm{sd}) \text { age }=64(16) \text { years; CABG: } \\
\mathrm{n}=23, \mathrm{M} / \mathrm{F}=20 / 3, \text { mean }(\mathrm{sd}) \text { age }=68(8) \text { years] } \\
\text { - Tranexamic acid group: } \mathrm{n}=41[\text { AVR: } \mathrm{n}=19, \mathrm{M} / \mathrm{F}=7 / 12 \text {, mean }(\mathrm{sd}) \text { age }=63 \text { (19) years; } \\
\text { CABG: } \mathrm{n}=22, \mathrm{M} / \mathrm{F}=19 / 3, \text { mean }(\mathrm{sd}) \text { age }=62(9) \text { years } \\
\text { - Control group: } \mathrm{n}=20[\mathrm{AVR}: \mathrm{n}=10, \mathrm{M} / \mathrm{F}=7 / 3 \text {, mean }(\mathrm{sd}) \text { age }=60(22) \text { years; CABG: } \mathrm{n}= \\
10, \mathrm{M} / \mathrm{F}=9 / 1, \text { mean }(\mathrm{sd}) \text { age }=66(3) \text { years }]\end{array}$ \\
\hline
\end{tabular}

Interventions

- Aprotinin group received 2 million kallikrien inactivator units (KIU) of aprotinin (280mg) after induction of anaesthesia, followed by an infusion of 500,000 KIU/hr $(70 \mathrm{mg} / \mathrm{hr})$ until chest closure, with a supplement to the oxygenator prime of 2 million KIU of aprotinin.

- Tranexamic acid group received $15 \mathrm{mg} / \mathrm{kg}$ of TXA between the injection of heparin (400IU/ $\mathrm{kg}$ ) and the beginning of extracorporeal circulation, plus $15 \mathrm{mg} / \mathrm{kg}$ after protamine injection $(1.3 \mathrm{mg} / 100 \mathrm{IU}$ of heparin).

- Control group did not receive any antifibrinolytic therapy.

Outcomes

Outcomes reported: Number of patients exposed to allogeneic blood, allogeneic blood usage (units) , blood loss.

$\begin{array}{ll}\text { Notes } & \begin{array}{l}\text { Quality assessment score (Schulz criteria): 1/7 } \\ \text { Transfusion protocol used }\end{array}\end{array}$

Transfusion protocol used

\section{Risk of bias}


Corbeau 1995 (Continued)

\begin{tabular}{lll}
\hline Item & Authors' judgement & Description \\
\hline Adequate sequence generation? & Unclear & Unclear \\
\hline Allocation concealment? & Unclear & Unclear \\
\hline $\begin{array}{l}\text { Blinding? } \\
\text { All outcomes }\end{array}$ & Unclear & Unclear \\
\hline
\end{tabular}

\section{Cosgrove 1992}

Methods

in the final analysis.
in the final analysis.
Method of randomisation and allocation concealment were not described. All subjects were included

Participants

169 patients undergoing isolated re-operative myocardial revascularisation were randomised to either one of three groups:

- Aprotinin group (High dose): $\mathrm{n}=57$, males $(87.7 \%)$, mean $(\mathrm{sd})$ age $=60.8(7.8)$ years

- Aprotinin group (Low dose): $\mathrm{n}=56$, males $(80.4 \%)$, mean (sd) age $=61.1(8.3)$ years

- Control group (Placebo): $n=56$, males (87.5\%), mean (sd) age $=63.0(8.8)$ years

\begin{tabular}{|c|c|c|}
\hline Interventions & \multicolumn{2}{|c|}{$\begin{array}{l}\text { - Aprotinin group (High dose) received } 70 \mathrm{mg} \text { of aprotinin in } 50 \mathrm{ml} \text { of } 0.9 \% \text { saline. After } \\
\text { induction of anaesthesia, a loading dose of } 200 \mathrm{ml} \text { of aprotinin solution was given intravenously } \\
\text { over } 20 \text { minutes. Immediately after this, a continuous infusion of } 50 \mathrm{ml} / \mathrm{hr} \text { was begun and } \\
\text { maintained until the patient left the operating room. An additional } 200 \mathrm{ml} \text { of aprotinin was added } \\
\text { to the prime volume of the cardiopulmonary bypass machine. } \\
\text { - Aprotinin group (Low dose) received } 35 \mathrm{mg} \text { of aprotinin in } 50 \mathrm{ml} \text { of } 0.9 \% \text { saline solution at } \\
\text { corresponding times as Group } 1 \text {. } \\
\text { - Control group received } 50 \mathrm{ml} \text { of saline solution at corresponding times as Group } 1 \text {. }\end{array}$} \\
\hline Outcomes & \multicolumn{2}{|c|}{$\begin{array}{l}\text { Outcomes reported: Number of patients exposed to allogeneic blood, allogeneic blood usage (units) } \\
\text {, mortality, myocardial infarction, re-operation for bleeding, fresh frozen plasma usage (units), } \\
\text { platelet usage (units). }\end{array}$} \\
\hline Notes & \multicolumn{2}{|c|}{$\begin{array}{l}\text { Quality assessment score (Schulz criteria): 4/7 } \\
\text { Transfusion protocol not specified }\end{array}$} \\
\hline \multicolumn{3}{|l|}{ Risk of bias } \\
\hline Item & Authors' judgement & Description \\
\hline Adequate sequence generation? & Unclear & Unclear \\
\hline Allocation concealment? & Unclear & Unclear \\
\hline $\begin{array}{l}\text { Blinding? } \\
\text { All outcomes }\end{array}$ & Yes & Double blind \\
\hline
\end{tabular}


Cvachovec 2001

\begin{tabular}{|c|c|c|}
\hline Methods & \multicolumn{2}{|c|}{$\begin{array}{l}\text { Method of randomisation and allocation concealment were not described in the abstract. [Czech } \\
\text { Republic] }\end{array}$} \\
\hline Participants & \multicolumn{2}{|c|}{$\begin{array}{l}42 \text { patients undergoing total hip arthroplasty were randomly allocated to one of two groups: } \\
\text { - Aprotinin group (Low dose): } \mathrm{n}=20, \mathrm{M} / \mathrm{F}=10 / 10 \\
\text { - Control group: } \mathrm{n}=22, \mathrm{M} / \mathrm{F}=8 / 14 \\
\text { NB: No age data were reported }\end{array}$} \\
\hline Interventions & \multicolumn{2}{|c|}{$\begin{array}{l}\text { - Aprotinin group (Low dose) received } 2 \text { million KIU }(280 \mathrm{mg}) \text { of aprotinin started pre- } \\
\text { operatively and continued in the course of the first hour of surgery. } \\
\text { - Control group did not receive aprotinin. }\end{array}$} \\
\hline Outcomes & \multicolumn{2}{|c|}{$\begin{array}{l}\text { Outcomes reported: Number of patients exposed to allogeneic blood, allogeneic blood usage (units) } \\
\text {, blood loss. }\end{array}$} \\
\hline Notes & \multicolumn{2}{|c|}{$\begin{array}{l}\text { Foreign language paper } \\
\text { Transfusion protocol used }\end{array}$} \\
\hline \multicolumn{3}{|l|}{ Risk of bias } \\
\hline Item & Authors' judgement & Description \\
\hline Adequate sequence generation? & Unclear & Unclear \\
\hline Allocation concealment? & Unclear & Unclear \\
\hline $\begin{array}{l}\text { Blinding? } \\
\text { All outcomes }\end{array}$ & Unclear & Unclear \\
\hline
\end{tabular}

\section{D’Ambra 1996}

Methods $\begin{aligned} & \text { A separate random code, using blocks of six, was generated for each site by the statistical department } \\ & \text { of Bayer Incorporated. The study medication for each patient was supplied for each patient in a } \\ & \text { case pack containing } 14 \text { vials. The loading dose vials, pump prime vials, and constant infusion vials } \\ & \text { were separately identified and packaged within the pack for each patient. Investigators were blinded } \\ & \text { to the identity and lot number of each case pack. }\end{aligned}$

Participants

213 patients undergoing cardiac surgery were enrolled and randomised at the five sites to one of three groups:

- Aprotinin group (High dose): $\mathrm{n}=65, \mathrm{M} / \mathrm{F}=31 / 34$, mean $(\mathrm{sd})$ age $=59.8$ (3.1) years

- Aprotinin group (Low dose): $\mathrm{n}=62, \mathrm{M} / \mathrm{F}=33 / 29$, mean (sd) age $=59.2$ (3.2) years

- Control group (Placebo): $\mathrm{n}=64, \mathrm{M} / \mathrm{F}=30 / 34$, mean $(\mathrm{sd})$ age $=60.0$ (3.1) years

NB: Of the 213 patients enrolled and randomised, 212 were included in the safety analysis and 191 were included in a primary analysis of efficacy.

Interventions

- Aprotinin group (High dose) received an intravenous loading dose of $280 \mathrm{mg}$ of aprotinin (2 million KIU) infused over 20-30 minutes followed by a continuous infusion of $70 \mathrm{mg} / \mathrm{hr}$ $(500,000 \mathrm{KIU} / \mathrm{hr})$ infused until chest closure. An additional dose of aprotinin equivalent to the 
loading dose was added to the pump prime.

- Aprotinin group (Low dose) received a loading dose of $140 \mathrm{mg}$ of aprotinin (1 million KIU) infused over 20-30 minutes followed by a continuous infusion of $35 \mathrm{mg}(250,000 \mathrm{KIU} / \mathrm{hr})$ of aprotinin, infused until chest closure. An additional dose of aprotinin, equivalent to the loading dose, was added to the pump prime.

- Control group received equivalent volumes of normal saline solution at corresponding times to the active treatments.

NB: Blood conservation measures were used for all groups. These measures included the reinfusion of post-operative mediastinal shed blood (cell salvage) and the pre-operative donation of autologous blood (PAD). Epsilon aminocaproic acid (EACA) and desmopressin (DDAVP) were used to treat active bleeding after the reversal of heparin.

Outcomes

Outcomes reported: Number of patients exposed to allogeneic blood, allogeneic blood usage (units) , fresh frozen plasma usage (units), platelet usage (units), mortality, myocardial infarction, reoperation, renal dysfunction, deep vein thrombosis, cardiovascular complications, cerebrovascular accident.

Notes Quality assessment score (Schulz criteria): 6/7

Transfusion protocol used

\section{Risk of bias}

\begin{tabular}{l|l|l}
\hline Item & Authors' judgement & Description \\
\hline Adequate sequence generation? & Yes & $\begin{array}{l}\text { Random code generated for each site by the sta- } \\
\text { tistical department of Bayer }\end{array}$ \\
\hline $\begin{array}{l}\text { Allocation concealment? } \\
\text { Blinding? }\end{array}$ & Yes & Adequate \\
\hline All outcomes & Yes & Double blind \\
\hline
\end{tabular}

\section{D’Ambrosio 1999}

\begin{tabular}{l|l} 
Methods & Method of randomisation and allocation concealment were not described. \\
\hline Participants & $\begin{array}{l}60 \text { patients undergoing total hip arthroplasty were randomised into one of four groups: } \\
\text { Comparison } 1:\end{array}$ \\
$\begin{array}{l}\text { - Aprotinin group: } \mathrm{n}=15, \mathrm{M} / \mathrm{F}=8 / 7, \text { mean }(\mathrm{sd}) \text { age }=61.5(9.2) \text { years } \\
\text { - Control group (Placebo): } \mathrm{n}=15, \mathrm{M} / \mathrm{F}=9 / 6, \text { mean }(\mathrm{sd}) \text { age }=66.7 \text { (7.3) years }\end{array}$ \\
$\begin{array}{l}\text { Comparison 2: } \\
\text { - Aprotinin group: } \mathrm{n}=15, \mathrm{M} / \mathrm{F}=7 / 8, \text { mean }(\mathrm{sd}) \text { age }=66.6(9.2) \text { years } \\
\text { - Control group (Placebo): } \mathrm{n}=15, \mathrm{M} / \mathrm{F}=7 / 8, \text { mean }(\mathrm{sd}) \text { age }=60.5(12.9) \text { years }\end{array}$ \\
\hline Comparison $1:$ \\
$\begin{array}{l}\text { - Aprotinin group (epidural + general anaesthesia) received } 500,000 \text { KIU of aprotinin } \\
\text { administered as a bolus before skin incision and } 500,000 \mathrm{KIU} \text { continuous infusion until the skin } \\
\text { was sutured. }\end{array}$
\end{tabular}


D’Ambrosio 1999 (Continued)

\begin{tabular}{|c|c|c|}
\hline & \multicolumn{2}{|c|}{$\begin{array}{l}\text { - Control group (epidural + general anaesthesia) received saline solution } 0.9 \% \text { in same } \\
\text { manner as aprotinin. } \\
\text { Comparison 2: } \\
\text { - Aprotinin group (general anaesthesia) 500,000 KIU was administered as a bolus before skin } \\
\text { incision and 500,000 KIU continuous until the skin was sutured. } \\
\text { - Control group (placebo) (general anaesthesia) saline solution } 0.9 \% \text { in the same manner as } \\
\text { aprotinin. } \\
\text { NB: All subjects were exposed to pre-operative autologous blood donation and cell savage. }\end{array}$} \\
\hline Outcomes & \multicolumn{2}{|c|}{$\begin{array}{l}\text { Outcomes reported: Number of patients exposed to allogeneic blood, blood loss, allogeneic } \& \\
\text { autologous blood usage (units). }\end{array}$} \\
\hline Notes & \multicolumn{2}{|c|}{$\begin{array}{l}\text { Quality assessment score (Schulz criteria): } 2 / 7 \\
\text { No transfusion protocol used }\end{array}$} \\
\hline \multicolumn{3}{|l|}{ Risk of bias } \\
\hline Item & Authors' judgement & Description \\
\hline Adequate sequence generation? & Unclear & Unclear \\
\hline Allocation concealment? & Unclear & Unclear \\
\hline $\begin{array}{l}\text { Blinding? } \\
\text { All outcomes }\end{array}$ & Yes & Double blind \\
\hline
\end{tabular}

\section{Daily 1994}

\begin{tabular}{l|l} 
Methods & $\begin{array}{l}\text { Method of randomisation was not described. Epsilon-aminocaproic acid (EACA) and placebo were } \\
\text { delivered to the operating room in numbered, but otherwise identical vials labelled "study drug". }\end{array}$ \\
\hline Participants & $\begin{array}{l}40 \text { patients undergoing first-time coronary artery bypass grafting without prior sternotomy were } \\
\text { randomised to one of two groups: } \\
\text { - Epsilon aminocaproic acid group: } \mathrm{n}=21, \mathrm{M} / \mathrm{F} 14 / 7, \text { mean }(\mathrm{sd}) \text { age }=63(9) \text { years } \\
\text { - Control group (Placebo): } \mathrm{n}=19, \mathrm{M} / \mathrm{F}=18 / 1, \text { mean }(\mathrm{sd}) \text { age }=67 \text { (10) years }\end{array}$ \\
\hline Interventions & $\begin{array}{l}\text { - Epsilon aminocaproic acid group }(\mathrm{EACA}) \text { received } 10 \mathrm{~g} \text { of EACA in } 40 \mathrm{ml} \text { of saline solution } \\
\text { given after induction of anaesthesia but before the skin incision. Another } 40 \mathrm{ml} \text { was given after } \\
\text { heparin administration in the pump, and a third } 40 \mathrm{ml} \text { dose was given after the administration of } \\
\text { protamine. } \\
\text { - Control group (Placebo) received equivalent volumes of saline solution. }\end{array}$ \\
\hline NB: Both groups were exposed to cell salvage.
\end{tabular}

Outcomes

Outcomes reported: Number of patients exposed to allogeneic blood, blood loss (12/24hrs), myocardial infarction, stroke (cerebrovascular accident), use of shed mediastinal blood.

Notes

Quality assessment score (Schulz criteria): 6/7

Transfusion protocol used 
Daily 1994 (Continued)

\begin{tabular}{lll} 
Risk of bias & Authors' judgement & Description \\
\hline Item & Unclear & Unclear \\
\hline Adequate sequence generation? & Uncequate \\
\hline Allocation concealment? & Yes & Adequable blind \\
\hline $\begin{array}{l}\text { Blinding? } \\
\text { All outcomes }\end{array}$ & Yes & Doul \\
\hline
\end{tabular}

Dalmau 1999

\begin{tabular}{|c|c|c|}
\hline Methods & \multicolumn{2}{|c|}{ Method of randomisation and allocation concealment were not described. } \\
\hline Participants & \multicolumn{2}{|c|}{$\begin{array}{l}124 \text { patients undergoing orthotopic liver transplantation were randomised to one of three groups: } \\
\text { - Epsilon aminocaproic acid group: } \mathrm{n}=42 \\
\text { - Tranexamic acid group: } \mathrm{n}=42 \\
\text { - Control group (Placebo) group: } \mathrm{n}=40 \\
\text { NB: No age or gender data were reported. }\end{array}$} \\
\hline Interventions & \multicolumn{2}{|c|}{$\begin{array}{l}\text { - Epsilon aminocaproic acid (EACA) group received a continuous infusion of EACA ( } 8 \mathrm{~g} \text { in } \\
480 \mathrm{~mL} \text { normal saline) at } 16 \mathrm{mg} / \mathrm{kg} \text { per hour. EACA was infused from induction of anaesthesia to } \\
\text { graft reperfusion. } \\
\text { - Tranexamic acid group (TXA) received a continuous infusion of TXA ( } 5 \mathrm{~g} \text { in } 450 \mathrm{~mL} \text { norma } \\
\text { saline) at } 10 \mathrm{mg} / \mathrm{kg} \text { per hour. TXA was infused from induction of anaesthesia to graft reperfusion } \\
\text { - Control group received an equal volume infusion of normal saline. Placebo was infused from } \\
\text { the induction of anaesthesia to graft reperfusion. }\end{array}$} \\
\hline Outcomes & \multicolumn{2}{|c|}{$\begin{array}{l}\text { Outcomes reported: Number of patients exposed to allogeneic blood, allogeneic blood usage (units } \\
\text {, fresh frozen plasma usage (units), platelet usage (units), thrombotic events, cryoprecipitate (units }\end{array}$} \\
\hline Notes & \multicolumn{2}{|c|}{$\begin{array}{l}\text { Quality assessment score (Schulz criteria): } 4 / 7 \\
\text { Transfusion protocol used }\end{array}$} \\
\hline \multicolumn{3}{|l|}{ Risk of bias } \\
\hline Item & Authors' judgement & Description \\
\hline Adequate sequence generation? & Unclear & Unclear \\
\hline Allocation concealment? & Unclear & Unclear \\
\hline $\begin{array}{l}\text { Blinding? } \\
\text { All outcomes }\end{array}$ & Yes & Double blind \\
\hline
\end{tabular}


Dalmau 2000

\begin{tabular}{|c|c|c|}
\hline Methods & \multicolumn{2}{|c|}{$\begin{array}{l}\text { Drugs were prepared then randomised to patients using a randomisation schedule provided in } \\
\text { sealed envelopes. }\end{array}$} \\
\hline Participants & \multicolumn{2}{|c|}{$\begin{array}{l}132 \text { patients undergoing orthotopic liver transplantation (OLT) were randomly assigned to one of } \\
\text { three groups: } \\
\text { - Epsilon aminocaproic acid group: } \mathrm{n}=42, \mathrm{M} / \mathrm{F}=26 / 16 \text {, median (range) age }=56 \text { (32-69) } \\
\text { years } \\
\text { - Tranexamic acid group: } \mathrm{n}=42, \mathrm{M} / \mathrm{F}=31 / 11 \text {, median (range) age }=58(22-69) \text { years } \\
\text { - Control group (Placebo): } \mathrm{n}=40, \mathrm{M} / \mathrm{F}=22 / 18 \text {, median (range) age }=60(18-67) \text { years }\end{array}$} \\
\hline Interventions & \multicolumn{2}{|c|}{$\begin{array}{l}\text { - Epsilon aminocaproic acid (EACA) group received a continuous infusion of EACA ( } 8 \mathrm{~g} \text { in } \\
480 \mathrm{ml} \text { of normal saline) at a rate of } 16 \mathrm{mg} / \mathrm{kg} / \mathrm{hr} \text { from the induction of anesthesia until the portal } \\
\text { vein was unclamped. } \\
\text { - Tranexamic acid (TXA) group received a continuous dose infusion of TXA ( } 5 \mathrm{~g} \text { in } 450 \mathrm{ml} \text { of } \\
\text { normal saline) at a rate of } 16 \mathrm{mg} / \mathrm{kg} / \mathrm{hour} \text { from the induction of anaesthesia until the portal vein } \\
\text { was unclamped. } \\
\text { - Control group received isotonic saline at an equal volume ( } 10 \mathrm{ml} / \mathrm{kg} / \mathrm{hour} \text { ) from the } \\
\text { induction of anaesthesia until the portal vein was unclamped. }\end{array}$} \\
\hline Outcomes & \multicolumn{2}{|c|}{$\begin{array}{l}\text { Outcomes reported: Number of patients exposed to allogeneic blood, allogeneic blood usage (units) } \\
\text {, fresh frozen plasma (units), platelets (units), other arterial thrombosis, } \\
\text { prophylactic DDAVP treatment, DDAVP treatment for bleeding, EACA treatment (clinical fibrol- } \\
\text { ysis). }\end{array}$} \\
\hline Notes & \multicolumn{2}{|c|}{$\begin{array}{l}\text { Quality assessment score (Schulz criteria): 4/7 } \\
\text { Transfusion protocol used }\end{array}$} \\
\hline \multicolumn{3}{|l|}{ Risk of bias } \\
\hline Item & Authors' judgement & Description \\
\hline Adequate sequence generation? & Unclear & Unclear \\
\hline Allocation concealment? & No & $\begin{array}{l}\text { Inadequate - sealed envelopes were used to con- } \\
\text { ceal treatment allocation }\end{array}$ \\
\hline $\begin{array}{l}\text { Blinding? } \\
\text { All outcomes }\end{array}$ & Yes & Double blind \\
\hline
\end{tabular}

Dalmau 2004

\begin{tabular}{l|l} 
Methods & $\begin{array}{l}\text { Drugs were prepared and then randomised to patients using a randomisation schedule provided in } \\
\text { sealed envelopes. Method of randomisation was not described. }\end{array}$ \\
\hline Participants & $\begin{array}{r}127 \text { patients undergoing orthotopic liver transplantation were randomised to one of two groups: } \\
\text { - Aprotinin group (Low dose) } \mathrm{n}=63, \mathrm{M} / \mathrm{F}=45 / 19, \text { mean (sd) age }=54(9) \text { years } \\
\text { - Tranexamic acid group: } \mathrm{n}=64, \mathrm{M} / \mathrm{F}=44 / 19, \text { mean (sd) age }=53(10) \text { years }\end{array}$
\end{tabular}


Dalmau 2004 (Continued)

\begin{tabular}{|c|c|c|}
\hline Interventions & \multicolumn{2}{|c|}{$\begin{array}{l}\text { - Aprotinin group (Low dose) received a bolus of } 2 \text { million KIU in } 250 \mathrm{ml} \text { of IV solution in } 30 \\
\text { minutes followed by a continuous infusion of } 500,000 \mathrm{KIU} / \mathrm{hr} \text {. Diluted in normal saline to be } \\
\text { administered at a rate of } 100 \mathrm{ml} / \mathrm{hr} \text { after the bolus dose. } \\
\text { - Tranexamic acid (TXA) group received a bolus of } 250 \mathrm{ml} \text { of normal saline in } 30 \text { minutes } \\
\text { followed by a continuous infusion of TXA at a dose of } 10 \mathrm{mg} / \mathrm{kg} / \mathrm{hr} \text {. Diluted in normal saline to } \\
\text { be administered at a rate of } 100 \mathrm{ml} / \mathrm{hour} \text { after the bolus dose. }\end{array}$} \\
\hline Outcomes & \multicolumn{2}{|c|}{$\begin{array}{l}\text { Outcomes reported: Allogeneic blood usage (units), fresh frozen plasma (units), platelets (units) } \\
\text {, mortality, myocardial infarction, DDAVP pre-operative administration, EACA intra-operative } \\
\text { administration, any thrombosis, re-operation for bleeding, renal failure. }\end{array}$} \\
\hline Notes & \multicolumn{2}{|c|}{$\begin{array}{l}\text { Quality assessment score (Schulz criteria): 4/7 } \\
\text { Transfusion protocol used }\end{array}$} \\
\hline \multicolumn{3}{|l|}{ Risk of bias } \\
\hline Item & Authors' judgement & Description \\
\hline Adequate sequence generation? & Unclear & Unclear \\
\hline Allocation concealment? & No & $\begin{array}{l}\text { Inadequate - sealed envelopes were used to con- } \\
\text { ceal treatment allocation }\end{array}$ \\
\hline $\begin{array}{l}\text { Blinding? } \\
\text { All outcomes }\end{array}$ & Yes & Double blind \\
\hline
\end{tabular}

Defraigne 2000

\begin{tabular}{|c|c|}
\hline Methods & $\begin{array}{l}\text { Randomisation was accomplished using a random number table. Sealed envelopes were used to } \\
\text { conceal treatment allocation. }\end{array}$ \\
\hline Participants & $\begin{array}{l}200 \text { patients undergoing cardiac surgery were randomly allocated to one of four groups: } \\
\text { - Aprotinin group: } \mathrm{n}=50, \mathrm{M} / \mathrm{F}=34 / 16, \text { mean }(\mathrm{sd}) \text { age }=62.8(13.4) \text { years } \\
\text { - Control group: } \mathrm{n}=50, \mathrm{M} / \mathrm{F}=36 / 14, \text { mean }(\mathrm{sd}) \text { age }=64.2(11.3) \text { years } \\
\text { - Aprotinin group: } \mathrm{n}=50, \mathrm{M} / \mathrm{F}=35 / 15, \text { mean }(\mathrm{sd}) \text { age }=64(13.4) \text { years } \\
\text { - Control group: } \mathrm{n}=50, \mathrm{M} / \mathrm{F}=34 / 16, \text { mean }(\mathrm{sd}) \text { age }=60.1(12.7) \text { years }\end{array}$ \\
\hline Interventions & $\begin{array}{l}\text { - Aprotinin group (Heparin coated CPB circuit with aprotinin administration - HCO-A) } \\
\text { received a loading dose of } 280 \mathrm{mg} \text { ( } 2 \text { million KIU) before surgery and } 280 \mathrm{mg} \text { in the pump prime } \\
\text { and a continuous infusion of } 500,000 \mathrm{KIU} / \text { hour IV. } \\
\text { - Control group (Heparin coated } \mathrm{CPB} \text { circuit without aprotinin - HCO). } \\
\text { - Aprotinin group (Uncoated CPB circuit with aprotinin) received a loading dose of } 280 \mathrm{mg}(2 \\
\text { million KIU) of aprotinin before surgery and } 280 \mathrm{mg} \text { in the prime solution and continuous } \\
\text { infusion of } 500,000 \mathrm{KIU} / \text { hour IV. } \\
\text { - Control group (Uncoated CPB circuit without aprotinin administration). }\end{array}$ \\
\hline
\end{tabular}

Outcomes

Outcomes reported: Allogeneic blood usage (units), fresh frozen plasma usage (units), platelet usage (units), blood loss. 
Defraigne 2000 (Continued)

\begin{tabular}{|c|c|c|}
\hline Notes & $\begin{array}{l}\text { Quality assessment score (Schulz criteria): 6/7 } \\
\text { Transfusion protocol used }\end{array}$ & \\
\hline \multicolumn{3}{|l|}{ Risk of bias } \\
\hline Item & Authors' judgement & Description \\
\hline Adequate sequence generation? & Yes & Random number table \\
\hline Allocation concealment? & No & $\begin{array}{l}\text { Inadequate - sealed envelopes were used to con- } \\
\text { ceal treatment allocation }\end{array}$ \\
\hline $\begin{array}{l}\text { Blinding? } \\
\text { All outcomes }\end{array}$ & No & Single blind \\
\hline
\end{tabular}

\section{Del Rossi 1989}

\begin{tabular}{|c|c|c|}
\hline Methods & \multicolumn{2}{|c|}{ Method of randomisation and allocation concealment were not described. } \\
\hline Participants & \multicolumn{2}{|c|}{$\begin{array}{l}350 \text { patients undergoing elective coronary artery bypass surgery, repair of myocardial aneurysms, } \\
\text { valve replacement or combined procedures were randomly assigned to one of two groups: } \\
\text { - Epsilon aminocaproic acid group: } \mathrm{n}=170, \mathrm{M} / \mathrm{F}=132 / 38 \text {, mean }(\mathrm{sd}) \text { age males }=58.9(2.1) \\
\text { years; mean }(\mathrm{sd}) \text { age females }=61.6(2.8) \text { years } \\
\text { - Control group (Placebo): } \mathrm{n}=180, \mathrm{M} / \mathrm{F}=144 / 66 \text {, mean (sd) age males = } 59.8 \text { (5.6) years; } \\
\text { mean (sd) age females }=60.2(4.2) \text { years }\end{array}$} \\
\hline Interventions & \multicolumn{2}{|c|}{$\begin{array}{l}\text { - Epsilon aminocaproic acid group received an initial priming dose of } 5 \mathrm{~g} \text { of EACA prior to } \\
\text { skin incision, followed by a continuous infusion of } 1 \mathrm{~g} / \mathrm{hr} \text { over the next } 6 \text { to } 8 \text { hours. } \\
\text { - Control group received saline solution in the same fashion. }\end{array}$} \\
\hline Outcomes & \multicolumn{2}{|c|}{$\begin{array}{l}\text { Outcomes reported: Allogeneic blood usage (units), blood loss ( } 24 \mathrm{hrs}) \text {, mortality, myocardial in- } \\
\text { farction, re-operation for bleeding, stroke, graft failure. }\end{array}$} \\
\hline Notes & \multicolumn{2}{|c|}{$\begin{array}{l}\text { Quality assessment score (Schulz criteria): 4/7 } \\
\text { Transfusion protocol used }\end{array}$} \\
\hline \multicolumn{3}{|l|}{ Risk of bias } \\
\hline Item & Authors' judgement & Description \\
\hline Adequate sequence generation? & Unclear & Unclear \\
\hline Allocation concealment? & Unclear & Unclear \\
\hline $\begin{array}{l}\text { Blinding? } \\
\text { All outcomes }\end{array}$ & Yes & Double blind \\
\hline
\end{tabular}


Deleuze 1991

\begin{tabular}{|c|c|c|}
\hline Methods & \multicolumn{2}{|c|}{$\begin{array}{l}\text { Pharmaceutical company supplied the study drugs in identical bottles, identifiable only by number. } \\
\text { The method of generating allocation sequences was not described. [French] }\end{array}$} \\
\hline Participants & \multicolumn{2}{|c|}{$\begin{array}{l}60 \text { coronary patients undergoing at least two aorto-coronary bypass grafts for the first time were } \\
\text { randomised to one of two groups: } \\
\text { - Aprotinin group: } n=30, M / F=24 / 6, \text { mean }(s d) \text { age }=60.3(8.0) \text { years } \\
\text { - Control group (Placebo): } n=30, M / F=25 / 5 \text {, mean (sd) age }=61.3(8.0) \text { years }\end{array}$} \\
\hline Interventions & \multicolumn{2}{|c|}{$\begin{array}{l}\text { - Aprotinin group received } 4 \text { flasks }(200 \mathrm{ml}) \text { of aprotinin intravenously, after the induction of } \\
\text { anaesthesia, over } 30 \text { minutes via a central venous catheter, then a continuous infusion of aprotinin } \\
\text { at } 50 \mathrm{ml} / \mathrm{hr} \text { until the end of surgery. A further } 4 \text { flasks were administered via the extracorporeal } \\
\text { circulation circuit. } \\
\text { - Control group received the equivalent volume of physiological serum over the same time } \\
\text { periods. } \\
\text { NB: One active flask contained } 70 \mathrm{mg}(500,000 \mathrm{KIU}) \text { of aprotinin in } 50 \mathrm{mls} \text { of physiological serum. } \\
\text { One placebo flask contained an equivalent quantity of physiological serum. }\end{array}$} \\
\hline Outcomes & \multicolumn{2}{|c|}{$\begin{array}{l}\text { Outcomes reported: Number of patients exposed to allogeneic blood, allogeneic blood usage (units) } \\
\text {, blood loss ( } 48 \mathrm{hrs} \text { ), re-operation. }\end{array}$} \\
\hline Notes & \multicolumn{2}{|c|}{$\begin{array}{l}\text { Quality assessment score (Schulz criteria): 4/7 } \\
\text { Transfusion protocol used } \\
\text { French article - translated }\end{array}$} \\
\hline \multicolumn{3}{|l|}{ Risk of bias } \\
\hline Item & Authors' judgement & Description \\
\hline Adequate sequence generation? & Unclear & Unclear \\
\hline Allocation concealment? & Yes & Adequate \\
\hline $\begin{array}{l}\text { Blinding? } \\
\text { All outcomes }\end{array}$ & Yes & Double blind \\
\hline
\end{tabular}

Demeyere 2006

\begin{tabular}{l|l}
\hline Methods & $\begin{array}{l}\text { Method of sequence generation and allocation concealment were not described. [Poster presenta- } \\
\text { tion] }\end{array}$ \\
\hline Participants & $\begin{array}{l}60 \text { patients undergoing cardiac surgery were randomised to one of three groups: } \\
\text { - Aprotinin group: } \mathrm{n}=20 \\
\text { - Tranexamic acid group: } \mathrm{n}=20 \\
\text { - Control group: } \mathrm{n}=20\end{array}$ \\
NB: Demographic data were not reported.
\end{tabular}




\begin{tabular}{|c|c|c|}
\hline & \multicolumn{2}{|c|}{$\begin{array}{l}\text { - Tranexamic acid group received } 100 \mathrm{mg} \text { loading dose then } 1 \mathrm{mg} / \mathrm{kg} / \mathrm{hr} \text { infusion. } \\
\text { - Control group received saline. }\end{array}$} \\
\hline Outcomes & \multicolumn{2}{|c|}{ Outcomes reported: Transfusion of blood products, blood loss, re-operation for bleeding } \\
\hline Notes & \multicolumn{2}{|c|}{$\begin{array}{l}\text { Quality assessment score (Schulz criteria): } 2 / 7 \\
\text { Transfusion protocol not reported }\end{array}$} \\
\hline \multicolumn{3}{|l|}{ Risk of bias } \\
\hline Item & Authors' judgement & Description \\
\hline Adequate sequence generation? & Unclear & Unclear \\
\hline Allocation concealment? & Unclear & Unclear \\
\hline $\begin{array}{l}\text { Blinding? } \\
\text { All outcomes }\end{array}$ & Unclear & Unclear \\
\hline
\end{tabular}

\section{Desai 2009}

\begin{tabular}{|c|c|c|}
\hline Methods & \multicolumn{2}{|c|}{ Methods of sequence generation and allocation concealment were unclear. } \\
\hline Participants & \multicolumn{2}{|c|}{$\begin{array}{l}75 \text { patients undergoing cardiac surgery were randomised to one of two groups: } \\
\text { - Aprotinin group: } \mathrm{n}=38 \\
\text { - Control group: } \mathrm{n}=37 \\
\text { NB: Demographic data were not reported. }\end{array}$} \\
\hline Interventions & \multicolumn{2}{|c|}{$\begin{array}{l}\text { - Aprotinin group (full-dose) } 10,000 \mathrm{KIU} \text { test dose, } 2 \text { million KIU via central line and } \\
\text { 500,000 KIU/hr IV until the end of surgery. } \\
\text { - Control group received saline. }\end{array}$} \\
\hline Outcomes & \multicolumn{2}{|c|}{$\begin{array}{l}\text { Outcomes reported: Number of patients receiving blood transfusion, blood loss, myocardial in- } \\
\text { farction, renal failure, mortality, re-operation for bleeding. }\end{array}$} \\
\hline Notes & \multicolumn{2}{|c|}{$\begin{array}{l}\text { Quality assessment score (Schulz criteria): } 3 / 7 \\
\text { Transfusion protocol used }\end{array}$} \\
\hline \multicolumn{3}{|l|}{ Risk of bias } \\
\hline Item & Authors' judgement & Description \\
\hline Adequate sequence generation? & Unclear & Unclear \\
\hline Allocation concealment? & Unclear & Unclear \\
\hline
\end{tabular}


Desai 2009 (Continued)

\begin{tabular}{|c|c|c|}
\hline $\begin{array}{l}\text { Blinding? } \\
\text { All outcomes }\end{array}$ & Yes & Double blind \\
\hline
\end{tabular}

\section{Dietrich 1990}

\begin{tabular}{l|l} 
Methods & $\begin{array}{l}\text { Method of randomisation was not described. Aprotinin and placebo were provided by the manu- } \\
\text { facturer (Bayer AG, Leverkusen, FRG) in identical packages, each containing } 12 \text { bottles that could } \\
\text { only be identified by the random number. One patient from the aprotinin trial arm was excluded } \\
\text { from the final analysis. }\end{array}$ \\
\hline Participants & $\begin{array}{l}40 \text { patients scheduled for elective primary myocardial revascularisation were randomised to one of } \\
\text { two groups: } \\
\text { - Aprotinin group: } \mathrm{n}=20, \text { mean }(\mathrm{sd}) \text { age }=58(10) \text { years } \\
\text { - Control group (Placebo): } \mathrm{n}=20 \text {, mean }(\mathrm{sd}) \text { age }=55(8) \text { years } \\
\text { NB: Gender data were not reported. }\end{array}$ \\
\hline
\end{tabular}

Interventions

- Aprotinin group received a loading dose of 2 million KIU $(280 \mathrm{mg})$ of aprotinin after induction of anaesthesia and before surgery, over a 15 minute period followed by a continuous infusion of 500,000 KIU/hr administered by infusion pump for the entire duration of surgery. An additional bolus of 2 million KIU of aprotinin was added to the pump prime of the heart-lung machine.

- Control group received an equal volume of saline solution.

\begin{tabular}{|c|c|c|}
\hline Outcomes & \multicolumn{2}{|c|}{$\begin{array}{l}\text { Outcomes reported: Number of patients exposed to allogeneic blood, allogeneic whole blood usage } \\
\text { (ml/units), fresh frozen plasma usage (units), platelet usage (units), blood loss, re-operation for } \\
\text { bleeding. }\end{array}$} \\
\hline Notes & \multicolumn{2}{|c|}{$\begin{array}{l}\text { Quality assessment score (Schulz criteria): 5/7 } \\
\text { Transfusion protocol used }\end{array}$} \\
\hline \multicolumn{3}{|l|}{ Risk of bias } \\
\hline Item & Authors' judgement & Description \\
\hline Adequate sequence generation? & Unclear & Unclear \\
\hline Allocation concealment? & Yes & Adequate \\
\hline $\begin{array}{l}\text { Blinding? } \\
\text { All outcomes }\end{array}$ & Yes & Double blind \\
\hline
\end{tabular}


Dietrich 1992

\begin{tabular}{|c|c|c|}
\hline Methods & \multicolumn{2}{|c|}{ Method of randomisation and allocation concealment were not described. No exclusions reported } \\
\hline Participants & \multicolumn{2}{|c|}{$\begin{array}{l}1784 \text { adult patients undergoing primary coronary artery bypass grafting, valve replacement (or } \\
\text { combined procedures), and cardiac reoperations, were randomly assigned to one of two groups: } \\
\text { - Aprotinin group: } n=902, \mathrm{M} / \mathrm{F}=667 / 239 \text {, mean (sd) age }=60(10) \text { years } \\
\text { - Control group: } n=882, \mathrm{M} / \mathrm{F}=653 / 229 \text {, mean (sd) age }=59 \text { (11) years }\end{array}$} \\
\hline Interventions & \multicolumn{2}{|c|}{$\begin{array}{l}\text { - Aprotinin group received a loading dose of } 2 \text { million KIU of aprotinin after induction of } \\
\text { anaesthesia and before surgery, over a } 15 \text {-minute period, followed by a continuous infusion of } \\
500,000 \mathrm{KIU} / \mathrm{hr} \text { administered by an infusion pump during the entire course of surgery. An } \\
\text { additional bolus of } 2 \text { million KIU of aprotinin was added to the pump prime of the heart-lung } \\
\text { machine. } \\
\text { - Control group received no aprotinin. } \\
\text { NB: Both groups were exposed to cell salvage. }\end{array}$} \\
\hline Outcomes & \multicolumn{2}{|c|}{$\begin{array}{l}\text { Outcomes reported: Number of patients exposed to allogeneic blood, allogeneic blood usage, } \\
\text { blood loss ( } 36 \mathrm{hrs}) \text {, mortality, intensive care unit length of stay (days), re-operation, renal failure, } \\
\text { hypotension. }\end{array}$} \\
\hline Notes & \multicolumn{2}{|c|}{$\begin{array}{l}\text { Quality assessment score (Schulz criteria): 2/7 } \\
\text { Transfusion protocol used }\end{array}$} \\
\hline \multicolumn{3}{|l|}{ Risk of bias } \\
\hline Item & Authors' judgement & Description \\
\hline Adequate sequence generation? & Unclear & Unclear \\
\hline Allocation concealment? & Unclear & Unclear \\
\hline $\begin{array}{l}\text { Blinding? } \\
\text { All outcomes }\end{array}$ & Unclear & Unclear \\
\hline
\end{tabular}

\section{Dietrich 1995}

Methods Patients were independently randomised, using a table of random numbers, to either aprotinin or control group. Aprotinin and placebo were provided by the manufacturer (Bayer AG, Leverkusen, Germany) in identical packages each containing 14 bottles, that could only be identified by the random number. No loss to follow-up reported.

\begin{tabular}{l|l} 
Participants & $\begin{array}{l}30 \text { male patients scheduled for elective primary coronary revascularisation were randomly assigned } \\
\text { to one of two groups: } \\
\text { - Aprotinin group: } \mathrm{n}=15, \text { mean }(\mathrm{sd}) \text { age }=62.93(6.77) \text { years } \\
\text { - Control group (Placebo): } \mathrm{n}=15, \text { mean }(\mathrm{sd}) \text { age }=62.07(10.01) \text { years }\end{array}$ \\
\hline
\end{tabular}

Interventions

- Aprotinin group received a loading dose of 2 million KIU $(280 \mathrm{mg})$ of aprotinin over a $15-$ minute period at the start of surgery, followed by a continuous infusion of 500,000 KIU/hr throughout the course of surgery. An additional bolus of 2 million KIU was added to the prime of 
Dietrich 1995 (Continued)

the heart-lung machine.

- Control group received an equal volume of saline.

NB: Both groups were exposed to cell salvage.

\begin{tabular}{|c|c|c|}
\hline Outcomes & \multicolumn{2}{|c|}{$\begin{array}{l}\text { Outcomes reported: Number of patients exposed to allogeneic blood, allogeneic blood usage (units) } \\
\text {, blood loss, complications, re-operation, mortality. }\end{array}$} \\
\hline Notes & \multicolumn{2}{|l|}{$\begin{array}{l}\text { Quality assessment score (Schulz criteria): 7/7 } \\
\text { Transfusion protocol used }\end{array}$} \\
\hline \multicolumn{3}{|l|}{ Risk of bias } \\
\hline Item & Authors' judgement & Description \\
\hline Adequate sequence generation? & Yes & Table of random numbers \\
\hline Allocation concealment? & Yes & Adequate \\
\hline $\begin{array}{l}\text { Blinding? } \\
\text { All outcomes }\end{array}$ & Yes & Double blind \\
\hline
\end{tabular}

\section{Dietrich 2008}

\begin{tabular}{l|l}
\hline Methods & Computer-generated randomisation list and central allocation were used. \\
\hline Participants & $\begin{array}{r}22 \text { patients undergoing cardiac surgery were randomly allocated to one of two groups: } \\
\text { - Aprotinin group: } \mathrm{n}=110, \mathrm{M} / \mathrm{F}=82 / 28, \text { mean }(\mathrm{sd}) \text { age }=67.3(10.6) \text { years } \\
\text { - Tranexamic acid group }(\mathrm{n}=110), \mathrm{M} / \mathrm{F}=72 / 38, \text { mean }(\mathrm{sd}) \text { age }=69.8(10.3) \text { years }\end{array}$ \\
\hline Interventions & $\begin{array}{l}\text { - Aprotonin group received a } 1 \mathrm{ml} \text { test dose, then } 1 \text { million KIU IV over } 10 \text { minutes, then } \\
\text { continuous infusion of } 500,000 \mathrm{KIU} / \mathrm{hr} \text { for the duration of surgery, additional } 2 \text { million KIU } \\
\text { added to } \mathrm{CPB} \text { circuit priming fluid. } \\
\text { - Tranexamic acid group received a } 2 \mathrm{~g} \text { bolus dose, followed by a continuous infusion of } 1 \mathrm{~g} / \mathrm{hr}, \\
\text { additional bolus added to } \mathrm{CPB} \text { circuit priming fluid. }\end{array}$ \\
\hline
\end{tabular}

Outcomes

Outcomes reported: Number of patients receiving blood transfusion, volume of blood transfused (units), mortality, renal failure, length of hospital stay (days).

Notes

Quality assessment score (Schulz criteria): 7/7

Transfusion protocol used

Risk of bias

\begin{tabular}{|c|c|c|}
\hline Item & Authors' judgement & Description \\
\hline Adequate sequence generation? & Yes & Computer-generated randomisation list \\
\hline
\end{tabular}


Dietrich 2008 (Continued)

\begin{tabular}{l|ll}
\hline Allocation concealment? & Yes & Adequate \\
\hline $\begin{array}{l}\text { Blinding? } \\
\text { All outcomes }\end{array}$ & Yes & Double blind \\
\hline
\end{tabular}

Dignan 2001

\begin{tabular}{|c|c|c|}
\hline Methods & \multicolumn{2}{|c|}{$\begin{array}{l}\text { Study drug was administered by the anesthesiologist as an infusion in a blinded fashion. Allocation } \\
\text { concealment was not described. }\end{array}$} \\
\hline Participants & \multicolumn{2}{|c|}{$\begin{array}{l}200 \text { participants undergoing elective cardiac surgery were randomly assigned to one of two groups: } \\
\text { - Aprotinin group (Low dose): } \mathrm{n}=101, \mathrm{M} / \mathrm{F}=75 / 26 \text {, mean (range) age }=62.8(35-80) \text { years } \\
\text { - Control group (Placebo): } \mathrm{n}=99, \mathrm{M} / \mathrm{F}=77 / 22 \text {, mean (range) age }=65.2(40-81) \text { years }\end{array}$} \\
\hline Interventions & \multicolumn{2}{|c|}{$\begin{array}{l}\text { - Aprotinin group received } 1 \text { million KIU of aprotinin in total }(140 \mathrm{mg})-500,000 \mathrm{KIU} \text { before } \\
\text { skin incision and 500,000 KIU during the initiation of CPB. } \\
\text { - Control group received the same volume of normal saline. }\end{array}$} \\
\hline Outcomes & \multicolumn{2}{|c|}{$\begin{array}{l}\text { Outcomes reported: Number of patients exposed to allogeneic blood, allogeneic whole blood usage } \\
\text { (units/mls), fresh frozen plasma usage (units), platelet usage (units), } \\
\text { blood loss, re-operation for bleeding, acute renal failure, stroke. }\end{array}$} \\
\hline Notes & \multicolumn{2}{|c|}{$\begin{array}{l}\text { Quality assessment score (Schulz criteria): 3/7 } \\
\text { Transfusion protocol used }\end{array}$} \\
\hline \multicolumn{3}{|l|}{ Risk of bias } \\
\hline Item & Authors' judgement & Description \\
\hline Adequate sequence generation? & Unclear & Unclear \\
\hline Allocation concealment? & Unclear & Unclear \\
\hline $\begin{array}{l}\text { Blinding? } \\
\text { All outcomes }\end{array}$ & Yes & Double blind \\
\hline
\end{tabular}

Diprose 2005

\begin{tabular}{ll} 
Methods & $\begin{array}{l}\text { Computer generated random numbers determined patient allocation to one of three treatment } \\
\text { groups. Sealed envelopes were used to conceal treatment allocation. }\end{array}$ \\
\hline Participants & $\begin{array}{l}186 \text { patients undergoing elective cardiac surgery were randomly assigned to one of three groups: } \\
\text { - Aprotinin group (High dose): } \mathrm{n}=63, \mathrm{M} / \mathrm{F}=52 / 8 \text {, median (interquartile range) age }=62 \\
\text { (55-69) years } \\
\text { - Tranexamic acid group: } \mathrm{n}=62, \mathrm{M} / \mathrm{F}=49 / 11 \text {, median (interquartile range) age }=65(58.5- \\
\text { 73.5) years } \\
\text { - Control group (Placebo): } \mathrm{n}=61, \mathrm{M} / \mathrm{F}=52 / 8 \text {, median (interquartile range) age }=65(60-70)\end{array}$
\end{tabular}




\begin{tabular}{|c|c|c|}
\hline Interventions & \multicolumn{2}{|c|}{$\begin{array}{l}\text { - Aprotinin group received } 2 \text { million } \mathrm{KIU}(280 \mathrm{mg}) \text { in } 200 \mathrm{ml} \text { volume at the start of surgery, } 2 \\
\text { million KIU of aprotinin was added to the pump prime, and a continuous infusion of } 500,000 \\
\text { KIU/hr was given throughout the operation. } \\
\text { - Tranexamic acid group received } 5 \mathrm{~g} \text { in } 200 \mathrm{ml} \text { normal saline, } 200 \mathrm{ml} \text { of normal saline added to } \\
\text { the pump prime, and a continuous infusion of } 50 \mathrm{ml} / \text { hour of normal saline throughout the } \\
\text { operation. } \\
\text { - Control group received normal saline as an IV bolus into the pump prime and a continuous } \\
\text { infusion of } 50 \mathrm{ml} / \text { hour of normal saline per hour throughout the operation. } \\
\text { NB: All groups received intra-operative cell salvage (Compact A; Dideco, Sorin Biomedica, Italy) } \\
\text { and each group received a test dose of } 5 \mathrm{ml} \text { of study solution. }\end{array}$} \\
\hline Outcomes & \multicolumn{2}{|c|}{$\begin{array}{l}\text { Outcomes reported: Number of patients exposed to allogeneic blood, allogeneic blood usage (units) } \\
\text {, blood loss, mortality, myocardial infarction, re-operation for bleeding, renal failure, respiratory } \\
\text { failure. }\end{array}$} \\
\hline Notes & \multicolumn{2}{|c|}{$\begin{array}{l}\text { Quality assessment score (Schulz criteria): 5/7 } \\
\text { Transfusion protocol used }\end{array}$} \\
\hline \multicolumn{3}{|l|}{ Risk of bias } \\
\hline Item & Authors' judgement & Description \\
\hline Adequate sequence generation? & Yes & $\begin{array}{l}\text { Computer generated random numbers deter- } \\
\text { mined patient allocation }\end{array}$ \\
\hline Allocation concealment? & No & Inadequate \\
\hline $\begin{array}{l}\text { Blinding? } \\
\text { All outcomes }\end{array}$ & Yes & Double blind \\
\hline
\end{tabular}

Dorman 2008

\begin{tabular}{l|l}
\hline Methods & Method of sequence generation and allocation concealment were not described. \\
\hline Participants & $\begin{array}{l}60 \text { patients undergoing cardiac surgery were randomly allocated to one of two groups: } \\
\text { - Aprotinin group: } \mathrm{n}=30, \mathrm{M} / \mathrm{F}=25 / 5, \text { mean }(\mathrm{SEM}) \text { age }=62.0(2.0) \text { years } \\
\text { - Epsilon aminocaproic acid group: } \mathrm{n}=30, \mathrm{M} / \mathrm{F}=20 / 10, \text { mean }(\mathrm{SEM}) \text { age }=60.0(2.0) \text { years }\end{array}$ \\
\hline Interventions & $\begin{array}{l}\text { - Aprotonin group received } 1 \text { million KIU IV at the start of surgery with an additional } 1 \\
\text { million KIU in the CPB circuit, and a continuous infusion of } 250,000 \text { KIU per hour until the } \\
\text { end of surgery. } \\
\text { - Epsilon aminocaproic acid group received } 5 \mathrm{~g} \text { IV concurrent with systemic heparinization } \\
\text { and an additional } 5 \mathrm{~g} \text { in the } \mathrm{CPB} \text { circuit, and another } 5 \mathrm{~g} \text { administered IV immediately after } \\
\text { discontinuation of CPB. }\end{array}$ \\
\hline
\end{tabular}




\begin{tabular}{|c|c|c|}
\hline Outcomes & \multicolumn{2}{|c|}{$\begin{array}{l}\text { Outcomes reported: Number of patients receiving blood transfusion, volume blood transfused } \\
\text { (units), blood loss. }\end{array}$} \\
\hline Notes & \multicolumn{2}{|l|}{$\begin{array}{l}\text { Quality assessment score (Schulz criteria): } 2 / 7 \\
\text { Transfusion protocol used }\end{array}$} \\
\hline \multicolumn{3}{|l|}{ Risk of bias } \\
\hline Item & Authors' judgement & Description \\
\hline Adequate sequence generation? & Unclear & Unclear \\
\hline Allocation concealment? & Unclear & Unclear \\
\hline $\begin{array}{l}\text { Blinding? } \\
\text { All outcomes }\end{array}$ & Unclear & Unclear \\
\hline
\end{tabular}

\section{Dryden 1997}

\begin{tabular}{|c|c|c|}
\hline Methods & \multicolumn{2}{|c|}{$\begin{array}{l}\text { Method of randomisation was not described. The "study drug" was mixed by independent Intensive } \\
\text { Care Unit (ICU) personnel. }\end{array}$} \\
\hline Participants & \multicolumn{2}{|c|}{$\begin{array}{l}41 \text { patients undergoing re-operative cardiac valve surgery were randomised to one of two groups: } \\
\text { - Tranexamic acid group: } \mathrm{n}=22, \mathrm{M} / \mathrm{F}=9 / 13 \text {, mean }(\mathrm{sd}) \text { age }=63(12.6) \text { years } \\
\text { - Control group (Placebo): } \mathrm{n}=19, \mathrm{M} / \mathrm{F}=8 / 11 \text {, mean (sd) age }=64(18) \text { years }\end{array}$} \\
\hline Interventions & \multicolumn{2}{|c|}{$\begin{array}{l}\text { - Tranexamic acid group received } 10 \mathrm{~g} \text { of TXA in } 500 \mathrm{ml} \text { of normal saline infused after the } \\
\text { induction of anaesthesia as an intravenous bolus over } 30 \text { minutes prior to skin incision. } \\
\text { - Control group received normal saline solution in the same volume. }\end{array}$} \\
\hline Outcomes & \multicolumn{2}{|c|}{$\begin{array}{l}\text { Outcomes reported: Allogeneic blood usage (mls), blood loss, total platelets transfused, total plasma } \\
\text { transfused, mortality, hospital length of stay (days), hospital complications, re-operation for active } \\
\text { bleeding. }\end{array}$} \\
\hline Notes & \multicolumn{2}{|c|}{$\begin{array}{l}\text { Quality assessment score (Schulz criteria): 5/7 } \\
\text { Transfusion protocol used }\end{array}$} \\
\hline \multicolumn{3}{|l|}{ Risk of bias } \\
\hline Item & Authors' judgement & Description \\
\hline Adequate sequence generation? & Unclear & Unclear \\
\hline Allocation concealment? & Yes & Adequate \\
\hline
\end{tabular}


Dryden 1997 (Continued)

\begin{tabular}{|c|c|c|}
\hline $\begin{array}{l}\text { Blinding? } \\
\text { All outcomes }\end{array}$ & No & Single blind \\
\hline
\end{tabular}

Eberle 1998

\begin{tabular}{|c|c|c|}
\hline Methods & \multicolumn{2}{|c|}{$\begin{array}{l}\text { Different treatment solutions were identical in appearance. Method of randomisation was not } \\
\text { described. }\end{array}$} \\
\hline Participants & \multicolumn{2}{|c|}{$\begin{array}{l}40 \text { patients undergoing cardiac surgery were randomised to one of three groups: } \\
\text { - Epsilon aminocaproic acid group: } \mathrm{n}=20 \text {, mean }(\mathrm{sd}) \text { age }=61(10) \text { years } \\
\text { - Aprotinin group: } \mathrm{n}=20 \text {, mean }(\mathrm{sd}) \text { age }=60(10) \text { years } \\
\text { - Non-randomised historical control group: } \mathrm{n}=10 \text {, mean }(\mathrm{sd}) \text { age }=57(14) \text { years } \\
\text { NB: No gender data were reported. }\end{array}$} \\
\hline Interventions & \multicolumn{2}{|c|}{$\begin{array}{l}\text { - Epsilon aminocaproic acid group received a test dose of } 1 \mathrm{~mL} \text { followed after } 10 \text { minutes by a } \\
\text { loading dose of } 200 \mathrm{~mL} \text { of solution given over } 30 \text { minutes. EACA was infused continuously at a } \\
\text { rate of } 50 \mathrm{ml} / \text { hour until the start of CPB. EACA- } 10 \mathrm{~g} \text { both as loading and pump prime dose at } \\
2.5 \mathrm{~g} / \text { hour as an infusion. } \\
\text { - Aprotinin group received the same volume regimen as EACA. } 2 \text { million } \mathrm{KIU}(280 \mathrm{mg}) \text { for } \\
\text { loading and pump prime followed by an infusion of } 500,000 \mathrm{KIU} / \mathrm{hour}(70 \mathrm{mg} / \mathrm{hr}) \text { from CPB } \\
\text { weaning until } 4 \text { hours after heparin reversal. } \\
\text { - Control group did not receive either EACA or aprotinin treatment. } \\
\text { NB: Both EACA and aprotinin groups received cell salvage. }\end{array}$} \\
\hline Outcomes & \multicolumn{2}{|c|}{$\begin{array}{l}\text { Outcomes reported: Number of patients exposed to allogeneic blood, allogeneic blood usage (units) } \\
\text {, blood loss, myocardial Infarction. }\end{array}$} \\
\hline Notes & \multicolumn{2}{|c|}{$\begin{array}{l}\text { Quality assessment score (Schulz criteria): 4/7 } \\
\text { Transfusion protocol used }\end{array}$} \\
\hline \multicolumn{3}{|l|}{ Risk of bias } \\
\hline Item & Authors' judgement & Description \\
\hline Adequate sequence generation? & Unclear & Unclear \\
\hline Allocation concealment? & Yes & Adequate \\
\hline $\begin{array}{l}\text { Blinding? } \\
\text { All outcomes }\end{array}$ & Yes & Double blind \\
\hline
\end{tabular}




\begin{tabular}{|c|c|c|}
\hline Methods & \multicolumn{2}{|c|}{$\begin{array}{l}\text { Each bottle of aprotinin provided by the pharmaceutical company was placed in a box with bottles } \\
\text { of normal saline solution. These bottles were indistinguishable from one another. An assistant, who } \\
\text { was only involved in randomisation of the medication, arranged these bottles into } 50 \text { pairs. Each } \\
\text { pair consisted of two bottles of aprotinin or two bottles of saline solution. Each of these pairs was } \\
\text { randomly assigned a number from } 1 \text { to } 50 \text {. Each patient was randomly assigned a number and } \\
\text { then given the corresponding bottles. After the study was completed, the randomisation code was } \\
\text { broken and the data were analysed. }\end{array}$} \\
\hline Participants & \multicolumn{2}{|c|}{$\begin{array}{l}50 \text { patients undergoing thoracic aortic operations with the use of profound hypothermic circulatory } \\
\text { arrest were randomly assigned to one of two groups: } \\
\text { - Aprotinin group: } \mathrm{n}=25, \mathrm{M} / \mathrm{F}=9 / 16, \text { mean (range) age }=70(58-80) \text { years } \\
\text { - Control group (Placebo): } \mathrm{n}=25, \mathrm{M} / \mathrm{F}=7 / 18 \text {, mean (range) age }=70(60-78) \text { years }\end{array}$} \\
\hline Interventions & \multicolumn{2}{|c|}{$\begin{array}{l}\text { - Aprotinin group received } 1 \text { million KIU of aprotinin }(140 \mathrm{mg}) \text { before the onset of } \\
\text { cardiopulmonary bypass. } \\
\text { - Control group received an equal volume of } 0.9 \% \text { saline solution as a placebo. }\end{array}$} \\
\hline Outcomes & \multicolumn{2}{|c|}{$\begin{array}{l}\text { Outcomes reported: Allogeneic blood usage (units), blood loss, mortality ( } 30 \text {-day), myocardial } \\
\text { infarction, renal dysfunction/renal failure, re-operation for bleeding, neurological deficit, stroke. }\end{array}$} \\
\hline Notes & \multicolumn{2}{|c|}{$\begin{array}{l}\text { Quality assessment score (Schulz criteria): 6/7 } \\
\text { Transfusion protocol used }\end{array}$} \\
\hline \multicolumn{3}{|l|}{ Risk of bias } \\
\hline Item & Authors' judgement & Description \\
\hline Adequate sequence generation? & Unclear & Unclear \\
\hline Allocation concealment? & Yes & Adequate \\
\hline $\begin{array}{l}\text { Blinding? } \\
\text { All outcomes }\end{array}$ & Yes & Double blind \\
\hline
\end{tabular}

\section{Ekback 2000}

\begin{tabular}{l|l}
\hline Methods & Method of randomisation and allocation concealment were not described. \\
\hline Participants & $\begin{array}{r}40 \text { patients undergoing elective adult surgery and were randomised to one of two groups: } \\
\text { - Tranexamic acid group: } \mathrm{n}=20, \mathrm{M} / \mathrm{F}=9 / 11, \text { mean }(\mathrm{sd}) \text { age }=66.4(9.0) \text { years } \\
\text { - Control group (Placebo): } \mathrm{n}=20, \mathrm{M} / \mathrm{F}=11 / 9, \text { mean }(\mathrm{sd}) \text { age }=65.6(8.8) \text { years }\end{array}$ \\
\hline Interventions & $\begin{array}{l}\text { - Tranexamic acid group received a bolus dose of } 10 \mathrm{mg} / \mathrm{kg} \text { before surgical incision. A } \\
\text { continuous infusion of } 1.0 \mathrm{mg} / \mathrm{kg} / \mathrm{hr} \text { during } 10 \text { hours was then started immediately after the first } \\
\text { bolus dose. A second bolus dose of } 1.0 \mathrm{mg} / \mathrm{kg} \text { was given three hours later to counteract potential } \\
\text { dilutive effects of intra-operative autotransfusion. } \\
\text { - Control group received physiological saline as a placebo. } \\
\text { NB: All study participants underwent pre-operative autologous blood donation }(2 \text { units autologous }\end{array}$
\end{tabular}


Ekback 2000 (Continued)

blood donated) on two occasions within a four week period. Both trial arms were equally exposed to cell salvage.

Outcomes

Outcomes reported: Number of patients exposed to allogeneic blood, blood loss, deep vein thrombosis.

$\begin{array}{ll}\text { Notes } & \text { Quality assessment score (Schulz criteria): 4/7 } \\ \text { Transfusion protocol used }\end{array}$

Risk of bias

\begin{tabular}{lll}
\hline Item & Authors' judgement & Description \\
\hline Adequate sequence generation? & Unclear & Unclear \\
\hline Allocation concealment? & Unclear & Unclear \\
\hline $\begin{array}{l}\text { Blinding? } \\
\text { All outcomes }\end{array}$ & Yes & Double blind \\
\hline
\end{tabular}

\section{Ellis 2001}

\begin{tabular}{|c|c|}
\hline Methods & $\begin{array}{l}\text { Randomisation was carried out using a computer generated randomisation table. Allocation con- } \\
\text { cealment was not described. }\end{array}$ \\
\hline Participants & $\begin{array}{l}30 \text { patients were randomly assigned to one of three groups: } \\
\text { - Tranexamic acid group: } \mathrm{n}=10, \mathrm{M} / \mathrm{F}=4 / 6 \text {, mean }(\mathrm{sd}) \text { age }=71(5) \text { years } \\
\text { - Desmopressin group: } \mathrm{n}=10, \mathrm{M} / \mathrm{F}=2 / 8, \text { mean }(\mathrm{sd}) \text { age }=72(6) \text { years } \\
\text { - Control group (Placebo): } \mathrm{n}=10, \mathrm{M} / \mathrm{F}=3 / 7 \text {, mean }(\mathrm{sd}) \text { age }=72(8) \text { years }\end{array}$ \\
\hline Interventions & $\begin{array}{l}\text { - Tranexamic acid group received TXA } 30 \text { minutes before deflating the limb tourniquet an IV } \\
\text { bolus dose }(15 \mathrm{mg} / \mathrm{kg}) \text { administered over a } 30 \text { minute period, thereafter a constant IV infusion of } \\
\text { TXA ( } 10 \mathrm{mg} / \mathrm{kg} / \mathrm{hr} \text { ) was administered until } 12 \text { hours after final deflation of the limb tourniquet. } \\
\text { - Desmopressin group } 30 \text { minutes before deflating the limb tourniquet an IV bolus dose of } \\
\text { DDAVP }(0.3 \mathrm{micrograms} / \mathrm{kg}) \text { was infused over a } 30 \text { minute period, thereafter a constant IV } \\
\text { infusion of saline was administered until } 12 \text { hours after final deflation of the limb tourniquet. } \\
\text { - Control group received an equal volume of saline. }\end{array}$ \\
\hline Outcomes & $\begin{array}{l}\text { Outcomes reported: Number of patients exposed to allogeneic blood, allogenic blood usage (units) } \\
\text {, hospital length of stay (days). }\end{array}$ \\
\hline Notes & $\begin{array}{l}\text { Quality assessment score (Schulz criteria): } 3 / 7 \\
\text { Transfusion protocol used }\end{array}$ \\
\hline
\end{tabular}

\section{Risk of bias}

Item

\section{Description}


Ellis 2001 (Continued)

\begin{tabular}{llll}
\hline Adequate sequence generation? & Yes & $\begin{array}{l}\text { Randomisation was carried out using a computer } \\
\text { generated randomisation table }\end{array}$ \\
\hline $\begin{array}{l}\text { Allocation concealment? } \\
\begin{array}{l}\text { Blinding? } \\
\text { All outcomes }\end{array}\end{array}$ & Unclear & Yes & Doublear \\
\hline
\end{tabular}

Engel 2001

\begin{tabular}{|c|c|c|}
\hline Methods & \multicolumn{2}{|c|}{ Method of randomisation and allocation concealment was not described. } \\
\hline Participants & \multicolumn{2}{|c|}{$\begin{array}{l}36 \text { patients were randomised to one of three groups: } \\
\text { - Tranexamic acid group: } \mathrm{n}=12, \mathrm{M} / \mathrm{F}=4 / 8 \text {, mean }(\mathrm{sd}) \text { age }=71(9) \text { years } \\
\text { - Aprotinin group: } \mathrm{n}=12, \mathrm{M} / \mathrm{F}=3 / 9 \text {, mean }(\mathrm{sd}) \text { age }=71(9) \text { years } \\
\text { - Control group: } \mathrm{n}=12, \mathrm{M} / \mathrm{F}=4 / 8, \text { mean (sd) age }=66(11) \text { years }\end{array}$} \\
\hline Interventions & \multicolumn{2}{|c|}{$\begin{array}{l}\text { - Tranexamic acid group received } 15 \mathrm{mg} / \mathrm{kg} \text { of TXA followed by a repeat dose of } 10 \mathrm{mg} / \mathrm{kg} \text { after } \\
3 \text { hours. } \\
\text { - Aprotinin group received } 1 \text { million KIU ( } 140 \mathrm{mg}) \text { of aprotinin immediately before deflating } \\
\text { the tourniquet followed by an infusion of } 500,000 \mathrm{KIU} \text { per hour for } 4 \text { hours. } \\
\text { - Control group received no antifibrinolytic treatment. }\end{array}$} \\
\hline Outcomes & \multicolumn{2}{|c|}{$\begin{array}{l}\text { Outcomes reported: Number of patients exposed to allogeneic blood, allogeneic blood usage (units) } \\
\text {, blood loss, deep vein thrombosis. }\end{array}$} \\
\hline Notes & \multicolumn{2}{|c|}{$\begin{array}{l}\text { Quality assessment score (Schulz criteria): 2/7 } \\
\text { Transfusion protocol used }\end{array}$} \\
\hline \multicolumn{3}{|l|}{ Risk of bias } \\
\hline Item & Authors' judgement & Description \\
\hline Adequate sequence generation? & Unclear & Unclear \\
\hline Allocation concealment? & Unclear & Unclear \\
\hline $\begin{array}{l}\text { Blinding? } \\
\text { All outcomes }\end{array}$ & No & Unblinded \\
\hline
\end{tabular}


Englberger 2002a

\begin{tabular}{|c|c|c|}
\hline Methods & \multicolumn{2}{|c|}{$\begin{array}{l}\text { Bottles of aprotinin and saline (placebo solution) were numbered continuously. Blinding of bottles } \\
\text { was performed by personal otherwise not involved in the study. Method of randomisation was not } \\
\text { described. }\end{array}$} \\
\hline Participants & \multicolumn{2}{|c|}{$\begin{array}{l}47 \text { patients undergoing elective 'off-pump' cardiac surgery were randomly allocated to one of two } \\
\text { groups: } \\
\text { - Aprotonin group (Low dose): } \mathrm{n}=22, \mathrm{M} / \mathrm{F}=16 / 6 \text {, mean }(\mathrm{sd}) \text { age }=63.9(10.8) \text { years } \\
\text { - Control group (Placebo): } \mathrm{n}=25, \mathrm{M} / \mathrm{F}=19 / 6 \text {, mean }(\mathrm{sd}) \text { age }=66.4(9.0) \text { years }\end{array}$} \\
\hline Interventions & \multicolumn{2}{|c|}{$\begin{array}{l}\text { - Aprotonin group received a loading dose of } 2 \text { million KIU }(280 \mathrm{mg}) \text { of aprotinin at the } \\
\text { beginning of surgery followed by a continuous infusion of } 500,000 \mathrm{KIU} / \mathrm{hr} \text { throughout surgery } \\
(70 \mathrm{mg} / \mathrm{hr}) \text {. } \\
\text { - Control group received the same volume of saline solution. }\end{array}$} \\
\hline Outcomes & \multicolumn{2}{|c|}{$\begin{array}{l}\text { Outcomes reported: Number of patients exposed to allogeneic blood, number of patients exposed to } \\
\text { fresh frozen plasma, blood loss, mortality, myocardial infarction, re-operation for bleeding, number } \\
\text { of patients exposed to autotransfusion, volume of blood autotransfused, hospital length of stay } \\
\text { (days), neurological deficit, renal dysfunction. }\end{array}$} \\
\hline Notes & \multicolumn{2}{|c|}{$\begin{array}{l}\text { Quality assessment score (Schulz criteria): 5/7 } \\
\text { Transfusion protocol used }\end{array}$} \\
\hline \multicolumn{3}{|l|}{ Risk of bias } \\
\hline Item & Authors' judgement & Description \\
\hline Adequate sequence generation? & Unclear & Unclear \\
\hline Allocation concealment? & Yes & Adequate \\
\hline $\begin{array}{l}\text { Blinding? } \\
\text { All outcomes }\end{array}$ & Yes & Double blind \\
\hline
\end{tabular}

\section{Englberger 2002b}

\begin{tabular}{l|l}
\hline Methods & Method of randomisation and allocation concealment were not described. \\
\hline Participants & $\begin{array}{r}29 \text { patients undergoing elective cardiac surgery were randomly allocated to one of two groups: } \\
\text { - Aprotinin group (Low dose - pump prime): } \mathrm{n}=15, \mathrm{M} / \mathrm{F}=13 / 2, \text { mean }(\mathrm{sd}) \text { age }=60.3(10) \\
\text { years } \\
- \text { Control group: } \mathrm{n}=14, \mathrm{M} / \mathrm{F}=10 / 4, \text { mean }(\mathrm{sd}) \text { age }=61.5(7.5) \text { years }\end{array}$ \\
\hline Interventions & $\begin{array}{l}\text { - Aprotonin group received } 2 \text { million KIU of aprotinin }(280 \mathrm{mg}) \text { added to the pump prime } \\
\text { only. } \\
\text { - Control group - treatment details were not reported. } \\
\text { NB: Both groups were exposed to cell salvage. }\end{array}$
\end{tabular}




\begin{tabular}{|c|c|c|}
\hline Outcomes & \multicolumn{2}{|c|}{$\begin{array}{l}\text { Outcomes reported: Number of patients exposed to allogeneic blood, allogeneic blood usage (units) } \\
\text {, number of patients exposed to fresh frozen plasma, fresh frozen plasma usage (units), blood loss, } \\
\text { mortality, myocardial infarction, re-operation for bleeding, neurological deficit, renal dysfunction, } \\
\text { hospital length of stay (days), number of patients exposed to autotransfusion, volume of blood } \\
\text { autotransfused. }\end{array}$} \\
\hline Notes & \multicolumn{2}{|c|}{$\begin{array}{l}\text { Quality assessment score (Schulz criteria): 2/7 } \\
\text { Transfusion protocol used }\end{array}$} \\
\hline \multicolumn{3}{|l|}{ Risk of bias } \\
\hline Item & Authors' judgement & Description \\
\hline Adequate sequence generation? & Unclear & Unclear \\
\hline Allocation concealment? & Unclear & Unclear \\
\hline $\begin{array}{l}\text { Blinding? } \\
\text { All outcomes }\end{array}$ & Unclear & Unclear \\
\hline
\end{tabular}

\section{Fauli 2005}

Methods $\quad$ Method of randomisation was not described. All patients received pre-prepared infusions of similar volume and appearance provided by the pharmaceutical company.

\begin{tabular}{l|l}
\hline Participants & $\begin{array}{l}60 \\
\text { patients undergoing cardiac surgery were randomised to one of three groups: } \\
\text { - Aprotinin group (High dose): } \mathrm{n}=20, \mathrm{M} / \mathrm{F}=15 / 5, \text { mean }(\mathrm{sd}) \text { age }=52.5(10.1) \text { years } \\
\text { - Aprotinin group (Low dose): } \mathrm{n}=20, \mathrm{M} / \mathrm{F}=17 / 3, \text { mean }(\mathrm{sd}) \text { age }=57.7(4.6) \text { years } \\
\text { - Control group (Placebo): } \mathrm{n}=20, \mathrm{M} / \mathrm{F}=14 / 6, \text { mean }(\mathrm{sd}) \text { age }=56.5(6.5) \text { years }\end{array}$ \\
\hline Interventions & $\begin{array}{l}\text { - Aprotinin group (High dose) received a loading dose of } 280 \mathrm{mg} \text { of aprotinin, followed by a } \\
\text { continuous infusion of } 70 \mathrm{mg} / \mathrm{hr} \text { of aprotinin until closure of sternotomy, and } 280 \mathrm{mg} \text { of aprotinin } \\
\text { was added to the pump prime. } \\
\text { - Aprotinin group (Low dose) received } 280 \mathrm{mg} \text { of aprotinin added to the pump prime. } \\
\text { - Control group (Placebo) received the same volume of saline. }\end{array}$ \\
\hline
\end{tabular}

Outcomes

Outcomes reported: Allogeneic blood usage (units), blood loss (24hrs), hospital length of stay (days).

Notes

Quality assessment score (Schulz criteria): 6/7

Transfusion protocol used

\section{Risk of bias}

\begin{tabular}{l|ll}
\hline Item & Authors' judgement & Description \\
\hline Adequate sequence generation? & Unclear & Unclear
\end{tabular}


Fauli 2005 (Continued)

\begin{tabular}{l|ll}
\hline Allocation concealment? & Yes & Adequate \\
\hline $\begin{array}{l}\text { Blinding? } \\
\text { All outcomes }\end{array}$ & Yes & Double blind \\
\hline
\end{tabular}

Feindt 1994

\begin{tabular}{l|l}
\hline Methods & Method of randomisation and allocation concealment were not described. \\
\hline Participants & $\begin{array}{r}20 \text { patients undergoing aortocoronary bypass surgery were randomly allocated to one of two groups: } \\
\text { - Aprotinin group (High-dose): } \mathrm{n}=10, \mathrm{M} / \mathrm{F}=\mathrm{NR}, \text { mean }(\mathrm{sd}) \text { age }=62.3(1.2) \text { years } \\
\text { - Control group (Placebo): } \mathrm{n}=10, \mathrm{M} / \mathrm{F}=\mathrm{NR}, \mathrm{mean}(\mathrm{sd}) \text { age }=66.4(2.4) \text { years }\end{array}$ \\
\hline Interventions & $\begin{array}{l}\text { - Aprotonin group received high-dose }(2 \text { million units kallikrein inhibitor at the induction of } \\
\text { anaesthesia, } 2 \text { million units added to the priming volume of the heart-lung machine and } 500,000 \\
\text { U/h during the operation). } \\
\text { - Control group - treatment details were not reported. }\end{array}$ \\
\hline
\end{tabular}

Outcomes Outcomes reported: Number of patients exposed to allogeneic blood transfusion, post-operative blood loss, mortality, parameters of thrombin activation and fibrinolysis.

$\begin{array}{ll}\text { Notes } & \text { Quality assessment score (Schulz criteria): 2/7 } \\ \text { Transfusion protocol used }\end{array}$

Risk of bias

\begin{tabular}{lll}
\hline Item & Authors' judgement & Description \\
\hline Adequate sequence generation? & Unclear & Unclear \\
\hline Allocation concealment? & Unclear & Unclear \\
\hline $\begin{array}{l}\text { Blinding? } \\
\text { All outcomes }\end{array}$ & Yes & $\begin{array}{l}\text { Double blind - study was described as being dou- } \\
\text { ble blind }\end{array}$ \\
\hline
\end{tabular}

Fergusson 2008

\begin{tabular}{l} 
Methods $\begin{array}{l}\text { The research pharmacist at each centre randomly assigned patients to receive one of three antifib- } \\
\text { rinolytic agents with the use of a voice-activated automated centralised program. An independent } \\
\text { biostatistician generated the randomisation scheme using a computer-generated randomisation list. } \\
\text { Researchers, patients, members of the clinical teams, and members of the data and safety monitor- } \\
\text { ing committee were all unaware of study-group assignment. }\end{array}$ \\
\hline $\begin{array}{l}\text { 2331 high-risk cardiac surgical patients were randomly allocated to one of three groups: } \\
\text { - Aprotinin group: } \mathrm{n}=781, \mathrm{M} / \mathrm{F}=543 / 238, \text { mean }(\mathrm{sd}) \text { age }=67.0(10.8) \text { years } \\
\text { - Tranexamic acid group: } \mathrm{n}=770, \mathrm{M} / \mathrm{F}=562 / 208, \text { mean }(\mathrm{sd}) \text { age }=66.9(11.4) \text { years } \\
\text { - Epsilon aminocaproic acid: } \mathrm{n}=780, \mathrm{M} / \mathrm{F}=569 / 211, \text { mean }(\mathrm{sd}) \text { age }=66.6(10.8) \text { years }\end{array}$
\end{tabular}




- Aprotonin group received high-dose aprotinin with a test dose of $40,000 \mathrm{KIU}$ administered
during a 10 minute period after the insertion of a central venous line and induction of anaesthesia.
In the absence of of anaphylaxis, the remainder of the loading dose (1.96 million KIU), after
which a maintenance infusion of $500,000 \mathrm{KIU}$ per hour was initiated and maintained during
surgery. An additional dose of 2 million $\mathrm{KIU}$ was added to the cardiopulmonary-bypass circuit.
- Tranexamic acid group received a $30 \mathrm{mg} / \mathrm{kg}$ loading dose, a $16 \mathrm{mg} / \mathrm{kg}$ maintenance dose, then
$2 \mathrm{mg} / \mathrm{kg}$ added to the bypass circuit.
- Epsilon aminocaproic acid group received a $10 \mathrm{~g}$ loading dose, then a $2 \mathrm{~g}$ maintenance
infusion.

Outcomes

Outcomes reported: Number of patients transfused allogeneic blood, massive post-operative bleeding, re-operation for bleeding, myocardial infarction, stroke, mortality, renal failure, length of stay (days).

Notes

Quality assessment score (Schulz criteria): 7/7

Transfusion protocol used

Risk of bias

\begin{tabular}{lll}
\hline Item & Authors' judgement & Description \\
\hline Adequate sequence generation? & Yes & Computer-generated randomisation list \\
\hline Allocation concealment? & Yes & Adequate \\
\hline $\begin{array}{l}\text { Blinding? } \\
\text { All outcomes }\end{array}$ & Yes & Double blind \\
\hline
\end{tabular}

Findlay 2001

\begin{tabular}{l|l}
\hline Methods & Method of randomisation and allocation concealment were not described. \\
\hline Participants & $\begin{array}{l}63 \text { patients undergoing orthotopic liver transplantation were randomised to one of two groups: } \\
\text { - Aprotinin group: } \mathrm{n}=33, \text { mean }(\mathrm{sd}) \text { age }=50(9) \text { years } \\
\text { - Control group (Placebo): } \mathrm{n}=30, \text { mean }(\mathrm{sd}) \text { age }=52(10) \text { years } \\
\text { NB: Gender data were not reported. }\end{array}$ \\
\hline
\end{tabular}

Interventions

- Aprotinin group received a loading dose of 1 million KIU over 30 minutes (after a test dose of 10,000 KIU) followed by an infusion of 250,000 KIU/hr until skin closure.

- Control group received an equivalent infusion of normal saline.

NB: Both groups were exposed to cell salvage.

Outcomes

Anti-fibrinolytic use for minimising perioperative allogeneic blood transfusion (Review)

Copyright (C) 20II The Cochrane Collaboration. Published by John Wiley \& Sons, Ltd. 
Findlay 2001 (Continued)

\begin{tabular}{|c|c|c|}
\hline Notes & \multicolumn{2}{|c|}{$\begin{array}{l}\text { Quality assessment score (Schulz criteria): } 2 / 7 \\
\text { Transfusion protocol used }\end{array}$} \\
\hline \multicolumn{3}{|l|}{ Risk of bias } \\
\hline Item & Authors' judgement & Description \\
\hline Adequate sequence generation? & Unclear & Unclear \\
\hline Allocation concealment? & Unclear & Unclear \\
\hline $\begin{array}{l}\text { Blinding? } \\
\text { All outcomes }\end{array}$ & Yes & Double blind \\
\hline
\end{tabular}

\section{Fraedrich 1989}

\begin{tabular}{|c|c|c|}
\hline Methods & \multicolumn{2}{|c|}{ Method of randomisation and allocation concealment were not described. } \\
\hline Participants & \multicolumn{2}{|c|}{$\begin{array}{l}80 \text { male patients undergoing primary coronary bypass surgery were randomised to one of two } \\
\text { groups: } \\
\text { - Aprotinin group: } \mathrm{n}=40 \text {, mean age }=60.6 \text { years } \\
\text { - Control group: } \mathrm{n}=40 \text {, mean age }=58.2 \text { years } \\
\text { NB: Four patients, two from the intervention and two from the control group were excluded. } \\
\text { Aprotinin group excluded two patients: one allergic reaction, one severe cardiac failure. Control } \\
\text { group excluded two patients: one surgical bleeding, one lethal cardiac failure. }\end{array}$} \\
\hline Interventions & \multicolumn{2}{|c|}{$\begin{array}{l}\text { - Aprotinin group received a loading dose of } 280 \mathrm{mg} \text { of aprotinin prior to sternotomy, } \\
\text { followed by a continuous intravenous infusion of } 70 \mathrm{mg} / \mathrm{hr} \text { until skin closure. An additional } \\
280 \mathrm{mg} \text { of aprotinin was added to the prime volume of the membrane oxygenator. } \\
\text { - Control group was not treated with aprotinin. }\end{array}$} \\
\hline Outcomes & \multicolumn{2}{|c|}{$\begin{array}{l}\text { Outcomes reported: Allogeneic blood usage, plasma usage, blood loss, volume of re-transfused } \\
\text { mediastinal blood. }\end{array}$} \\
\hline Notes & \multicolumn{2}{|c|}{$\begin{array}{l}\text { Quality assessment score (Schulz criteria): 2/7 } \\
\text { Transfusion protocol not specified }\end{array}$} \\
\hline \multicolumn{3}{|l|}{ Risk of bias } \\
\hline Item & Authors' judgement & Description \\
\hline Adequate sequence generation? & Unclear & Unclear \\
\hline Allocation concealment? & Unclear & Unclear \\
\hline $\begin{array}{l}\text { Blinding? } \\
\text { All outcomes }\end{array}$ & No & Single blind \\
\hline
\end{tabular}


Garcia-Enguita 1998

\begin{tabular}{|c|c|c|}
\hline Methods & \multicolumn{2}{|c|}{ Method of randomisation and allocation concealment were not described. [Abstract only] } \\
\hline Participants & \multicolumn{2}{|c|}{$\begin{array}{l}30 \text { patients undergoing elective orthopaedic surgery were randomly allocated to one of two groups } \\
\text { - Aprotinin group (Low dose): } \mathrm{n}=15 \\
\text { - Control group (Placebo): } \mathrm{n}=15 \\
\text { NB: Gender and age data were reported. }\end{array}$} \\
\hline Interventions & \multicolumn{2}{|c|}{$\begin{array}{l}\text { - Aprotinin group received a loading dose of } 2 \text { million KIU }(280 \mathrm{mg}) \text { of aprotinin before the } \\
\text { induction of anaesthesia administered over } 30 \text { minutes followed by } 500,000 \mathrm{KIU} / \mathrm{hr} \text { for the } \\
\text { duration of surgery. } \\
\text { - Control group received } 200 \mathrm{ml} \text { of normal saline over } 30 \text { minutes followed by } 50 \mathrm{~mL} / \mathrm{hr} \text { of } \\
\text { saline. }\end{array}$} \\
\hline Outcomes & \multicolumn{2}{|c|}{ Outcomes reported: Allogenic blood usage (units), blood loss. } \\
\hline Notes & \multicolumn{2}{|c|}{$\begin{array}{l}\text { Quality assessment score (Schulz criteria): 2/7 } \\
\text { Transfusion protocol was not used }\end{array}$} \\
\hline \multicolumn{3}{|l|}{ Risk of bias } \\
\hline Item & Authors' judgement & Description \\
\hline Adequate sequence generation? & Unclear & Unclear \\
\hline Allocation concealment? & Unclear & Unclear \\
\hline $\begin{array}{l}\text { Blinding? } \\
\text { All outcomes }\end{array}$ & Unclear & Unclear \\
\hline
\end{tabular}

\section{Garcia-Huete 1997}

\begin{tabular}{l|l}
\hline Methods & Method of randomisation and allocation concealment were not described. \\
\hline Participants & $\begin{array}{l}80 \text { consecutive patients undergoing elective orthotopic liver transplantation were randomised to } \\
\text { one of two groups: } \\
\text { - Aprotinin group: } \mathrm{n}=39, \mathrm{M} / \mathrm{F}=24 / 15, \text { mean (range) age }=50(15-64) \text { years } \\
\text { - Control group (Placebo): } \mathrm{n}=41, \mathrm{M} / \mathrm{F}=27 / 14, \text { mean (range) age }=50(17-65) \text { years }\end{array}$ \\
\hline Interventions & $\begin{array}{l}\text { - Aprotinin group received an initial dose of } 2 \text { million kallikrein inactivator units (KIU) of } \\
\text { aprotinin in the induction phase of anaesthesia followed by an infusion of } 500,000 \text { KIU/hr of } \\
\text { aprotinin until the end of the procedure. } \\
\text { - Control group received an equal volume of saline solution. }\end{array}$ \\
\hline Outcomes & $\begin{array}{l}\text { Outcomes reported: } \text { Number of patients exposed to allogeneic blood, allogeneic blood usage (units) } \\
\text { mortality, allergic reactions, re-operation for bleeding, re-transplantation. }\end{array}$ \\
\hline Notes & $\begin{array}{l}\text { Quality assessment score (Schulz criteria): } 4 / 7 \\
\text { Transfusion protocol used }\end{array}$ \\
\hline
\end{tabular}


Garcia-Huete 1997 (Continued)

\begin{tabular}{lll} 
Risk of bias & & \\
\hline Item & Authors' judgement & Description \\
\hline Adequate sequence generation? & Unclear & Unclear \\
\hline Allocation concealment? & Unclear & Unclear \\
\hline $\begin{array}{l}\text { Blinding? } \\
\text { All outcomes }\end{array}$ & Yes & Double blind \\
\hline
\end{tabular}

\section{Garneti 2004}

\begin{tabular}{|c|c|c|}
\hline Methods & \multicolumn{2}{|c|}{$\begin{array}{l}\text { Patients were randomised using a list of random numbers. Allocation concealment was not de- } \\
\text { scribed. }\end{array}$} \\
\hline Participants & \multicolumn{2}{|c|}{$\begin{array}{l}50 \text { patients undergoing total hip arthroplasty were randomised to one of two groups: } \\
\text { - Tranexamic acid group: } n=25 \text { ), mean (sd) age }=69.6(11.99) \text { years } \\
\text { - Control group (Placebo): } n=25 \text {, mean (sd) age }=67.6(11.4) \text { years }\end{array}$} \\
\hline Interventions & \multicolumn{2}{|c|}{$\begin{array}{l}\text { - Tranexamic acid group received } 10 \mathrm{mg} / \mathrm{kg} \text { of intravenous TXA as a bolus at time of } \\
\text { anaesthesia. } \\
\text { - Control group received a similar volume of normal saline as a bolus at time of anaesthesia. }\end{array}$} \\
\hline Outcomes & \multicolumn{2}{|c|}{$\begin{array}{l}\text { Outcomes reported: Number of patients exposed to allogeneic blood, allogeneic blood usage (units) } \\
\text {, blood loss (48hrs), deep vein thrombosis, pulmonary embolus. }\end{array}$} \\
\hline Notes & \multicolumn{2}{|c|}{$\begin{array}{l}\text { Quality assessment score (Schulz criteria): 5/7 } \\
\text { Transfusion protocol not specified }\end{array}$} \\
\hline \multicolumn{3}{|l|}{ Risk of bias } \\
\hline Item & Authors' judgement & Description \\
\hline Adequate sequence generation? & Yes & List of random numbers \\
\hline Allocation concealment? & Unclear & Unclear \\
\hline $\begin{array}{l}\text { Blinding? } \\
\text { All outcomes }\end{array}$ & Yes & Double blind \\
\hline
\end{tabular}


Gherli 1992

\begin{tabular}{|c|c|c|}
\hline Methods & \multicolumn{2}{|c|}{ Method of randomisation and allocation concealment were not described. [Italian] } \\
\hline Participants & \multicolumn{2}{|c|}{$\begin{array}{l}31 \text { patients undergoing cardiopulmonary bypass were randomly divided into one of three groups: } \\
\text { - Aprotinin group (High dose): } \mathrm{n}=9, \mathrm{M} / \mathrm{F}=7 / 2 \text {, mean (range) age }=61.5(44-71) \text { years } \\
\text { - Aprotinin group (Low dose): } \mathrm{n}=9, \mathrm{M} / \mathrm{F}=8 / 1 \text {, mean (range) age }=58.2(47-71) \text { years } \\
\text { - Control group: } \mathrm{n}=13, \mathrm{M} / \mathrm{F}=10 / 3 \text {, mean (range) age }=60.4(52-66) \text { years }\end{array}$} \\
\hline Interventions & \multicolumn{2}{|c|}{$\begin{array}{l}\text { - Aprotinin group (High dose) received } 2 \text { million kallikrein inactivator units }(\mathrm{KIU}) \text { of } \\
\text { aprotinin over } 15 \text { minutes, followed by a continuous infusion of } 1 \text { million KIU/hr of aprotinin. } \\
\text { An additional } 2 \text { million KIU of aprotinin was added to the pump prime. } \\
\text { - Aprotinin group (Low dose) received } 1 \text { million kallikrein inactivator units (KIU) of } \\
\text { aprotinin over } 15 \text { minutes, followed by a continuous infusion of 500,000 KIU/hr of aprotinin. } \\
\text { An additional } 1 \text { million KIU of aprotinin was added to the pump prime. } \\
\text { - Control group did not receive aprotinin. }\end{array}$} \\
\hline Outcomes & \multicolumn{2}{|c|}{$\begin{array}{l}\text { Outcomes reported: Number of patients exposed to allogeneic blood, allogeneic blood usage (units) } \\
\text {, blood loss, myocardial infarction, renal failure, blood products used, haemoglobin levels. }\end{array}$} \\
\hline Notes & \multicolumn{2}{|c|}{$\begin{array}{l}\text { Quality assessment score (Schulz criteria): 2/7 } \\
\text { Transfusion protocol used }\end{array}$} \\
\hline \multicolumn{3}{|l|}{ Risk of bias } \\
\hline Item & Authors' judgement & Description \\
\hline Adequate sequence generation? & Unclear & Unclear \\
\hline Allocation concealment? & Unclear & Unclear \\
\hline $\begin{array}{l}\text { Blinding? } \\
\text { All outcomes }\end{array}$ & Unclear & Unclear \\
\hline
\end{tabular}

\section{Gill 2009}

\begin{tabular}{l|l} 
Methods & Patients were allocated according to a computer-generated randomisation schedule. \\
\hline Participants & $\begin{array}{l}\text { 10 patients undergoing orthopaedic (hip) surgery were randomised to one of two groups: } \\
\text { - Tranexamic acid group: } \mathrm{n}=5, \text { mean (range) age }=66.6(53-83) \text { years } \\
\text { - Control group (Placebo): } \mathrm{n}=5, \text { mean (range) age }=61.4(36-73) \text { years }\end{array}$ \\
\hline Interventions & $\begin{array}{l}\text { - Tranexamic acid group received } 10 \mathrm{mg} / \mathrm{kg} \text { bolus before anaesthesia then } 1 \mathrm{mg} / \mathrm{kg} / \mathrm{hr} \text { at start of } \\
\text { surgery until wound closure. } \\
\text { - Control group (placebo) received saline. }\end{array}$ \\
\hline
\end{tabular}

Outcomes

Outcomes reported: Number of patients receiving blood transfusion, blood loss, volume blood transfused (units). 
Gill 2009 (Continued)

\begin{tabular}{|c|c|c|}
\hline Notes & $\begin{array}{l}\text { Quality assessment score (Schulz criteria): 5/7 } \\
\text { Transfusion protocol used }\end{array}$ & \\
\hline \multicolumn{3}{|l|}{ Risk of bias } \\
\hline Item & Authors' judgement & Description \\
\hline Adequate sequence generation? & Yes & Computer-generated randomisation schedule \\
\hline Allocation concealment? & Yes & Adequate \\
\hline $\begin{array}{l}\text { Blinding? } \\
\text { All outcomes }\end{array}$ & Yes & Double blind \\
\hline
\end{tabular}

\section{Golanski 2000}

\begin{tabular}{|c|c|c|}
\hline Methods & \multicolumn{2}{|c|}{ Method of randomisation and allocation concealment were not described. [Polish] } \\
\hline Participants & \multicolumn{2}{|c|}{$\begin{array}{l}54 \text { patients undergoing elective cardiac surgery were randomly allocated to one of two groups: } \\
\text { - Aprotinin group (Low dose): } \mathrm{n}=30, \mathrm{M} / \mathrm{F}=29 / 1 \text {, mean }(\mathrm{sd}) \text { age }=56.2(10.5) \text { years } \\
\text { - Control group: } \mathrm{n}=24, \mathrm{M} / \mathrm{F}=22 / 2 \text {, mean (sd) age }=54.7 \text { (8.1) years }\end{array}$} \\
\hline Interventions & \multicolumn{2}{|c|}{$\begin{array}{l}\text { - Aprotinin group received an infusion of } 3 \text { million kallikrein inactivator units (KIU) of } \\
\text { aprotinin intra-operatively. } \\
\text { - Control did not receive aprotinin. }\end{array}$} \\
\hline Outcomes & \multicolumn{2}{|c|}{ Outcomes reported: Allogeneic blood usage (units), blood loss, mortality, myocardial infarction. } \\
\hline Notes & \multicolumn{2}{|c|}{ Quality assessment score (Schulz criteria): 0/7 } \\
\hline \multicolumn{3}{|l|}{ Risk of bias } \\
\hline Item & Authors' judgement & Description \\
\hline Adequate sequence generation? & Unclear & Unclear \\
\hline Allocation concealment? & Unclear & Unclear \\
\hline $\begin{array}{l}\text { Blinding? } \\
\text { All outcomes }\end{array}$ & Unclear & Unclear \\
\hline
\end{tabular}




\begin{tabular}{|c|c|c|}
\hline Methods & \multicolumn{2}{|c|}{$\begin{array}{l}\text { Coded ampoules of TXA or saline placebo prepared by the drug company were randomised in } \\
\text { blocks of } 10 \text { (five saline, five TXA) by means of computer generated numbers. Four patients were } \\
\text { withdrawn from the final analysis before the randomisation code was broken. }\end{array}$} \\
\hline Participants & \multicolumn{2}{|c|}{$\begin{array}{l}55 \text { patients undergoing total knee replacement surgery were randomised to one of two groups: } \\
\text { - Tranexamic acid group: } n=27, M / F=9 / 18 \text {, median (IQR) age }=72(46-83) \text { years } \\
\text { - Control group (Placebo): } n=24, M / F=6 / 18 \text {, median (IQR) age }=72(50-84) \text { years }\end{array}$} \\
\hline Interventions & \multicolumn{2}{|c|}{$\begin{array}{l}\text { - Tranexamic acid group received } 10 \mathrm{mg} / \mathrm{kg} \text { of intravenous TXA at the end of the surgical } \\
\text { procedure, just before the release of the tourniquet (maximum dose of } 1000 \mathrm{mg} \text { ). The dose of } \\
\text { TXA was repeated after } 3 \text { hours. } \\
\text { - Control group received saline placebo solution at corresponding times as the TXA group. }\end{array}$} \\
\hline Outcomes & \multicolumn{2}{|c|}{$\begin{array}{l}\text { Outcomes reported: Number of patients exposed to allogeneic blood, allogeneic blood usage (units) } \\
\text {, blood loss, deep vein thrombosis, infection. }\end{array}$} \\
\hline Notes & \multicolumn{2}{|c|}{$\begin{array}{l}\text { Quality assessment score (Schulz criteria): 6/7 } \\
\text { Transfusion protocol used }\end{array}$} \\
\hline \multicolumn{3}{|l|}{ Risk of bias } \\
\hline Item & Authors' judgement & Description \\
\hline Adequate sequence generation? & Yes & Computer generated numbers \\
\hline Allocation concealment? & Yes & Adequate \\
\hline $\begin{array}{l}\text { Blinding? } \\
\text { All outcomes }\end{array}$ & Yes & Double blind \\
\hline
\end{tabular}

\section{Gott 1998}

\begin{tabular}{l|l}
\hline Methods & Method of randomisation and allocation concealment were not described. \\
\hline Participants & 400 cardiac surgery patients were randomly allocated to one of three groups: \\
& - Aprotinin group: $\mathrm{n}=109$ \\
- Control/standard: $\mathrm{n}=112$ & - Leukocyte depletion: $\mathrm{n}=112$ \\
- Heparin-bonded circuitry: $\mathrm{n}=67$ \\
NB: No demographic data were reported.
\end{tabular}

Interventions

- Aprotonin group received low-dose - standard treatment plus a half-Hammersmith aprotonin protocol.

- Control - standard treatment.

- Leukocyte depletion - based on the standard CPB protocol with addition of leukocyte filtration of arterial line and cardioplegia delivery line.

- Heparin-bonded circuitry, membrane oxygenator and a centrifugal pump. 


\section{Gott 1998 (Continued)}

\begin{tabular}{|c|c|c|}
\hline Outcomes & \multicolumn{2}{|c|}{$\begin{array}{l}\text { Outcomes reported: Total amount of allogeneic blood transfused (units), mortality, length of } \\
\text { hospital stay, renal dysfunction, lung function. }\end{array}$} \\
\hline Notes & \multicolumn{2}{|l|}{$\begin{array}{l}\text { Quality assessment score (Schulz criteria): 0/7 } \\
\text { Transfusion protocol not used }\end{array}$} \\
\hline \multicolumn{3}{|l|}{ Risk of bias } \\
\hline Item & Authors' judgement & Description \\
\hline Adequate sequence generation? & Unclear & Unclear \\
\hline Allocation concealment? & Unclear & Unclear \\
\hline $\begin{array}{l}\text { Blinding? } \\
\text { All outcomes }\end{array}$ & Unclear & Unclear \\
\hline
\end{tabular}

Green 1995

Methods Study was described as an open label randomised controlled trial conducted in two phases. Patients were assigned to groups by means of computer generated table of random numbers.

\begin{tabular}{|c|c|}
\hline Participants & $\begin{array}{l}84 \text { consecutive patients undergoing primary coronary artery bypass graft surgery or re-operations } \\
\text { were randomly assigned to one of two groups: } \\
\text { - Aprotinin group: } \mathrm{n}=48, \mathrm{M} / \mathrm{F}=39 / 9 \text {, mean }(\mathrm{sd}) \text { age }=62(9) \text { years } \\
\text { - Control group: } \mathrm{n}=36, \mathrm{M} / \mathrm{F}=31 / 5 \text {, mean }(\mathrm{sd}) \text { age }=63(8) \text { years } \\
\text { Two aprotinin dose regimens were studied: } \\
\text { - Dosage level } 1 \text { - Aprotinin group: } \mathrm{n}=24, \mathrm{M} / \mathrm{F}=20 / 4 \text {, mean }(\mathrm{sd}) \text { age }=64(8) \text { years } \\
\text { - Dosage level } 1 \text { - Control group: } \mathrm{n}=18, \mathrm{M} / \mathrm{F} 16 / 2, \text { mean }(\mathrm{sd}) \text { age }=63(8) \text { years } \\
\text { - Dosage level } 2 \text { - Aprotinin group: } \mathrm{n}=24, \mathrm{M} / \mathrm{F}=19 / 5 \text {, mean }(\mathrm{sd}) \text { age }=60(9) \text { years } \\
\text { - Dosage level } 2 \text { - Control group: } \mathrm{n}=18, \mathrm{M} / \mathrm{F}=15 / 3 \text {, mean }(\mathrm{sd}) \text { age }=64(8) \text { years }\end{array}$ \\
\hline Interventions & $\begin{array}{l}\text { - Phase } 1 \text {, patients assigned to recombinant }(\mathrm{r}) \text { aprotinin (treatment group) received } 2 \mathrm{mg} / \mathrm{kg} \\
\text { (14,300 kallikrein inactivation units } / \mathrm{kg} \text { ) as an intravenous bolus given in } 20 \text { minutes after the } \\
\text { induction of anaesthesia, an intravenous infusion of } 0.5 \mathrm{mg} / \mathrm{kg} / \mathrm{hr} \text { until the patient left the } \\
\text { operating room, and } 1 \mathrm{mg} / \mathrm{kg} \text { added to each litre of lactated Ringers solution for priming of the } \\
\text { membrane oxygenator. } \\
\text { - Phase } 2 \text {, each dose was doubled. Studies of dosage level } 1 \text { were performed in Chicago ( } 42 \\
\text { patients) and studies of dosage level } 2 \text { were conducted both in Chicago ( } 26 \text { patients) and in } \\
\text { Temple, Texas ( } 16 \text { patients). Patients were stratified according to centre, surgeon, and type of } \\
\text { surgery. } \\
\text { The study also compared patients who underwent primary operations }(\mathrm{n}=60) \text { with those patients } \\
\text { who underwent re-operations }(\mathrm{n}=24) \text {. } \\
\text { NB: Both groups were exposed to cell salvage. }\end{array}$ \\
\hline
\end{tabular}
loss, autotransfusion device erythrocytes, myocardial infarction, mortality, renal function (BUN + creatinine levels), pre-operative and post-operative haemoglobin levels. 
Green 1995 (Continued)

\begin{tabular}{|c|c|c|}
\hline Notes & $\begin{array}{l}\text { Quality assessment score (Schulz criteria): 3/7 } \\
\text { Transfusion protocol used }\end{array}$ & \\
\hline \multicolumn{3}{|l|}{ Risk of bias } \\
\hline Item & Authors' judgement & Description \\
\hline Adequate sequence generation? & Yes & $\begin{array}{l}\text { Patients were assigned to groups by means of } \\
\text { computer generated table of random numbers }\end{array}$ \\
\hline Allocation concealment? & Unclear & Unclear \\
\hline $\begin{array}{l}\text { Blinding? } \\
\text { All outcomes }\end{array}$ & No & \\
\hline
\end{tabular}

Greilich 2001

\begin{tabular}{l|l}
\hline Methods & Method of randomisation and allocation concealment were not described. \\
\hline Participants & $\begin{array}{l}72 \text { patients undergoing elective cardiac surgery were randomised to one of three groups: } \\
\text { - Epsilon aminocaproic acid group: } \mathrm{n}=23, \text { mean }(\mathrm{sd}) \text { age }=63(8) \text { years } \\
\text { - Aprotinin group: } \mathrm{n}=24, \text { mean }(\mathrm{sd}) \text { age }=64(9) \text { years } \\
\text { - Control group (Placebo) }(\mathrm{n}=25), \text { mean }(\mathrm{sd}) \text { age }=62(7) \text { years }\end{array}$ \\
\hline
\end{tabular}

\begin{tabular}{|c|c|}
\hline Interventions & $\begin{array}{l}\text { - Epsilon aminocaproic acid group received } 100 \mathrm{mg} / \mathrm{kg} \text { loading dose, } 5 \mathrm{~g} \text { added to the pump } \\
\text { prime, and } 30 \mathrm{mg} / \mathrm{kg} / \mathrm{hr} \text { as a continuous infusion. } \\
\text { - Aprotinin group received } 2 \text { million KIU of aprotinin added to the pump prime, and } \\
500,000 \mathrm{KIU} / \mathrm{hr} \text { ( } 70 \mathrm{mg} / \mathrm{hour} \text { ) as a continuous infusion. } \\
\text { - Control group received } 200 \mathrm{ml} \text { normal saline as a loading dose, } 200 \mathrm{ml} \text { of normal saline } \\
\text { added to the pump prime, and } 50 \mathrm{ml} / \mathrm{hr} \text { of saline as a continuous infusion. }\end{array}$ \\
\hline
\end{tabular}

Outcomes

Outcomes reported: Number of patients exposed to allogeneic blood, blood loss, intensive care unit length of stay (days), mechanical ventilation (hours).

\begin{tabular}{l|ll}
\hline Notes & $\begin{array}{l}\text { Quality assessment score (Schulz criteria): 2/7 } \\
\text { Transfusion protocol used }\end{array}$ & \\
\hline Risk of bias & Authors' judgement & Description \\
\hline Item & Unclear & Unclear \\
\hline Adequate sequence generation? & Unclear
\end{tabular}


Greilich 2001 (Continued)

\begin{tabular}{|c|c|c|}
\hline $\begin{array}{l}\text { Blinding? } \\
\text { All outcomes }\end{array}$ & Yes & Double blind \\
\hline
\end{tabular}

\section{Greilich 2003}

\begin{tabular}{l|l}
\hline Methods & Method of randomisation and allocation concealment were not described. \\
\hline Participants & $\begin{array}{l}60 \text { male patients undergoing cardiac surgery were randomised to one of three groups: } \\
\text { - Aprotinin group (High dose): } \mathrm{n}=20 \text {, mean }(\mathrm{sd}) \text { age }=64(9) \text { years } \\
\text { - Epsilon aminocaproic acid }(\mathrm{n}=20), \mathrm{mean}(\mathrm{sd}) \text { age }=62(9) \text { years } \\
\text { - Control group (Placebo) }(\mathrm{n}=20), \text { mean }(\mathrm{sd}) \text { age }=63(8) \text { years }\end{array}$ \\
\hline Interventions & $\begin{array}{l}\text { - Aprotinin group (High dose) received } 2 \text { million } \mathrm{KIU}(280 \mathrm{mg}) \text { of aprotinin as a loading dose } \\
\text { and a continuous infusion of } 500,000 \mathrm{KIU} / \mathrm{hr} \text { of aprotinin. An additional } 2 \text { million KIU } \\
\text { (280mg) of aprotinin was added to the pump prime solution. } \\
\text { - Epsilon aminocaproic acid group received } 100 \mathrm{mg} / \mathrm{kg} \text { of EACA as a loading dose and a } \\
\text { continuous infusion of } 30 \mathrm{mg} / \mathrm{kg} / \mathrm{hr} \text {. An additional } 5 \mathrm{~g} \text { of EACA was added to the pump prime. } \\
\text { - Control group (placebo) received normal saline in equivalent volumes. }\end{array}$ \\
\hline $\mathrm{NB}$ : All groups were exposed to cell salvage.
\end{tabular}

Outcomes

Outcomes reported: Allogeneic blood usage (units), platelet usage (units), blood loss, plasma levels of Interleukin-6 and Interleukin-8 during and after CPB.

$\begin{array}{ll}\text { Notes } & \text { Quality assessment score (Schulz criteria): 4/7 } \\ \text { Transfusion protocol used }\end{array}$

Risk of bias

\begin{tabular}{lll}
\hline Item & Authors' judgement & Description \\
\hline Adequate sequence generation? & Unclear & Unclear \\
\hline Allocation concealment? & Unclear & Unclear \\
\hline $\begin{array}{l}\text { Blinding? } \\
\text { All outcomes }\end{array}$ & Yes & Double blind \\
\hline
\end{tabular}

\section{Greilich 2004}

\begin{tabular}{l|l}
\hline Methods & Method of randomisation and allocation concealment were not described. \\
\hline Participants & $\begin{aligned} 36 & \text { male patients undergoing cardiac surgery were randomised to one of three groups: } \\
& \text { - Aprotinin group (High dose): } \mathrm{n}=12, \text { mean }(\mathrm{sd}) \text { age }=62(8) \text { years } \\
& \text { - Epsilon aminocaproic acid group: } \mathrm{n}=12, \text { mean }(\mathrm{sd}) \text { age }=64(9) \text { years } \\
& \text { - Control group (Placebo): } \mathrm{n}=12, \text { mean }(\mathrm{sd}) \text { age }=65(8) \text { years }\end{aligned}$
\end{tabular}




\section{Greilich 2004 (Continued)}

\begin{tabular}{|c|c|c|}
\hline Interventions & \multicolumn{2}{|c|}{$\begin{array}{l}\text { - Aprotinin group (High dose) received a loading dose of } 2 \text { million KIU ( } 280 \mathrm{mg} \text { ) of aprotinin } \\
\text { and a continuous infusion of } 500,000 \mathrm{KIU} / \mathrm{hr} \text {. An additional } 2 \text { million KIU of aprotinin was } \\
\text { added to the pump prime. } \\
\text { - Epsilon aminocaproic acid group received a loading dose of } 100 \mathrm{mg} / \mathrm{kg} \text { of EACA and a } \\
\text { continuous infusion of } 30 \mathrm{mg} / \mathrm{kg} / \mathrm{hr} \text {. An additional } 5 \mathrm{~g} \text { of EACA was added to the pump prime. } \\
\text { - Control group received normal saline solution using similar volumes as aprotinin and EACA } \\
\text { treatments. }\end{array}$} \\
\hline Outcomes & \multicolumn{2}{|c|}{$\begin{array}{l}\text { Outcomes reported: Allogeneic blood usage (units), blood loss ( } 24 \mathrm{hrs}) \text {, biochemical markers of } \\
\text { plasmin activity (D-dimer), biochemical markers of platelet (CD62P), activation, biochemical } \\
\text { markers of leukocyte (CD11b) activation, biochemical markers of leukocyte-platelet conjugate } \\
\text { formation. }\end{array}$} \\
\hline Notes & \multicolumn{2}{|c|}{$\begin{array}{l}\text { Quality assessment score (Schulz criteria):3/7 } \\
\text { Transfusion protocol used }\end{array}$} \\
\hline \multicolumn{3}{|l|}{ Risk of bias } \\
\hline Item & Authors' judgement & Description \\
\hline Adequate sequence generation? & Unclear & Unclear \\
\hline Allocation concealment? & Unclear & Unclear \\
\hline $\begin{array}{l}\text { Blinding? } \\
\text { All outcomes }\end{array}$ & Yes & Double blind \\
\hline
\end{tabular}

\section{Greilich 2009}

\begin{tabular}{ll} 
Methods & $\begin{array}{l}\text { A table of random numbers was used to generate the allocation sequence. Central (pharmacy) } \\
\text { allocation was used. }\end{array}$ \\
\hline Participants & $\begin{array}{l}78 \text { male patients undergoing cardiac surgery were randomised to one of three groups: } \\
\text { - Aprotonin group: } \mathrm{n}=26, \text { mean }(\mathrm{sd}) \text { age }=65(9) \text { years } \\
\text { - EACA group: } \mathrm{n}=25, \text { mean }(\mathrm{sd}) \text { age }=62(8) \text { years } \\
\text { - Control group (Placebo): } \mathrm{n}=27, \text { mean }(\mathrm{sd}) \text { age }=62(7) \text { years }\end{array}$ \\
\hline
\end{tabular}

Interventions

- Aprotonin group received full-dose - loading dose 2 million KIU over 15 minutes plus 2 million added to pump prime and infusion of 500,000 KIU/hr until patient arrival at ICU.

- EACA group - high dose $-100 \mathrm{mg} / \mathrm{kg}$ initial loading dose, $5 \mathrm{~g}$ in pump prime solution, $30 \mathrm{mg} / \mathrm{kg} / \mathrm{hr}$.

- Control group received saline.

Outcomes

Outcomes reported: Number of patients transfused allogeneic blood, mortality, stroke, myocardial infarction, renal failure, length of hospital stay (days). 
Greilich 2009 (Continued)

\begin{tabular}{l|l|l}
\hline Risk of bias & Authors' judgement & Description \\
\hline Item & Yes & $\begin{array}{l}\text { A table of random numbers was used to generate } \\
\text { the allocation sequence }\end{array}$ \\
\hline Adequate sequence generation? & Adequate \\
\hline $\begin{array}{l}\text { Allocation concealment? } \\
\begin{array}{l}\text { Blinding? } \\
\text { All outcomes }\end{array}\end{array}$ & Yes & Double blind \\
\hline
\end{tabular}

\section{Groh 1993}

\begin{tabular}{|c|c|c|}
\hline Methods & \multicolumn{2}{|c|}{ Method of randomisation and allocation concealment was not described. } \\
\hline Participants & \multicolumn{2}{|c|}{$\begin{array}{l}20 \text { patients undergoing orthotopic liver transplantation were randomised to one of two groups: } \\
\text { - Aprotinin group: } \mathrm{n}=9 \text {, mean (range) age }=51(28-66) \text { years } \\
\text { - Control group (Placebo): } \mathrm{n}=9 \text {, mean (range) age }=49(31-59) \text { years } \\
\text { NB: Two patients were excluded from the final analysis }\end{array}$} \\
\hline Interventions & \multicolumn{2}{|c|}{$\begin{array}{l}\text { - Aprotinin group received a loading dose of } 2 \text { million KIU of aprotinin after the induction of } \\
\text { anaesthesia, followed by a continuous infusion of } 500,000 \mathrm{KIU} / \mathrm{hr} \text { of aprotinin until the end of } \\
\text { the procedure. } \\
\text { - Control group received an unspecified placebo. } \\
\text { NB: Both groups were exposed to cell salvage. }\end{array}$} \\
\hline Outcomes & \multicolumn{2}{|c|}{$\begin{array}{l}\text { Outcomes reported: Allogeneic blood usage (units), fresh frozen plasma usage (units), platelet usage } \\
\text { (units) }\end{array}$} \\
\hline Notes & \multicolumn{2}{|c|}{$\begin{array}{l}\text { Quality assessment score (Schulz criteria):3/7 } \\
\text { Transfusion protocol used }\end{array}$} \\
\hline \multicolumn{3}{|l|}{ Risk of bias } \\
\hline Item & Authors' judgement & Description \\
\hline Adequate sequence generation? & Unclear & Unclear \\
\hline Allocation concealment? & Unclear & Unclear \\
\hline $\begin{array}{l}\text { Blinding? } \\
\text { All outcomes }\end{array}$ & Yes & Double blind \\
\hline
\end{tabular}


Harder 1991

\begin{tabular}{|c|c|c|}
\hline Methods & \multicolumn{2}{|c|}{$\begin{array}{l}\text { Method of randomisation was not described. The randomisation code was only known by the } \\
\text { hospital pharmacy. Allocation concealment was by means of coded vials collected from the hospital } \\
\text { pharmacy in the morning before the operation. No exclusions were reported. }\end{array}$} \\
\hline Participants & \multicolumn{2}{|c|}{$\begin{array}{l}80 \text { male patients scheduled for elective coronary artery bypass grafting with cardiopulmonary bypass } \\
\text { were randomised to one of two groups: } \\
\text { - Aprotinin group: } n=40, \text { mean }(\mathrm{sd}) \text { age }=57.6(8.8) \text { years } \\
\text { - Control group (Placebo): } \mathrm{n}=40 \text {, mean }(\mathrm{sd}) \text { age }=57.0(8.8) \text { years }\end{array}$} \\
\hline Interventions & \multicolumn{2}{|c|}{$\begin{array}{l}\text { - Aprotinin group received a bolus of } 200 \mathrm{ml} \text { ( } 2 \text { million KIU) of aprotinin just after the Swan- } \\
\text { Ganz pulmonary artery catheter was introduced, followed by a continuous infusion of } 500,000 \\
\text { KIU/hr }(50 \mathrm{ml}) \text { via an infusion pump. An additional } 2 \text { million KIU of aprotinin was added to the } \\
\text { pump prime. The total amount of aprotinin delivered by infusion was } 4 \text { million KIU before and } \\
\text { during bypass. } \\
\text { - Control group received saline solution in equivalent volumes. }\end{array}$} \\
\hline Outcomes & \multicolumn{2}{|c|}{$\begin{array}{l}\text { Outcomes reported: Number of patients exposed to allogeneic blood, allogeneic blood usage (units) } \\
\text {, blood loss. }\end{array}$} \\
\hline Notes & \multicolumn{2}{|c|}{$\begin{array}{l}\text { Quality assessment score (Schulz criteria): 6/7 } \\
\text { Transfusion protocol used }\end{array}$} \\
\hline \multicolumn{3}{|l|}{ Risk of bias } \\
\hline Item & Authors' judgement & Description \\
\hline Adequate sequence generation? & Unclear & Unclear \\
\hline Allocation concealment? & Yes & Adequate \\
\hline $\begin{array}{l}\text { Blinding? } \\
\text { All outcomes }\end{array}$ & Yes & Double blind \\
\hline
\end{tabular}

Hardy 1993

Methods

Randomisation was performed by the pharmacy department with each successive block of four patients being randomised (random allocation of two patients to Group A and two patients to Group C). Method used to generate allocation sequences was not described.

Participants

44 patients scheduled for repeat myocardial revascularisation, repeat value surgery, or a combined procedure (primary or repeat) were randomised to one of two groups:

- Aprotinin group: $\mathrm{n}=22, \mathrm{M} / \mathrm{F}=16 / 6$, mean (sd) age $=62$ (9) years

- Control group (Placebo): $\mathrm{n}=19, \mathrm{M} / \mathrm{F}=12 / 12$, mean (sd) age = 58 (11) years

NB: Three patients in the control group were excluded: one patient died in the operating room and one died upon arrival in the ICU. The third patient was excluded when the surgeon proceeded to a single valve replacement instead of the planned combined procedure. 
Hardy 1993 (Continued)

\begin{tabular}{|c|c|c|}
\hline Interventions & \multicolumn{2}{|c|}{$\begin{array}{l}\text { - Aprotinin group received a bolus of } 200,000 \mathrm{KIU} \text { of aprotinin after the induction of } \\
\text { anaesthesia, but before skin incision, over a period of } 20 \text { minutes, followed by an infusion of } \\
100,000 \mathrm{KIU} / \mathrm{hr} \text { during the entire surgical procedure and in the intensive care unit (ICU), for a } \\
\text { total dose of } 1 \text { million KIU. } \\
\text { - Control group received an equal volume of sodium chloride } 0.9 \% \text { throughout surgery and } \\
\text { recovery. }\end{array}$} \\
\hline Outcomes & \multicolumn{2}{|c|}{$\begin{array}{l}\text { Outcomes reported: Number of patients exposed to allogeneic blood, allogeneic blood usage (units) } \\
\text {, fresh frozen plasma usage (units), platelet usage (units), mortality, re-exploration for bleeding. }\end{array}$} \\
\hline Notes & \multicolumn{2}{|c|}{$\begin{array}{l}\text { Quality assessment score (Schulz criteria): } 5 / 7 \\
\text { Transfusion protocol used }\end{array}$} \\
\hline \multicolumn{3}{|l|}{ Risk of bias } \\
\hline Item & Authors' judgement & Description \\
\hline Adequate sequence generation? & Unclear & Unclear \\
\hline Allocation concealment? & Yes & Adequate \\
\hline $\begin{array}{l}\text { Blinding? } \\
\text { All outcomes }\end{array}$ & Yes & Double blind \\
\hline
\end{tabular}

\section{Hardy 1997}

Methods

Each successive block of four patients was randomised by the Department of Pharmacy (random allocation of two patients to the treatment group and two patients to the control group). Method used to generate allocation sequences was not described.

Participants
$\begin{aligned} & 52 \text { patients undergoing primary or repeat myocardial revascularisation, or repeat valve surgery were } \\ & \text { randomised to one of two groups: } \\ & \text { - Aprotinin group: } \mathrm{n}=26, \mathrm{M} / \mathrm{F}=15 / 11 \text {, mean }(\mathrm{sd}) \text { age }=59(11) \text { years } \\ & \text { - Control group (placebo) }(\mathrm{n}=26), \mathrm{M} / \mathrm{F}=19 / 7 \text {, mean (sd) age }=59 \text { (10) years }\end{aligned}$

Interventions

- Aprotinin group received 1 million KIU of aprotinin added to the priming solution of the cardiopulmonary bypass circuit.

- Control group (placebo) received an equal volume of sodium chloride $0.9 \%$ added to the priming solution of the cardiopulmonary bypass circuit.

Outcomes

Outcomes reported: Number of patients exposed to allogeneic blood, allogeneic blood usage (units) , re-operation, blood loss, haemoglobin concentrations, coagulation factors.

Notes

Quality assessment score (Schulz criteria): 6/7

Transfusion protocol used

\section{Risk of bias}


Hardy 1997 (Continued)

\begin{tabular}{lll}
\hline Item & Authors' judgement & Description \\
\hline Adequate sequence generation? & Unclear & Unclear \\
\hline Allocation concealment? & Yes & Adequate \\
\hline $\begin{array}{l}\text { Blinding? } \\
\text { All outcomes }\end{array}$ & Yes & Double blind \\
\hline
\end{tabular}

\section{Hardy 1998}

Methods Study participants were randomised by the pharmacy department. Each successive block of nine patients were randomised to ensure a comparable number of patients in all groups and a similar distribution of patients over time. All bags were coded by the Department of Pharmacy and identical volumes of solution were infused.

Participants

134 patients undergoing scheduled elective coronary artery bypass graft surgery were randomised to one of three groups:

- Control group (Placebo): $\mathrm{n}=45, \mathrm{M} / \mathrm{F}=38 / 7$

- Tranexamic acid group: $\mathrm{n}=43, \mathrm{M} / \mathrm{F}=27 / 16$

- Epsilon aminocaproic acid group: $\mathrm{n}=46, \mathrm{M} / \mathrm{F}=35 / 11$

NB: Age data were not reported

Interventions

- Control group (placebo) received a bolus plus an infusion of placebo solution ( $0.9 \%$ normal saline solution).

- Tranexamic acid group received a 10g bolus of TXA over 20 minutes, followed by a placebo infusion. The placebo consisted of $0.9 \%$ normal saline solution.

- Epsilon aminocaproic acid group received a $15 \mathrm{~g}$ bolus over 20 minutes, followed by an infusion of $1 \mathrm{~g} / \mathrm{hr}$.

Outcomes

Outcomes reported: Number of patients exposed to allogeneic blood, allogeneic blood usage (units), fresh frozen plasma usage (units), platelet usage (units), blood loss, mortality, myocardial infarction, cerebrovascular accident, re-operation for bleeding.

Notes Quality assessment score (Schulz criteria): 6/7

Transfusion protocol used

\section{Risk of bias}

\begin{tabular}{l|ll}
\hline Item & Authors' judgement & Description \\
\hline Adequate sequence generation? & Yes & Adequate \\
\hline Allocation concealment? & Yes & Adequate \\
\hline $\begin{array}{l}\text { Blinding? } \\
\text { All outcomes }\end{array}$ & Yes & Double blind \\
\hline
\end{tabular}


Harley 2002

\begin{tabular}{|c|c|c|}
\hline Methods & \multicolumn{2}{|c|}{ Method of randomisation and allocation concealment were not described. } \\
\hline Participants & \multicolumn{2}{|c|}{$\begin{array}{l}35 \text { patients undergoing elective orthopaedic surgery were randomised to one of two groups: } \\
\text { - Epsilon aminocaproic acid group: } \mathrm{n}=26, \mathrm{M} / \mathrm{F}=10 / 16 \text {, mean }(\mathrm{sd}) \text { age }=69(11) \text { years } \\
\text { - Control group (Placebo): } \mathrm{n}=29, \mathrm{M} / \mathrm{F}=11 / 18 \text {, mean (sd) age }=69(10) \text { years }\end{array}$} \\
\hline Interventions & \multicolumn{2}{|c|}{$\begin{array}{l}\text { - Epsilon aminocaproic acid group received a loading dose of } 150 \mathrm{mg} / \mathrm{kg} \text { administered as a } \\
\text { bolus dose over } 20 \text { minutes on the patients arrival in OR. An hourly EACA infusion of } 12.5 \mathrm{mg} / \\
\mathrm{kg} \text { was subsequently administered for an additional } 5 \text { hours. } \\
\text { - Control group received a placebo of saline solution }\end{array}$} \\
\hline Outcomes & \multicolumn{2}{|c|}{$\begin{array}{l}\text { Outcomes reported: Number of patients exposed to allogeneic blood, allogeneic blood usage (units) } \\
\text {, blood loss, deep vein thrombosis, pulmonary embolus. }\end{array}$} \\
\hline Notes & \multicolumn{2}{|c|}{$\begin{array}{l}\text { Quality assessment score (Schulz criteria): 3/7 } \\
\text { Transfusion protocol used }\end{array}$} \\
\hline \multicolumn{3}{|l|}{ Risk of bias } \\
\hline Item & Authors' judgement & Description \\
\hline Adequate sequence generation? & Unclear & Unclear \\
\hline Allocation concealment? & Unclear & Unclear \\
\hline $\begin{array}{l}\text { Blinding? } \\
\text { All outcomes }\end{array}$ & Yes & Double blind \\
\hline
\end{tabular}

\section{Harmon 2004}

\begin{tabular}{l|l} 
Methods & $\begin{array}{l}\text { A computer-generated randomisation sequence was used to allocate patients. The sequence was } \\
\text { concealed (numbered containers) until treatment was assigned. }\end{array}$ \\
\hline Participants & $\begin{array}{l}36 \text { patients undergoing cardiac surgery were randomised to one of two groups: } \\
\text { - Aprotinin group (High dose): } \mathrm{n}=17, \mathrm{M} / \mathrm{F}=17 / 3, \text { mean }(\mathrm{sd}) \text { age }=63.4(5.4) \text { years } \\
\text { - Control group (Placebo): } \mathrm{n}=18, \mathrm{M} / \mathrm{F}=14 / 4, \text { mean (sd) age }=60.1 \text { (9.5) years } \\
\text { NB: One patient was excluded from the final analysis }\end{array}$ \\
\hline Interventions & $\begin{array}{l}\text { - Aprotinin group (High dose) received a loading dose of } 2 \text { million KIU (280mg) of aprotinin } \\
\text { after the induction of anaesthesia and a continuous infusion of } 500,000 \mathrm{KIU} / \mathrm{hr} \text { of aprotinin } \\
\text { during surgery. An additional } 2 \text { million KIU was added to the CPB circuit prime. } \\
\text { - Control group (placebo) receive an unspecified solution. }\end{array}$ \\
\hline Outcomes & $\begin{array}{l}\text { Outcomes reported: Cognitive deficit, number of patients exposed to allogeneic blood, blood loss, } \\
\text { hospital length of stay (days), serious adverse events. }\end{array}$ \\
\hline Notes & $\begin{array}{l}\text { Quality assessment score (Schulz criteria): } 5 / 7 \\
\text { Transfusion protocol used }\end{array}$
\end{tabular}


Harmon 2004 (Continued)

\begin{tabular}{|c|c|c|}
\hline Item & Authors' judgement & Description \\
\hline Adequate sequence generation? & Yes & Computer-generated randomisation \\
\hline Allocation concealment? & Yes & Adequate \\
\hline $\begin{array}{l}\text { Blinding? } \\
\text { All outcomes }\end{array}$ & No & Single blind \\
\hline
\end{tabular}

\section{Havel 1992}

\begin{tabular}{|c|c|c|}
\hline Methods & \multicolumn{2}{|c|}{$\begin{array}{l}\text { Patients were randomly allocated to receive the test compound or a placebo by use of sealec } \\
\text { envelopes. Method of randomisation was not described. }\end{array}$} \\
\hline Participants & \multicolumn{2}{|c|}{$\begin{array}{l}20 \text { male patients undergoing orthotopic heart transplantation were randomised to one of two } \\
\text { groups: } \\
\text { - Aprotinin group (Low dose): } \mathrm{n}=10 \\
\text { - Control group (Placebo): } \mathrm{n}=10 \\
\text { NB: Demographic data not reported. }\end{array}$} \\
\hline Interventions & \multicolumn{2}{|c|}{$\begin{array}{l}\text { - Aprotinin group received } 280 \mathrm{mg} \text { of aprotinin over } 20 \text { minutes after anaesthesia prior to } \\
\text { surgery. In addition } 280 \mathrm{mg} \text { was added to the priming volume of the heart lung machine. } \\
\text { - Control group received a corresponding volume of normal saline solution. }\end{array}$} \\
\hline Outcomes & \multicolumn{2}{|c|}{$\begin{array}{l}\text { Outcomes reported: Number of patients exposed to allogeneic blood, allogeneic blood usage (units) } \\
\text {, blood loss ( } 24 \mathrm{hrs})\end{array}$} \\
\hline Notes & \multicolumn{2}{|c|}{$\begin{array}{l}\text { Quality assessment score (Schulz criteria): } 5 / 7 \\
\text { Transfusion protocol not specified }\end{array}$} \\
\hline \multicolumn{3}{|l|}{ Risk of bias } \\
\hline Item & Authors' judgement & Description \\
\hline Adequate sequence generation? & Unclear & Unclear \\
\hline Allocation concealment? & No & $\begin{array}{l}\text { Inadequate - used sealed envelopes to conceal } \\
\text { treatment allocation }\end{array}$ \\
\hline $\begin{array}{l}\text { Blinding? } \\
\text { All outcomes }\end{array}$ & Yes & Double blind \\
\hline
\end{tabular}


Havel 1994

\begin{tabular}{|c|c|c|}
\hline Methods & \multicolumn{2}{|c|}{$\begin{array}{l}\text { Method used to generate random sequences was not described. Bottles of aprotinin and placebo } \\
\text { were indistinguishable from each other. The preparation of each patient was individually packaged } \\
\text { with } 12 \text { bottles each; each individual bottle, as well as the carton, was marked with a label carrying } \\
\text { the patient number (the randomisation number). Each study package contained a total of } 12 \text { bottles, } \\
\text { of which eight carried the label "Infusion" and four the label "Pump". }\end{array}$} \\
\hline Participants & \multicolumn{2}{|c|}{$\begin{array}{l}45 \text { male patients undergoing cardiac surgery were randomised to one of three groups: } \\
\text { - Aprotinin group (High dose): } \mathrm{n}=15 \text {, mean }(\mathrm{sd}) \text { age }=60(8) \text { years } \\
\text { - Aprotinin group (Low dose): } \mathrm{n}=15 \text {, mean }(\mathrm{sd}) \text { age }=59(8) \text { years } \\
\text { - Control group (Placebo): } \mathrm{n}=15 \text {, mean }(\mathrm{sd}) \text { age }=60(9) \text { years }\end{array}$} \\
\hline Interventions & \multicolumn{2}{|c|}{$\begin{array}{l}\text { - Aprotinin group (High dose) received } 2 \text { million KIU of aprotinin as a bolus over } 30 \text { minutes } \\
\text { after the institution of anaesthesia but before skin incision, followed by a continuous infusion of } 2 \\
\text { million KIU/hr of aprotinin over } 4 \text { hours, and an additional } 2 \text { million KIU of aprotinin was } \\
\text { added to the pump prime. } \\
\text { - Aprotinin group (Low dose) received } 2 \text { million KIU of aprotinin added to the pump prime } \\
\text { only. } \\
\text { - Control group received } 0.9 \% \text { saline solution. }\end{array}$} \\
\hline Outcomes & \multicolumn{2}{|c|}{ Outcomes reported: Allogeneic blood usage (units), blood loss, graft patency rates. } \\
\hline Notes & \multicolumn{2}{|c|}{$\begin{array}{l}\text { Quality assessment score (Schulz criteria): 6/7 } \\
\text { Transfusion protocol not used }\end{array}$} \\
\hline \multicolumn{3}{|l|}{ Risk of bias } \\
\hline Item & Authors' judgement & Description \\
\hline Adequate sequence generation? & Unclear & Unclear \\
\hline Allocation concealment? & Yes & Adequate \\
\hline $\begin{array}{l}\text { Blinding? } \\
\text { All outcomes }\end{array}$ & Yes & Double blind \\
\hline
\end{tabular}

\section{Hayashida 1997}

\begin{tabular}{l|l} 
Methods & $\begin{array}{l}\text { Patients were randomised by means of computer-generated randomisation table. Method of allo- } \\
\text { cation concealment was not described. }\end{array}$ \\
\hline Participants & $\begin{array}{l}167 \text { patients undergoing primary isolated coronary artery bypass graft surgery were randomised to } \\
\text { one of three groups: } \\
\text { - Aprotinin group (Minimal dose) }: \mathrm{n}=55, \mathrm{M} / \mathrm{F}=43 / 12, \text { mean }(\mathrm{sd}) \text { age }=64.4(8.8) \text { years }\end{array}$ \\
& $\begin{array}{l}\text { - Aprotinin group (Low dose) }: \mathrm{n}=55, \mathrm{M} / \mathrm{F}=35 / 20, \text { mean }(\mathrm{sd}) \text { age }=63.2(8.2) \text { years } \\
\text { - Control group: } \mathrm{n}=57, \mathrm{M} / \mathrm{F}=41 / 16, \text { mean }(\mathrm{sd}) \text { age }=61.2(9.8) \text { years }\end{array}$
\end{tabular}




\section{Hayashida 1997 (Continued)}

Interventions
cardiopulmonary bypass priming solution.
- Aprotinin group (Low dose) received $30,000 \mathrm{KIU} / \mathrm{kg}$ of aprotinin in the priming solution
and a continuous infusion of aprotinin at a rate of $7,500 \mathrm{KIU} / \mathrm{kg}$ every hour during
cardiopulmonary bypass. The mean dose of aprotinin in the low dose group was $2.7 \mathrm{million} \mathrm{KIU}$
(range 1.4 million KIU to 4.0 million KIU).
- Control group received no aprotinin treatment.
NB: All groups were exposed to pre-operative autologous blood donation (PAD).

Outcomes

Outcomes reported: Number of patients exposed to allogeneic blood, allogeneic blood usage (units) , fresh frozen plasma usage (units), all blood product requirements (units), blood loss, mortality, myocardial infarction, allergic reaction, parameters of clotting and fibrinolysis, renal function, early graft pattency rates.

$\begin{array}{ll}\text { Notes } & \begin{array}{l}\text { Quality assessment score (Schulz criteria): 4/7 } \\ \text { Transfusion protocol used }\end{array}\end{array}$

Transfusion protocol used

\section{Risk of bias}

\begin{tabular}{lll}
\hline Item & Authors' judgement & Description \\
\hline Adequate sequence generation? & Yes & Computer-generated randomisation table \\
\hline Allocation concealment? & Unclear & Unclear \\
\hline $\begin{array}{l}\text { Blinding? } \\
\text { All outcomes }\end{array}$ & No & Single blind \\
\hline
\end{tabular}

\section{Hayes 1996}

\begin{tabular}{l|l}
\hline Methods & Method of randomisation and allocation concealment were not described. \\
\hline Participants & $\begin{array}{l}40 \text { patients scheduled for total hip replacement surgery were randomly allocated to one of two } \\
\text { groups: } \\
\text { - Aprotinin group: } \mathrm{n}=20, \mathrm{M} / \mathrm{F}=8 / 12, \text { mean }(\mathrm{sd}) \text { age }=70.0(7.9) \text { years } \\
\text { - Control group: } \mathrm{n}=20, \mathrm{M} / \mathrm{F}=7 / 13 \text {, mean }(\mathrm{sd}) \text { age }=72.9(10.3) \text { years }\end{array}$ \\
\hline
\end{tabular}

\begin{tabular}{l|l} 
Interventions & $\begin{array}{c}\text { - Aprotinin group received } 2 \text { million KIU of } \\
\text { surgical incision. } \\
\bullet \text { Control group received an equal volume of }\end{array}$ \\
\hline Outcomes & $\begin{array}{l}\text { Outcomes reported: Allogeneic blood usage (u } \\
\text { parameters, complications. }\end{array}$ \\
\hline Notes & $\begin{array}{l}\text { Quality assessment score (Schulz criteria): 4/7 } \\
\text { Transfusion protocol used }\end{array}$
\end{tabular}


Hayes 1996 (Continued)

\begin{tabular}{l|ll} 
Risk of bias & & \\
\hline Item & Authors' judgement & Description \\
\hline Adequate sequence generation? & Unclear & Unclear \\
\hline Allocation concealment? & Unclear & Unclear \\
\hline $\begin{array}{l}\text { Blinding? } \\
\text { All outcomes }\end{array}$ & Yes & Double blind \\
\hline
\end{tabular}

\section{Hei 2005}

\begin{tabular}{|c|c|c|}
\hline Methods & \multicolumn{2}{|c|}{ Method of randomisation and allocation concealment were not described. [Chinese language] } \\
\hline Participants & \multicolumn{2}{|c|}{$\begin{array}{l}40 \text { patients with severe hepatitis undergoing liver transplantation were randomly assigned to one } \\
\text { of two groups: } \\
\text { - Aprotinin group: } \mathrm{n}=20 \\
\text { - Control group (Placebo): } \mathrm{n}=20 \\
\text { Demographic data: } \mathrm{M} / \mathrm{F}=38 / 2 \text {, age range }=31-67 \text { years. }\end{array}$} \\
\hline Interventions & \multicolumn{2}{|c|}{$\begin{array}{l}\text { - Aprotinin group received a continuous infusion of } 400,000 \mathrm{KIU} \text { of aprotinin commenced at } \\
\text { the induction of anaesthesia and ceased at the end of surgery. } \\
\text { - Control group received normal saline at the same volumes as the aprotinin regimen. }\end{array}$} \\
\hline Outcomes & \multicolumn{2}{|c|}{$\begin{array}{l}\text { Outcomes reported: Allogeneic blood usage (units), fresh frozen plasma usage, platelet usage, blood } \\
\text { loss. }\end{array}$} \\
\hline Notes & \multicolumn{2}{|c|}{ Transfusion protocol used } \\
\hline \multicolumn{3}{|l|}{ Risk of bias } \\
\hline Item & Authors' judgement & Description \\
\hline Adequate sequence generation? & Unclear & Unclear \\
\hline Allocation concealment? & Unclear & Unclear \\
\hline $\begin{array}{l}\text { Blinding? } \\
\text { All outcomes }\end{array}$ & Unclear & Unclear \\
\hline
\end{tabular}


Hekmat 2004

\begin{tabular}{|c|c|c|}
\hline Methods & \multicolumn{2}{|c|}{$\begin{array}{l}\text { Randomisation was based on a computer-generated code and sealed in sequentially numbered, } \\
\text { opaque envelopes. }\end{array}$} \\
\hline Participants & \multicolumn{2}{|c|}{$\begin{array}{l}120 \text { patients undergoing elective cardiac surgery were randomly assigned to one of two groups: } \\
\text { - Aprotinin group (High dose): } \mathrm{n}=60, \mathrm{M} / \mathrm{F}=51 / 9 \text {, mean }(\mathrm{sd}) \text { age }=63 \text { (8) years } \\
\text { - Tranexamic acid group: } \mathrm{n}=60, \mathrm{M} / \mathrm{F}=51 / 7 \text {, mean (sd) age }=63 \text { (8) years }\end{array}$} \\
\hline Interventions & \multicolumn{2}{|c|}{$\begin{array}{l}\text { - Aprotinin group (High dose - "Full Hammersmith" regimen) received a loading dose of } 2 \\
\text { million kallikrein inactivation units (KIU) of aprotinin, } 2 \text { million KIU of aprotinin added to the } \\
\mathrm{CPB} \text { pump prime, and a continuous infusion of } 500,000 \mathrm{KIU} / \mathrm{hr} \text { during } \mathrm{CPB} \text {. } \\
\text { - Tranexamic acid group received } 500 \mathrm{mg} \text { of TXA as a loading dose, } 500 \mathrm{mg} \text { added to the CPB } \\
\text { pump prime, and } 1 \mathrm{~g} \text { was given post } \mathrm{CPB} \text { (a total of } 2 \mathrm{~g} \text { of TXA). } \\
\text { NB: Cell Salvage was used during surgery in both groups using a cell saver (Brat2, Cobe Cardio- } \\
\text { vascular Inc, Arvada, CO.). }\end{array}$} \\
\hline Outcomes & \multicolumn{2}{|c|}{$\begin{array}{l}\text { Outcomes reported: Allogeneic blood usage (units), fresh frozen plasma usage (units), platelet } \\
\text { usage (units), blood loss, mortality, myocardial Infarction, hospital length of stay (days), intensive } \\
\text { care unit length of stay (days), intubation time (hours), number of patients requiring Intra-aortic } \\
\text { balloon pump (IABP) therapy. }\end{array}$} \\
\hline Notes & \multicolumn{2}{|c|}{$\begin{array}{l}\text { Quality assessment score (Schulz criteria): 5/7 } \\
\text { Transfusion protocol used }\end{array}$} \\
\hline \multicolumn{3}{|l|}{ Risk of bias } \\
\hline Item & Authors' judgement & Description \\
\hline Adequate sequence generation? & Yes & Computer-generated code \\
\hline Allocation concealment? & No & Inadequate - sealed envelopes \\
\hline $\begin{array}{l}\text { Blinding? } \\
\text { All outcomes }\end{array}$ & Yes & Double blind \\
\hline
\end{tabular}

\section{Hendrice 1995}

\begin{tabular}{l|l}
\hline Methods & Method of randomisation and allocation concealment were not described. \\
\hline Participants & $\begin{array}{l}\text { 26 patients undergoing primary coronary artery bypass surgery were randomly allocated to one of } \\
\text { two groups: } \\
\text { - Aprotinin group: } \mathrm{n}=12, \text { mean }(\mathrm{sd}) \text { age }=59.8(7.9) \text { years } \\
\text { - Control group: } \mathrm{n}=14, \text { mean }(\mathrm{sd}) \text { age }=58.1(17.3) \text { years }\end{array}$ \\
\hline Nb: Gender data not provided.
\end{tabular}




\section{Hendrice 1995 (Continued)}

- Control group did not receive aprotinin.

\begin{tabular}{|c|c|c|}
\hline Outcomes & \multicolumn{2}{|c|}{$\begin{array}{l}\text { Outcomes reported: Allogeneic blood usage, blood loss ( } 24 \mathrm{hrs}) \text {, haemoglobin levels, coagulation } \\
\text { factors. }\end{array}$} \\
\hline Notes & \multicolumn{2}{|c|}{$\begin{array}{l}\text { Quality assessment score (Schulz criteria): 2/7 } \\
\text { Transfusion protocol not specified }\end{array}$} \\
\hline \multicolumn{3}{|l|}{ Risk of bias } \\
\hline Item & Authors' judgement & Description \\
\hline Adequate sequence generation? & Unclear & Unclear \\
\hline Allocation concealment? & Unclear & Unclear \\
\hline $\begin{array}{l}\text { Blinding? } \\
\text { All outcomes }\end{array}$ & Unclear & Unclear \\
\hline
\end{tabular}

\section{Hiipala 1995}

Methods

Concealment of treatment allocation was by means of a ticket drawn from an envelope containing an equal number of treatment and placebo tickets. The method used to generate allocation sequences was not described.

Participants

29 patients undergoing total knee arthroplasty were randomly allocated to one of two groups:

- Tranexamic acid group: $\mathrm{n}=15, \mathrm{M} / \mathrm{F}=2 / 13$, mean (range) age $=70$ (56-82) years

- Control group (Placebo): $\mathrm{n}=13, \mathrm{M} / \mathrm{F}=3 / 10$, mean (range) age $=70(63-78)$ years

Interventions

- Tranexamic acid group received a bolus of $15 \mathrm{mg} / \mathrm{kg}$ of TXA 2-5 minutes before deflation of limb tourniquet.

- Control group received an equal volume of sodium chloride solution $(0.9 \%)$.

Outcomes

Outcomes reported: Number of patients exposed to allogeneic blood, allogeneic blood usage (units) , blood loss, myocardial infarction, deep vein thrombosis, pulmonary embolus, minor non-thromboembolic complications.

$\begin{array}{ll}\text { Notes } & \begin{array}{l}\text { Quality assessment score (Schulz criteria): 4/7 } \\ \text { Transfusion protocol used }\end{array}\end{array}$

\section{Risk of bias}

\begin{tabular}{lll}
\hline Item & Authors' judgement & Description \\
\hline Adequate sequence generation? & Unclear & Unclear \\
\hline Allocation concealment? & No & Inadequate
\end{tabular}


Hiipala 1995 (Continued)

\begin{tabular}{|c|c|c|}
\hline $\begin{array}{l}\text { Blinding? } \\
\text { All outcomes }\end{array}$ & Yes & Double blind \\
\hline
\end{tabular}

Hiipala 1997

Methods A ticket indicating the group was drawn and enclosed in an envelope. The envelopes were opened after the study was completed. Injection syringes were prepared by a person outside the surgical team.

Participants

75 patients scheduled for 77 total knee arthroplasties were randomly allocated to one of two groups:

- Tranexamic acid group: $\mathrm{n}=39, \mathrm{M} / \mathrm{F}=4 / 35$, mean (sd) age $=70$ (7) years

- Control group (Placebo): $\mathrm{n}=38, \mathrm{M} / \mathrm{F}=8 / 30$, mean $(\mathrm{sd})$ age $=69(5)$ years

NB: Three patients were excluded from the final analysis for miscellaneous reasons.

\begin{tabular}{l|l}
\hline Interventions & $\begin{array}{l}\text { - Tranexamic acid group received } 15 \mathrm{mg} / \mathrm{kg} \text { of TXA before the removal of the limb tourniquet, } \\
\text { followed by two } 10 \mathrm{mg} / \mathrm{kg} \text { additional doses. } \\
\text { - Control group received equal volumes of normal saline. }\end{array}$ \\
\hline
\end{tabular}

Outcomes Outcomes reported: Number of patients exposed to allogeneic blood, allogeneic blood usage (units) , blood loss, mortality, deep vein thrombosis, pulmonary embolus, non-thrombotic complications.

\begin{tabular}{|c|c|c|}
\hline Notes & $\begin{array}{l}\text { Quality assessment score (Schulz criteria): 4/7 } \\
\text { Transfusion protocol used }\end{array}$ & \\
\hline \multicolumn{3}{|l|}{ Risk of bias } \\
\hline Item & Authors' judgement & Description \\
\hline Adequate sequence generation? & Unclear & Unclear \\
\hline Allocation concealment? & No & Inadequate \\
\hline $\begin{array}{l}\text { Blinding? } \\
\text { All outcomes }\end{array}$ & Yes & Double blind \\
\hline
\end{tabular}

Hill 1998

Methods

Patients were randomised according to a computer-generated sequence. Method of allocation concealment was not described.

Participants

20 adult patients scheduled for first-time myocardial revascularisation were randomly assigned to one of two groups:

- Aprotinin group: $\mathrm{n}=10$, mean $(\mathrm{sd})$ age $=64(7.9)$ years

- Control group: $\mathrm{n}=10$, mean $(\mathrm{sd})$ age $=62(7.9)$ years

NB: Gender data not reported. 


\section{Hill 1998 (Continued)}

\begin{tabular}{|c|c|c|}
\hline Interventions & \multicolumn{2}{|c|}{$\begin{array}{l}\text { - Aprotinin group received } 280 \mathrm{mg} \text { of aprotinin ( } 2 \text { million KIU) intravenously as a loading } \\
\text { dose followed by } 70 \mathrm{mg}(500,000 \mathrm{KIU}) \text { of aprotinin per hour as a constant intravenous infusion } \\
\text { until chest closure. In addition } 280 \mathrm{mg} \text { of aprotinin ( } 2 \text { million KIU) was added to the "pump } \\
\text { prime". } \\
\text { - Control group did not receive aprotinin. }\end{array}$} \\
\hline Outcomes & \multicolumn{2}{|c|}{ Outcomes reported: Allogeneic blood usage (units), interleukin-10 (IL-10) levels. } \\
\hline Notes & \multicolumn{2}{|c|}{$\begin{array}{l}\text { Quality assessment score (Schulz criteria): 3/7 } \\
\text { Transfusion protocol not specified }\end{array}$} \\
\hline \multicolumn{3}{|l|}{ Risk of bias } \\
\hline Item & Authors' judgement & Description \\
\hline Adequate sequence generation? & Yes & Computer-generated sequence \\
\hline Allocation concealment? & Unclear & Unclear \\
\hline $\begin{array}{l}\text { Blinding? } \\
\text { All outcomes }\end{array}$ & Unclear & Unclear \\
\hline
\end{tabular}

Horrow 1990

\begin{tabular}{l|l}
\hline Methods & $\begin{array}{l}\text { Patients were randomised using a random number table. Sealed envelopes ensured that only the } \\
\text { pharmacist, who prepared the encoded infusions, knew whether a patient received drug or placebo. }\end{array}$ \\
\hline Participants & $\begin{array}{l}49 \text { patients undergoing cardiac surgery were randomised to one of two groups: } \\
\text { - Tranexamic acid group: } \mathrm{n}=18, \text { mean }(\mathrm{sd}) \text { age }=66(10) \\
\text { - Control group (Placebo): } \mathrm{n}=20, \text { mean }(\mathrm{sd}) \text { age }=62 \text { (9) years }\end{array}$ \\
\hline Interventions & $\begin{array}{l}\text { - Tranexamic acid group received a } 10 \mathrm{mg} / \mathrm{kg} \text { infusion of TXA over a 20-minute period } \\
\text { followed by an infusion of } 1 \mathrm{mg} / \mathrm{kg} \text { for } 10 \text { hours. } \\
\text { - Control group received equivalent infusions of saline (100ml total volume). } \\
\text { NB: Both groups received cell salvage. }\end{array}$ \\
\hline Outcomes & $\begin{array}{l}\text { Outcomes reported: } \text { Allogeneic blood usage (units), number of participants exposed to fresh frozen } \\
\text { plasma, number of participants exposed to platelets, blood loss (12 hrs), deep vein thrombosis, } \\
\text { pulmonary embolus, stroke, number of patients receiving cell salvage, volume of cell salvage auto- } \\
\text { transfused. }\end{array}$ \\
\hline Notes & $\begin{array}{l}\text { Quality assessment score (Schulz criteria): } 4 / 7 \\
\text { Transfusion protocol not specified }\end{array}$ \\
\hline
\end{tabular}

\section{Risk of bias}


Horrow 1990 (Continued)

\begin{tabular}{lll}
\hline Adequate sequence generation? & Yes & Random number table \\
\hline Allocation concealment? & No & Inadequate \\
\hline $\begin{array}{l}\text { Blinding? } \\
\text { All outcomes }\end{array}$ & Yes & Double blind \\
\hline
\end{tabular}

\section{Horrow 1991}

Methods

A table of random numbers determined patient allocation to one of four groups. Coded infusion bags and sealed envelopes prepared by a pharmacist not involved in the study provided double blinded conditions.

Participants
$\begin{aligned} & \text { repair of atrial septal defect, were randomly allocated to one of four groups: } \\ & \text { - Control group (Placebo): } \mathrm{n}=44 \text {, mean }(\mathrm{sd}) \text { age }=64(10) \text { years } \\ & \text { - Tranexamic acid group: } \mathrm{n}=37 \text {, mean }(\mathrm{sd}) \text { age }=65(11) \text { years } \\ & \text { - Desmopressin group: } \mathrm{n}=38 \text {, mean }(\mathrm{sd}) \text { age }=63(11) \text { years } \\ & \text { - Tranexamic acid + Desmopressin group: } \mathrm{n}=40 \text {, mean }(\mathrm{sd}) \text { age }=63 \text { (9) years } \\ & \text { NB: Gender data were not reported. }\end{aligned}$

Interventions
$\begin{aligned} & \text { - Control group received saline solutions. } \\ & \text { before skin incision (loading dose }-10 \mathrm{mg} / \mathrm{kg} \text { over } 30 \text { minutes) followed by a } 12 \text { hour infusion of } \\ & 1 \mathrm{mg} / \mathrm{kg} / \mathrm{hr} \text {. } \\ & \text { - Desmopressin group received desmopressin acetate }(0.3 \mathrm{ug} / \mathrm{kg} \text { over } 20 \text { minutes) beginning } \\ & \text { after extracorporeal circulation following completion of protamine infusion. } \\ & \text { - Tranexamic acid + Desmopressin group received both tranexamic acid and desmopressin in } \\ & \text { an identical fashion to groups } 2 \text { and } 3 \text {. } \\ & \text { NB: All patients received cell salvaged autologous blood if available. }\end{aligned}$

Outcomes $\quad$ Outcomes reported: Number of patients exposed to allogeneic blood, allogeneic blood usage (units) , fresh frozen plasma usage (units), platelet usage (units), blood loss (12hrs), myocardial infarction, stroke, deep venous thrombosis, re-operation for bleeding, rash, ventricular dysfunction, pulmonary oedema, ventricular tachycardia.

$\begin{array}{ll}\text { Notes } & \begin{array}{l}\text { Quality assessment score (Schulz criteria): 4/7 } \\ \text { Transfusion protocol used }\end{array}\end{array}$

Risk of bias

\begin{tabular}{lll}
\hline Item & Authors' judgement & Description \\
\hline Adequate sequence generation? & Yes & Table of random numbers \\
\hline Allocation concealment? & No & Inadequate
\end{tabular}


Horrow 1991 (Continued)

\begin{tabular}{|c|c|c|}
\hline $\begin{array}{l}\text { Blinding? } \\
\text { All outcomes }\end{array}$ & No & Single blind \\
\hline
\end{tabular}

Horrow 1995

Methods

Coded infusion bags for both loading and infusion doses and sealed envelopes prepared by a pharmacist provided allocation concealment. Randomisation was determined by a table of random numbers.

\begin{tabular}{|c|c|}
\hline Participants & $\begin{array}{l}148 \text { patients undergoing elective cardiac operations were randomised to one of six groups: } \\
\text { - Control group (Placebo): } \mathrm{n}=27, \mathrm{M} / \mathrm{F}=23 / 4, \mathrm{mean}(\mathrm{sd}) \text { age }=63(10.4) \text { years } \\
\text { - Tranexamic acid group (Quarter dose): } \mathrm{n}=24 ; \mathrm{M} / \mathrm{F}=18 / 6 ; \text { mean }(\mathrm{sd}) \text { age }=67(9.8) \text { years } \\
\text { - Tranexamic acid group (Half dose): } \mathrm{n}=22, \mathrm{M} / \mathrm{F}=19 / 3 \text {, mean }(\mathrm{sd}) \text { age }=61(9.4) \text { years } \\
\text { - Tranexamic acid group (Whole dose): } \mathrm{n}=21, \mathrm{M} / \mathrm{F}=18 / 3 \text {, mean (sd) age }=66(9.2) \text { years } \\
\text { - Tranexamic acid group (Double dose): } \mathrm{n}=27, \mathrm{M} / \mathrm{F}=22 / 5 \text {, mean (sd) age }=63(10.4) \text { years } \\
\text { - Tranexamic acid group (Fourfold dose) }: \mathrm{n}=27, \mathrm{M} / \mathrm{F}=21 / 6 \text {, mean (sd) age }=65(10.4 \text { ) years }\end{array}$ \\
\hline Interventions & $\begin{array}{l}\text { - Control group received saline infusions. } \\
\text { - Tranexamic acid group (Quarter dose) received a loading dose of } 2.5 \mathrm{mg} / \mathrm{kg} \text { of TXA after the } \\
\text { induction of anaesthesia over a period of } 30 \text { minutes followed by a } 12 \text { hour continuous infusion } \\
\text { of } 0.25 \mathrm{mg} / \mathrm{kg} / \mathrm{hr} \text { of TXA. } \\
\text { - Tranexamic acid group (Half dose) received a loading dose of } 5.0 \mathrm{mg} / \mathrm{kg} \text { of TXA after the } \\
\text { induction of anaesthesia over a period of } 30 \text { minutes followed by a } 12 \text { hour continuous infusion } \\
\text { of } 0.5 \mathrm{mg} / \mathrm{kg} / \mathrm{hr} \text { of TXA. } \\
\text { - Tranexamic acid group (Whole dose) received a loading dose of } 10 \mathrm{mg} / \mathrm{kg} \text { of TXA after the } \\
\text { induction of anaesthesia over a period of } 30 \text { minutes followed by a } 12 \text { hour continuous infusion } \\
\text { of } 1.0 \mathrm{mg} / \mathrm{kg} / \mathrm{hr} \text { of TXA. } \\
\text { - Tranexamic acid group (Double dose) received a loading dose of } 20 \mathrm{mg} / \mathrm{kg} \text { of TXA after the } \\
\text { induction of anaesthesia over a period of } 30 \text { minutes followed by a } 12 \text { hour continuous infusion } \\
\text { of } 2.0 \mathrm{mg} / \mathrm{kg} / \mathrm{hr} \text { of TXA. } \\
\text { - Tranexamic acid group (Fourfold dose) received a loading dose of } 40 \mathrm{mg} / \mathrm{kg} \text { of TXA after the } \\
\text { induction of anaesthesia over a period of } 30 \text { minutes followed by a } 12 \text { hour continuous infusion } \\
\text { of } 4.0 \mathrm{mg} / \mathrm{kg} / \mathrm{hr} \text { of TXA. } \\
\text { NB: All groups were exposed to cell salvage. }\end{array}$ \\
\hline
\end{tabular}

Outcomes

Outcomes reported: Number of patients exposed to allogeneic blood, allogeneic blood usage (units) , number of patients exposed to fresh frozen plasma, number of patients exposed to platelets, blood loss (12 hrs), mortality, hypotension.

Notes

Quality assessment score (Schulz criteria): 5/7

Transfusion protocol used

Risk of bias

\begin{tabular}{l|ll}
\hline Item & Authors' judgement & Description \\
\hline Adequate sequence generation? & Yes & Table of random numbers
\end{tabular}


Horrow 1995 (Continued)

\begin{tabular}{l|ll}
\hline Allocation concealment? & No & Inadequate \\
\hline $\begin{array}{l}\text { Blinding? } \\
\text { All outcomes }\end{array}$ & Yes & Double blind \\
\hline
\end{tabular}

Husted 2003

\begin{tabular}{|c|c|c|}
\hline Methods & \multicolumn{2}{|c|}{$\begin{array}{l}\text { Randomisation was performed by computer. The drugs were packed in numbered envelopes by a } \\
\text { person not connected with the surgical procedure and handled by the anaesthetist. The randomi- } \\
\text { sation code was not broken until the study was completed. }\end{array}$} \\
\hline Participants & \multicolumn{2}{|c|}{$\begin{array}{l}40 \text { patients undergoing primary total hip arthroplasty were randomised to one of two groups: } \\
\text { - Tranexamic acid group: } \mathrm{n}=20, \mathrm{M} / \mathrm{F}=7 / 13 \text {, mean age }=65 \text { years } \\
\text { - Control group (Placebo): } \mathrm{n}=20, \mathrm{M} / \mathrm{F}=6 / 14 \text {, mean age }=67 \text { years }\end{array}$} \\
\hline Interventions & \multicolumn{2}{|c|}{$\begin{array}{l}\text { - Tranexamic acid group received a bolus dose of } 10 \mathrm{mg} / \mathrm{kg} \text { of TXA (maximum } 1 \mathrm{~g} \text { ) during } 10 \\
\text { minutes about } 15 \text { minutes before the incision, followed by a continuous infusion of } 1 \mathrm{mg} / \mathrm{kg} / \mathrm{hr} \\
\text { dissolved in } 1 \text { litre of saline for } 10 \text { hours (maximum } 1 \mathrm{~g} \text { over } 10 \text { hours). } \\
\text { - Control group received saline as a bolus injection of } 20 \mathrm{ml} \text { about } 15 \text { minutes before the } \\
\text { operation followed by a continuous infusion of } 1 \text { litre of saline during } 10 \text { hours. }\end{array}$} \\
\hline Outcomes & \multicolumn{2}{|c|}{$\begin{array}{l}\text { Outcomes reported: Number of patients exposed to allogeneic blood, allogeneic blood usage (units) } \\
\text {, blood loss, deep vein thrombosis, pulmonary embolus, infection, haemoglobin levels. }\end{array}$} \\
\hline Notes & \multicolumn{2}{|c|}{$\begin{array}{l}\text { Quality assessment score (Schulz criteria): 6/7 } \\
\text { Transfusion protocol used }\end{array}$} \\
\hline \multicolumn{3}{|l|}{ Risk of bias } \\
\hline Item & Authors' judgement & Description \\
\hline Adequate sequence generation? & Yes & Randomisation was performed by computer \\
\hline Allocation concealment? & Yes & Adequate \\
\hline $\begin{array}{l}\text { Blinding? } \\
\text { All outcomes }\end{array}$ & Yes & Double blind \\
\hline
\end{tabular}

\section{Ickx 2006}

Methods

Participants
Method of randomisation and allocation concealment were not described.

51 patients undergoing primary liver transplantation were randomised to one of two groups:

- Aprotinin group (Low dose): $\mathrm{n}=24, \mathrm{M} / \mathrm{F}=20 / 4$, mean $(\mathrm{sd})$ age $=50$ (10) years

- Tranexamic acid group $(\mathrm{n}=27), \mathrm{M} / \mathrm{F}=25 / 2$, mean $(\mathrm{sd})$ age $=53(7)$ years 


- Aprotinin group (Low dose) received $280 \mathrm{mg}$ of aprotinin as a slow bolus over 30 minutes
followed by a continuous infusion of $70 \mathrm{mg} / \mathrm{hr}$. The infusion was initiated during the anhepatic
phase, 30 minutes before the expected reperfusion time, and maintained until 2 hours after
reperfusion.
- Tranexamic acid group received a slow bolus of $40 \mathrm{mg} / \mathrm{kg}$ of TXA over 30 minutes followed
by a continuous infusion at a rate of $40 \mathrm{mg} / \mathrm{kg} / \mathrm{hr}$. The infusion was initiated during the anhepatic
phase, 30 minutes before the expected reperfusion time, and maintained until 2 hours after
reperfusion.

Outcomes

Outcomes reported: Number of patients exposed to allogeneic blood, number of patients exposed to fresh frozen plasma, number of patients exposed to platelets, allogeneic blood usage (units), mortality, hospital length of stay (days), intensive care unit length of stay (days).

$\begin{array}{ll}\text { Notes } & \text { Quality assessment score (Schulz criteria): 2/7 } \\ \text { Transfusion protocol used }\end{array}$

\section{Risk of bias}

\begin{tabular}{|c|c|c|}
\hline Item & Authors' judgement & Description \\
\hline Adequate sequence generation? & Unclear & Unclear \\
\hline Allocation concealment? & Unclear & Unclear \\
\hline $\begin{array}{l}\text { Blinding? } \\
\text { All outcomes }\end{array}$ & Unclear & Unclear \\
\hline
\end{tabular}

Isetta 1993

Methods

Method of randomisation and allocation concealment were not described. Exclusions or loss to follow-up were not reported. [Abstract]

Participants

240 patients undergoing cardiac surgery were randomised to one of four groups:

- Tranexamic acid group: $\mathrm{n}=70$

- Aprotinin group (Low dose): $\mathrm{n}=70$

- Aprotinin group (High dose): $\mathrm{n}=70$

- Control group: $\mathrm{n}=70$

NB: Demographic data were not reported. 


\begin{tabular}{|c|c|c|}
\hline Interventions & \multicolumn{2}{|c|}{$\begin{array}{l}\text { - Tranexamic acid group received } 15 \mathrm{mg} / \mathrm{kg} \text { of TXA before the injection of heparin prior to } \\
\text { cardiopulmonary bypass (CPB). } \\
\text { - Aprotinin group (Low dose) received } 500,000 \mathrm{KIU} \text { of aprotinin during } 20 \text { minutes after } \\
\text { induction, followed by a continuous infusion of } 500,000 \mathrm{KIU} / \mathrm{hr} \text { until the end of CPB. } \\
\text { - Aprotinin group (High dose) received } 2 \text { million } \mathrm{KIU} \text { of aprotinin over a } 45 \text { minute period } \\
\text { after induction followed by a continuous infusion of } 500,000 \mathrm{KIU} / \mathrm{hr} \text { until the end of CPB, the } \\
\text { priming volume of the CPB circuit included } 2 \text { million KIU of aprotinin. } \\
\text { - Control group received no antifibrinolytic treatment. } \\
\text { NB: All groups were exposed to cell salvage. }\end{array}$} \\
\hline Outcomes & \multicolumn{2}{|c|}{$\begin{array}{l}\text { Outcomes reported: Number of patients exposed to allogeneic blood, blood loss, haematocrit } \\
\text { values. }\end{array}$} \\
\hline Notes & \multicolumn{2}{|c|}{$\begin{array}{l}\text { Quality assessment score (Schulz criteria): 0/7 (Abstract) } \\
\text { Transfusion protocol used }\end{array}$} \\
\hline \multicolumn{3}{|l|}{ Risk of bias } \\
\hline Item & Authors' judgement & Description \\
\hline Adequate sequence generation? & Unclear & Unclear \\
\hline Allocation concealment? & Unclear & Unclear \\
\hline $\begin{array}{l}\text { Blinding? } \\
\text { All outcomes }\end{array}$ & Unclear & Unclear \\
\hline
\end{tabular}

Jamieson 1997

\begin{tabular}{l|l}
\hline Methods & Method of randomisation and allocation concealment were not described. \\
\hline Participants & $\begin{array}{l}50 \text { patients undergoing re-operative cardiac surgery were randomised to one of two groups: } \\
\text { - Aprotinin group (High dose): } \mathrm{n}=24, \mathrm{M} / \mathrm{F}=11 / 13, \text { median (range) age }=54(34-77) \text { years } \\
\text { - Control group (Placebo): } \mathrm{n}=36, \mathrm{M} / \mathrm{F}=12 / 12, \text { median (range) age }=53(28-78) \text { years }\end{array}$ \\
\hline Interventions & $\begin{array}{l}\text { - Aprotinin group (High dose) received a loading dose of 280mg of aprotinin infused after } \\
\text { induction of anaesthesia, } 280 \mathrm{mg} \text { in the cardiopulmonary prime solution, and } 70 \mathrm{mg} / \mathrm{hr} \text { of } \\
\text { aprotinin for a period of } 6 \text { hours. } \\
\text { - Control group received a placebo of normal saline. }\end{array}$ \\
\hline NB: Both groups were exposed to cell salvage. \\
\hline Outcomes & $\begin{array}{l}\text { Outcomes reported: } \text { Number of patients exposed to allogeneic blood, allogeneic blood usage (units) } \\
\text { fresh frozen plasma usage (units), platelet usage (units), blood loss, mortality, hospital length of } \\
\text { stay (days), total blood products transfused. }\end{array}$ \\
\hline Notes & $\begin{array}{l}\text { Quality assessment score (Schulz criteria): 4/7 } \\
\text { Transfusion protocol used }\end{array}$ \\
\hline
\end{tabular}

\section{Risk of bias}


Jamieson 1997 (Continued)

\begin{tabular}{lll}
\hline Item & Authors' judgement & Description \\
\hline Adequate sequence generation? & Unclear & Unclear \\
\hline Allocation concealment? & Unclear & Unclear \\
\hline $\begin{array}{l}\text { Blinding? } \\
\text { All outcomes }\end{array}$ & Yes & Double blind \\
\hline
\end{tabular}

Jansen 1999

Methods

Randomisation was performed using a computer-generated random number list. Method of allocation concealment was not described.

Participants

42 patients undergoing unilateral bicondylar cemented total knee arthroplasty were randomised to one of two groups:

- Tranexamic acid group: $\mathrm{n}=21, \mathrm{M} / \mathrm{F}=5 / 16$, mean (range) age $=70.7(62-80)$ years

- Control group (Placebo) $(\mathrm{n}=21), \mathrm{M} / \mathrm{F}=3 / 18$, mean (range) age = $71.0(64-84)$ years

Interventions

- Tranexamic acid group received $15 \mathrm{mg} / \mathrm{kg}$ of intravenous TXA before inflation of the tourniquet and surgery and repeated every 8 hours for 3 days.

- Control group received an equivalent volume of normal saline.

Outcomes

Outcomes reported: Number of patients exposed to allogeneic blood, allogeneic blood usage (units) , blood loss, deep venous thrombosis.

$\begin{array}{ll}\text { Notes } & \text { Quality assessment score (Schulz criteria): 5/7 } \\ & \text { Transfusion protocol used }\end{array}$

\section{Risk of bias}

\begin{tabular}{l|l|l}
\hline Item & Authors' judgement & Description \\
\hline Adequate sequence generation? & Yes & $\begin{array}{l}\text { Randomisation was performed using a com- } \\
\text { puter-generated random number list }\end{array}$ \\
\hline $\begin{array}{l}\text { Allocation concealment? } \\
\begin{array}{l}\text { Blinding? } \\
\text { All outcomes }\end{array}\end{array}$ & Unclear & Unclear \\
\hline
\end{tabular}


Janssens 1994

\begin{tabular}{|c|c|c|}
\hline Methods & \multicolumn{2}{|c|}{ Method of randomisation and allocation concealment were not described. } \\
\hline Participants & \multicolumn{2}{|c|}{$\begin{array}{l}40 \text { patients undergoing primary total hip replacement were randomised to one of two groups: } \\
\text { - Aprotinin group: } \mathrm{n}=20, \mathrm{M} / \mathrm{F}=10 / 10 \text {, mean }(\mathrm{sd}) \text { age }=64.9(13.2) \text { years } \\
\text { - Control group (Placebo): } \mathrm{n}=20 \text {, mean }(\mathrm{sd}) \text { age }=65.3(15.3) \text { years }\end{array}$} \\
\hline Interventions & \multicolumn{2}{|c|}{$\begin{array}{l}\text { - Aprotinin group received a bolus injection of } 2 \text { million KIU of aprotinin over } 30 \text { minutes } \\
\text { after the induction of anaesthesia, followed by an infusion of } 500,000 \mathrm{KIU} / \mathrm{hr} \text { until the end of } \\
\text { surgery with a maximum dose of } 3.5 \text { million KIU of aprotinin. } \\
\text { - Control group received the same volume of normal saline according to the same protocol as } \\
\text { aprotinin. }\end{array}$} \\
\hline Outcomes & \multicolumn{2}{|c|}{$\begin{array}{l}\text { Outcomes reported: Blood loss, number of patients exposed to allogeneic/autologous blood, allo- } \\
\text { geneic blood usage (units), autologous blood usage (units), hospital length of stay (days), deep vein } \\
\text { thrombosis. }\end{array}$} \\
\hline Notes & \multicolumn{2}{|c|}{$\begin{array}{l}\text { Quality assessment score (Schulz criteria): 4/7 } \\
\text { Transfusion protocol used }\end{array}$} \\
\hline \multicolumn{3}{|l|}{ Risk of bias } \\
\hline Item & Authors' judgement & Description \\
\hline Adequate sequence generation? & Unclear & Unclear \\
\hline Allocation concealment? & Unclear & Unclear \\
\hline $\begin{array}{l}\text { Blinding? } \\
\text { All outcomes }\end{array}$ & Yes & Double blind \\
\hline
\end{tabular}

Jares 2003

\begin{tabular}{l|l}
\hline Methods & Method of randomisation and allocation concealment were not described. \\
\hline Participants & $\begin{array}{l}47 \text { patients undergoing 'off pump' coronary artery bypass graft surgery were randomised to one of } \\
\text { two groups: } \\
\text { - Tranexamic acid group: } \mathrm{n}=22, \mathrm{M} / \mathrm{F}=20 / 2 \\
\text { - Control group: } \mathrm{n}=25, \mathrm{M} / \mathrm{F}=15 / 10\end{array}$ \\
\hline NB: No age data were reported.
\end{tabular}

Outcomes

Outcomes reported: Number of patients exposed to allogeneic blood, blood loss, pulmonary embolus, aspirin use $<5$ days, re-operation for bleeding, stroke. 
Jares 2003 (Continued)

\begin{tabular}{|c|c|c|}
\hline Notes & $\begin{array}{l}\text { Quality assessment score (Schulz criteria): 1/7 } \\
\text { Transfusion protocol used }\end{array}$ & \\
\hline \multicolumn{3}{|l|}{ Risk of bias } \\
\hline Item & Authors' judgement & Description \\
\hline Adequate sequence generation? & Unclear & Unclear \\
\hline Allocation concealment? & Unclear & Unclear \\
\hline $\begin{array}{l}\text { Blinding? } \\
\text { All outcomes }\end{array}$ & No & No blinding \\
\hline
\end{tabular}

Jeserschek 2003

\begin{tabular}{|c|c|c|}
\hline Methods & \multicolumn{2}{|c|}{ Method of randomisation and allocation concealment were not described. } \\
\hline Participants & \multicolumn{2}{|c|}{$\begin{array}{l}18 \text { patients undergoing elective orthopaedic surgery were randomised to one of two groups: } \\
\text { - Aprotinin group (Low dose): } n=9, M / F=3 / 6 \text {, mean }(\mathrm{sd}) \text { age }=67(12.0) \text { years } \\
\text { - Control group (Placebo): } n=9, M / F=5 / 4 \text {, mean (sd) age }=72.7(7.8) \text { years }\end{array}$} \\
\hline Interventions & \multicolumn{2}{|c|}{$\begin{array}{l}\text { - Aprotinin group (Low dose) received at the begining of the operation } 1 \text { million KIU of } \\
\text { aprotinin }(140 \mathrm{mg} \text { ) as a loading dose followed by a continuous infusion of 500,000 KIU/hr. } \\
\text { - Control group received the same volume of normal saline. } \\
\text { NB: Both groups were exposed to intra-operative cell salvage. }\end{array}$} \\
\hline Outcomes & \multicolumn{2}{|c|}{$\begin{array}{l}\text { Outcomes reported: Number of patients exposed to allogeneic blood, allogeneic blood usage (units) } \\
\text {, blood loss. }\end{array}$} \\
\hline Notes & \multicolumn{2}{|c|}{$\begin{array}{l}\text { Quality assessment score (Schulz criteria): 4/7 } \\
\text { Transfusion protocol not used }\end{array}$} \\
\hline \multicolumn{3}{|l|}{ Risk of bias } \\
\hline Item & Authors' judgement & Description \\
\hline Adequate sequence generation? & Unclear & Unclear \\
\hline Allocation concealment? & Unclear & Unclear \\
\hline $\begin{array}{l}\text { Blinding? } \\
\text { All outcomes }\end{array}$ & Yes & Double blind \\
\hline
\end{tabular}


Jimenez 2007

Methods

Patients were assigned to treatment group by independent pharmacists using a list of pseudorandomised numbers to receive coded infusions of either TXA or placebo. The cose was revealed once recruitment, data collection, and laboratory analyses were completed.

\begin{tabular}{l|l}
\hline Participants & $\begin{array}{l}50 \\
\text { patients undergoing cardiac surgery were randomised to one of two groups: } \\
\end{array}$ \\
$\begin{array}{ll}\text { - Tranexamic acid group: } \mathrm{n}=24, \mathrm{M} / \mathrm{F}=12 / 12, \text { mean }(95 \% \mathrm{CI}) \text { age }=66(63-70) \text { years } \\
\text { Interventions }\end{array}$ & $\begin{array}{l}\text { - Trantrol group (Placebo): } \mathrm{n}=26, \mathrm{M} / \mathrm{F}=15 / 11, \text { mean }(95 \% \mathrm{CI}) \text { age }=67(62-71) \text { years } \\
\text { - Control group received saline. }\end{array}$ \\
\hline
\end{tabular}

Outcomes

Outcomes reported: Number of patients receiving blood transfusion, volume of blood transfused (units), blood loss, mortality, hospital length of stay, mechanical ventilation hours, inflammatory response, d-dimer levels.

$\begin{array}{ll}\text { Notes } & \text { Quality assessment score (Schulz criteria): 7/7 } \\ \text { Use of a transfusion protocol was not reported. }\end{array}$

\section{Risk of bias}

\begin{tabular}{lll}
\hline Item & Authors' judgement & Description \\
\hline Adequate sequence generation? & Yes & Randomised number list \\
\hline Allocation concealment? & Yes & Adeaquate \\
\hline $\begin{array}{l}\text { Blinding? } \\
\text { All outcomes }\end{array}$ & Yes & Double blind \\
\hline
\end{tabular}

Johansson 2005

Methods

Patients were randomised by computer in blocks of 10 . Coded ampoules were prepared by the pharmaceutical company. All personnel and patients were blinded as to the treatment until the randomisation code was broken which took place after all patients had been evaluated.

\begin{tabular}{l} 
Participants \\
$\begin{array}{l}119 \text { patients undergoing total hip arthroplasty were randomised to one of two groups: } \\
\text { - Tranexamic acid group: } \mathrm{n}=47, \mathrm{M} / \mathrm{F}=25 / 22, \text { mean }(\mathrm{sd}) \text { age }=69 \text { (7) years } \\
\text { - Control group (Placebo): } \mathrm{n}=53, \mathrm{M} / \mathrm{F}=28 / 25, \text { mean }(\mathrm{sd}) \text { age }=68(8) \text { years } \\
\text { NB: Before the randomisation code was broken } 19 \text { patients were excluded due to violation of the } \\
\text { study protocol. }\end{array}$ \\
\hline $\begin{array}{l}\text { - Tranexamic acid group received a bolus infusion of } 15 \mathrm{mg} / \mathrm{kg} \text { of TXA mixed in } 100 \mathrm{ml} \text { of } \\
\text { normal saline immediately before the start of the operation. } \\
\text { - Control group received normal saline. }\end{array}$
\end{tabular}

Outcomes

Outcomes reported: Number of patients exposed to allogeneic blood, allogeneic blood usage (units) , blood loss, deep vein thrombosis. 


\begin{tabular}{l|ll}
\hline Notes & $\begin{array}{l}\text { Quality assessment score (Schulz criteria): 5/7 } \\
\text { Transfusion protocol used }\end{array}$ \\
\hline Risk of bias & Authors' judgement & Description \\
\hline Item & Yes & Computer randomisation \\
\hline Adequate sequence generation? & Adequate \\
\hline Allocation concealment? & Yes & Double blind \\
\hline $\begin{array}{l}\text { Blinding? } \\
\text { All outcomes }\end{array}$ & Yes & \\
\hline
\end{tabular}

\section{Kahveci 1996}

\begin{tabular}{|c|c|c|}
\hline Methods & \multicolumn{2}{|c|}{ Method of randomisation and allocation concealment were not described. [Turkish language] } \\
\hline Participants & \multicolumn{2}{|c|}{$\begin{array}{l}28 \text { patients undergoing coronary artery bypass surgery or cardiac valvular surgery were randomised } \\
\text { to one of two groups: } \\
\text { - Aprotinin group (Low dose): } \mathrm{n}=14, \mathrm{M} / \mathrm{F}=6 / 8 \text {, mean }(\mathrm{sd}) \text { age }=45.5(12.8) \text { years } \\
\text { - Control group: } \mathrm{n}=14, \mathrm{M} / \mathrm{F}=8 / 6 \text {, mean }(\mathrm{sd}) \text { age }=48(10.5) \text { years }\end{array}$} \\
\hline Interventions & \multicolumn{2}{|c|}{$\begin{array}{l}\text { - Aprotinin group (Low dose) received a bolus of } 2 \text { million KIU of aprotinin }(280 \mathrm{mg} \text { ) before } \\
\text { the induction of anaesthesia followed by a continuous infusion of 500,000 KIU/hr. } \\
\text { - Control group did not receive aprotinin. }\end{array}$} \\
\hline Outcomes & \multicolumn{2}{|c|}{ Outcomes reported: Allogeneic blood usage (units), fresh frozen plasma usage (units), blood loss. } \\
\hline Notes & \multicolumn{2}{|c|}{ Transfusion protocol not used } \\
\hline \multicolumn{3}{|l|}{ Risk of bias } \\
\hline Item & Authors' judgement & Description \\
\hline Adequate sequence generation? & Unclear & Unclear \\
\hline Allocation concealment? & Unclear & Unclear \\
\hline $\begin{array}{l}\text { Blinding? } \\
\text { All outcomes }\end{array}$ & Unclear & Unclear \\
\hline
\end{tabular}


Kalangos 1994

\begin{tabular}{|c|c|c|}
\hline Methods & \multicolumn{2}{|c|}{$\begin{array}{l}\text { Method of randomisation and allocation concealment were not described. No exclusions were } \\
\text { reported. }\end{array}$} \\
\hline Participants & \multicolumn{2}{|c|}{$\begin{array}{l}165 \text { adult patients undergoing elective primary aortocoronary bypass operations were randomised } \\
\text { to one of three groups: } \\
\text { - Aprotinin group (High dose): } \mathrm{n}=55, \mathrm{M} / \mathrm{F}=47 / 8 \text {, mean }(\mathrm{sd}) \text { age }=58.2(5.6) \text { years } \\
\text { - Aprotinin group (Low dose): } \mathrm{n}=55, \mathrm{M} / \mathrm{F}=44 / 11 \text {, mean }(\mathrm{sd}) \text { age }=57.7(6.6) \text { years } \\
\text { - Control (Placebo): } \mathrm{n}=55, \mathrm{M} / \mathrm{F}=49 / 6, \text { mean }(\mathrm{sd}) \text { age }=60.5(6.8) \text { years }\end{array}$} \\
\hline Interventions & \multicolumn{2}{|c|}{$\begin{array}{l}\text { - Aprotinin group (High dose) received an intravenous bolus of } 2 \text { million KIU of aprotinin at } \\
\text { induction of anaesthesia. Another } 2 \text { million KIU of aprotinin was added to the pump prime } \\
\text { volume. A continuous infusion of } 500,000 \mathrm{KIU} / \mathrm{hr} \text { of aprotinin was maintained until the end of } \\
\text { the operation. } \\
\text { - Aprotinin group (Low dose) received } 25,000 \mathrm{KIU} / \mathrm{kg} \text { added to the pump prime solution } \\
\text { (mean dosage } 1.78 \text { million } \mathrm{KIU} \text {; range } 1.375 \text { million KIU to } 2.3 \text { million KIU) and saline was } \\
\text { administered at all other corresponding times. } \\
\text { - Control group received identical volumes of saline at all corresponding times. }\end{array}$} \\
\hline Outcomes & \multicolumn{2}{|c|}{$\begin{array}{l}\text { Outcomes reported: Number of patients exposed to allogeneic blood, allogeneic blood usage (units) } \\
\text {, blood loss, myocardial infarction, creatine phosphokinase - myocardial band (CK-MB) levels. }\end{array}$} \\
\hline Notes & \multicolumn{2}{|c|}{$\begin{array}{l}\text { Quality assessment score (Schulz criteria): 4/7 } \\
\text { Transfusion protocol used }\end{array}$} \\
\hline \multicolumn{3}{|l|}{ Risk of bias } \\
\hline Item & Authors' judgement & Description \\
\hline Adequate sequence generation? & Unclear & Unclear \\
\hline Allocation concealment? & Unclear & Unclear \\
\hline $\begin{array}{l}\text { Blinding? } \\
\text { All outcomes }\end{array}$ & Yes & Double blind \\
\hline
\end{tabular}

\section{Karski 1995}

Methods

Randomisation was performed by the pharmacy department. The method used to generate allocation sequences was not described.

Participants

150 patients undergoing cardiac operations were randomised to one of three groups:

- Tranexamic acid group (TA-10): $\mathrm{n}=50$, mean (sd) age $=59(21.2)$ years

- Tranexamic acid group (TA-20): $\mathrm{n}=50$, mean $(\mathrm{sd})$ age $=63(7.0)$ years

- Control group (Placebo): $\mathrm{n}=50$, mean (sd) age $=58$ (14.1) years

NB: Gender data were not reported. 
Karski 1995 (Continued)

\begin{tabular}{|c|c|c|}
\hline Interventions & \multicolumn{2}{|c|}{$\begin{array}{l}\text { - Tranexamic acid group (TA-10) received an infusion of } 10 \mathrm{~g} \text { of TXA intravenously over } 20 \\
\text { minutes after induction of anaesthesia and a placebo infusion ( } 0.9 \% \text { normal saline) over the } \\
\text { subsequent } 5 \text { hours. } \\
\text { - Tranexamic acid group (TA-20) received } 10 \mathrm{~g} \text { of TXA over } 20 \text { minutes and then a further } \\
10 \mathrm{~g} \text { infused over } 5 \text { hours. } \\
\text { - Control group received a placebo bolus ( } 0.9 \% \text { normal saline) and a placebo infusion }(0.9 \% \\
\text { normal saline) over } 5 \text { hours. } \\
\text { NB: All groups were exposed to cell salvage (autotransfusion). Patients with defined 'excessive bleed- } \\
\text { ing' were treated with } 10-40 \mathrm{~g} \text { of intravenous epsilon aminocaproic acid (EACA) or desmopressin } \\
\text { (DDAVP). }\end{array}$} \\
\hline Outcomes & \multicolumn{2}{|c|}{$\begin{array}{l}\text { Outcomes reported: Number of patients exposed to allogeneic blood, allogeneic blood usage (units) } \\
\text {, blood loss. }\end{array}$} \\
\hline Notes & \multicolumn{2}{|c|}{$\begin{array}{l}\text { Quality assessment score (Schulz criteria): } 4 / 7 \\
\text { Transfusion protocol used }\end{array}$} \\
\hline \multicolumn{3}{|l|}{ Risk of bias } \\
\hline Item & Authors' judgement & Description \\
\hline Adequate sequence generation? & Unclear & Unclear \\
\hline Allocation concealment? & Yes & Adequate \\
\hline $\begin{array}{l}\text { Blinding? } \\
\text { All outcomes }\end{array}$ & No & Single blind \\
\hline
\end{tabular}

Karski 2005

\begin{tabular}{l|l}
\hline Methods & $\begin{array}{l}\text { A computer-generated randomisation code in blocks of four was used to assign patients to treatment } \\
\text { or control in a double-blinded fashion. The hospital pharmacy prepared identical bags of solution. }\end{array}$ \\
\hline Participants & $\begin{array}{r}312 \text { patients undergoing cardiac surgery were randomised to one of two groups: } \\
\text { - Tranexamic acid group: } \mathrm{n}=147, \mathrm{M} / \mathrm{F}=128 / 19, \mathrm{mean}(\mathrm{sd}) \text { age }=59.9(8.9) \text { years } \\
\text { - Control group (Placebo): } \mathrm{n}=165, \mathrm{M} / \mathrm{F}=147 / 18, \text { mean }(\mathrm{sd}) \text { age }=60(8.3) \text { years }\end{array}$ \\
\hline Interventions & $\begin{array}{l}\text { - Tranexamic acid group received } 100 \mathrm{mg} / \mathrm{kg} \text { of TXA in } 100 \mathrm{ml} \text { solution over } 20 \text { minutes after } \\
\text { the induction of anaesthesia. } \\
\text { - Control group received } 5 \% \text { dextrose. }\end{array}$ \\
\hline Outcomes & $\begin{array}{l}\text { Outcomes reported: } \text { Number of patinets exposed to allogeneic blood, number of patients exposed } \\
\text { to fresh frozen plasma, number of patients exposed to platelets, mortality, myocardial infarction, } \\
\text { stroke, cardiac arrest, atrial fibrillation. }\end{array}$ \\
\hline Notes & $\begin{array}{l}\text { Quality assessment score (Schulz criteria): } 7 / 7 \\
\text { Transfusion protocol not specified } \\
\text { NB: Open-labeled tranexamic acid was administered to } 4 \text { patients in the TXA group and } 24 \text { patients }\end{array}$ \\
\hline
\end{tabular}


Karski 2005 (Continued)

in the control group.

\section{Risk of bias}

\begin{tabular}{lll}
\hline Item & Authors' judgement & Description \\
\hline Adequate sequence generation? & Yes & Computer-generated randomisation code \\
\hline Allocation concealment? & Yes & Adequate \\
\hline $\begin{array}{l}\text { Blinding? } \\
\text { All outcomes }\end{array}$ & Yes & Double blind \\
\hline
\end{tabular}

Kaspar 1997

\begin{tabular}{|c|c|c|}
\hline Methods & \multicolumn{2}{|c|}{$\begin{array}{l}\text { Study infusions were prepared by the hospital pharmacy using a computer generated randomisation } \\
\text { schedule. }\end{array}$} \\
\hline Participants & \multicolumn{2}{|c|}{$\begin{array}{l}32 \text { consecutive patients undergoing orthotopic liver transplantation were randomly allocated to } \\
\text { one of two groups: } \\
\text { - Tranexamic acid group: } \mathrm{n}=16 \\
\text { - Control group (Placebo): } \mathrm{n}=16 \\
\text { NB: Demographic data were not reported. }\end{array}$} \\
\hline Interventions & \multicolumn{2}{|c|}{$\begin{array}{l}\text { - Tranexamic acid group received a continuous small dose infusion of TXA ( } 1 \mathrm{~g} \text { in } 500 \mathrm{ml} \text { of } \\
\text { normal saline) at a dose of } 2 \mathrm{mg} / \mathrm{kg} / \mathrm{hr} \text {. } \\
\text { - Control group received an equal volume of normal saline. } \\
\text { NB: Both groups were exposed to cell salvage. }\end{array}$} \\
\hline Outcomes & \multicolumn{2}{|c|}{$\begin{array}{l}\text { Outcomes reported: Allogeneic blood usage (units), fresh frozen plasma (units), platelets (units), } \\
\text { mortality, hepatic arterial thrombosis, epsilon aminocaproic acid 'rescue', cryoprecipitate. }\end{array}$} \\
\hline Notes & \multicolumn{2}{|c|}{$\begin{array}{l}\text { Quality assessment score (Schulz criteria): } 7 / 7 \\
\text { Transfusion protocol not specified }\end{array}$} \\
\hline \multicolumn{3}{|l|}{ Risk of bias } \\
\hline Item & Authors' judgement & Description \\
\hline Adequate sequence generation? & Yes & Computer generated randomisation schedule \\
\hline Allocation concealment? & Yes & Adequate \\
\hline $\begin{array}{l}\text { Blinding? } \\
\text { All outcomes }\end{array}$ & Yes & Double blind \\
\hline
\end{tabular}


Katoh 1997

\begin{tabular}{|c|c|c|}
\hline Methods & \multicolumn{2}{|c|}{ Method of randomisation and allocation concealment were not described. } \\
\hline Participants & \multicolumn{2}{|c|}{$\begin{array}{l}93 \text { patients undergoing either coronary artery bypass grafting or heart valve operations were ran- } \\
\text { domly divided into one of three groups: } \\
\text { - Tranexamic acid group }(\mathrm{TA}-1): \mathrm{n}=31, \mathrm{M} / \mathrm{F}=22 / 9 \text {, mean }(\mathrm{sd}) \text { age }=63.7(8.3) \text { years } \\
\text { - Tranexamic acid group }(\mathrm{TA}-2): \mathrm{n}=31, \mathrm{M} / \mathrm{F}=21 / 10 \text {, mean }(\mathrm{sd}) \text { age }=62.9(9.5) \text { years } \\
\text { - Control group: } \mathrm{n}=31, \mathrm{M} / \mathrm{F}=22 / 9 \text {, mean }(\mathrm{sd}) \text { age }=64.7(11.7) \text { years }\end{array}$} \\
\hline Interventions & \multicolumn{2}{|c|}{$\begin{array}{l}\text { - Tranexamic acid group (TA-1) received an infusion of tranexamic acid (TXA) of } 100 \mathrm{mg} / \mathrm{kg} \\
\text { intravenously (IV) over } 20 \text { minutes soon after induction of anaesthesia and before } \\
\text { cardiopulmonary bypass (CPB). } \\
\text { - Tranexamic acid group (TA-2) received a } 100 \mathrm{mg} / \mathrm{kg} \text { dose of TXA intravenously (IV) over } 20 \\
\text { minutes soon after induction of anaesthesia and before CPB, and an additional dose of } 50 \mathrm{mg} / \mathrm{kg} \\
\text { infused IV over } 20 \text { minutes soon after being weaned from CPB. } \\
\text { - Control group did not receive tranexamic acid. }\end{array}$} \\
\hline Outcomes & \multicolumn{2}{|c|}{$\begin{array}{l}\text { Outcomes reported: Number of patients exposed to allogeneic blood, allogeneic blood usage (units) } \\
\text {, blood loss, mortality, myocardial infarction, stroke, pulmonary embolism, deep vein thrombosis. }\end{array}$} \\
\hline Notes & \multicolumn{2}{|c|}{$\begin{array}{l}\text { Quality assessment score (Schulz criteria): 2/7 } \\
\text { Transfusion protocol used }\end{array}$} \\
\hline \multicolumn{3}{|l|}{ Risk of bias } \\
\hline Item & Authors' judgement & Description \\
\hline Adequate sequence generation? & Unclear & Unclear \\
\hline Allocation concealment? & Unclear & Unclear \\
\hline $\begin{array}{l}\text { Blinding? } \\
\text { All outcomes }\end{array}$ & Unclear & Unclear \\
\hline
\end{tabular}

\section{Katsaros 1996}

Methods

Participants

Interventions
Method of randomisation was not described. Allocation concealment was by coded infusions. One patient was eliminated from the study due to improper data collection.

211 patients scheduled for open heart operations were randomised to one of two groups:

- Tranexamic acid group: $\mathrm{n}=104, \mathrm{M} / \mathrm{F}=68 / 36$, mean (sd) age $=65$ (9.3) years

- Control group (Placebo): $\mathrm{n}=106, \mathrm{M} / \mathrm{F}=80 / 26$, mean (sd) age $=63$ (12.3) years

- Tranexamic acid group received $10 \mathrm{~g}$ of TXA (diluted to $250 \mathrm{ml}$ with normal saline solution) intravenously over 20 minutes. No incision was made until the completion of the infusions.

- Control group received $250 \mathrm{ml}$ of normal saline solution.

NB: Both groups were exposed to cell salvage. 
Katsaros 1996 (Continued)

\begin{tabular}{|c|c|c|}
\hline Outcomes & \multicolumn{2}{|c|}{$\begin{array}{l}\text { Outcomes reported: Number of patients exposed to allogeneic blood, bood loss ( } 24 \mathrm{hrs}) \text {, mortality, } \\
\text { myocardial infarctions, deep vein thrombosis, pulmonary embolus, } \\
\text { re-operation for bleeding, cerebrovascular accident, renal failure, central nervous system complica- } \\
\text { tions. }\end{array}$} \\
\hline Notes & \multicolumn{2}{|c|}{$\begin{array}{l}\text { Quality assessment score (Schulz criteria): 5/7 } \\
\text { Transfusion protocol used }\end{array}$} \\
\hline \multicolumn{3}{|l|}{ Risk of bias } \\
\hline Item & Authors' judgement & Description \\
\hline Adequate sequence generation? & Unclear & Unclear \\
\hline Allocation concealment? & Yes & Adequate \\
\hline $\begin{array}{l}\text { Blinding? } \\
\text { All outcomes }\end{array}$ & Yes & Double blind \\
\hline
\end{tabular}

\section{Katzel 1998}

\begin{tabular}{|c|c|c|}
\hline Methods & \multicolumn{2}{|c|}{ Method of randomisation and allocation concealment were not described. [German language] } \\
\hline Participants & \multicolumn{2}{|c|}{$\begin{array}{l}24 \text { male patients undergoing thoracic surgery for malignant lung disease were randomised to one } \\
\text { of two groups: } \\
\text { - Aprotinin group (Low dose): } \mathrm{n}=12 \text {, mean }(\mathrm{sd}) \text { age }=57.1(8.2) \text { years } \\
\text { - Control group (Placebo): } \mathrm{n}=12 \text {, mean }(\mathrm{sd}) \text { age }=59.4(9.0) \text { years }\end{array}$} \\
\hline Interventions & \multicolumn{2}{|c|}{$\begin{array}{l}\text { - Aprotinin group (Low dose) received a bolus of } 280 \mathrm{mg} \text { of aprotinin ( } 2 \text { million KIU) } \\
\text { followed by 500,000 KIU of aprotinin during surgery until } 1 \text { hour after surgery. } \\
\text { - Control group was infused with isotonic saline. }\end{array}$} \\
\hline Outcomes & \multicolumn{2}{|c|}{$\begin{array}{l}\text { Outcomes reported: Number of patients exposed to allogeneic blood, blood loss, transient ischaemic } \\
\text { attack. }\end{array}$} \\
\hline Notes & \multicolumn{2}{|c|}{$\begin{array}{l}\text { Quality assessment score (Schulz criteria): 4/7 } \\
\text { Transfusion protocol used }\end{array}$} \\
\hline \multicolumn{3}{|l|}{ Risk of bias } \\
\hline Item & Authors' judgement & Description \\
\hline Adequate sequence generation? & Unclear & Unclear \\
\hline Allocation concealment? & Unclear & Unclear \\
\hline
\end{tabular}


Katzel 1998 (Continued)

\begin{tabular}{|c|c|c|}
\hline $\begin{array}{l}\text { Blinding? } \\
\text { All outcomes }\end{array}$ & Yes & Double blind \\
\hline
\end{tabular}

Kazemi 2010

\begin{tabular}{|c|c|c|}
\hline Methods & \multicolumn{2}{|c|}{ Methods of sequence generation and allocation concealment were not described. } \\
\hline Participants & \multicolumn{2}{|c|}{$\begin{array}{l}64 \text { patients undergoing orthopaedic surgery were randomised to one of two groups: } \\
\text { - Tranexamic acid group: } \mathrm{n}=32, \mathrm{M} / \mathrm{F}=23 / 9 \text {, mean }(\mathrm{sd}) \text { age }=46.6(16.2) \text { years } \\
\text { - Control group: } \mathrm{n}=32, \mathrm{M} / \mathrm{F}=20 / 12 \text {, mean }(\mathrm{sd}) \text { age }=45.4(17.2) \text { years }\end{array}$} \\
\hline Interventions & \multicolumn{2}{|c|}{$\begin{array}{l}\text { - Tranexamic acid group received } 15 \mathrm{mg} / \mathrm{kg} \text { given five minutes pre-operatively. } \\
\text { - Control group received saline. }\end{array}$} \\
\hline Outcomes & \multicolumn{2}{|c|}{$\begin{array}{l}\text { Outcomes reported: Volume of blood transfused (units), blood loss, deep vein thrombosis, length } \\
\text { of stay (days). }\end{array}$} \\
\hline Notes & \multicolumn{2}{|c|}{ Transfusion protocol was used. } \\
\hline \multicolumn{3}{|l|}{ Risk of bias } \\
\hline Item & Authors' judgement & Description \\
\hline Adequate sequence generation? & Unclear & Unclear \\
\hline Allocation concealment? & Unclear & Unclear \\
\hline $\begin{array}{l}\text { Blinding? } \\
\text { All outcomes }\end{array}$ & Yes & Double blind \\
\hline
\end{tabular}

Kikura 2006

\begin{tabular}{l|l}
\hline Methods & Method of randomisation and allocation concealment were not described. \\
\hline Participants & $\begin{array}{l}100 \text { patients undergoing cardiac surgery were randomised to one of two groups: } \\
\text { - Epsilon aminocaproic acid: } \mathrm{n}=50, \mathrm{M} / \mathrm{F}=38 / 12, \text { mean }(\mathrm{sd}) \text { age }=63(10) \text { years } \\
\text { - Control group (Placebo): } \mathrm{n}=50, \mathrm{M} / \mathrm{F}=40 / 10, \text { mean }(\mathrm{sd}) \text { age }=62(11) \text { years }\end{array}$ \\
\hline Interventions & $\begin{array}{l}\text { - Epsilon aminocaproic acid group received } 100 \mathrm{mg} / \mathrm{kg} \text { of EACA as a loading dose over } 20-30 \\
\text { minutes, after endotracheal intubation, followed by a continuous infusion of } 1 \mathrm{~g} / \mathrm{hr} \text { of EACA } \\
\text { during the operation, and a loading dose of } 10 \mathrm{~g} \text { given into the } \mathrm{CPB} \text { circuit prime solution. The } \\
\text { infusion was discontinued on completion of surgery. } \\
\text { - Control group (placebo) received identical appearing normal saline in identical volumes at } \\
\text { the same times as EACA treatment. } \\
\text { NB: Both groups were exposed to intra-operative cell salvage. }\end{array}$
\end{tabular}


Kikura 2006 (Continued)

\begin{tabular}{|c|c|c|}
\hline Outcomes & \multicolumn{2}{|c|}{$\begin{array}{l}\text { Outcomes reported: Number of patients exposed to allogeneic blood, allogeneic blood usage (units) } \\
\text {, number of patients exposed to fresh frozen plasma, number of patients exposed to platelets, blooc } \\
\text { loss (24hrs). }\end{array}$} \\
\hline Notes & \multicolumn{2}{|c|}{$\begin{array}{l}\text { Quality assessment score (Schulz criteria): 4/7 } \\
\text { Transfusion protocol used }\end{array}$} \\
\hline \multicolumn{3}{|l|}{ Risk of bias } \\
\hline Item & Authors' judgement & Description \\
\hline Adequate sequence generation? & Unclear & Unclear \\
\hline Allocation concealment? & Unclear & Unclear \\
\hline $\begin{array}{l}\text { Blinding? } \\
\text { All outcomes }\end{array}$ & Yes & Double blind \\
\hline
\end{tabular}

Kipfer 2003

\begin{tabular}{|c|c|c|}
\hline Methods & \multicolumn{2}{|c|}{ Method of randomisation and allocation concealment were not described. } \\
\hline Participants & \multicolumn{2}{|c|}{$\begin{array}{l}30 \text { adult patients undergoing elective cardiac surgery were randomised into one of two groups: } \\
\text { - Aprotinin group (Low dose): } \mathrm{n}=15, \mathrm{M} / \mathrm{F}=12 / 3 \text {, mean }(\mathrm{sd}) \text { age }=62.3(7) \text { years } \\
\text { - Control group: } \mathrm{n}=15, \mathrm{M} / \mathrm{F}=12 / 3 \text {, mean }(\mathrm{sd}) \text { age }=61.3(7) \text { years }\end{array}$} \\
\hline Interventions & \multicolumn{2}{|c|}{$\begin{array}{l}\text { - Aprotinin group (Low dose - pump prime) received } 2 \text { million KIU }(280 \mathrm{mg}) \text { of aprotinin } \\
\text { added to the prime volume of the CPB. } \\
\text { - Control group did not receive aprotonin. }\end{array}$} \\
\hline Outcomes & \multicolumn{2}{|c|}{$\begin{array}{l}\text { Outcomes reported: Number of patients exposed to allogeneic blood, allogeneic blood usage (units), } \\
\text { blood loss, fresh frozen plasma, myocardial infarction, mortality, myocardial infarction, retransfused } \\
\text { mediastinal shed blood, re-operation for bleeding, renal dysfunction, neurological deficit, hospital } \\
\text { length of stay (days). }\end{array}$} \\
\hline Notes & \multicolumn{2}{|c|}{$\begin{array}{l}\text { Quality assessment score (Schulz criteria): 2/7 } \\
\text { Transfusion protocol used }\end{array}$} \\
\hline \multicolumn{3}{|l|}{ Risk of bias } \\
\hline Item & Authors' judgement & Description \\
\hline Adequate sequence generation? & Unclear & Unclear \\
\hline Allocation concealment? & Unclear & Unclear \\
\hline
\end{tabular}


Kipfer 2003 (Continued)

\begin{tabular}{l|l|l}
\hline $\begin{array}{l}\text { Blinding? } \\
\text { All outcomes }\end{array}$ & Unclear & Unclear \\
\hline
\end{tabular}

\section{Klein 1998}

\begin{tabular}{|c|c|c|}
\hline Methods & \multicolumn{2}{|c|}{ Method of randomisation and allocation concealment were not described. } \\
\hline Participants & \multicolumn{2}{|c|}{$\begin{array}{l}109 \text { patients undergoing elective cardiac surgery were randomised to one of three groups: } \\
\text { - Aprotinin group (High dose }+\mathrm{ASA}): \mathrm{n}=40), \mathrm{M} / \mathrm{F}=33 / 7 \text {, mean }(\mathrm{sd}) \text { age }=64.0(6.3) \text { years } \\
\text { - Aprotinin group (High dose) } \mathrm{n}=38, \mathrm{M} / \mathrm{F}=34 / 4 \text {, mean }(\mathrm{sd}) \text { age }=62.1(7.3) \text { years } \\
\text { - Control group (Placebo): } \mathrm{n}=31, \mathrm{M} / \mathrm{F}=28 / 3 \text {, mean }(\mathrm{sd}) \text { age }=63.0(9.3) \text { years }\end{array}$} \\
\hline Interventions & \multicolumn{2}{|c|}{$\begin{array}{l}\text { - Aprotinin group (High dose }+ \text { ASA) received a loading dose of } 2 \text { million KIU ( } 280 \mathrm{mg} \text { ) } \\
\text { followed by a continuous infusion of } 500,000 \mathrm{KIU} / \mathrm{hr} \text { until chest closure for a } 6 \text { hour maximum } \\
\text { period. In addition, } 2 \text { million KIU of aprotinin was added to the pump prime. Patients } \\
\text { underwent a minimum } 10 \text {-day run-in period on ASA (100mg/day) until surgery. } \\
\text { - Aprotinin group (High dose) received a loading dose of } 2 \text { million KIU ( } 280 \mathrm{mg} \text { ) followed by } \\
\text { a continuous infusion of } 500,000 \mathrm{KIU} / \mathrm{hr} \text { until chest closure for a } 6 \text { hour maximum period. In } \\
\text { addition, } 2 \text { million KIU of aprotinin was added to the pump prime. } \\
\text { - Control group received an unspecified placebo. }\end{array}$} \\
\hline Outcomes & \multicolumn{2}{|c|}{$\begin{array}{l}\text { Outcomes reported: Allogeneic blood usage (units), blood loss, fresh frozen plasma, myocardial } \\
\text { infarction. }\end{array}$} \\
\hline Notes & \multicolumn{2}{|c|}{$\begin{array}{l}\text { Quality assessment score (Schulz criteria): } 4 / 7 \\
\text { Transfusion protocol used }\end{array}$} \\
\hline \multicolumn{3}{|l|}{ Risk of bias } \\
\hline Item & Authors' judgement & Description \\
\hline Adequate sequence generation? & Unclear & Unclear \\
\hline Allocation concealment? & Unclear & Unclear \\
\hline $\begin{array}{l}\text { Blinding? } \\
\text { All outcomes }\end{array}$ & Yes & Double blind \\
\hline
\end{tabular}

\section{Kluger 2003}

Methods

Patients underwent permuted block randomisation using random number tables. All patients received four syringes, labelled A,B,C,and D. Patients, clinicians, and investigators were all blinded to group allocation.

Participants

90 patients undergoing cardiac surgery were randomised to one of three groups:

- Epsilon aminocaproic acid group (Post-heparin): $\mathrm{n}=30, \mathrm{M} / \mathrm{F}=24 / 6$, mean (sd) age $=65$

(8.1) years 


\begin{tabular}{|c|c|}
\hline & $\begin{array}{l}\text { - Epsilon aminocaproic acid group (Pre-incision): } \mathrm{n}=28, \mathrm{M} / \mathrm{F}=23 / 5 \text {, mean }(\mathrm{sd}) \text { age }=66 \\
\text { (8.1) years } \\
\text { - Control group (Placebo): } \mathrm{n}=30, \mathrm{M} / \mathrm{F}=22 / 8 \text {, mean }(\mathrm{sd}) \text { age }=67(6.5) \text { years } \\
\text { NB: Two patients were excluded from the final analysis. }\end{array}$ \\
\hline Interventions & $\begin{array}{l}\text { - Epsilon aminocaproic acid group (Post-heparin) received an initial bolus of normal saline } \\
\text { prior to skin incision, followed by a normal saline infusion. Three minutes after heparin } \\
\text { administration patients received a bolus of } 150 \mathrm{mg} / \mathrm{kg} \text { of EACA over } 10 \text { minutes and then an } \\
\text { infusion of } 15 \mathrm{mg} / \mathrm{kg} / \mathrm{hr} \text {. } \\
\text { - Epsilon aminocaproic acid group (Pre-incision) received a bolus of } 150 \mathrm{mg} / \mathrm{kg} \text { of EACA over } \\
10 \mathrm{minutes} \text { after the induction of anaesthesia but before skin incision, followed by an infusion of } \\
15 \mathrm{mg} / \mathrm{kg} / \mathrm{hr} \text {. Three minutes after heparin administration, to maintain blinding, this group } \\
\text { received a bolus of normal saline over } 10 \text { minutes, followed by a resumption of the EACA } \\
\text { infusion until the termination of CPB. } \\
\text { - Control group received normal saline boluses and infusions throughout. } \\
\text { NB: All groups were exposed to acute normovolaemic haemodilution (ANH). }\end{array}$ \\
\hline
\end{tabular}

Outcomes

Outcomes reported: Number of patients exposed to allogeneic blood, number of patients exposed to fresh frozen plasma and platelets, mortality, myocardial infarction,

re-operation for bleeding, stroke.

$\begin{array}{ll}\text { Notes } & \begin{array}{l}\text { Quality assessment score (Schulz criteria): 6/7 } \\ \text { Transfusion protocol used }\end{array}\end{array}$

\section{Risk of bias}

\begin{tabular}{|c|c|c|}
\hline Item & Authors' judgement & Description \\
\hline Adequate sequence generation? & Yes & Random number table \\
\hline Allocation concealment? & Yes & Adequate \\
\hline $\begin{array}{l}\text { Blinding? } \\
\text { All outcomes }\end{array}$ & Yes & Double blind \\
\hline
\end{tabular}

\section{Koster 2004}

\begin{tabular}{l|l}
\hline Methods & Allocation of patients was blinded to the surgeon. Method of randomisation was not described. \\
\hline Participants & $\begin{array}{r}200 \text { patients undergoing elective cardiac surgery were randomised to one of two groups: } \\
\text { - Aprotinin group (Low dose }+ \text { heparin): } \mathrm{n}=100, \mathrm{M} / \mathrm{F}=56 / 44, \text { mean }(\mathrm{sd}) \text { age }=64(15) \text { years } \\
\text { - Control group (Heparin alone): } \mathrm{n}=100, \mathrm{M} / \mathrm{F}=57 / 43, \text { mean }(\mathrm{sd}) \text { age }=66(17) \text { years }\end{array}$ \\
\hline Interventions & $\begin{array}{l}\text { - Aprotinin group (Low dose }+ \text { heparin) received a bolus of } 1 \text { million KIU of aprotinin } \\
\text { immediately before initiation of CPB and a continuous infusion of } 250,000 \mathrm{KIU} / \mathrm{hr} \text { during the } \\
\text { period of CPB. In addition, } 1 \text { million KIU of aprotinin was added to the CPB pump prime. } \\
\text { - Control group received standard care without aprotinin treatment. } \\
\text { NB: Both groups were exposed to cell salvage. }\end{array}$
\end{tabular}


Koster 2004 (Continued)

\begin{tabular}{|c|c|c|}
\hline Outcomes & \multicolumn{2}{|c|}{$\begin{array}{l}\text { Outcomes reported: Allogeneic blood usage (units), fresh frozen plasma, blood loss, duration of } \\
\text { ventilation (hours). }\end{array}$} \\
\hline Notes & \multicolumn{2}{|l|}{$\begin{array}{l}\text { Quality assessment score (Schulz criteria): 0/7 } \\
\text { Transfusion protocol used }\end{array}$} \\
\hline \multicolumn{3}{|l|}{ Risk of bias } \\
\hline Item & Authors' judgement & Description \\
\hline Adequate sequence generation? & Unclear & Unclear \\
\hline Allocation concealment? & Unclear & Unclear \\
\hline $\begin{array}{l}\text { Blinding? } \\
\text { All outcomes }\end{array}$ & No & Single blind \\
\hline
\end{tabular}

\section{Kratzer 1997}

\begin{tabular}{|c|c|c|}
\hline Methods & \multicolumn{2}{|c|}{$\begin{array}{l}\text { Method of randomisation was by means of a random number generator. Method used to conceal } \\
\text { treatment allocation was not described. [German language] }\end{array}$} \\
\hline Participants & \multicolumn{2}{|c|}{$\begin{array}{l}18 \text { patients undergoing orthotopic liver transplantation were randomised to one of two groups: } \\
\text { - Aprotinin group: } \mathrm{n}=9 \text {, mean age } 47.9 \text { years } \\
\text { - Control group (Placebo): } \mathrm{n}=9 \text {, mean age } 49.4 \text { years } \\
\text { NB: Gender data were not reported. }\end{array}$} \\
\hline Interventions & \multicolumn{2}{|c|}{$\begin{array}{l}\text { - Aprotinin group received an intravenous bolus of } 2 \text { million KIU }(280 \mathrm{mg}) \text { of aprotinin at } \\
\text { induction of anaesthesia and a continuous infusion of } 500,000 \mathrm{KIU} / \mathrm{hr} \text { of aprotinin until the end } \\
\text { of the operation. } \\
\text { - Control group received physiological saline solution. }\end{array}$} \\
\hline Outcomes & \multicolumn{2}{|c|}{ Outcomes reported: Allogeneic blood usage (units), coagulation parameters, blood loss. } \\
\hline Notes & \multicolumn{2}{|c|}{ Transfusion protocol used } \\
\hline \multicolumn{3}{|l|}{ Risk of bias } \\
\hline Item & Authors' judgement & Description \\
\hline Adequate sequence generation? & Unclear & Unclear \\
\hline Allocation concealment? & Unclear & Unclear \\
\hline $\begin{array}{l}\text { Blinding? } \\
\text { All outcomes }\end{array}$ & Unclear & Unclear \\
\hline
\end{tabular}


Kreisler 2005

\begin{tabular}{|c|c|c|}
\hline Methods & \multicolumn{2}{|c|}{$\begin{array}{l}\text { Randomisation was accomplished through the use of a computer-generated table of random num- } \\
\text { bers. Method used to conceal treatment allocation was not described. }\end{array}$} \\
\hline Participants & \multicolumn{2}{|c|}{$\begin{array}{l}71 \text { patients undergoing cardiac surgery were randomised to one of three groups: } \\
\text { - Epsilon aminocaproic acid: } \mathrm{n}=22, \mathrm{M} / \mathrm{F}=17 / 5 \text {, mean }(\mathrm{sd}) \text { age }=63.4(7.2) \text { years } \\
\text { - Heparin-coated CPB circuit: } \mathrm{n}=20,17 / 3 \text {, mean }(\mathrm{sd}) \text { age }=59.6(10.4) \text { years } \\
\text { - Control group (Placebo): } \mathrm{n}=25, \mathrm{M} / \mathrm{F}=17 / 8 \text {, mean }(\mathrm{sd}) \text { age }=61.4(8.8) \text { years }\end{array}$} \\
\hline Interventions & \multicolumn{2}{|c|}{$\begin{array}{l}\text { - Epsilon aminocaproic acid group received non-heparin coated circuits and EACA. A loading } \\
\text { dose of } 75 \mathrm{mg} / \mathrm{kg} \text { of EACA was given over } 10 \text { minutes after the induction of anaesthesia and prior } \\
\text { to skin incision followed by a maintenance infusion of EACA of } 12.5 \mathrm{mg} / \mathrm{kg} / \mathrm{hr} \text { continued for } 2 \\
\text { hours after the arrival of the patient in the intensive care unit. An additional } 5 \mathrm{~g} \text { of EACA was } \\
\text { added to the CPB priming fluid. } \\
\text { - Heparin coated (bonded) CPB circuit group were treated with tip-to-tip heparin-coated } \\
\mathrm{CPB} \text { circuits, including the cardiotomy reservoir, arterial filter, aortic and venous cannulas, and a } \\
\text { placebo infusion of normal saline. } \\
\text { - Control group received non-heparin coated circuits and a } 0.9 \% \text { normal saline load and } \\
\text { maintenance infusion given in the same manner as EACA-treated patients. } \\
\text { NB: All groups were exposed to cell savage. }\end{array}$} \\
\hline Outcomes & \multicolumn{2}{|c|}{$\begin{array}{l}\text { Outcomes reported: Number of patients exposed to allogeneic blood, number of patients exposed } \\
\text { to platelets, hospital length of stay (days), intensive care length of stay (hours), cell saver volume } \\
\text { autotransfused. }\end{array}$} \\
\hline Notes & \multicolumn{2}{|c|}{$\begin{array}{l}\text { Quality assessment score (Schulz criteria): 4/7 } \\
\text { Transfusion protocol used }\end{array}$} \\
\hline \multicolumn{3}{|l|}{ Risk of bias } \\
\hline Item & Authors' judgement & Description \\
\hline Adequate sequence generation? & Yes & Computer-generated table of random numbers \\
\hline Allocation concealment? & Unclear & Unclear \\
\hline $\begin{array}{l}\text { Blinding? } \\
\text { All outcomes }\end{array}$ & Yes & Double blind \\
\hline
\end{tabular}

\section{Kuepper 2003}

\begin{tabular}{ll} 
Methods & $\begin{array}{l}\text { Group assignment was by sealed envelopes. Sealed envelopes were opened after induction of anaes- } \\
\text { thesia by the unblinded investigator who was not part of the operating team. }\end{array}$ \\
\hline Participants & $\begin{array}{l}120 \text { patients undergoing elective cardiac surgery were randomised to one of two groups: } \\
\text { - Aprotinin group (Low dose): } \mathrm{n}=60, \mathrm{M} / \mathrm{F}=40 / 20, \text { mean }(\mathrm{sd}) \text { age }=65.5(7.8) \text { years } \\
\text { - Control group: } \mathrm{n}=59, \mathrm{M} / \mathrm{F}=40 / 19, \text { mean }(\mathrm{sd}) \text { age }=65.6(8.8) \text { years }\end{array}$
\end{tabular}


Kuepper 2003 (Continued)

\begin{tabular}{|c|c|c|}
\hline Interventions & \multicolumn{2}{|c|}{$\begin{array}{l}\text { - Aprotonin group (Low dose) received a single loading dose of } 2 \text { million KIU ( } 280 \mathrm{mg} \text { ) of } \\
\text { aprotinin given after the induction of anaesthesia but before skin incision. } \\
\text { - Control group did not receive aprotinin. }\end{array}$} \\
\hline Outcomes & \multicolumn{2}{|c|}{$\begin{array}{l}\text { Outcomes reported: Allogeneic blood usage (units), blood loss, fresh frozen plasma, platelets (units) } \\
\text {, mortality. }\end{array}$} \\
\hline Notes & \multicolumn{2}{|c|}{$\begin{array}{l}\text { Quality assessment score (Schulz criteria): } 3 / 7 \\
\text { Transfusion threshold for RBC not reported }\end{array}$} \\
\hline \multicolumn{3}{|l|}{ Risk of bias } \\
\hline Item & Authors' judgement & Description \\
\hline Adequate sequence generation? & Unclear & Unclear \\
\hline Allocation concealment? & No & Inadequate \\
\hline $\begin{array}{l}\text { Blinding? } \\
\text { All outcomes }\end{array}$ & No & Single blind \\
\hline
\end{tabular}

Kuitunen 2005

\begin{tabular}{l|l}
\hline Methods & $\begin{array}{l}\text { Study drugs were prepared by the hospital pharmacy. Randomisation was carried out using closed } \\
\text { envelopes. }\end{array}$ \\
\hline Participants & $\begin{array}{l}60 \text { patients undergoing primary coronary artery bypass graft surgery were randomised to one of } \\
\text { three groups: } \\
\text { - Aprotinin group (High dose): } \mathrm{n}=20 \text {, mean }(\mathrm{sd}) \text { age }=61 \text { (8.9) years } \\
\text { - Tranexamic acid group: } \mathrm{n}=20 \text {, mean }(\mathrm{sd}) \text { age }=63(8.9) \text { years } \\
\text { - Control group (Placebo): } \mathrm{n}=20 \text {, mean }(\mathrm{sd}) \text { age }=65(8.9) \text { years }\end{array}$ \\
\hline Interventions & $\begin{array}{l}\text { - Aprotinin group (High dose) received } 2 \text { million } \mathrm{KIU}(280 \mathrm{mg}) \text { of aprotinin after the } \\
\text { induction of anaesthesia, followed by an infusion of } 500,000 \mathrm{KIU} / \mathrm{hr} \text { (70mg/hr) until the end of } \\
\text { surgery. In addition, } 2 \text { million KIU (280mg) of aprotinin was added to the pump prime of the } \\
\text { CPB circuit. } \\
\text { - Tranexamic acid group received } 15 \mathrm{mg} / \mathrm{kg} \text { after the induction of anaesthesia, followed by an } \\
\text { infusion of } 15 \mathrm{mg} / \mathrm{kg} \text { until the end of surgery. In addition, } 15 \mathrm{mg} / \mathrm{kg} \text { was added to the pump prime } \\
\text { of the CPB circuit. } \\
\text { - Control group received normal saline. } \\
\text { NB: All groups were exposed to cell salvage. }\end{array}$ \\
\hline
\end{tabular}

Outcomes

Outcomes reported: Number of patients exposed to allogeneic blood, number of patients exposed to fresh frozen plasma, number of participants exposed to platelets,

blood loss (16hrs), mortality, myocardial infarction, re-operation for bleeding, stroke. 
Kuitunen 2005 (Continued)

\begin{tabular}{|c|c|c|}
\hline Notes & $\begin{array}{l}\text { Quality assessment score (Schulz criteria): 5/7 } \\
\text { Transfusion protocol used }\end{array}$ & \\
\hline \multicolumn{3}{|l|}{ Risk of bias } \\
\hline Item & Authors' judgement & Description \\
\hline Adequate sequence generation? & No & Closed envelopes \\
\hline Allocation concealment? & No & Inadequate \\
\hline $\begin{array}{l}\text { Blinding? } \\
\text { All outcomes }\end{array}$ & Yes & Double blind \\
\hline
\end{tabular}

Kuitunen 2006

\begin{tabular}{|c|c|c|}
\hline Methods & \multicolumn{2}{|c|}{ Method of randomisation and allocation concealment were not described. } \\
\hline Participants & \multicolumn{2}{|c|}{$\begin{array}{l}30 \text { patients undergoing elective primary cardiac surgery were randomised to one of two groups: } \\
\text { - Tranexamic acid group: } \mathrm{n}=15, \mathrm{M} / \mathrm{F}=12 / 3 \text {, mean }(\mathrm{sd}) \text { age }=57(16) \text { years } \\
\text { - Control group: } \mathrm{n}=15, \mathrm{M} / \mathrm{F}=11 / 4 \text {, mean (sd) age }=61 \text { (11) years }\end{array}$} \\
\hline Interventions & \multicolumn{2}{|c|}{$\begin{array}{l}\text { - Tranexamic acid group received } 1 \mathrm{~g} \text { after administration of } 15 \mathrm{ml} / \mathrm{kg} \text { of } 6 \% \mathrm{HES} \text { in the } \\
\text { immediate post-operative period. } \\
\text { - Control group received saline after administration of } 15 \mathrm{ml} / \mathrm{kg} \text { of } 6 \% \mathrm{HES} \text { in the immediate } \\
\text { post-operative period. }\end{array}$} \\
\hline Outcomes & \multicolumn{2}{|c|}{$\begin{array}{l}\text { Outcomes reported: Number of patients exposed to allogeneic blood transfusion, number of pa- } \\
\text { tients exposed to fresh frozen plasma and platelets, blood loss ( } 24 \mathrm{hrs}) \text {, re-operation for bleeding }\end{array}$} \\
\hline Notes & \multicolumn{2}{|c|}{ Transfusion protocol used. } \\
\hline \multicolumn{3}{|l|}{ Risk of bias } \\
\hline Item & Authors' judgement & Description \\
\hline Adequate sequence generation? & Unclear & Unclear \\
\hline Allocation concealment? & Unclear & Unclear \\
\hline $\begin{array}{l}\text { Blinding? } \\
\text { All outcomes }\end{array}$ & Yes & Double blind \\
\hline
\end{tabular}


Kunt 2005

\begin{tabular}{|c|c|c|}
\hline Methods & \multicolumn{2}{|c|}{ Method of randomisation and allocation concealment were not described. } \\
\hline Participants & \multicolumn{2}{|c|}{$\begin{array}{l}86 \text { patients undergoing routine cardiac surgery were randomised to one of two groups: } \\
\text { - Aprotinin group: } \mathrm{n}=40, \mathrm{M} / \mathrm{F}=30 / 10 \text {, mean (sd) age }=63(12) \text { years } \\
\text { - Control group: } \mathrm{n}=46, \text { mean (sd) age }=60(7) \text { years }\end{array}$} \\
\hline Interventions & \multicolumn{2}{|c|}{$\begin{array}{l}\text { - Aprotinin group received } 500,000 \mathrm{KIU}(70 \mathrm{mg}) \text { of aprotinin in the pump prime only. } \\
\text { - Control group received "no aprotinin." }\end{array}$} \\
\hline Outcomes & \multicolumn{2}{|c|}{$\begin{array}{l}\text { Outcomes reported: Allogeneic blood usage (units), blood loss ( } 24 \mathrm{hrs} \text { ), mortality, hospital length } \\
\text { of stay (days), intensive care unit length of stay (hours), re-operation for bleeding. }\end{array}$} \\
\hline Notes & \multicolumn{2}{|c|}{$\begin{array}{l}\text { Quality assessment score (Schulz criteria): } 2 / 7 \\
\text { Transfusion protocol not used }\end{array}$} \\
\hline \multicolumn{3}{|l|}{ Risk of bias } \\
\hline Item & Authors' judgement & Description \\
\hline Adequate sequence generation? & Unclear & Unclear \\
\hline Allocation concealment? & Unclear & Unclear \\
\hline $\begin{array}{l}\text { Blinding? } \\
\text { All outcomes }\end{array}$ & Unclear & Unclear \\
\hline
\end{tabular}

\section{Kyriss 2001}

Methods

Randomisation carried out using a computer-generated random list. Allocation concealment not specified.

\begin{tabular}{l|l} 
Participants & $\begin{array}{c}38 \text { patients undergoing elective thoracic surgery were randomised to one of two groups: } \\
\text { - Aprotinin group (Low dose): } \mathrm{n}=18, \mathrm{M} / \mathrm{F}=12 / 6, \text { mean age }=51.8 \text { years } \\
\text { - Control group (Placebo): } \mathrm{n}=20, \mathrm{M} / \mathrm{F}=12 / 8, \text { mean age }=50.8 \text { years }\end{array}$ \\
\hline Interventions & $\begin{array}{l}\text { - Aprotonin group (Low dose) received a test dose of } 10,000 \mathrm{KIU} \text { during induction of } \\
\text { anaesthesia followed by an initial bolus dose of } 2 \mathrm{million} \mathrm{KIU} \text { (280 } \mathrm{mg} \text { ) of aprotinin over } 20 \\
\text { minutes and a continuous infusion of } 500,000 \mathrm{KIU} / \mathrm{hr} \text { during the surgical procedure. } \\
\bullet \text { Control group received a corresponding volume of saline solution. }\end{array}$ \\
\hline Outcomes & $\begin{array}{l}\text { Outcomes reported: Number of patients exposed to allogeneic blood, allogeneic blood usage (units) } \\
\text { blood loss, fresh frozen plasma, blood loss, mortality, blood loss, re-operation for bleeding. }\end{array}$ \\
\hline Notes & $\begin{array}{l}\text { Quality assessment score (Schulz criteria): } 5 / 7 \\
\text { No transfusion protocol }\end{array}$ \\
\hline
\end{tabular}

\section{Risk of bias}


Kyriss 2001 (Continued)

\begin{tabular}{lll}
\hline Item & Authors' judgement & Description \\
\hline Adequate sequence generation? & Yes & Computer-generated random list \\
\hline Allocation concealment? & Unclear & Unclear \\
\hline $\begin{array}{l}\text { Blinding? } \\
\text { All outcomes }\end{array}$ & Yes & Double blind \\
\hline
\end{tabular}

Landymore 1997

Methods

Method of randomisation was not described. The study drugs were prepared by pharmacy, given an identification number, and then sent to the operating room.

Participants
$\begin{aligned} & \text { groups: } \\ & \text { - Aprotinin group (Low dose): } \mathrm{n}=48 \\ & \text { - Epsilon-aminocaproic acid group: } \mathrm{n}=44 \\ & \text { - Tranexamic acid group: } \mathrm{n}=56 \\ & \text { - Control group: } \mathrm{n}=50 \text { (not included in randomisation process) } \\ & \text { NB: Demographic data were not reported. }\end{aligned}$

Interventions

- Aprotinin group (Low dose) received a loading dose of 200,000 KIU of aprotinin administered before cardiopulmonary bypass (CPB), followed by a maintenance dose of 200,000 $\mathrm{KIU} / \mathrm{hr}$ of aprotinin continued until the termination of CPB.

- Epsilon-aminocaproic acid group received a loading dose of $5 \mathrm{~g}$ administered before $\mathrm{CPB}$, followed by a maintenance dose of $1 \mathrm{~g} / \mathrm{hr}$ of EACA continued until the termination of CPB.

- Tranexamic acid group received a loading dose of $10 \mathrm{mg} / \mathrm{kg}$ of TXA administered before $\mathrm{CPB}$, followed by a maintenance dose of $1 \mathrm{mg} / \mathrm{kg} / \mathrm{hr}$ of TXA continued until the termination of $\mathrm{CPB}$.

- Control group did not receive antifibrinolytic treatment.

Outcomes

Outcomes reported: Allogeneic blood usage (units), blood loss, thrombosis, deep vein thrombosis, pulmonary embolus.

Notes

Quality assessment score (Schulz criteria):2/7

Transfusion protocol used

Risk of bias

\begin{tabular}{lll}
\hline Item & Authors' judgement & Description \\
\hline Adequate sequence generation? & Unclear & Unclear \\
\hline Allocation concealment? & Unclear & Unclear
\end{tabular}




\begin{tabular}{l|l|l}
\hline $\begin{array}{l}\text { Blinding? } \\
\text { All outcomes }\end{array}$ & Unclear & Unclear \\
\hline
\end{tabular}

\section{Lass 1995}

Methods

Method of randomisation and allocation concealment were not described. Four aprotinin $(7.8 \%)$ and eight control (14.5\%) patients were excluded from the final analysis.

\begin{tabular}{l|l} 
Participants & $\begin{array}{l}110 \text { male patients undergoing elective primary coronary bypass surgery were randomised to one of } \\
\text { two groups: } \\
\text { - Aprotinin group (High dose): } \mathrm{n}=55 \\
\text { - Control (Placebo): } \mathrm{n}=55\end{array}$ \\
NB: Demographic data were not reported. \\
\hline Interventions \\
$\begin{array}{l}\text { - Aprotinin group (High dose) received } 2 \text { million KIU of aprotinin as a loading dose before } \\
\text { sternotomy followed by an infusion of } 500,000 \mathrm{KIU} / \mathrm{hr} \text { until the end of surgery. An additional } 2 \\
\text { million KIU was added to the priming volume. } \\
\text { - Control group received saline solution as a matching placebo in identical form by the same } \\
\text { administration scheme. }\end{array}$
\end{tabular}

\begin{tabular}{|c|c|c|}
\hline Outcomes & \multicolumn{2}{|c|}{$\begin{array}{l}\text { Outcomes reported: Number of patients exposed to allogeneic blood, allogeneic blood usage (units) } \\
\text {, graft patency, blood loss, mortality, myocardial infarction, acute heart failure, post-operative } \\
\text { complications, re-operation. }\end{array}$} \\
\hline Notes & \multicolumn{2}{|c|}{$\begin{array}{l}\text { Quality assessment score (Schulz criteria): 3/7 } \\
\text { Transfusion protocol used }\end{array}$} \\
\hline \multicolumn{3}{|l|}{ Risk of bias } \\
\hline Item & Authors' judgement & Description \\
\hline Adequate sequence generation? & Unclear & Unclear \\
\hline Allocation concealment? & Unclear & Unclear \\
\hline $\begin{array}{l}\text { Blinding? } \\
\text { All outcomes }\end{array}$ & Yes & Double blind \\
\hline
\end{tabular}

\section{Later 2009}

Methods

Allocated according to a computer-generated randomisation sequence, allocation concealed by use of sealed, opaque envelopes.

Participants

298 patients undergoing cardiac surgery were randomised to one of three groups:

- Aprotonin group: $\mathrm{n}=96, \mathrm{M} / \mathrm{F}=73 / 23$, mean (sd) age $=66.5$ (10.7) years

- Tranexamic acid group: $\mathrm{n}=99, \mathrm{M} / \mathrm{F}=73 / 26$, mean $(\mathrm{sd})$ age $=64.1$ (13.0) years

- Control group: $\mathrm{n}=103, \mathrm{M} / \mathrm{F}=68 / 35$, mean (sd) age $=65$ (11.2) years 


\begin{tabular}{|c|c|c|}
\hline Interventions & \multicolumn{2}{|c|}{$\begin{array}{l}\text { - Aporinin group (high dose) received } 2 \text { million KIU pre-CPB, } 2 \text { million KIU at pump prime } \\
\text { and } 500,000 \mathrm{KIU} / \mathrm{hr} \text { during } \mathrm{CPB} \text {. } \\
\text { - Tranexamic acid group received } 1 \mathrm{~g} \text { loading dose, } 500 \mathrm{mg} \text { added to CBP system prime, and a } \\
\text { continuous infusion of } 400 \mathrm{mg} / \mathrm{hr} \text {. } \\
\text { - Control group received saline. }\end{array}$} \\
\hline Outcomes & \multicolumn{2}{|c|}{$\begin{array}{l}\text { Outcomes reported: Number of patienst exposed to allogeneic blood, myocardial infarction, renal } \\
\text { failure, hospital length of stay (days), re-operation for bleeding. }\end{array}$} \\
\hline Notes & \multicolumn{2}{|c|}{$\begin{array}{l}\text { Quality assessment score (Schulz criteria): 5/7 } \\
\text { Transfusion protocol used. }\end{array}$} \\
\hline \multicolumn{3}{|l|}{ Risk of bias } \\
\hline Item & Authors' judgement & Description \\
\hline Adequate sequence generation? & Unclear & Computer-generated randomisation sequence \\
\hline Allocation concealment? & No & Inadequate - sealed envelopes \\
\hline $\begin{array}{l}\text { Blinding? } \\
\text { All outcomes }\end{array}$ & Yes & Double blind \\
\hline
\end{tabular}

Laub 1994

\begin{tabular}{l|l}
\hline Methods & Method of randomisation and allocation concealment were not described. \\
\hline Participants & $\begin{array}{l}47 \text { patients undergoing isolated coronary revascularisation were randomised to one of two groups: } \\
\text { - Aprotinin group: } \mathrm{n}=16, \mathrm{M} / \mathrm{F}=12 / 4, \text { mean }(\mathrm{sd}) \text { age }=65.3(11.2) \text { years }\end{array}$ \\
$\begin{array}{l}\text { - Control group (Placebo): } \mathrm{n}=16, \mathrm{M} / \mathrm{F}=13 / 3, \text { mean }(\mathrm{sd}) \text { age }=63.6(10) \text { years } \\
\text { NB: The study group consisted of } 32 \text { patients in total. Fifteen of the originally enrolled patients } \\
\text { were not included in the final analysis due to: adverse reactions while receiving the study medication } \\
(\mathrm{n}=2), \text { inability to obtain or a technically inadequate CT scan }(\mathrm{n}=7) \text {, refusal to come for follow- } \\
\text { up examinations }(\mathrm{n}=4) \text {, or died }(\mathrm{n}=2) .\end{array}$
\end{tabular}

Interventions

- Aprotinin group received $500 \mathrm{KIU}$ of aprotinin as a test dose after the induction of anaesthesia, followed by 2 million KIU ( $280 \mathrm{mg})$ of aprotinin as a bolus. An infusion of 0.5 million KIU of aprotinin was commenced after the bolus was given and 2 million KIU of aprotinin was added to the pump prime.

- Control group received an identical volume of placebo.

NB: Autologous blood salvage with reinfusion of washed RBCs was used for all patients. Shed mediastinal and pleural blood was filtered and reinfused using an autotransfusion system.

Outcomes

Outcomes reported: Number of patients exposed to allogeneic blood, volume of allogeneic blood transfused, blood loss, volume of platelets and fresh frozen plasma, re-operation for bleeding, postoperative $\mathrm{Hb}$ levels, graft occlusions, any blood product usage, haematologic variables, coagulation profiles, renal function. 
Laub 1994 (Continued)

\begin{tabular}{|c|c|c|}
\hline Notes & \multicolumn{2}{|c|}{$\begin{array}{l}\text { Quality assessment score (Schulz criteria): } 2 / 7 \\
\text { Transfusion protocol used }\end{array}$} \\
\hline \multicolumn{3}{|l|}{ Risk of bias } \\
\hline Item & Authors' judgement & Description \\
\hline Adequate sequence generation? & Unclear & Unclear \\
\hline Allocation concealment? & Unclear & Unclear \\
\hline $\begin{array}{l}\text { Blinding? } \\
\text { All outcomes }\end{array}$ & Yes & Double blind \\
\hline
\end{tabular}

Lavee 1993

\begin{tabular}{|c|c|c|}
\hline Methods & \multicolumn{2}{|c|}{ Method of randomisation and allocation concealment were not described. } \\
\hline Participants & \multicolumn{2}{|c|}{$\begin{array}{l}30 \text { patients undergoing various cardiopulmonary bypass procedures were randomised to one of two } \\
\text { groups: } \\
\text { - Aprotinin group (Low dose): } \mathrm{n}=15, \mathrm{M} / \mathrm{F}=13 / 2 \text {, mean }(\mathrm{sd}) \text { age }=62(11) \text { years } \\
\text { - Control group (Placebo): } \mathrm{n}=11, \mathrm{M} / \mathrm{F}=11 / 4 \text {, mean (sd) age }=60 \text { (9) years }\end{array}$} \\
\hline Interventions & \multicolumn{2}{|c|}{$\begin{array}{l}\text { - Aprotinin group received } 2 \text { million KIU of aprotinin added to the priming volume of the } \\
\text { oxygenator. No additional aprotinin doses were given to the patients. } \\
\text { - Control group received an equivalent volume of placebo solution (saline solution } 0.9 \% \text { ) } \\
\text { added to the priming volume of the oxygenator. }\end{array}$} \\
\hline Outcomes & \multicolumn{2}{|c|}{$\begin{array}{l}\text { Outcomes reported: Allogeneic blood usage (units), blood loss (24hrs), platelets (units), platelet } \\
\text { aggregation. }\end{array}$} \\
\hline Notes & \multicolumn{2}{|c|}{$\begin{array}{l}\text { Quality assessment score (Schulz criteria): } 2 / 7 \\
\text { Transfusion protocol used }\end{array}$} \\
\hline \multicolumn{3}{|l|}{ Risk of bias } \\
\hline Item & Authors' judgement & Description \\
\hline Adequate sequence generation? & Unclear & Unclear \\
\hline Allocation concealment? & Unclear & Unclear \\
\hline $\begin{array}{l}\text { Blinding? } \\
\text { All outcomes }\end{array}$ & Yes & Double blind \\
\hline
\end{tabular}


Leijdekkers 2006

\begin{tabular}{|c|c|c|}
\hline Methods & \multicolumn{2}{|c|}{$\begin{array}{l}\text { Patients were randomised to receive either placebo or aprotinin using a standard randomisation list } \\
\text { stored in the pharmacy department, only to be opened after the study was closed for inclusion. }\end{array}$} \\
\hline Participants & \multicolumn{2}{|c|}{$\begin{array}{l}35 \text { patients undergoing cardiac surgery were randomised to one of two groups: } \\
\text { - Aprotonin group: } \mathrm{n}=16, \mathrm{M} / \mathrm{F}=14 / 2 \text {, mean (sd) age }=68(9.5) \text { years } \\
\text { - Control group: } \mathrm{n}=19, \mathrm{M} / \mathrm{F}=14 / 5 \text {, mean (sd) age }=68(6.8) \text { years }\end{array}$} \\
\hline Interventions & \multicolumn{2}{|c|}{$\begin{array}{l}\text { - Aporinin group received } 2 \text { million KIU starting dose followed by } 500,000 \mathrm{KIU} / \mathrm{hr} \text { during } \\
\text { surgery. } \\
\text { - Control group received saline. }\end{array}$} \\
\hline Outcomes & \multicolumn{2}{|c|}{$\begin{array}{l}\text { Outocmes reported: Volume blood transfused (units), blood loss, mortality, re-operation for bleed- } \\
\text { ing. }\end{array}$} \\
\hline Notes & \multicolumn{2}{|c|}{$\begin{array}{l}\text { Quality assessment score (Schulz criteria): } 7 / 7 \\
\text { Transfusion protocol used. }\end{array}$} \\
\hline \multicolumn{3}{|l|}{ Risk of bias } \\
\hline Item & Authors' judgement & Description \\
\hline Adequate sequence generation? & Yes & Randomisation list \\
\hline Allocation concealment? & Yes & Adequate \\
\hline $\begin{array}{l}\text { Blinding? } \\
\text { All outcomes }\end{array}$ & Yes & Double blind \\
\hline
\end{tabular}

Lemay 2004

\begin{tabular}{l|l}
\hline Methods & $\begin{array}{l}\text { Method of randomisation was not described. Study drugs were prepared by the hospital pharmacist. } \\
\text { Patient caregivers and the investigator collecting the data were blinded to the solution used. }\end{array}$ \\
\hline Participants & $\begin{array}{l}40 \text { patients undergoing total hip replacement were randomised to one of two groups: } \\
\text { - Tranexamic acid group: } \mathrm{n}=20, \mathrm{M} / \mathrm{F}=12 / 8, \text { mean }(\mathrm{sd}) \text { age }=59.7(10.3) \text { years } \\
\text { - Control group (Placebo): } \mathrm{n}=19, \mathrm{M} / \mathrm{F}=13 / 6, \text { mean }(\mathrm{sd}) \text { age }=53.6(12.8) \text { years }\end{array}$ \\
\hline NB: One patient was excluded from the final analysis.
\end{tabular}

Outcomes

Outcomes reported: Number of patients exposed to allogeneic blood, allogeneic blood usage (units) , blood loss, deep vein thrombosis, changes in haemoglobin levels. 
Lemay 2004 (Continued)

\begin{tabular}{|c|c|c|}
\hline Notes & \multicolumn{2}{|c|}{$\begin{array}{l}\text { Quality assessment score (Schulz criteria): 5/7 } \\
\text { Transfusion protocol used }\end{array}$} \\
\hline \multicolumn{3}{|l|}{ Risk of bias } \\
\hline Item & Authors' judgement & Description \\
\hline Adequate sequence generation? & Unclear & Unclear \\
\hline Allocation concealment? & Yes & Adequate \\
\hline $\begin{array}{l}\text { Blinding? } \\
\text { All outcomes }\end{array}$ & Yes & Double blind \\
\hline
\end{tabular}

\section{Lemmer 1994}

Methods $\quad$ Metho used to generate allocation sequences was not described. Aprotinin and an identically appearing placebo was supplied by Bayer AG, Leverkusen, Germany. Enrolled patients were stratified as to whether they were undergoing primary procedures $(n=151$ patients: Lemmer 1$)$ or repeat procedures ( $\mathrm{n}=65$ patients: Lemmer 2$)$.

Participants 151 patients undergoing isolated primary coronary artery bypass graft operations were randomised to one of two groups:

- Aprotinin group: $\mathrm{n}=74, \mathrm{M} / \mathrm{F}=51 / 16$, mean age $=64$ years

- Control group (Placebo): $\mathrm{n}=74, \mathrm{M} / \mathrm{F}=61 / 13$, mean age $=62$ years

65 patients undergoing repeat coronary artery bypass graft operations were randomised to one of two groups:

- Aprotinin group (High dose): $\mathrm{n}=29, \mathrm{M} / \mathrm{F}=21 / 2$, mean age $=66$ years

- Control group (Placebo): $\mathrm{n}=36, \mathrm{M} / \mathrm{F}=29 / 3$, mean age $=65$ years

Interventions

- Aprotinin group received a loading dose of $280 \mathrm{mg}$ of aprotinin followed by a continuous infusion of $70 \mathrm{mg} / \mathrm{hr}$, and $280 \mathrm{mg}$ of aprotinin was added to the oxygenator prime solution. The continuous infusion was discontinued on the patients' arrival to the intensive care unit.

- Control group received identical volumes of $0.9 \%$ sodium chloride solution.

NB: Both groups were exposed to cell salvage.

Outcomes

Outcomes reported: Number of patients exposed to allogeneic blood, allogeneic blood usage (units) , fresh frozen plasma (units), platelets (units), blood loss, mortality,

myocardial infarction, re-operation for bleeding, allergic reactions, renal failure, renal failure + dialysis.

Notes

Quality assessment score (Schulz criteria): 5/7

Transfusion protocol used.

Of the 151 patients undergoing primary CABG, 141 (74 in the aprotinin treated group and 67 in the placebo treated group) fulfilled the criteria for efficacy analysis. Patients were eliminated from efficacy analysis before the random code was broken.

Of the 65 patients undergoing repeat CABG surgery 55 (23 in the aprotinin treated group and 
32 in the placebo treated group) fulfilled the criteria for efficacy analysis. Patients were eliminated from efficacy analysis before the random code was broken.

\section{Risk of bias}

\begin{tabular}{lll}
\hline Item & Authors' judgement & Description \\
\hline Adequate sequence generation? & Unclear & Unclear \\
\hline Allocation concealment? & Yes & Adequate \\
\hline $\begin{array}{l}\text { Blinding? } \\
\text { All outcomes }\end{array}$ & Yes & Double blind \\
\hline
\end{tabular}

\section{Lemmer 1996}

\begin{tabular}{|c|c|}
\hline Methods & Method of randomisation and allocation concealment were not described. \\
\hline Participants & $\begin{array}{l}704 \text { first time coronary artery bypass grafting patients were randomised to one of four groups: } \\
\text { - Control group (Placebo): } \mathrm{n}=178, \mathrm{M} / \mathrm{F}=151 / 27 \text {, mean }(\mathrm{sd}) \text { age }=62.5(10.67) \text { years } \\
\text { - Aprotinin group (High dose): } \mathrm{n}=173, \mathrm{M} / \mathrm{F}=145 / 28, \text { mean }(\mathrm{sd}) \text { age }=61.3(10.5) \text { years } \\
\text { - Aprotinin group (Low dose): } \mathrm{n}=180, \mathrm{M} / \mathrm{F}=155 / 25, \text { mean }(\mathrm{sd}) \text { age }=61.7(10.7) \text { years } \\
\text { - Aprotinin group (Pump prime dose): } \mathrm{n}=173, \mathrm{M} / \mathrm{F}=151 / 22 \text {, mean }(\mathrm{sd}) \text { age }=62.1(10.5) \\
\text { years }\end{array}$ \\
\hline Interventions & $\begin{array}{l}\text { - Control group received equivalent volumes of } 0.9 \% \text { sodium chloride at the same time } \\
\text { periods. } \\
\text { - Aprotinin group (High dose) received a loading dose of } 280 \mathrm{mg} \text { of aprotinin, a continuous } \\
\text { infusion dose of } 70 \mathrm{mg} / \mathrm{hr} \text { until the end of the operation, and } 280 \mathrm{mg} \text { of aprotinin was added to } \\
\text { the pump prime solution. } \\
\text { - Aprotinin group (Low dose) received a loading dose of } 140 \mathrm{mg} \text { of aprotinin, a continuous } \\
\text { infusion dose of } 35 \mathrm{mg} / \mathrm{hr} \text { until the end of the operation, and } 140 \mathrm{mg} \text { of aprotinin was added to } \\
\text { the pump prime solution. } \\
\text { - Aprotinin group (Pump prime dose) received a loading dose of placebo }(0.9 \% \text { sodium } \\
\text { chloride), a continuous infusion of placebo until the end of the operation, and } 280 \mathrm{mg} \text { of } \\
\text { aprotinin was added to the pump prime. } \\
\text { NB: All groups were exposed to cell salvage autotransfusion. }\end{array}$ \\
\hline Outcomes & $\begin{array}{l}\text { Outcomes reported: Total blood product exposures per patient, number of patients exposed to } \\
\text { allogeneic blood, allogeneic blood usage (units), platelet (units), fresh frozen plasma (units), cryo- } \\
\text { precipitate (units), blood loss, re-operation for diffuse bleeding, myocardial infarction. }\end{array}$ \\
\hline Notes & $\begin{array}{l}\text { Quality assessment score (Schulz criteria): 2/7 } \\
\text { Transfusion protocol used }\end{array}$ \\
\hline
\end{tabular}

\section{Risk of bias}

\section{Description}


Lemmer 1996 (Continued)

\begin{tabular}{lll}
\hline Adequate sequence generation? & Unclear & Unclear \\
\hline Allocation concealment? & Unclear & Unclear \\
\hline $\begin{array}{l}\text { Blinding? } \\
\text { All outcomes }\end{array}$ & Yes & Double blind \\
\hline
\end{tabular}

Lemmer'1 1994

\begin{tabular}{|c|c|c|}
\hline Methods & \multicolumn{2}{|l|}{ Refer to Lemmer 1994} \\
\hline Participants & \multicolumn{2}{|c|}{$\begin{array}{l}151 \text { patients undergoing isolated primary coronary artery bypass graft operations were randomised } \\
\text { to one of two groups: } \\
\text { - Aprotinin group: } \mathrm{n}=74, \mathrm{M} / \mathrm{F}=51 / 16 \text {, mean age }=64 \text { years } \\
\text { - Control group (Placebo): } \mathrm{n}=74, \mathrm{M} / \mathrm{F}=61 / 13 \text {, mean age }=62 \text { years }\end{array}$} \\
\hline Interventions & \multicolumn{2}{|c|}{$\begin{array}{l}\text { - Aprotinin group received a loading dose of } 280 \mathrm{mg} \text { of aprotinin followed by a continuous } \\
\text { infusion of } 70 \mathrm{mg} / \mathrm{hr} \text {, and } 280 \mathrm{mg} \text { of aprotinin was added to the oxygenator prime solution. The } \\
\text { continuous infusion was discontinued on the patients arrival to the intensive care unit. } \\
\text { - Control group received identical volumes of } 0.9 \% \text { sodium chloride solution. } \\
\text { NB: Both groups were exposed to cell salvage. }\end{array}$} \\
\hline Outcomes & \multicolumn{2}{|c|}{$\begin{array}{l}\text { Outcomes reported: Number of patients exposed to allogeneic blood, allogeneic blood usage (units) } \\
\text {, fresh frozen plasma (units), platelets (units), blood loss, mortality, } \\
\text { myocardial infarction, re-operation for bleeding, allergic reactions, renal failure, renal failure }+ \\
\text { dialysis. }\end{array}$} \\
\hline Notes & \multicolumn{2}{|c|}{$\begin{array}{l}\text { Quality assessment score (Schulz criteria): } 5 / 7 \\
\text { Transfusion protocol used. } \\
\text { Of the } 151 \text { patients undergoing primary CABG, } 141 \text { ( } 74 \text { in the aprotinin treated group and } 67 \text { in } \\
\text { the placebo treated group) fulfilled the criteria for efficacy analysis. Patients were eliminated from } \\
\text { efficacy analysis before the random code was broken. }\end{array}$} \\
\hline \multicolumn{3}{|l|}{ Risk of bias } \\
\hline Item & Authors' judgement & Description \\
\hline Adequate sequence generation? & Unclear & Unclear \\
\hline Allocation concealment? & Yes & Adequate \\
\hline $\begin{array}{l}\text { Blinding? } \\
\text { All outcomes }\end{array}$ & Yes & Double blind \\
\hline
\end{tabular}


Lemmer·2 1994

\begin{tabular}{l|l}
\hline Methods & Refer to Lemmer 1994 \\
\hline Participants & $\begin{array}{l}65 \text { patients undergoing repeat coronary artery bypass graft operations were randomised to one of } \\
\text { two groups: } \\
\text { - Aprotinin group (High dose): } \mathrm{n}=29, \mathrm{M} / \mathrm{F}=21 / 2, \text { mean age }=66 \text { years } \\
\text { - Control group (Placebo): } \mathrm{n}=36, \mathrm{M} / \mathrm{F}=29 / 3, \text { mean age }=65 \text { years }\end{array}$ \\
\hline Interventions & $\begin{array}{l}\text { - Aprotinin group (High dose) received a loading dose of } 280 \mathrm{mg} \text { of aprotinin followed by a } \\
\text { continuous infusion of } 70 \mathrm{mg} / \mathrm{hr} \text {, and } 280 \mathrm{mg} \text { of aprotinin was added to the oxygenator prime } \\
\text { solution. The continuous infusion was discontinued on the patients arrival to the intensive care } \\
\text { unit. } \\
\text { - Control group received an identical volume of } 0.9 \% \text { sodium chloride solution. }\end{array}$ \\
\hline NB: Both groups were exposed to cell salvage.
\end{tabular}

Risk of bias

\begin{tabular}{lll}
\hline Item & Authors' judgement & Description \\
\hline Adequate sequence generation? & Unclear & Unclear \\
\hline Allocation concealment? & Yes & Adequate \\
\hline $\begin{array}{l}\text { Blinding? } \\
\text { All outcomes }\end{array}$ & Yes & Yes \\
\hline
\end{tabular}

Lentschener 1997

Methods

Generation of allocation sequences was by means of computer-generated random codes. Method of allocation concealment was not described.

Participants

97 patients scheduled for elective liver resection performed through subcostal incision were randomly assigned to one of two groups:

- Aprotinin group: $\mathrm{n}=48, \mathrm{M} / \mathrm{F}=23 / 24$, mean $(\mathrm{sd})$ age $=53$ (15) years

- Control group (Placebo): $\mathrm{n}=49, \mathrm{M} / \mathrm{F}=26 / 21$, mean $(\mathrm{sd})$ age $=54$ (15) years

Interventions

- Aprotinin group received a loading dose of 2 million KIU over 20 minutes after the induction of anaesthesia, followed by a continuous infusion of 500,000 KIU/hr administered by infusion pump until skin closure. An additional bolus of 500,000 KIU of aprotinin was infused 
Lentschener 1997 (Continued)

\begin{tabular}{|c|c|c|}
\hline & \multicolumn{2}{|c|}{$\begin{array}{l}\text { for every three units of } \mathrm{RBC} \text { transfused. } \\
\text { - Control group received equivalent volumes of the placebo ( } 0.9 \% \text { saline solution) at the } \\
\text { respective times. }\end{array}$} \\
\hline Outcomes & \multicolumn{2}{|c|}{$\begin{array}{l}\text { Outcomes reported: Blood loss, number of patients exposed to allogeneic blood transfusion, fresh } \\
\text { frozen plasma transfused, platelet units transfused. }\end{array}$} \\
\hline Notes & \multicolumn{2}{|c|}{$\begin{array}{l}\text { Quality assessment score (Schulz criteria): } 3 / 7 \\
\text { Transfusion protocol used }\end{array}$} \\
\hline \multicolumn{3}{|l|}{ Risk of bias } \\
\hline Item & Authors' judgement & Description \\
\hline Adequate sequence generation? & Yes & Computer-generated random codes \\
\hline Allocation concealment? & Unclear & Unclear \\
\hline $\begin{array}{l}\text { Blinding? } \\
\text { All outcomes }\end{array}$ & Yes & Double blind \\
\hline
\end{tabular}

\section{Lentschener 1999}

\begin{tabular}{l|l} 
Methods & $\begin{array}{l}\text { Patients were randomised in a double blind fashion by using a computer generated random code. } \\
\text { Randomisation was both stratified by the number of fused levels and blocked in groups of four } \\
\text { before the induction of anesthesia. Allocation concealment was not described. }\end{array}$ \\
\hline Participants & $\begin{array}{l}72 \text { patients undergoing posterior lumbar spine fusion were randomly assigned to one of two groups: } \\
\text { - Aprotinin group: } \mathrm{n}=35, \mathrm{M} / \mathrm{F}=18 / 17, \mathrm{mean}(\mathrm{sd}) \text { age }=46(9) \text { years } \\
\text { - Control group (Placebo): } \mathrm{n}=37, \mathrm{M} / \mathrm{F}=19 / 18, \mathrm{mean}(\mathrm{sd}) \text { age }=51 \text { (11) years }\end{array}$ \\
\hline Interventions & $\begin{array}{l}\text { - Aprotinin group received a loading dose of } 2 \text { million } \mathrm{KIU}(280 \mathrm{mg}) \text { over } 20 \text { minutes after } \\
\text { induction of anaesthesia, followed by a continuous infusion of } 500,000 \mathrm{KIU} / \mathrm{hr} \text { administered by } \\
\text { infusion pump until skin closure. An additional bolus of } 500,000 \mathrm{KIU} \text { of aprotinin was infused } \\
\text { every three units of RBC transfused. } \\
\text { - Control group received equivalent volumes of the placebo }(0.9 \% \text { saline solution) at the } \\
\text { respective times. }\end{array}$ \\
\hline
\end{tabular}

Outcomes

Outcomes reported: Number of patients exposed to allogeneic blood, allogeneic blood (units), autologous transfusion, blood loss (24hrs), post-operative total autologous units (total).

$\begin{array}{ll}\text { Notes } & \text { Quality assessment score (Schulz criteria): 5/7 } \\ \text { Transfusion protocol used }\end{array}$

\section{Risk of bias}

Item

Authors' judgement

\section{Description}


Lentschener 1999 (Continued)

\begin{tabular}{lll}
\hline Adequate sequence generation? & Yes & Computer generated random code \\
\hline Allocation concealment? & Unclear & Unclear \\
\hline $\begin{array}{l}\text { Blinding? } \\
\text { All outcomes }\end{array}$ & Yes & Double blind \\
\hline
\end{tabular}

\section{Levy 1995}

Methods Method of randomisation and allocation concealment were not described. Eleven medical centres participated. Study performed efficacy and safety analysis. Exclusions defined by protocol.

Participants

287 patients undergoing repeat coronary artery bypass graft surgery were randomly assigned to one of four groups:

- Aprotinin group (High dose): $\mathrm{n}=73$ (safety analysis), $\mathrm{n}=61$ (efficacy analysis), $\mathrm{M} / \mathrm{F}=55 / 6$ mean $($ sd) age $=64(7.8)$ years

- Aprotinin group (Low dose): $\mathrm{n}=70$ (safety analysis), $\mathrm{n}=59$ (efficacy analysis), M/F = 52/7; mean $($ sd) age $=65+/-7.7$ years

- Aprotinin group (Pump-prime): $\mathrm{n}=72$ (safety analysis), $\mathrm{n}=68$ (efficacy analysis), $\mathrm{M} / \mathrm{F}=$ $62 / 6 ;$ mean $(\mathrm{sd})$ age $=66(8.2)$ years

- Control group (Placebo): $\mathrm{n}=72$ (safety analysis), $\mathrm{n}=65$ (efficacy analysis), M/F = 59/6; mean $(\mathrm{sd})$ age $=64(8.0)$ years

Interventions

- Aprotinin group (High dose) received a loading dose of 2 million KIU of aprotinin, plus an additional 2 million KIU was added to the cardiopulmonary bypass (CPB) circuit prime, followed by a continuous infusion of 500,000 KIU/hr during surgery.

- Aprotinin group (Low dose) received a loading dose of 1 million KIU of aprotinin, plus 1 million KIU was added to the CPB circuit prime, followed by a continuous infusion of 250,000 $\mathrm{KIU} / \mathrm{hr}$ during surgery.

- Aprotinin group (Pump-prime) received 2 million KIU of aprotinin added to the CPB circuit prime.

- Control group received equivalent volumes of $0.9 \%$ sodium chloride.

NB: All groups were exposed to cell salvage.

Outcomes Outcomes reported: Number of patients exposed to allogeneic blood, allogeneic blood usage (units) , fresh frozen plasma usage (units), platelet usage (units), blood loss.

Notes Quality assessment score (Schulz criteria): 2/7

Transfusion protocol used

Risk of bias

\begin{tabular}{lll}
\hline Item & Authors' judgement & Description \\
\hline Adequate sequence generation? & Unclear & Unclear \\
\hline Allocation concealment? & Unclear & Unclear
\end{tabular}


Levy 1995 (Continued)

\begin{tabular}{|c|c|c|}
\hline $\begin{array}{l}\text { Blinding? } \\
\text { All outcomes }\end{array}$ & Yes & Double blind \\
\hline
\end{tabular}

Li 2005

\begin{tabular}{|c|c|}
\hline Methods & Method of randomisation and allocation concealment were not described. \\
\hline Participants & $\begin{array}{l}70 \text { patients undergoing elective cardiac surgery were randomised to one of four groups: } \\
\text { - Control group: } \mathrm{n}=10, \mathrm{M} / \mathrm{F}=9 / 1 \text {, mean }(\mathrm{sd}) \text { age }=59(9) \text { years } \\
\text { - Platelet-rich plasmapheresis }+ \text { acute normovolaemic haemodilution + cell salvage group: } \mathrm{n}= \\
\text { 20, } \mathrm{M} / \mathrm{F}=17 / 3 \text {, mean }(\mathrm{sd}) \text { age }=59(6) \text { years } \\
\text { - Aprotinin group: } \mathrm{n}=22, \mathrm{M} / \mathrm{F}=9 / 1 \text {, mean }(\mathrm{sd}) \text { age }=61(7) \text { years } \\
\text { - Combined group: } \mathrm{n}=18, \mathrm{M} / \mathrm{F}=16 / 2 \text {, mean }(\mathrm{sd}) \text { age } 62(8) \text { years }\end{array}$ \\
\hline Interventions & $\begin{array}{l}\text { - Control received standard care with no active intervention. } \\
\text { - PRP+ ANH + CS group: After the induction of anaesthesia, blood was withdrawn via the 9- } \\
\text { French central venous catheter at a rate of } 35-45 \mathrm{ml} / \mathrm{min} \text { and collected in the } 125 \mathrm{~mL} \text { centrifugal } \\
\text { bowl of an autotransfusion unit (Cell Saver } 5 \text {, Haemonetics Corp., Braintree, MA). No systemic } \\
\text { heparin was administered at this time but calcium in the blood was sequested with citrate by } \\
\text { mixing the blood and ACD (adenosine, citrate, dextrose) agent (Perfect, Beijing, China) at a } \\
\text { volume ratio of 8:1. The withdrawn blood volume was replaced with a mixing (1:2) of plasma } \\
\text { substitute (Gelofusion) and crystalloid (Lactate Ringer Injection) at a volume ratio of } 1: 2-3 \text { to } \\
\text { maintain a steady PCWP. The withdrawn blood was centrifuged at } 2400 \text { rpm to separate the } \\
\text { RBC's from the plasma and platelets. After removing the RBC's the plasma was continuously } \\
\text { centrifuged at } 2400 \text { rpm to separate the PRP from the platelet-poor plasma. An average of } 30 \\
\text { minutes and three to four passes were required to complete blood withdrawal and separation of } \\
\text { the PRP and PPP. A volume of blood was withdrawn to obtain approximately } 300 \mathrm{ml} \text { of PRP from } \\
\text { each study patient. In addition, the autotransfusion device at the same machine (Cell saver } 5 \text {, } \\
\text { Haemonetics Corp., Braintree, MA) was also used to retrieve RBC's that were lost throughout the } \\
\text { course of the operation. Saline ( } 0.9 \% \text { NaCl) was used to irrigate all the sponges with blood in the } \\
\text { surgical field and then suctioned to the cell saver for further washing. Autologous RBC's both } \\
\text { obtained during initial blood withdrawal and obtained via the autotransfusion device during the } \\
\text { operation were reinfused as necessary. After reversal of heparin, the autologous PPP and PRP were } \\
\text { reinfused back to the patients as were any remaining autologous RBC's. } \\
\text { - Aprotinin group (High dose) received a loading dose of } 2 \text { million KIU before CPB and } 2 \\
\text { million KIU added to the pump prime and a continuous infusion of } 1 \text { million KIU/hr } \\
\text { administered until skin closure or until a total dose of } 5 \text { million KIU was achieved. } \\
\text { - Combined group (PRP + ANH +CS + Aprotinin) received treatment combining } \\
\text { interventions of Group } 2 \text { and Group } 3 \text {. }\end{array}$ \\
\hline
\end{tabular}

Outcomes

Outcomes reported: Number of patients exposed to allogeneic blood, allogeneic blood usage (units) , number of patients receiving fresh frozen plasma, number of patients receiving platelets, blood loss.

Notes

Quality assessment score (Schulz criteria): 2/7

Transfusion protocol used

Risk of bias

Anti-fibrinolytic use for minimising perioperative allogeneic blood transfusion (Review)

Copyright (C) 201 I The Cochrane Collaboration. Published by John Wiley \& Sons, Ltd. 


\begin{tabular}{lll}
\hline Item & Authors' judgement & Description \\
\hline Adequate sequence generation? & Unclear & Unclear \\
\hline Allocation concealment? & Unclear & Unclear \\
\hline $\begin{array}{l}\text { Blinding? } \\
\text { All outcomes }\end{array}$ & Unclear & Unclear \\
\hline
\end{tabular}

Liu 1993

\begin{tabular}{|c|c|c|}
\hline Methods & \multicolumn{2}{|c|}{$\begin{array}{l}\text { Allocation sequences were generated by a computer generated random list. The trial drug and } \\
\text { placebo were supplied in identical packs. Exclusions or loss to follow-up were reported. }\end{array}$} \\
\hline Participants & \multicolumn{2}{|c|}{$\begin{array}{l}40 \text { patients undergoing elective myocardial revascularisation were randomised to one of two groups: } \\
\text { - Aprotinin group: } \mathrm{n}=20, \mathrm{M} / \mathrm{F}=13 / 7 \text {, mean }(\mathrm{sd}) \text { age }=64.7(2.0) \text { years } \\
\text { - Control group (Placebo): } \mathrm{n}=20, \mathrm{M} / \mathrm{F}=17 / 3 \text {, mean }(\mathrm{sd}) \text { age }=66.7(1.3) \text { years }\end{array}$} \\
\hline Interventions & \multicolumn{2}{|c|}{$\begin{array}{l}\text { - Aprotinin group received a loading dose of } 1 \text { million KIU of aprotinin intravenously after } \\
\text { the induction of anaesthesia, } 1 \text { million KIU in the priming volume of the heart-lung machine and } \\
250,000 \mathrm{KIU} / \mathrm{hr} \text { after the loading dose to the end of skin closure, or up to } 1 \text { million KIU of } \\
\text { aprotinin if the operation exceeded } 4 \text { hours in duration. } \\
\text { - Control group received a corresponding volume of placebo (substance used was not } \\
\text { specified). } \\
\text { NB: Both groups were exposed to cell salvage. }\end{array}$} \\
\hline Outcomes & \multicolumn{2}{|c|}{$\begin{array}{l}\text { Outcomes reported: Number of patients exposed to allogeneic blood, allogeneic blood usage (units) } \\
\text {, fresh frozen plasma, blood loss, total post-operative autotransfusion from the chest drainage, } \\
\text { mortality, re-operation for bleeding, allergic reaction, hospital length of stay (days). }\end{array}$} \\
\hline Notes & \multicolumn{2}{|c|}{$\begin{array}{l}\text { Quality assessment score (Schulz criteria): 5/7 } \\
\text { Transfusion protocol not specified }\end{array}$} \\
\hline \multicolumn{3}{|l|}{ Risk of bias } \\
\hline Item & Authors' judgement & Description \\
\hline Adequate sequence generation? & Yes & $\begin{array}{l}\text { Allocation sequences were generated by a com- } \\
\text { puter generated random list }\end{array}$ \\
\hline Allocation concealment? & Yes & Adequate \\
\hline $\begin{array}{l}\text { Blinding? } \\
\text { All outcomes }\end{array}$ & Yes & Double blind \\
\hline
\end{tabular}


Liu 1998

\begin{tabular}{|c|c|c|}
\hline Methods & \multicolumn{2}{|c|}{ Method of randomisation and allocation concealment were not described. } \\
\hline Participants & \multicolumn{2}{|c|}{$\begin{array}{l}60 \text { patients undergoing open heart surgery were randomly assigned to one of three groups: } \\
\text { - Epsilon aminocaproic group: } \mathrm{n}=20 \text {, mean }(\mathrm{sd}) \text { age }=65(9) \text { years } \\
\text { - Epsilon aminocaproic }+ \text { platelet-rich plasmapheresis group: } \mathrm{n}=20 \text {, mean }(\mathrm{sd}) \text { age }=67 \text { (12) } \\
\text { years } \\
\text { - Control group: } \mathrm{n}=20 \text {, mean }(\mathrm{sd}) \text { age }=64(11) \text { years } \\
\text { NB: Gender data were not reported. }\end{array}$} \\
\hline Interventions & \multicolumn{2}{|c|}{$\begin{array}{l}\text { - Epsilon aminocaproic acid group received } 150 \mathrm{mg} / \mathrm{kg} \text { before CPB. } \\
\text { - Epsilon aminocaproic + platelet-rich plasmapheresis group received } 150 \mathrm{mg} / \mathrm{kg} \text { of EACA } \\
\text { before } \mathrm{CPB} \text { and platelet-rich plasma (PRP) at } 10 \mathrm{ml} / \mathrm{kg} \text { salvaged from each patient with a plasma } \\
\text { saver before CPB which was then reinfused. PRP was reinfused at the end of CPB after protamine } \\
\text { administration. } \\
\text { - Control group received standard care. }\end{array}$} \\
\hline Outcomes & \multicolumn{2}{|c|}{$\begin{array}{l}\text { Outcomes reported: Allogeneic blood usage (units), fresh frozen plasma usage (units), platelets } \\
\text { usage (units), blood loss. }\end{array}$} \\
\hline Notes & \multicolumn{2}{|c|}{$\begin{array}{l}\text { Quality assessment score (Schulz criteria): 0/7 } \\
\text { Transfusion protocol used }\end{array}$} \\
\hline \multicolumn{3}{|l|}{ Risk of bias } \\
\hline Item & Authors' judgement & Description \\
\hline Adequate sequence generation? & Unclear & Unclear \\
\hline Allocation concealment? & Unclear & Unclear \\
\hline $\begin{array}{l}\text { Blinding? } \\
\text { All outcomes }\end{array}$ & Unclear & Unclear \\
\hline
\end{tabular}

\section{Llau 1998}

\begin{tabular}{l|l}
\hline Methods & Method of randomisation and allocation concealment were not described. [Abstract] \\
\hline Participants & $\begin{array}{l}\text { 20 patients undergoing elective orthopaedic surgery were randomly allocated to one of two groups: } \\
\text { - Aprotinin group (Low dose): } \mathrm{n}=10, \text { mean (sd) age }=68 \text { (8) years } \\
\text { - Control group (Placebo): } \mathrm{n}=10, \text { mean (sd) age }=67 \text { (7) years }\end{array}$ \\
\hline Interventions & $\begin{array}{l}\text { - Aprotinin group (Low dose) received } 2 \text { million KIU of aprotinin } 30 \text { minutes immediately } \\
\text { after the induction of anaesthesia. } \\
\text { immediately after the induction of anaesthesia. }\end{array}$ \\
\hline Outcomes & $\begin{array}{l}\text { Outcomes reported: } \text { Number of patients exposed to allogeneic blood, allogeneic blood usage (units) } \\
\text {, blood loss, deep vein thrombosis, change in haematocrit levels - baseline to } 24 \text { hrs post-operative, } \\
\text { change in haemoglobin levels - baseline to } 24 \text { hrs post-operative. }\end{array}$ \\
\hline
\end{tabular}




\section{Llau 1998 (Continued)}

\begin{tabular}{l|ll}
\hline Notes & Transfusion protocol used. & \\
\hline Risk of bias & & Description \\
\hline Item & Authors' judgement & Unclear \\
\hline Adequate sequence generation? & Unclear & Unclear \\
\hline Allocation concealment? & Unclear & Unclear \\
\hline $\begin{array}{l}\text { Blinding? } \\
\text { All outcomes }\end{array}$ & Unclear & \\
\hline
\end{tabular}

Locatelli 1990

\begin{tabular}{l|l}
\hline Methods & Method of randomisation and allocation concealment were not described. [Italian language] \\
\hline Participants & $\begin{array}{l}38 \text { patients undergoing myocardial revascularisation were randomly allocated to one of three groups: } \\
\text { - Aprotinin group (High dose): } \mathrm{n}=12 \\
\text { - Aprotinin group (Low dose): } \mathrm{n}=13 \\
\text { - Control group: } \mathrm{n}=13\end{array}$ \\
NB: Demographic data were not reported.
\end{tabular}

Outcomes

Outcomes reported: Number of patients exposed to allogeneic blood, allogeneic blood usage (units) , blood loss (28 hrs), adverse reactions.

$\begin{array}{ll}\text { Notes } & \begin{array}{l}\text { Quality assessment score (Schulz criteria): 2/7 } \\ \text { Transfusion protocol used }\end{array}\end{array}$

\section{Risk of bias}

\begin{tabular}{lll}
\hline Item & Authors' judgement & Description \\
\hline Adequate sequence generation? & Unclear & Unclear \\
\hline Allocation concealment? & Unclear & Unclear
\end{tabular}


Locatelli 1990 (Continued)

\begin{tabular}{l|l|l}
\hline $\begin{array}{l}\text { Blinding? } \\
\text { All outcomes }\end{array}$ & Unclear & Unclear \\
\hline
\end{tabular}

\section{Luo 1998}

\begin{tabular}{|c|c|c|}
\hline Methods & \multicolumn{2}{|c|}{ Method of randomisation and allocation concealment were not described. } \\
\hline Participants & \multicolumn{2}{|c|}{$\begin{array}{l}20 \text { patients undergoing cardiac surgery were randomised to one of two groups } \\
\text { - Aprotonin group: } \mathrm{n}=10, \mathrm{M} / \mathrm{F}=7 / 3 \text {, mean }(\mathrm{sd}) \text { age }=36.9(15.97) \text { years } \\
\text { - Control group: } \mathrm{n}=10, \mathrm{M} / \mathrm{F}=7 / 3 \text {, mean (sd) age }=42.8(13.31) \text { years }\end{array}$} \\
\hline Interventions & \multicolumn{2}{|c|}{$\begin{array}{l}\text { - Aprotinin group received } 3 \text { million KIU of aprotinin. } \\
\text { - Control group did not receive aprotinin. no intervention. }\end{array}$} \\
\hline Outcomes & \multicolumn{2}{|c|}{ Outcomes reported: volume of blood transfused, duration of $\mathrm{CPB}$. } \\
\hline Notes & \multicolumn{2}{|c|}{$\begin{array}{l}\text { Quality assessment score (Schulz criteria): 2/7 } \\
\text { Transfusion protocol not reported. }\end{array}$} \\
\hline \multicolumn{3}{|l|}{ Risk of bias } \\
\hline Item & Authors' judgement & Description \\
\hline Adequate sequence generation? & Unclear & Unclear \\
\hline Allocation concealment? & Unclear & Unclear \\
\hline $\begin{array}{l}\text { Blinding? } \\
\text { All outcomes }\end{array}$ & Unclear & Unclear \\
\hline
\end{tabular}

\section{Maccario 1994}

\begin{tabular}{l|l}
\hline Methods & Method of randomisation and allocation concealment were not described. [Italian language] \\
\hline Participants & 99 patients undergoing coronary artery bypass graft surgery and valvular cardiac surgery were \\
randomised to one of three groups: \\
- Aprotinin group (High dose): $\mathrm{n}=33$, mean $(\mathrm{sd})$ age $=64.0(8.51)$ years \\
- Aprotinin group (Low dose): $\mathrm{n}=33$, mean $(\mathrm{sd})$ age $=63.5(8.37)$ years \\
- Control group: $\mathrm{n}=33$, mean $(\mathrm{sd})$ age $=62.9(9.7)$ years \\
NB: Gender data were not reported.
\end{tabular}

Interventions

- Aprotinin group (High dose) received a loading dose of 2 million KIU of aprotinin intravenously (IV) over a period of 30 minutes, followed by 500,000 KIU/hr until the termination of the operation. An additional 2 million KIU was added to pump prime.

- Aprotinin group (Low dose) received 2 million KIU added to the pump prime.

- Control group did not receive aprotinin.

NB: All groups were exposed to acute normovolemic haemodilution and cell salvage. 


\begin{tabular}{ll} 
Outcomes & $\begin{array}{l}\text { Outcomes reported: } \text { Number of patients exposed to allogeneic blood, allogeneic blood usage (units) } \\
\text {, blood loss (24hrs), allergic reactions. }\end{array}$ \\
\hline Notes & $\begin{array}{l}\text { Quality assessment score (Schulz criteria): } 2 / 7 \\
\text { Transfusion protocol used. } \\
\text { Four patients were excluded from the study due to surgical bleeding (one from the control group, } \\
\text { one from the high-dose aprotinin group, and two from the low-dose aprotinin group). One patient } \\
\text { from the low-dose aprotinin group died and was excluded from analysis. }\end{array}$ \\
\hline
\end{tabular}

\section{Risk of bias}

\begin{tabular}{lll}
\hline Item & Authors' judgement & Description \\
\hline Adequate sequence generation? & Unclear & Unclear \\
\hline Allocation concealment? & Unclear & Unclear \\
\hline $\begin{array}{l}\text { Blinding? } \\
\text { All outcomes }\end{array}$ & No & Single blind \\
\hline
\end{tabular}

\section{MacGillivray 2010}

\begin{tabular}{|c|c|}
\hline Methods & $\begin{array}{l}\text { Tranexamic acid or placebo for infusion was prepared by the institution's pharmacy in two identical } \\
50 \mathrm{~mL} \text { bags (identified only by random number) with the constituents unknown to the administering } \\
\text { anesthesiologist or surgeon. Method of randomisation was not described. }\end{array}$ \\
\hline Participants & $\begin{array}{l}60 \text { patients undergoing orthopaedic (knee) surgery were randomised to one of three groups: } \\
\text { - Tranexamic acid group } \# 1: \mathrm{n}=20, \mathrm{M} / \mathrm{F}=7 / 13 \text {, mean }(\mathrm{sd}) \text { age }=62(4.3) \text { years } \\
\text { - Tranexamic acid group } \# 2: \mathrm{n}=20, \mathrm{M} / \mathrm{F}=8 / 12 \text {, mean }(\mathrm{sd}) \text { age }=65(4.3) \text { years } \\
\text { - Control group: } \mathrm{n}=20, \mathrm{M} / \mathrm{F}=5 / 15, \text { mean }(\mathrm{sd}) \text { age }=66(7.3) \text { years }\end{array}$ \\
\hline Interventions & $\begin{array}{l}\text { - Tranexamic acid group \#1 received two doses of } 10 \mathrm{mg} / \mathrm{kg} \text {. Patients received the first infusion } \\
\text { over } 10 \text { minutes before deflation of the first tourniquet and the second (over } 10 \text { minutes) } 3 \text { hours } \\
\text { after the first. } \\
\text { - Tranexamic acid group } \# 2 \text { received two doses of } 15 \mathrm{mg} / \mathrm{kg} \text {. Patients received the first infusion } \\
\text { over } 10 \text { minutes before deflation of the first tourniquet and the second (over } 10 \text { minutes) } 3 \text { hours } \\
\text { after the first. } \\
\text { - Control group received normal saline. Patients received the first infusion of saline over } 10 \\
\text { minutes before deflation of the first tourniquet and the second (over } 10 \text { minutes) } 3 \text { hours after the } \\
\text { first. } \\
\text { NB: Patients received re-infusion of autotransfused blood from the intra-articular drains. }\end{array}$ \\
\hline Outcomes & $\begin{array}{l}\text { Outcomes reported: number of patients exposed to allogeneic blood transfusion, blood loss, number } \\
\text { of allogeneic units transfused, adverse events. }\end{array}$ \\
\hline Notes & $\begin{array}{l}\text { Quality assessment score (Schulz criteria): 6/7 } \\
\text { Transfusion protocol used. }\end{array}$ \\
\hline
\end{tabular}


MacGillivray 2010 (Continued)

\begin{tabular}{|c|c|c|}
\hline Item & Authors' judgement & Description \\
\hline Adequate sequence generation? & Unclear & Unclear \\
\hline Allocation concealment? & Yes & Adequate \\
\hline $\begin{array}{l}\text { Blinding? } \\
\text { All outcomes }\end{array}$ & Yes & Double blind \\
\hline
\end{tabular}

Maddali 2007

\begin{tabular}{|c|c|c|}
\hline Methods & \multicolumn{2}{|c|}{$\begin{array}{l}\text { A computer-generated randomisation code was used to allocate participants. Allocation was con- } \\
\text { cealed by using sequentially-numbered, sealed opaque envelopes. }\end{array}$} \\
\hline Participants & \multicolumn{2}{|c|}{$\begin{array}{l}222 \text { patients undergoing cardiac surgery were randomised to one of two groups: } \\
\text { - Tranexamic acid group: } \mathrm{n}=111, \mathrm{M} / \mathrm{F}=80 / 31 \text {, mean }(\mathrm{sd}) \text { age }=57.1(8.9) \text { years } \\
\text { - Control group: } \mathrm{n}=111, \mathrm{M} / \mathrm{F}=72 / 38 \text {, mean }(\mathrm{sd}) \text { age }=58.2(8.3) \text { years }\end{array}$} \\
\hline Interventions & \multicolumn{2}{|c|}{$\begin{array}{l}\text { - Tranexamic acid group received loading dose of } 10 \mathrm{mg} / \mathrm{kg} \text { before incision, then a continuous } \\
\text { infusion of } 1 \mathrm{mg} / \mathrm{kg} / \mathrm{hr} \text { until end of CPB. } \\
\text { - Control group received saline. }\end{array}$} \\
\hline Outcomes & \multicolumn{2}{|c|}{$\begin{array}{l}\text { Outcomes reported: Volume blood transfused, blood loss, mortality, stroke, re-operation for bleed- } \\
\text { ing. }\end{array}$} \\
\hline Notes & \multicolumn{2}{|c|}{$\begin{array}{l}\text { Quality assessment score (Schulz criteria): } 4 / 7 \\
\text { Transfusion protocol used }\end{array}$} \\
\hline \multicolumn{3}{|l|}{ Risk of bias } \\
\hline Item & Authors' judgement & Description \\
\hline Adequate sequence generation? & Yes & Computer-generated randomisation code \\
\hline Allocation concealment? & No & Inadequate - sealed envelopes \\
\hline $\begin{array}{l}\text { Blinding? } \\
\text { All outcomes }\end{array}$ & Yes & Double blind \\
\hline
\end{tabular}


Maineri 2000

\begin{tabular}{|c|c|c|}
\hline Methods & \multicolumn{2}{|c|}{ Method of randomisation and allocation concealment were not described. } \\
\hline Participants & \multicolumn{2}{|c|}{$\begin{array}{l}48 \text { patients undergoing elective cardiac surgery were randomised to one of two groups: } \\
\text { - Epsilon aminocaproic acid group: } \mathrm{n}=24 \text {, mean }(\mathrm{sd}) \text { age }=59.9(10) \text { years } \\
\text { - Tranexamic acid group }(\mathrm{n}=24), \text { mean }(\mathrm{sd}) \text { age }=64.2(9) \text { years } \\
\text { NB: Gender data were not reported. }\end{array}$} \\
\hline Interventions & \multicolumn{2}{|c|}{$\begin{array}{l}\text { - Epsilon aminocaproic acid group received } 10 \mathrm{~g} \text { of EACA as a standard dose in } 30 \text { minutes } \\
\text { following the induction of anaesthesia, and a maintenance infusion of } 2 \mathrm{~g} / \mathrm{hr} \text { was given } \\
\text { throughout the operation. } \\
\text { - Tranexamic acid group received a loading dose of } 20 \mathrm{mg} / \mathrm{kg} \text { of TXA given in } 60 \text { minutes, } \\
\text { followed by a maintenance infusion of } 2 \mathrm{mg} / \mathrm{kg} / \mathrm{hr} \text {. }\end{array}$} \\
\hline Outcomes & \multicolumn{2}{|c|}{$\begin{array}{l}\text { Outcomes reported: Number of patients exposed to allogeneic blood, allogeneic blood usage (units) } \\
\text {, blood loss, pulmonary embolus, post -operative Hct, stroke. }\end{array}$} \\
\hline Notes & \multicolumn{2}{|c|}{$\begin{array}{l}\text { Quality assessment score (Schulz criteria): 0/7 } \\
\text { Transfusion protocol used }\end{array}$} \\
\hline \multicolumn{3}{|l|}{ Risk of bias } \\
\hline Item & Authors' judgement & Description \\
\hline Adequate sequence generation? & Unclear & Unclear \\
\hline Allocation concealment? & Unclear & Unclear \\
\hline $\begin{array}{l}\text { Blinding? } \\
\text { All outcomes }\end{array}$ & Unclear & Unclear \\
\hline
\end{tabular}

\section{Mansour 2004}

$\begin{array}{ll}\text { Methods } & \begin{array}{l}\text { Randomisation of patients was performed with the help of a computer -generated random number } \\ \text { sequence programme. To ensure proper blinding the three studied solutions were prepared by the } \\ \text { pharmacy as bottles. }\end{array}\end{array}$

Participants

60 patients undergoing elective cardiac surgery (off pump CABG) were randomly assigned to one of three groups:

- Aprotinin group: $\mathrm{n}=20, \mathrm{M} / \mathrm{F}=1 / 5$, mean $(\mathrm{sd})$ age $=56.4(9.1)$ years

- Tranexamic acid group: $\mathrm{n}=20, \mathrm{M} / \mathrm{F}=17 / 3$, mean (sd) age $=57.5(8.4)$ years

- Control group (Placebo): $\mathrm{n}=20, \mathrm{M} / \mathrm{F}=19 / 1$, mean $(\mathrm{sd})$ age $=57.7$ (8.4) years

Interventions

- Aprotinin group received 2 million KIU of aprotinin after skin incision, followed by a continuous infusion of 3 million KIU throughout surgery at a rate of 500,000 KIU/hr.

- Tranexamic acid group received $1.5 \mathrm{~g}$ of TXA $(15 \mathrm{mg} / \mathrm{kg})$ after skin incision followed by a continuous infusion of $1 \mathrm{~g}$ throughout surgery at a rate of $2 \mathrm{mg} / \mathrm{kg} / \mathrm{hr}$.

- Control group received normal saline at the same time and volumes as aprotinin and TXA. NB: Loading dose was administered over 20 minutes in all groups. Infusion dose was infused at a rate of $50 \mathrm{ml} / \mathrm{hr}$ in all groups. 


\begin{tabular}{|c|c|c|}
\hline Outcomes & \multicolumn{2}{|c|}{$\begin{array}{l}\text { Outcomes reported: Number of patients exposed to allogeneic blood, allogeneic blood usage (units) } \\
\text {, fresh frozen plasma usage (units), platelet usage (units), blood loss ( } 24 \mathrm{hrs} \text { ), myocardial infarction, } \\
\text { number of patients exposed to fresh frozen plasma, number of patients exposed to platelets, re- } \\
\text { operation for bleeding, renal dysfunction, hospital length of stay (days), renal dysfunction, neuro- } \\
\text { logical deficit. }\end{array}$} \\
\hline Notes & \multicolumn{2}{|c|}{$\begin{array}{l}\text { Quality assessment score (Schulz criteria): 7/7 } \\
\text { Transfusion protocol used }\end{array}$} \\
\hline \multicolumn{3}{|l|}{ Risk of bias } \\
\hline Item & Authors' judgement & Description \\
\hline Adequate sequence generation? & Yes & $\begin{array}{l}\text { Computer -generated random number sequence } \\
\text { programme }\end{array}$ \\
\hline Allocation concealment? & Yes & Adequate \\
\hline $\begin{array}{l}\text { Blinding? } \\
\text { All outcomes }\end{array}$ & Yes & Double blind \\
\hline
\end{tabular}

\section{Marcel 1996}

Methods Patients were randomised by a computer program. Method of allocation concealment was not described.

\begin{tabular}{|c|c|}
\hline Participants & $\begin{array}{l}44 \text { consecutive patients undergoing orthotopic liver transplantation were randomised to one of } \mathrm{t} \\
\text { groups: } \\
\text { - Aprotinin group: } \mathrm{n}=21 \\
\text { - Control group (Placebo): } \mathrm{n}=23 \\
\text { NB: Demographic data were not reported. }\end{array}$ \\
\hline Interventions & $\begin{array}{l}\text { - Aprotinin group received 200,000 KIU per hour via an intravenous infusion which was } \\
\text { started immediately after the induction of anaesthesia. } \\
\text { - Control group received normal saline. }\end{array}$ \\
\hline
\end{tabular}

Outcomes

Outcomes reported: Allogeneic blood usage (units), fresh frozen plasma usage (units), platelet usage (units), blood loss (24hrs).

$\begin{array}{ll}\text { Notes } & \text { Quality assessment score (Schulz criteria): 5/7 } \\ \text { Transfusion protocol used }\end{array}$

\section{Risk of bias}

Item

Authors' judgement

Description 
Marcel 1996 (Continued)

\begin{tabular}{lll}
\hline Adequate sequence generation? & Yes & Randomised by a computer program \\
\hline Allocation concealment? & Unclear & Unclear \\
\hline $\begin{array}{l}\text { Blinding? } \\
\text { All outcomes }\end{array}$ & Yes & Double blind \\
\hline
\end{tabular}

Mehr-Aein 2007

\begin{tabular}{|c|c|c|}
\hline Methods & \multicolumn{2}{|c|}{$\begin{array}{l}\text { Method of randomisation was not reported. Concealment of allocation was achieved by using } \\
\text { pharmacy prepared coded infusion syringes. }\end{array}$} \\
\hline Participants & \multicolumn{2}{|c|}{$\begin{array}{l}66 \text { patients undergoing cardiac surgery were randomised to one of two groups: } \\
\text { - Tranexamic acid group: } \mathrm{n}=33 \text {, mean }(\mathrm{sd}) \text { age }=44(10) \text { years } \\
\text { - Control group: } \mathrm{n}=33 \text {, mean }(\mathrm{sd}) \text { age }=45(10) \text { years } \\
\text { NDB: Gender data were not reported. }\end{array}$} \\
\hline Interventions & \multicolumn{2}{|c|}{$\begin{array}{l}\text { - Tranexamic acid group received loading dose of } 15 \mathrm{mg} / \mathrm{kg} \text { at beginning of surgery, same dose } \\
\text { before infusion of heparin at end of surgery, and again after protamine infusion. } \\
\text { - Control group received saline. }\end{array}$} \\
\hline Outcomes & \multicolumn{2}{|c|}{$\begin{array}{l}\text { Outcomes reported: Number of patient exposed to allogeneic blood transfusion, volume of blood } \\
\text { transfused (units), blood loss, re-operation for bleeding, mortality, myocardial infarction, renal } \\
\text { failure, length of hospital stay (days). }\end{array}$} \\
\hline Notes & \multicolumn{2}{|c|}{$\begin{array}{l}\text { Quality assessment score (Schulz criteria): 6/7 } \\
\text { Transfusion protocol used }\end{array}$} \\
\hline \multicolumn{3}{|l|}{ Risk of bias } \\
\hline Item & Authors' judgement & Description \\
\hline Adequate sequence generation? & Unclear & Unclear \\
\hline Allocation concealment? & Yes & Adequate \\
\hline $\begin{array}{l}\text { Blinding? } \\
\text { All outcomes }\end{array}$ & Yes & Double blind \\
\hline
\end{tabular}

Mengistu 2008

\begin{tabular}{l|l}
\hline Methods & Method of randomisation and allocation concealment were not described. \\
\hline Participants & $\begin{aligned} 50 & \text { patients undergoing cardiac surgery were randomised to one of two groups: } \\
& \text { - Aprotinin group: } \mathrm{n}=25, \mathrm{M} / \mathrm{F}=20 / 5, \text { mean }(\mathrm{sd}) \text { age }=69(9) \text { years } \\
& \text { - Tranexamic acid group: } \mathrm{n}=25, \mathrm{M} / \mathrm{F}=18 / 7, \text { mean }(\mathrm{sd}) \text { age }=70 \text { (9) years }\end{aligned}$
\end{tabular}




\begin{tabular}{|c|c|c|}
\hline Interventions & \multicolumn{2}{|c|}{$\begin{array}{l}\text { - Aprotinin group received } 2 \text { million KIU pre-CPB, } 2 \text { million KIU at pump prime, and } \\
\text { 500,000 KIU/hr until arrival at ICU. } \\
\text { - Tranexamic group received } 2 \mathrm{~g} \text { administered after induction of anaesthesia and } 6 \mathrm{mg} / \mathrm{kg} / \mathrm{hr} \\
\text { given continuously until arrival at ICU, and } 1 \mathrm{~g} \text { added to CBP system prime. }\end{array}$} \\
\hline Outcomes & \multicolumn{2}{|c|}{$\begin{array}{l}\text { Outcomes reported: number of patients exposed to allogeneic blood transfusion, volume of allo- } \\
\text { geneic blood transfused, blood loss. }\end{array}$} \\
\hline Notes & \multicolumn{2}{|c|}{$\begin{array}{l}\text { Quality assessment score (Schulz criteria): } 3 / 7 \\
\text { Transfusion protocol used }\end{array}$} \\
\hline \multicolumn{3}{|l|}{ Risk of bias } \\
\hline Item & Authors' judgement & Description \\
\hline Adequate sequence generation? & Unclear & Unclear \\
\hline Allocation concealment? & Unclear & Unclear \\
\hline $\begin{array}{l}\text { Blinding? } \\
\text { All outcomes }\end{array}$ & No & Single blind \\
\hline
\end{tabular}

\section{Menichetti 1996}

\begin{tabular}{l|l}
\hline Methods & Method of randomisation and allocation concealment were not described. \\
\hline Participants & $\begin{array}{l}9 \text { consecutive patients undergoing coronary artery bypass surgery were randomised to one of four } \\
\text { groups: } \\
\text { - Aprotinin group: } \mathrm{n}=24, \mathrm{M} / \mathrm{F}=12 / 12, \mathrm{mean}(\mathrm{sd}) \text { age }=60.4(5.1) \text { years } \\
\text { - Epsilon aminocaproic acid group: } \mathrm{n}=24, \mathrm{M} / \mathrm{F}=14 / 10, \text { mean }(\mathrm{sd}) \text { age }=56.6(6.7) \text { years } \\
\text { - Tranexamic acid group: } \mathrm{n}=24, \mathrm{M} / \mathrm{F}=12 / 12, \mathrm{mean}(\mathrm{sd}) \text { age }=55.2(8.6) \text { years } \\
\text { - Control group: } \mathrm{n}=24, \mathrm{M} / \mathrm{F}=13 / 11, \mathrm{mean}(\mathrm{sd}) \text { age }=61.0(9.7) \text { years }\end{array}$ \\
\hline Interventions & $\begin{array}{l}\text { - Aprotinin group received a loading dose of } 2 \text { million } \mathrm{KIU} \text { of aprotinin followed by a } \\
\text { continuous infusion of } 500,000 \mathrm{KIU} / \mathrm{hr} \text {. An additional } 2 \text { million KIU of aprotinin was added to } \\
\text { the CPB prime solution. } \\
\text { - Epsilon aminocaproic acid group received } 80 \mathrm{mg} \text { bolus of EACA intravenously and after } 30 \\
\text { minutes a continuous infusion of } 30 \mathrm{mg} / \mathrm{kg} \text { of EACA. An additional } 80 \mathrm{mg} / \mathrm{kg} \text { of EACA was } \\
\text { added to the CPB prime solution. } \\
\text { - Tranexamic acid group received a } 10 \mathrm{mg} / \mathrm{kg} \text { bolus of TXA followed by a continuous infusion } \\
\text { of } 3 \mathrm{mg} / \mathrm{kg} / \mathrm{hr} \text {. An additional } 10 \mathrm{mg} / \mathrm{kg} \text { of TXA was added to the CPB prime solution. } \\
\text { - Control group received usual care. }\end{array}$ \\
\hline
\end{tabular}
ation for bleeding, haemoglobin levels, activated clotting times (ACT), prothrombin times, activated partial thromboplastin times (APTT), plasminogen levels, factor VIII levels, TAT complex/values. 
Menichetti 1996 (Continued)

\begin{tabular}{|c|c|c|}
\hline Notes & \multicolumn{2}{|c|}{$\begin{array}{l}\text { Quality assessment score (Schulz criteria): 2/7 } \\
\text { Transfusion protocol used }\end{array}$} \\
\hline \multicolumn{3}{|l|}{ Risk of bias } \\
\hline Item & Authors' judgement & Description \\
\hline Adequate sequence generation? & Unclear & Unclear \\
\hline Allocation concealment? & Unclear & Unclear \\
\hline $\begin{array}{l}\text { Blinding? } \\
\text { All outcomes }\end{array}$ & Unclear & Unclear \\
\hline
\end{tabular}

\section{Misfeld 1998}

\begin{tabular}{|c|c|c|}
\hline Methods & \multicolumn{2}{|c|}{ Method of randomisation and allocation concealment were not described. } \\
\hline Participants & \multicolumn{2}{|c|}{$\begin{array}{l}42 \text { patients undergoing elective cardiac surgery were randomised to one of three groups: } \\
\text { - Aprotinin group: } \mathrm{n}=14, \mathrm{M} / \mathrm{F}=14 / 0 \text {, mean }(\mathrm{sd}) \text { age }=63(6) \text { years } \\
\text { - Tranexamic acid group: } \mathrm{n}=14, \mathrm{M} / \mathrm{F}=14 / 0, \text { mean }(\mathrm{sd}) \text { age }=56(7) \text { years } \\
\text { - Control group: } \mathrm{n}=14, \mathrm{M} / \mathrm{F}=11 / 3 \text {, mean }(\mathrm{sd}) \text { age }=59(10) \text { years }\end{array}$} \\
\hline Interventions & \multicolumn{2}{|c|}{$\begin{array}{l}\text { - Aprotinin group received a test dose of } 30,000 \mathrm{KIU} \text { at anesthesia induction and } 1 \text { million } \\
\text { KIU of aprotinin was added to the pump prime. After protamine administration further } \\
\text { aprotinin was administered in a dose of } 200,000 \mathrm{KIU} / \mathrm{hr} \text { for another } 5 \text { hours. } \\
\text { - Tranexamic acid group received } 10 \mathrm{mg} / \mathrm{kg} \text { as a bolus after heparinization followed by a } \\
\text { continuous intravenous infusion of } 1 \mathrm{mg} / \mathrm{kg} / \mathrm{hr} \text { over } 10 \text { hours. } \\
\text { - Control treatment was not specified. }\end{array}$} \\
\hline Outcomes & \multicolumn{2}{|c|}{$\begin{array}{l}\text { Outcomes reported: Number of patients exposed to allogeneic blood, allogeneic blood usage (units) } \\
\text {, blood loss ( } 6 \mathrm{hrs}) \text {, mortality, change in haemoglobin levels - baseline to } 24 \mathrm{hrs} \text { post-operative. }\end{array}$} \\
\hline Notes & \multicolumn{2}{|c|}{$\begin{array}{l}\text { Quality assessment score (Schulz criteria): 1/7 } \\
\text { Transfusion protocol used }\end{array}$} \\
\hline \multicolumn{3}{|l|}{ Risk of bias } \\
\hline Item & Authors' judgement & Description \\
\hline Adequate sequence generation? & Unclear & Unclear \\
\hline Allocation concealment? & Unclear & Unclear \\
\hline $\begin{array}{l}\text { Blinding? } \\
\text { All outcomes }\end{array}$ & No & Single blind \\
\hline
\end{tabular}


Mohr 1992

\begin{tabular}{|c|c|c|}
\hline Methods & \multicolumn{2}{|c|}{$\begin{array}{l}\text { Method of randomisation and allocation concealment were not described. No loss to follow-up } \\
\text { reported. }\end{array}$} \\
\hline Participants & \multicolumn{2}{|c|}{$\begin{array}{l}50 \text { patients undergoing primary coronary artery bypass graft surgery }(\mathrm{CABG}) \text {, repeat CABG, valve } \\
\text { replacement, or valve replacement }+\mathrm{CABG} \text { surgery were randomised to one of three groups: } \\
\text { - Aprotinin group (High dose): } \mathrm{n}=17, \mathrm{M} / \mathrm{F}=14 / 3 \text {, mean }(\mathrm{sd}) \text { age }=58(10) \text { years } \\
\text { - Aprotinin group (Low dose): } \mathrm{n}=17, \mathrm{M} / \mathrm{F}=14 / 3 \text {, mean }(\mathrm{sd}) \text { age }=62(10) \text { years } \\
\text { - Control group (Placebo): } \mathrm{n}=16, \mathrm{M} / \mathrm{F}=11 / 5 \text {, mean }(\mathrm{sd}) \text { age }=63(11) \text { years }\end{array}$} \\
\hline Interventions & \multicolumn{2}{|c|}{$\begin{array}{l}\text { - Aprotinin group (High dose) received a loading dose of } 2 \text { million KIU of aprotinin for } 20 \\
\text { minutes before sternotomy. An additional } 2 \text { million KIU of aprotinin was added to the priming } \\
\text { volume of the bubble oxygenator, and a continuous infusion of } 500,000 \mathrm{KIU} / \mathrm{hr} \text { was given after } \\
\text { the loading dose throughout surgery until skin closure or a total of } 6 \text { million KIU of aprotinin } \\
\text { was achieved. } \\
\text { - Aprotinin group (Low dose) received placebo (saline } 0.9 \% \text { ) as a loading dose, } 2 \text { million KIU } \\
\text { of aprotinin in the pump prime, and placebo in the continuous infusion phase. } \\
\text { - Control group received equal volumes of placebo solution ( } 0.9 \% \text { saline) at the respective } \\
\text { times. }\end{array}$} \\
\hline Outcomes & \multicolumn{2}{|c|}{$\begin{array}{l}\text { Outcomes reported: Number of patients exposed to allogeneic blood, allogeneic blood usage (units) } \\
\text {, blood loss ( } 24 \mathrm{hrs} \text { ), re-operation for bleeding, post-operative platelet counts, platelet aggregation } \\
\text { evaluation by scanning electron microscopy. }\end{array}$} \\
\hline Notes & \multicolumn{2}{|c|}{$\begin{array}{l}\text { Quality assessment score (Schulz criteria): 4/7 } \\
\text { Transfusion protocol used }\end{array}$} \\
\hline \multicolumn{3}{|l|}{ Risk of bias } \\
\hline Item & Authors' judgement & Description \\
\hline Adequate sequence generation? & Unclear & Unclear \\
\hline Allocation concealment? & Unclear & Unclear \\
\hline $\begin{array}{l}\text { Blinding? } \\
\text { All outcomes }\end{array}$ & Yes & Double blind \\
\hline
\end{tabular}

\section{Mongan 1998}

\begin{tabular}{l|l}
\hline Methods & $\begin{array}{l}\text { Patients were randomised using a computer-generated random sequence. Method used to conceal } \\
\text { treatment allocation was not described. }\end{array}$ \\
\hline Participants & $\begin{array}{l}150 \text { patients undergoing primary coronary artery bypass graft surgery were randomised to one of } \\
\text { two groups: } \\
\text { - Aprotinin group }(\text { High dose) }: \mathrm{n}=75, \mathrm{M} / \mathrm{F}=61 / 14, \text { mean }(\mathrm{sd}) \text { age }=62(10) \text { years } \\
\text { - Tranexamic acid }(\mathrm{n}=75), \mathrm{M} / \mathrm{F}=59 / 16, \text { mean }(\mathrm{sd}) \text { age }=61(11) \text { years }\end{array}$ \\
\hline
\end{tabular}




\begin{tabular}{|c|c|c|}
\hline Interventions & \multicolumn{2}{|c|}{$\begin{array}{l}\text { - Aprotinin group (High dose) received a loading dose of } 2 \text { million KIU }(280 \mathrm{mg} \text { in } 200 \mathrm{ml}) \\
\text { administered before skin incision and a continuous infusion of } 500,000 \mathrm{KIU} / \mathrm{hr}(3 \mathrm{million} \mathrm{KIU} \\
\text { in } 300 \mathrm{ml}) \text { limited to the subsequent } 6 \text { hours. An additional } 2 \text { million KIU (280mg in } 200 \mathrm{ml}) \text { was } \\
\text { added to the pump prime. } \\
\text { - Tranexamic acid group received a loading dose of } 1 \mathrm{~g}(15 \mathrm{mg} / \mathrm{kg}) \text { administered before skin } \\
\text { incision and a continuous infusion of } 1 \mathrm{~g} \text { infused at } 50 \mathrm{ml} / \mathrm{hr}(2 \mathrm{mg} / \mathrm{kg} / \mathrm{hr} \text { in } 300 \mathrm{ml}) \text { limited to the } \\
\text { subsequent } 6 \text { hours. Normal saline solution }(300 \mathrm{ml}) \text { was added to the pump prime. }\end{array}$} \\
\hline Outcomes & \multicolumn{2}{|c|}{$\begin{array}{l}\text { Outcomes reported: Number of patients exposed to allogeneic blood, allogeneic blood usage (units) } \\
\text {, mortality, myocardial infarction, re-operation for bleeding, stroke. }\end{array}$} \\
\hline Notes & \multicolumn{2}{|c|}{$\begin{array}{l}\text { Quality assessment score (Schulz criteria): 5/7 } \\
\text { Transfusion protocol used }\end{array}$} \\
\hline \multicolumn{3}{|l|}{ Risk of bias } \\
\hline Item & Authors' judgement & Description \\
\hline Adequate sequence generation? & Yes & Computer-generated random sequence \\
\hline Allocation concealment? & Unclear & Unclear \\
\hline $\begin{array}{l}\text { Blinding? } \\
\text { All outcomes }\end{array}$ & Yes & Double blind \\
\hline
\end{tabular}

\section{Moran 2000}

\begin{tabular}{|c|c|}
\hline Methods & $\begin{array}{l}\text { Patients were randomly assigned by a computer-generated code. Method used to conceal treatment } \\
\text { allocation was not described. }\end{array}$ \\
\hline Participants & $\begin{array}{l}42 \text { patients undergoing coronary artery bypass surgery were randomised to one of three groups: } \\
\text { - Aprotinin group (High dose): } \mathrm{n}=12, \mathrm{M} / \mathrm{F}=11 / 1 \text {, mean }(\mathrm{sd}) \text { age }=58.0(8.4) \text { years } \\
\text { - Aprotinin group (Low dose): } \mathrm{n}=12, \mathrm{M} / \mathrm{F}=12 / 4 \text {, mean }(\mathrm{sd}) \text { age }=59.6(10.7) \text { years } \\
\text { - Control group (placebo) }(\mathrm{n}=14), \mathrm{M} / \mathrm{F}=11 / 3 \text {, mean }(\mathrm{sd}) \text { age }=59.7(8.6) \text { years } \\
\text { NB: Four patients were excluded from the final efficacy analysis. All } 42 \text { patients were included in } \\
\text { the safety analysis. }\end{array}$ \\
\hline Interventions & $\begin{array}{l}\text { - Aprotinin group (High dose) received a total dose of } 6 \text { million KIU }(840 \mathrm{mg}) \text { of aprotinin. } \\
\text { Prior to anaesthesia } 2 \text { million KIU ( } 280 \mathrm{mg} \text { ) of aprotinin was administered and another } 2 \text { million } \\
\text { KIU ( } 280 \mathrm{mg} \text { ) was added to the pump prime. An additional } 2 \text { million KIU }(280 \mathrm{mg}) \text { was } \\
\text { administered after the completion of CPB. } \\
\text { - Aprotinin group (Low dose) received a total dose of } 4 \text { million KIU }(560 \mathrm{mg}) \text { of aprotinin. } \\
\text { Prior to anaesthesia } 2 \text { million KIU ( } 280 \mathrm{mg} \text { ) of aprotinin was administered and another } 2 \text { million } \\
\text { KIU ( } 280 \mathrm{mg} \text { ) was added to the pump prime. } \\
\text { - Control group received } 600 \mathrm{ml} \text { of normal saline solution. }\end{array}$ \\
\hline
\end{tabular}
myocardial infarction, re-operation for bleeding, stroke. 
Moran 2000 (Continued)

\begin{tabular}{|c|c|c|}
\hline Notes & $\begin{array}{l}\text { Quality assessment score (Schulz criteria): 4/7 } \\
\text { Transfusion protocol used }\end{array}$ & \\
\hline \multicolumn{3}{|l|}{ Risk of bias } \\
\hline Item & Authors' judgement & Description \\
\hline Adequate sequence generation? & Yes & Computer-generated code \\
\hline Allocation concealment? & Unclear & Unclear \\
\hline $\begin{array}{l}\text { Blinding? } \\
\text { All outcomes }\end{array}$ & Yes & Double blind \\
\hline
\end{tabular}

\section{Murkin 1994}

Methods

Method of allocation concealment was not described. Randomisation was by means of computergenerated random code.

Participants
$\begin{aligned} & 54 \text { patients undergoing first-time coronary artery bypass or valvular heart operations requiring } \\ & \text { cardiopulmonary bypass }(\mathrm{CPB}) \text { were randomised to one of two groups: } \\ & \text { - Aprotinin group: } \mathrm{n}=29, \mathrm{M} / \mathrm{F}=22 / 7, \text { mean }(\mathrm{sd}) \text { age }=62(9.7) \text { years } \\ & \text { - Control group (Placebo): } \mathrm{n}=25, \mathrm{M} / \mathrm{F}=16 / 9 \text {, mean }(\mathrm{sd}) \text { age }=65.8(7.5) \text { years }\end{aligned}$
$\begin{aligned} & \text { NB: Three of the } 57 \text { enrolled patients were deemed ineligible because of cancellation of the operation } \\ & (\mathrm{n}=2) \text { and non-use of } \mathrm{CPB}(\mathrm{n}=1) \text {. All } 54 \text { remaining patients were included for analysis. }\end{aligned}$

Interventions

- Aprotinin group received 2 million KIU of aprotinin $(200 \mathrm{ml})$ as a loading dose including an initial $5 \mathrm{ml}$ dose given after establishment of full monitoring and anaesthesia, 2 million KIU of aprotinin was added to the $\mathrm{CPB}$ pump prime, and a continuous infusion of 500,000 KIU/hr of aprotinin was given throughout the operation and for 1 hour after the patient had returned to the intensive care unit (ICU). The maximum dose of aprotinin was 7 million KIU.

- Control group received equal volumes of placebo (substance not specified).

NB: Both groups were exposed to cell salvage.

Outcomes

Outcomes reported: Number of patients exposed to allogeneic blood, allogeneic blood usage (units) , fresh frozen plasma usage (units), platelet usage (units), blood loss (36hrs), myocardial infarction, pulmonary embolic events, hospital length of stay (days).

Notes $\quad$ Quality assessment score (Schulz criteria): 4/7

Transfusion protocol used

\section{Risk of bias}

\begin{tabular}{l|ll}
\hline Item & Authors' judgement & Description \\
\hline Adequate sequence generation? & Yes & Computer-generated random code
\end{tabular}


Murkin 1994 (Continued)

\begin{tabular}{l|ll}
\hline Allocation concealment? & Unclear & Unclear \\
\hline $\begin{array}{l}\text { Blinding? } \\
\text { All outcomes }\end{array}$ & Yes & Double blind \\
\hline
\end{tabular}

\section{Murkin 1995}

\begin{tabular}{|c|c|c|}
\hline Methods & \multicolumn{2}{|c|}{$\begin{array}{l}\text { Randomisation was by means of computer generated codes. Method of allocation concealment was } \\
\text { not described. }\end{array}$} \\
\hline Participants & \multicolumn{2}{|c|}{$\begin{array}{l}53 \text { consecutive patients undergoing revision total hip arthroplasty or primary bilateral total hip } \\
\text { arthroplasty were randomly assigned to one of two groups: } \\
\text { - Aprotinin group: } \mathrm{n}=29, \mathrm{M} / \mathrm{F}=9 / 20 \text {, mean }(\text { sd) age }=66.9(15) \text { years } \\
\text { - Control group (Placebo): } \mathrm{n}=24, \mathrm{M} / \mathrm{F}=11 / 13 \text {, mean }(\mathrm{sd}) \text { age }=65.5 \text { (16.6) years }\end{array}$} \\
\hline Interventions & \multicolumn{2}{|c|}{$\begin{array}{l}\text { - Aprotinin group received } 2 \text { million KIU }(200 \mathrm{ml}) \text { of aprotinin over } 15 \text { minutes followed by } \\
\text { an infusion of } 500,000 \mathrm{KIU}(50 \mathrm{ml}) \text { per hour. Those patients weighing less than } 60 \mathrm{~kg} \text { and more } \\
\text { than } 80 \mathrm{~kg} \text { received a loading dose of } 2.8 \mathrm{ml} / \mathrm{kg}(10,000 \mathrm{KIU} / \mathrm{ml}) \text { and an infusion of } 0.7 \mathrm{ml} / \mathrm{kg} / \mathrm{hr} \text {. } \\
\text { - Control group received an equivalent volume of } 0.9 \% \text { saline. }\end{array}$} \\
\hline Outcomes & \multicolumn{2}{|c|}{$\begin{array}{l}\text { Outcomes reported: Number of patients exposed to allogeneic blood, allogeneic blood usage (units) } \\
\text {, blood loss, cerebrovascular accident, deep vein thrombosis, } \\
\text { hospital length of stay (days). }\end{array}$} \\
\hline Notes & \multicolumn{2}{|c|}{$\begin{array}{l}\text { Quality assessment score (Schulz criteria): 5/7 } \\
\text { Transfusion protocol used }\end{array}$} \\
\hline \multicolumn{3}{|l|}{ Risk of bias } \\
\hline Item & Authors' judgement & Description \\
\hline Adequate sequence generation? & Yes & Computer generated codes \\
\hline Allocation concealment? & Unclear & Unclear \\
\hline $\begin{array}{l}\text { Blinding? } \\
\text { All outcomes }\end{array}$ & Yes & Double blind \\
\hline
\end{tabular}

Murkin 2000

Methods

Labels on all medication vials were the same except for the patient executive number. Patients were stratified on the basis of whether or not pre-operative autologous blood donations had been made.

Participants

301 undergoing elective primary unilateral total hip replacement were randomised to one of four groups:

- Aprotinin group (Low dose): $\mathrm{n}=69, \mathrm{M} / \mathrm{F}=34 / 35$, mean age $=63.7$ years

- Aprotinin group (Medium dose) $\mathrm{n}=68, \mathrm{M} / \mathrm{F}=27 / 41$, mean age $=65.5$ years 
Murkin 2000 (Continued)

\begin{tabular}{|c|c|}
\hline & $\begin{array}{l}\text { - Aprotinin group (High dose): } \mathrm{n}=75, \mathrm{M} / \mathrm{F}=46 / 29 \text {, mean age }=63.4 \text { years } \\
\text { - Control group (Placebo): } \mathrm{n}=68, \mathrm{M} / \mathrm{F}=32 / 36, \text { mean age }=63.2 \text { years }\end{array}$ \\
\hline Interventions & $\begin{array}{l}\text { - Aprotinin group (Low dose) received a loading dose of } 500,000 \mathrm{KIU}(70 \mathrm{mg}) \text { of aprotinin. } \\
\text { - Aprotinin group (Medium dose) received a loading dose of } 1 \text { million KIU (140mg) of } \\
\text { aprotinin and a continuous infusion of } 250,000 \mathrm{KIU} / \mathrm{hr} \text {. } \\
\text { - Aprotinin group (High dose) received a loading dose of } 2 \text { million KIU }(280 \mathrm{mg}) \text { of aprotinin } \\
\text { and a continuous infusion of } 500,000 \mathrm{KIU} / \mathrm{hr} \text {. } \\
\text { - Control group received an unspecified placebo. } \\
\text { NB: Epsilon aminocaproic acid and desmopressin were used if deemed necessary. Data regarding } \\
\text { the use of these two drugs to minimise blood loss were not reported. All groups used pre-operative } \\
\text { autologous donation. }\end{array}$ \\
\hline
\end{tabular}

Outcomes

Outcomes reported: Number of patients exposed to allogeneic blood, blood loss, myocardial infarction, mortality, deep venous thrombosis, pre-operative autologous blood donation.

$\begin{array}{ll}\text { Notes } & \text { Quality assessment score (Schulz criteria): 6/7 } \\ \text { Transfusion protocol used }\end{array}$

Transfusion protocol used

\section{Risk of bias}

\begin{tabular}{lll}
\hline Item & Authors' judgement & Description \\
\hline Adequate sequence generation? & Unclear & Unclear \\
\hline Allocation concealment? & Yes & Adequate \\
\hline $\begin{array}{l}\text { Blinding? } \\
\text { All outcomes }\end{array}$ & Yes & Double blind \\
\hline
\end{tabular}

\section{Murphy 2006}

\begin{tabular}{l|l}
\hline Methods & Allocation was generated by a card system and concealed in sealed, opaque envelopes. \\
\hline Participants & $\begin{array}{l}100 \text { off-pump coronary artery bypass grafting surgical patients were randomly allocated to one of } \\
\text { two groups: } \\
\text { - Tranexamic acid group: } \mathrm{n}=50, \mathrm{M} / \mathrm{F}=42 / 8, \text { mean }(\mathrm{sd}) \text { age }=64.9(7.0) \text { years } \\
\text { - Control group (Placebo): } \mathrm{n}=50, \mathrm{M} / \mathrm{F}=37 / 13, \text { mean }(\mathrm{sd}) \text { age }=65.8(8.7) \text { years }\end{array}$ \\
\hline Interventions & $\begin{array}{l}\text { - Tranexamic acid group received } 2 \mathrm{~g} \text { as an intravenous bolus before sternotomy. } \\
\text { - Control group received a bolus of normal saline. }\end{array}$ \\
\hline NB: All patients underwent peri-operative cell salvage with autotransfusion of washed salvaged red \\
blood cells at the completion of the operative procedure.
\end{tabular}

Outcomes

Outcomes reported: number of patients exposed to allogeneic blood transfusion, blood loss, mortality, stroke, renal dysfunction, myocardial infarction, length of stay. 


\begin{tabular}{|c|c|c|}
\hline Notes & $\begin{array}{l}\text { Quality assessment score (Schulz criteria): 5/7 } \\
\text { Transfusion protocol used }\end{array}$ & \\
\hline \multicolumn{3}{|l|}{ Risk of bias } \\
\hline Item & Authors' judgement & Description \\
\hline Adequate sequence generation? & Unclear & Unclear \\
\hline Allocation concealment? & No & Inadequate - sealed envelopes \\
\hline $\begin{array}{l}\text { Blinding? } \\
\text { All outcomes }\end{array}$ & Yes & Double blind \\
\hline
\end{tabular}

\section{Niskanen 2005}

\begin{tabular}{|c|c|c|}
\hline Methods & \multicolumn{2}{|c|}{$\begin{array}{l}\text { Patients were randomised into two groups by an envelope method in a double-blind manner. The } \\
\text { randomisation and preparation of the drug were done in the absence of other personnel by two } \\
\text { anaesthesia nurses not engaged in the study. The code was broken after the last patient had been } \\
\text { treated. }\end{array}$} \\
\hline Participants & \multicolumn{2}{|c|}{$\begin{array}{l}40 \text { patients undergoing cemented hip arthroplasty were randomised to one of two groups: } \\
\text { - Tranexamic acid group: } \mathrm{n}=19, \mathrm{M} / \mathrm{F}=6 / 13 \text {, mean }(\mathrm{sd}) \text { age }=66(9.1) \text { years } \\
\text { - Control group (Placebo): } \mathrm{n}=20, \mathrm{M} / \mathrm{F}=7 / 13 \text {, mean }(\mathrm{sd}) \text { age }=65(8.2) \text { years } \\
\text { NB: One patient was excluded from the final analysis. }\end{array}$} \\
\hline Interventions & \multicolumn{2}{|c|}{$\begin{array}{l}\text { - Tranexamic acid group received } 10 \mathrm{mg} / \mathrm{kg} \text { of intravenous TXA over } 5-10 \text { minutes, } \\
\text { immediately before the operation. The next two doses were given } 8 \text { hours and } 16 \text { hours after the } \\
\text { first injection. } \\
\text { - Control group received corresponding doses of saline. }\end{array}$} \\
\hline Outcomes & \multicolumn{2}{|c|}{$\begin{array}{l}\text { Outcomes reported: Number of patients exposed to allogeneic blood, allogeneic blood usage (units) } \\
\text {, deep vein thrombosis. }\end{array}$} \\
\hline Notes & \multicolumn{2}{|c|}{$\begin{array}{l}\text { Quality assessment score (Schulz criteria): 6/7 } \\
\text { Transfusion protocol used }\end{array}$} \\
\hline \multicolumn{3}{|l|}{ Risk of bias } \\
\hline Item & Authors' judgement & Description \\
\hline Adequate sequence generation? & Unclear & Unclear \\
\hline Allocation concealment? & Yes & Adequate \\
\hline $\begin{array}{l}\text { Blinding? } \\
\text { All outcomes }\end{array}$ & Yes & Double blind \\
\hline
\end{tabular}


Norman 2009

\begin{tabular}{|c|c|c|}
\hline Methods & \multicolumn{2}{|c|}{$\begin{array}{l}\text { Patients were allocated according to a randomisation schedule based on study accession number. } \\
\text { Pharmacy controlled allocation. }\end{array}$} \\
\hline Participants & \multicolumn{2}{|c|}{$\begin{array}{l}20 \text { undergoing extrapleural pneumonectomy for mesothelioma were randomised to one of two } \\
\text { groups: } \\
\text { - Aprotinin group: } n=11, M / F=0 / 9 \text {, mean }(s d) \text { age }=63.5(6.2) \text { years } \\
\text { - Control group (Placebo): } n=9, M / F=8 / 3 \text {, mean (sd) age }=62(7.6) \text { years }\end{array}$} \\
\hline Interventions & \multicolumn{2}{|c|}{$\begin{array}{l}\text { - Aprotinin group (High dose) received a loading dose of } 2 \text { million KIU infused over } 1 \text { hour, } \\
\text { followed by maintenance infusion of 500,000 KIU/hr until ICU admission. } \\
\text { - Control group received a saline placebo. }\end{array}$} \\
\hline Outcomes & \multicolumn{2}{|c|}{$\begin{array}{l}\text { Outcomes reported: Number of patients exposed to allogeneic blood transfusion, blood loss, mor- } \\
\text { tality. }\end{array}$} \\
\hline Notes & \multicolumn{2}{|c|}{$\begin{array}{l}\text { Quality assessment score (Schulz criteria): } 7 / 7 \\
\text { Use of transfusion protocol not reported }\end{array}$} \\
\hline \multicolumn{3}{|l|}{ Risk of bias } \\
\hline Item & Authors' judgement & Description \\
\hline Adequate sequence generation? & Yes & $\begin{array}{l}\text { Randomisation schedule based on study acces- } \\
\text { sion number }\end{array}$ \\
\hline Allocation concealment? & Yes & Adequate \\
\hline $\begin{array}{l}\text { Blinding? } \\
\text { All outcomes }\end{array}$ & Yes & Double blind \\
\hline
\end{tabular}

Nurözler 2008

Methods $\quad$ Patients were allocated according to a list of random treatment codes. Method used to conceal treatment allocation was not described.

\begin{tabular}{l|l}
\hline Participants & $\begin{array}{l}51 \text { undergoing cardiac surgery were randomised to one of two groups: } \\
\text { - Aprotinin group: } \mathrm{n}=25, \mathrm{M} / \mathrm{F}=19 / 6, \text { mean }(\mathrm{sd}) \text { age }=63.1(8.8) \text { years } \\
\text { - Control group (Placebo): } \mathrm{n}=26, \mathrm{M} / \mathrm{F}=18 / 8, \text { mean }(\mathrm{sd}) \text { age }=64.6(6.7) \text { years }\end{array}$ \\
\hline Interventions & $\begin{array}{l}\text { - Aprotinin group (low dose) received bolus } 1 \text { million KIU infused over } 30 \text { minutes, then } \\
\text { continuous infusion of } 500,000 \mathrm{KIU} / \mathrm{hr} \text { until end of surgery. } \\
\text { - Control group received a saline placebo. }\end{array}$
\end{tabular}

Outcomes

Outcomes reported: Number of patients exposed to allogeneic blood transfusion, volume blood transfused (units), blood loss, re-operation for bleeding, myocardial infarction, stroke, length of hospital stay (days). 
Nurözler 2008 (Continued)

\begin{tabular}{|c|c|c|}
\hline Notes & $\begin{array}{l}\text { Quality assessment score (Schulz criteria): 3/7 } \\
\text { Transfusion protocol used }\end{array}$ & \\
\hline \multicolumn{3}{|l|}{ Risk of bias } \\
\hline Item & Authors' judgement & Description \\
\hline Adequate sequence generation? & Yes & List of random treatment codes \\
\hline Allocation concealment? & Unclear & Unclear \\
\hline $\begin{array}{l}\text { Blinding? } \\
\text { All outcomes }\end{array}$ & Yes & Double blind \\
\hline
\end{tabular}

Nuttall 2000

Methods Patients were randomly assigned by a computer-generated random number sequence. Method used to conceal treatment allocation was not described.

Participants

168 patients undergoing cardiac surgery were randomised to one of four groups:

- Aprotinin group (High dose) $: \mathrm{n}=40, \mathrm{M} / \mathrm{F}=28 / 12$, median (range) age $=70.5$ (45-86) years

- Tranexamic acid group: $\mathrm{n}=45, \mathrm{M} / \mathrm{F}=31 / 14$, median (range) age $=71(43-83)$ years

- Tranexamic acid + acute normovolaemic haemodilution (ANH) group: $\mathrm{n}=32, \mathrm{M} / \mathrm{F}=28 / 4$, median (range) age $=67.5(42-91)$ years

- Control group (Placebo): $\mathrm{n}=43, \mathrm{M} / \mathrm{F}=35 / 8$, median (range) age $=63$ (29-83) years

NB: Eight patients were excluded from the final analysis.

Interventions

- Aprotinin group (High dose) received a test dose of $1.4 \mathrm{mg}(1 \mathrm{ml})$ followed by a loading dose of $280 \mathrm{mg}$ of aprotinin over 20-30 minutes. In addition, patients received a continuous infusion of $70 \mathrm{mg} / \mathrm{hr}(50 \mathrm{ml} / \mathrm{hr})$ of aprotinin and $280 \mathrm{mg}(200 \mathrm{ml})$ was added to the pump prime.

- Tranexamic acid group received a loading dose of $10 \mathrm{mg} / \mathrm{kg}$ and a continuous infusion of $1 \mathrm{mg} / \mathrm{kg} / \mathrm{hr}$ commenced after central venous cannulation and continued for 2 hours into treatment in intensive care.

- Tranexamic acid + ANH group received a loading dose of $10 \mathrm{mg} / \mathrm{kg}$ and a continuous infusion of $1 \mathrm{mg} / \mathrm{kg} / \mathrm{hr}$ commenced after central venous cannulation and continued for 2 hours into treatment in intensive care. In addition, patients received intra-operative autologous blood (12.5\% of whole blood volume withdrawn before $\mathrm{CPB}$ and within 10 mins after central venous cannulation).

- Control group received a normal saline infusion.

Outcomes

Outcomes reported: Number of patients exposed to allogeneic blood, allogeneic blood usage (units) , mortality, re-operation for bleeding.

$\begin{array}{ll}\text { Notes } & \text { Quality assessment score (Schulz criteria): 4/7 } \\ \text { Transfusion protocol used }\end{array}$

Risk of bias 
Nuttall 2000 (Continued)

\begin{tabular}{|c|c|c|}
\hline Item & Authors' judgement & Description \\
\hline Adequate sequence generation? & Yes & Computer-generated random number sequence \\
\hline Allocation concealment? & Unclear & Unclear \\
\hline $\begin{array}{l}\text { Blinding? } \\
\text { All outcomes }\end{array}$ & Yes & Double blind \\
\hline
\end{tabular}

\section{Okita 1996}

\begin{tabular}{|c|c|c|}
\hline Methods & \multicolumn{2}{|c|}{ Method of randomisation and allocation concealment were not described. } \\
\hline Participants & \multicolumn{2}{|c|}{$\begin{array}{l}60 \text { patients undergoing aortic surgery under deep hypothermic circulatory arrest were randomly } \\
\text { allocated to one of two groups: } \\
\text { - Aprotinin group: } \mathrm{n}=39, \mathrm{M} / \mathrm{F}=26 / 13 \text {, mean (sd) age }=63.5(8.9) \text { years } \\
\text { - Control group: } \mathrm{n}=21, \mathrm{M} / \mathrm{F}=16 / 5 \text {, mean (sd) age }=67.9(9.4) \text { years }\end{array}$} \\
\hline Interventions & \multicolumn{2}{|c|}{$\begin{array}{l}\text { - Aprotinin group received } 2 \text { million KIU of aprotinin administered in the pump prime only. } \\
\text { - Control group did not receive aprotinin. }\end{array}$} \\
\hline Outcomes & \multicolumn{2}{|c|}{$\begin{array}{l}\text { Outcomes reported: Number of patients exposed to allogeneic blood, allogeneic blood usage, blood } \\
\text { loss ( } 24 \text { hrs), mortality, myocardial infarction, stroke, renal failure, } \\
\text { respiratory failure + pneumonia. }\end{array}$} \\
\hline Notes & \multicolumn{2}{|c|}{$\begin{array}{l}\text { Quality assessment score (Schulz criteria): } 2 / 7 \\
\text { Transfusion protocol not specified }\end{array}$} \\
\hline \multicolumn{3}{|l|}{ Risk of bias } \\
\hline Item & Authors' judgement & Description \\
\hline Adequate sequence generation? & Unclear & Unclear \\
\hline Allocation concealment? & Unclear & Unclear \\
\hline $\begin{array}{l}\text { Blinding? } \\
\text { All outcomes }\end{array}$ & Unclear & Unclear \\
\hline
\end{tabular}


Orpen 2006

\begin{tabular}{|c|c|c|}
\hline Methods & \multicolumn{2}{|c|}{$\begin{array}{l}\text { A pharmacist not involved with the study carried out randomisation in the pharmacy by a sealed } \\
\text { envelope method and prepared the contents of the administered solution. The operating team was } \\
\text { blinded to the contents of the administered solution for every patient although allowance was made } \\
\text { for the code to be broken should an adverse drug reaction occur. }\end{array}$} \\
\hline Participants & \multicolumn{2}{|c|}{$\begin{array}{l}30 \text { patients undergoing total knee arthroplasty were randomised to one of two groups: } \\
\text { - Tranexamic acid group: } \mathrm{n}=15, \mathrm{M} / \mathrm{F}=8 / 7 \text {, mean }(95 \% \mathrm{CI}) \text { age }=73(70-78) \text { years } \\
\text { - Control group: } \mathrm{n}=14, \mathrm{M} / \mathrm{F}=3 / 11 \text {, mean }(95 \% \mathrm{CI}) \text { age }=69(63-74) \text { years } \\
\text { NB: One patient was excluded from the final analysis. }\end{array}$} \\
\hline Interventions & \multicolumn{2}{|c|}{$\begin{array}{l}\text { - Tranexamic acid group received } 15 \mathrm{mg} / \mathrm{kg} \text { of TXA intravenously at the time that cement } \\
\text { mixing commenced. } \\
\text { - Control group received an equivalent volume of normal saline given intravenously at the } \\
\text { time that cement mixing commenced. }\end{array}$} \\
\hline Outcomes & \multicolumn{2}{|c|}{$\begin{array}{l}\text { Outcomes reported: Number of patients exposed to allogeneic blood, blood loss, deep vein throm- } \\
\text { bosis, change in haemoglobin levels. }\end{array}$} \\
\hline Notes & \multicolumn{2}{|c|}{$\begin{array}{l}\text { Quality assessment score (Schulz criteria): 5/7 } \\
\text { Transfusion protocol used }\end{array}$} \\
\hline \multicolumn{3}{|l|}{ Risk of bias } \\
\hline Item & Authors' judgement & Description \\
\hline Adequate sequence generation? & Unclear & Unclear \\
\hline Allocation concealment? & Yes & Adequate \\
\hline $\begin{array}{l}\text { Blinding? } \\
\text { All outcomes }\end{array}$ & Yes & Double blind \\
\hline
\end{tabular}

Palmer 2003

\begin{tabular}{l|l}
\hline Methods & $\begin{array}{l}\text { A computer generated randomisation schedule was used to randomly assign patients into the } \\
\text { treatment groups. The vials used for each group were only identifiable by the patient study number. }\end{array}$ \\
\hline Participants & $\begin{array}{l}95 \text { patients undergoing elective neurological surgery were divided into two subsets: } \\
\text { Meningioma subset: } \mathrm{n}=56 \\
\text { - Aprotinin group: } \mathrm{n}=30, \mathrm{M} / \mathrm{F}=7 / 23, \text { mean }(\mathrm{sd}) \text { age }=58.4(13.0) \text { years } \\
\text { - Control group }(\mathrm{Placebo}): \mathrm{n}=26, \mathrm{M} / \mathrm{F}=9 / 17, \text { mean }(\mathrm{sd}) \text { age }=58.5(2.8) \text { years } \\
\text { Vestibular Schwannoma subset: } \mathrm{n}=39 \\
\text { - Aprotinin group: } \mathrm{n}=17, \mathrm{M} / \mathrm{F}=11 / 6, \text { mean }(\mathrm{sd}) \text { age }=48.6(10.9) \text { years } \\
\text { - Control group }(\mathrm{Placebo}): \mathrm{n}=17, \mathrm{M} / \mathrm{F}=11 / 16, \text { mean }(\mathrm{sd}) \text { age }=54.1(12.0) \text { years }\end{array}$ \\
\hline Interventions & $\begin{array}{l}\text { - Aprotinin group (Low dose) received a loading dose of } 30,000 \mathrm{KIU} / \mathrm{kg} \text { of aprotinin infused } \\
\text { over } 15-20 \text { minutes administered before the start of surgery and followed by a continuous } \\
\text { infusion of } 10,000 \mathrm{KIU} / \mathrm{kg} / \mathrm{hr} \text { until the patient was transferred to the Intensive Care Unit. }\end{array}$
\end{tabular}


Palmer 2003 (Continued)

- Control group received $0.9 \%$ sodium chloride solution.

\begin{tabular}{|c|c|c|}
\hline Outcomes & \multicolumn{2}{|c|}{$\begin{array}{l}\text { Outcomes reported: Number of patients exposed to allogeneic blood, allogeneic blood usage (units) } \\
\text {, blood loss, mortality (7-day \& 30-day). }\end{array}$} \\
\hline Notes & \multicolumn{2}{|c|}{$\begin{array}{l}\text { Quality assessment score (Schulz criteria): 6/7 } \\
\text { Transfusion protocol used }\end{array}$} \\
\hline \multicolumn{3}{|l|}{ Risk of bias } \\
\hline Item & Authors' judgement & Description \\
\hline Adequate sequence generation? & Yes & Computer generated randomisation schedule \\
\hline Allocation concealment? & Yes & Adequate \\
\hline $\begin{array}{l}\text { Blinding? } \\
\text { All outcomes }\end{array}$ & Yes & Double blind \\
\hline
\end{tabular}

\section{Parvizi 2007}

\begin{tabular}{|c|c|c|}
\hline Methods & \multicolumn{2}{|c|}{$\begin{array}{l}\text { Patients were allocated according to a computer-generated randomisation list. Adequacy of alloca- } \\
\text { tion concealment was unclear. }\end{array}$} \\
\hline Participants & \multicolumn{2}{|c|}{$\begin{array}{l}162 \text { undergoing cardiac surgery were randomised to one of two groups: } \\
\text { - Aprotinin group: } \mathrm{n}=81), \mathrm{M} / \mathrm{F}=49 / 32, \text { mean }(\mathrm{sd}) \text { age }=52.6(13.8) \text { years } \\
\text { - Control group (Placebo): } \mathrm{n}=81, \mathrm{M} / \mathrm{F}=49 / 32 \text {, mean }(\mathrm{sd}) \text { age }=54.1(11.4) \text { years }\end{array}$} \\
\hline Interventions & \multicolumn{2}{|c|}{$\begin{array}{l}\text { - Aprotinin group received 500,000 KIU infused before and 500,000 KIU during CPB. } \\
\text { - Control group received a saline placebo. }\end{array}$} \\
\hline Outcomes & \multicolumn{2}{|c|}{$\begin{array}{l}\text { Outcomes reported: Number of patients exposed to allogeneic blood transfusion, volume of blood } \\
\text { transfused, blood loss, myocardial infarction, re-operation for bleeding. }\end{array}$} \\
\hline Notes & \multicolumn{2}{|c|}{$\begin{array}{l}\text { Quality assessment score (Schulz criteria): 5/7 } \\
\text { Transfusion protocol used }\end{array}$} \\
\hline \multicolumn{3}{|l|}{ Risk of bias } \\
\hline Item & Authors' judgement & Description \\
\hline Adequate sequence generation? & Yes & Computer-generated randomisation list \\
\hline Allocation concealment? & Unclear & Unclear \\
\hline $\begin{array}{l}\text { Blinding? } \\
\text { All outcomes }\end{array}$ & Yes & Double blind \\
\hline
\end{tabular}


Penta de Peppo 1995

\begin{tabular}{|c|c|c|}
\hline Methods & \multicolumn{2}{|c|}{ Method of randomisation and allocation concealment were not described. } \\
\hline Participants & \multicolumn{2}{|c|}{$\begin{array}{l}60 \text { consecutive patients undergoing elective open-heart surgery were randomised to one of four } \\
\text { groups: } \\
\text { - Control group: } \mathrm{n}=15, \mathrm{M} / \mathrm{F}=13 / 2, \text { mean }(\mathrm{sd}) \text { age }=63(7) \text { years } \\
\text { - Epsilon aminocaproic acid group: } \mathrm{n}=15, \mathrm{M} / \mathrm{F}=13 / 2 \text {, mean }(\mathrm{sd}) \text { age }=62(7) \text { years } \\
\text { - Tranexamic acid group: } \mathrm{n}=15, \mathrm{M} / \mathrm{F}=12 / 3 \text {, mean }(\mathrm{sd}) \text { age }=60(12) \text { years } \\
\text { - Aprotinin group (High dose): } \mathrm{n}=15, \mathrm{M} / \mathrm{F}=12 / 3 \text {, mean (sd) age = } 64 \text { (10) years }\end{array}$} \\
\hline Interventions & \multicolumn{2}{|c|}{$\begin{array}{l}\text { - Control group received no antifibrinolytic treatment. } \\
\text { - Epsilon aminocaproic acid group received } 10 \mathrm{~g} \text { of EACA intravenously (IV) at the induction } \\
\text { of anaesthesia followed by an infusion of } 2 \mathrm{~g} / \mathrm{hr} \text { for } 5 \text { hours. } \\
\text { - Tranexamic acid group received } 10 \mathrm{mg} / \mathrm{kg} \text { of TXA IV within } 30 \text { minutes after the induction } \\
\text { of anaesthesia, followed by an infusion of } 1 \mathrm{mg} / \mathrm{kg} \text { per hour for } 10 \text { hours. } \\
\text { - Aprotinin group (High dose) received } 2 \text { million KIU of aprotinin IV at the induction of } \\
\text { anaesthesia followed by an infusion of } 500,000 \mathrm{KIU} / \mathrm{hr} \text { during surgery and } 2 \text { million KIU of } \\
\text { aprotinin added to the extracorporeal circuit. } \\
\text { NB: All groups were exposed to cell salvage. }\end{array}$} \\
\hline Outcomes & \multicolumn{2}{|c|}{$\begin{array}{l}\text { Outcomes reported: Number of patients exposed to allogeneic blood, allogeneic blood usage (units) } \\
\text {, blood loss, re-operation for bleeding, }\end{array}$} \\
\hline Notes & \multicolumn{2}{|c|}{$\begin{array}{l}\text { Quality assessment score (Schulz criteria): } 2 / 7 \\
\text { Transfusion protocol used }\end{array}$} \\
\hline \multicolumn{3}{|l|}{ Risk of bias } \\
\hline Item & Authors' judgement & Description \\
\hline Adequate sequence generation? & Unclear & Unclear \\
\hline Allocation concealment? & Unclear & Unclear \\
\hline $\begin{array}{l}\text { Blinding? } \\
\text { All outcomes }\end{array}$ & Unclear & Unclear \\
\hline
\end{tabular}

\section{Petsatodis 2006}

\begin{tabular}{l|l} 
Methods & $\begin{array}{l}\text { Patients were randomised using an envelope technique. Method of allocation concealment was not } \\
\text { described. }\end{array}$ \\
\hline Participants & $\begin{array}{l}50 \text { patients undergoing total hip arthroplasty were randomised to one of two groups: } \\
\text { - Aprotinin group: } \mathrm{n}=25, \text { mean }(\mathrm{sd}) \text { age }=58.4(12.5) \text { years } \\
\text { - Control group (Placebo): } \mathrm{n}=25, \text { mean }(\mathrm{sd}) \text { age }=59.6(10.9) \text { years }\end{array}$ \\
\hline Interventions & $\begin{array}{l}\text { - Aprotinin group received a bolus of } 20,000 \mathrm{KIU} / \mathrm{kg} \text { of aprotinin at the time of anaesthesia } \\
\text { followed by an infusion of } 50,000 \mathrm{KIU} / \mathrm{hr} . \\
\text { - Control group received normal saline in the same volumes. }\end{array}$
\end{tabular}




\section{Petsatodis 2006 (Continued)}

\begin{tabular}{|c|c|c|}
\hline Outcomes & $\begin{array}{l}\text { Outcomes reported: Number of patients expose } \\
\text {, blood loss, deep vein thrombosis. }\end{array}$ & to allogeneic blood, allogeneic blood usage (units) \\
\hline Notes & $\begin{array}{l}\text { Quality assessment score (Schulz criteria): 5/7 } \\
\text { Transfusion protocol not used }\end{array}$ & \\
\hline \multicolumn{3}{|l|}{ Risk of bias } \\
\hline Item & Authors' judgement & Description \\
\hline Adequate sequence generation? & Unclear & Unclear \\
\hline Allocation concealment? & Unclear & Inadequate - sealed envelopes \\
\hline $\begin{array}{l}\text { Blinding? } \\
\text { All outcomes }\end{array}$ & Yes & Double blind \\
\hline
\end{tabular}

\section{Pinosky 1997}

\begin{tabular}{l|l}
\hline Methods & Method of randomisation and allocation concealment were not described. \\
\hline Participants & $\begin{array}{l}59 \text { patients undergoing cardiac surgery were randomly assigned to one of three groups: } \\
\text { - Epsilon aminocaproic acid group: } \mathrm{n}=20, \mathrm{M} / \mathrm{F}=12 / 8, \text { mean }(\mathrm{sd}) \text { age }=62.6(9.4) \text { years } \\
\text { - Tranexamic acid group: } \mathrm{n}=20, \mathrm{M} / \mathrm{F}=12 / 18, \text { mean }(\mathrm{sd}) \text { age }=62.6(9.4) \text { years } \\
\text { - Control group (Placebo): } \mathrm{n}=19, \mathrm{M} / \mathrm{F}=15 / 4, \text { mean }(\mathrm{sd}) \text { age }=60.6(10.9) \text { years }\end{array}$ \\
\hline Interventions & $\begin{array}{l}\text { - Epsilon aminocaproic acid group received an intravenous loading dose of } 150 \mathrm{mg} / \mathrm{kg} \text { and a } \\
\text { continuous infusion of } 10 \mathrm{mg} / \mathrm{kg} / \mathrm{hr} \text { for } 6 \text { hours. EACA was given immediately following the } \\
\text { induction of anaesthesia. } \\
\text { - Tranexamic acid group received a loading dose of } 15 \mathrm{mg} / \mathrm{kg} \text { followed by a continuous infusion } \\
\text { of } 1 \mathrm{mg} / \mathrm{kg} / \mathrm{hr} \text { for } 6 \text { hours. TXA was given immediately following the induction of anaesthesia. } \\
\text { - Control group received a bolus of normal saline and a continuous infusion of normal saline } \\
\text { for } 6 \text { hours. } \\
\text { NB: Both groups were exposed to cell salvage. }\end{array}$ \\
\hline
\end{tabular}

Outcomes

Outcomes reported: Number of patients exposed to allogeneic blood, blood loss, aspirin use, number of patients exposed to platelets and fresh frozen plasma.

$\begin{array}{ll}\text { Notes } & \text { Quality assessment score (Schulz criteria): 2/7 } \\ \text { Transfusion protocol used }\end{array}$

\section{Risk of bias}

\begin{tabular}{l|ll}
\hline Item & Authors' judgement & Description \\
\hline Adequate sequence generation? & Unclear & Unclear
\end{tabular}


Pinosky 1997 (Continued)

\begin{tabular}{l|ll}
\hline Allocation concealment? & Unclear & Unclear \\
\hline $\begin{array}{l}\text { Blinding? } \\
\text { All outcomes }\end{array}$ & Yes & Double blind \\
\hline
\end{tabular}

\section{Pleym 2003}

\begin{tabular}{|c|c|c|}
\hline Methods & \multicolumn{2}{|c|}{$\begin{array}{l}\text { Randomisation was by mean of a computer programme. Study medications were delivered in } \\
\text { identical } 50 \mathrm{~mL} \text { syringes. }\end{array}$} \\
\hline Participants & \multicolumn{2}{|c|}{$\begin{array}{l}79 \text { patients undergoing elective cardiac surgery were randomised to one of two groups: } \\
\text { - Tranexamic acid group: } \mathrm{n}=40, \mathrm{M} / \mathrm{F}=34 / 6 \text {, mean }(\mathrm{sd}) \text { age }=63.6(9.9) \text { years } \\
\text { - Control group (Placebo): } \mathrm{n}=39, \mathrm{M} / \mathrm{F}=32 / 7 \text {, mean (sd) age }=62(9.2) \text { years }\end{array}$} \\
\hline Interventions & \multicolumn{2}{|c|}{$\begin{array}{l}\text { - Tranexamic acid group received } 30 \mathrm{mg} / \mathrm{kg} \text { of TXA as a bolus injection given over } 5 \text { minutes } \\
\text { immediately before the start of CPB. } \\
\text { - Control group received a bolus injection of the corresponding volume of } 0.9 \% \text { sodium } \\
\text { chloride solution given } 5 \text { minutes immediately before the start of CPB. } \\
\text { NB: Both groups were exposed to post-operative cell salvage, tranexamic acid, and desmopressin. }\end{array}$} \\
\hline Outcomes & \multicolumn{2}{|c|}{$\begin{array}{l}\text { Outcomes reported: Number of patients exposed to allogeneic blood, allogeneic blood usage (units) } \\
\text {, blood loss, re-operation for bleeding, fresh frozen plasma usage (units), platelet usage (units) } \\
\text {, pulmonary embolus, retransfused mediastinal shed blood, post-operative TXA, post-operative } \\
\text { DDAVP, ASA } 75 \mathrm{mg} / \text { day, ASA } 160 \mathrm{mg} / \text { day. }\end{array}$} \\
\hline Notes & \multicolumn{2}{|c|}{$\begin{array}{l}\text { Quality assessment score (Schulz criteria): 6/7 } \\
\text { Transfusion protocol used }\end{array}$} \\
\hline \multicolumn{3}{|l|}{ Risk of bias } \\
\hline Item & Authors' judgement & Description \\
\hline Adequate sequence generation? & Yes & Computer programme \\
\hline Allocation concealment? & Yes & Adequate \\
\hline $\begin{array}{l}\text { Blinding? } \\
\text { All outcomes }\end{array}$ & Yes & Double blind \\
\hline
\end{tabular}




The trial drug was provided double-blind by the manufacturer in blocks of 12 identical case packs.
Each case pack contained all bottles for one patient, identifiable only by the sequence number.
Each block of 12 case packs contained four packs of each dosage group, randomly assigned to the
sequence numbers 1 to 12 . Patients received the next available case pack of each block. Centres were
provided with sealed cards with the randomisation codes to enable an individual patient's code to
be broken in an emergency. A separate set of the sealed randomisation cards was kept at the Central
Data Centre. At the end of the study all cards with randomisation codes were sent to the Central
Data Centre.

\begin{tabular}{|c|c|}
\hline Participants & $\begin{array}{l}141 \text { patients undergoing orthotopic liver transplantation were randomised to one of three groups: } \\
\text { - Aprotinin group (High dose): } \mathrm{n}=46, \mathrm{M} / \mathrm{F}=34 / 12 \text {, median (range) age }=52(18-66) \text { years } \\
\text { - Aprotinin group (Low dose): } \mathrm{n}=43, \mathrm{M} / \mathrm{F}=34 / 19 \text {, median (range) age }=49(18-69) \text { years } \\
\text { - Control group (Placebo): } \mathrm{n}=48, \mathrm{M} / \mathrm{F}=36 / 12 \text {, median (range) age }=53(19-68) \text { years } \\
\text { NB: Four patients were excluded from the final analysis. }\end{array}$ \\
\hline
\end{tabular}

Interventions

- Aprotinin group (High dose) received a loading dose of 2 million KIU (280mg) of aprotinin over 20 minutes before and during the induction of anaesthesia, followed by a continuous infusion of 1 million KIU/hr (140mg/hr) until 2 hours after graft reperfusion. An additional dose of 1 million KIU was administered 30 minutes before graft reperfusion.

- Aprotinin group (Low dose) received a loading dose of 2 million KIU (280mg) of aprotinin over 20 minutes before and during the induction of anaesthesia, followed by a continuous infusion of 500,000 KIU/hr until 2 hours after graft reperfusion.

- Control group received $0.9 \%$ normal saline in an identical time schedule and volume.

Outcomes

Outcomes reported: Allogeneic blood usage (units), blood loss, number of patients exposed to platelets and cryoprecipitate.

\begin{tabular}{lll}
\hline Notes & $\begin{array}{l}\text { Quality assessment score (Schulz criteria): 5/7 } \\
\text { Transfusion protocol used }\end{array}$ \\
\hline Risk of bias & Authors' judgement & Description \\
\hline Item & Unclear & Unclear \\
\hline Adequate sequence generation? & Yes & Adequate \\
\hline Allocation concealment? & Yes & Double blind \\
\hline $\begin{array}{l}\text { Blinding? } \\
\text { All outcomes }\end{array}$ & & \\
\hline
\end{tabular}

Poston 2006

\begin{tabular}{l|l} 
Methods & $\begin{array}{l}\text { Study drug or placebo was delivered to the operating room in unlabeled bottles to maintain blinding. } \\
\text { Method of randomisation was not specified. }\end{array}$ \\
\hline Participants & $\begin{array}{l}70 \text { patients undergoing 'off-pump' coronary artery bypass graft surgery were randomised to one of } \\
\text { two groups: } \\
\text { - Aprotinin group (Low dose): } \mathrm{n}=29\end{array}$ \\
\hline
\end{tabular}




\begin{tabular}{|c|c|c|}
\hline & \multicolumn{2}{|c|}{$\begin{array}{l}\text { - Control group (Placebo): } \mathrm{n}=31 \\
\text { NB: Demographic data were not reported. }\end{array}$} \\
\hline Interventions & \multicolumn{2}{|c|}{$\begin{array}{l}\text { - Aprotinin group (Low dose) received } 10,000 \mathrm{KIU} \text { of aprotinin as a test dose followed by } 2 \\
\text { million KIU ( } 280 \mathrm{mg} \text { ) of aprotinin as a bolus before sternotomy, and } 500,000 \mathrm{KIU} / \mathrm{hr}(70 \mathrm{mg} / \mathrm{hr} \text { ) } \\
\text { of aprotinin as a continuous infusion until the end of surgery. } \\
\text { - Control group received normal saline. } \\
\text { NB: Both groups were exposed to cell salvage. }\end{array}$} \\
\hline Outcomes & \multicolumn{2}{|c|}{$\begin{array}{l}\text { Outcomes reported: Number of patients exposed to allogeneic blood, allogeneic blood usage (units), } \\
\text { fresh frozen plasma usage (units), platelet usage (units), myocardial infarction, deep vein thrombosis, } \\
\text { stroke, hospital length of stay (days), Intensive Care Unit length of stay (days). }\end{array}$} \\
\hline Notes & \multicolumn{2}{|c|}{$\begin{array}{l}\text { Quality assessment score (Schulz criteria): 4/7 } \\
\text { Transfusion protocol used }\end{array}$} \\
\hline \multicolumn{3}{|l|}{ Risk of bias } \\
\hline Item & Authors' judgement & Description \\
\hline Adequate sequence generation? & Unclear & Unclear \\
\hline Allocation concealment? & Yes & Adequate \\
\hline $\begin{array}{l}\text { Blinding? } \\
\text { All outcomes }\end{array}$ & Yes & Double blind \\
\hline
\end{tabular}

\section{Prendergast 11996}

\begin{tabular}{l|l}
\hline Methods & Method of randomisation and allocation concealment were not described. \\
\hline Participants & $\begin{array}{l}38 \text { patients undergoing primary sternotomy for heart transplantation were randomised to one of } \\
\text { two groups: } \\
\text { - Aprotinin group: } \mathrm{n}=18, \mathrm{M} / \mathrm{F}=15 / 3, \text { mean }(\mathrm{sd}) \text { age }=45.4(10.2) \text { years } \\
\text { - Control group: } \mathrm{n}=20, \mathrm{M} / \mathrm{F}=14 / 6, \text { mean }(\mathrm{sd}) \text { age }=49.3 \text { (6.7) years }\end{array}$ \\
\hline Interventions & $\begin{array}{l}\text { - Aprotinin group received } 200 \mathrm{ml} \text { of aprotinin as a loading dose intravenously followed by a } \\
\text { continuous infusion of aprotinin of } 50 \mathrm{ml} / \mathrm{hr} \text { until the end of the operation. In addition, 200ml of } \\
\text { aprotinin was added to the cardiopulmonary bypass circuit. } \\
\text { - Control group did not receive aprotinin. }\end{array}$ \\
\hline NB: Precise dose of aprotinin (KIU or mg) was not reported.
\end{tabular}


Prendergast'1 1996 (Continued)

\begin{tabular}{l|ll} 
Risk of bias & & \\
\hline Item & Authors' judgement & Description \\
\hline Adequate sequence generation? & Unclear & Unclear \\
\hline Allocation concealment? & Unclear & Unclear \\
\hline $\begin{array}{l}\text { Blinding? } \\
\text { All outcomes }\end{array}$ & Unclear & Unclear \\
\hline
\end{tabular}

\section{Prendergast 21996}

\begin{tabular}{|c|c|c|}
\hline Methods & \multicolumn{2}{|c|}{ Method of randomisation and allocation concealment were not described. } \\
\hline Participants & \multicolumn{2}{|c|}{$\begin{array}{l}32 \text { patients undergoing re-operative heart transplantation were randomised to one of two groups: } \\
\text { - Aprotinin group: } \mathrm{n}=16, \mathrm{M} / \mathrm{F}=14 / 2 \text {, mean }(\mathrm{sd}) \text { age }=54.4(6.9) \text { years } \\
\text { - Control group: } \mathrm{n}=16, \mathrm{M} / \mathrm{F}=13 / 3 \text {, mean }(\mathrm{sd}) \text { age }=55 \text { (10.6) years }\end{array}$} \\
\hline Interventions & \multicolumn{2}{|c|}{$\begin{array}{l}\text { - Aprotinin received a } 200 \mathrm{ml} \text { loading dose of aprotinin intravenously followed by a } \\
\text { continuous infusion of } 50 \mathrm{ml} / \mathrm{hr} \text { until the end of the operation. In addition, } 200 \mathrm{ml} \text { of aprotinin } \\
\text { was added to the cardiopulmonary bypass circuit. } \\
\text { - Control group received no aprotinin. } \\
\text { NB: Precise dose of aprotinin (KIU or } \mathrm{mg} \text { ) was not reported. }\end{array}$} \\
\hline Outcomes & \multicolumn{2}{|c|}{$\begin{array}{l}\text { Outcomes reported: Allogeneic blood usage (units), blood loss ( } 24 \mathrm{hrs} \text { ), haemoglobin levels, crea- } \\
\text { tinine levels. }\end{array}$} \\
\hline Notes & \multicolumn{2}{|c|}{$\begin{array}{l}\text { Quality assessment score (Schulz criteria): } 2 / 7 \\
\text { Transfusion protocol not specified }\end{array}$} \\
\hline \multicolumn{3}{|l|}{ Risk of bias } \\
\hline Item & Authors' judgement & Description \\
\hline Adequate sequence generation? & Unclear & Unclear \\
\hline Allocation concealment? & Unclear & Unclear \\
\hline $\begin{array}{l}\text { Blinding? } \\
\text { All outcomes }\end{array}$ & Unclear & Unclear \\
\hline
\end{tabular}


Pugh 1995

\begin{tabular}{|c|c|c|}
\hline Methods & \multicolumn{2}{|c|}{ Method of randomisation and allocation concealment were not described. } \\
\hline Participants & \multicolumn{2}{|c|}{$\begin{array}{l}75 \text { patients scheduled for routine primary cardiac surgery were randomly allocated to one of three } \\
\text { groups: } \\
\text { - Control group: } \mathrm{n}=23, \mathrm{M} / \mathrm{F}=16 / 7 \text {, mean }(\mathrm{sd}) \text { age }(+/-\mathrm{SD})=66(9.3) \text { years } \\
\text { - Tranexamic acid group: } \mathrm{n}=22, \mathrm{M} / \mathrm{F}=17 / 5 \text {, mean }(\mathrm{sd}) \text { age }=58(10) \text { years } \\
\text { - Aprotinin group: } \mathrm{n}=21, \mathrm{M} / \mathrm{F}=15 / 6 \text {, mean }(\mathrm{sd}) \text { age }=62(9.7) \text { years } \\
\text { NB: Nine patients were withdrawn from the trial: two from the control group, three from the } \\
\text { tranexamic acid group, and four from the aprotinin group. }\end{array}$} \\
\hline Interventions & \multicolumn{2}{|c|}{$\begin{array}{l}\text { - Control group received neither trial drug nor placebo preparation. } \\
\text { - Tranexamic acid group received } 2.5 \mathrm{~g} \text { of TXA before skin incision, with a further } 2.5 \mathrm{~g} \text { of } \\
\text { TXA added to the cardiopulmonary bypass }(\mathrm{CPB}) \text { solution. } \\
\text { - Aprotinin group received } 1 \text { million KIU of aprotinin before skin incision, with a further } 1 \\
\text { million KIU added to the priming solution. }\end{array}$} \\
\hline Outcomes & \multicolumn{2}{|c|}{$\begin{array}{l}\text { Outcomes reported: Number of patients exposed to allogeneic blood, allogeneic blood usage (units) } \\
\text {, number of patinets exposed to fresh frozen plasma, blood loss, } \\
\text { re-operation for bleeding. }\end{array}$} \\
\hline Notes & \multicolumn{2}{|c|}{$\begin{array}{l}\text { Quality assessment score (Schulz criteria): 1/7 } \\
\text { Transfusion protocol used }\end{array}$} \\
\hline \multicolumn{3}{|l|}{ Risk of bias } \\
\hline Item & Authors' judgement & Description \\
\hline Adequate sequence generation? & Unclear & Unclear \\
\hline Allocation concealment? & Unclear & Unclear \\
\hline $\begin{array}{l}\text { Blinding? } \\
\text { All outcomes }\end{array}$ & Unclear & Unclear \\
\hline
\end{tabular}

\section{Ranaboldo 1997}

Methods

Allocation concealment was by the use of identical coded bottles containing active drug or placebo. The method of randomisation was not described.

Participants
$\begin{aligned} & \text { 136 patients undergoing elective aortic surgery were randomised to one of two groups: } \\ & \text { - Aprotinin group: } \mathrm{n}=66, \mathrm{M} / \mathrm{F}=55 / 11, \text { median age }=68 \text { years } \\ & \text { NB: Eight patients were excluded from the final analysis. Four deaths occurred within } 7 \text { days of } \\ & \text { surgery (two in each group). Four patients were found at operation not to be suitable for the planned } \\ & \text { reconstructive surgery. }\end{aligned}$

Interventions

- Aprotinin group received 2 million KIU of aprotinin as a loading dose over a 20 minute period, followed by a maintenance infusion of $500,000 \mathrm{KIU} / \mathrm{hr}$.

- Control group received equal volumes of $0.9 \%$ normal saline. 
Ranaboldo 1997 (Continued)

\begin{tabular}{|c|c|c|}
\hline Outcomes & \multicolumn{2}{|c|}{$\begin{array}{l}\text { Outcomes reported: Number of patients exposed to allogeneic blood, allogeneic blood usage (units) } \\
\text {, blood loss ( } 24 \mathrm{hrs}) \text {, mortality ( } 30 \text { day), myocardial infarction, stroke, pulmonary embolus, deep } \\
\text { vein thrombosis, chest infection, hepatitis, sepsis, renal failure, urinary tract infection. }\end{array}$} \\
\hline Notes & \multicolumn{2}{|c|}{$\begin{array}{l}\text { Quality assessment score (Schulz criteria): 4/7 } \\
\text { Transfusion protocol used }\end{array}$} \\
\hline \multicolumn{3}{|l|}{ Risk of bias } \\
\hline Item & Authors' judgement & Description \\
\hline Adequate sequence generation? & Unclear & Unclear \\
\hline Allocation concealment? & Yes & Adequate \\
\hline $\begin{array}{l}\text { Blinding? } \\
\text { All outcomes }\end{array}$ & Yes & Double blind \\
\hline
\end{tabular}

\section{Rao 1999}

\begin{tabular}{|c|c|c|}
\hline Methods & \multicolumn{2}{|c|}{ Method of randomisation and allocation concealment were not described. } \\
\hline Participants & \multicolumn{2}{|c|}{$\begin{array}{l}30 \text { patients undergoing elective cardiac surgery were randomised to one of two groups: } \\
\text { - Epsilon aminocaproic acid group: } \mathrm{n}=15, \mathrm{M} / \mathrm{F}=13 / 2 \text {, mean age }=53 \text { years } \\
\text { - Control group: } \mathrm{n}=15, \mathrm{M} / \mathrm{F}=13 / 2 \text {, mean age }=55 \text { years }\end{array}$} \\
\hline Interventions & \multicolumn{2}{|c|}{$\begin{array}{l}\text { - Epsilon aminocaproic acid group received } 100 \mathrm{mg} / \mathrm{kg} \text { of EACA as a loading dose slowly after } \\
\text { the induction of anaesthesia and a continuous infusion of EACA at } 1 \mathrm{~g} / \mathrm{hr} \text { for a further } 6 \text { hours. } \\
\text { - Control group received no EACA treatment. }\end{array}$} \\
\hline Outcomes & \multicolumn{2}{|c|}{$\begin{array}{l}\text { Outcomes reported: Allogeneic blood usage (units), blood loss ( } 24 \mathrm{hrs}) \text {, myocardial infarction, } \\
\text { fresh frozen plasma usage (units), platelet usage (units), ASA treatment until surgery }(185 \mathrm{mg}) \text {, ASA } \\
\text { treatment until surgery }(375 \mathrm{mg}) \text {, stroke, re-operation for bleeding. }\end{array}$} \\
\hline Notes & \multicolumn{2}{|c|}{$\begin{array}{l}\text { Quality assessment score (Schulz criteria): 0/7 } \\
\text { Transfusion protocol not used }\end{array}$} \\
\hline \multicolumn{3}{|l|}{ Risk of bias } \\
\hline Item & Authors' judgement & Description \\
\hline Adequate sequence generation? & Unclear & Unclear \\
\hline Allocation concealment? & Unclear & Unclear \\
\hline
\end{tabular}


Rao 1999 (Continued)

\begin{tabular}{l|l|l}
\hline $\begin{array}{l}\text { Blinding? } \\
\text { All outcomes }\end{array}$ & Unclear & Unclear \\
\hline
\end{tabular}

\section{Ray 1997}

\begin{tabular}{|c|c|c|}
\hline Methods & \multicolumn{2}{|c|}{ Method of randomisation and allocation concealment were not described. } \\
\hline Participants & \multicolumn{2}{|c|}{$\begin{array}{l}106 \text { patients undergoing aortic or mitral valve replacement or both were randomly assigned to one } \\
\text { of two groups: } \\
\text { - Aprotinin group: } \mathrm{n}=54, \mathrm{M} / \mathrm{F}=35 / 19 \text {, median age }=54 \text { years } \\
\text { - Control group (Placebo): } \mathrm{n}=52, \mathrm{M} / \mathrm{F}=28 / 24 \text {, median age }=58 \text { years }\end{array}$} \\
\hline Interventions & \multicolumn{2}{|c|}{$\begin{array}{l}\text { - Aprotinin group received } 2 \text { million KIU of aprotinin }(280 \mathrm{mg}) \text { over } 20 \text { minutes after the } \\
\text { induction of anaesthesia followed by } 500,000 \mathrm{KIU} / \mathrm{hr}(70 \mathrm{mg} / \mathrm{hr}) \text { until the patient was returned to } \\
\text { the post-operative ward. In addition, } 2 \text { million KIU }(280 \mathrm{mg}) \text { was added to the oxygenator prime. } \\
\text { - Control group received an equivalent volume of normal saline. } \\
\text { NB: Both groups were exposed to cell salvage. }\end{array}$} \\
\hline Outcomes & \multicolumn{2}{|c|}{$\begin{array}{l}\text { Outcomes reported: Number of patients exposed to allogeneic blood, re-operation, platelet usage } \\
\text { (units), fresh frozen plasma usage (units). }\end{array}$} \\
\hline Notes & \multicolumn{2}{|c|}{$\begin{array}{l}\text { Quality assessment score (Schulz criteria): } 3 / 7 \\
\text { Transfusion protocol used }\end{array}$} \\
\hline \multicolumn{3}{|l|}{ Risk of bias } \\
\hline Item & Authors' judgement & Description \\
\hline Adequate sequence generation? & Unclear & Unclear \\
\hline Allocation concealment? & Unclear & Unclear \\
\hline $\begin{array}{l}\text { Blinding? } \\
\text { All outcomes }\end{array}$ & Yes & Double blind \\
\hline
\end{tabular}

\section{Ray 1999}

\begin{tabular}{l|l}
\hline Methods & Method of randomisation and allocation concealment were not described. \\
\hline Participants & $\begin{aligned} \text { 150 patients in elective adult cardiac surgery were randomly assigned to one of three groups: } \\
\text { - Aprotinin group (High dose): } \mathrm{n}=50 \\
\text { - Aprotinin group (Low dose): } \mathrm{n}=50 \\
\text { - Control group (Placebo): } \mathrm{n}=50\end{aligned}$ \\
NB: Gender or age data were not reported.
\end{tabular}




\section{Ray 1999 (Continued)}

- Aprotinin group (High dose) received a loading dose of $2 \mathrm{million}$ KIU over 20 minutes after
the induction of anaesthesia followed by $500,000 \mathrm{KIU} / \mathrm{hr}(70 \mathrm{mg} / \mathrm{hr})$ until the patient was
returned to the post-operative ward. In addition, 2 million KIU (280mg) was added to the pump
prime.
- Aprotinin group (Low dose) received a loading dose of $140 \mathrm{mg}(1 \mathrm{million}$ KIU) infused over
20 minutes after the induction of anaesthesia and a pump prime dose of $140 \mathrm{mg}(1 \mathrm{million} \mathrm{KIU})$.
- Control group received a volume of saline solution equivalent to the volume admitted in the
low dose aprotinin.

Outcomes

Outcomes reported: Number of patients exposed to allogeneic blood, re-operation for bleeding.

$\begin{array}{ll}\text { Notes } & \text { Quality assessment score (Schulz criteria): 4/7 } \\ \text { Transfusion protocol not used }\end{array}$

\section{Risk of bias}

\begin{tabular}{lll}
\hline Item & Authors' judgement & Description \\
\hline Adequate sequence generation? & Unclear & Unclear \\
\hline Allocation concealment? & Unclear & Unclear \\
\hline $\begin{array}{l}\text { Blinding? } \\
\text { All outcomes }\end{array}$ & Yes & Double blind \\
\hline
\end{tabular}

\section{Ray 2001}

\begin{tabular}{|c|c|}
\hline Methods & Method of randomisation and allocation concealment were not described. \\
\hline Participants & $\begin{array}{l}100 \text { patients undergoing elective cardiac surgery were randomly assigned to one of two groups: } \\
\text { - Epsilon aminocaproic acid group: } \mathrm{n}=51 \\
\text { - Aprotinin group: } \mathrm{n}=49 \\
\text { NB: Gender or age data were not reported. }\end{array}$ \\
\hline Interventions & $\begin{array}{l}\text { - Epsilon aminocaproic acid group received a test dose of } 250 \mathrm{mg} \text { at least } 10 \text { minutes before } \\
\text { the loading dose of } 5 \mathrm{~g} \text { given over a } 20 \text { minute period after the induction of anaesthesia and } 1.25 \mathrm{~g} / \\
\text { hr continuous infusion until } 4 \text { hours after bypass. In addition, } 5 \mathrm{~g} \text { of EACA was added to the } \\
\text { pump prime before cross clamping. } \\
\text { - Aprotinin group received a test dose of } 10,000 \mathrm{KIU} \text { before the loading dose ( } 1 \text { million KIU) } \\
\text { given over a } 20 \text { minute period after the induction of anaesthesia and } 1 \text { million KIU was added to } \\
\text { the pump prime. }\end{array}$ \\
\hline
\end{tabular}

Outcomes

Outcomes reported: Number of patients exposed to allogeneic blood, re-operation for bleeding, aspirin use within 10 days, Intensive Care Unit length of stay (hours), neurologic events.

Notes

Quality assessment score (Schulz criteria): 1/7

Transfusion protocol was not used 
Ray 2001 (Continued)

\begin{tabular}{l|ll} 
Risk of bias & & \\
\hline Item & Authors' judgement & Description \\
\hline Adequate sequence generation? & Unclear & Unclear \\
\hline Allocation concealment? & Unclear & Unclear \\
\hline $\begin{array}{l}\text { Blinding? } \\
\text { All outcomes }\end{array}$ & No & Single blind \\
\hline
\end{tabular}

\section{Ray 2005}

\begin{tabular}{|c|c|c|}
\hline Methods & \multicolumn{2}{|c|}{$\begin{array}{l}\text { Method of randomisation and allocation concealment were not described. Allocation of the ran- } \\
\text { domised drug was performed by a nurse not otherwise connected with the study. }\end{array}$} \\
\hline Participants & \multicolumn{2}{|c|}{$\begin{array}{l}45 \text { patients undergoing elective primary total hip arthroplasty were randomly allocated to one of } \\
\text { three groups: } \\
\text { - Aprotinin group: } \mathrm{n}=15 \text {, mean (interquartile range) age }=69(58-74) \text { years } \\
\text { - Epsilon aminocaproic acid group: } \mathrm{n}=15 \text {, mean (interquartile range) age }=72(59-77) \text { years } \\
\text { - Control group (Placebo): } \mathrm{n}=15, \text { mean (interquartile range) age }=72(59-77) \text { years }\end{array}$} \\
\hline Interventions & \multicolumn{2}{|c|}{$\begin{array}{l}\text { - Aprotinin group received a 10,000 KIU test dose of aprotinin followed by a bolus of } 2 \\
\text { million KIU given over } 30 \text { minutes after the induction of anaesthesia followed by a continuous } \\
\text { infusion of } 500,000 \mathrm{KIU} / \mathrm{hr} \text { for } 3 \text { hours. } \\
\text { - Epsilon aminocaproic acid group received } 10 \mathrm{~g} \text { of EACA in } 250 \mathrm{~mL} \text { of IV saline given over } 30 \\
\text { minutes after the induction of anaesthesia followed by } 5 \mathrm{~g} \text { in } 250 \mathrm{~mL} \text { of IV saline over } 3 \text { hours. } \\
\text { - Control group received normal saline in the same manner as the other trial arms. }\end{array}$} \\
\hline Outcomes & \multicolumn{2}{|c|}{$\begin{array}{l}\text { Outcomes reported: Number of patients exposed to allogeneic blood, allogeneic blood usage (units) } \\
\text {, blood loss ( } 24 \mathrm{hrs}) \text {, deep vein thrombosis, pre-operative apirin use. }\end{array}$} \\
\hline Notes & \multicolumn{2}{|c|}{$\begin{array}{l}\text { Quality assessment score (Schulz criteria): } 2 / 7 \\
\text { Transfusion protocol not used }\end{array}$} \\
\hline \multicolumn{3}{|l|}{ Risk of bias } \\
\hline Item & Authors' judgement & Description \\
\hline Adequate sequence generation? & Unclear & Unclear \\
\hline Allocation concealment? & Unclear & Unclear \\
\hline $\begin{array}{l}\text { Blinding? } \\
\text { All outcomes }\end{array}$ & Yes & Double blind \\
\hline
\end{tabular}




\section{Rhydderch 1993}

\begin{tabular}{|c|c|c|}
\hline Methods & \multicolumn{2}{|c|}{ Methods of sequence generation and allocation concealment were unclear. } \\
\hline Participants & \multicolumn{2}{|c|}{$\begin{array}{l}43 \text { undergoing cardiac surgery were randomised to one of two groups: } \\
\text { - Aprotinin group }(\mathrm{n}=20), \mathrm{M} / \mathrm{F}=14 / 6 \text {, mean }(\mathrm{SD}) \text { age }=42(15) \text { years } \\
\text { - Control group (placebo) }(\mathrm{n}=23), \mathrm{M} / \mathrm{F}=15 / 8 \text {, mean }(\mathrm{SD}) \text { age }=37(17) \text { years }\end{array}$} \\
\hline Interventions & \multicolumn{2}{|c|}{$\begin{array}{l}\text { - Aprotinin group received } 2 \text { million KIU added to the pump prime. } \\
\text { - Control group received a saline placebo. }\end{array}$} \\
\hline Outcomes & \multicolumn{2}{|c|}{ Outcomes reported: Volume blood transfused, re-operation for bleeding. } \\
\hline Notes & \multicolumn{2}{|c|}{$\begin{array}{l}\text { Quality assessment score (Schulz criteria): 3/7 } \\
\text { Transfusion protocol used }\end{array}$} \\
\hline \multicolumn{3}{|l|}{ Risk of bias } \\
\hline Item & Authors' judgement & Description \\
\hline Adequate sequence generation? & Unclear & Unclear \\
\hline Allocation concealment? & Unclear & Unclear \\
\hline $\begin{array}{l}\text { Blinding? } \\
\text { All outcomes }\end{array}$ & Yes & Double blind \\
\hline
\end{tabular}

\section{Rocha 1994}

\begin{tabular}{l|l} 
Methods & Method of randomisation and allocation concealment were not described. \\
\hline Participants & 109 of 122 eligible patients scheduled for coronary artery bypass graft surgery, valvular surgery, or \\
mixed cardiac surgery were randomised to one of four groups: \\
- Aprotinin group: $\mathrm{n}=28, \mathrm{M} / \mathrm{F}=16 / 12$, mean $(\mathrm{sd})$ age $=58.9(10.0)$ years \\
- Desmopressin group: $\mathrm{n}=25, \mathrm{M} / \mathrm{F}=14 / 11$, mean $(\mathrm{sd})$ age $=56.6(8.8)$ years \\
- Desmopressin group: $\mathrm{n}=28, \mathrm{M} / \mathrm{F}=20 / 8$, mean $(\mathrm{sd})$ age $=57.3(7.6)$ years \\
- Control group: $\mathrm{n}=28, \mathrm{M} / \mathrm{F}=22 / 6$, mean $(\mathrm{sd})$ age $=56.3(10.1)$ years
\end{tabular}

Interventions

- Aprotinin group received a bolus infusion of 2 million KIU of aprotinin within 30 minutes after the induction of anaesthesia followed by a continuous infusion of 500,000 KIU/hr of aprotinin until the patient left the operating room. In addition, a bolus of 2 million KIU of aprotinin was added to the pump prime by replacement of crystalloid.

- Desmopressin group received $0.3 \mathrm{ug} / \mathrm{kg}$ of desmopressin (DDAVP) in $50 \mathrm{ml}$ of saline solution over a period of 20 minutes, given intravenously on completion of cardiopulmonary bypass $(\mathrm{CPB})$ and immediately after administration of protamine.

- Desmopressin group received two doses of DDAVP $(2 \times 0.3 \mathrm{ug} / \mathrm{ml})$ and an additional dose 6 hours after surgery.

- Control group did not receive aprotinin or DDAVP. 
Rocha 1994 (Continued)

\begin{tabular}{|c|c|c|}
\hline Outcomes & \multicolumn{2}{|c|}{$\begin{array}{l}\text { Outcomes reported: Number of patients exposed to allogeneic blood, allogeneic blood usage, blood } \\
\text { loss ( } 72 \mathrm{hrs}) \text {, mortality, thrombosis. }\end{array}$} \\
\hline Notes & \multicolumn{2}{|c|}{$\begin{array}{l}\text { Quality assessment score (Schulz criteria): 1/7 } \\
\text { Transfusion protocol not used }\end{array}$} \\
\hline \multicolumn{3}{|l|}{ Risk of bias } \\
\hline Item & Authors' judgement & Description \\
\hline Adequate sequence generation? & Unclear & Unclear \\
\hline Allocation concealment? & Unclear & Unclear \\
\hline $\begin{array}{l}\text { Blinding? } \\
\text { All outcomes }\end{array}$ & No & Single blind \\
\hline
\end{tabular}

\section{Rodrigus 1996}

\begin{tabular}{|c|c|c|}
\hline Methods & \multicolumn{2}{|c|}{ Method of randomisation and allocation concealment were not described. } \\
\hline Participants & \multicolumn{2}{|c|}{$\begin{array}{l}99 \text { adult patients undergoing elective primary coronary artery bypass graft, or valvular surgery, with } \\
\text { cardiopulmonary bypass were randomised to one of two groups: } \\
\text { - Aprotinin group: } \mathrm{n}=46, \mathrm{M} / \mathrm{F}=39 / 7 \text {, mean }(\mathrm{sd}) \text { age }=60.4(8.8) \text { years } \\
\text { - Control group (Placebo): } \mathrm{n}=47, \mathrm{M} / \mathrm{F}=34 / 13 \text {, mean }(\mathrm{sd}) \text { age }=59(7.8) \text { years } \\
\text { NB: Six of the } 99 \text { patients randomised were excluded from the study. Ninety-three patients remained } \\
\text { in the study for analysis. }\end{array}$} \\
\hline Interventions & \multicolumn{2}{|c|}{$\begin{array}{l}\text { - Aprotinin group received aprotinin as an infusion of } 2 \text { million } \mathrm{KIU} \text { in } 200 \mathrm{ml} \text { of normal } \\
\text { saline after induction, followed by a continuous infusion of } 500,000 \mathrm{KIU} / \mathrm{hr} \text { and } 2 \text { million KIU } \\
\text { in the priming volume of the extracorporeal circuit. } \\
\text { - Control group received the same volume of normal saline. }\end{array}$} \\
\hline Outcomes & \multicolumn{2}{|c|}{$\begin{array}{l}\text { Outcomes reported: Number of patients exposed to allogeneic blood, allogeneic blood usage (units) } \\
\text {, fresh frozen plasma usage (units), platelet usage (units), blood loss ( } 24 \mathrm{hrs}) \text {, mortality, myocardia } \\
\text { infarction [definite \& possible], re-operation for bleeding, atrial fibrillation. }\end{array}$} \\
\hline Notes & \multicolumn{2}{|c|}{$\begin{array}{l}\text { Quality assessment score (Schulz criteria): } 3 / 7 \\
\text { Transfusion protocol not specified }\end{array}$} \\
\hline \multicolumn{3}{|l|}{ Risk of bias } \\
\hline Item & Authors' judgement & Description \\
\hline Adequate sequence generation? & Unclear & Unclear \\
\hline Allocation concealment? & Unclear & Unclear \\
\hline
\end{tabular}


Rodrigus 1996 (Continued)

\begin{tabular}{|c|c|c|}
\hline $\begin{array}{l}\text { Blinding? } \\
\text { All outcomes }\end{array}$ & Yes & Double blind \\
\hline
\end{tabular}

\section{Rossi 1997}

\begin{tabular}{l|l}
\hline Methods & Method of randomisation and allocation concealment were not described. \\
\hline Participants & $\begin{array}{l}43 \text { patients scheduled for elective primary myocardial revascularisation were randomly allocated to } \\
\text { one of two groups: } \\
\text { - Aprotinin group: } \mathrm{n}=21, \text { mean }(\mathrm{sd}) \text { age }=58(8) \text { years } \\
\text { - Control group }(\mathrm{n}=22) \text {, mean }(\mathrm{sd}) \text { age }=56(12) \text { years }\end{array}$ \\
NB: Gender data were not reported.
\end{tabular}

Interventions

- Aprotinin group received 2 million KIU of aprotinin in the cardiopulmonary bypass prime.

- Control group did not receive aprotinin.

NB: Both groups were exposed to acute normovolaemic haemodilution (ANH).

Outcomes

Outcomes reported: Number of patients exposed to allogeneic blood, blood loss (24hrs), re-operation for bleeding, side effects.

$\begin{array}{ll}\text { Notes } & \begin{array}{l}\text { Quality assessment score (Schulz criteria): 2/7 } \\ \text { Transfusion protocol used }\end{array}\end{array}$

\section{Risk of bias}

\begin{tabular}{lll}
\hline Item & Authors' judgement & Description \\
\hline Adequate sequence generation? & Unclear & Unclear \\
\hline Allocation concealment? & Unclear & Unclear \\
\hline $\begin{array}{l}\text { Blinding? } \\
\text { All outcomes }\end{array}$ & Unclear & Unclear \\
\hline
\end{tabular}

\section{Royston 1987}

\begin{tabular}{l|l} 
Methods & $\begin{array}{l}\text { Patients were randomly allocated to receive test compound by means of sealed envelopes. Method } \\
\text { of randomisation was not described. }\end{array}$ \\
\hline Participants & $\begin{array}{l}22 \text { patients undergoing repeat cardiac surgery through previous median sternotomy wound were } \\
\text { randomised to one of two groups: } \\
\text { - Aprotinin group: } \mathrm{n}=11, \text { mean }(\mathrm{sd}) \text { age }=53(15) \text { years } \\
\text { - Control group: } \mathrm{n}=11 \text {, mean }(\mathrm{sd}) \text { age }=57(13) \text { years } \\
\text { NB: Gender data were not reported. }\end{array}$
\end{tabular}




\section{Royston 1987 (Continued)}

\begin{tabular}{|c|c|c|}
\hline Interventions & \multicolumn{2}{|c|}{$\begin{array}{l}\text { - Aprotinin group received a loading dose of } 280 \mathrm{mg} \text { of aprotinin via central venous access over } \\
20 \text { minutes before the opening of the previous median sternotomy wound, followed by a } \\
\text { continuous infusion of } 70 \mathrm{mg} / \mathrm{hr} \text { until skin closure at the end of the operation. An additional } \\
280 \mathrm{mg} \text { of aprotinin was added to the prime volume of the oxygenator. } \\
\text { - Control group did not receive aprotinin. } \\
\text { NB: Both groups were exposed to acute normovolaemic haemodilution (ANH). }\end{array}$} \\
\hline Outcomes & \multicolumn{2}{|c|}{$\begin{array}{l}\text { Outcomes reported: Number of patients exposed to allogeneic blood, allogeneic blood usage (units) } \\
\text {, blood loss ( } 24 \mathrm{hrs}) \text {, mortality, total haemoglobin loss, } \\
\text { time for wound closure (mins), platelet counts. }\end{array}$} \\
\hline Notes & \multicolumn{2}{|c|}{$\begin{array}{l}\text { Quality assessment score (Schulz criteria):3/7 } \\
\text { Transfusion protocol used }\end{array}$} \\
\hline \multicolumn{3}{|l|}{ Risk of bias } \\
\hline Item & Authors' judgement & Description \\
\hline Adequate sequence generation? & Unclear & Unclear \\
\hline Allocation concealment? & No & Inadequate - sealed envelopes \\
\hline $\begin{array}{l}\text { Blinding? } \\
\text { All outcomes }\end{array}$ & Unclear & Unclear \\
\hline
\end{tabular}

Sadeghi 2007

Methods Patients were randomised using a random number technique. The correct treatment option was assured by means of coded infusion syringes prepared by hospital pharmacy not involved otherwise in the study.

\begin{tabular}{l|l}
\hline Participants & $\begin{array}{l}\text { 67 undergoing orthopaedic surgery for hip fractures were randomised to one of two groups: } \\
\text { - Tranexamic acid group: } \mathrm{n}=32, \mathrm{M} / \mathrm{F}=17 / 15, \text { mean }(\mathrm{sd}) \text { age }=51.81(25.7) \text { years } \\
\text { - Control group (Placebo): } \mathrm{n}=35, \mathrm{M} / \mathrm{F}=24 / 11, \text { mean }(\mathrm{sd}) \text { age }=44.4(26.16) \text { years }\end{array}$ \\
\hline Interventions & $\begin{array}{l}\text { - Tranexamic acid group received a bolus of } 15 \mathrm{mg} / \mathrm{kg} \text { at the beginning of surgery. } \\
\text { - Control group received saline solution. }\end{array}$ \\
\hline Outcomes & $\begin{array}{l}\text { Outcomes reported: } \text { Number of patients exposed to allogeneic blood transfusion, mortality, blood } \\
\text { loss, volume blood transfused (units), length of hospital stay (days). }\end{array}$ \\
\hline Notes & $\begin{array}{l}\text { Quality assessment score (Schulz criteria):7/7 } \\
\text { Transfusion protocol not used }\end{array}$ \\
\hline
\end{tabular}

Risk of bias

$\begin{array}{lll}\text { Item Authors' judgement } & \text { Description }\end{array}$


Sadeghi 2007 (Continued)

\begin{tabular}{lll}
\hline Adequate sequence generation? & Yes & Random number technique \\
\hline Allocation concealment? & Yes & Adequate \\
\hline $\begin{array}{l}\text { Blinding? } \\
\text { All outcomes }\end{array}$ & Yes & Double blind \\
\hline
\end{tabular}

Samama 2002

Centres were provided with sealed envelopes with the randomisation codes to enable an individual
patient's code to be broken in an emergency. A separate set of sealed randomisation tables were kept
at the central data centre. To maintain masking, all patients received identical volumes of solution
and an identical number of bottles for the identical dose and for the continuous infusion, regardless
of treatment group.

Participants
- Aprotients undergoing elective orthopaedic surgery were randomised to one of three groups:
- Aprotinin group (Low dose): $\mathrm{n}=22$, mean $(\mathrm{sd})$ age $=48$ (19) years
- Control group (Placebo): $\mathrm{n}=18$, mean $(\mathrm{sd})$ age $=44(22)$ years
NB: Gender data were not reported.

- Aprotinin group (High dose) received a loading dose of 4 million KIU ( $560 \mathrm{mg}$ ) given over
20 minutes before and during the induction of anaesthesia followed by a continuous infusion of 1
million KIU until skin closure.
- Aprotinin group (Low dose) received a loading dose of 2 million KIU ( $280 \mathrm{mg}$ ) given over 20
minutes before and during the induction of anaesthesia followed by a continuous infusion of
$500,000 \mathrm{KIU}$ until skin closure.
- Control group received saline in an identical time schedule and volume.

Outcomes

Outcomes reported: Number of patients exposed to allogeneic blood, allogeneic blood usage (units) , blood loss, deep vein thrombosis, pulmonary embolus, trauma cases, cell salvage used, autologous transfusion.

$\begin{array}{ll}\text { Notes } & \begin{array}{l}\text { Quality assessment score (Schulz criteria):6/7 } \\ \text { Transfusion protocol used }\end{array}\end{array}$

\section{Risk of bias}

\begin{tabular}{lll}
\hline Item & Authors' judgement & Description \\
\hline Adequate sequence generation? & Yes & Randomisation codes \\
\hline $\begin{array}{l}\text { Allocation concealment? } \\
\text { Blinding? }\end{array}$ & No & Inadequate - sealed envelopes \\
\hline All outcomes & Ies & Double blind \\
\hline
\end{tabular}


Santamaria 2000

\begin{tabular}{|c|c|c|}
\hline Methods & \multicolumn{2}{|c|}{ Method of randomisation and allocation concealment were not described. } \\
\hline Participants & \multicolumn{2}{|c|}{$\begin{array}{l}84 \text { patients undergoing elective coronary artery bypass graft surgery were randomly allocated to } \\
\text { one of two groups: } \\
\text { - Aprotinin group (High dose): } \mathrm{n}=28, \mathrm{M} / \mathrm{F}=27 / 1 \text {, mean (range) age }=58(38-78) \text { years } \\
\text { - Aprotinin group (Low dose): } \mathrm{n}=28, \mathrm{M} / \mathrm{F}=24 / 4 \text {, mean (range) age }=61(40-75) \text { years } \\
\text { - Control group (Placebo): } \mathrm{n}=28, \mathrm{M} / \mathrm{F}=24 / 4 \text {, mean (range) age = } 59(41-76) \text { years }\end{array}$} \\
\hline Interventions & \multicolumn{2}{|c|}{$\begin{array}{l}\text { - Aprotinin group (High dose) received a bolus of } 2 \text { million KIU as a loading dose followed by } \\
\text { a continuous infusion of } 500,000 \mathrm{KIU} / \mathrm{hr} \text { during } \mathrm{CPB} \text {. In addition, } 2 \text { million KIU ( } 280 \mathrm{mg} \text { ) of } \\
\text { aprotinin was added to the pump prime. } \\
\text { - Aprotonin group (Low dose - pump prime only) received a bolus of saline as a loading dose } \\
\text { followed by a continuous infusion of saline during CPB. Two million KIU ( } 280 \mathrm{mg} \text { ) of aprotinin } \\
\text { was added to the prime solution. } \\
\text { - Control group received a bolus of saline. Saline was added to the priming solution and a } \\
\text { continuous infusion of saline was administered during CPB. }\end{array}$} \\
\hline Outcomes & \multicolumn{2}{|c|}{$\begin{array}{l}\text { Outcomes reported: Number of patients exposed to allogeneic blood, allogeneic blood usage (units) } \\
\text {, blood loss, myocardial infarction, stroke, hypertension, A-V block. }\end{array}$} \\
\hline Notes & \multicolumn{2}{|c|}{$\begin{array}{l}\text { Quality assessment score (Schulz criteria):4/7 } \\
\text { Transfusion protocol used }\end{array}$} \\
\hline \multicolumn{3}{|l|}{ Risk of bias } \\
\hline Item & Authors' judgement & Description \\
\hline Adequate sequence generation? & Unclear & Unclear \\
\hline Allocation concealment? & Unclear & Unclear \\
\hline $\begin{array}{l}\text { Blinding? } \\
\text { All outcomes }\end{array}$ & Yes & Double blind \\
\hline
\end{tabular}

Santos 2006

\begin{tabular}{ll} 
Methods & $\begin{array}{l}\text { Groups were randomised by means of sequentially numbered sealed envelopes opened by a nurse in } \\
\text { the operating room. Only the nurse, who prepared the infusions, knew whether a patient received } \\
\text { drug or placebo. Study drugs were delivered in identical volumes. Staff in the operating room and } \\
\text { in the intensive care unit were not aware of the treatment. }\end{array}$ \\
\hline Participants & $\begin{array}{l}65 \text { patients undergoing primary coronary artery bypass graft surgery were randomised to one of } \\
\text { two groups: } \\
\text { - Tranexamic acid group: } \mathrm{n}=29, \mathrm{M} / \mathrm{F}=18 / 11, \text { mean }(\mathrm{sd}) \text { age }=62(9.2) \text { years } \\
\text { - Control group (placebo) }(\mathrm{n}=31), \mathrm{M} / \mathrm{F}=25 / 6, \text { mean }(\mathrm{sd}) \text { age }=59(8.7) \text { years } \\
\text { NB: Five patients were excluded from the final analysis. }\end{array}$
\end{tabular}




\begin{tabular}{|c|c|c|}
\hline Interventions & \multicolumn{2}{|c|}{$\begin{array}{l}\text { - Tranexamic acid group received a loading dose of } 10 \mathrm{mg} / \mathrm{kg} \text { of TXA before skin incision, } \\
\text { followed by a continuous infusion of } 1 \mathrm{mg} / \mathrm{kg} / \mathrm{hr} \text { for } 5 \text { hours. } \\
\text { - Control group received a bolus of normal saline solution in an identical syringe and a } \\
\text { continuous infusion of normal saline for } 5 \text { hours. }\end{array}$} \\
\hline Outcomes & \multicolumn{2}{|c|}{$\begin{array}{l}\text { Outcomes reported: Number of patients exposed to allogeneic blood, allogeneic blood usage (units) } \\
\text {, fresh frozen plasma usage (units), blood loss, mortality, re-operation for bleeding. }\end{array}$} \\
\hline Notes & \multicolumn{2}{|c|}{$\begin{array}{l}\text { Quality assessment score (Schulz criteria): 4/7 } \\
\text { Transfusion protocol used }\end{array}$} \\
\hline \multicolumn{3}{|l|}{ Risk of bias } \\
\hline Item & Authors' judgement & Description \\
\hline Adequate sequence generation? & Unclear & Unclear \\
\hline Allocation concealment? & No & Inadequate - sealed envelopes \\
\hline $\begin{array}{l}\text { Blinding? } \\
\text { All outcomes }\end{array}$ & Yes & Double blind \\
\hline
\end{tabular}

\section{Schmartz 2003}

Methods Sixty patients were divided into three groups by means of computerised randomisation. Allocation concealment was not specified.

\begin{tabular}{|c|c|}
\hline Participants & $\begin{array}{l}60 \text { male patients undergoing primary elective cardiac surgery were ranc } \\
\text { groups: } \\
\text { - Aprotinin group (High dose): } \mathrm{n}=20 \text {, mean }(\mathrm{sd}) \text { age }=62(9) \text { years } \\
\text { - Aprotinin group (Low dose): } \mathrm{n}=20 \text {, mean }(\mathrm{sd}) \text { age }=59(11) \text { years } \\
\text { - Control group (Placebo): } \mathrm{n}=20 \text {, mean }(\mathrm{sd}) \text { age }=61(11) \text { years }\end{array}$ \\
\hline
\end{tabular}

Interventions
a continuous infusion of 500,000 KIU/hr. In addition, 2 million KIU (280mg) of aprotinin was
added to the pump prime.
- Aprotinin group (Low dose) received a loading dose of 1 million KIU (140mg) followed by a
continuous infusion of $250,000 \mathrm{KIU} / \mathrm{hr}$. In addition, 1 million KIU (140mg) of aprotinin was
added to the pump prime.
- Control group received an unspecified placebo.

Outcomes

Outcomes reported: Allogeneic blood usage (units), blood markers of inflammation during and after CPB.

Notes

Quality assessment score (Schulz criteria): 4/7

Transfusion protocol not used 
Schmartz 2003 (Continued)

\begin{tabular}{lll} 
Risk of bias & & \\
\hline Item & Authors' judgement & Description \\
\hline Adequate sequence generation? & Yes & Computerised randomisation \\
\hline Allocation concealment? & Unclear & Unclear \\
\hline $\begin{array}{l}\text { Blinding? } \\
\text { All outcomes }\end{array}$ & Yes & Double blinding \\
\hline
\end{tabular}

\section{Schweizer 2000}

\begin{tabular}{|c|c|c|}
\hline Methods & \multicolumn{2}{|c|}{$\begin{array}{l}\text { Concealment of treatment allocation was not described. Patients were allocated randomly in a } \\
\text { double-blind manner. Method of randomisation was not described. }\end{array}$} \\
\hline Participants & \multicolumn{2}{|c|}{$\begin{array}{l}60 \text { patients undergoing elective coronary artery bypass graft surgery, aortic valve replacement and } \\
\text { mitral valve replacement and repair were randomised to one of two groups: } \\
\text { - Aprotinin group (Low dose): } \mathrm{n}=28), \mathrm{M} / \mathrm{F}=21 / 7 \text {, mean (range) age }=66(35-85) \text { years } \\
\text { - Control group (Placebo): } \mathrm{n}=29, \mathrm{M} / \mathrm{F}=21 / 8 \text {, mean (range) age }=64(33-81) \text { years }\end{array}$} \\
\hline Interventions & \multicolumn{2}{|c|}{$\begin{array}{l}\text { - Aprotinin group (Low dose) received a mean dose of } 4.1 \text { million KIU of aprotinin, } \\
\text { consisting of a loading dose of } 280 \mathrm{mg}(2 \text { million KIU) over } 30 \text { minutes, } 140 \mathrm{mg} \text { ( } 1 \text { million KIU) } \\
\text { added to the pump prime and a continuous infusion of } 500,000 \mathrm{KIU} / \mathrm{hr} \text { from the start of surgery } \\
\text { until skin closure. } \\
\text { - Control group received a similar volume of normal saline. }\end{array}$} \\
\hline Outcomes & \multicolumn{2}{|c|}{$\begin{array}{l}\text { Outcomes reported: Allogeneic blood usage (units), blood loss, mortality, myocardial infarction, } \\
\text { re-operation for bleeding. }\end{array}$} \\
\hline Notes & \multicolumn{2}{|c|}{$\begin{array}{l}\text { Quality assessment score (Schulz criteria):3/7 } \\
\text { Transfusion protocol used }\end{array}$} \\
\hline \multicolumn{3}{|l|}{ Risk of bias } \\
\hline Item & Authors' judgement & Description \\
\hline Adequate sequence generation? & Unclear & Unclear \\
\hline Allocation concealment? & Unclear & Unclear \\
\hline $\begin{array}{l}\text { Blinding? } \\
\text { All outcomes }\end{array}$ & Yes & Double blind \\
\hline
\end{tabular}


Shore-Lesserson 1996

\begin{tabular}{l} 
Methods \\
$\begin{array}{l}\text { Patients were randomly assigned to treatment or placebo by computer generated table. The phar- } \\
\text { macist who prepared the infusions knew whether the patient received active treatment or placebo } \\
\text { in the event of an adverse response. }\end{array}$ \\
\hline Participants \\
$\begin{array}{l}31 \text { patients undergoing repeat open heart surgery were randomised to one of two groups: } \\
\text { - Tranexamic acid group: } \mathrm{n}=17, \mathrm{M} / \mathrm{F}=10 / 7 \text {, mean }(\mathrm{sd}) \text { age }=68 \text { (13) years } \\
\text { - Control group (Placebo): } \mathrm{n}=13, \mathrm{M} / \mathrm{F}=10 / 3 \text {, mean }(\mathrm{sd}) \text { age }=63(6) \text { years } \\
\text { NB: One patient from the placebo group was withdrawn from the study due to excessive post- } \\
\text { operative bleeding and requiring intra-aortic balloon counter pulsation. }\end{array}$ \\
$\begin{array}{l}\text { - Tranexamic acid group received an initial dose of TXA, } 20 \mathrm{mg} / \mathrm{kg} \text { over } 20 \text { minutes, followed } \\
\text { by a continuous infusion of } 2 \mathrm{mg} / \mathrm{kg} / \mathrm{hr} \text {. This infusion was terminated at the completion of the } \\
\text { surgical procedure. } \\
\text { - Control group received an equal volume of saline. } \\
\text { NB: Both groups were exposed to cell salvage. }\end{array}$ \\
\hline
\end{tabular}

Outcomes

Outcomes reported: Number of patients exposed to allogeneic blood, allogeneic blood usage, fresh frozen plasma usage, platelet usage, blood loss, mortality, myocardial infarction, pulmonary complications, re-operation, renal impairment, cerebral ischemia, embolic stroke.

\begin{tabular}{|c|c|c|}
\hline Notes & $\begin{array}{l}\text { Quality assessment score (Schulz criteria): 6/7 } \\
\text { Transfusion protocol used }\end{array}$ & \\
\hline \multicolumn{3}{|l|}{ Risk of bias } \\
\hline Item & Authors' judgement & Description \\
\hline Adequate sequence generation? & Yes & Computer generated table \\
\hline Allocation concealment? & Yes & Adequate \\
\hline $\begin{array}{l}\text { Blinding? } \\
\text { All outcomes }\end{array}$ & Yes & Double blind \\
\hline
\end{tabular}

\section{Sorin 1999}

\begin{tabular}{l|l}
\hline Methods & Method of randomisation and allocation concealment were not described. [Abstract] \\
\hline Participants & $\begin{array}{l}\text { 42 patients undergoing total knee replacement were randomly assigned to one of two groups: } \\
\text { - Tranexamic acid group: } \mathrm{n}=21 \\
\text { - Control group (Placebo): } \mathrm{n}=21\end{array}$ \\
NB: Demographic data were not reported.
\end{tabular}

Interventions

- Tranexamic acid group received $15 \mathrm{mg} / \mathrm{kg}$ of TXA 30 minutes before surgery and subsequently every 8 hours over the following 3 days.

- Control group received an equal volume of saline. 
Sorin 1999 (Continued)

\begin{tabular}{|c|c|c|}
\hline Outcomes & \multicolumn{2}{|c|}{$\begin{array}{l}\text { Outcomes reported: Number of patients exposed to allogeneic blood, allogeneic blood usage (units) } \\
\text {, blood loss, deep vein thrombosis. }\end{array}$} \\
\hline Notes & \multicolumn{2}{|l|}{ Transfusion protocol used } \\
\hline \multicolumn{3}{|l|}{ Risk of bias } \\
\hline Item & Authors' judgement & Description \\
\hline Adequate sequence generation? & Unclear & Unclear \\
\hline Allocation concealment? & Unclear & Unclear \\
\hline $\begin{array}{l}\text { Blinding? } \\
\text { All outcomes }\end{array}$ & Unclear & Unclear \\
\hline
\end{tabular}

Speekenbrink 1995

\begin{tabular}{l|l}
\hline Methods & Method of randomisation and allocation concealment were not described. \\
\hline Participants & $\begin{array}{l}60 \text { patients scheduled for elective primary coronary artery bypass grafting were randomly assigned } \\
\text { to one of four groups: } \\
\text { - Aprotinin group: } \mathrm{n}=15, \mathrm{M} / \mathrm{F}=13 / 2, \text { mean }(\mathrm{sd}) \text { age }=62 \text { (10) years } \\
\text { - Tranexamic acid group: } \mathrm{n}=15, \mathrm{M} / \mathrm{F}=14 / 1, \mathrm{mean}(\mathrm{sd}) \text { age }=61 \text { (11) years } \\
\text { - Dipyridamole group: } \mathrm{n}=15, \mathrm{M} / \mathrm{F}=13 / 2, \text { mean }(\mathrm{sd}) \text { age }=60 \text { (9) years } \\
\text { - Control group: } \mathrm{n}=15, \mathrm{M} / \mathrm{F}=14 / 1, \mathrm{mean}(\mathrm{sd}) \text { age }=57 \text { (12) years }\end{array}$ \\
\hline Interventions & $\begin{array}{l}\text { - Aprotinin group received single dose of } 2 \text { million } \mathrm{KIU} \text { of aprotinin added to the pump } \\
\text { prime. } \\
\text { of } 1 \text { Trangexamic acid group received a bolus of } 10 \mathrm{mg} / \mathrm{kg} \text { over } 20 \text { minutes and continued at a rate } \\
\text { - Dipyridamole (Persantin) group received } 100 \mathrm{mg} \text { four times a day (oral), } 36 \text { hours before the } \\
\text { operation. After induction of anaesthesia treatment was continued with intravenous dipyridamole } \\
\text { at a rate of } 0.24 \mathrm{mg} / \mathrm{kg} / \mathrm{hr} \text { for } 24 \text { hours. } \\
\text { - Control group received usual care. }\end{array}$ \\
\hline Risk of bias & $\begin{array}{l}\text { Outcomes reported: Number of patients exposed to allogeneic blood, allogeneic blood usage (units) } \\
\text { fresh frozen plasma usage (units), blood loss (6hrs), myocardial infarction, haemorrhage from } \\
\text { chest. }\end{array}$ \\
\hline Notes & $\begin{array}{l}\text { Quality assessment score (Schulz criteria): } 1 / 7 \\
\text { Transfusion protocol not specified }\end{array}$ \\
\hline
\end{tabular}

Item

Authors' judgement

Description 
Speekenbrink 1995 (Continued)

\begin{tabular}{l|ll}
\hline Adequate sequence generation? & Unclear & Unclear \\
\hline Allocation concealment? & Unclear & Unclear \\
\hline $\begin{array}{l}\text { Blinding? } \\
\text { All outcomes }\end{array}$ & Unclear & Unclear \\
\hline
\end{tabular}

\section{Speekenbrink 1996}

\begin{tabular}{l|l} 
Methods & $\begin{array}{l}\text { Study medications were supplied in boxes containing } 12 \text { bottles with } 50 \mathrm{~mL} \text { solution. The ran- } \\
\text { domisation code was kept by supplied. The codes were broken after data acquisition were complete } \\
\text { and verified. }\end{array}$ \\
\hline Participants & $\begin{array}{l}\text { 115 patients scheduled for elective coronary artery bypass graft surgery were randomised to one of } \\
\text { three groups: } \\
\text { - Control group (Placebo): } \mathrm{n}=37, \mathrm{M} / \mathrm{F}=29 / 8, \text { mean (sd) age }=57 \text { (8) years } \\
\text { - Aprotinin group (Low dose): } \mathrm{n}=37, \mathrm{M} / \mathrm{F}=33 / 4, \text { mean }(\mathrm{sd}) \text { age }=62(9) \text { years } \\
\text { - Aprotinin group (High dose): } \mathrm{n}=38, \mathrm{M} / \mathrm{F}=30 / 8, \text { mean }(\mathrm{sd}) \text { age }=62(9) \text { years }\end{array}$ \\
\hline Interventions & $\begin{array}{l}\text { - Control group received equivalent volumes of normal saline. } \\
\text { - Aprotinin group (Low dose) received } 500,000 \mathrm{KIU} \text { of aprotinin in the prime solution. } \\
\text { by an infusion of } 500,000 \mathrm{KIU} / \mathrm{hr} \text {. In addition, } 500,000 \mathrm{KIU} \text { of aprotinin was added to the } \\
\text { prime solution. }\end{array}$ \\
\hline Outcomes & $\begin{array}{l}\text { Outcomes reported: } \text { Number of patients exposed to allogeneic blood, allogeneic blood usage (units) } \\
\text { blood loss (24hrs), mortality, myocardial infarction, renal failure, re-operation for bleeding. }\end{array}$ \\
\hline Notes & $\begin{array}{l}\text { Quality assessment score (Schulz criteria): } 5 / 7 \\
\text { Transfusion protocol used } \\
\text { Three patients were excluded from the final analysis: Two from the placebo group (one for excessive } \\
\text { postoperative bleeding caused by a broken suture and one for a small left ventricular aneurysm } \\
\text { requiring resection), one from the high dose aprotinin group who had dense pericardial adhesions } \\
\text { resembling those found in reoperation. }\end{array}$ \\
\hline
\end{tabular}

\section{Risk of bias}

\begin{tabular}{lll}
\hline Item & Authors' judgement & Description \\
\hline Adequate sequence generation? & Unclear & Unclear \\
\hline Allocation concealment? & Yes & Adequate \\
\hline $\begin{array}{l}\text { Blinding? } \\
\text { All outcomes }\end{array}$ & Yes & Double blind \\
\hline
\end{tabular}


Stammers 1997

\begin{tabular}{|c|c|c|}
\hline Methods & \multicolumn{2}{|c|}{$\begin{array}{l}\text { Method of randomisation was not described. All drugs were drawn up by a pharmacist and placed } \\
\text { in a } 500 \mathrm{~mL} \text { glass bottle which was labelled with the patient's name, registration number and date. } \\
\text { No other clinician knew of the treatment received by the patient. }\end{array}$} \\
\hline Participants & \multicolumn{2}{|c|}{$\begin{array}{l}20 \text { patients undergoing first time coronary artery bypass grafting were randomly assigned to one of } \\
\text { two groups: } \\
\text { - Aprotinin group: } \mathrm{n}=8, \mathrm{M} / \mathrm{F}=6 / 2, \text { mean }(\mathrm{sd}) \text { age }=66.3(5.8) \text { years } \\
\text { - Control group (Placebo): } \mathrm{n}=12, \mathrm{M} / \mathrm{F}=10 / 2 \text {, mean }(\mathrm{sd}) \text { age }=63.9(9.2) \text { years }\end{array}$} \\
\hline Interventions & \multicolumn{2}{|c|}{$\begin{array}{l}\text { - Aprotinin group received a loading dose of } 2 \text { million KIU }(280 \mathrm{mg}) \text { of aprotinin } \\
\text { administered intravenously immediately following the induction of anaesthesia, } 2 \text { million KIU of } \\
\text { aprotinin placed in the priming volume of the extracorporeal circuit, and a constant infusion of } \\
500,000 \mathrm{KIU} / \mathrm{hr}(70 \mathrm{mg} / \mathrm{hr}) \text { until chest closure. } \\
\text { - Control group received an equal volume of saline administered in the same manner. }\end{array}$} \\
\hline Outcomes & \multicolumn{2}{|c|}{$\begin{array}{l}\text { Outcomes reported: Allogeneic blood usage, Intensive care ventilator time (hrs), renal failure, } \\
\text { neurological injury, hospital length of stay (days), blood loss ( } 24 \mathrm{hrs}) \text {. }\end{array}$} \\
\hline Notes & \multicolumn{2}{|c|}{$\begin{array}{l}\text { Quality assessment score (Schulz criteria): 6/7 } \\
\text { Transfusion protocol used }\end{array}$} \\
\hline \multicolumn{3}{|l|}{ Risk of bias } \\
\hline Item & Authors' judgement & Description \\
\hline Adequate sequence generation? & Unclear & Unclear \\
\hline Allocation concealment? & Yes & Adequate \\
\hline $\begin{array}{l}\text { Blinding? } \\
\text { All outcomes }\end{array}$ & Yes & Double blind \\
\hline
\end{tabular}

\section{Stewart 2001}

\begin{tabular}{l|l} 
Methods & $\begin{array}{l}\text { Randomisation using numbers chosen randomly from a computer generated table. Study drug and } \\
\text { placebo bottles were identifiable only by the random number. }\end{array}$ \\
\hline Participants & $\begin{array}{l}30 \text { patients undergoing elective orthognathic surgery (maxillary Le Fort I and mandibular sagittal } \\
\text { split osteotomies) were randomised to one of two groups: } \\
\text { - Aprotinin group (Low dose): } \mathrm{n}=15 \\
\text { - Control group (Placebo): } \mathrm{n}=15\end{array}$ \\
NB: Gender and age data were not reported.
\end{tabular}

Interventions

- Aprotinin group (Low dose) received a loading dose of 280mg (2 million KIU) given after the induction of anaesthesia and before the operation started for over 20 minutes, followed by a continuous infusion at a rate of $500,000 \mathrm{KIU} / \mathrm{hr}$ was infused until the end of the procedure.

- Control group received normal saline at the same time and volumes as aprotinin. 


\section{Stewart 2001 (Continued)}

\begin{tabular}{|c|c|c|}
\hline Outcomes & \multicolumn{2}{|c|}{$\begin{array}{l}\text { Outcomes reported: Number of patients exposed to allogeneic blood, allogeneic blood usage (units) } \\
\text {, blood loss. }\end{array}$} \\
\hline Notes & \multicolumn{2}{|l|}{$\begin{array}{l}\text { Quality assessment score (Schulz criteria): 6/7 } \\
\text { Transfusion protocol used }\end{array}$} \\
\hline \multicolumn{3}{|l|}{ Risk of bias } \\
\hline Item & Authors' judgement & Description \\
\hline Adequate sequence generation? & Yes & Computer generated table \\
\hline Allocation concealment? & Yes & Adequate \\
\hline $\begin{array}{l}\text { Blinding? } \\
\text { All outcomes }\end{array}$ & Yes & Double blind \\
\hline
\end{tabular}

\section{Swart 1994}

\begin{tabular}{|c|c|c|}
\hline Methods & \multicolumn{2}{|c|}{$\begin{array}{l}\text { Method of randomisation was not described. Intervention and placebo solutions were supplied by } \\
\text { Bayer AG (Germany). }\end{array}$} \\
\hline Participants & \multicolumn{2}{|c|}{$\begin{array}{l}50 \text { patients undergoing primary coronary artery bypass surgery and } 50 \text { patients undergoing valve } \\
\text { surgery were randomised to one of two groups: } \\
\text { - Aprotinin group: } \mathrm{n}=49, \mathrm{M} / \mathrm{F}=33 / 16 \text {, mean (range) age }=53.1(18-78) \text { years } \\
\text { - Control group (Placebo) }(\mathrm{n}=49), \mathrm{M} / \mathrm{F}=32 / 17 \text {, mean (range) age }=51.6(18-76) \text { years }\end{array}$} \\
\hline Interventions & \multicolumn{2}{|c|}{$\begin{array}{l}\text { - Aprotinin group received } 2 \text { million KIU of aprotinin at the start of the operation, infused } \\
\text { over a period of } 30 \text { minutes followed by a continuous infusion of aprotinin at 500,000 KIU/hr } \\
\text { for } 4 \text { hours or until the end of the operation. In addition, } 2 \text { million KIU was added to the } \\
\text { priming solution of the extracorporeal circuit. } \\
\text { - Control group received similar volumes of saline. }\end{array}$} \\
\hline Outcomes & \multicolumn{2}{|c|}{$\begin{array}{l}\text { Outcomes reported: Number of patients exposed to allogeneic blood, blood loss (48hrs), mortality, } \\
\text { biochemistry and haematology values. }\end{array}$} \\
\hline Notes & \multicolumn{2}{|c|}{$\begin{array}{l}\text { Quality assessment score (Schulz criteria): 5/7 } \\
\text { Transfusion protocol used }\end{array}$} \\
\hline \multicolumn{3}{|l|}{ Risk of bias } \\
\hline Item & Authors' judgement & Description \\
\hline Adequate sequence generation? & Unclear & Unclear \\
\hline Allocation concealment? & Yes & Adequate \\
\hline
\end{tabular}




\section{Swart 1994 (Continued)}

\begin{tabular}{|c|c|c|}
\hline $\begin{array}{l}\text { Blinding? } \\
\text { All outcomes }\end{array}$ & Yes & Double blind \\
\hline
\end{tabular}

\section{Tabuchi 1994}

\begin{tabular}{|c|c|}
\hline Methods & $\begin{array}{l}\text { Method of randomisation was not described. The study solution was prepared by the pharmacy } \\
\text { department according to a randomised code, which was kept blind to all clinicians and investigators } \\
\text { until all data were obtained. }\end{array}$ \\
\hline Participants & $\begin{array}{l}40 \text { patients undergoing elective coronary artery bypass grafting were randomised to one of two } \\
\text { groups: } \\
\text { - Aprotinin group: } \mathrm{n}=19, \text { mean }(\mathrm{sd}) \text { age }=60.9(8.7) \text { years } \\
\text { - Control group (Placebo): } \mathrm{n}=17 \text {, mean }(\mathrm{sd}) \text { age }=60.2(8.6) \text { years } \\
\text { NB: Gender data were not reported. Four patients were excluded from the final analysis; three from } \\
\text { the placebo group for surgical bleeding requiring repeat thoracotomy, and one from the aprotinin } \\
\text { group for haemothorax. }\end{array}$ \\
\hline
\end{tabular}

- Aprotinin group received $325 \mathrm{mg}$ of aspirin orally 10 hours before operation and 2 million
KIU of aprotinin $(280 \mathrm{mg})$ added to the pump prime solution.
- Control group received $325 \mathrm{mg}$ of aspirin orally 10 hours before operation and $200 \mathrm{ml}$ of
placebo solution.

Outcomes

Outcomes reported: Number of patients exposed to allogeneic blood, allogeneic blood usage (units) , fresh frozen plasma usage (units), platelet usage (units), re-operation for bleeding, haemothorax.

\begin{tabular}{ll}
\hline Notes & $\begin{array}{l}\text { Quality assessment score (Schulz criteria): 5/7 } \\
\text { Transfusion protocol used }\end{array}$ \\
\hline
\end{tabular}

\section{Risk of bias}

\begin{tabular}{lll}
\hline Item & Authors' judgement & Description \\
\hline Adequate sequence generation? & Unclear & Unclear \\
\hline Allocation concealment? & Yes & Adequate \\
\hline $\begin{array}{l}\text { Blinding? } \\
\text { All outcomes }\end{array}$ & Yes & Double blind \\
\hline
\end{tabular}

\section{Taggart 2003}

Methods

A pre-determined randomisation scheme was generated by the pharmaceutical company supplying the trial drug. Sealed code break cards were available if necessary. The study was analysed on an intention-to-treat (ITT) basis and included those patients who received open-label aprotinin. 
Taggart 2003 (Continued)

\begin{tabular}{|c|c|c|}
\hline Participants & \multicolumn{2}{|c|}{$\begin{array}{l}74 \text { patients undergoing cardiac surgery with total arterial grafting were randomised to one of two } \\
\text { groups: } \\
\text { - Aprotinin group (High dose): } \mathrm{n}=37, \mathrm{M} / \mathrm{F}=33 / 3 \text {, mean }(\mathrm{sd}) \text { age }=60(8) \text { years } \\
\text { - Control group (Placebo): } \mathrm{n}=34, \mathrm{M} / \mathrm{F}=32 / 2 \text {, mean }(\mathrm{sd}) \text { age }=61 \text { (8) years } \\
\text { NB: Four patients were excluded from the final analysis. }\end{array}$} \\
\hline Interventions & \multicolumn{2}{|c|}{$\begin{array}{l}\text { - Aprotinin group (High dose) received a } 5 \mathrm{~mL}(1.4 \mathrm{mg} / \mathrm{mL}) \text { test dose of aprotinin after the } \\
\text { induction of anaesthesia and before sternotomy. The remaining } 195 \mathrm{~mL} \text { of the loading dose was } \\
\text { administered over } 20-30 \text { minutes using an infusion pump. After the completion of the loading } \\
\text { dose, a maintenance infusion of } 50 \mathrm{ml} / \mathrm{hr} \text { was continued for } 4 \text { hours. A further } 200 \mathrm{~mL} \text { was added } \\
\text { to the pump prime of the bypass circuit. } \\
\text { - Control group received an unspecified solution. }\end{array}$} \\
\hline Outcomes & \multicolumn{2}{|c|}{$\begin{array}{l}\text { Outcomes reported: } \text { Number of patients exposed to allogeneic blood, allogeneic blood usage (units) } \\
\text {, number of participants exposed to fresh frozen plasma and platelets, blood loss, myocardial } \\
\text { infarction, re-operation for bleeding, hospital length of stay. }\end{array}$} \\
\hline Notes & \multicolumn{2}{|c|}{$\begin{array}{l}\text { Quality assessment score (Schulz criteria): } 3 / 7 \\
\text { Transfusion protocol used } \\
\text { NB: Nine patients in the control group (placebo) received open-label aprotinin whilst two patients } \\
\text { in the aprotinin group received open-label aprotinin. }\end{array}$} \\
\hline \multicolumn{3}{|l|}{ Risk of bias } \\
\hline Item & Authors' judgement & Description \\
\hline Adequate sequence generation? & Unclear & Unclear \\
\hline Allocation concealment? & Yes & Adequate \\
\hline $\begin{array}{l}\text { Blinding? } \\
\text { All outcomes }\end{array}$ & No & Single blind \\
\hline
\end{tabular}

Taghaddomi 2009

Methods Table of random numbers was used to generate the allocation sequence. An independent anesthesiologist prepared coded infusions with tranexamic acid and placebo and was not directly involved in the clinical treatment of randomised patients. Both operating room staff and the intensive care unit staff were blinded regarding the study group.

$\begin{array}{ll}\text { Participants } & 100 \text { patients undergoing cardiac surgery were randomised to one of two groups: } \\ & \text { - Tranexamic acid group: } \mathrm{n}=50, \mathrm{M} / \mathrm{F}=38 / 12, \text { mean }(\mathrm{sd}) \text { age }=54.7 \text { (10.9) years } \\ \text { - Control group (Placebo): } \mathrm{n}=50, \mathrm{M} / \mathrm{F}=34 / 16, \text { mean }(\mathrm{sd}) \text { age }=60.3(10.2) \text { years }\end{array}$

Interventions

- Tranexamic acid group received a bolus of $1 \mathrm{~g}$ was given 20 minutes before incision then a maintenance dose of $400 \mathrm{mg} / \mathrm{hr}$ during the entire surgical procedure.

- Control group received normal saline. 
Taghaddomi 2009 (Continued)

\begin{tabular}{|c|c|c|}
\hline Outcomes & \multicolumn{2}{|c|}{$\begin{array}{l}\text { Outcomes reported: Number of patients exposed to allogeneic blood transfusion, blood loss, stroke, } \\
\text { renal failure, myocardial infarction. }\end{array}$} \\
\hline Notes & \multicolumn{2}{|l|}{$\begin{array}{l}\text { Quality assessment score (Schulz criteria): 6/7 } \\
\text { Transfusion protocol used }\end{array}$} \\
\hline \multicolumn{3}{|l|}{ Risk of bias } \\
\hline Item & Authors' judgement & Description \\
\hline Adequate sequence generation? & Yes & Table of random numbers \\
\hline Allocation concealment? & Yes & Adequate \\
\hline $\begin{array}{l}\text { Blinding? } \\
\text { All outcomes }\end{array}$ & Yes & Double blind \\
\hline
\end{tabular}

Tanaka 2001

\begin{tabular}{|c|c|}
\hline Methods & $\begin{array}{l}\text { Ampoules containing either tranexamic acid or placebo were numbered and placed in envelopes at } \\
\text { random by a pharmacologist. }\end{array}$ \\
\hline Participants & $\begin{array}{l}99 \text { patients undergoing elective knee arthroplasty were randomised to one of four groups: } \\
\text { - Control group (Placebo): } \mathrm{n}=26, \mathrm{M} / \mathrm{F}=9 / 17, \text { mean }(\text { range) age }=65(58-70) \text { years } \\
\text { - Tranexamic acid group (Pre-operative TXA): } \mathrm{n}=24, \mathrm{M} / \mathrm{F}=7 / 17 \text {, mean (range) age }=65 \\
(59-70) \text { years } \\
\text { - Tranexamic acid group (Intra-operative TXA): } \mathrm{n}=22, \mathrm{M} / \mathrm{F}=7 / 15 \text {, mean (range) age }=65 \\
\text { (60-71) years } \\
\text { - Tranexamic acid group (Pre-and-intra-operative TXA): } \mathrm{n}=27, \mathrm{M} / \mathrm{F}=8 / 19 \text {, mean (range) } \\
\text { age }=65(59-69) \text { years }\end{array}$ \\
\hline Interventions & $\begin{array}{l}\text { - Control group received saline twice, } 10 \text { minutes before surgery and on deflation of the } \\
\text { tourniquet. } \\
\text { - Tranexamic acid group (Pre-operative TXA) received } 20 \mathrm{mg} / \mathrm{kg} \text { of TXA } 10 \text { minutes before } \\
\text { surgery and saline } 10 \text { minutes before the deflation of the tourniquet. } \\
\text { - Tranexamic acid group (Intra-operative TXA) received saline } 10 \text { minutes before surgery and } \\
\text { 20mg/kg of TXA } 10 \text { minutes before deflation of the tourniquet. } \\
\text { - Tranexamic acid group (Pre-and-intra-operative TXA) received } 10 \mathrm{mg} \text { of TXA } 10 \text { minutes } \\
\text { before surgery and } 10 \mathrm{mg} / \mathrm{kg} 10 \text { minutes before deflation of the tourniquet. }\end{array}$ \\
\hline Outcomes & $\begin{array}{l}\text { Outcomes reported: Number of patients exposed to allogeneic blood, allogeneic blood usage (units) } \\
\text {, blood loss. }\end{array}$ \\
\hline Notes & $\begin{array}{l}\text { Quality assessment score (Schulz criteria): 6/7 } \\
\text { Transfusion protocol not used }\end{array}$ \\
\hline
\end{tabular}

\section{Risk of bias}


Tanaka 2001 (Continued)

\begin{tabular}{lll}
\hline Item & Authors' judgement & Description \\
\hline Adequate sequence generation? & Unclear & Unclear \\
\hline Allocation concealment? & Yes & Adequate \\
\hline $\begin{array}{l}\text { Blinding? } \\
\text { All outcomes }\end{array}$ & Yes & Double blind \\
\hline
\end{tabular}

Tassani 2000

\begin{tabular}{|c|c|c|}
\hline Methods & \multicolumn{2}{|c|}{$\begin{array}{l}\text { Study performed in a double blind, placebo controlled manner. Method of randomisation and } \\
\text { allocation concealment were not described. }\end{array}$} \\
\hline Participants & \multicolumn{2}{|c|}{$\begin{array}{l}20 \text { patients undergoing elective cardiac surgery were randomly assigned to one of two groups: } \\
\text { - Aprotinin group: } \mathrm{n}=10 \\
\text { - Control group (Placebo): } \mathrm{n}=10 \\
\text { NB: Gender and age data were not reported. }\end{array}$} \\
\hline Interventions & \multicolumn{2}{|c|}{$\begin{array}{l}\text { - Aprotinin group received a loading dose of } 2 \text { million KIU of aprotinin, a priming dose of } 2 \\
\text { million KIU, and a continuous infusion of 500,000 KIU/hr during surgery. } \\
\text { - Control group received an unspecified placebo. }\end{array}$} \\
\hline Outcomes & \multicolumn{2}{|c|}{$\begin{array}{l}\text { Outcomes reported: Number of patients exposed to allogeneic blood, allogeneic blood usage (units) } \\
\text {, blood loss. }\end{array}$} \\
\hline Notes & \multicolumn{2}{|c|}{$\begin{array}{l}\text { Quality assessment score (Schulz criteria): } 2 / 7 \\
\text { Transfusion protocol not used }\end{array}$} \\
\hline \multicolumn{3}{|l|}{ Risk of bias } \\
\hline Item & Authors' judgement & Description \\
\hline Adequate sequence generation? & Unclear & Unclear \\
\hline Allocation concealment? & Unclear & Unclear \\
\hline $\begin{array}{l}\text { Blinding? } \\
\text { All outcomes }\end{array}$ & Yes & Double blind \\
\hline
\end{tabular}

\section{Thorpe 1994}

\begin{tabular}{l|l}
\hline Methods & Method of randomisation and allocation concealment were not described. \\
\hline Participants & $\begin{array}{l}17 \text { patients undergoing elective knee replacement surgery were randomly allocated to one of two } \\
\text { groups: } \\
\begin{array}{l}\text { - Aprotinin group: } \mathrm{n}=8 \\
\text { A }\end{array}\end{array}$
\end{tabular}




\section{Thorpe 1994 (Continued)}

- Control group: $\mathrm{n}=9$

NB: Demographic data were not reported.

Interventions

- Aprotinin group received 0.5 million KIU of aprotinin over 20 minutes immediately before inflation of the tourniquet, another 0.5 million KIU of aprotinin over 20 minutes before deflation of the tourniquet followed by an infusion of 1 million KIU over the next 2 hours.

- Control group did not receive aprotinin.

Outcomes

Outcomes reported: Number of patients exposed to allogeneic blood, allogeneic blood usage (units) , blood loss, femoral thrombosis.

\begin{tabular}{ll}
\hline Notes & $\begin{array}{l}\text { Quality assessment score (Schulz criteria): } 1 / 7 \\
\text { Transfusion protocol not specified }\end{array}$ \\
\hline
\end{tabular}

\section{Risk of bias}

\begin{tabular}{l|ll}
\hline Item & Authors' judgement & Description \\
\hline Adequate sequence generation? & Unclear & Unclear \\
\hline Allocation concealment? & Unclear & Unclear \\
\hline $\begin{array}{l}\text { Blinding? } \\
\text { All outcomes }\end{array}$ & Unclear & Unclear \\
\hline
\end{tabular}

\section{Trinh-Duc 1992}

\begin{tabular}{l|l} 
Methods & Method of randomisation and allocation concealment were not described. [French language] \\
\hline Participants & $\begin{aligned} & 60 \text { patients undergoing cardiac surgery were randomised to one of two groups: } \\
& \text { - Aprotinin group (High dose): } \mathrm{n}=29, \mathrm{M} / \mathrm{F}=19 / 10, \text { mean }(\mathrm{sd}) \text { age }=54.89(14.92) \text { years } \\
& \text { - Epsilon aminocaproic acid group }(\mathrm{n}=27), \mathrm{M} / \mathrm{F}=20 / 7, \text { mean }(\mathrm{sd}) \text { age }=61.07(10.65) \text { years } \\
& \text { NB: Four patients were excluded from the final analysis. }\end{aligned}$ \\
\hline
\end{tabular}

Interventions

- Aprotinin group received 2 million KIU $(280 \mathrm{mg})$ of aprotinin after the induction of anaesthesia followed by a continuous infusion of 500,000 KIU/hr of aprotinin until skin closure. In addition, 2 million KIU $(280 \mathrm{mg})$ of aprotinin was added to the pump prime.

- Epsilon aminocaproic acid group received $5 \mathrm{~g}$ of EACA as a bolus after the induction of anaesthesia followed by a continuous infusion of $2 \mathrm{~g} / \mathrm{hr}$ until skin closure. In addition, $5 \mathrm{~g}$ of EACA was added to the pump prime.

NB: Both groups received cell salvage and acute normovolaemic haemodilution (ANH).

Outcomes

Notes
Outcomes reported: Number of patients exposed to allogeneic blood, allogeneic blood usage (units) , fresh frozen plasma usage (units), blood loss (48hrs), mortality, minor stroke, respiratory problems, severe hypotension. 
Trinh-Duc 1992 (Continued)

\begin{tabular}{lll} 
Risk of bias & & \\
\hline Item & Authors' judgement & Description \\
\hline Adequate sequence generation? & Unclear & Unclear \\
\hline Allocation concealment? & Unclear & Unclear \\
\hline $\begin{array}{l}\text { Blinding? } \\
\text { All outcomes }\end{array}$ & Unclear & Unclear \\
\hline
\end{tabular}

\section{Troianos 1999}

\begin{tabular}{|c|c|c|}
\hline Methods & \multicolumn{2}{|c|}{ Method of randomisation and allocation concealment were not described. } \\
\hline Participants & \multicolumn{2}{|c|}{$\begin{array}{l}72 \text { patients undergoing primary coronary artery bypass surgery were randomised to one of two } \\
\text { groups: } \\
\text { - Epsilon aminocaproic acid group: } \mathrm{n}=38, \mathrm{M} / \mathrm{F}=27 / 11 \text {, mean }(\mathrm{sd}) \text { age }=66(9) \text { years } \\
\text { - Control group (Placebo): } \mathrm{n}=36, \mathrm{M} / \mathrm{F}=24 / 12 \text {, mean }(\mathrm{sd}) \text { age }=65(9) \text { years }\end{array}$} \\
\hline Interventions & \multicolumn{2}{|c|}{$\begin{array}{l}\text { - Epsilon aminocaproic acid group received a bolus dose of } 0.5 \mathrm{ml} / \mathrm{kg} \text { of EACA administered } \\
\text { immediately after systemic heparization }(125 \mathrm{mg} / \mathrm{kg}) \text {, and an infusion of EACA commenced at } \\
0.05 \mathrm{ml} / \mathrm{kg} / \mathrm{hr}(12.5 \mathrm{mg} / \mathrm{kg} / \mathrm{hr}) \text { and continued until after the administration of protamine and } \\
\text { before the patient left the operating room. } \\
\text { - Control group received saline solution. }\end{array}$} \\
\hline Outcomes & \multicolumn{2}{|c|}{$\begin{array}{l}\text { Outcomes reported: Number of patients exposed to allogeneic blood, allogeneic blood usage (units) } \\
\text {, number of patients exposed to fresh frozen plasma and platelets, blood loss (6hrs \& 48hrs), re- } \\
\text { exploration for bleeding, haemoglobin loss. }\end{array}$} \\
\hline Notes & \multicolumn{2}{|c|}{$\begin{array}{l}\text { Quality assessment score (Schulz criteria): 3/7 } \\
\text { Transfusion protocol used }\end{array}$} \\
\hline \multicolumn{3}{|l|}{ Risk of bias } \\
\hline Item & Authors' judgement & Description \\
\hline Adequate sequence generation? & Unclear & Unclear \\
\hline Allocation concealment? & Unclear & Unclear \\
\hline $\begin{array}{l}\text { Blinding? } \\
\text { All outcomes }\end{array}$ & Yes & Double blind \\
\hline
\end{tabular}


Turkoz 2001

\begin{tabular}{|c|c|c|}
\hline Methods & \multicolumn{2}{|c|}{ Method of randomisation and allocation concealment were not described. } \\
\hline Participants & \multicolumn{2}{|c|}{$\begin{array}{l}30 \text { patients undergoing elective cardiac surgery were allocated randomly to one of three groups: } \\
\text { - Aprotonin group (High dose): } \mathrm{n}=10, \mathrm{M} / \mathrm{F}=9 / 1 \text {, mean }(\mathrm{sd}) \text { age }=60.2(3.4) \text { years } \\
\text { - Methylprednisolone group: } \mathrm{n}=10, \mathrm{M} / \mathrm{F}=8 / 2 \text {, mean }(\mathrm{sd}) \text { age }=58.3(3.0) \text { years } \\
\text { - Control group: } \mathrm{n}=10, \mathrm{M} / \mathrm{F}=9 / 1 \text {, mean }(\mathrm{sd}) \text { age }=63.8(1.9) \text { years }\end{array}$} \\
\hline Interventions & \multicolumn{2}{|c|}{$\begin{array}{l}\text { - Aprotinin group (High dose) received a loading dose of } 280 \mathrm{mg} \text { ( } 2 \text { million KIU) of aprotinin } \\
\text { followed by a continuous infusion of } 70 \mathrm{mg} / \mathrm{hr}(500,000 \mathrm{KIU} / \mathrm{hr} \text { ) administered during the } \\
\text { operation. In addition, } 280 \mathrm{mg} \text { ( } 2 \mathrm{million} \mathrm{KIU}) \text { of aprotinin was added to the pump prime. } \\
\text { - Methylprednisolone group received } 30 \mathrm{mg} / \mathrm{kg} \text { of methylprednisolone intravenously } 5 \\
\text { minutes before surgery. } \\
\text { - Control group received standard care. }\end{array}$} \\
\hline Outcomes & \multicolumn{2}{|c|}{ Outcomes reported: Allogeneic blood usage (units), blood loss. } \\
\hline Notes & \multicolumn{2}{|c|}{$\begin{array}{l}\text { Quality assessment score (Schulz criteria): 2/7 } \\
\text { Transfusion protocol used }\end{array}$} \\
\hline \multicolumn{3}{|l|}{ Risk of bias } \\
\hline Item & Authors' judgement & Description \\
\hline Adequate sequence generation? & Unclear & Unclear \\
\hline Allocation concealment? & Unclear & Unclear \\
\hline $\begin{array}{l}\text { Blinding? } \\
\text { All outcomes }\end{array}$ & Unclear & Unclear \\
\hline
\end{tabular}

\section{Uozaki 2001}

\begin{tabular}{l|l}
\hline Methods & Method of randomisation and allocation concealment were not described. \\
\hline Participants & $\begin{array}{r}\text { 14 patients undergoing elective cardiac surgery were allocated to one of two groups: } \\
\text { - Tranexamic acid group: } \mathrm{n}=14, \mathrm{M} / \mathrm{F}=5 / 1, \text { mean }(\mathrm{sd}) \text { age }=72.3 \text { (4.1) years } \\
\text { - Control group: } \mathrm{n}=7, \mathrm{M} / \mathrm{F}=3 / 3, \mathrm{mean}(\mathrm{sd}) \text { age }=63.3(5.3) \text { years }\end{array}$ \\
\hline Interventions & $\begin{array}{l}\text { - Tranexamic acid group received } 50 \mathrm{mg} / \mathrm{kg} \text { of intravenous TXA before skin incision and after } \\
\text { the start of CPB. }\end{array}$ \\
\hline Outcomes & Outcomes reported: Allogeneic blood usage (units), blood loss (24hrs), re-operation for bleeding. \\
\hline Notes & $\begin{array}{l}\text { Quality assessment score (Schulz criteria): 0/7 } \\
\text { Transfusion protocol not used }\end{array}$ \\
\hline
\end{tabular}

\section{Risk of bias}


Uozaki 2001 (Continued)

\begin{tabular}{lll}
\hline Item & Authors' judgement & Description \\
\hline Adequate sequence generation? & Unclear & Unclear \\
\hline $\begin{array}{l}\text { Allocation concealment? } \\
\begin{array}{l}\text { Blinding? } \\
\text { All outcomes }\end{array}\end{array}$ & Unclear & Unclear \\
\hline
\end{tabular}

Urban 2001

\begin{tabular}{|c|c|c|}
\hline Methods & \multicolumn{2}{|c|}{$\begin{array}{l}\text { Patients were randomised by means of a random number generator. Method used to conceal } \\
\text { treatment allocation was not described. }\end{array}$} \\
\hline Participants & \multicolumn{2}{|c|}{$\begin{array}{l}60 \text { patients undergoing complex reconstructive spinal surgery were randomised to one of three } \\
\text { groups: } \\
\text { - Aprotinin group: } \mathrm{n}=20 \text {, mean }(\mathrm{sd}) \text { age }=47.2 \text { years } \\
\text { - Epsilon aminocaproic acid group: } \mathrm{n}=17, \text { mean }(\mathrm{sd}) \text { age }=46.6 \text { years } \\
\text { - Control group: } \mathrm{n}=18 \text {, mean }(\mathrm{sd}) \text { age }=47.3 \text { years } \\
\text { NB: Gender data were not reported. Five patients were excluded from the final analysis. }\end{array}$} \\
\hline Interventions & \multicolumn{2}{|c|}{$\begin{array}{l}\text { - Aprotinin group received } 1 \text { million KIU of aprotinin as a loading dose over } 30 \text { minutes } \\
\text { followed by } 250,000 \mathrm{KIU} / \mathrm{hr} \text {. } \\
\text { - Epsilon aminocaproic acid group received a } 5 \mathrm{~g} \text { loading dose over } 30 \text { minutes followed by } \\
15 \mathrm{mg} / \mathrm{kg} / \mathrm{hr} \text {. } \\
\text { - Control group received no antifibrinolytic treatment. } \\
\text { NB: All groups were exposed to cell salvage and pre-operative autologous blood donation (PAD). }\end{array}$} \\
\hline Outcomes & \multicolumn{2}{|c|}{$\begin{array}{l}\text { Outcomes reported: Allogeneic \& autologous blood usage (units), blood loss, respiratory compli- } \\
\text { cations. }\end{array}$} \\
\hline Notes & \multicolumn{2}{|c|}{$\begin{array}{l}\text { Quality assessment score (Schulz criteria): 2/7 } \\
\text { Transfusion protocol used }\end{array}$} \\
\hline \multicolumn{3}{|l|}{ Risk of bias } \\
\hline Item & Authors' judgement & Description \\
\hline Adequate sequence generation? & Yes & Random number generator \\
\hline Allocation concealment? & Unclear & Unclear \\
\hline $\begin{array}{l}\text { Blinding? } \\
\text { All outcomes }\end{array}$ & Unclear & Unclear \\
\hline
\end{tabular}


Utada 1997

\begin{tabular}{|c|c|c|}
\hline Methods & \multicolumn{2}{|c|}{ Method of randomisation and allocation concealment were not described. [Japanese language] } \\
\hline Participants & \multicolumn{2}{|c|}{$\begin{array}{l}21 \text { patients undergoing primary total hip replacement were randomised to one of two groups: } \\
\text { - Aprotinin group (Low dose): } \mathrm{n}=11, \mathrm{M} / \mathrm{F}=1 / 10 \text {, mean }(\mathrm{sd}) \text { age }=63(11) \text { years } \\
\text { - Control group (Placebo): } \mathrm{n}=10, \mathrm{M} / \mathrm{F}=2 / 8 \text {, mean }(\mathrm{sd}) \text { age }=64 \text { (5) years }\end{array}$} \\
\hline Interventions & \multicolumn{2}{|c|}{$\begin{array}{l}\text { - Aprotinin group (Low dose) received } 2 \text { million KIU }(280 \mathrm{mg}) \text { of aprotinin as a continuous } \\
\text { infusion throughout the surgical procedure. } \\
\text { - Control group received normal saline solution. }\end{array}$} \\
\hline Outcomes & \multicolumn{2}{|c|}{ Outcomes reported: Allogeneic blood usage (units), blood loss, changes in haemoglobin levels. } \\
\hline Notes & \multicolumn{2}{|c|}{ Transfusion protocol not used } \\
\hline \multicolumn{3}{|l|}{ Risk of bias } \\
\hline Item & Authors' judgement & Description \\
\hline Adequate sequence generation? & Unclear & Unclear \\
\hline Allocation concealment? & Unclear & Unclear \\
\hline $\begin{array}{l}\text { Blinding? } \\
\text { All outcomes }\end{array}$ & Unclear & Unclear \\
\hline
\end{tabular}

\section{Van der Linden 2005}

\begin{tabular}{ll} 
Methods & $\begin{array}{l}\text { Random assignment was conducted using unmasked envelopes, each containing a card indicating } \\
\text { treatment with aprotinin or placebo. A nurse, assigned to another department of the hospital } \\
\text { was responsible for the preparation of placebo and treatment solutions, which were identical in } \\
\text { appearance and packing. }\end{array}$ \\
\hline Participants & $\begin{array}{l}75 \text { patients scheduled for urgent or acute isolated coronary artery bypass graft surgery were ran- } \\
\text { domised to one of two groups: } \\
\text { - Aprotinin group (High dose): } \mathrm{n}=37, \mathrm{M} / \mathrm{F}=31 / 6, \text { mean }(\mathrm{sd}) \text { age }=66.4(10) \text { years } \\
\text { - Control group (Placebo): } \mathrm{n}=38, \mathrm{M} / \mathrm{F}=25 / 13, \text { mean }(\mathrm{sd}) \text { age }=68.3(10) \text { years }\end{array}$ \\
\hline Interventions & $\begin{array}{l}\text { - Aprotinin group (High dose) received a } 1 \mathrm{ml} \text { test dose of aprotinin after the induction of } \\
\text { anaesthesia then received } 2 \mathrm{million} \mathrm{KIU}(280 \mathrm{mg}) \text { of aprotinin as a bolus before the start of } \\
\text { surgery. Another } 2 \text { million KIU of aprotinin was added to the pump prime and a continuous } \\
\text { infusion of } 500,000 \mathrm{KIU} / \mathrm{hr} \text { was infused during surgery. } \\
\text { - Control group received an equal volume of saline solution at the same time periods as the } \\
\text { aprotinin regimen. }\end{array}$ \\
\hline
\end{tabular}

Outcomes

Outcomes reported: Number of patients exposed to allogeneic blood, allogeneic blood usage (units) , fresh frozen plasma usage (units), platelets usage (units), mortality, myocardial infarction, stroke, atrial fibrillation, number of patients receiving TXA treatment. 
Van der Linden 2005 (Continued)

\begin{tabular}{|c|c|c|}
\hline Notes & \multicolumn{2}{|c|}{$\begin{array}{l}\text { Quality assessment score (Schulz criteria): 4/7 } \\
\text { Transfusion protocol used }\end{array}$} \\
\hline \multicolumn{3}{|l|}{ Risk of bias } \\
\hline Item & Authors' judgement & Description \\
\hline Adequate sequence generation? & Unclear & Unclear \\
\hline Allocation concealment? & No & Inadequate - sealed envelopes \\
\hline $\begin{array}{l}\text { Blinding? } \\
\text { All outcomes }\end{array}$ & Yes & Double blind \\
\hline
\end{tabular}

Van Oeveren 1987

\begin{tabular}{|c|c|c|}
\hline Methods & \multicolumn{2}{|c|}{ Method of randomisation and allocation concealment were not described. } \\
\hline Participants & \multicolumn{2}{|c|}{$\begin{array}{l}22 \text { patients undergoing coronary artery bypass graft surgery were randomised to one of two groups: } \\
\text { - Aprotinin group: } \mathrm{n}=11 \text {, mean }(\mathrm{sd}) \text { age }=56.2(3.9) \text { years } \\
\text { - Control group: } \mathrm{n}=11 \text {, mean }(\mathrm{sd}) \text { age }=57.5(5.1) \text { years }\end{array}$} \\
\hline Interventions & \multicolumn{2}{|c|}{$\begin{array}{l}\text { - Aprotinin group received an infusion of } 2 \text { million KIU ( } 280 \mathrm{mg} \text { ) of aprotinin over } 20-30 \\
\text { minutes and a continuous infusion of } 500,000 \mathrm{KIU} / \mathrm{hr} \text { until the end of the operation. In } \\
\text { addition, for each litre of transfused whole blood given during the operation, an additional } \\
500,000 \mathrm{KIU} \text { of aprotinin was administered by a separate bolus infusion. } \\
\text { - Control group did not receive aprotinin. }\end{array}$} \\
\hline Outcomes & \multicolumn{2}{|c|}{ Outcomes reported: Allogeneic blood usage, blood loss, biochemical markers. } \\
\hline Notes & \multicolumn{2}{|c|}{$\begin{array}{l}\text { Quality assessment score (Schulz criteria): 0/7 } \\
\text { Transfusion protocol not used }\end{array}$} \\
\hline \multicolumn{3}{|l|}{ Risk of bias } \\
\hline Item & Authors' judgement & Description \\
\hline Adequate sequence generation? & Unclear & Unclear \\
\hline Allocation concealment? & Unclear & Unclear \\
\hline $\begin{array}{l}\text { Blinding? } \\
\text { All outcomes }\end{array}$ & Unclear & Unclear \\
\hline
\end{tabular}


Vander-Salm 1996

\begin{tabular}{|c|c|c|}
\hline Methods & \multicolumn{2}{|c|}{$\begin{array}{l}\text { Random assignment was by means of a random number table and drug preparation was performed } \\
\text { by the hospital pharmacy. }\end{array}$} \\
\hline Participants & \multicolumn{2}{|c|}{$\begin{array}{l}103 \text { patients undergoing coronary artery bypass graft surgery or valvular surgery were randomly } \\
\text { assigned to one of two groups: } \\
\text { - Epsilon aminocaproic acid group: } \mathrm{n}=51, \mathrm{M} / \mathrm{F}=35 / 16 \text {, mean }(\mathrm{sd}) \text { age }=64.7(12.1) \text { years } \\
\text { - Control group (Placebo): } \mathrm{n}=52, \mathrm{M} / \mathrm{F}=40 / 12 \text {, mean }(\mathrm{sd}) \text { age }=64.2(12.4) \text { years }\end{array}$} \\
\hline Interventions & \multicolumn{2}{|c|}{$\begin{array}{l}\text { - Epsilon aminocaproic acid group received } 10 \mathrm{mg} \text { of EACA intravenously before skin } \\
\text { incision, } 10 \mathrm{~g} \text { of EACA after heparin administration, and } 10 \mathrm{~g} \text { of EACA at discontinuation of } \\
\text { cardiopulmonary bypass }(\mathrm{CPB}) \text { but before protamine administration. } \\
\text { - Control group received saline solution in the same volumes and with the same timing as the } \\
\text { EACA treated group. }\end{array}$} \\
\hline Outcomes & \multicolumn{2}{|c|}{$\begin{array}{l}\text { Outcomes reported: Number of patients exposed to allogeneic blood, allogeneic blood usage (units) } \\
\text {, number of patients exposed to fresh frozen plasma, blood loss (12hrs \& 24hrs), mortality, cere- } \\
\text { brovascular accident, re-operation for bleeding. }\end{array}$} \\
\hline Notes & \multicolumn{2}{|c|}{$\begin{array}{l}\text { Quality assessment score (Schulz criteria): 7/7 } \\
\text { Transfusion protocol used }\end{array}$} \\
\hline \multicolumn{3}{|l|}{ Risk of bias } \\
\hline Item & Authors' judgement & Description \\
\hline Adequate sequence generation? & Yes & Random number table \\
\hline Allocation concealment? & Yes & Adequate \\
\hline $\begin{array}{l}\text { Blinding? } \\
\text { All outcomes }\end{array}$ & Yes & Double blind \\
\hline
\end{tabular}

Vanek 2005

Methods

An envelope method with random numbers was used to randomise patients. An independent pharmacologist not directly involved in the clinical treatment of randomised patients prepared coded infusions with the study drug and placebo.

Participants

91 patients undergoing cardiac surgery were randomised to one of three groups:

- Tranexamic acid group: $\mathrm{n}=32, \mathrm{M} / \mathrm{F}=16 / 16$, mean $(95 \% \mathrm{CI})$ age $=68.4(64.6-72.2)$ years

- Aprotinin group: $\mathrm{n}=29, \mathrm{M} / \mathrm{F}=20 / 9$, mean $(95 \% \mathrm{CI})$ age $=67.3(64.2-70.4)$ years

- Control group: $\mathrm{n}=30, \mathrm{M} / \mathrm{F}=22 / 8$, mean $(95 \% \mathrm{CI})$ age $=68.9(65.8-72.0)$ years

Interventions

- Tranexamic acid group received $1 \mathrm{~g}$ TXA before skin incision and a continuous infusion of $200 \mathrm{mg} / \mathrm{hr}$ during the whole surgical procedure.

- Aprotinin group received 1 million KIU of aprotinin before skin incision and a continuous infusion of 250,000 KIU/hr during the whole surgical procedure.

- Control group received normal saline $0.9 \%$ before skin incision and a continuous infusion 
during the whole surgical procedure.

\begin{tabular}{|c|c|c|}
\hline Outcomes & \multicolumn{2}{|c|}{$\begin{array}{l}\text { Outcomes reported: Number of patients exposed to allogeneic blood, blood loss, number of patients } \\
\text { exposed to fresh frozen plasma. }\end{array}$} \\
\hline Notes & \multicolumn{2}{|l|}{$\begin{array}{l}\text { Quality assessment score (Schulz criteria): 6/7 } \\
\text { Transfusion protocol used }\end{array}$} \\
\hline \multicolumn{3}{|l|}{ Risk of bias } \\
\hline Item & Authors' judgement & Description \\
\hline Adequate sequence generation? & Yes & Random numbers \\
\hline Allocation concealment? & Yes & Adequate \\
\hline $\begin{array}{l}\text { Blinding? } \\
\text { All outcomes }\end{array}$ & Yes & Double blind \\
\hline
\end{tabular}

\section{Vedrinne 1992}

\begin{tabular}{|c|c|}
\hline Methods & Method of randomisation and allocation concealment were not described. \\
\hline Participants & $\begin{array}{l}90 \text { consecutive patients undergoing cardiac surgery were randomly allocated to one of three groups: } \\
\text { - Aprotinin group: } \mathrm{n}=30, \mathrm{M} / \mathrm{F}=23 / 7, \text { mean }(\mathrm{sd}) \text { age }=58(8) \text { years } \\
\text { - Auto-transfusion group: } \mathrm{n}=30, \mathrm{M} / \mathrm{F}=20 / 10 \text {, mean }(\mathrm{sd}) \text { age }=57(7) \text { years } \\
\text { - Control group: } \mathrm{n}=30, \mathrm{M} / \mathrm{F}=24 / 6 \text {, mean }(\mathrm{sd}) \text { age }=59(10) \text { years }\end{array}$ \\
\hline Interventions & $\begin{array}{l}\text { - Aprotinin group received } 2 \mathrm{million} \mathrm{KIU} \text { of aprotinin at the induction of anaesthesia infused } \\
\text { over } 20-30 \text { minutes ( } 10,000 \mathrm{KIU} / \mathrm{ml} \text { of pure aprotinin without additives) followed by a } \\
\text { continuous infusion of } 500,000 \mathrm{KIU} / \mathrm{hr} \text { of aprotinin administered throughout the operation. In } \\
\text { addition, } 2 \text { million KIU of aprotinin was added to the priming solution of the extracorporeal } \\
\text { circuit. } \\
\text { - Auto-transfusion group had } 400 \mathrm{ml} \text { of autologous blood withdrawn into citrate-phosphate- } \\
\text { dextrose during electrocardiographic and haemodynamic monitoring. Blood was withdrawn after } \\
\text { the induction of anaesthesia and before skin incision. Withdrawn blood was concomitantly } \\
\text { replaced by } 500 \mathrm{ml} \text { of } 4 \% \text { albumin. Autologous blood was kept at room temperature (18-20 } \\
\text { degrees) and was transfused after the completion of cardiopulmonary bypass, but before the } \\
\text { patients were transferred to the Intensive Care Unit (ICU). } \\
\text { - Control group patients underwent routine management without autologous transfusion or } \\
\text { aprotinin treatment. }\end{array}$ \\
\hline Outcomes & $\begin{array}{l}\text { Outcomes reported: Number of patients exposed to allogeneic blood, allogeneic blood usage (units) } \\
\text {, number of patients exposed to fresh frozen plasma and platelets, } \\
\text { blood loss (6hrs \& } 48 \mathrm{hrs} \text { ), re-operation for bleeding, haemoglobin loss. }\end{array}$ \\
\hline Notes & $\begin{array}{l}\text { Quality assessment score (Schulz criteria): 2/7 } \\
\text { Transfusion protocol used }\end{array}$ \\
\hline
\end{tabular}


Vedrinne 1992 (Continued)

\begin{tabular}{l|ll} 
Risk of bias & & \\
\hline Item & Authors' judgement & Description \\
\hline Adequate sequence generation? & Unclear & Unclear \\
\hline Allocation concealment? & Unclear & Unclear \\
\hline $\begin{array}{l}\text { Blinding? } \\
\text { All outcomes }\end{array}$ & Unclear & Unclear \\
\hline
\end{tabular}

\section{Veien 2002}

\begin{tabular}{|c|c|}
\hline Methods & $\begin{array}{l}\text { Patients were randomised using a computer generated randomisation table to treatment groups. } \\
\text { Method of allocation concealment was not described. }\end{array}$ \\
\hline Participants & $\begin{array}{l}30 \text { patients undergoing elective orthopaedic surgery were randomly assigned to one of two groups: } \\
\text { - Tranexamic acid group: } \mathrm{n}=15, \mathrm{M} / \mathrm{F}=4 / 11 \text {, mean }(\mathrm{sd}) \text { age }=70.5(9.5) \text { years } \\
\text { - Control group: } \mathrm{n}=15, \mathrm{M} / \mathrm{F}=1 / 14 \text {, mean (sd) age }=69.5(9.0) \text { years }\end{array}$ \\
\hline Interventions & $\begin{array}{l}\text { - Tranexamic acid group received } 10 \mathrm{mg} / \mathrm{kg} \text { of TXA administered just before the release of the } \\
\text { tourniquet, and } 10 \mathrm{mg} / \mathrm{kg} \text { of TXA given } 3 \text { hours later in the recovery room. Although a maximum } \\
\text { of } 1 \mathrm{~g} \text { was given each time. } \\
\text { - Control group received standard care without TXA treatment. } \\
\text { NB: All groups were exposed to cell salvage. }\end{array}$ \\
\hline
\end{tabular}

Outcomes

Outcomes reported: Number of patients exposed to allogeneic blood, allogeneic blood usage (units) , cell salvage autologous blood returned, thrombo-embolic events.

Notes

Quality assessment score (Schulz criteria):3/7

Transfusion protocol used

\section{Risk of bias}

\begin{tabular}{l|ll}
\hline Item & Authors' judgement & Description \\
\hline Adequate sequence generation? & Unclear & Computer generated randomisation table \\
\hline Allocation concealment? & Unclear & Unclear \\
\hline $\begin{array}{l}\text { Blinding? } \\
\text { All outcomes }\end{array}$ & Unclear & Unclear \\
\hline
\end{tabular}




\begin{tabular}{|c|c|c|}
\hline Methods & \multicolumn{2}{|c|}{ Method of allocation concealment and randomisation were not described. } \\
\hline Participants & \multicolumn{2}{|c|}{$\begin{array}{l}112 \text { patients undergoing 'off-pump' coronary artery bypass graft surgery were randomised to one } \\
\text { of two groups: } \\
\text { - Aprotinin group: } \mathrm{n}=36, \mathrm{M} / \mathrm{F}=28 / 8 \text {, mean }(\mathrm{sd}) \text { age }=61.4(7.5) \text { years } \\
\text { - Tranexamic acid group: } \mathrm{n}=36, \mathrm{M} / \mathrm{F}=28 / 8 \text {, mean }(\mathrm{sd}) \text { age }=62.8(7.9) \text { years } \\
\text { - Control group (Placebo): } \mathrm{n}=40, \mathrm{M} / \mathrm{F}=32 / 8 \text {, mean }(\mathrm{sd}) \text { age }=60.7(8.0) \text { years }\end{array}$} \\
\hline Interventions & \multicolumn{2}{|c|}{$\begin{array}{l}\text { - Aprontinin group received } 1 \text { million KIU loading dose at beginning of surgery, followed by } \\
\text { continuous infusion of } 500000 \mathrm{KIU} \text { per hour during surgery. } \\
\text { - Tranexamic acid group received a loading dose of } 0.75 \mathrm{~g} \text { of TXA over } 20 \text { minutes at the } \\
\text { beginning of surgery followed by a continuous infusion of } 0.25 \mathrm{~g} / \mathrm{hr} \text { throughout surgery. } \\
\text { - Control group received the same volume of saline solution. }\end{array}$} \\
\hline Outcomes & \multicolumn{2}{|c|}{$\begin{array}{l}\text { Outcomes reported: Number of patients exposed to allogeneic blood, allogeneic blood usage (units) } \\
\text {, fresh frozen plasma usage (units), number of patients exposed to fresh frozen plasma, blood loss } \\
\text { ( } 24 \mathrm{hrs} \text { ), hospital length of stay (days), Intensive Care Unit length of stay (days). }\end{array}$} \\
\hline Notes & \multicolumn{2}{|c|}{$\begin{array}{l}\text { Quality assessment score (Schulz criteria): } 4 / 7 \\
\text { Transfusion protocol used } \\
\text { Results for aprotinin versus control and TXA versus control - reported in separate publications. }\end{array}$} \\
\hline \multicolumn{3}{|l|}{ Risk of bias } \\
\hline Item & Authors' judgement & Description \\
\hline Adequate sequence generation? & Unclear & Unclear \\
\hline Allocation concealment? & Unclear & Unclear \\
\hline $\begin{array}{l}\text { Blinding? } \\
\text { All outcomes }\end{array}$ & Yes & Double blind \\
\hline
\end{tabular}

\section{Wendel 1995}

Methods

Method of randomisation was not described. Aprotinin and placebo were provided by the manufacturer in identical bottles that differed only in the random numbers on their labels.

Participants

40 patients undergoing aorto-coronary artery bypass graft surgery were randomised to one of two groups:

- Aprotinin group: $\mathrm{n}=20$, mean $(\mathrm{sd})$ age $=62.4(7.4)$ years

- Control group (Placebo): $\mathrm{n}=20$, mean $(\mathrm{sd})$ age $=60.6(7.7)$ years

NB: Gender data were not reported.

Interventions

- Aprotinin group received 30,000 KIU/kg of aprotinin as a loading dose over 20 minutes, followed by a continuous infusion of 7,000 KIU/ $\mathrm{kg} / \mathrm{per} / \mathrm{hr}$. In addition, 30,000 KIU $/ \mathrm{kg}$ of aprotinin was added to the priming solution after 5 minutes of extracorporeal circulation (ECC).

- Control group received physiologic saline solution. 
Wendel 1995 (Continued)

\begin{tabular}{|c|c|c|}
\hline Outcomes & \multicolumn{2}{|c|}{$\begin{array}{l}\text { Outcomes reported: Number of patients exposed to allogeneic blood, allogeneic blood usage (units) } \\
\text {, blood loss, myocardial infarction, infarctional biomarkers. }\end{array}$} \\
\hline Notes & \multicolumn{2}{|c|}{$\begin{array}{l}\text { Quality assessment score (Schulz criteria): 5/7 } \\
\text { Transfusion protocol not specified }\end{array}$} \\
\hline \multicolumn{3}{|l|}{ Risk of bias } \\
\hline Item & Authors' judgement & Description \\
\hline Adequate sequence generation? & Unclear & Unclear \\
\hline Allocation concealment? & Yes & Adequate \\
\hline $\begin{array}{l}\text { Blinding? } \\
\text { All outcomes }\end{array}$ & Yes & Double blind \\
\hline
\end{tabular}

\section{Wong 2000}

Methods The randomisation and preparation of study drugs was performed by the hospitals department of pharmacy. There was no attempt to stratify the randomisation process.

Participants
- Tranexamic acid group: $\mathrm{n}=40$, mean $(\mathrm{sd})$ age $=66.0(10.9)$ years
- Aprotinin group (High dose): $\mathrm{n}=40$, mean $(\mathrm{sd})$ age $=65.4$ (8.6) years
NB: Gender data were not reported.

Interventions

- Tranexamic acid group received a bolus of $10 \mathrm{~g}$ of TXA over 20 minutes after the induction of anaesthesia and before skin incision. Normal saline $0.9 \%$ was used during the other time periods similar to the aprotinin regimen. A test dose of $1 \mathrm{~mL}$ was given.

- Aprotinin group (High dose) received an infusion of 2 million KIU $(280 \mathrm{mg})$ of aprotinin infused over 20 minutes after the induction of anaesthesia and before skin incision, followed by a continuous infusion of 500,000 KIU/hr administered throughout the operation until skin closure. In addition, 2 million KIU ( $280 \mathrm{mg}$ ) was added to the pump prime.

NB: Both groups were exposed to cell salvage.

Outcomes

Outcomes reported: Number of patients exposed to allogeneic blood, allogeneic blood usage (units) , blood loss (24hrs), myocardial infarction, mortality, fresh frozen plasma usage, platelet usage (units), re-operation for bleeding, stroke.

$\begin{array}{ll}\text { Notes } & \text { Quality assessment score (Schulz criteria): 5/7 } \\ \text { Transfusion protocol used }\end{array}$

Transfusion protocol used

Risk of bias

\section{Description}


Wong 2000 (Continued)

\begin{tabular}{llll}
\hline Adequate sequence generation? & Unclear & Unclear \\
\hline Allocation concealment? & Yes & Adequate \\
\hline $\begin{array}{l}\text { Blinding? } \\
\text { All outcomes }\end{array}$ & Yes & Double blind \\
\hline
\end{tabular}

\section{Wong 2008}

\begin{tabular}{|c|c|c|}
\hline Methods & \multicolumn{2}{|c|}{$\begin{array}{l}\text { A computer-generated randomisation list was used for sequence generation. The randomisation } \\
\text { schedule was kept inaccessible throughout the study period. Patient assignments were placed into } \\
\text { sequentially numbered opaque sealed envelopes. A research pharmacist, not involved with care } \\
\text { of the patient prepared the placebo and treatment medications that were identical in appearance } \\
\text { The research personnel, anaesthesiologists, surgeons, and operating room staff were blinded to the } \\
\text { randomisation. }\end{array}$} \\
\hline Participants & \multicolumn{2}{|c|}{$\begin{array}{l}151 \text { patients undergoing orthopaedic (spinal) surgery were randomised to one of two groups: } \\
\text { - Tranexamic acid group: } \mathrm{n}=73, \mathrm{M} / \mathrm{F}=21 / 52 \text {, mean }(\mathrm{sds}) \text { age }=56.8(16.2) \text { years } \\
\text { - Control group (Placebo): } \mathrm{n}=74, \mathrm{M} / \mathrm{F}=26 / 48 \text {, mean (sd) age }=50.0(16.2) \text { years }\end{array}$} \\
\hline Interventions & \multicolumn{2}{|c|}{$\begin{array}{l}\text { - Tranexamic acid group received a bolus of } 10 \mathrm{mg} / \mathrm{kg} \mathrm{IV} \text {, then maintenance infusion of } 1 \mathrm{mg} / \\
\mathrm{kg} / \mathrm{hr} \text { until skin closure. } \\
\text { - Control group received the same volume of saline solution. }\end{array}$} \\
\hline Outcomes & \multicolumn{2}{|c|}{$\begin{array}{l}\text { Outcomes reported: number of patients exposed to allogeneic blood transfusion, blood loss, volume } \\
\text { blood transfused (units), deep vein thrombosis. }\end{array}$} \\
\hline Notes & \multicolumn{2}{|c|}{ Transfusion protocol used } \\
\hline \multicolumn{3}{|l|}{ Risk of bias } \\
\hline Item & Authors' judgement & Description \\
\hline Adequate sequence generation? & Yes & Computer-generated randomisation list \\
\hline Allocation concealment? & Yes & Adequate \\
\hline $\begin{array}{l}\text { Blinding? } \\
\text { All outcomes }\end{array}$ & Yes & Double blind \\
\hline
\end{tabular}


Wu 2006

\begin{tabular}{|c|c|c|}
\hline Methods & \multicolumn{2}{|c|}{$\begin{array}{l}\text { Method of randomisation was not described. Sealed envelopes were used to conceal treatment } \\
\text { allocation. }\end{array}$} \\
\hline Participants & \multicolumn{2}{|c|}{$\begin{array}{l}217 \text { patients undergoing liver tumor resection were randomised to one of two groups: } \\
\text { - Tranexamic acid group: } \mathrm{n}=106, \mathrm{M} / \mathrm{F}=77 / 29 \text {, mean (range) age }=62(22-88) \text { years } \\
\text { - Control group (Placebo): } \mathrm{n}=108, \mathrm{M} / \mathrm{F}=80 / 28 \text {, mean (range) age }=57(28-84) \text { years } \\
\text { NB: Three patients were excluded from the final analysis. }\end{array}$} \\
\hline Interventions & \multicolumn{2}{|c|}{$\begin{array}{l}\text { - Tranexamic acid group received } 500 \mathrm{mg} \text { of intravenous TXA administered just before the } \\
\text { operation, then received } 250 \mathrm{mg} \text { of intravenous TXA every } 6 \text { hours for } 3 \text { days. } \\
\text { - Control group group received a similar volume of normal saline at the same time intervals as } \\
\text { the TXA drug regimen. }\end{array}$} \\
\hline Outcomes & \multicolumn{2}{|c|}{$\begin{array}{l}\text { Outcomes reported: Number of patients exposed to allogeneic blood, blood loss, hospital length } \\
\text { of stay (days), wound infection. }\end{array}$} \\
\hline Notes & \multicolumn{2}{|c|}{$\begin{array}{l}\text { Quality assessment score (Schulz criteria): } 4 / 7 \\
\text { Transfusion protocol used }\end{array}$} \\
\hline \multicolumn{3}{|l|}{ Risk of bias } \\
\hline Item & Authors' judgement & Description \\
\hline Adequate sequence generation? & Unclear & Unclear \\
\hline Allocation concealment? & No & Inadequate - sealed envelopes \\
\hline $\begin{array}{l}\text { Blinding? } \\
\text { All outcomes }\end{array}$ & Yes & Double blind \\
\hline
\end{tabular}

Yamasaki 2004

\begin{tabular}{l|l} 
Methods & $\begin{array}{l}\text { Randomisation was carried out by a person not involved in the operation using a ticket drawn from } \\
\text { an envelope containing an equal number of tranexamic acid and placebo tickets. }\end{array}$ \\
\hline Participants & $\begin{array}{l}40 \text { patients undergoing cementless total hip arthroplasty were randomised to one of two groups: } \\
\text { - Tranexamic acid group: } \mathrm{n}=20, \mathrm{M} / \mathrm{F}=19 / 1, \text { mean }(\mathrm{sd}) \text { age }=55.5(14.2) \text { years } \\
\text { - Control group: } \mathrm{n}=20, \mathrm{M} / \mathrm{F}=18 / 2, \mathrm{mean}(\mathrm{sd}) \text { age }=61.2(6.9) \text { years }\end{array}$ \\
\hline Interventions & $\begin{array}{l}\text { - Tranexamic acid group received } 1,000 \mathrm{mg} \text { of TXA administered intravenously } 5 \text { minutes } \\
\text { before the start of the operation. } \\
\text { - Control group did not receive TXA treatment. } \\
\text { NB: Both groups received pre-operatively donated autologous blood (PAD). }\end{array}$ \\
\hline
\end{tabular}

Outcomes

Outcomes reported: Number of patients exposed to allogeneic blood, blood loss (24hrs), thromboembolic events. 


\begin{tabular}{l|ll}
\hline Notes & $\begin{array}{l}\text { Quality assessment score (Schulz criteria): 2/7 } \\
\text { Transfusion protocol not used }\end{array}$ \\
\hline Risk of bias & Authors' judgement & Description \\
\hline Item & Yes & Adequate \\
\hline Adequate sequence generation? & No & Inadequate \\
\hline Allocation concealment? & Unclear & Unclear \\
\hline $\begin{array}{l}\text { Blinding? } \\
\text { All outcomes }\end{array}$ & & \\
\hline
\end{tabular}

\section{Yassen 1993}

\begin{tabular}{|c|c|c|}
\hline Methods & \multicolumn{2}{|c|}{ Method of randomisation and allocation concealment were not described. } \\
\hline Participants & \multicolumn{2}{|c|}{$\begin{array}{l}20 \text { patients undergoing orthotopic liver transplantation were randomly allocated to one of two } \\
\text { groups: } \\
\text { - Tranexamic acid group: } \mathrm{n}=10, \mathrm{M} / \mathrm{F}=5 / 5 \text {, mean }(\mathrm{sd}) \text { age }=44.8(12.2) \text { years } \\
\text { - Control group (Placebo): } \mathrm{n}=10, \mathrm{M} / \mathrm{F}=4 / 6 \text {, mean }(\mathrm{sd}) \text { age }=49.6(14.2) \text { years }\end{array}$} \\
\hline Interventions & \multicolumn{2}{|c|}{$\begin{array}{l}\text { - Tranexamic acid group received } 10 \mathrm{mg} / \mathrm{kg} \text { loading dose of TXA at the start of the anhepatic } \\
\text { phase of the operation, followed by an infusion of } 3 \mathrm{mg} / \mathrm{kg} / \mathrm{hr} \text { until the patient was transferred to } \\
\text { the Intensive Care Unit (ICU). } \\
\text { - Control group received a similar volume of normal saline as a bolus followed by an infusion. } \\
\text { NB: Both groups were exposed to cell salvage. }\end{array}$} \\
\hline Outcomes & \multicolumn{2}{|c|}{$\begin{array}{l}\text { Outcomes reported: Allogeneic blood usage (units), fresh frozen plasma usage (units), platelets } \\
\text { usage (units), blood loss, any thrombosis. }\end{array}$} \\
\hline Notes & \multicolumn{2}{|c|}{$\begin{array}{l}\text { Quality assessment score (Schulz criteria): 2/7 } \\
\text { Transfusion protocol used }\end{array}$} \\
\hline \multicolumn{3}{|l|}{ Risk of bias } \\
\hline Item & Authors' judgement & Description \\
\hline Adequate sequence generation? & Unclear & Unclear \\
\hline Allocation concealment? & Unclear & Unclear \\
\hline $\begin{array}{l}\text { Blinding? } \\
\text { All outcomes }\end{array}$ & Yes & Double blind \\
\hline
\end{tabular}




\section{Zabeeda 2002}

\begin{tabular}{|c|c|c|}
\hline Methods & \multicolumn{2}{|c|}{$\begin{array}{l}\text { Method of randomisation and allocation concealment were not described. The surgeon was blinded } \\
\text { with respect to whether tranexamic acid or placebo was infused. }\end{array}$} \\
\hline Participants & \multicolumn{2}{|c|}{$\begin{array}{l}50 \text { patients undergoing coronary artery bypass graft surgery were randomised to one of two groups: } \\
\text { - Tranexamic acid group: } \mathrm{n}=25, \mathrm{M} / \mathrm{F}=20 / 5 \text {, mean }(\mathrm{sd}) \text { age }=65.6(9) \text { years } \\
\text { - Control group (Placebo): } \mathrm{n}=25, \mathrm{M} / \mathrm{F}=18 / 7 \text {, mean (sd) age }=65(13) \text { years }\end{array}$} \\
\hline Interventions & \multicolumn{2}{|c|}{$\begin{array}{l}\text { - Tranexamic acid group received } 10 \mathrm{mg} / \mathrm{kg} \text { of TXA for more than } 15 \text { minutes in a volume of } \\
10 \mathrm{ml} \text { after the induction of anaesthesia followed by a continuous infusion of } 1 \mathrm{mg} / \mathrm{kg} / \mathrm{hr} \text { in a } \\
\text { volume of } 10 \mathrm{ml} \text { for the duration of the procedure. } \\
\text { - Control group received a } 10 \mathrm{ml} \text { bolus of } 0.9 \% \text { saline solution followed by a continuous } \\
\text { infusion of saline }(10 \mathrm{ml} / \mathrm{hr}) \text {. }\end{array}$} \\
\hline Outcomes & \multicolumn{2}{|c|}{$\begin{array}{l}\text { Outcomes reported: Number of patients exposed to allogeneic blood, allogeneic blood usage (units) } \\
\text {, blood loss ( } 24 \mathrm{hrs}) \text {, stroke, mediastinal infection, pre-operative aspirin use. }\end{array}$} \\
\hline Notes & \multicolumn{2}{|c|}{$\begin{array}{l}\text { Quality assessment score (Schulz criteria): 4/7 } \\
\text { Transfusion protocol used }\end{array}$} \\
\hline \multicolumn{3}{|l|}{ Risk of bias } \\
\hline Item & Authors' judgement & Description \\
\hline Adequate sequence generation? & Unclear & Unclear \\
\hline Allocation concealment? & Unclear & Unclear \\
\hline $\begin{array}{l}\text { Blinding? } \\
\text { All outcomes }\end{array}$ & Yes & Double blind \\
\hline
\end{tabular}

\section{Zhang 2007}

\begin{tabular}{l|l}
\hline Methods & Methods of sequence generation and allocation concealment were not described. [Chinese language] \\
\hline Participants & $\begin{array}{r}102 \text { patients undergoing orthopaedic knee surgery were randomised to one of two groups: } \\
\text { - Tranexamic acid group: } \mathrm{n}=51 \\
\text { - Control group (Placebo): } \mathrm{n}=51\end{array}$ \\
\hline NB: Randomised subjects were aged between $59-77$ years of age. Gender: M/F = 43/59
\end{tabular}

\section{Risk of bias}


Zhang 2007 (Continued)

\begin{tabular}{lll}
\hline Item & Authors' judgement & Description \\
\hline Adequate sequence generation? & Unclear & Unclear \\
\hline Allocation concealment? & Unclear & Unclear \\
\hline $\begin{array}{l}\text { Blinding? } \\
\text { All outcomes }\end{array}$ & Unclear & Unclear \\
\hline
\end{tabular}

Zohar 2004

\begin{tabular}{|c|c|c|}
\hline Methods & \multicolumn{2}{|c|}{$\begin{array}{l}\text { Patients were randomly allocated to treatment groups using a computer generated randomisation } \\
\text { table. Method used to conceal treatment allocation was not described. }\end{array}$} \\
\hline Participants & \multicolumn{2}{|c|}{$\begin{array}{l}40 \text { patients undergoing total knee replacement were randomised to one of two groups: } \\
\text { - Tranexamic acid group: } \mathrm{n}=20, \mathrm{M} / \mathrm{F}=6 / 14 \text {, mean }(\mathrm{sd}) \text { age }=73 \text { (8) years } \\
\text { - Control group: } \mathrm{n}=20, \mathrm{M} / \mathrm{F}=7 / 13 \text {, mean }(\mathrm{sd}) \text { age }=73 \text { (7) years }\end{array}$} \\
\hline Interventions & \multicolumn{2}{|c|}{$\begin{array}{l}\text { - Tranexamic acid group received } 15 \mathrm{mg} / \mathrm{kg} \text { of TXA as an intravenous bolus } 30 \text { minutes before } \\
\text { the limb tourniquet was deflated administered over } 30 \text { minutes. Thereafter a constant infusion of } \\
10 \mathrm{mg} / \mathrm{kg} / \mathrm{hr} \text { was administered until } 12 \text { hours after final deflation of the limb tourniquet. } \\
\text { - Control group received usual care with no TXA treatment. }\end{array}$} \\
\hline Outcomes & \multicolumn{2}{|c|}{$\begin{array}{l}\text { Outcomes reported: Number of patients exposed to allogeneic blood, allogeneic blood usage, blood } \\
\text { loss ( } 12 \mathrm{hrs}) \text {, thrombo-embolic events ( } 30 \text {-day), hospital length of stay (days). }\end{array}$} \\
\hline Notes & \multicolumn{2}{|c|}{$\begin{array}{l}\text { Quality assessment score (Schulz criteria): 1/7 } \\
\text { Transfusion protocol used }\end{array}$} \\
\hline \multicolumn{3}{|l|}{ Risk of bias } \\
\hline Item & Authors' judgement & Description \\
\hline Adequate sequence generation? & Yes & Computer generated randomisation table \\
\hline Allocation concealment? & Unclear & Unclear \\
\hline $\begin{array}{l}\text { Blinding? } \\
\text { All outcomes }\end{array}$ & Unclear & Unclear \\
\hline
\end{tabular}


Characteristics of excluded studies [ordered by study ID]

\begin{tabular}{ll}
\hline Study & Reason for exclusion \\
\hline Fejer 1998 & $\begin{array}{l}\text { Study was excluded on the basis there was uncertainty regarding the age of study participants. As the study } \\
\text { involved thoracolumbar transpedicular (TLT) fixation of the spine for spondylolisthesis subjects less than } 18 \text { years } \\
\text { of age may have been included. }\end{array}$ \\
\hline Langdown 2000 & $\begin{array}{l}\text { Study did not report the number of patients randomised to each trial arm rather reported the total number of } \\
\text { patients randomised. Study was excluded on the basis there was uncertainty regarding the number of patients in } \\
\text { each trial arm. }\end{array}$ \\
\hline Montesano 1996 & $\begin{array}{l}\text { Abstract refers to patients as being randomly selected but methods section of paper states study was retrospective. } \\
\text { Study was excluded on the basis there was uncertainty regarding trial design. }\end{array}$ \\
\hline Zufferey 2010 & Patients undergoing surgery for hip fractures - not elective. \\
\hline
\end{tabular}

Characteristics of ongoing studies [ordered by study ID]

\section{Myles 2008}

\begin{tabular}{ll}
\hline Trial name or title & ATACAS trial \\
\hline Methods & Multi-centre, randomised, blinded 2x2 factorial trial. \\
\hline Participants & Ne4600, patients undergoing elective CABG surgery. \\
\hline Interventions & $\begin{array}{l}\text { Patients will be allocated to one of four groups } \\
\text { (1) Aspirin } \\
\text { (2) Tranexamic acid } \\
\text { (3) Tranexamic acid plus aspirin } \\
\text { (4) Placebo }\end{array}$ \\
\hline Outcomes & $\begin{array}{l}\text { Mortality } \\
\text { Myocardial infarction } \\
\text { Stroke } \\
\text { Pulmonary embolism } \\
\text { Renal failure } \\
\text { Bowel infarction } \\
\text { Re-operation for bleeding } \\
\text { Blood transfusion }\end{array}$ \\
\hline Contact information & \\
\hline Notes & \\
\hline
\end{tabular}


Verma 2010

\begin{tabular}{ll} 
Trial name or title & \\
\hline Methods & Single-centre, randomised, double-blinded control study \\
\hline Participants & Patient undergoing corrective spinal surgery. \\
\hline Interventions & $\begin{array}{l}\text { Patients will be allocated to one of three groups } \\
\text { (1) Tranexamic acid } \\
\text { (2) EACA } \\
\text { (3) Saline }\end{array}$ \\
\hline Outcomes & $\begin{array}{l}\text { Perioperative blood loss } \\
\text { Renal failure }\end{array}$ \\
\hline Starting date & ClinicalTrials.gov ID: NCT00958581 \\
\hline Contact information & \\
\hline Notes &
\end{tabular}


DATA AND ANALYSES

Comparison 1. Aprotinin versus Control (Blood Transfusion \& Blood Loss)

\begin{tabular}{|c|c|c|c|c|}
\hline Outcome or subgroup title & $\begin{array}{l}\text { No. of } \\
\text { studies }\end{array}$ & $\begin{array}{c}\text { No. of } \\
\text { participants }\end{array}$ & Statistical method & Effect size \\
\hline 1 No. Exposed to Allogeneic Blood & 108 & 11172 & Risk Ratio (M-H, Random, 95\% CI) & $0.66[0.60,0.72]$ \\
\hline $\begin{array}{l}2 \text { No. Exposed to Allogeneic } \\
\text { Blood - Type of Surgery }\end{array}$ & 108 & 11172 & Risk Ratio (M-H, Random, 95\% CI) & $0.66[0.60,0.72]$ \\
\hline 2.1 Cardiac surgery & 84 & 9497 & Risk Ratio (M-H, Random, 95\% CI) & $0.68[0.63,0.73]$ \\
\hline 2.2 Orthopaedic surgery & 15 & 1146 & Risk Ratio (M-H, Random, 95\% CI) & $0.68[0.52,0.89]$ \\
\hline 2.3 Thoracic surgery & 3 & 78 & Risk Ratio (M-H, Random, 95\% CI) & $0.29[0.14,0.59]$ \\
\hline 2.4 Vascular surgery & 2 & 188 & Risk Ratio (M-H, Random, 95\% CI) & $1.00[0.97,1.03]$ \\
\hline 2.5 Liver surgery & 2 & 177 & Risk Ratio (M-H, Random, 95\% CI) & $0.58[0.37,0.90]$ \\
\hline 2.6 Neuro surgery & 1 & 56 & Risk Ratio (M-H, Random, 95\% CI) & $0.73[0.40,1.35]$ \\
\hline 2.7 Orthognathic surgery & 1 & 30 & Risk Ratio (M-H, Random, 95\% CI) & $0.11[0.02,0.77]$ \\
\hline $\begin{array}{l}3 \text { No. Exposed to Allogeneic } \\
\text { Blood - Transfusion Protocol }\end{array}$ & 108 & 11172 & Risk Ratio (M-H, Random, 95\% CI) & $0.66[0.60,0.72]$ \\
\hline 3.1 Transfusion Protocol & 87 & 9974 & Risk Ratio (M-H, Random, 95\% CI) & $0.65[0.59,0.71]$ \\
\hline 3.2 No Transfusion Protocol & 21 & 1198 & Risk Ratio (M-H, Random, 95\% CI) & $0.71[0.61,0.84]$ \\
\hline $\begin{array}{l}4 \text { No. Exposed to Allogeneic } \\
\text { Blood - Dose }\end{array}$ & 107 & 12116 & Risk Ratio (M-H, Random, 95\% CI) & $0.67[0.62,0.73]$ \\
\hline 4.1 Prime Dose & 16 & 1251 & Risk Ratio (M-H, Random, 95\% CI) & $0.83[0.71,0.96]$ \\
\hline 4.2 Low Dose & 50 & 3601 & Risk Ratio (M-H, Random, 95\% CI) & $0.65[0.55,0.77]$ \\
\hline 4.3 High Dose & 61 & 7264 & Risk Ratio (M-H, Random, 95\% CI) & $0.66[0.61,0.71]$ \\
\hline $\begin{array}{l}5 \text { No. Exposed to Allogeneic } \\
\text { Blood - Dose (Cardiac Surgery) }\end{array}$ & 83 & 10423 & Risk Ratio (M-H, Random, 95\% CI) & $0.69[0.65,0.74]$ \\
\hline 5.1 Prime Dose & 15 & 1191 & Risk Ratio (M-H, Random, 95\% CI) & $0.81[0.69,0.96]$ \\
\hline 5.2 Low Dose & 29 & 2372 & Risk Ratio (M-H, Random, 95\% CI) & $0.69[0.60,0.80]$ \\
\hline 5.3 High Dose & 58 & 6860 & Risk Ratio (M-H, Random, 95\% CI) & $0.67[0.62,0.72]$ \\
\hline $\begin{array}{l}6 \text { Trial Methodological Quality - } \\
\text { Allocation Concealment }\end{array}$ & 108 & 11172 & Risk Ratio (M-H, Random, 95\% CI) & $0.66[0.60,0.72]$ \\
\hline $\begin{array}{l}6.1 \text { Allocation concealment - } \\
\text { Yes }\end{array}$ & 33 & 2755 & Risk Ratio (M-H, Random, 95\% CI) & $0.64[0.53,0.79]$ \\
\hline $\begin{array}{l}\text { 6.2 Allocation concealment - } \\
\text { Unclear }\end{array}$ & 63 & 7489 & Risk Ratio (M-H, Random, 95\% CI) & $0.69[0.64,0.75]$ \\
\hline $\begin{array}{l}6.3 \text { Allocation concealment - } \\
\text { No }\end{array}$ & 12 & 928 & Risk Ratio (M-H, Random, 95\% CI) & $0.63[0.54,0.75]$ \\
\hline $\begin{array}{l}7 \text { Units of Allogeneic Blood } \\
\text { Transfused - Transfused } \\
\text { Patients }\end{array}$ & 40 & 3563 & Mean Difference (IV, Random, 95\% CI) & $-0.98[-1.29,-0.66]$ \\
\hline $\begin{array}{l}8 \text { Units of Allogeneic Blood } \\
\text { Transfused - All Patients }\end{array}$ & 74 & 7820 & Mean Difference (IV, Random, 95\% CI) & $-1.02[-1.26,-0.79]$ \\
\hline 9 Blood loss - Intra-operative & 16 & 883 & Mean Difference (IV, Random, 95\% CI) & $\begin{array}{l}-191.87[-280.45,- \\
103.28]\end{array}$ \\
\hline 9.1 Cardiac surgery & 7 & 470 & Mean Difference (IV, Random, 95\% CI) & $\begin{array}{l}-148.18[-240.21,- \\
56.14]\end{array}$ \\
\hline 9.2 Orthopaedic surgery & 5 & 201 & Mean Difference (IV, Random, 95\% CI) & $\begin{array}{l}-151.05[-317.63 \\
15.52]\end{array}$ \\
\hline
\end{tabular}

Anti-fibrinolytic use for minimising perioperative allogeneic blood transfusion (Review) 


\begin{tabular}{|c|c|c|c|c|}
\hline 9.3 Thoracic surgery & 2 & 40 & Mean Difference (IV, Random, 95\% CI) & $\begin{array}{l}-577.06[-893.71,- \\
260.41]\end{array}$ \\
\hline 9.4 Liver surgery & 2 & 137 & Mean Difference (IV, Random, 95\% CI) & $\begin{array}{l}-1200.40[-2943.39, \\
542.59]\end{array}$ \\
\hline 9.5 Vascular surgery & 1 & 35 & Mean Difference (IV, Random, 95\% CI) & $\begin{array}{l}-102.00[-1004.32, \\
796.32]\end{array}$ \\
\hline 10 Blood loss - Post-operative & 87 & 7896 & Mean Difference (IV, Random, 95\% CI) & $\begin{array}{l}-345.88[-383.47,- \\
308.29]\end{array}$ \\
\hline 10.1 Cardiac surgery & 75 & 7371 & Mean Difference (IV, Random, 95\% CI) & $\begin{array}{l}-369.62[-408.95,- \\
330.29]\end{array}$ \\
\hline 10.2 Orthopaedic surgery & 7 & 318 & Mean Difference (IV, Random, 95\% CI) & $\begin{array}{l}-113.58[-223.69,- \\
3.46]\end{array}$ \\
\hline 10.3 Thoracic surgery & 2 & 83 & Mean Difference (IV, Random, 95\% CI) & $\begin{array}{l}-359.31[-460.15,- \\
258.48]\end{array}$ \\
\hline 10.4 Orthognathic surgery & 1 & 30 & Mean Difference (IV, Random, 95\% CI) & $\begin{array}{l}-513.0[-717.21,- \\
308.79]\end{array}$ \\
\hline 10.5 Liver surgery & 1 & 44 & Mean Difference (IV, Random, 95\% CI) & $\begin{array}{l}-105.0[-194.36,- \\
15.64]\end{array}$ \\
\hline 10.6 Vascular surgery & 1 & 50 & Mean Difference (IV, Random, 95\% CI) & $\begin{array}{l}-203.00[-404.93,- \\
1.07]\end{array}$ \\
\hline $\begin{array}{l}11 \text { Blood loss - Post-operative - } \\
\text { Dose (Cardiac Surgery) }\end{array}$ & 75 & 8181 & Mean Difference (IV, Random, 95\% CI) & $\begin{array}{l}-367.69[-403.50,- \\
331.87]\end{array}$ \\
\hline 11.1 Prime Dose & 15 & 1158 & Mean Difference (IV, Random, 95\% CI) & $\begin{array}{l}-343.08[-458.13,- \\
228.04]\end{array}$ \\
\hline 11.2 Low Dose & 24 & 2038 & Mean Difference (IV, Random, 95\% CI) & $\begin{array}{l}-274.58[-316.48,- \\
232.67]\end{array}$ \\
\hline 11.3 High Dose & 52 & 4985 & Mean Difference (IV, Random, 95\% CI) & $\begin{array}{l}-418.59[-470.96,- \\
366.22]\end{array}$ \\
\hline 12 Blood loss - Total & 17 & 1789 & Mean Difference (IV, Random, 95\% CI) & $\begin{array}{l}-415.95[-520.38,- \\
311.51]\end{array}$ \\
\hline 12.1 Cardiac surgery & 7 & 1359 & Mean Difference (IV, Random, 95\% CI) & $\begin{array}{l}-448.86[-612.82,- \\
284.91]\end{array}$ \\
\hline 12.2 Orthopaedic surgery & 10 & 430 & Mean Difference (IV, Random, 95\% CI) & $\begin{array}{l}-399.09[-562.81,- \\
235.37]\end{array}$ \\
\hline
\end{tabular}

\section{Comparison 2. Tranexamic Acid versus Control (Blood Transfusion \& Blood Loss)}

\begin{tabular}{lccll} 
Outcome or subgroup title & $\begin{array}{c}\text { No. of } \\
\text { studies }\end{array}$ & $\begin{array}{c}\text { No. of } \\
\text { participants }\end{array}$ & Statistical method & Effect size \\
\hline 1 No. Exposed to Allogeneic Blood & 65 & 4842 & Risk Ratio (M-H, Random, 95\% CI) & $0.61[0.53,0.70]$ \\
$\begin{array}{l}\text { 2 No. Exposed to Allogeneic } \\
\quad\end{array}$ & 65 & 4842 & Risk Ratio (M-H, Random, 95\% CI) & $0.61[0.53,0.70]$ \\
$\quad$ Blood - Type of Surgery & 34 & 3006 & Risk Ratio (M-H, Random, 95\% CI) & $0.68[0.57,0.81]$ \\
$\quad$ 2.1 Cardiac surgery & 27 & 1381 & Risk Ratio (M-H, Random, 95\% CI) & $0.49[0.39,0.62]$ \\
2.2 Orthopaedic surgery & 2 & 296 & Risk Ratio (M-H, Random, 95\% CI) & Not estimable \\
2.3 Liver surgery & 1 & 59 & Risk Ratio (M-H, Random, 95\% CI) & $0.56[0.33,0.96]$ \\
2.4 Vascular surgery & 1 & 100 & Risk Ratio (M-H, Random, 95\% CI) & $1.5[0.75,3.01]$ \\
2.5 Gynaecological surgery & 100
\end{tabular}

Anti-fibrinolytic use for minimising perioperative allogeneic blood transfusion (Review)

Copyright (C) 201 I The Cochrane Collaboration. Published by John Wiley \& Sons, Ltd. 
3 No. Exposed to Allogeneic Blood - Transfusion Protocol 3.1 Transfusion Protocol 3.2 No Transfusion Protocol

4 No. Exposed to Allogeneic Blood - Dose (Cardiac Surgery)

4.1 Total dose $<2.0$ grams

4.2 Total dose $2.0-10.0$ grams

5 Trial Methodological Quality Allocation Concealment

5.1 Allocation concealment - 28 Yes

5.2 Allocation concealment Unclear

5.3 Allocation concealment No

6 Units Allogeneic Blood

Transfused - Transfused

Patients

7 Units of Allogeneic Blood

Transfused - All Patients

8 Blood loss - Intra-operative

8.1 Cardiac surgery

8.2 Orthopaedic surgery

8.3 Gynaecological surgery

8.4 Head \& neck surgery

9 Blood loss - Post-operative

9.1 Cardiac surgery

9.2 Orthopaedic surgery

9.3 Gynaecological surgery

9.4 Head \& neck surgery

10 Blood loss - Post-operative -

Dose (Cardiac Surgery)

10.1 Total dose $<2.0$ grams

10.2 Total dose $2.0-10.0$

grams

11 Blood loss - Total

11.1 Cardiac surgery
Risk Ratio (M-H, Random, 95\% CI)

Risk Ratio (M-H, Random, 95\% CI) Risk Ratio (M-H, Random, 95\% CI) Risk Ratio (M-H, Random, 95\% CI)

Risk Ratio (M-H, Random, 95\% CI) Risk Ratio (M-H, Random, 95\% CI)

Risk Ratio (M-H, Random, 95\% CI)

Risk Ratio (M-H, Random, 95\% CI)

Risk Ratio (M-H, Random, 95\% CI)

Risk Ratio (M-H, Random, 95\% CI)

Mean Difference (IV, Random, 95\% CI)

Mean Difference (IV, Random, 95\% CI)

Mean Difference (IV, Random, 95\% CI)

Mean Difference (IV, Random, 95\% CI)

Mean Difference (IV, Random, 95\% CI)

Mean Difference (IV, Random, 95\% CI)

Mean Difference (IV, Random, 95\% CI)

Mean Difference (IV, Random, 95\% CI)

Mean Difference (IV, Random, 95\% CI)

Mean Difference (IV, Random, 95\% CI)

Mean Difference (IV, Random, 95\% CI)

Mean Difference (IV, Random, 95\% CI)

Mean Difference (IV, Random, 95\% CI)

Mean Difference (IV, Random, 95\% CI)

Mean Difference (IV, Random, 95\% CI)

Mean Difference (IV, Random, 95\% CI)

Mean Difference (IV, Random, 95\% CI)
$0.61[0.53,0.70]$

$0.57[0.48,0.67]$

$0.76[0.61,0.96]$

$0.68[0.58,0.80]$

$0.70[0.58,0.84]$

$0.67[0.52,0.86]$

$0.61[0.53,0.70]$

$0.59[0.51,0.69]$

$0.53[0.37,0.76]$

$0.73[0.62,0.86]$

$-0.34[-0.80,0.11]$

$-0.87[-1.20,-0.53]$

$-121.41[-180.19,-$ 62.63]

-166.76 [-331.24, 2.27]

-115.52 [-187.88, 43.16]

$-164.00[-366.45$, 34.45]

Not estimable -247.17 [-294.76, 199.58]

$-272.87[-328.85,-$ 216.89]

-228.52 [-321.76, 135.27]

$-63.0[-118.89$, -

7.11]

Not estimable -272.87 [-328.85, 216.89]

-245.03 [-329.76, 160.29] $-297.94[-364.49,-$ 231.39]

-414.06 [-525.19, 302.92] -300.47 [-470.74, 130.21] 


\begin{tabular}{|c|c|c|c|c|}
\hline 11.2 Orthopaedic surgery & 20 & 1201 & Mean Difference (IV, Random, 95\% CI) & $\begin{array}{l}-446.19[-554.61,- \\
337.78]\end{array}$ \\
\hline 11.3 Liver surgery & 1 & 20 & Mean Difference (IV, Random, 95\% CI) & $\begin{array}{l}-6552.0[-14329.54, \\
1225.54]\end{array}$ \\
\hline 11.4 Gynaecological surgery & 1 & 100 & Mean Difference (IV, Random, 95\% CI) & $\begin{array}{l}-243.0[-460.02,- \\
25.98]\end{array}$ \\
\hline 11.5 Head \& neck surgery & 0 & 0 & Mean Difference (IV, Random, 95\% CI) & Not estimable \\
\hline
\end{tabular}

\section{Comparison 3. Epsilon Aminocaproic Acid versus Control (Blood Transfusion \& Blood Loss)}

\begin{tabular}{|c|c|c|c|c|}
\hline Outcome or subgroup title & $\begin{array}{l}\text { No. of } \\
\text { studies }\end{array}$ & $\begin{array}{c}\text { No. of } \\
\text { participants }\end{array}$ & Statistical method & Effect size \\
\hline 1 No. Exposed to Allogeneic Blood & 16 & 1035 & Risk Ratio (M-H, Random, 95\% CI) & $0.81[0.67,0.99]$ \\
\hline $\begin{array}{l}2 \text { No. Exposed to Allogeneic } \\
\text { Blood - Type of Surgery }\end{array}$ & 16 & 1035 & Risk Ratio (M-H, Random, 95\% CI) & $0.81[0.67,0.99]$ \\
\hline 2.1 Cardiac Surgery & 11 & 649 & Risk Ratio (M-H, Random, 95\% CI) & $0.70[0.52,0.93]$ \\
\hline 2.2 Orthopaedic Surgery & 4 & 304 & Risk Ratio (M-H, Random, 95\% CI) & $1.00[0.93,1.08]$ \\
\hline 2.3 Liver Surgery & 1 & 82 & Risk Ratio (M-H, Random, 95\% CI) & $0.93[0.80,1.08]$ \\
\hline $\begin{array}{l}3 \text { No. Exposed to Allogeneic } \\
\text { Blood - Transfusion Protocol }\end{array}$ & 16 & 1035 & Risk Ratio (M-H, Random, 95\% CI) & $0.81[0.67,0.99]$ \\
\hline 3.1 Transfusion Protocol & 15 & 1005 & Risk Ratio (M-H, Random, 95\% CI) & $0.80[0.65,0.98]$ \\
\hline 3.2 No Transfusion Protocol & 1 & 30 & Risk Ratio (M-H, Random, 95\% CI) & $1.33[0.36,4.97]$ \\
\hline $\begin{array}{l}4 \text { Trial Methodological Quality - } \\
\text { Allocation Concealment }\end{array}$ & 16 & 1035 & Risk Ratio (M-H, Random, 95\% CI) & $0.81[0.67,0.99]$ \\
\hline $\begin{array}{l}4.1 \text { Allocation concealment - } \\
\text { Yes }\end{array}$ & 5 & 452 & Risk Ratio (M-H, Random, 95\% CI) & $0.82[0.58,1.16]$ \\
\hline $\begin{array}{l}4.2 \text { Allocation concealment - } \\
\text { Unclear }\end{array}$ & 9 & 455 & Risk Ratio (M-H, Random, 95\% CI) & $0.68[0.46,1.03]$ \\
\hline $\begin{array}{l}4.3 \text { Allocation concealment - } \\
\text { No }\end{array}$ & 2 & 128 & Risk Ratio (M-H, Random, 95\% CI) & $0.93[0.81,1.08]$ \\
\hline $\begin{array}{l}5 \text { Units of Allogeneic Blood } \\
\text { Transfused - Transfused } \\
\text { Patients }\end{array}$ & 3 & 119 & Mean Difference (IV, Random, 95\% CI) & $0.22[-0.34,0.79]$ \\
\hline $\begin{array}{l}6 \text { Units of Allogeneic Blood } \\
\text { Transfused - All Patients }\end{array}$ & 6 & 432 & Mean Difference (IV, Random, 95\% CI) & $-1.30[-2.14,-0.45]$ \\
\hline 7 Blood loss - Intra-operative & 5 & 353 & Mean Difference (IV, Random, 95\% CI) & $\begin{array}{l}-156.63[-276.92,- \\
36.33]\end{array}$ \\
\hline 7.1 Cardiac surgery & 2 & 79 & Mean Difference (IV, Random, 95\% CI) & $\begin{array}{l}-213.58[-310.03,- \\
117.13]\end{array}$ \\
\hline 7.2 Orthopaedic surgery & 3 & 274 & Mean Difference (IV, Random, 95\% CI) & $\begin{array}{l}-40.66[-236.71 \\
155.38]\end{array}$ \\
\hline 8 Blood loss - Post-operative & 14 & 1174 & Mean Difference (IV, Random, 95\% CI) & $\begin{array}{l}-207.49[-276.43,- \\
138.54]\end{array}$ \\
\hline 8.1 Cardiac surgery & 12 & 946 & Mean Difference (IV, Random, 95\% CI) & $\begin{array}{l}-200.27[-273.44,- \\
127.09]\end{array}$ \\
\hline 8.2 Orthopaedic surgery & 2 & 228 & Mean Difference (IV, Random, 95\% CI) & $\begin{array}{l}-285.06[-452.73,- \\
117.39]\end{array}$ \\
\hline
\end{tabular}




\begin{tabular}{lllll}
9 Blood loss - Total & 2 & 92 & Mean Difference (IV, Random, 95\% CI) & $-299.69[-522.54,-$ \\
& & & & $76.84]$ \\
9.1 Orthopaedic surgery & 2 & 92 & Mean Difference (IV, Random, 95\% CI) & $\begin{array}{l}-299.69[-522.54,- \\
76.84]\end{array}$ \\
\hline
\end{tabular}

\section{Comparison 4. Aprotinin versus Tranexamic Acid (Blood Transfusion \& Blood Loss)}

\begin{tabular}{|c|c|c|c|c|}
\hline Outcome or subgroup title & $\begin{array}{l}\text { No. of } \\
\text { studies }\end{array}$ & $\begin{array}{c}\text { No. of } \\
\text { participants }\end{array}$ & Statistical method & Effect size \\
\hline 1 No. Exposed to Allogeneic Blood & 21 & 4185 & Risk Ratio (M-H, Random, 95\% CI) & $0.90[0.81,1.01]$ \\
\hline $\begin{array}{l}2 \text { No. Exposed to Allogeneic } \\
\text { Blood - Type of Surgery }\end{array}$ & 21 & 4185 & Risk Ratio (M-H, Random, 95\% CI) & $0.90[0.81,1.01]$ \\
\hline 2.1 Cardiac surgery & 18 & 3983 & Risk Ratio (M-H, Random, 95\% CI) & $0.87[0.76,0.99]$ \\
\hline 2.2 Liver surgery & 2 & 178 & Risk Ratio (M-H, Random, 95\% CI) & $1.01[0.91,1.11]$ \\
\hline 2.3 Orthopaedic surgery & 1 & 24 & Risk Ratio (M-H, Random, 95\% CI) & $11.00[0.67,179.29]$ \\
\hline $\begin{array}{l}3 \text { No. Exposed to Allogeneic } \\
\text { Blood - Transfusion Protocol }\end{array}$ & 21 & 4185 & Risk Ratio (M-H, Random, 95\% CI) & $0.90[0.81,1.01]$ \\
\hline 3.1 Transfusion Protocol & 20 & 4155 & Risk Ratio (M-H, Random, 95\% CI) & $0.90[0.80,1.01]$ \\
\hline 3.2 No Transfusion Protocol & 1 & 30 & Risk Ratio (M-H, Random, 95\% CI) & $0.92[0.67,1.27]$ \\
\hline $\begin{array}{l}4 \text { Trial Methodological Quality - } \\
\text { Allocation Concealment }\end{array}$ & 21 & 4185 & Risk Ratio (M-H, Random, 95\% CI) & $0.90[0.81,1.01]$ \\
\hline $\begin{array}{l}4.1 \text { Allocation concealment - } \\
\text { Yes }\end{array}$ & 4 & 1871 & Risk Ratio (M-H, Random, 95\% CI) & $0.80[0.69,0.92]$ \\
\hline $\begin{array}{l}\text { 4.2 Allocation concealment - } \\
\text { Unclear }\end{array}$ & 13 & 1832 & Risk Ratio (M-H, Random, 95\% CI) & $0.97[0.88,1.07]$ \\
\hline $\begin{array}{l}4.3 \text { Allocation concealment - } \\
\text { No }\end{array}$ & 4 & 482 & Risk Ratio (M-H, Random, 95\% CI) & $0.93[0.62,1.39]$ \\
\hline $\begin{array}{l}5 \text { Units Allogeneic Blood } \\
\text { Transfused - Transfused } \\
\text { Patients }\end{array}$ & 6 & 207 & Mean Difference (IV, Random, 95\% CI) & $-0.07[-0.44,0.30]$ \\
\hline $\begin{array}{l}6 \text { Units Allogeneic Blood } \\
\text { Transfused - All Patients }\end{array}$ & 10 & 992 & Mean Difference (IV, Random, 95\% CI) & $-0.24[-0.45,-0.04]$ \\
\hline 7 Blood loss & 14 & 1041 & Mean Difference (IV, Random, 95\% CI) & $\begin{array}{l}-136.44[-198.40,- \\
74.47]\end{array}$ \\
\hline $\begin{array}{l}7.1 \text { Cardiac surgery - } \\
\text { Post-operative }\end{array}$ & 13 & 831 & Mean Difference (IV, Random, 95\% CI) & $\begin{array}{l}-145.81[-209.99,- \\
81.62]\end{array}$ \\
\hline 7.2 Cardiac surgery - Total & 1 & 210 & Mean Difference (IV, Random, 95\% CI) & $\begin{array}{l}6.0[-171.38 \\
183.38]\end{array}$ \\
\hline
\end{tabular}


Comparison 5. Aprotinin versus Epsilon Aminocaproic Acid (Blood Transfusion \& Blood Loss)

\begin{tabular}{|c|c|c|c|c|}
\hline Outcome or subgroup title & $\begin{array}{l}\text { No. of } \\
\text { studies }\end{array}$ & $\begin{array}{c}\text { No. of } \\
\text { participants }\end{array}$ & Statistical method & Effect size \\
\hline 1 No. Exposed to Allogeneic Blood & 12 & 2200 & Risk Ratio (M-H, Random, 95\% CI) & $0.82[0.76,0.89]$ \\
\hline $\begin{array}{l}2 \text { No. Exposed to Allogeneic } \\
\text { Blood - Type of Surgery }\end{array}$ & 12 & 2200 & Risk Ratio (M-H, Random, 95\% CI) & $0.82[0.76,0.89]$ \\
\hline 2.1 Cardiac surgery & 10 & 2125 & Risk Ratio (M-H, Random, 95\% CI) & $0.82[0.76,0.89]$ \\
\hline 2.2 Orthopaedic surgery & 2 & 75 & Risk Ratio (M-H, Random, 95\% CI) & $0.82[0.48,1.40]$ \\
\hline $\begin{array}{l}3 \text { No. Exposed to Allogeneic } \\
\text { Blood - Transfusion Protocol }\end{array}$ & 12 & 2200 & Risk Ratio (M-H, Random, 95\% CI) & $0.82[0.76,0.89]$ \\
\hline 3.1 Transfusion Protocol & 9 & 2014 & Risk Ratio (M-H, Random, 95\% CI) & $0.82[0.76,0.89]$ \\
\hline 3.2 No Transfusion Protocol & 3 & 186 & Risk Ratio (M-H, Random, 95\% CI) & $0.78[0.47,1.31]$ \\
\hline $\begin{array}{l}4 \text { Trial Methodological Quality - } \\
\text { Allocation Concealment }\end{array}$ & 12 & 2200 & Risk Ratio (M-H, Random, 95\% CI) & $0.82[0.76,0.89]$ \\
\hline $\begin{array}{l}4.1 \text { Allocation concealment - } \\
\text { Yes }\end{array}$ & 3 & 1651 & Risk Ratio (M-H, Random, 95\% CI) & $0.86[0.71,1.05]$ \\
\hline $\begin{array}{l}\text { 4.2 Allocation concealment - } \\
\text { Unclear }\end{array}$ & 8 & 504 & Risk Ratio (M-H, Random, 95\% CI) & $0.76[0.58,0.99]$ \\
\hline $\begin{array}{l}4.3 \text { Allocation concealment - } \\
\text { No }\end{array}$ & 1 & 45 & Risk Ratio (M-H, Random, 95\% CI) & $0.88[0.49,1.55]$ \\
\hline $\begin{array}{l}5 \text { Units of Allogeneic Blood } \\
\text { Transfused - Transfused } \\
\text { Patients }\end{array}$ & 2 & 63 & Mean Difference (IV, Random, 95\% CI) & $-0.18[-0.63,0.28]$ \\
\hline $\begin{array}{c}6 \text { Units of Allogeneic Blood } \\
\text { Transfused - All Patients }\end{array}$ & 5 & 329 & Mean Difference (IV, Random, 95\% CI) & $-0.21[-0.55,0.14]$ \\
\hline 7 Blood loss & 8 & 499 & Mean Difference (IV, Random, 95\% CI) & $\begin{array}{l}-106.01[-212.50 \\
0.47]\end{array}$ \\
\hline $\begin{array}{l}\text { 7.1 Cardiac surgery - } \\
\text { Post-operative }\end{array}$ & 7 & 454 & Mean Difference (IV, Random, 95\% CI) & $\begin{array}{l}-111.43[-220.64,- \\
2.21]\end{array}$ \\
\hline $\begin{array}{l}7.2 \text { Orthopaedic surgery - } \\
\text { Total }\end{array}$ & 1 & 45 & Mean Difference (IV, Random, 95\% CI) & $\begin{array}{l}100.0[-515.06 \\
715.06]\end{array}$ \\
\hline
\end{tabular}

Comparison 6. Tranexamic Acid versus Epsilon Aminocaproic Acid (Blood Transfusion \& Blood Loss)

\begin{tabular}{|c|c|c|c|c|}
\hline Outcome or subgroup title & $\begin{array}{l}\text { No. of } \\
\text { studies }\end{array}$ & $\begin{array}{c}\text { No. of } \\
\text { participants }\end{array}$ & Statistical method & Effect size \\
\hline 1 No. Exposed to Allogeneic Blood & 8 & 2003 & Risk Ratio (M-H, Random, 95\% CI) & $0.97[0.77,1.21]$ \\
\hline $\begin{array}{c}2 \text { No. Exposed to Allogeneic } \\
\text { Blood - Type of Surgery }\end{array}$ & 8 & 2003 & Risk Ratio (M-H, Random, 95\% CI) & $0.97[0.77,1.21]$ \\
\hline 2.1 Cardiac surgery & 6 & 1852 & Risk Ratio (M-H, Random, 95\% CI) & $1.07[0.79,1.46]$ \\
\hline 2.2 Orthopaedic surgery & 1 & 67 & Risk Ratio (M-H, Random, 95\% CI) & $0.23[0.03,1.94]$ \\
\hline 2.3 Liver surgery & 1 & 84 & Risk Ratio (M-H, Random, 95\% CI) & $0.81[0.64,1.02]$ \\
\hline $\begin{array}{l}3 \text { No. Exposed to Allogeneic } \\
\text { Blood - Transfusion Protocol }\end{array}$ & 8 & 2003 & Risk Ratio (M-H, Random, 95\% CI) & $0.97[0.77,1.21]$ \\
\hline 3.1 Transfusion Protocol & 8 & 2003 & Risk Ratio (M-H, Random, 95\% CI) & $0.97[0.77,1.21]$ \\
\hline
\end{tabular}

Anti-fibrinolytic use for minimising perioperative allogeneic blood transfusion (Review)

Copyright (C) 201 I The Cochrane Collaboration. Published by John Wiley \& Sons, Ltd. 


\begin{tabular}{|c|c|c|c|c|}
\hline $\begin{array}{l}4 \text { Trial Methodological Quality - } \\
\text { Allocation Concealment }\end{array}$ & 8 & 2003 & Risk Ratio (M-H, Random, 95\% CI) & $0.97[0.77,1.21]$ \\
\hline $\begin{array}{l}4.1 \text { Allocation concealment - } \\
\text { Yes }\end{array}$ & 1 & 1550 & Risk Ratio (M-H, Random, 95\% CI) & $1.00[0.93,1.07]$ \\
\hline $\begin{array}{l}\text { 4.2 Allocation concealment - } \\
\text { Unclear }\end{array}$ & 5 & 302 & Risk Ratio (M-H, Random, 95\% CI) & $1.16[0.68,1.98]$ \\
\hline $\begin{array}{l}4.3 \text { Allocation concealment - } \\
\text { No }\end{array}$ & 2 & 151 & Risk Ratio (M-H, Random, 95\% CI) & $0.63[0.22,1.84]$ \\
\hline $\begin{array}{l}5 \text { Units of Allogeneic Blood } \\
\text { Transfused - Transfused } \\
\text { Patients }\end{array}$ & 4 & 133 & Mean Difference (IV, Random, 95\% CI) & $-0.34[-0.74,0.07]$ \\
\hline $\begin{array}{l}6 \text { Units of Allogeneic Blood } \\
\text { Transfused - All Patients }\end{array}$ & 3 & 268 & Mean Difference (IV, Random, 95\% CI) & $-0.28[-0.59,0.03]$ \\
\hline 7 Blood loss & 7 & 469 & Mean Difference (IV, Random, 95\% CI) & $\begin{array}{l}-4.20[-147.29 \\
138.89]\end{array}$ \\
\hline $\begin{array}{l}7.1 \text { Cardiac surgery - } \\
\text { Post-operative }\end{array}$ & 6 & 402 & Mean Difference (IV, Random, 95\% CI) & $\begin{array}{l}-4.36[-163.35 \\
154.63]\end{array}$ \\
\hline $\begin{array}{l}7.2 \text { Orthopaedic surgery - } \\
\text { Total }\end{array}$ & 1 & 67 & Mean Difference (IV, Random, 95\% CI) & $\begin{array}{l}-9.0[-270.16 \\
252.16]\end{array}$ \\
\hline $\begin{array}{l}7.3 \text { Gynaecological surgery - } \\
\text { Total }\end{array}$ & 0 & 0 & Mean Difference (IV, Random, 95\% CI) & Not estimable \\
\hline
\end{tabular}

\section{Comparison 7. Aprotinin versus Lysine Analogues (Blood Transfusion)}

\begin{tabular}{|c|c|c|c|c|}
\hline Outcome or subgroup title & $\begin{array}{l}\text { No. of } \\
\text { studies }\end{array}$ & $\begin{array}{c}\text { No. of } \\
\text { participants }\end{array}$ & Statistical method & Effect size \\
\hline 1 No. Exposed to Allogeneic Blood & 29 & 5566 & Risk Ratio (M-H, Random, 95\% CI) & $0.90[0.81,0.99]$ \\
\hline $\begin{array}{l}2 \text { No. Exposed to Allogeneic } \\
\text { Blood - Type of Surgery }\end{array}$ & 29 & 5469 & Risk Ratio (M-H, Random, 95\% CI) & $0.89[0.80,0.98]$ \\
\hline 2.1 Cardiac surgery & 24 & 5192 & Risk Ratio (M-H, Random, 95\% CI) & $0.86[0.76,0.96]$ \\
\hline 2.2 Orthopaedic surgery & 3 & 99 & Risk Ratio (M-H, Random, 95\% CI) & $1.06[0.32,3.48]$ \\
\hline 2.3 Liver surgery & 2 & 178 & Risk Ratio (M-H, Random, 95\% CI) & $1.01[0.91,1.11]$ \\
\hline $\begin{array}{l}3 \text { No. Exposed to Allogeneic } \\
\text { Blood - Transfusion Protocol }\end{array}$ & 29 & 5429 & Risk Ratio (M-H, Random, 95\% CI) & $0.89[0.81,0.98]$ \\
\hline 3.1 Transfusion Protocol & 25 & 5213 & Risk Ratio (M-H, Random, 95\% CI) & $0.89[0.80,0.99]$ \\
\hline 3.2 No Transfusion Protocol & 4 & 216 & Risk Ratio (M-H, Random, 95\% CI) & $0.85[0.65,1.12]$ \\
\hline $\begin{array}{l}4 \text { Units of Allogeneic Blood } \\
\text { Transfused - Transfused } \\
\text { Patients }\end{array}$ & 7 & 251 & Mean Difference (IV, Random, 95\% CI) & $-0.11[-0.42,0.21]$ \\
\hline $\begin{array}{l}5 \text { Units of Allogeneic Blood } \\
\text { Transfused - All Patients }\end{array}$ & 14 & 1254 & Mean Difference (IV, Random, 95\% CI) & $-0.25[-0.42,-0.09]$ \\
\hline $\begin{array}{l}6 \text { Trial Methodological Quality - } \\
\text { Allocation Concealment }\end{array}$ & 29 & 5566 & Risk Ratio (M-H, Random, 95\% CI) & $0.90[0.81,0.99]$ \\
\hline $\begin{array}{l}6.1 \text { Allocation concealment - } \\
\text { Yes }\end{array}$ & 6 & 2742 & Risk Ratio (M-H, Random, 95\% CI) & $0.82[0.71,0.95]$ \\
\hline $\begin{array}{l}\text { 6.2 Allocation concealment - } \\
\text { Unclear }\end{array}$ & 18 & 2297 & Risk Ratio (M-H, Random, 95\% CI) & $0.95[0.86,1.04]$ \\
\hline
\end{tabular}

Anti-fibrinolytic use for minimising perioperative allogeneic blood transfusion (Review)

Copyright (C) 201 I The Cochrane Collaboration. Published by John Wiley \& Sons, Ltd. 
Comparison 8. Adverse Events and Other Outcomes (Active versus Control)

\begin{tabular}{|c|c|c|c|c|}
\hline Outcome or subgroup title & $\begin{array}{l}\text { No. of } \\
\text { studies }\end{array}$ & $\begin{array}{c}\text { No. of } \\
\text { participants }\end{array}$ & Statistical method & Effect size \\
\hline 1 Re-operation for bleeding & 85 & & Risk Ratio (M-H, Random, 95\% CI) & Subtotals only \\
\hline 1.1 Aprotinin versus Control & 61 & 6117 & Risk Ratio (M-H, Random, 95\% CI) & $0.46[0.34,0.62]$ \\
\hline 1.2 Tranexamic Acid versus & 27 & 2386 & Risk Ratio (M-H, Random, 95\% CI) & $0.80[0.55,1.17]$ \\
\hline \multicolumn{5}{|l|}{ Control } \\
\hline 1.3 Epsilon Aminocaproic & 8 & 922 & Risk Ratio (M-H, Random, 95\% CI) & $0.32[0.11,0.99]$ \\
\hline 2 Mortality & 92 & & Risk Ratio (M-H, Random, 95\% CI) & Subtotals only \\
\hline 2.1 Aprotinin versus Control & 63 & 8876 & Risk Ratio (M-H, Random, 95\% CI) & $0.81[0.63,1.06]$ \\
\hline 2.2 Tranexamic Acid versus & 30 & 2917 & Risk Ratio (M-H, Random, 95\% CI) & $0.60[0.33,1.10]$ \\
\hline \multicolumn{5}{|l|}{ Control } \\
\hline 2.3 Epsilon Aminocaproic & 8 & 988 & Risk Ratio (M-H, Random, 95\% CI) & $1.07[0.44,2.57]$ \\
\hline \multicolumn{5}{|l|}{ Acid versus Control } \\
\hline 3 Myocardial Infarction (MI) & 71 & & Risk Ratio (M-H, Random, 95\% CI) & Subtotals only \\
\hline 3.1 Aprotinin versus Control & 49 & 7137 & Risk Ratio (M-H, Random, 95\% CI) & $0.87[0.69,1.11]$ \\
\hline 3.2 Tranexamic Acid versus & 21 & 2186 & Risk Ratio (M-H, Random, 95\% CI) & $0.79[0.41,1.52]$ \\
\hline \multicolumn{5}{|l|}{ Control } \\
\hline 3.3 Epsilon aminocaproic & 7 & 896 & Risk Ratio (M-H, Random, 95\% CI) & $0.88[0.48,1.63]$ \\
\hline \multicolumn{5}{|l|}{ Acid versus Control } \\
\hline 4 Stroke (CVA) & 45 & & Risk Ratio (M-H, Random, 95\% CI) & Subtotals only \\
\hline 4.1 Aprotinin versus Control & 23 & 3122 & Risk Ratio (M-H, Random, 95\% CI) & $0.82[0.44,1.52]$ \\
\hline 4.2 Tranexamic Acid versus & 18 & 2027 & Risk Ratio (M-H, Random, 95\% CI) & $1.23[0.49,3.07]$ \\
\hline \multicolumn{5}{|l|}{ Control } \\
\hline 4.3 Epsilon Aminocaproic & 8 & 936 & Risk Ratio (M-H, Random, 95\% CI) & $0.62[0.16,2.36]$ \\
\hline \multicolumn{5}{|l|}{ Acid versus Control } \\
\hline 5 Deep Vein Thrombosis (DVT) & 40 & & Risk Ratio (M-H, Random, 95\% CI) & Subtotals only \\
\hline 5.1 Aprotinin versus Control & 16 & 1456 & Risk Ratio (M-H, Random, 95\% CI) & $0.78[0.47,1.29]$ \\
\hline 5.2 Tranexamic Acid versus & 23 & 1472 & Risk Ratio (M-H, Random, 95\% CI) & $0.71[0.35,1.43]$ \\
\hline \multicolumn{5}{|l|}{ Control } \\
\hline 5.3 Epsilon Aminocaproic & 4 & 304 & Risk Ratio (M-H, Random, 95\% CI) & $0.78[0.20,3.03]$ \\
\hline \multicolumn{5}{|l|}{ Acid versus Control } \\
\hline 6 Pulmonary Embolism (PE) & 20 & & Risk Ratio (M-H, Random, 95\% CI) & Subtotals only \\
\hline 6.1 Aprotinin versus Control & 4 & 585 & Risk Ratio (M-H, Random, 95\% CI) & $1.49[0.42,5.29]$ \\
\hline 6.2 Tranexamic Acid versus & 14 & 1006 & Risk Ratio (M-H, Random, 95\% CI) & $0.67[0.23,1.99]$ \\
\hline \multicolumn{5}{|l|}{ Control } \\
\hline 6.3 Epsilon Aminocaproic & 3 & 274 & Risk Ratio (M-H, Random, 95\% CI) & $0.34[0.06,2.13]$ \\
\hline \multicolumn{5}{|l|}{ Acid versus Control } \\
\hline 7 Other Thrombosis & 19 & & Risk Ratio (M-H, Random, 95\% CI) & Subtotals only \\
\hline 7.1 Aprotinin versus Control & 9 & 736 & Risk Ratio (M-H, Random, 95\% CI) & $0.73[0.25,2.15]$ \\
\hline 7.2 Tranexamic Acid versus & 9 & 484 & Risk Ratio (M-H, Random, 95\% CI) & $2.10[0.49,8.99]$ \\
\hline Control & & & & \\
\hline
\end{tabular}




\begin{tabular}{|c|c|c|c|c|}
\hline $\begin{array}{l}\text { 7.3 Epsilon Aminocaproic } \\
\text { Acid versus Control }\end{array}$ & 2 & 264 & Risk Ratio (M-H, Random, 95\% CI) & $0.51[0.15,1.72]$ \\
\hline 8 Coronary artery graft occlusion & 2 & 728 & Risk Ratio (M-H, Random, 95\% CI) & $0.76[0.10,5.67]$ \\
\hline 8.1 Aprotinin versus Control & 2 & 728 & Risk Ratio (M-H, Random, 95\% CI) & $0.76[0.10,5.67]$ \\
\hline 9 Renal Failure / Dysfunction & 34 & & Risk Ratio (M-H, Random, 95\% CI) & Subtotals only \\
\hline 9.1 Aprotinin versus Control & 27 & 5185 & Risk Ratio (M-H, Random, 95\% CI) & $1.10[0.79,1.54]$ \\
\hline $\begin{array}{l}9.2 \text { Tranexamic Acid versus } \\
\text { Control }\end{array}$ & 9 & 912 & Risk Ratio (M-H, Random, 95\% CI) & $0.89[0.33,2.37]$ \\
\hline $\begin{array}{l}\text { 9.3 Epsilon Aminocaproic } \\
\text { Acid versus control }\end{array}$ & 2 & 235 & Risk Ratio (M-H, Random, 95\% CI) & $0.41[0.14,1.22]$ \\
\hline 10 Hospital Length of Stay & 31 & & Mean Difference (IV, Random, 95\% CI) & Subtotals only \\
\hline 10.1 Aprotinin versus Control & 23 & 2017 & Mean Difference (IV, Random, 95\% CI) & $-0.25[-0.71,0.20]$ \\
\hline $\begin{array}{l}\text { 10.2 Tranexamic Acid versus } \\
\text { Control }\end{array}$ & 10 & 772 & Mean Difference (IV, Random, 95\% CI) & $-0.34[-0.82,0.13]$ \\
\hline $\begin{array}{l}\text { 10.3 Epsilon Aminocaproic } \\
\text { Acid versus Control }\end{array}$ & 2 & 228 & Mean Difference (IV, Random, 95\% CI) & $0.58[-3.17,4.33]$ \\
\hline
\end{tabular}

\section{Comparison 9. Adverse Events and Other Outcomes (Active versus Active)}

\begin{tabular}{|c|c|c|c|c|}
\hline Outcome or subgroup title & $\begin{array}{l}\text { No. of } \\
\text { studies }\end{array}$ & $\begin{array}{c}\text { No. of } \\
\text { participants }\end{array}$ & Statistical method & Effect size \\
\hline $\begin{array}{l}1 \text { Re-operation for bleeding - } \\
\text { Aprotinin versus Tranexamic } \\
\text { Acid }\end{array}$ & 17 & 4010 & Risk Ratio (M-H, Random, 95\% CI) & $0.69[0.51,0.93]$ \\
\hline $\begin{array}{l}2 \text { Re-operation for bleeding - } \\
\text { Aprotinin versus Epsilon } \\
\text { Aminocaproic Acid }\end{array}$ & 6 & 2075 & Risk Ratio (M-H, Random, 95\% CI) & $0.70[0.49,1.00]$ \\
\hline $\begin{array}{l}3 \text { Re-operation for bleeding - } \\
\text { Tranexamic Acid versus Epsilon } \\
\text { Aminocaproic Acid }\end{array}$ & 5 & 1853 & Risk Ratio (M-H, Random, 95\% CI) & $1.00[0.73,1.39]$ \\
\hline $\begin{array}{l}4 \text { Mortality - Aprotinin versus } \\
\text { Tranexamic Acid }\end{array}$ & 17 & 4130 & Risk Ratio (M-H, Random, 95\% CI) & $1.35[0.94,1.93]$ \\
\hline $\begin{array}{l}5 \text { Mortality - Aprotinin versus } \\
\text { Epsilon Aminocaproic Acid }\end{array}$ & 5 & 1891 & Risk Ratio (M-H, Random, 95\% CI) & $1.51[0.99,2.30]$ \\
\hline $\begin{array}{l}6 \text { Mortality - Tranexamic Acid } \\
\text { versus Epsilon Aminocaproic } \\
\text { Acid }\end{array}$ & 5 & 1958 & Risk Ratio (M-H, Random, 95\% CI) & $0.93[0.59,1.47]$ \\
\hline $\begin{array}{l}7 \text { Mortality - Aprotinin versus } \\
\text { Lysine Analogues }\end{array}$ & 19 & 5127 & Risk Ratio (M-H, Random, 95\% CI) & $1.39[1.02,1.89]$ \\
\hline $\begin{array}{l}8 \text { Myocardial Infarction - } \\
\text { Aprotinin versus Tranexamic } \\
\text { Acid }\end{array}$ & 13 & 3574 & Risk Ratio (M-H, Random, 95\% CI) & $1.00[0.71,1.42]$ \\
\hline $\begin{array}{l}9 \text { Myocardial Infarction - } \\
\text { Aprotinin versus Epsilon } \\
\text { Aminocaproic Acid }\end{array}$ & 4 & 1676 & Risk Ratio (M-H, Random, 95\% CI) & $1.42[0.90,2.22]$ \\
\hline $\begin{array}{l}10 \text { Myocardial Infarction - } \\
\text { Tranexamic Acid versus Epsilon } \\
\text { Aminocaproic Acid }\end{array}$ & 3 & 1687 & Risk Ratio (M-H, Random, 95\% CI) & $1.33[0.80,2.23]$ \\
\hline
\end{tabular}

Anti-fibrinolytic use for minimising perioperative allogeneic blood transfusion (Review)

Copyright (C) 201 I The Cochrane Collaboration. Published by John Wiley \& Sons, Ltd. 
12 Stroke (CVA) - Aprotinin

14 Stroke (CVA) - Tranexamic

15 Deep Vein Thrombosis (DVT) Acid

16 Deep Vein Thrombosis (DVT) - Aprotinin versus Epsilon Aminocaproic Acid

17 Deep Vein Thrombosis (DVT) - Tranexamic Acid versus Epsilon Aminocaproic Acid

18 Pulmonary Embolism (PE) Aprotinin versus Tranexamic Acid

19 Pulmonary Embolism (PE) - Aprotinin versus Epsilon Aminocaproic Acid

22 Other Thrombosis - Aprotinin versus Epsilon Aminocaproic Acid

23 Other Thrombosis Tranexamic Acid versus Epsilon Aminocaproic Acid

24 Renal Failure / Dysfunction Aprotinin versus Tranexamic Acid

25 Renal Failure / Dysfunction - Aprotinin versus Epsilon Aminocaproic Acid

26 Renal Failure / Dysfunction Tranexamic Acid versus Epsilon Aminocaproic Acid Aprotinin versus Tranexamic Acid

Not estimable 


\section{Comparison 10. Adverse Events and Other Outcomes (Active versus Control) - Cardiac Surgery}

\begin{tabular}{|c|c|c|c|c|}
\hline Outcome or subgroup title & $\begin{array}{l}\text { No. of } \\
\text { studies }\end{array}$ & $\begin{array}{c}\text { No. of } \\
\text { participants }\end{array}$ & Statistical method & Effect size \\
\hline 1 Re-operation for bleeding & 78 & 8895 & Risk Ratio (M-H, Random, 95\% CI) & $0.56[0.44,0.71]$ \\
\hline 1.1 Aprotinin versus Control & 56 & 5827 & Risk Ratio (M-H, Random, 95\% CI) & $0.46[0.34,0.63]$ \\
\hline 1.2 Tranexamic Acid versus & 26 & 2328 & Risk Ratio (M-H, Random, 95\% CI) & $0.79[0.54,1.17]$ \\
\hline \multicolumn{5}{|l|}{ Control } \\
\hline 1.3 Epsilon Aminocaproic & 7 & 740 & Risk Ratio (M-H, Random, 95\% CI) & $0.35[0.11,1.17]$ \\
\hline \multicolumn{5}{|l|}{ Acid versus Control } \\
\hline 2 Mortality & 76 & 11240 & Risk Ratio (M-H, Random, 95\% CI) & $0.84[0.65,1.07]$ \\
\hline 2.1 Aprotinin versus Control & 55 & 8174 & Risk Ratio (M-H, Random, 95\% CI) & $0.84[0.64,1.10]$ \\
\hline 2.2 Tranexamic Acid versus & 23 & 2342 & Risk Ratio (M-H, Random, 95\% CI) & $0.58[0.26,1.28]$ \\
\hline \multicolumn{5}{|l|}{ Control } \\
\hline 2.3 Epsilon Aminocaproic & 6 & 724 & Risk Ratio (M-H, Random, 95\% CI) & $1.65[0.50,5.43]$ \\
\hline \multicolumn{5}{|l|}{ Acid versus Control } \\
\hline 3 Myocardial Infarction & 65 & 9472 & Risk Ratio (M-H, Random, 95\% CI) & $0.88[0.71,1.09]$ \\
\hline 3.1 Aprotinin versus Control & 46 & 6658 & Risk Ratio (M-H, Random, 95\% CI) & $0.90[0.71,1.14]$ \\
\hline 3.2 Tranexamic Acid versus & 19 & 2100 & Risk Ratio (M-H, Random, 95\% CI) & $0.74[0.37,1.47]$ \\
\hline \multicolumn{5}{|l|}{ Control } \\
\hline 3.3 Epsilon Aminocaproic & 6 & 714 & Risk Ratio (M-H, Random, 95\% CI) & $0.88[0.48,1.63]$ \\
\hline \multicolumn{5}{|l|}{ Acid versus Control } \\
\hline 4 Stroke & 38 & 4850 & Risk Ratio (M-H, Random, 95\% CI) & $0.95[0.55,1.63]$ \\
\hline 4.1 Aprotinin versus Control & 18 & 2127 & Risk Ratio (M-H, Random, 95\% CI) & $0.81[0.40,1.67]$ \\
\hline 4.2 Tranexamic Acid versus & 17 & 1969 & Risk Ratio (M-H, Random, 95\% CI) & $1.44[0.53,3.91]$ \\
\hline \multicolumn{5}{|l|}{ Control } \\
\hline 4.3 Epsilon Aminocaproic & 7 & 754 & Risk Ratio (M-H, Random, 95\% CI) & $0.70[0.16,3.10]$ \\
\hline \multicolumn{5}{|l|}{ Acid versus Control } \\
\hline 5 Deep Vein Thrombosis (DVT) & 7 & 1046 & Risk Ratio (M-H, Random, 95\% CI) & $0.95[0.31,2.87]$ \\
\hline 5.1 Aprotinin versus Control & 3 & 624 & Risk Ratio (M-H, Random, 95\% CI) & $1.29[0.36,4.58]$ \\
\hline 5.2 Tranexamic Acid versus & 4 & 422 & Risk Ratio (M-H, Random, 95\% CI) & $0.37[0.04,3.47]$ \\
\hline \multicolumn{5}{|l|}{ Control } \\
\hline 6 Pulmonary Embolism & 7 & 921 & Risk Ratio (M-H, Random, 95\% CI) & $0.63[0.14,2.74]$ \\
\hline 6.1 Tranexamic Acid versus & 6 & 569 & Risk Ratio (M-H, Random, 95\% CI) & $0.33[0.04,3.15]$ \\
\hline \multicolumn{5}{|l|}{ Control } \\
\hline 6.2 Aprotinin versus Control & 1 & 352 & Risk Ratio (M-H, Random, 95\% CI) & $1.01[0.14,7.10]$ \\
\hline 7 Other Thrombosis & 5 & & Risk Ratio (M-H, Random, 95\% CI) & Subtotals only \\
\hline 7.1 Aprotinin versus Control & 4 & 426 & Risk Ratio (M-H, Random, 95\% CI) & $0.62[0.11,3.36]$ \\
\hline 7.2 Tranexamic Acid versus & 1 & 100 & Risk Ratio (M-H, Random, 95\% CI) & Not estimable \\
\hline \multicolumn{5}{|l|}{ Control } \\
\hline 8 Renal Failure / Dysfunction & 30 & 5912 & Risk Ratio (M-H, Random, 95\% CI) & $0.97[0.71,1.33]$ \\
\hline
\end{tabular}




$\begin{array}{lccll}\text { 8.1 Aprotinin versus Control } & 24 & 4947 & \text { Risk Ratio (M-H, Random, 95\% CI) } & 1.07[0.76,1.51] \\ \begin{array}{l}\text { 8.2 Tranexamic Acid versus } \\ \text { Control }\end{array} & 9 & 912 & \text { Risk Ratio (M-H, Random, 95\% CI) } & 0.89[0.33,2.37] \\ \begin{array}{l}\text { 8.3 Epsilon Aminocaproic } \\ \text { Acid versus control }\end{array} & 1 & 53 & \text { Risk Ratio (M-H, Random, 95\% CI) } & 0.35[0.11,1.14] \\ \text { Hospital Length of Stay } & 19 & & \text { Mean Difference (IV, Random, 95\% CI) } & \text { Subtotals only } \\ \begin{array}{l}\text { 9.1 Aprotinin versus Control } \\ 9.2 \text { Tranexamic Acid versus }\end{array} & 17 & 1756 & \text { Mean Difference (IV, Random, 95\% CI) } & -0.22[-0.73,0.29] \\ \text { Control } & 5 & 434 & \text { Mean Difference (IV, Random, 95\% CI) } & -0.08[-0.34,0.18] \\ \end{array}$

Analysis I.I. Comparison I Aprotinin versus Control (Blood Transfusion \& Blood Loss), Outcome I No. Exposed to Allogeneic Blood.

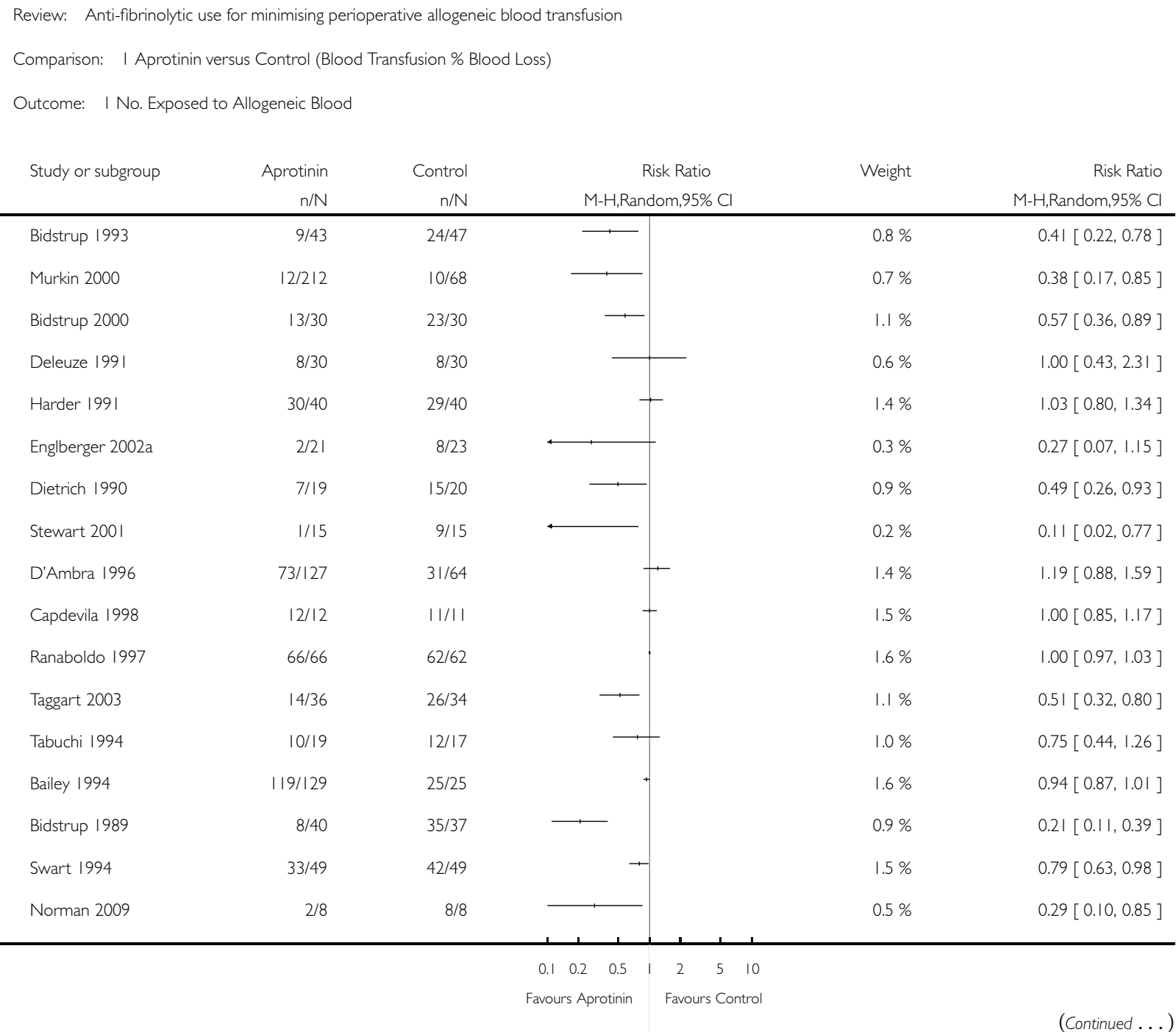




\begin{tabular}{|c|c|c|c|c|c|}
\hline Study or subgroup & $\begin{array}{r}\text { Aprotinin } \\
\mathrm{n} / \mathrm{N}\end{array}$ & $\begin{array}{r}\text { Control } \\
n / N\end{array}$ & $\begin{array}{c}\text { Risk Ratio } \\
\text { M-H. Random.95\% Cl }\end{array}$ & Weight & $\begin{array}{r}\text { (... Continued }) \\
\text { Risk Ratio } \\
\text { M-H.Random.95\% Cl }\end{array}$ \\
\hline Hardy 1993 & $17 / 22$ & $16 / 19$ & - & $1.4 \%$ & $0.92[0.68,1.24]$ \\
\hline Dietrich 1995 & $11 / 15$ & $15 / 15$ & $\rightarrow$ & $1.3 \%$ & $0.74[0.54,1.02]$ \\
\hline Lemmer'। 1994 & $28 / 74$ & $35 / 67$ & $\rightarrow$ & $1.2 \%$ & $0.72[0.50,1.05]$ \\
\hline Palmer 2003 & $11 / 30$ & $13 / 26$ & - & $0.9 \%$ & $0.73[0.40,1.35]$ \\
\hline Mansour 2004 & 1/20 & $12 / 20$ & - & $0.2 \%$ & $0.08[0.01,0.58]$ \\
\hline Harmon 2004 & $3 / 17$ & $4 / 18$ & & $0.3 \%$ & $0.79[0.21,3.04]$ \\
\hline Lemmer'2 1994 & $7 / 23$ & $23 / 32$ & & $0.8 \%$ & $0.42[0.22,0.82]$ \\
\hline Wendel 1995 & $10 / 20$ & $15 / 18$ & & $1.1 \%$ & $0.60[0.37,0.97]$ \\
\hline Speekenbrink 1996 & $55 / 75$ & 29/37 & + & $1.5 \%$ & $0.94[0.75,1.16]$ \\
\hline Colwell 2007 & $20 / 175$ & $39 / 177$ & 一 & $1.1 \%$ & $0.52[0.32,0.85]$ \\
\hline Liu 1993 & $3 / 20$ & $12 / 20$ & & $0.4 \%$ & $0.25[0.08,0.75]$ \\
\hline Vanek 2005 & 1/29 & $6 / 30$ & & $0.2 \%$ & $0.17[0.02,1.35]$ \\
\hline Greilich 2009 & $18 / 26$ & $17 / 27$ & + & $1.2 \%$ & $1.10[0.75,1.62]$ \\
\hline Poston 2006 & $10 / 29$ & $|8 / 3|$ & - & $0.9 \%$ & $0.59[0.33,1.07]$ \\
\hline Hardy 1997 & $23 / 26$ & $23 / 26$ & - & $1.5 \%$ & $1.00[0.82,1.22]$ \\
\hline Cohen 1998 & $17 / 56$ & $37 / 59$ & - & $1.1 \%$ & $0.48[0.31,0.75]$ \\
\hline Lass 1995 & $25 / 51$ & $37 / 47$ & - & $1.3 \%$ & $0.62[0.45,0.85]$ \\
\hline Thorpe 1994 & 1/8 & $6 / 9$ & & $0.2 \%$ & $0.19[0.03,1.24]$ \\
\hline Cvachovec 200I & $8 / 20$ & $16 / 22$ & & $0.9 \%$ & $0.55[0.30,1.00]$ \\
\hline Cicek 1996b & $11 / 29$ & $18 / 28$ & + & $1.0 \%$ & $0.59[0.34,1.01]$ \\
\hline Alderman 1998 & $|52 / 40|$ & $213 / 395$ & - & $1.5 \%$ & $0.70[0.60,0.82]$ \\
\hline Penta de Peppo 1995 & $0 / 15$ & $3 / 15$ & & $0.1 \%$ & $0.14[0.01,2.55]$ \\
\hline Murkin 1995 & $18 / 29$ & $17 / 24$ & $\rightarrow$ & $1.2 \%$ & $0.88[0.60,1.29]$ \\
\hline D'Ambrosio 1999 & 1/30 & 0/30 & & $0.1 \%$ & $3.00[0.13,70.83]$ \\
\hline Blauhut 1994 & $3 / 14$ & $9 / 14$ & & $0.5 \%$ & $0.33[0.11,0.98]$ \\
\hline Cosgrove 1992 & $55 / 113$ & $44 / 56$ & + & $1.5 \%$ & $0.62[0.49,0.78]$ \\
\hline Tassani 2000 & $4 / 10$ & $5 / 10$ & 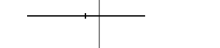 & $0.5 \%$ & $0.80[0.30,2.13]$ \\
\hline Bidstrup 1990 & $15 / 26$ & $16 / 18$ & $\rightarrow$ & $1.3 \%$ & $0.65[0.45,0.94]$ \\
\hline Carrera 1994 & $42 / 51$ & $50 / 51$ & + & $1.6 \%$ & $0.84[0.74,0.96]$ \\
\hline Englberger $2002 b$ & $2 / 15$ & $7 / 14$ & & $0.3 \%$ & $0.27[0.07,1.07]$ \\
\hline Lemmer 1996 & 173/487 & $87 / 157$ & + & $1.5 \%$ & $0.64[0.53,0.77]$ \\
\hline
\end{tabular}

$\begin{array}{lllllll}0.1 & 0.2 & 0.5 & 1 & 2 & 5 & 10\end{array}$

(Continued...)

Anti-fibrinolytic use for minimising perioperative allogeneic blood transfusion (Review)

Copyright (C) 201 I The Cochrane Collaboration. Published by John Wiley \& Sons, Ltd. 


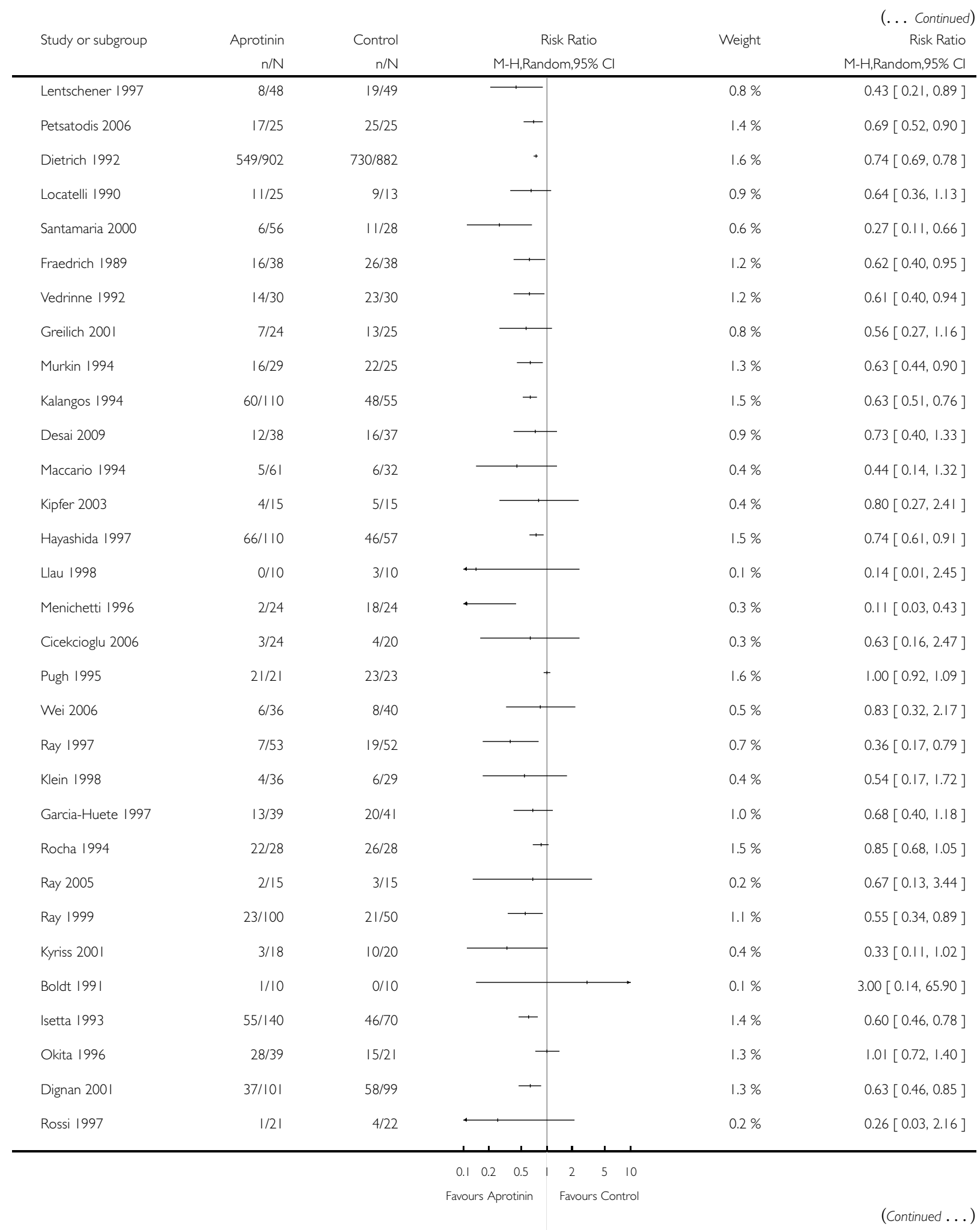

Anti-fibrinolytic use for minimising perioperative allogeneic blood transfusion (Review) 


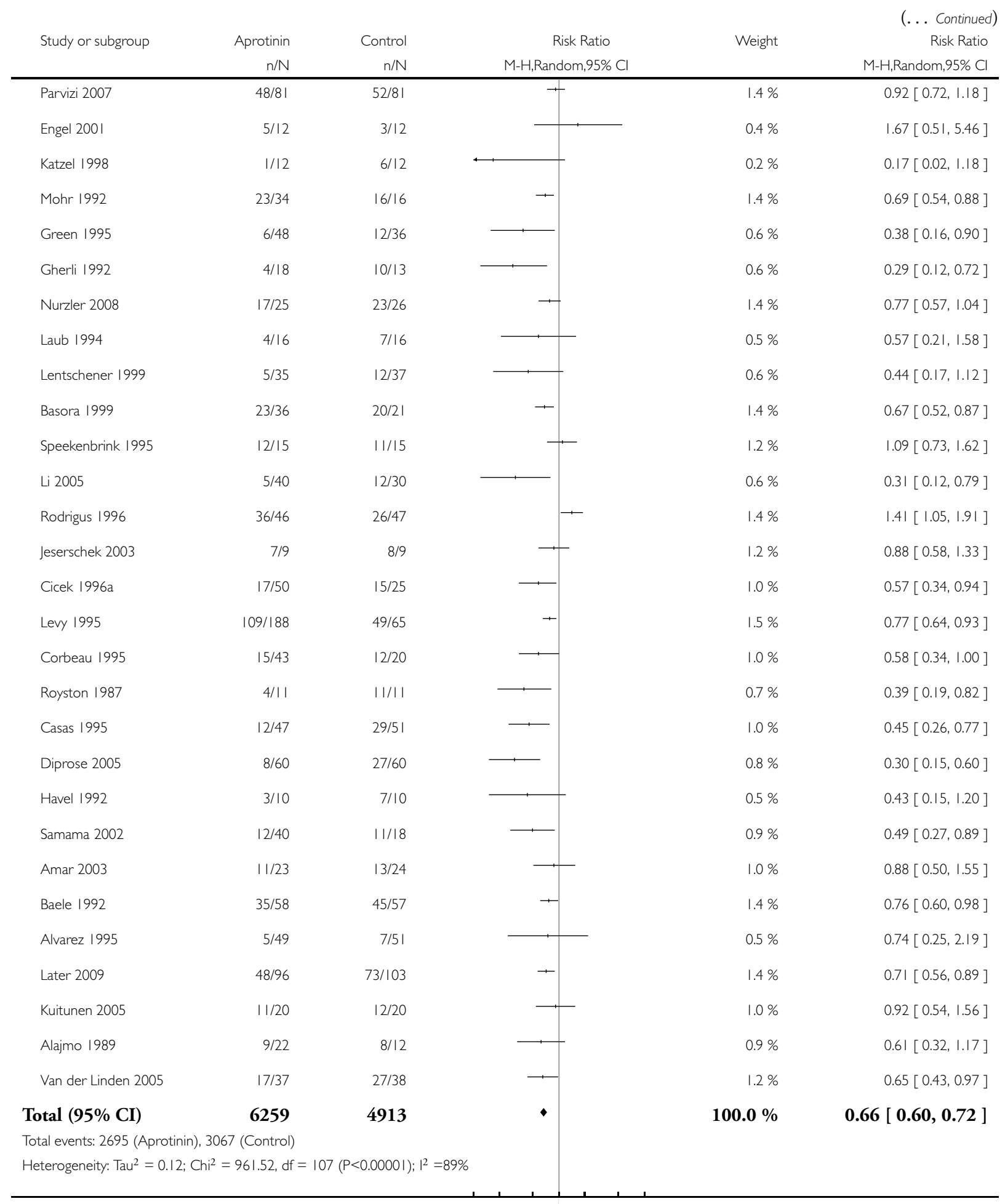

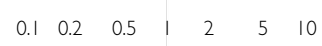

(Continued ....)

Anti-fibrinolytic use for minimising perioperative allogeneic blood transfusion (Review)

Copyright (C) 201 I The Cochrane Collaboration. Published by John Wiley \& Sons, Ltd. 


\begin{tabular}{|c|c|c|c|c|c|}
\hline & & & & & (... Continued) \\
\hline \multirow[t]{2}{*}{ Study or subgroup } & Aprotinin & Control & Risk Ratio & Weight & Risk Ratio \\
\hline & $n / N$ & $n / N$ & M-H,Random, $95 \% \mathrm{Cl}$ & & M-H,Random,95\% Cl \\
\hline
\end{tabular}

Test for overall effect: $Z=9.54(P<0.00001)$

$\begin{array}{lllllll}0.1 & 0.2 & 0.5 & 1 & 2 & 5 & 10\end{array}$

Favours Aprotinin Favours Control

Analysis I.2. Comparison I Aprotinin versus Control (Blood Transfusion \& Blood Loss), Outcome 2 No. Exposed to Allogeneic Blood - Type of Surgery.

Review: Anti-fibrinolytic use for minimising perioperative allogeneic blood transfusion

Comparison: I Aprotinin versus Control (Blood Transfusion \% Blood Loss)

Outcome: 2 No. Exposed to Allogeneic Blood - Type of Surgery

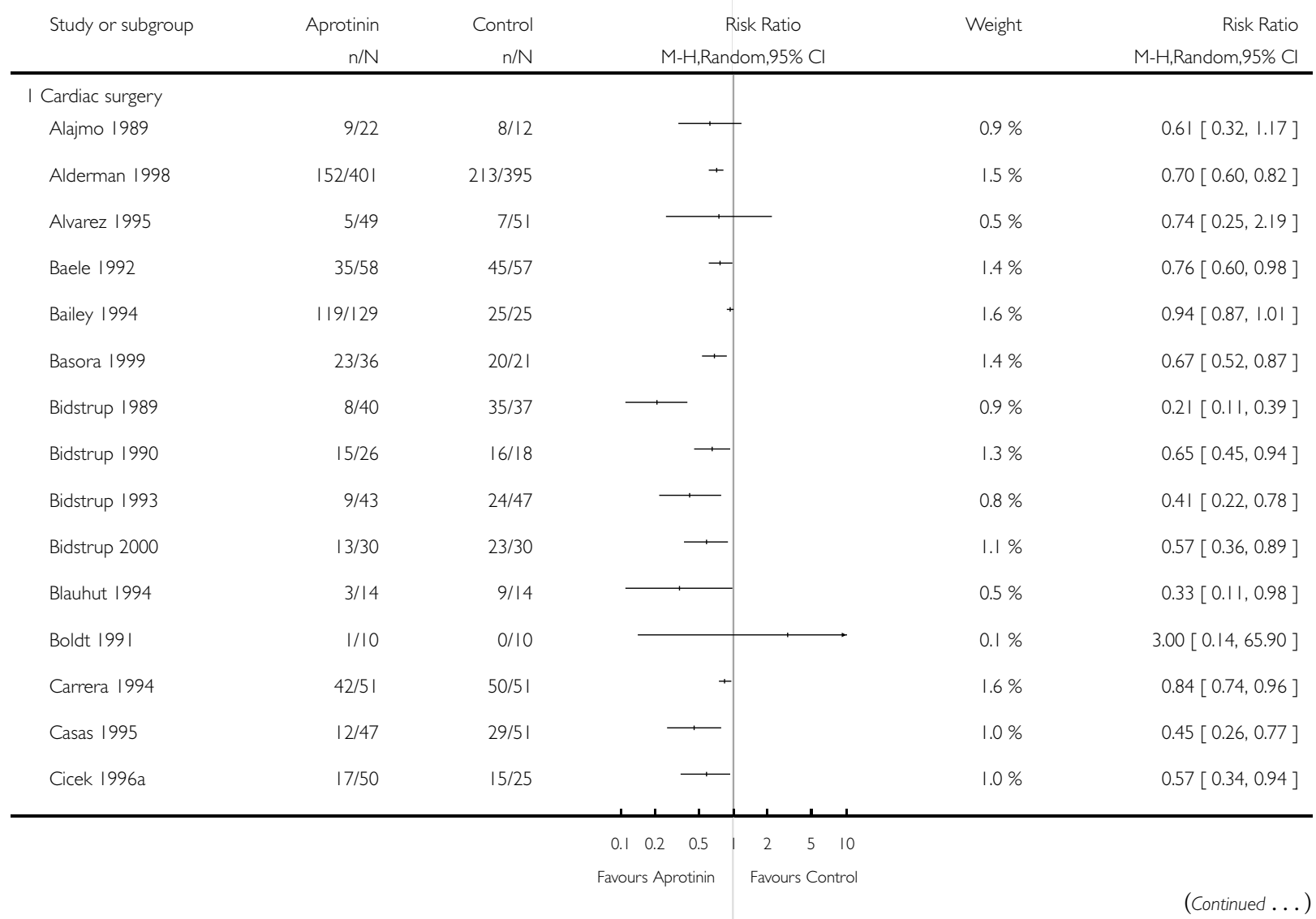




\begin{tabular}{|c|c|c|c|c|c|}
\hline Study or subgroup & $\begin{array}{r}\text { Aprotinin } \\
\mathrm{n} / \mathrm{N}\end{array}$ & $\begin{array}{r}\text { Control } \\
n / N\end{array}$ & $\begin{array}{c}\text { Risk Ratio } \\
\text { M-H.Random.95\% C। }\end{array}$ & Weight & $\begin{array}{r}\text { (... Continued }) \\
\text { Risk Ratio } \\
\text { M-H.Random.95\% Cl }\end{array}$ \\
\hline Cicek 1996b & $11 / 29$ & $18 / 28$ & . & $1.0 \%$ & $0.59[0.34,1.01]$ \\
\hline Cicekcioglu 2006 & $3 / 24$ & $4 / 20$ & & $0.3 \%$ & $0.63[0.16,2.47]$ \\
\hline Cohen 1998 & $17 / 56$ & $37 / 59$ & $\longrightarrow$ & $1.1 \%$ & $0.48[0.31,0.75]$ \\
\hline Corbeau 1995 & $15 / 43$ & $12 / 20$ & G. & $1.0 \%$ & $0.58[0.34,1.00]$ \\
\hline Cosgrove 1992 & $55 / 113$ & $44 / 56$ & + & $1.5 \%$ & $0.62[0.49,0.78]$ \\
\hline D'Ambra 1996 & $73 / 127$ & $31 / 64$ & T & $1.4 \%$ & $1.19[0.88,1.59]$ \\
\hline Deleuze 199| & $8 / 30$ & $8 / 30$ & 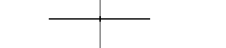 & $0.6 \%$ & $1.00[0.43,2.31]$ \\
\hline Desai 2009 & $12 / 38$ & $16 / 37$ & - & $0.9 \%$ & $0.73[0.40,1.33]$ \\
\hline Dietrich 1990 & $7 / 19$ & $15 / 20$ & 1 & $0.9 \%$ & $0.49[0.26,0.93]$ \\
\hline Dietrich 1992 & $549 / 902$ & $730 / 882$ & + & $1.6 \%$ & $0.74[0.69,0.78]$ \\
\hline Dietrich 1995 & $11 / 15$ & $15 / 15$ & + & $1.3 \%$ & $0.74[0.54,1.02]$ \\
\hline Dignan 200I & $37 / 101$ & $58 / 99$ & $一$ & $1.3 \%$ & $0.63[0.46,0.85]$ \\
\hline Diprose 2005 & $8 / 60$ & $27 / 60$ & - & $0.8 \%$ & $0.30[0.15,0.60]$ \\
\hline Englberger 2002a & $2 / 21$ & $8 / 23$ & & $0.3 \%$ & $0.27[0.07,1.15]$ \\
\hline Englberger $2002 b$ & $2 / 15$ & $7 / 14$ & & $0.3 \%$ & $0.27[0.07,1.07]$ \\
\hline Fraedrich 1989 & $16 / 38$ & $26 / 38$ & & $1.2 \%$ & $0.62[0.40,0.95]$ \\
\hline Gherli 1992 & $4 / 18$ & $10 / 13$ & & $0.6 \%$ & $0.29[0.12,0.72]$ \\
\hline Green 1995 & $6 / 48$ & $12 / 36$ & & $0.6 \%$ & $0.38[0.16,0.90]$ \\
\hline Greilich 200I & $7 / 24$ & $13 / 25$ & & $0.8 \%$ & $0.56[0.27,1.16]$ \\
\hline Greilich 2009 & $18 / 26$ & $17 / 27$ & + & $1.2 \%$ & $1.10[0.75,1.62]$ \\
\hline Harder 1991 & $30 / 40$ & $29 / 40$ & - & $1.4 \%$ & $1.03[0.80,1.34]$ \\
\hline Hardy 1993 & $17 / 22$ & $16 / 19$ & & $1.4 \%$ & $0.92[0.68,1.24]$ \\
\hline Hardy 1997 & $23 / 26$ & $23 / 26$ & - & $1.5 \%$ & $1.00[0.82,1.22]$ \\
\hline Harmon 2004 & $3 / 17$ & $4 / 18$ & & $0.3 \%$ & $0.79[0.21,3.04]$ \\
\hline Havel 1992 & $3 / 10$ & $7 / 10$ & & $0.5 \%$ & $0.43[0.15,1.20]$ \\
\hline Hayashida 1997 & $66 / 110$ & $46 / 57$ & + & $1.5 \%$ & $0.74[0.61,0.91]$ \\
\hline Isetta 1993 & $55 / / 40$ & $46 / 70$ & 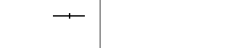 & $1.4 \%$ & $0.60[0.46,0.78]$ \\
\hline Kalangos 1994 & $60 / 110$ & $48 / 55$ & + & $1.5 \%$ & $0.63[0.5 ।, 0.76]$ \\
\hline Kipfer 2003 & $4 / 15$ & $5 / 15$ & & $0.4 \%$ & $0.80[0.27,2.41]$ \\
\hline Klein 1998 & $4 / 36$ & $6 / 29$ & - & $0.4 \%$ & $0.54[0.17,1.72]$ \\
\hline Kuitunen 2005 & $11 / 20$ & $12 / 20$ & - & $1.0 \%$ & $0.92[0.54,1.56]$ \\
\hline
\end{tabular}

$\begin{array}{lllllll}0.1 & 0.2 & 0.5 & 1 & 2 & 5 & 10\end{array}$

Favours Aprotinin Favours Control

(Continued....)

Anti-fibrinolytic use for minimising perioperative allogeneic blood transfusion (Review)

Copyright (C) 201 I The Cochrane Collaboration. Published by John Wiley \& Sons, Ltd. 


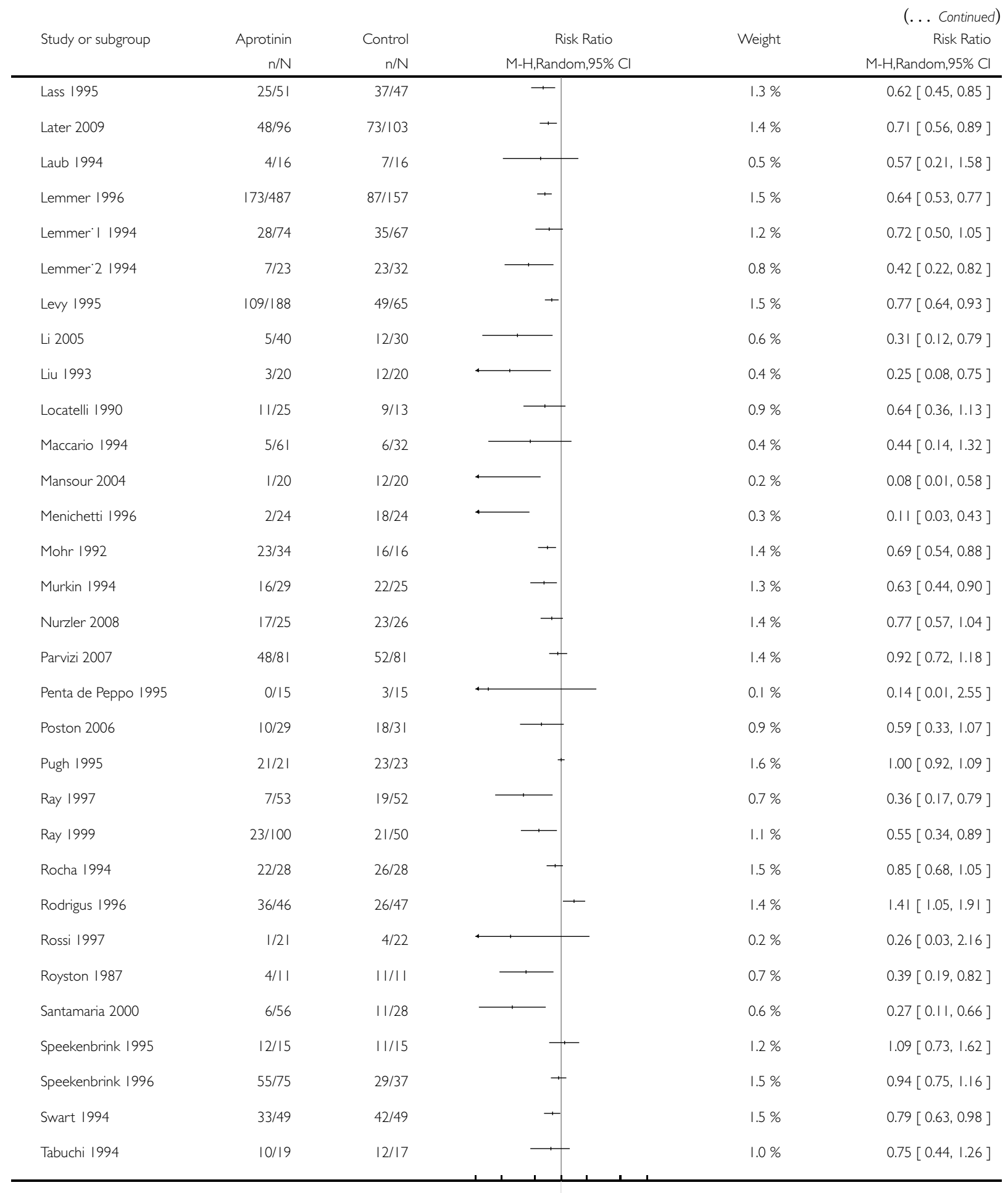

$\begin{array}{lllllll}0.1 & 0.2 & 0.5 & 1 & 2 & 5 & 10\end{array}$

Favours Aprotinin Favours Control

(Continued....)

Anti-fibrinolytic use for minimising perioperative allogeneic blood transfusion (Review)

Copyright (C) 201 I The Cochrane Collaboration. Published by John Wiley \& Sons, Ltd. 


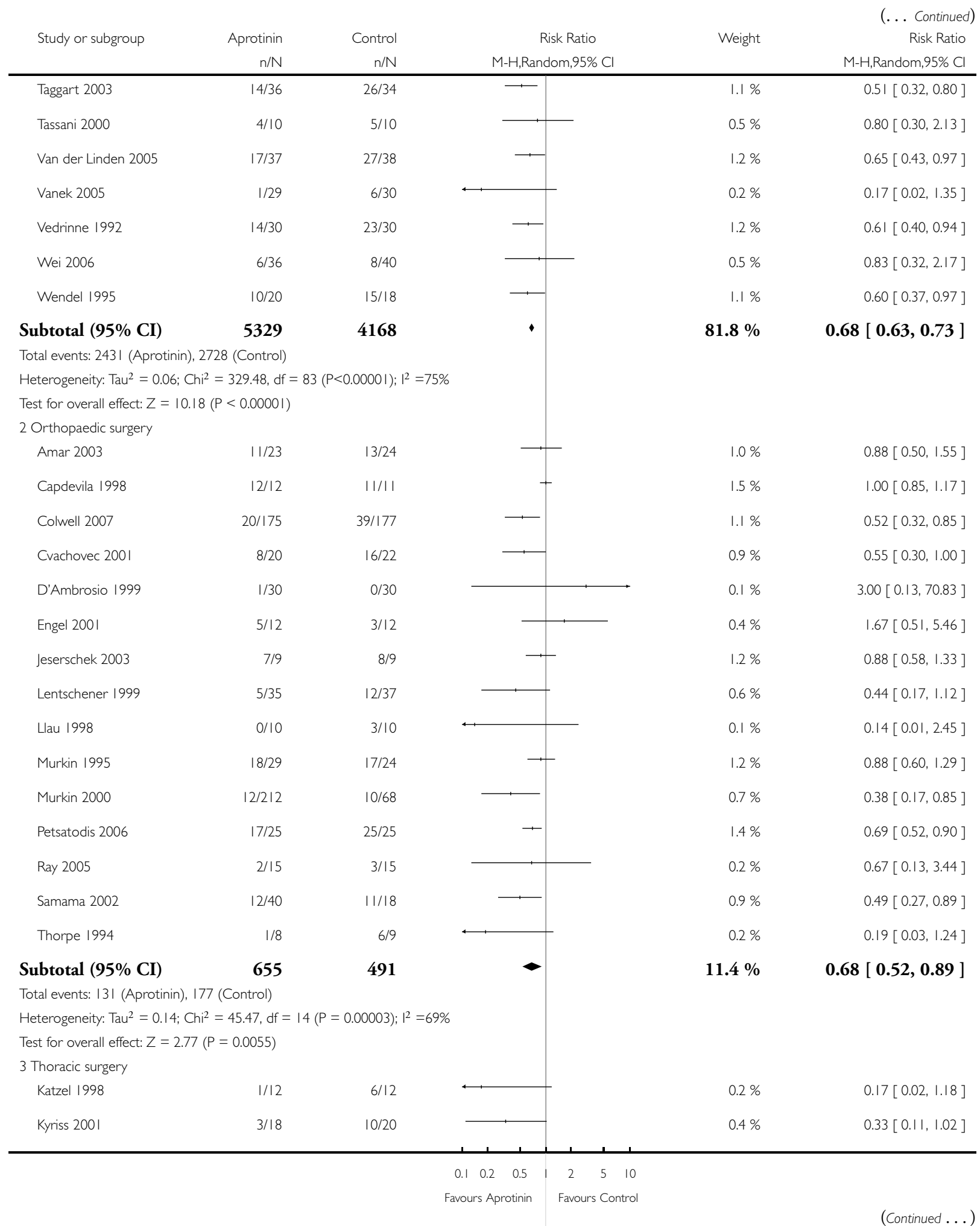

Anti-fibrinolytic use for minimising perioperative allogeneic blood transfusion (Review) 


\begin{tabular}{|c|c|c|c|c|c|}
\hline \multirow[t]{2}{*}{ Study or subgroup } & Aprotinin & Control & Risk Ratio & Weight & $\begin{array}{r}\text { (. . C Continued) } \\
\text { Risk Ratio } \\
\text { M-H,Random,95\% Cl }\end{array}$ \\
\hline & $\mathrm{n} / \mathrm{N}$ & $\mathrm{n} / \mathrm{N}$ & \multicolumn{2}{|l|}{ M-H,Random, $95 \% \mathrm{Cl}$} & $\mathrm{M}-\mathrm{H}$, Random, $95 \% \mathrm{Cl}$ \\
\hline Norman 2009 & $2 / 8$ & $8 / 8$ & ב- & $0.5 \%$ & $0.29[0.10,0.85]$ \\
\hline Subtotal $(95 \% \mathrm{CI})$ & 38 & 40 & & $1.1 \%$ & $0.29[0.14,0.59]$ \\
\hline \multicolumn{6}{|c|}{ Total events: 6 (Aprotinin), 24 (Control) } \\
\hline \multicolumn{6}{|c|}{ Heterogeneity: $\mathrm{Tau}^{2}=0.0 ; \mathrm{Chi}^{2}=0.37, \mathrm{df}=2(\mathrm{P}=0.83) ; \mathrm{I}^{2}=0.0 \%$} \\
\hline \multicolumn{6}{|c|}{ Test for overall effect: $Z=3.4 \mathrm{I}(P=0.00066)$} \\
\hline \multicolumn{6}{|l|}{4 Vascular surgery } \\
\hline Okita 1996 & 28/39 & $15 / 21$ & t & $1.3 \%$ & $1.01[0.72,1.40]$ \\
\hline Ranaboldo 1997 & $66 / 66$ & $62 / 62$ & & $1.6 \%$ & $1.00[0.97,1.03]$ \\
\hline Subtotal $(95 \% \mathrm{CI})$ & 105 & 83 & & $2.9 \%$ & $1.00[0.97,1.03]$ \\
\hline \multicolumn{6}{|c|}{ Total events: 94 (Aprotinin), 77 (Control) } \\
\hline \multicolumn{6}{|c|}{ Heterogeneity: $\mathrm{Tau}^{2}=0.0 ; \mathrm{Chi}^{2}=0.01, \mathrm{df}=\mathrm{I}(\mathrm{P}=0.94) ; \mathrm{I}^{2}=0.0 \%$} \\
\hline \multicolumn{6}{|c|}{ Test for overall effect: $Z=0.00(P=1.0)$} \\
\hline \multicolumn{6}{|l|}{5 Liver surgery } \\
\hline Garcia-Huete 1997 & 13/39 & $20 / 41$ & & $1.0 \%$ & $0.68[0.40,1.18]$ \\
\hline Lentschener 1997 & $8 / 48$ & $19 / 49$ & 而 & $0.8 \%$ & $0.43[0.21,0.89]$ \\
\hline Subtotal $(95 \% \mathrm{CI})$ & 87 & 90 & $\longrightarrow$ & $1.7 \%$ & $0.58[0.37,0.90]$ \\
\hline \multicolumn{6}{|c|}{ Total events: 21 (Aprotinin), 39 (Control) } \\
\hline \multicolumn{6}{|c|}{ Heterogeneity: $\mathrm{Tau}^{2}=0.00 ; \mathrm{Chi}^{2}=1.03, \mathrm{df}=1(P=0.31) ; 1^{2}=3 \%$} \\
\hline \multicolumn{6}{|c|}{ Test for overall effect: $Z=2.44(P=0.015)$} \\
\hline \multicolumn{6}{|l|}{6 Neuro surgery } \\
\hline Palmer 2003 & $11 / 30$ & $13 / 26$ & & $0.9 \%$ & $0.73[0.40,1.35]$ \\
\hline Subtotal $(95 \% \mathrm{CI})$ & 30 & 26 & & $0.9 \%$ & $0.73[0.40,1.35]$ \\
\hline \multicolumn{6}{|c|}{ Total events: II (Aprotinin), 13 (Control) } \\
\hline \multicolumn{6}{|c|}{ Heterogeneity: not applicable } \\
\hline \multicolumn{6}{|c|}{ Test for overall effect: $Z=1.00(P=0.32)$} \\
\hline \multicolumn{6}{|l|}{7 Orthognathic surgery } \\
\hline Stewart 2001 & $1 / 15$ & $9 / 15$ & & $0.2 \%$ & $0.11[0.02,0.77]$ \\
\hline Subtotal $(95 \% \mathrm{CI})$ & 15 & 15 & & $0.2 \%$ & $0.11[0.02,0.77]$ \\
\hline \multicolumn{6}{|c|}{ Total events: I (Aprotinin), 9 (Control) } \\
\hline \multicolumn{6}{|c|}{ Heterogeneity: not applicable } \\
\hline \multicolumn{6}{|c|}{ Test for overall effect: $Z=2.22(P=0.026)$} \\
\hline Total $(95 \% \mathrm{CI})$ & 6259 & 4913 & - & $100.0 \%$ & $0.66[0.60,0.72]$ \\
\hline \multicolumn{6}{|c|}{ Total events: 2695 (Aprotinin), 3067 (Control) } \\
\hline \multicolumn{6}{|c|}{ Heterogeneity: $\mathrm{Tau}^{2}=0.12 ; \mathrm{Chi}^{2}=961.52, \mathrm{df}=107(\mathrm{P}<0.0000 \mathrm{I}) ; \mathrm{I}^{2}=89 \%$} \\
\hline Test for overall effect: $Z=$ & $<0.00001)$ & & & & \\
\hline
\end{tabular}


Analysis I.3. Comparison I Aprotinin versus Control (Blood Transfusion \& Blood Loss), Outcome 3 No. Exposed to Allogeneic Blood - Transfusion Protocol.

Review: Anti-fibrinolytic use for minimising perioperative allogeneic blood transfusion

Comparison: I Aprotinin versus Control (Blood Transfusion \% Blood Loss)

Outcome: 3 No. Exposed to Allogeneic Blood - Transfusion Protocol

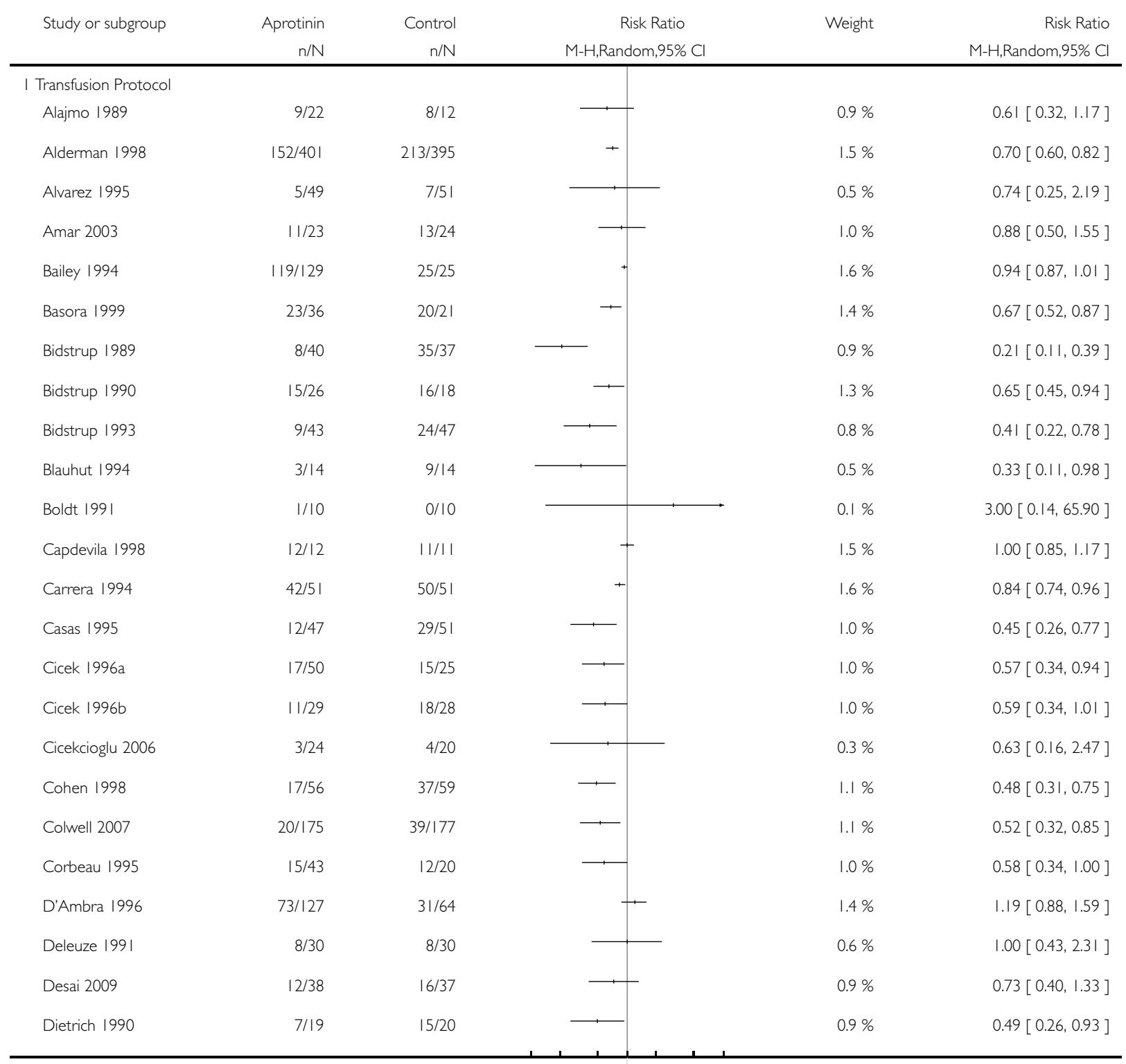

$\begin{array}{lllllll}0.1 & 0.2 & 0.5 & 1 & 2 & 5 & 10\end{array}$

Favours Aprotinin Favours Control

(Continued ...) 


\begin{tabular}{|c|c|c|c|c|c|}
\hline \multirow[t]{2}{*}{ Study or subgroup } & Aprotinin & Control & Risk Ratio & \multirow[t]{2}{*}{ Weight } & \multirow{2}{*}{$\begin{array}{r}\text { (... Continued } \\
\text { Risk Ratio } \\
\text { M-H,Random, } 95 \% \mathrm{Cl}\end{array}$} \\
\hline & $\mathrm{n} / \mathrm{N}$ & $\mathrm{n} / \mathrm{N}$ & M-H,Random, $95 \% \mathrm{Cl}$ & & \\
\hline Dietrich 1992 & $549 / 902$ & $730 / 882$ & + & $1.6 \%$ & $0.74[0.69,0.78]$ \\
\hline Dietrich 1995 & $11 / 15$ & $15 / 15$ & $\rightarrow$ & $1.3 \%$ & $0.74[0.54,1.02]$ \\
\hline Dignan 200। & $37 / 101$ & $58 / 99$ & $\leftarrow$ & $1.3 \%$ & $0.63[0.46,0.85]$ \\
\hline Diprose 2005 & $8 / 60$ & $27 / 60$ & 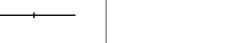 & $0.8 \%$ & $0.30[0.15,0.60]$ \\
\hline Engel 2001 & $5 / 12$ & $3 / 12$ & & $0.4 \%$ & I.67 [0.5I, 5.46] \\
\hline Englberger $2002 a$ & $2 / 21$ & $8 / 23$ & & $0.3 \%$ & $0.27[0.07,1.15]$ \\
\hline Englberger $2002 b$ & $2 / 15$ & $7 / 14$ & & $0.3 \%$ & $0.27[0.07,1.07]$ \\
\hline Garcia-Huete 1997 & $13 / 39$ & $20 / 41$ & & $1.0 \%$ & $0.68[0.40,1.18]$ \\
\hline Gherli 1992 & $4 / 18$ & $10 / 13$ & & $0.6 \%$ & $0.29[0.12,0.72]$ \\
\hline Green 1995 & $6 / 48$ & $12 / 36$ & & $0.6 \%$ & $0.38[0.16,0.90]$ \\
\hline Greilich 200I & $7 / 24$ & $13 / 25$ & & $0.8 \%$ & $0.56[0.27,1.16]$ \\
\hline Greilich 2009 & $18 / 26$ & $17 / 27$ & - & $1.2 \%$ & $1.10[0.75,1.62]$ \\
\hline Harder 1991 & $30 / 40$ & $29 / 40$ & - & $1.4 \%$ & $1.03[0.80,1.34]$ \\
\hline Hardy 1993 & $17 / 22$ & $16 / 19$ & $\rightarrow$ & $1.4 \%$ & $0.92[0.68,1.24]$ \\
\hline Hardy 1997 & $23 / 26$ & $23 / 26$ & $t$ & $1.5 \%$ & $1.00[0.82,1.22]$ \\
\hline Harmon 2004 & $3 / 17$ & $4 / 18$ & 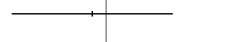 & $0.3 \%$ & $0.79[0.21,3.04]$ \\
\hline Hayashida 1997 & $66 / 110$ & $46 / 57$ & - & $1.5 \%$ & $0.74[0.61,0.91]$ \\
\hline Isetta 1993 & $55 / / 40$ & $46 / 70$ & + & $1.4 \%$ & $0.60[0.46,0.78]$ \\
\hline Kalangos 1994 & $60 / 110$ & $48 / 55$ & + & $1.5 \%$ & $0.63[0.51,0.76]$ \\
\hline Katzel 1998 & $1 / 12$ & $6 / 12$ & 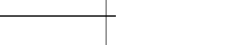 & $0.2 \%$ & $0.17[0.02,1.18]$ \\
\hline Kipfer 2003 & $4 / 15$ & $5 / 15$ & & $0.4 \%$ & $0.80[0.27,2.41]$ \\
\hline Klein 1998 & $4 / 36$ & $6 / 29$ & - & $0.4 \%$ & $0.54[0.17,1.72]$ \\
\hline Kuitunen 2005 & $11 / 20$ & $12 / 20$ & 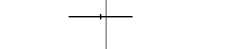 & $1.0 \%$ & $0.92[0.54,1.56]$ \\
\hline Lass 1995 & $25 / 51$ & $37 / 47$ & $\rightarrow$ & $1.3 \%$ & $0.62[0.45,0.85]$ \\
\hline Later 2009 & $48 / 96$ & $73 / 103$ & - & $1.4 \%$ & $0.71[0.56,0.89]$ \\
\hline Laub 1994 & $4 / 16$ & $7 / 16$ & 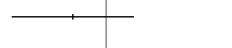 & $0.5 \%$ & $0.57[0.21,1.58]$ \\
\hline Lemmer 1996 & 173/487 & $87 / 157$ & + & $1.5 \%$ & $0.64[0.53,0.77]$ \\
\hline Lemmer'| 1994 & $28 / 74$ & $35 / 67$ & 7 & $1.2 \%$ & $0.72[0.50,1.05]$ \\
\hline Lemmer 21994 & $7 / 23$ & $23 / 32$ & 巨" & $0.8 \%$ & $0.42[0.22,0.82]$ \\
\hline Lentschener 1997 & $8 / 48$ & $19 / 49$ & 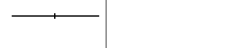 & $0.8 \%$ & $0.43[0.21,0.89]$ \\
\hline Lentschener 1999 & $5 / 35$ & $12 / 37$ & 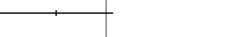 & $0.6 \%$ & $0.44[0.17,1.12]$ \\
\hline
\end{tabular}

$\begin{array}{lllllll}0.1 & 0.2 & 0.5 & 1 & 2 & 5 & 10\end{array}$

Favours Aprotinin Favours Control

(Continued....) 


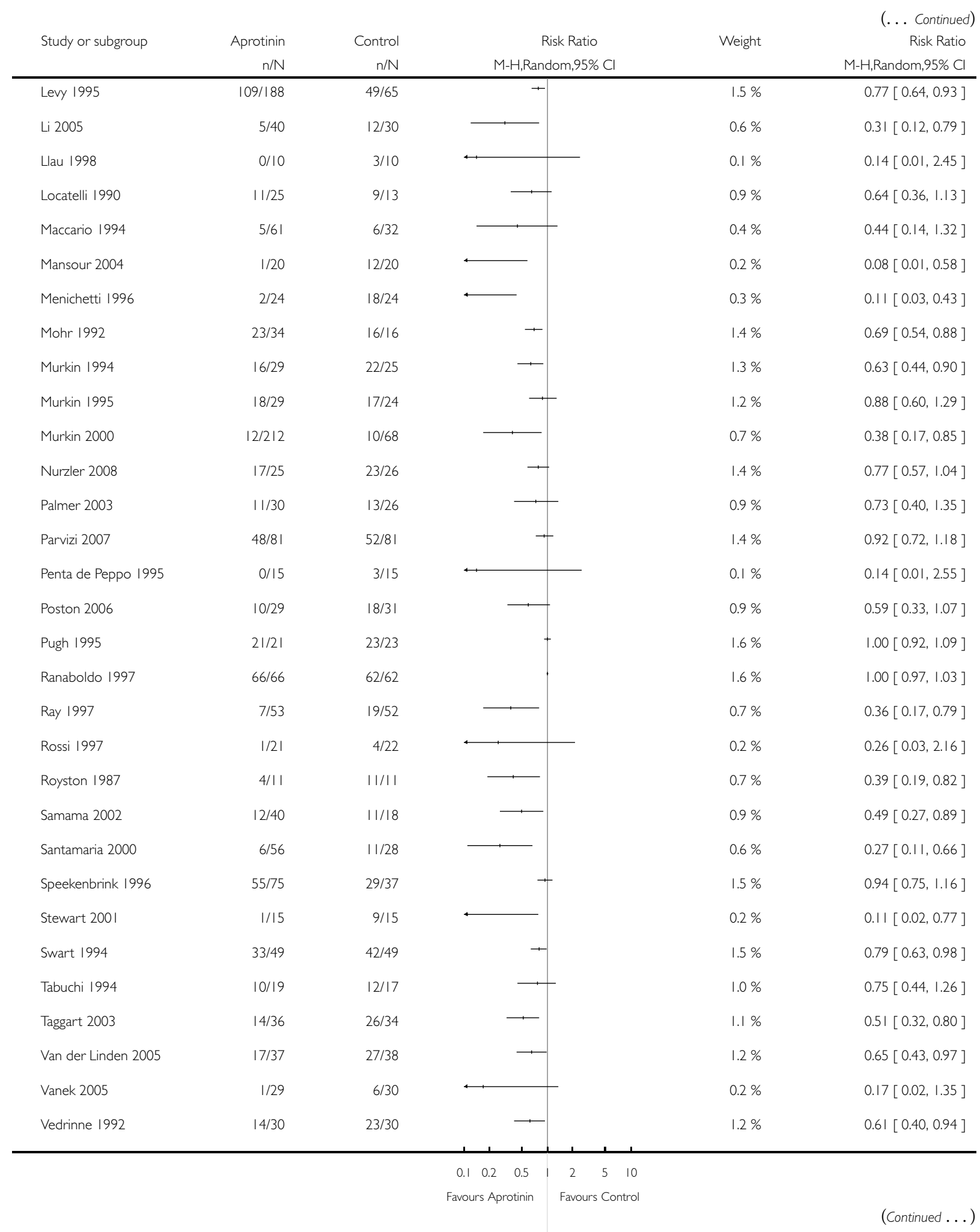

Anti-fibrinolytic use for minimising perioperative allogeneic blood transfusion (Review) 


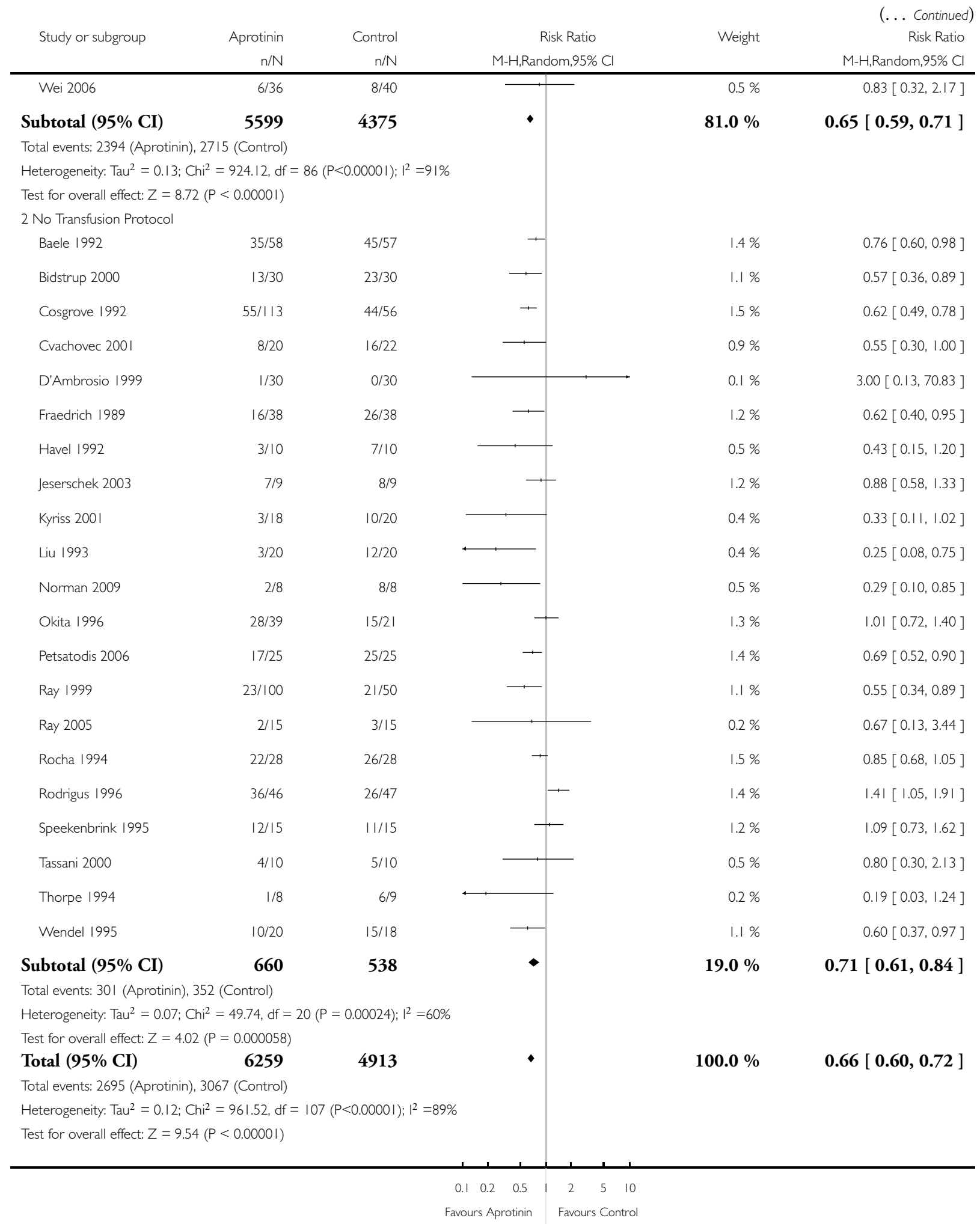

Anti-fibrinolytic use for minimising perioperative allogeneic blood transfusion (Review) 
Analysis I.4. Comparison I Aprotinin versus Control (Blood Transfusion \& Blood Loss), Outcome 4 No. Exposed to Allogeneic Blood - Dose.

Review: Anti-fibrinolytic use for minimising perioperative allogeneic blood transfusion

Comparison: I Aprotinin versus Control (Blood Transfusion \% Blood Loss)

Outcome: 4 No. Exposed to Allogeneic Blood - Dose

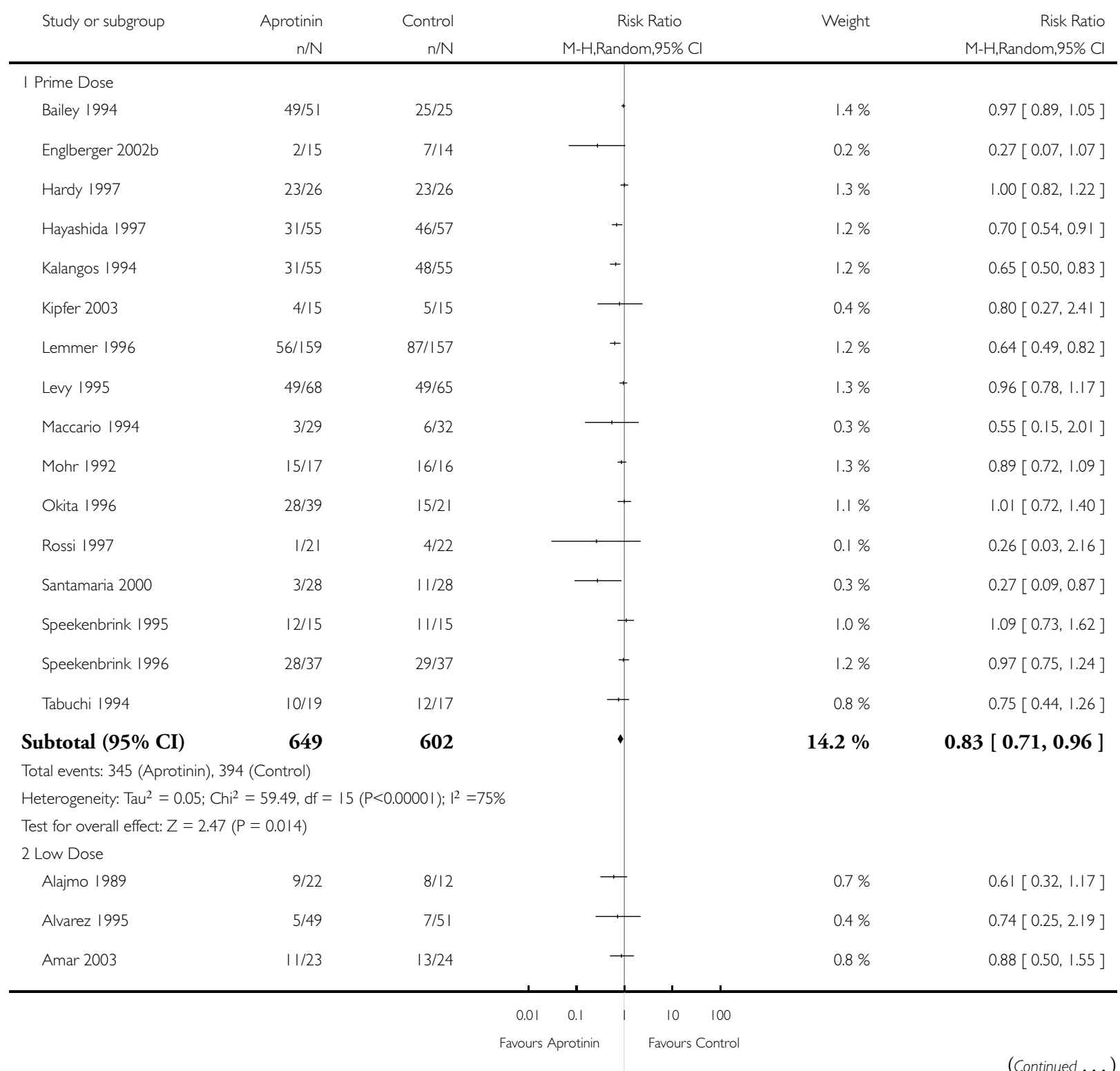




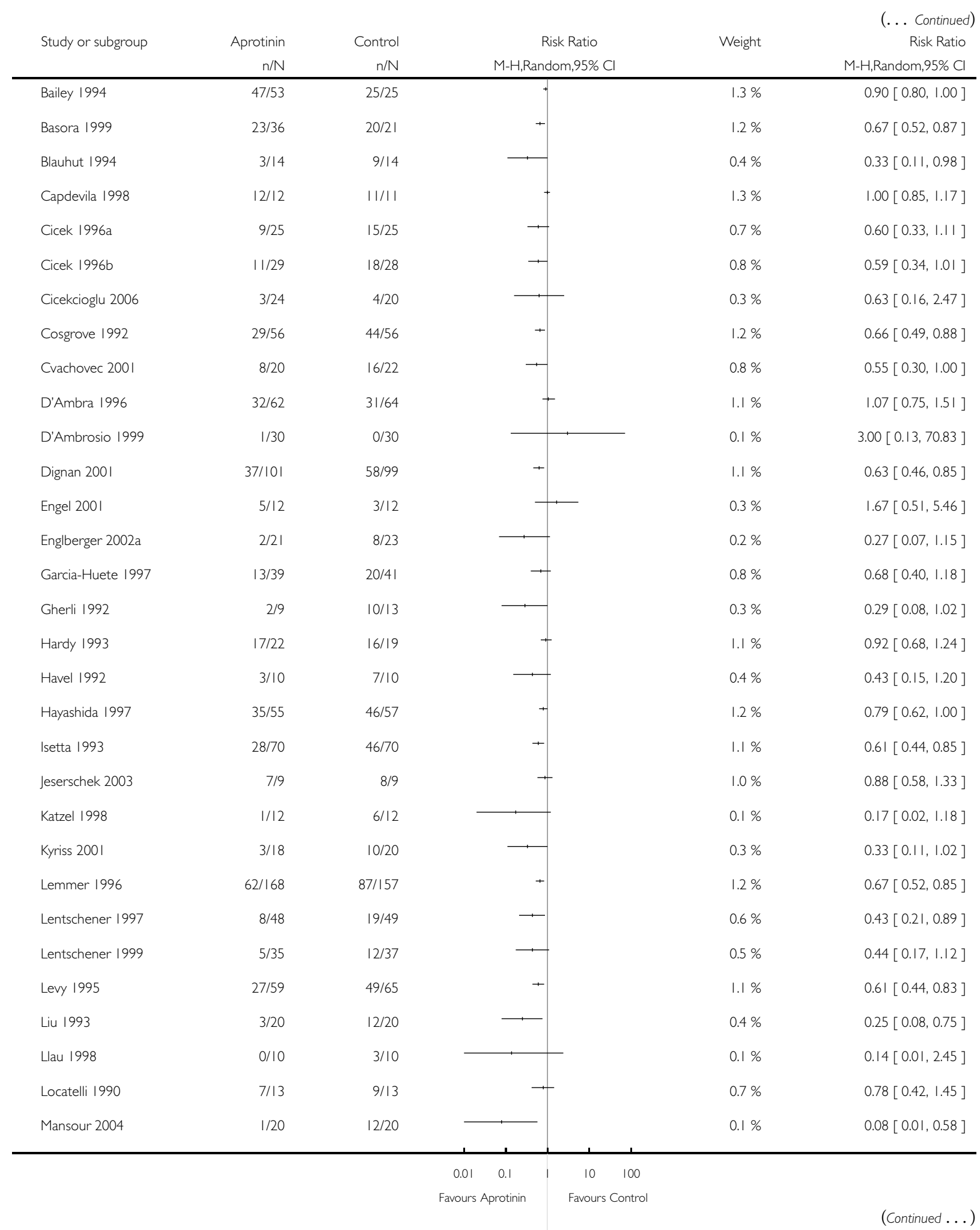

Anti-fibrinolytic use for minimising perioperative allogeneic blood transfusion (Review) 


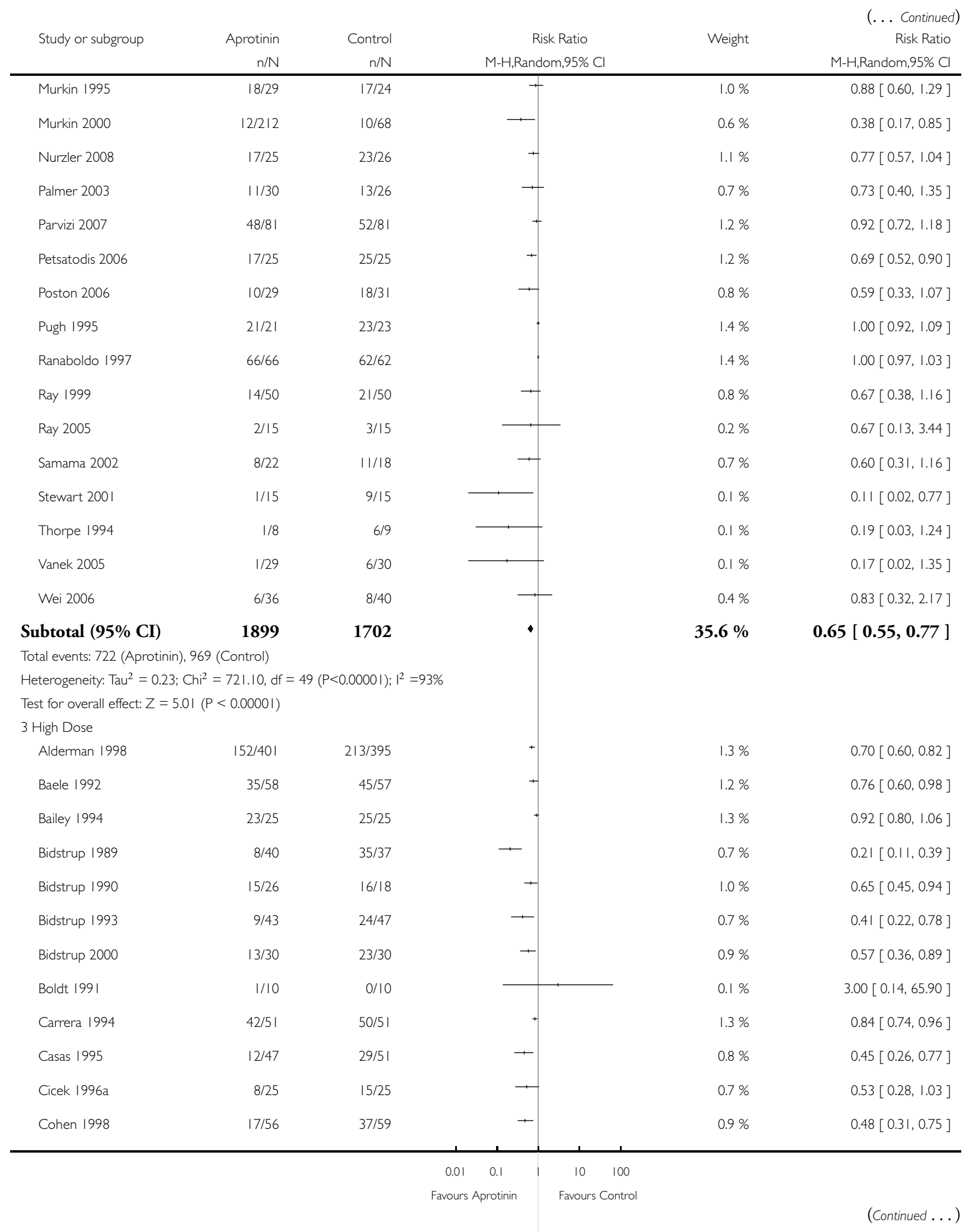

Anti-fibrinolytic use for minimising perioperative allogeneic blood transfusion (Review)

Copyright (C) 201 I The Cochrane Collaboration. Published by John Wiley \& Sons, Ltd. 


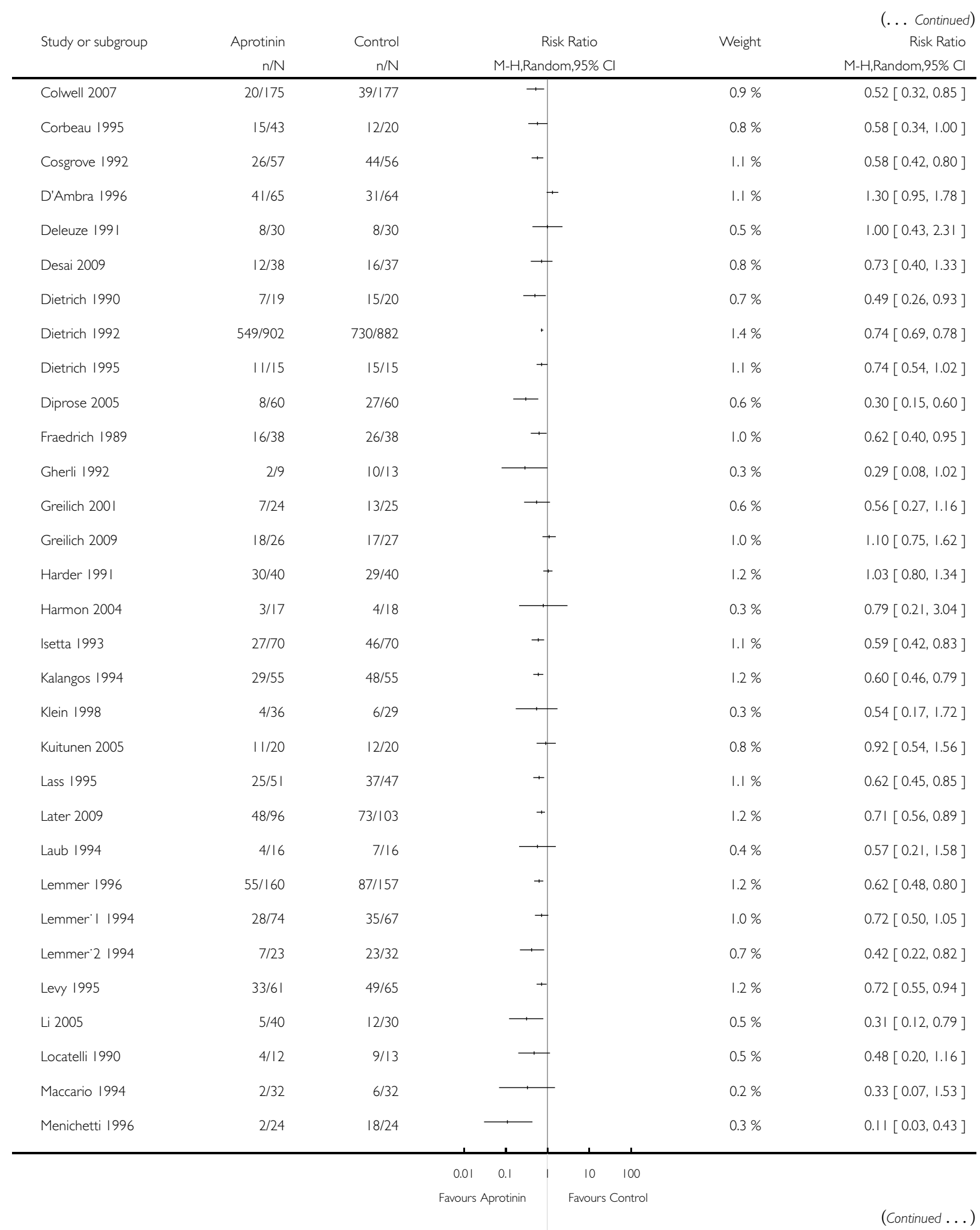

Anti-fibrinolytic use for minimising perioperative allogeneic blood transfusion (Review) 


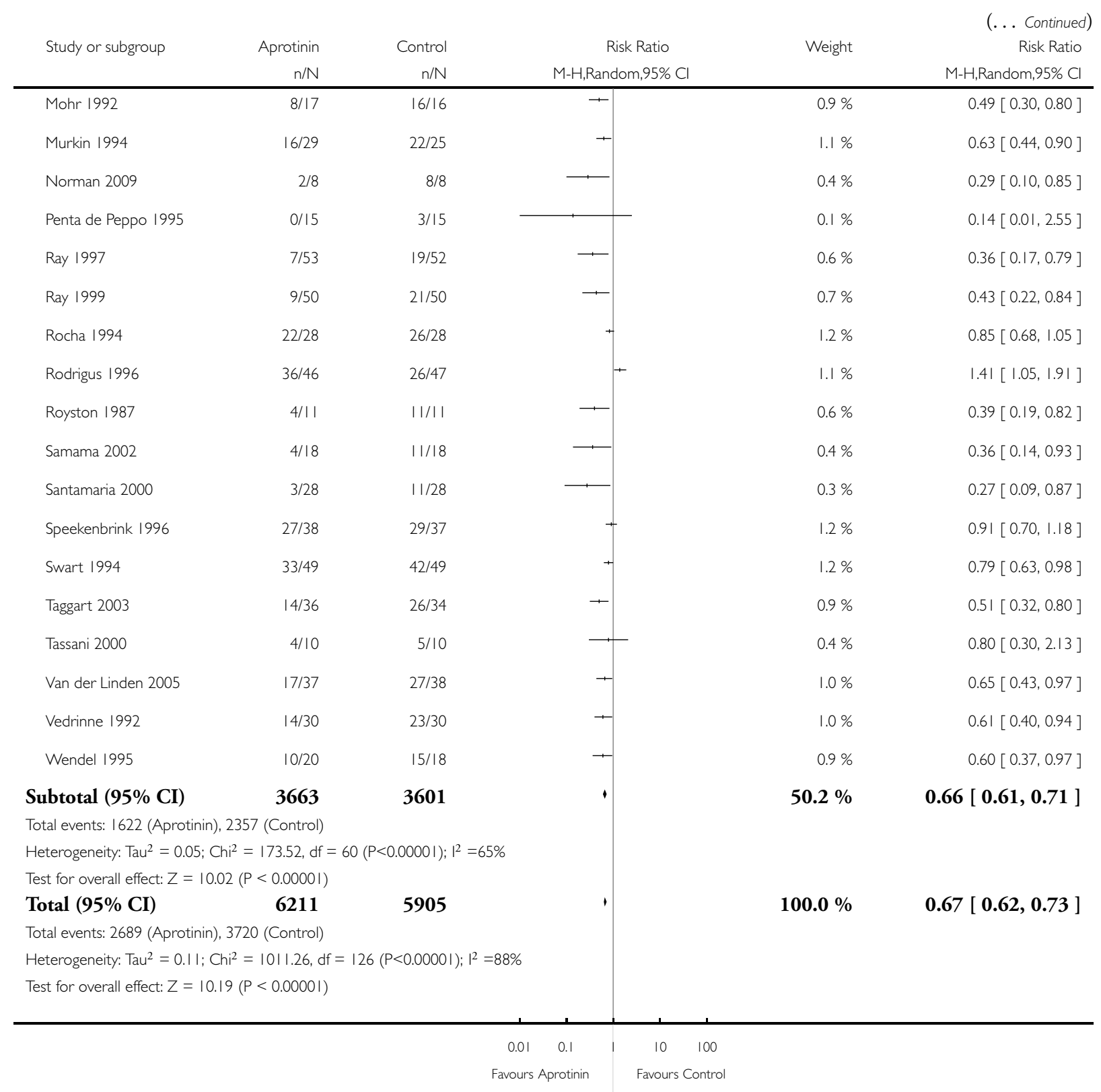


Analysis I.5. Comparison I Aprotinin versus Control (Blood Transfusion \& Blood Loss), Outcome 5 No. Exposed to Allogeneic Blood - Dose (Cardiac Surgery).

Review: Anti-fibrinolytic use for minimising perioperative allogeneic blood transfusion

Comparison: I Aprotinin versus Control (Blood Transfusion \% Blood Loss)

Outcome: 5 No. Exposed to Allogeneic Blood - Dose (Cardiac Surgery)

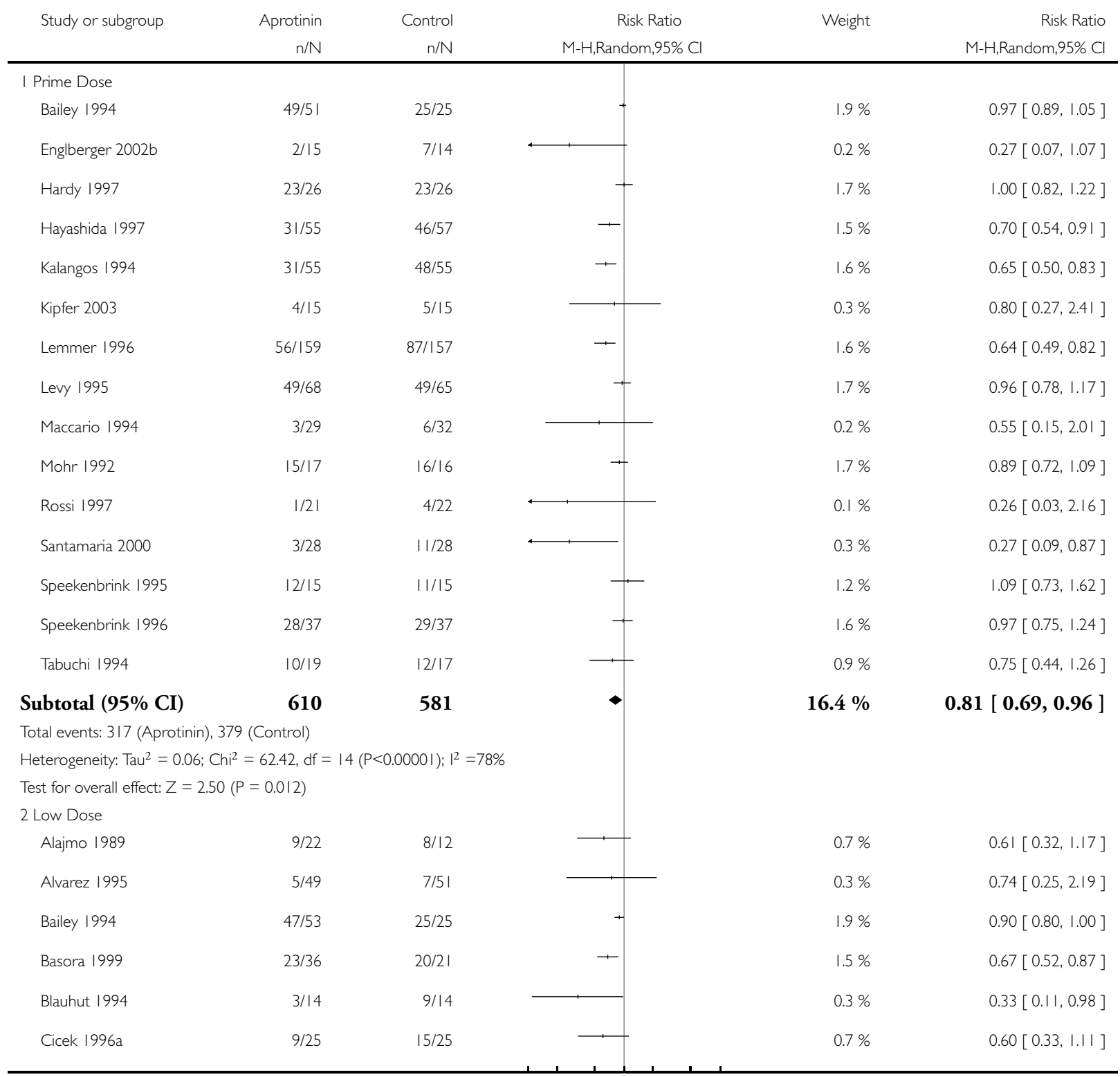

$\begin{array}{lllllll}0.1 & 0.2 & 0.5 & 1 & 2 & 5 & 10\end{array}$

(Continued ....) 


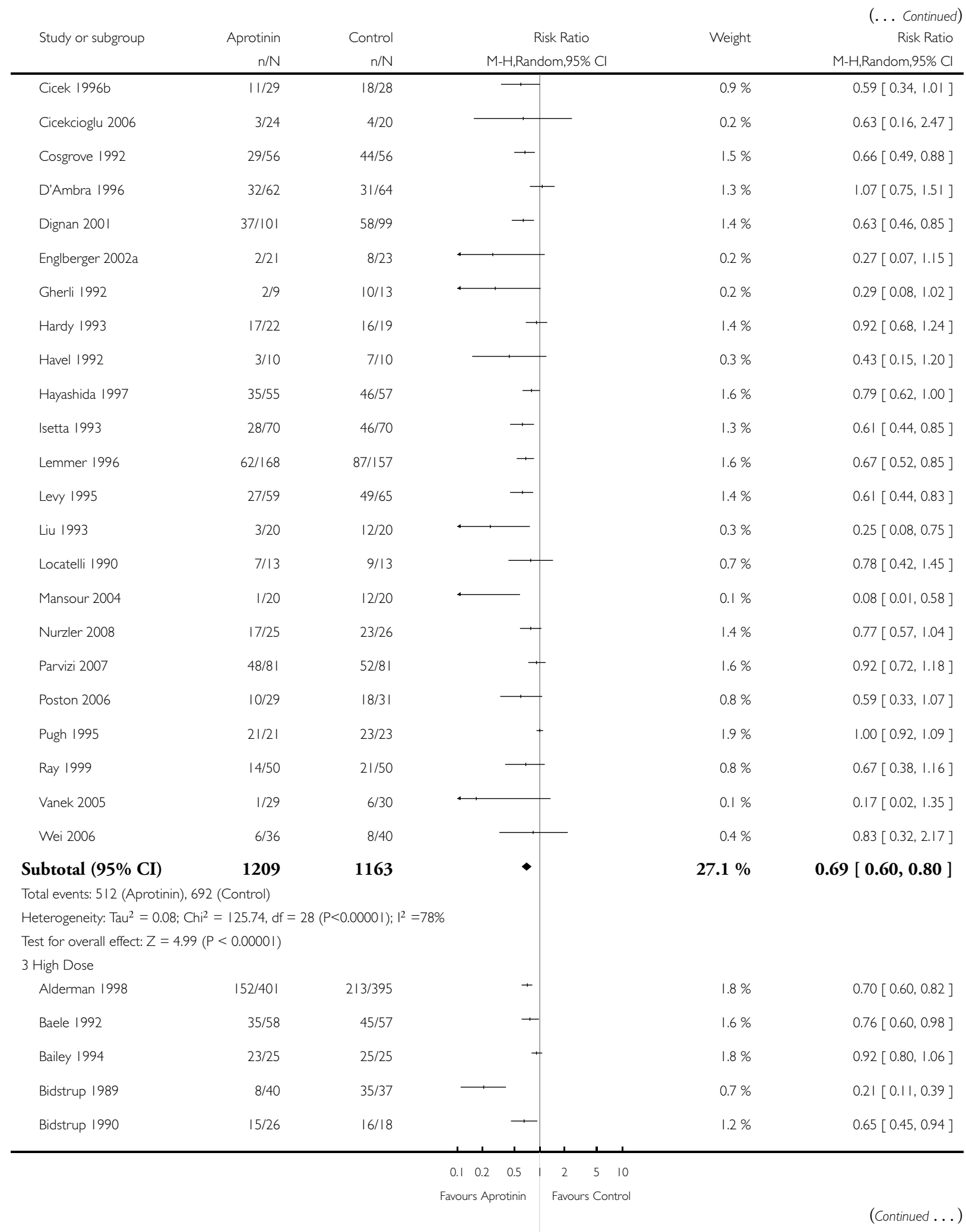

Anti-fibrinolytic use for minimising perioperative allogeneic blood transfusion (Review) 


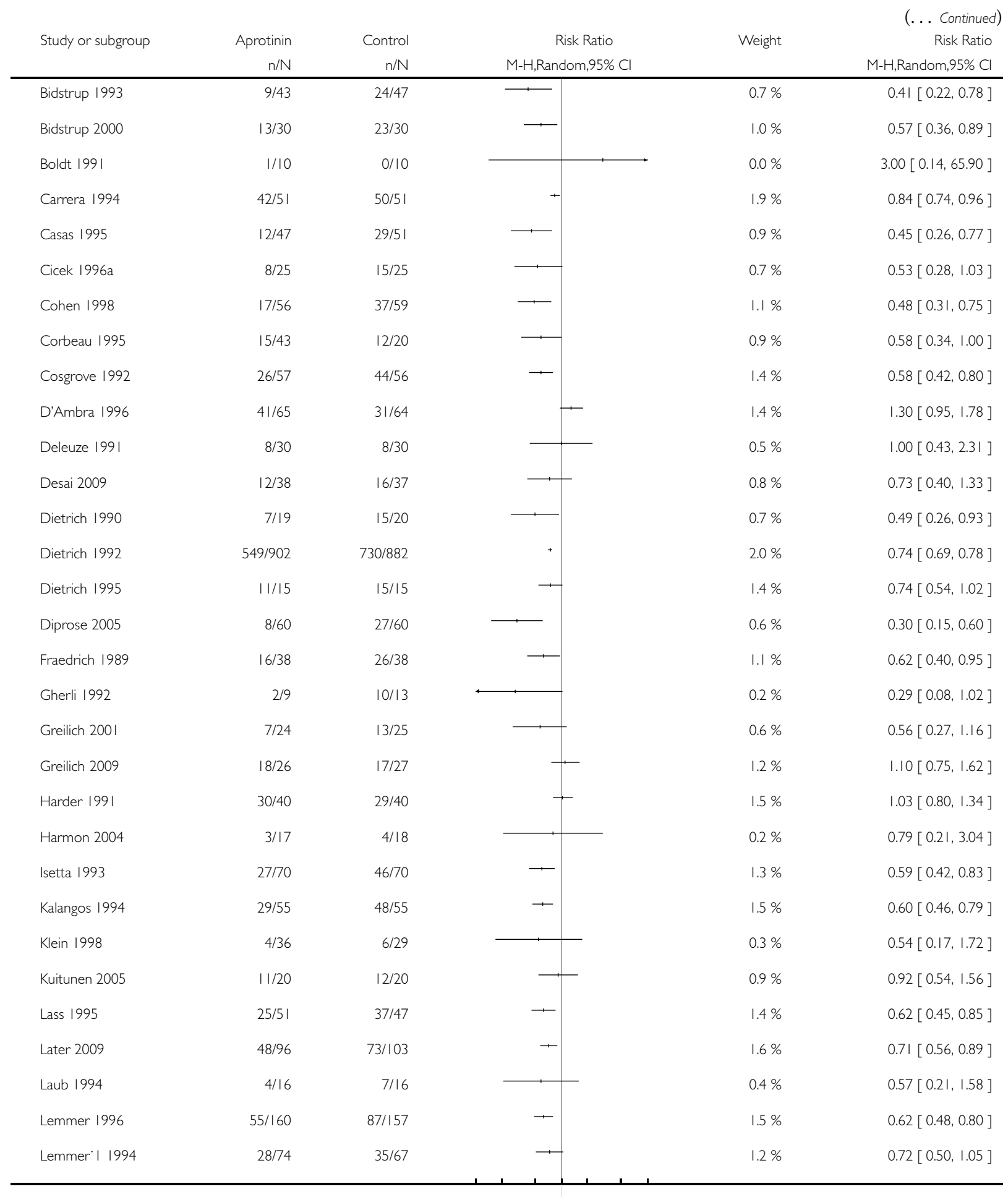

$\begin{array}{lllllll}0.1 & 0.2 & 0.5 & 1 & 2 & 5 & 10\end{array}$

Favours Aprotinin Favours Control

(Continued....)

Anti-fibrinolytic use for minimising perioperative allogeneic blood transfusion (Review)

Copyright (C) 201 I The Cochrane Collaboration. Published by John Wiley \& Sons, Ltd. 


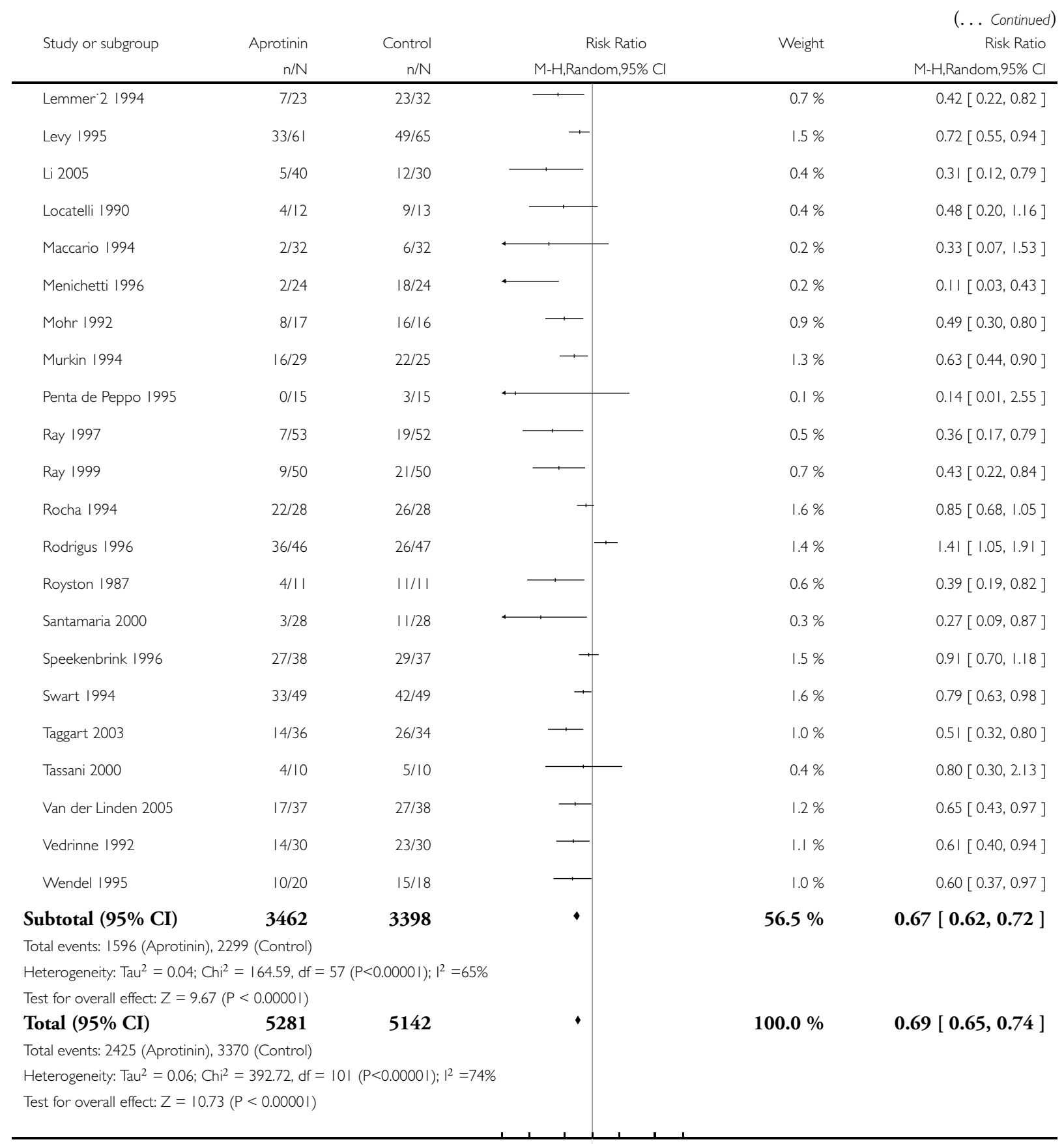

$\begin{array}{lllllll}0.1 & 0.2 & 0.5 & 1 & 2 & 5 & 10\end{array}$

Favours Aprotinin Favours Control 
Analysis I.6. Comparison I Aprotinin versus Control (Blood Transfusion \& Blood Loss), Outcome 6 Trial Methodological Quality - Allocation Concealment.

Review: Anti-fibrinolytic use for minimising perioperative allogeneic blood transfusion

Comparison: I Aprotinin versus Control (Blood Transfusion \% Blood Loss)

Outcome: 6 Trial Methodological Quality - Allocation Concealment

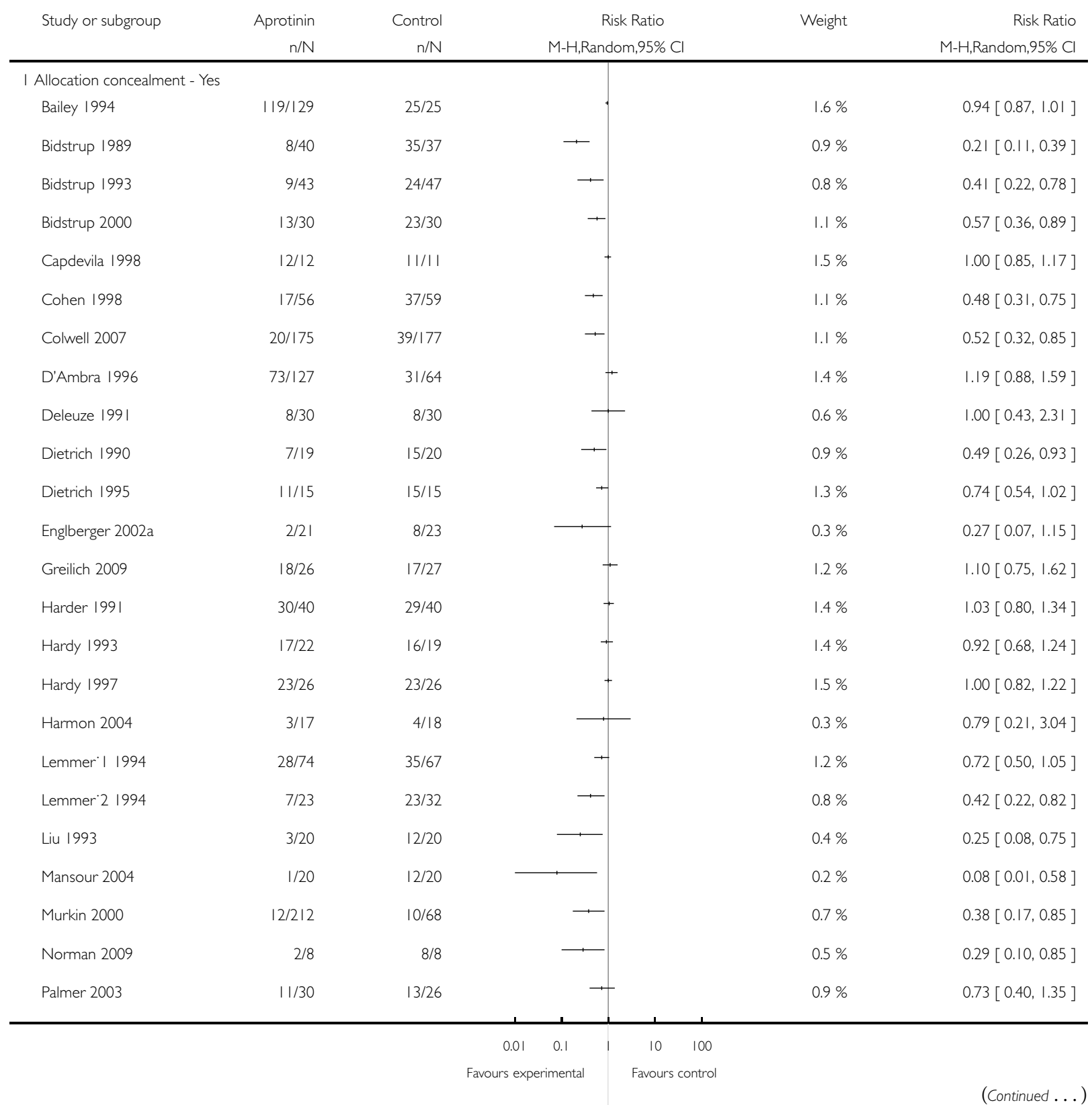




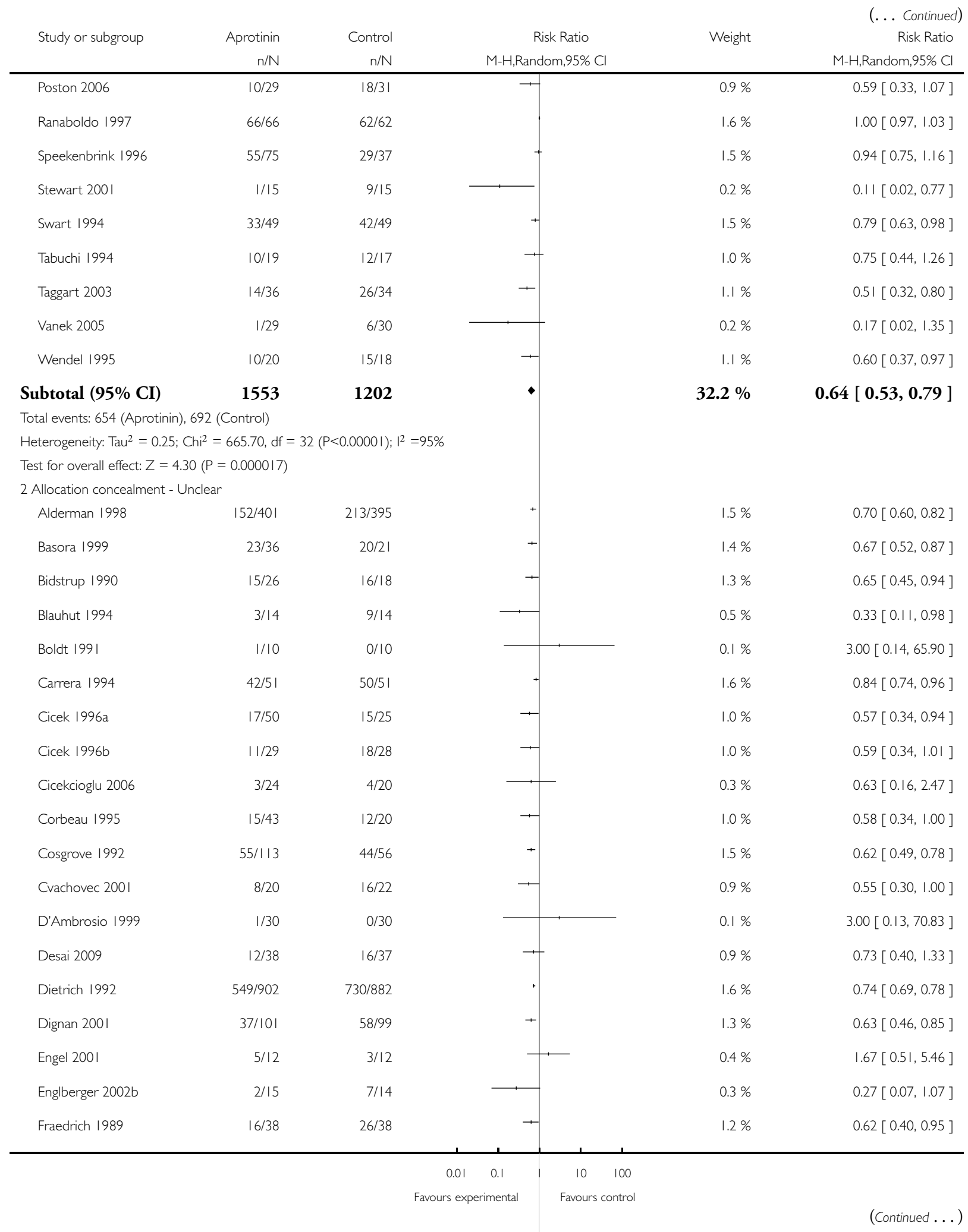

Anti-fibrinolytic use for minimising perioperative allogeneic blood transfusion (Review)

Copyright (C) 201 I The Cochrane Collaboration. Published by John Wiley \& Sons, Ltd. 


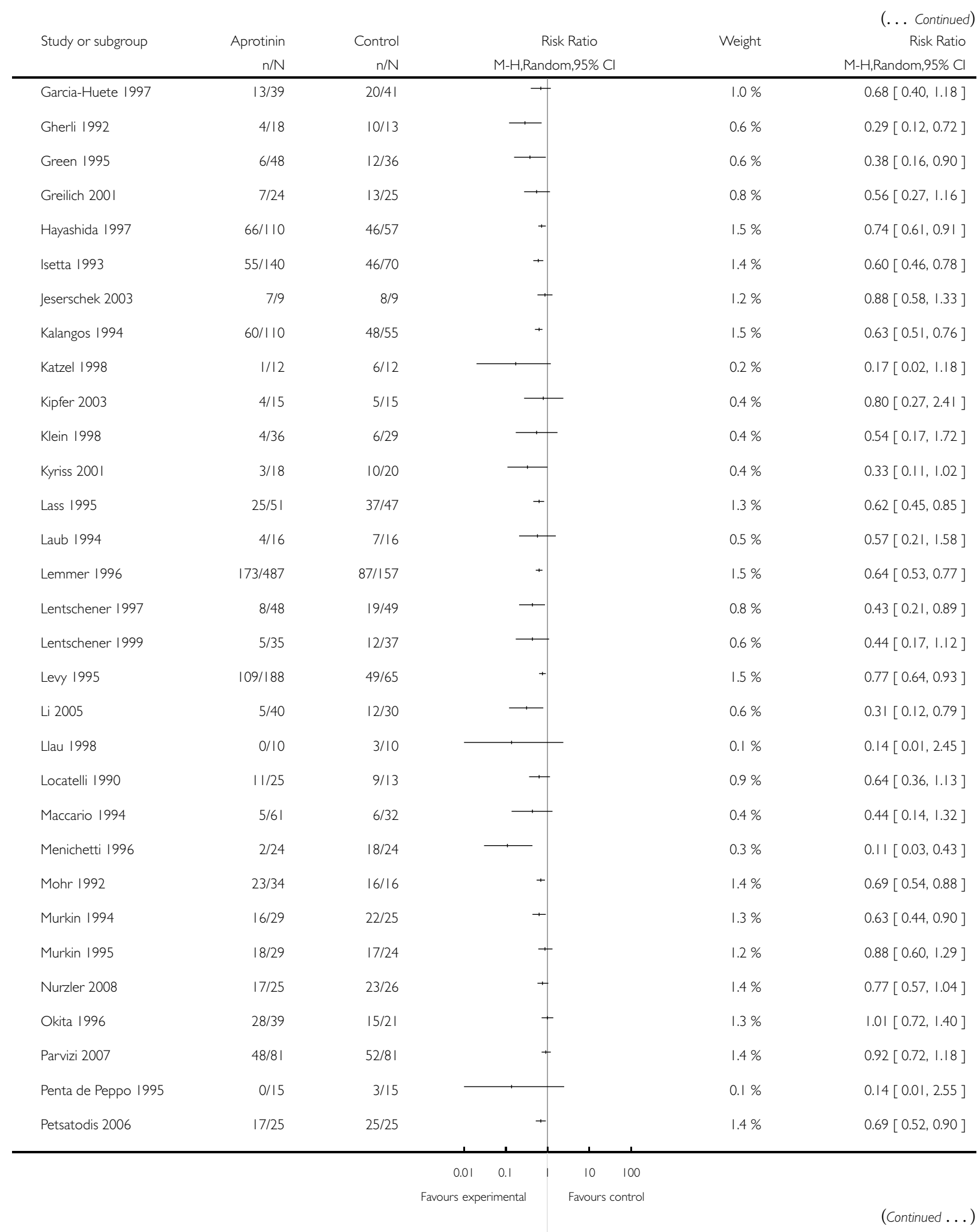

Anti-fibrinolytic use for minimising perioperative allogeneic blood transfusion (Review) 


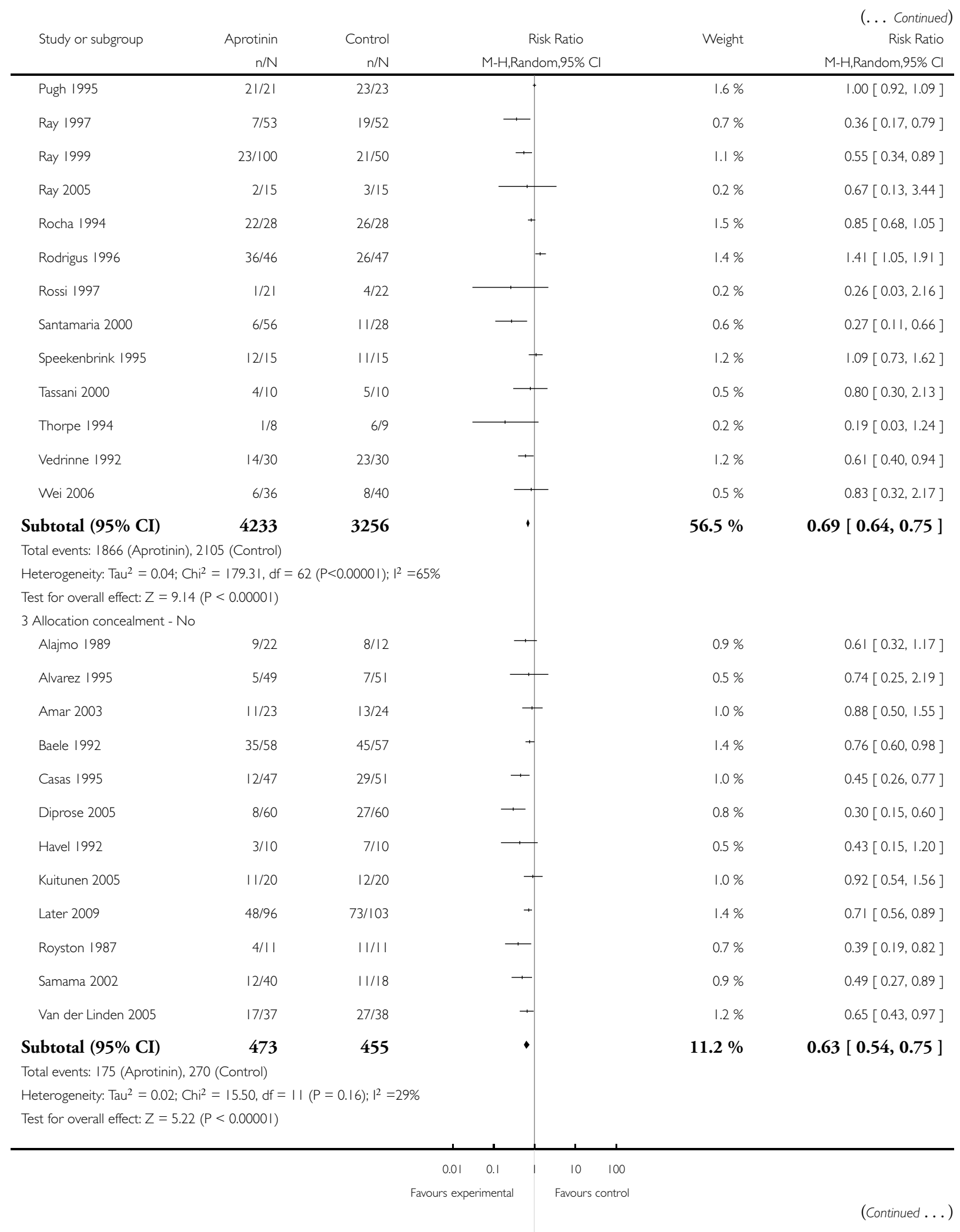

Anti-fibrinolytic use for minimising perioperative allogeneic blood transfusion (Review)

Copyright (C) 201 I The Cochrane Collaboration. Published by John Wiley \& Sons, Ltd. 


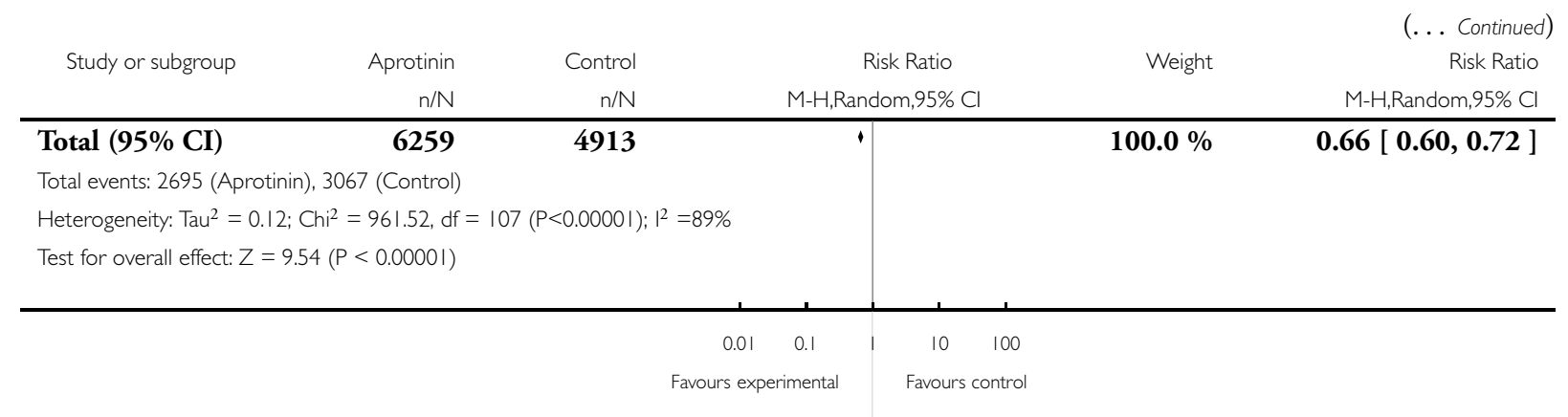

Analysis I.7. Comparison I Aprotinin versus Control (Blood Transfusion \& Blood Loss), Outcome 7 Units of Allogeneic Blood Transfused - Transfused Patients.

Review: Anti-fibrinolytic use for minimising perioperative allogeneic blood transfusion

Comparison: I Aprotinin versus Control (Blood Transfusion \% Blood Loss)

Outcome: 7 Units of Allogeneic Blood Transfused - Transfused Patients

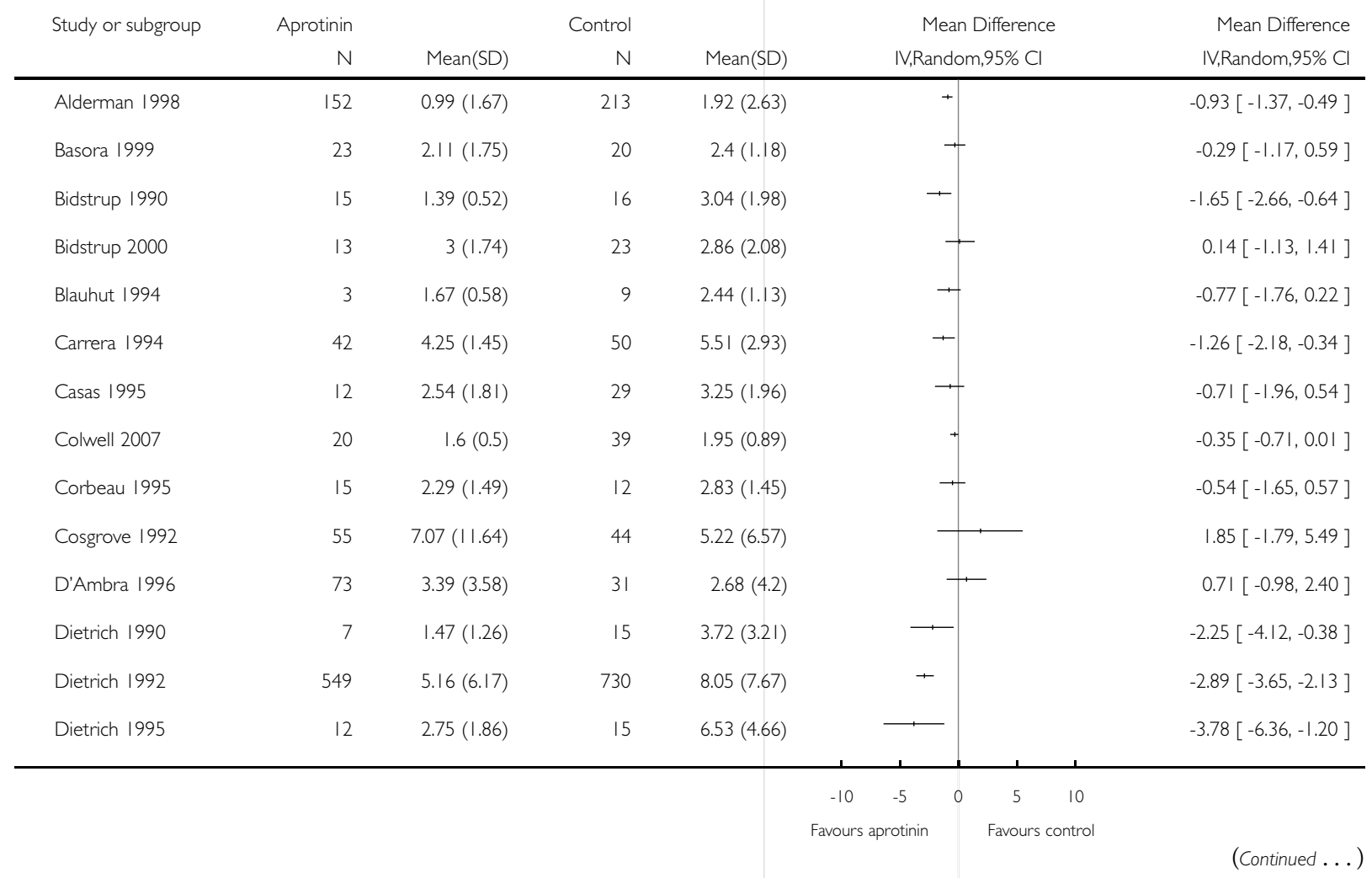




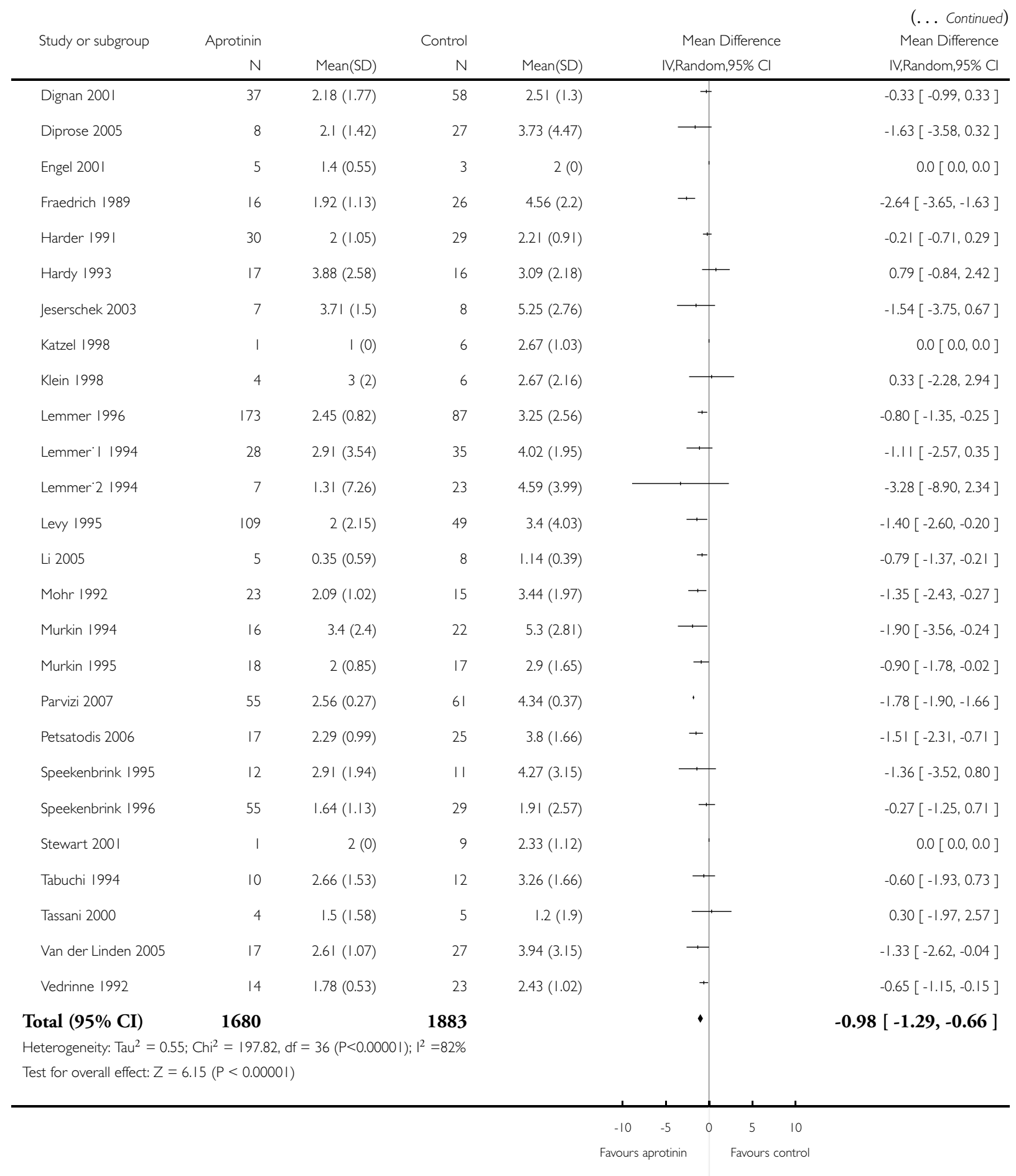

Anti-fibrinolytic use for minimising perioperative allogeneic blood transfusion (Review) 
Analysis I.8. Comparison I Aprotinin versus Control (Blood Transfusion \& Blood Loss), Outcome 8 Units of Allogeneic Blood Transfused - All Patients.

Review: Anti-fibrinolytic use for minimising perioperative allogeneic blood transfusion

Comparison: I Aprotinin versus Control (Blood Transfusion \% Blood Loss)

Outcome: 8 Units of Allogeneic Blood Transfused - All Patients

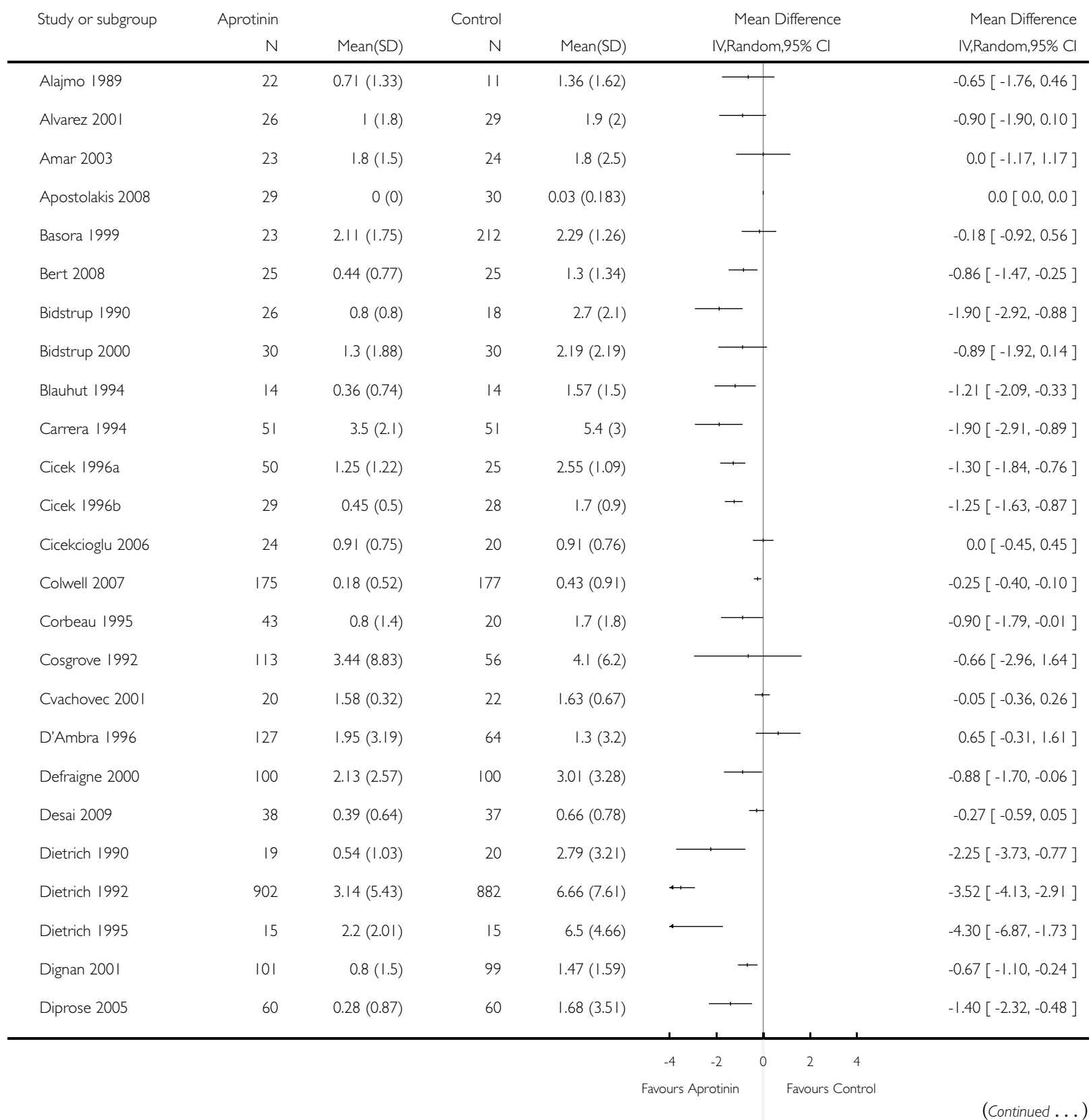




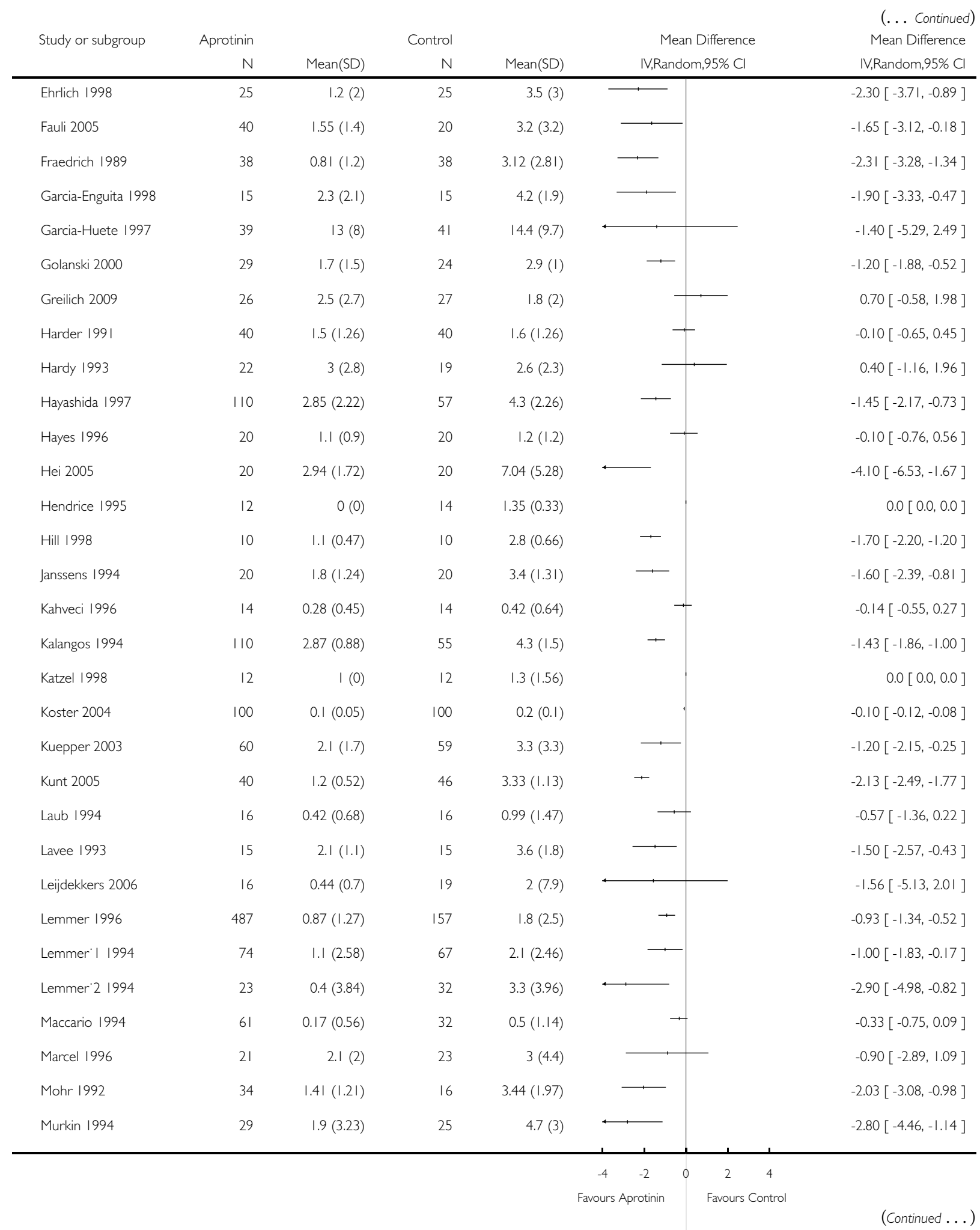

Anti-fibrinolytic use for minimising perioperative allogeneic blood transfusion (Review) 


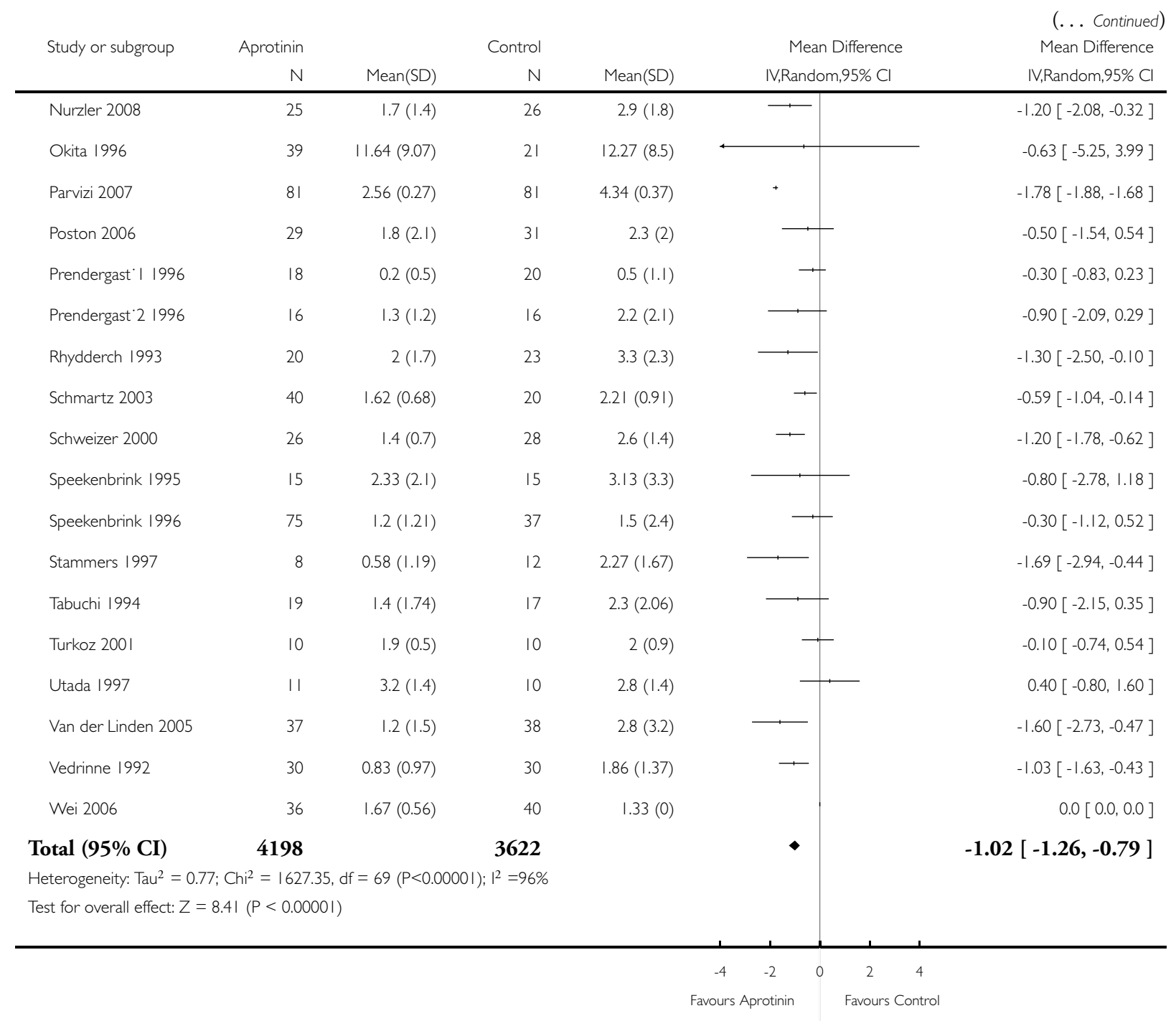


Analysis I.9. Comparison I Aprotinin versus Control (Blood Transfusion \& Blood Loss), Outcome 9 Blood loss - Intra-operative.

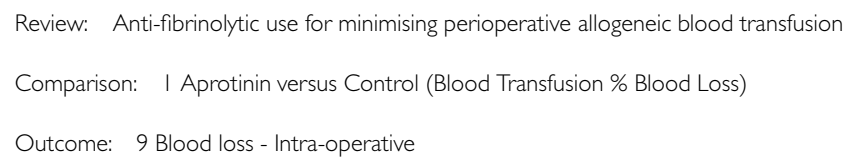

\begin{tabular}{|c|c|c|c|c|c|c|c|c|}
\hline \multicolumn{9}{|l|}{ I Cardiac surgery } \\
\hline Desai 2009 & 38 & $794(465)$ & 37 & 1034 (659) & $\longrightarrow$ & & $6.7 \%$ & $-240.00[-498.74,18.74]$ \\
\hline Dietrich 1990 & 19 & $363(159)$ & 20 & $636(322)$ & $\rightarrow$ & & $10.4 \%$ & $-273.00[-431.20,-114.80]$ \\
\hline Fauli 2005 & 40 & $958.5(385.12)$ & 20 & $829(421)$ & & $\mp$ & $8.0 \%$ & $129.50[-90.24,349.24]$ \\
\hline Harmon 2004 & 17 & $568(355)$ & 18 & $840(296)$ & $\longrightarrow$ & & $8.1 \%$ & $-272.00[-489.20,-54.80]$ \\
\hline Hendrice 1995 & 12 & $225(121)$ & 14 & $401(207)$ & $\rightarrow$ & & $11.8 \%$ & $-176.00[-304.24,-47.76]$ \\
\hline Koster 2004 & 100 & $261(107)$ & 100 & $355(127)$ & $=$ & + & $15.3 \%$ & $-94.00[-126.55,-61.45]$ \\
\hline Leijdekkers 2006 & 16 & $2362(1340)$ & 19 & $2466(1370)$ & & & $0.9 \%$ & $-104.00[-1004.32,796.32]$ \\
\hline Subtotal (95\% CI) & 242 & & 228 & & 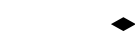 & & $61.1 \%$ & $-148.18[-240.21,-56.14]$ \\
\hline \multicolumn{9}{|c|}{ Heterogeneity: $\mathrm{Tau}^{2}=6875.87 ; \mathrm{Chi}^{2}=13.63, \mathrm{df}=6(\mathrm{P}=0.03) ; \mathrm{I}^{2}=56 \%$} \\
\hline \multicolumn{9}{|c|}{ Test for overall effect: $Z=3.16(P=0.0016)$} \\
\hline \multicolumn{9}{|l|}{2 Orthopaedic surgery } \\
\hline Amar 2003 & 23 & $1200(1000)$ & 24 & $1000(900)$ & & & $2.3 \%$ & $200.00[-344.67,744.67]$ \\
\hline Hayes 1996 & 20 & $725(150)$ & 20 & $768(235)$ & & - & $12.0 \%$ & $-43.00[-165.18,79.18]$ \\
\hline Janssens 1994 & 20 & $793(332)$ & 20 & $1113(494)$ & 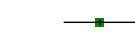 & & $6.6 \%$ & $-320.00[-580.85,-59.15]$ \\
\hline Murkin 1995 & 29 & $996(436.2)$ & 24 & $1318(710.35)$ & & & $5.0 \%$ & $-322.00[-647.53,3.53]$ \\
\hline Utada 1997 & 11 & $1573(462)$ & 10 & $1760(623)$ & & & $2.9 \%$ & $-187.00[-659.90,285.90]$ \\
\hline Subtotal (95\% CI) & 103 & & 98 & & & & $28.8 \%$ & $-151.05[-317.63,15.52]$ \\
\hline \multicolumn{9}{|c|}{ Heterogeneity: $\mathrm{Tau}^{2}=13580.14 ; \mathrm{Chi}^{2}=6.62, \mathrm{df}=4(P=0.16) ;\left.\right|^{2}=40 \%$} \\
\hline \multicolumn{9}{|c|}{ Test for overall effect: $Z=1.78(P=0.076)$} \\
\hline \multicolumn{9}{|l|}{3 Thoracic surgery } \\
\hline Katzel 1998 & 12 & $324(159)$ & 12 & $856(563)$ & & & $4.9 \%$ & $-532.00[-863.00,-201.00]$ \\
\hline Norman 2009 & 8 & $769(630.4)$ & & | $832(1436.39)$ & & & $0.6 \%$ & $-1063.00[-2149.99,23.99]$ \\
\hline Subtotal (95\% CI) & 20 & & 20 & & & & $5.5 \%$ & $-577.06[-893.71,-260.41]$ \\
\hline \multicolumn{9}{|c|}{ Heterogeneity: $\operatorname{Tau}^{2}=0.0 ; \mathrm{Chi}^{2}=0.84, \mathrm{df}=\mathrm{I}(\mathrm{P}=0.36) ; \mathrm{I}^{2}=0.0 \%$} \\
\hline \multicolumn{9}{|c|}{ Test for overall effect: $Z=3.57(P=0.00035)$} \\
\hline \multicolumn{9}{|l|}{4 Liver surgery } \\
\hline Hei 2005 & 20 & $3107(|28|)$ & 20 & $5342(3013)$ & $\longleftarrow$ & & $0.4 \%$ & $-2235.00[-3669.87,-800.13]$ \\
\hline \multirow[t]{3}{*}{ Lentschener 1997} & 48 & $1217(966)$ & 49 & $1653(1221)$ & & & $3.2 \%$ & $-436.00[-873.67,1.67]$ \\
\hline & & & & & $000 \quad-500$ & 500 & 1000 & \\
\hline & & & & Favou & urs Aprotinin & Favours & Control & \\
\hline
\end{tabular}




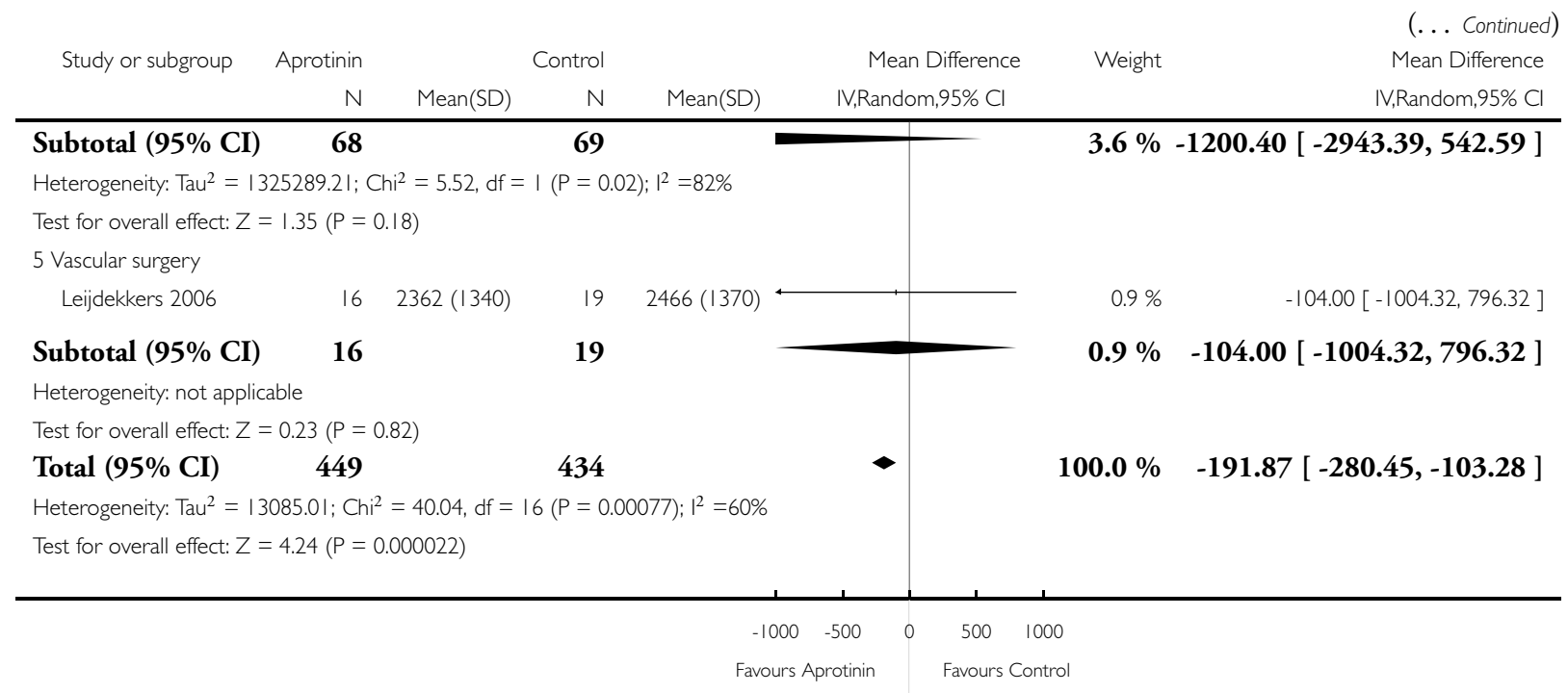

\section{Analysis I.10. Comparison I Aprotinin versus Control (Blood Transfusion \& Blood Loss), Outcome 10} Blood loss - Post-operative.

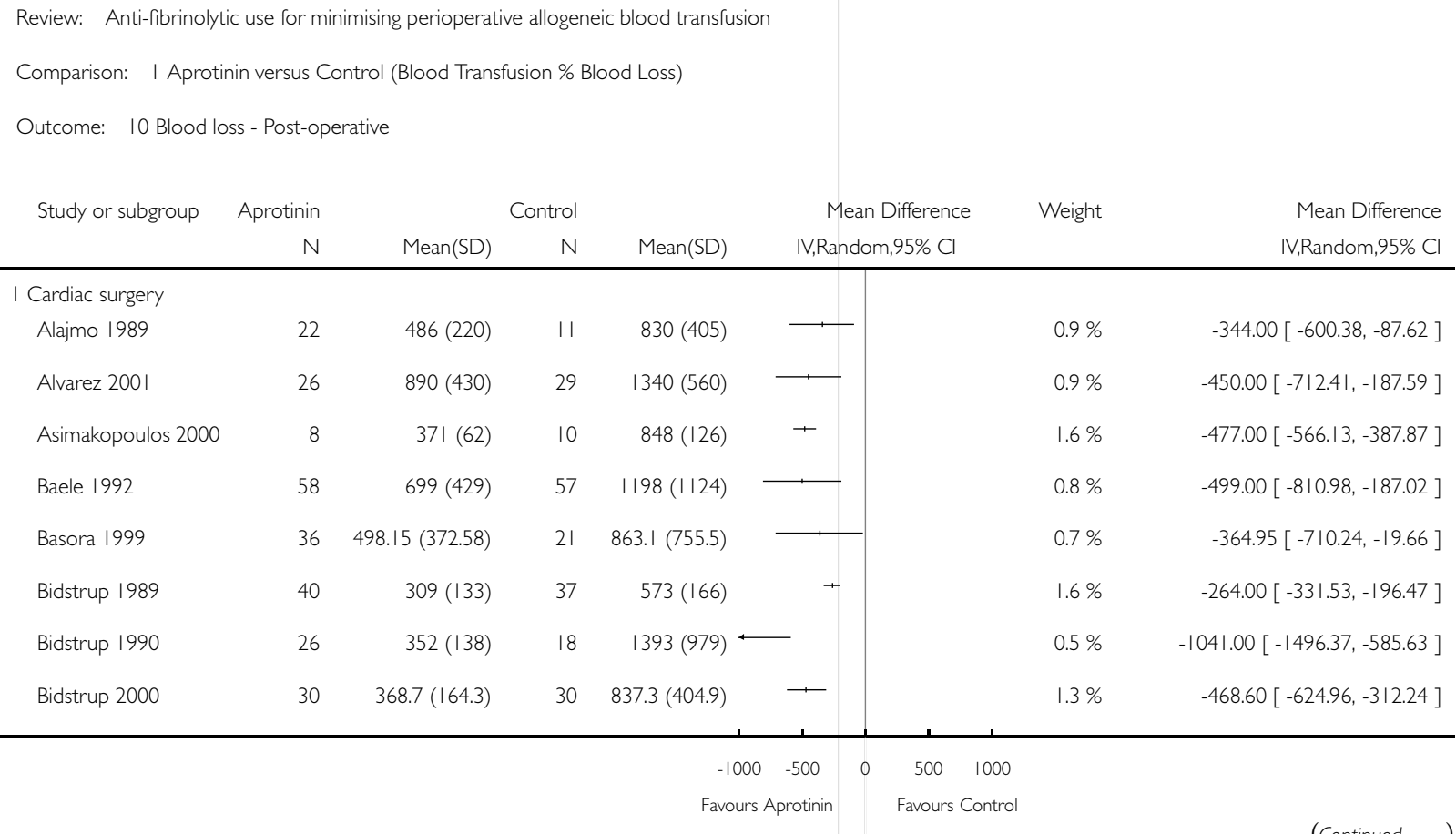




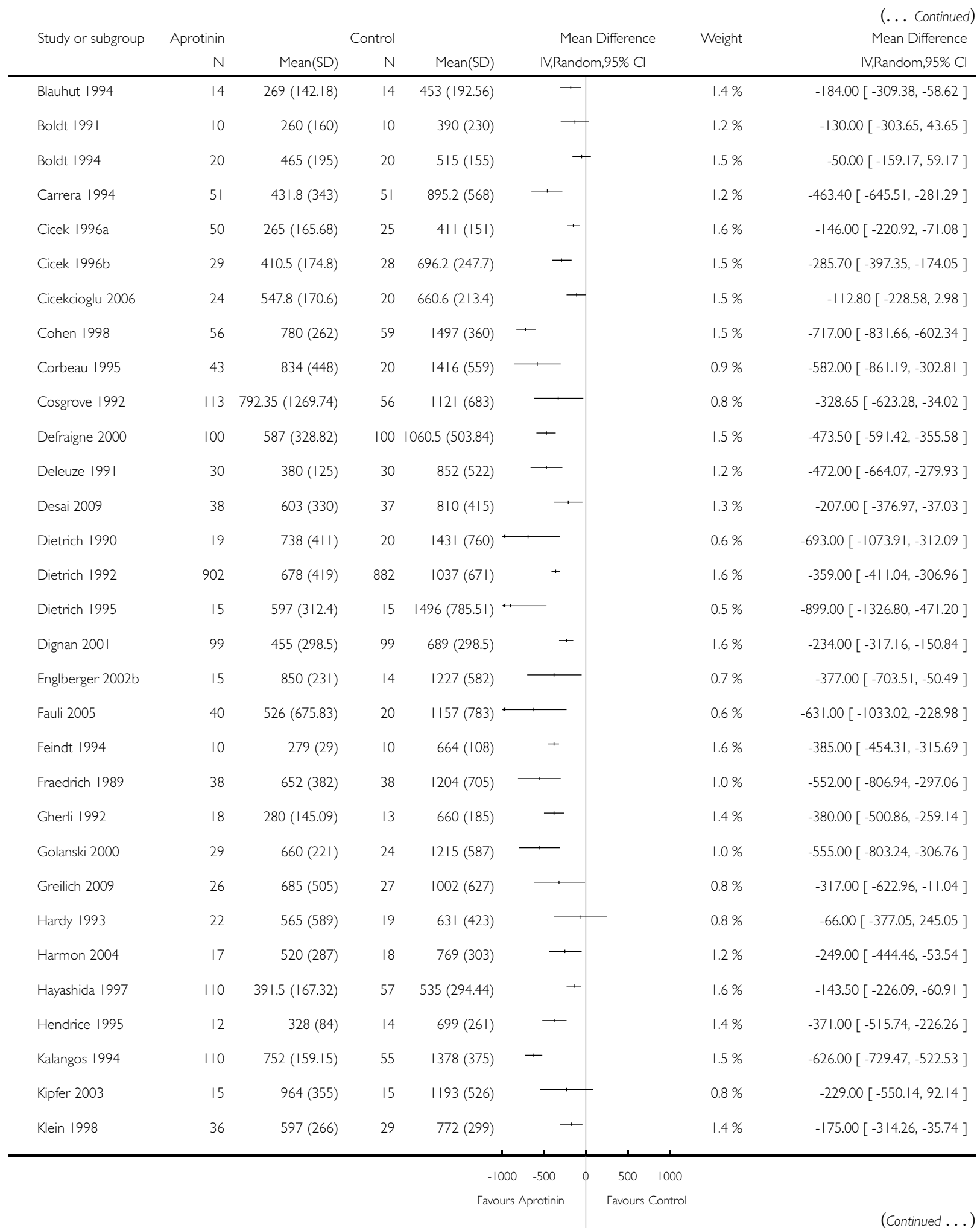

Anti-fibrinolytic use for minimising perioperative allogeneic blood transfusion (Review) 


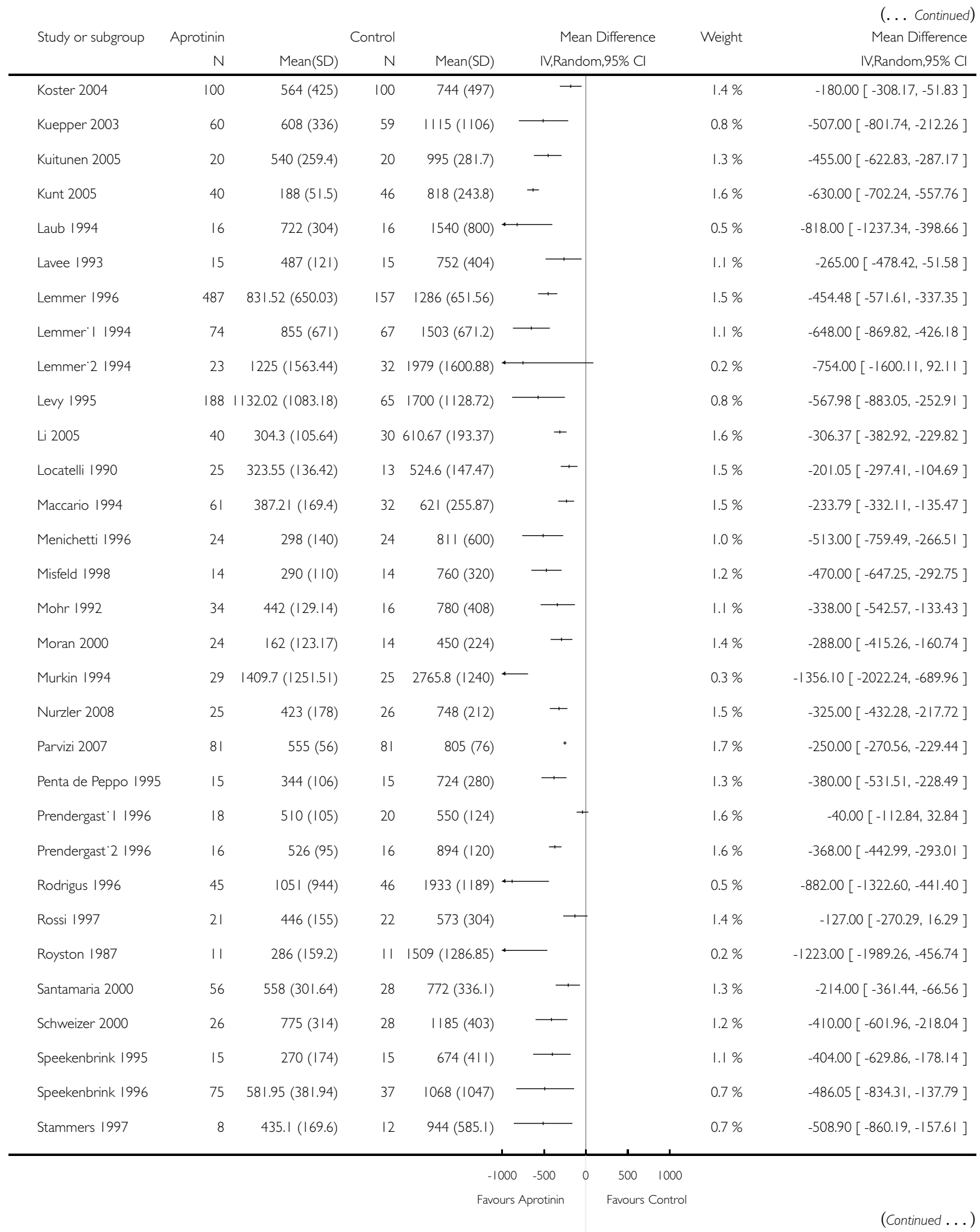

Anti-fibrinolytic use for minimising perioperative allogeneic blood transfusion (Review) 


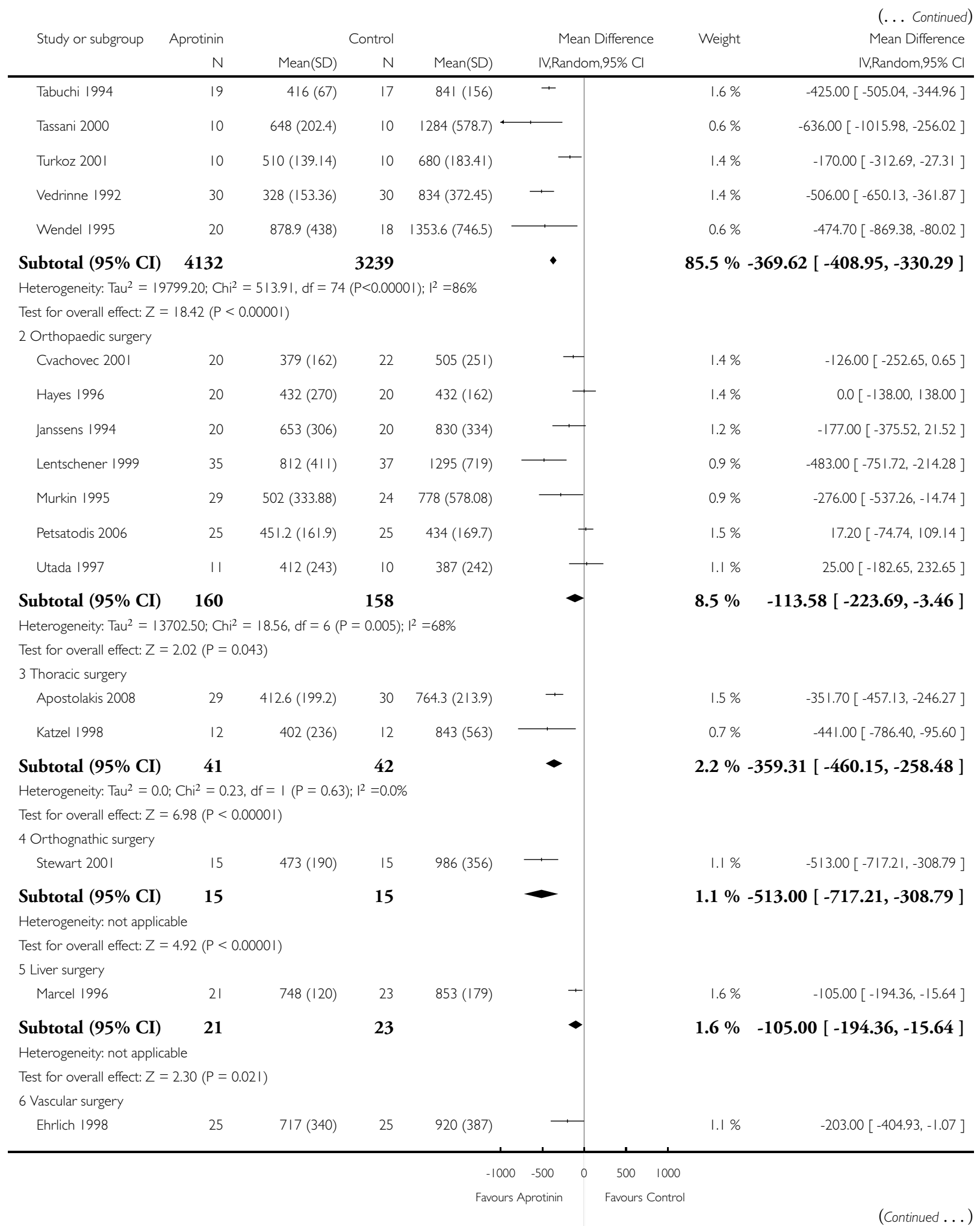

Anti-fibrinolytic use for minimising perioperative allogeneic blood transfusion (Review)

Copyright (C) 201 I The Cochrane Collaboration. Published by John Wiley \& Sons, Ltd. 


\begin{tabular}{|c|c|c|c|c|c|c|c|}
\hline \multirow[t]{2}{*}{ Study or subgroup } & \multirow{2}{*}{$\begin{array}{r}\text { Aprotinin } \\
\mathrm{N}\end{array}$} & \multicolumn{2}{|r|}{ Control } & & \multirow{2}{*}{$\begin{array}{l}\text { Mean Difference } \\
\text { IV,Random,95\% Cl }\end{array}$} & \multirow[t]{2}{*}{ Weight } & \multirow{2}{*}{$\begin{array}{c}\text { (... . Continued }) \\
\text { Mean Difference } \\
\text { IV,Random, } 95 \% \mathrm{Cl}\end{array}$} \\
\hline & & Mean(SD) & $\mathrm{N}$ & Mean(SD) & & & \\
\hline Subtotal (95\% CI) & 25 & & 25 & & $\longrightarrow$ & $1.1 \%$ & $-203.00[-404.93,-1.07]$ \\
\hline \multicolumn{8}{|c|}{ Heterogeneity: not applicable } \\
\hline \multicolumn{8}{|c|}{ Test for overall effect: $Z=1.97(P=0.049)$} \\
\hline Total (95\% CI) & 4394 & & 3502 & & - & \multicolumn{2}{|c|}{$100.0 \%-345.88[-383.47,-308.29]$} \\
\hline \multicolumn{8}{|c|}{ Heterogeneity: $\operatorname{Tau}^{2}=21627.05 ; \mathrm{Chi}^{2}=620.49, \mathrm{df}=86(\mathrm{P}<0.0000 \mathrm{I}) ; \mathrm{I}^{2}=86 \%$} \\
\hline \multicolumn{8}{|c|}{ Test for overall effect: $Z=\mid 8.03(P<0.0000 \mid)$} \\
\hline & & & & -1000 & -500 & 1000 & \\
\hline \multicolumn{8}{|c|}{ Favours Aprotinin $\quad$ Favours Control } \\
\hline
\end{tabular}

\section{Analysis I.I I. Comparison I Aprotinin versus Control (Blood Transfusion \& Blood Loss), Outcome II Blood loss - Post-operative - Dose (Cardiac Surgery).}

Review: Anti-fibrinolytic use for minimising perioperative allogeneic blood transfusion

Comparison: I Aprotinin versus Control (Blood Transfusion \% Blood Loss)

Outcome: I I Blood loss - Post-operative - Dose (Cardiac Surgery)

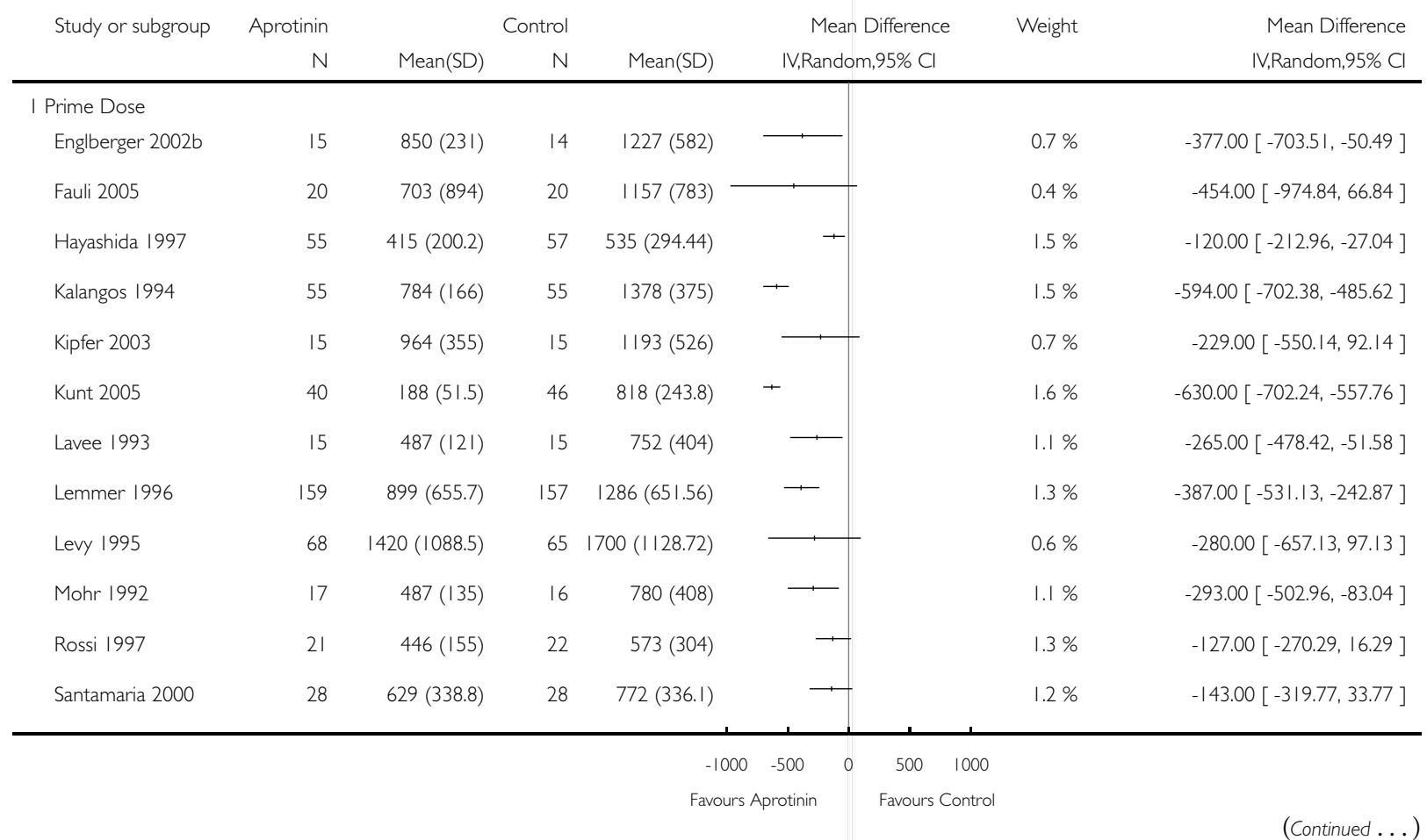




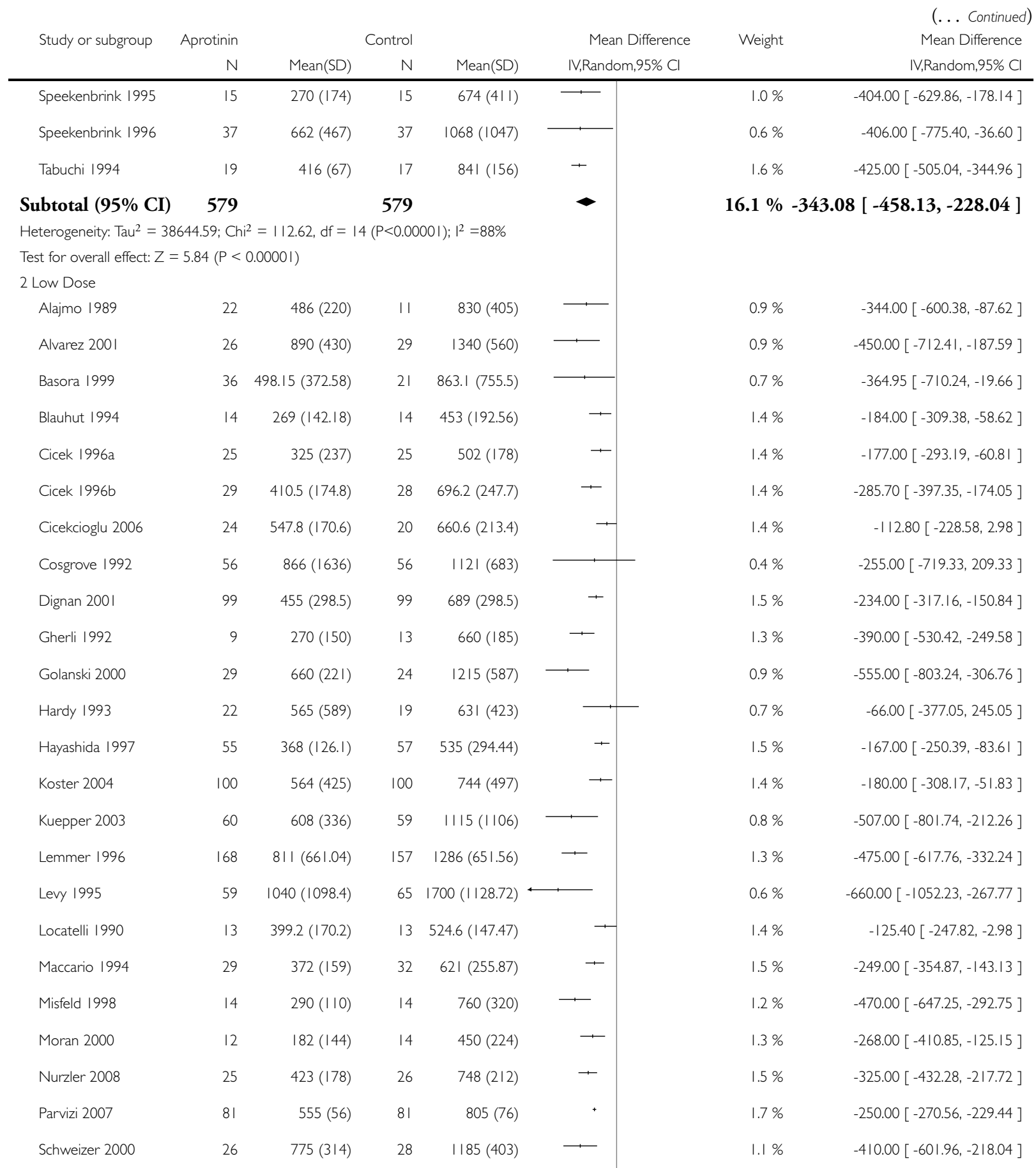




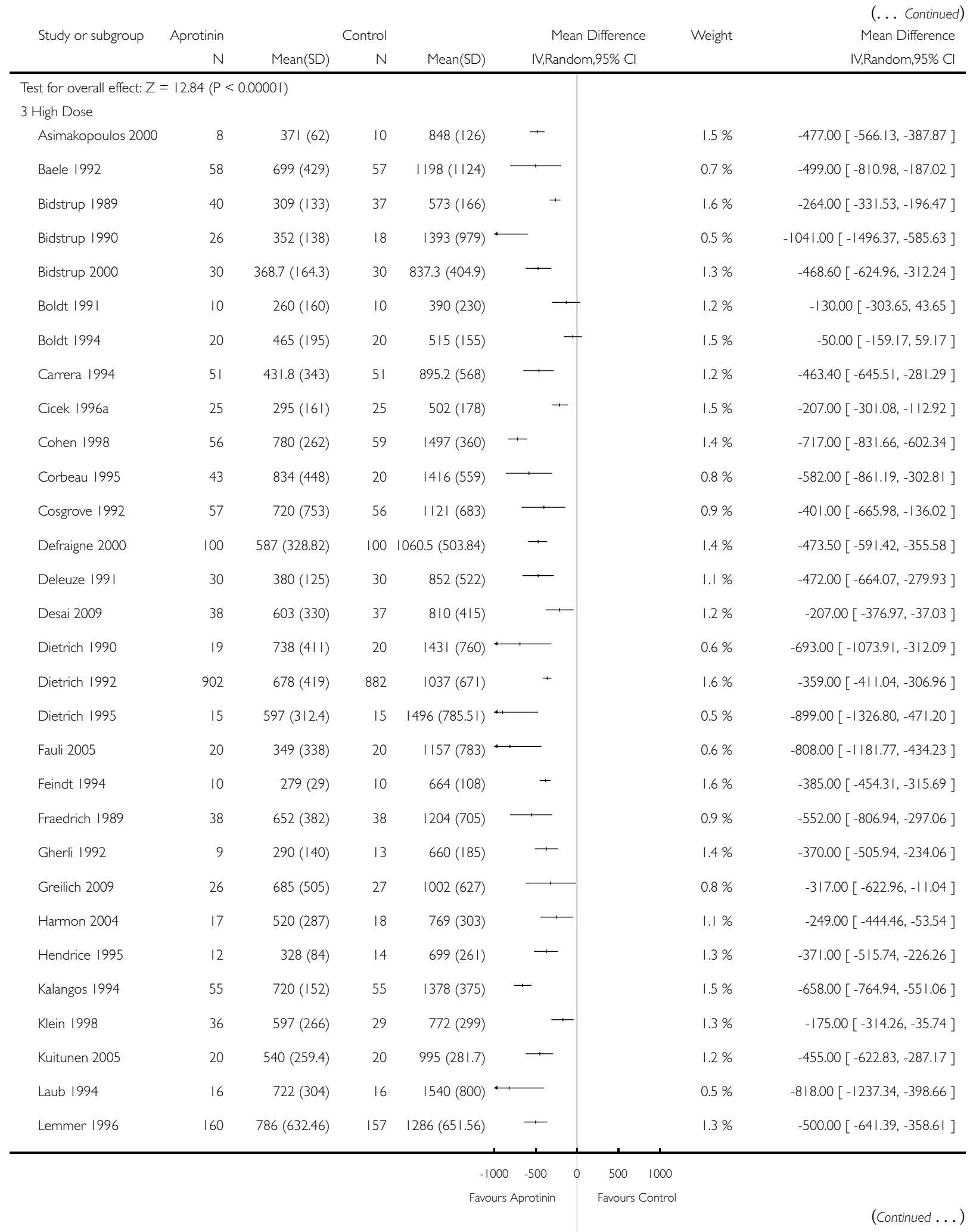

Anti-fibrinolytic use for minimising perioperative allogeneic blood transfusion (Review) 


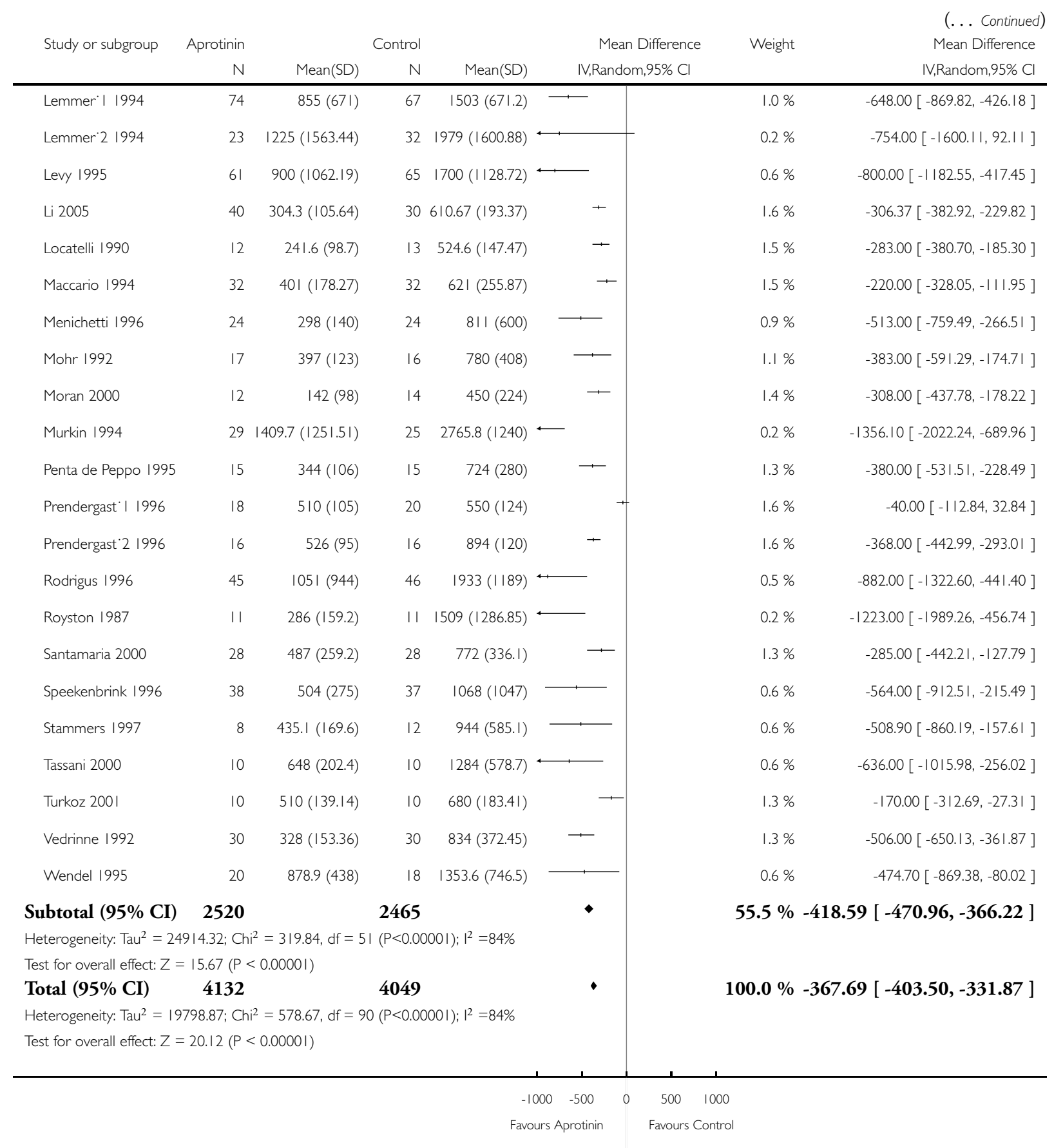

Anti-fibrinolytic use for minimising perioperative allogeneic blood transfusion (Review) 
Analysis I.12. Comparison I Aprotinin versus Control (Blood Transfusion \& Blood Loss), Outcome 12 Blood loss - Total.

Review: Anti-fibrinolytic use for minimising perioperative allogeneic blood transfusion

Comparison: I Aprotinin versus Control (Blood Transfusion \% Blood Loss)

Outcome: 12 Blood loss - Total

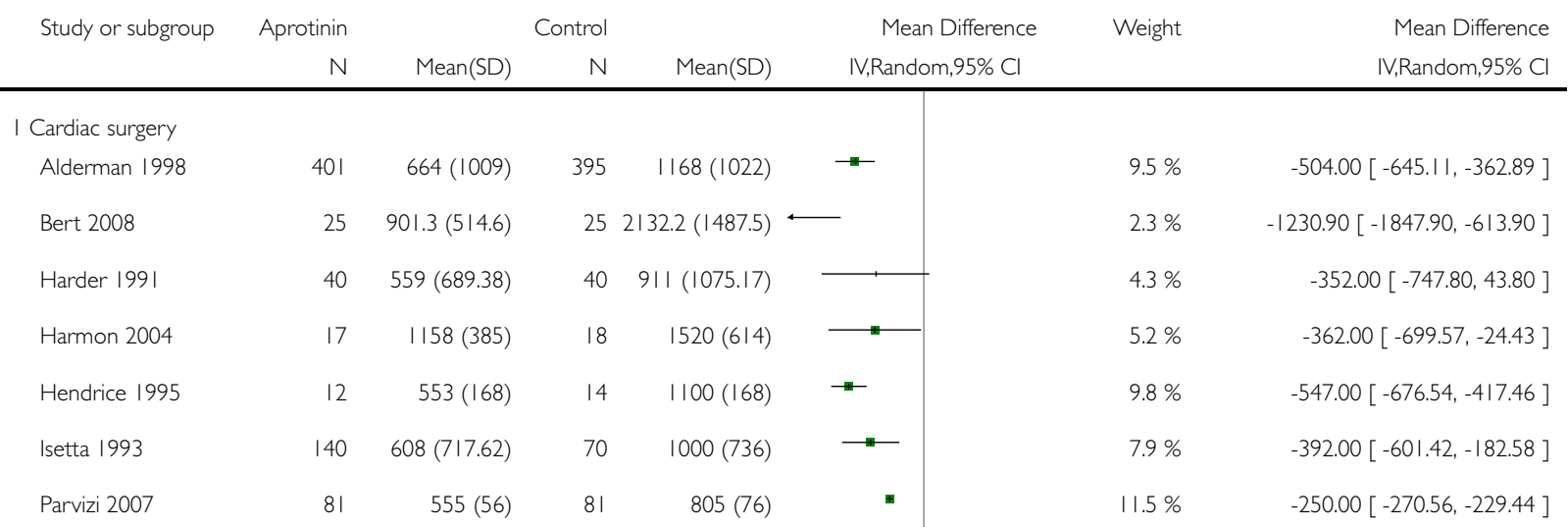

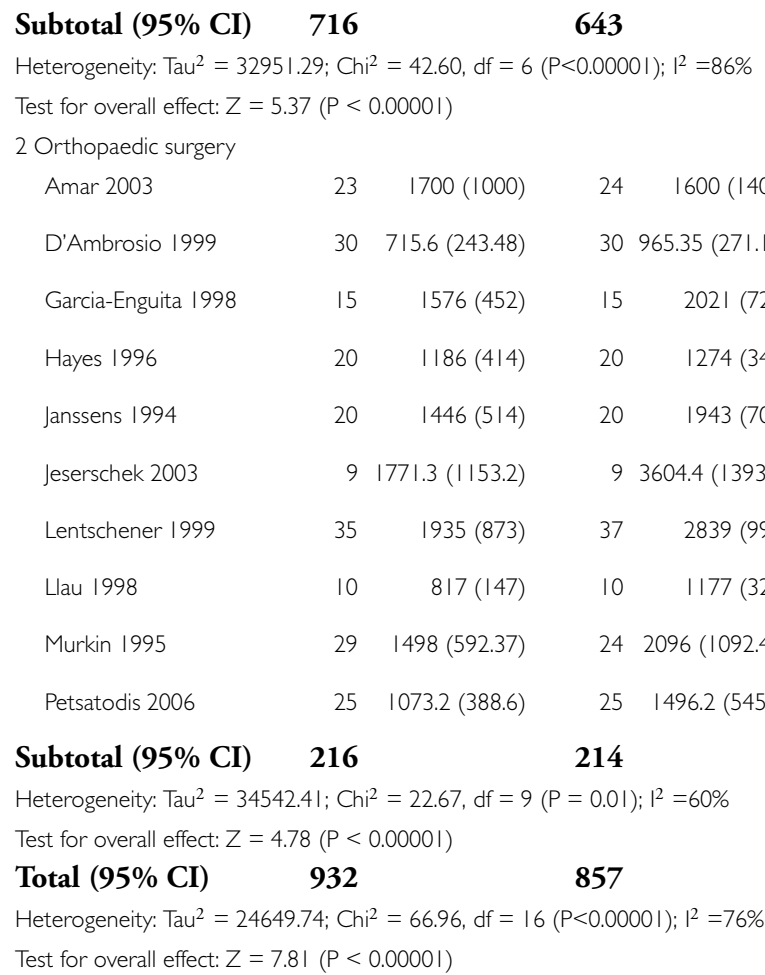

Heterogeneity: Tau $^{2}=32951.29 ; \mathrm{Chi}^{2}=42.60, \mathrm{df}=6(\mathrm{P}<0.0000 \mathrm{I}) ; \mathrm{I}^{2}=86 \%$

Test for overall effect: $Z=5.37(P<0.0000 \mathrm{I})$

2 Orthopaedic surgery

Subtotal (95\% CI) 216

214

Heterogeneity: $\operatorname{Tau}^{2}=34542.4 \mathrm{I} ; \mathrm{Chi}^{2}=22.67, \mathrm{df}=9(\mathrm{P}=0.0 \mathrm{l}) ; \mathrm{I}^{2}=60 \%$

Test for overall effect: $Z=4.78(P<0.0000 \mathrm{I})$

Total (95\% CI) $\quad 932 \quad 857$

Heterogeneity: $\mathrm{Tau}^{2}=24649.74 ; \mathrm{Chi}^{2}=66.96, \mathrm{df}=16(\mathrm{P}<0.0000 \mathrm{I}) ; \mathrm{I}^{2}=76 \%$

Test for overall effect: $Z=7.81(P<0.00001)$

$50.5 \%-448.86[-612.82,-284.91]$

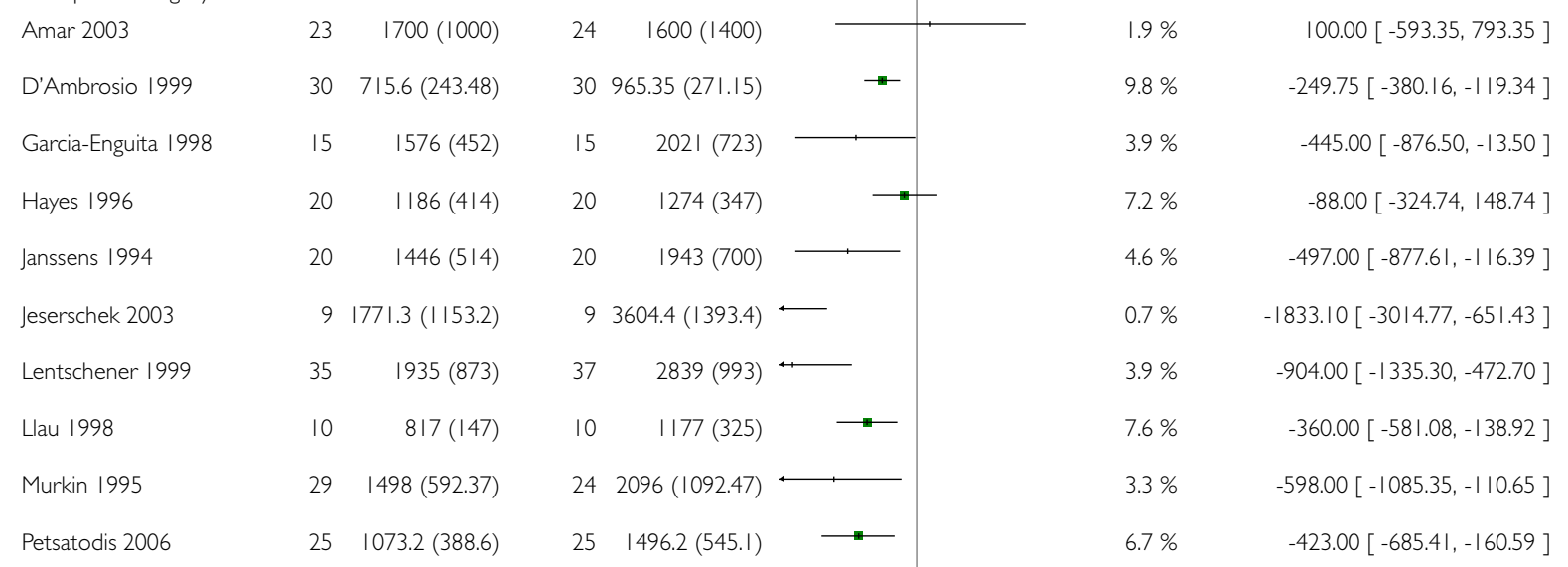

$49.5 \% \quad-399.09[-562.81,-235.37]$

$100.0 \%-415.95[-520.38,-311.51]$ 
Analysis 2.1. Comparison 2 Tranexamic Acid versus Control (Blood Transfusion \& Blood Loss), Outcome I No. Exposed to Allogeneic Blood.

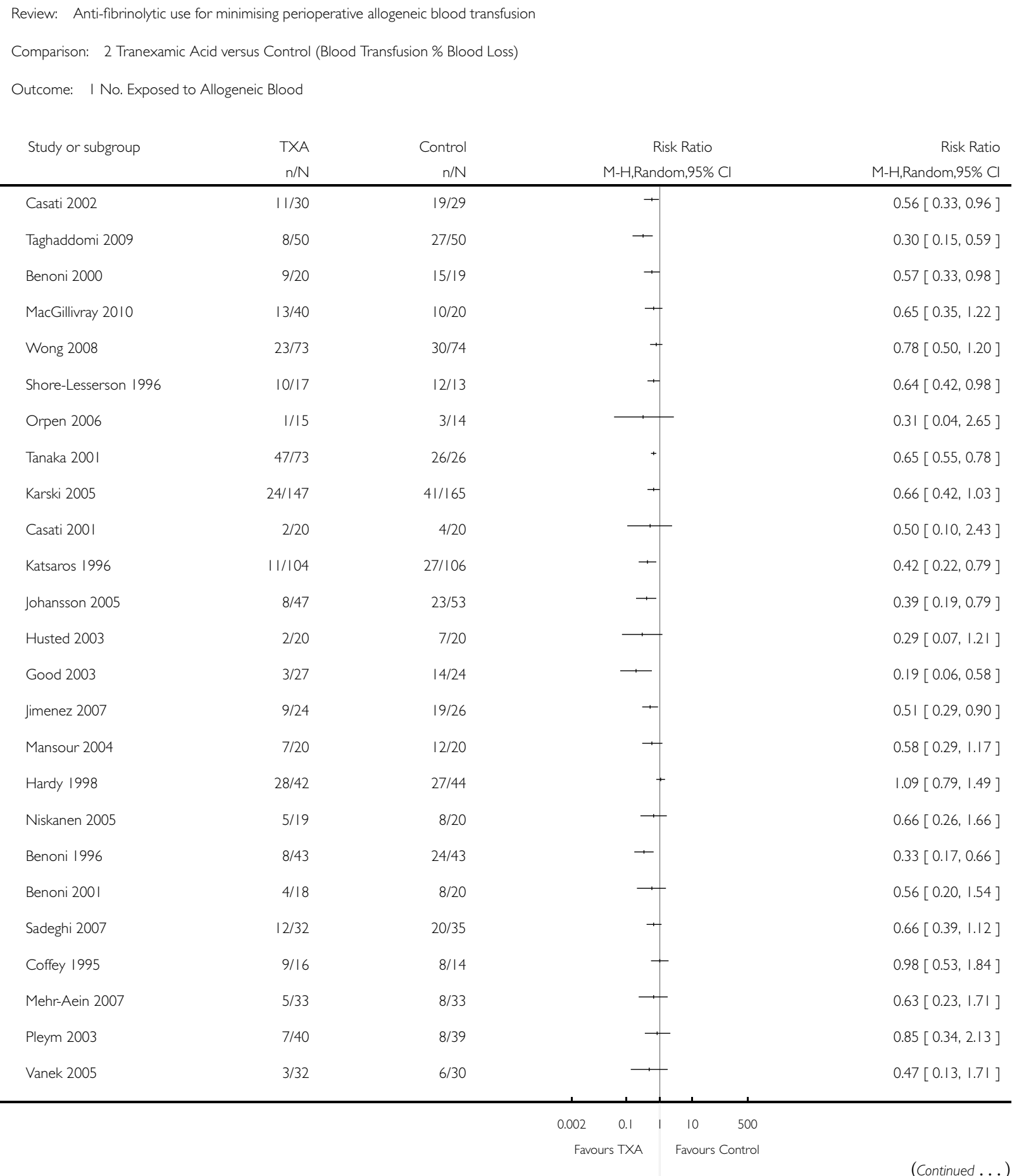




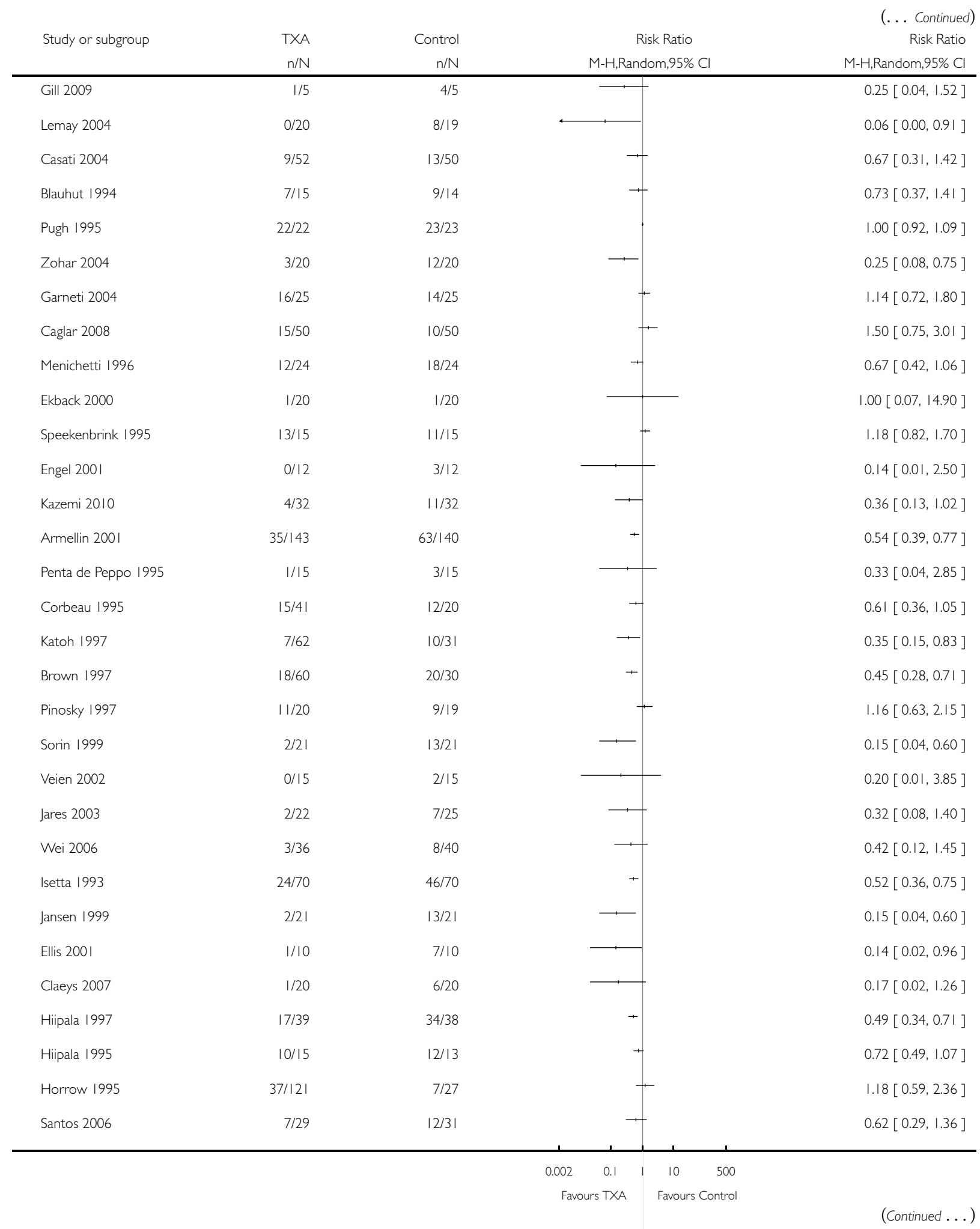

Anti-fibrinolytic use for minimising perioperative allogeneic blood transfusion (Review)

Copyright @ 201 I The Cochrane Collaboration. Published by John Wiley \& Sons, Ltd. 


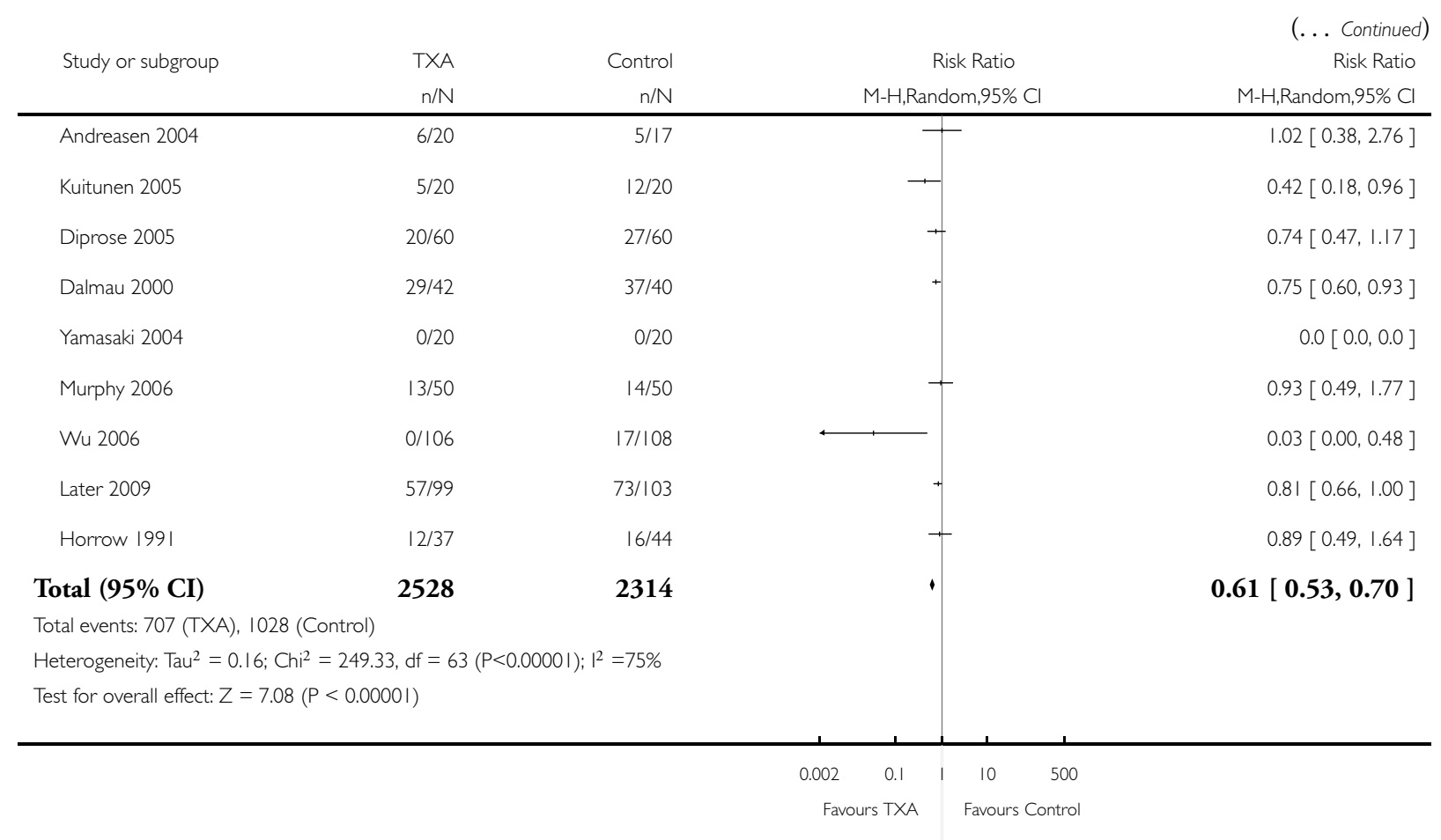


Analysis 2.2. Comparison 2 Tranexamic Acid versus Control (Blood Transfusion \& Blood Loss), Outcome 2 No. Exposed to Allogeneic Blood - Type of Surgery.

Review: Anti-fibrinolytic use for minimising perioperative allogeneic blood transfusion

Comparison: 2 Tranexamic Acid versus Control (Blood Transfusion \% Blood Loss)

Outcome: 2 No. Exposed to Allogeneic Blood - Type of Surgery

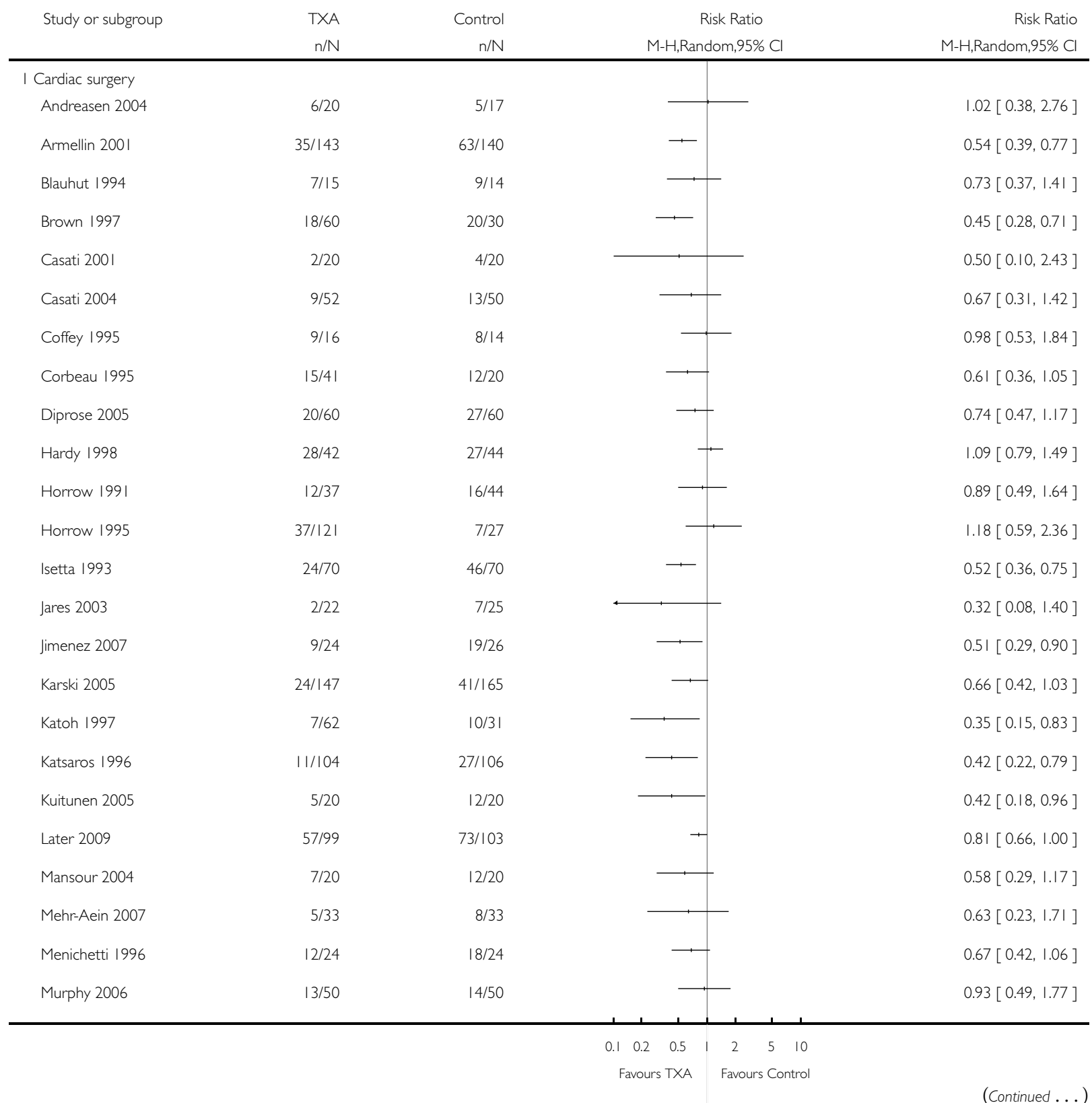




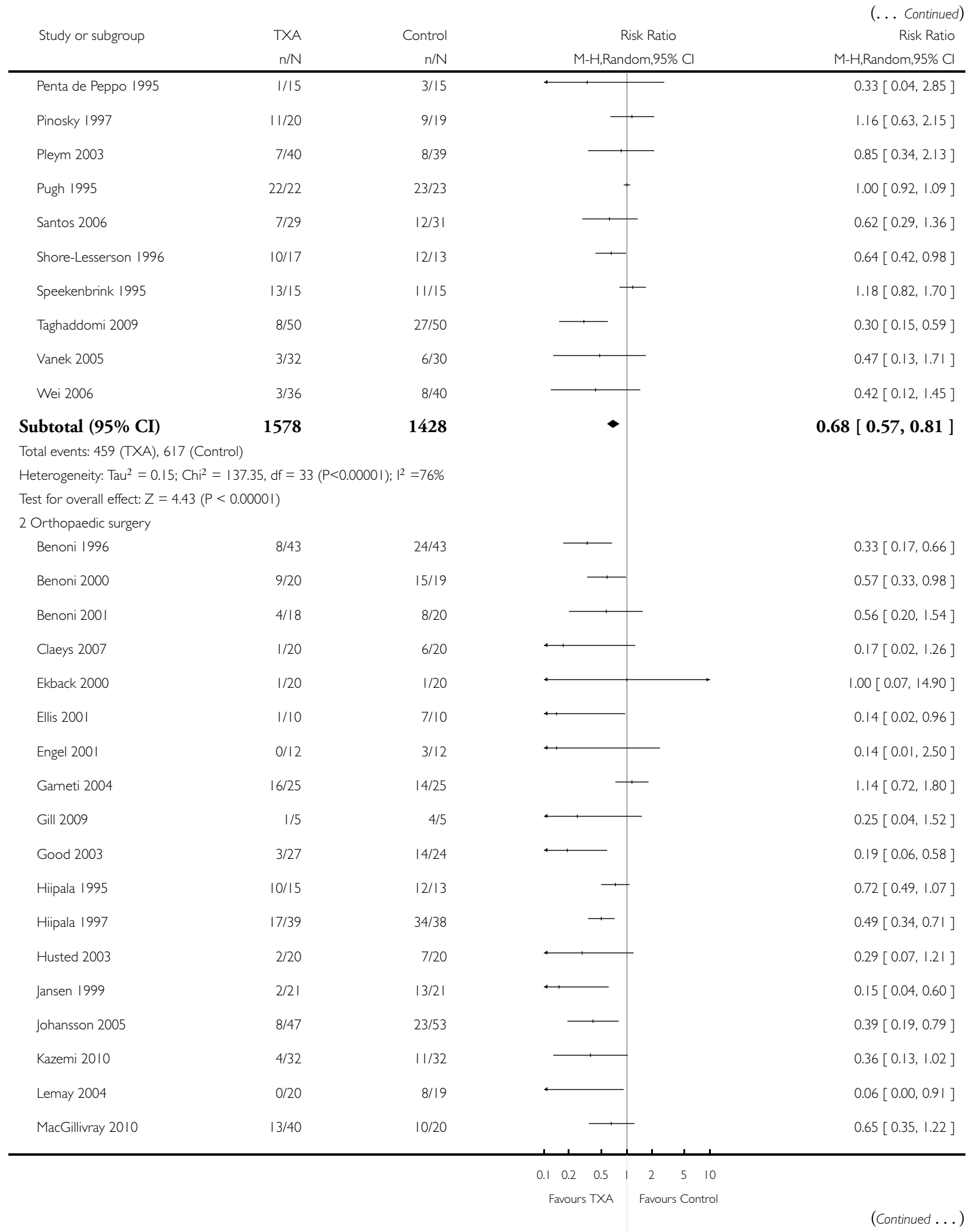

Anti-fibrinolytic use for minimising perioperative allogeneic blood transfusion (Review)

Copyright (C) 201 I The Cochrane Collaboration. Published by John Wiley \& Sons, Ltd. 


\begin{tabular}{|c|c|c|c|c|}
\hline \multirow{3}{*}{ Study or subgroup } & \multirow{3}{*}{$\begin{array}{r}\text { TXA } \\
\mathrm{n} / \mathrm{N} \\
\end{array}$} & \multirow{3}{*}{$\begin{array}{r}\text { Control } \\
\mathrm{n} / \mathrm{N}\end{array}$} & \multirow{3}{*}{$\begin{array}{c}\text { Risk Ratio } \\
\text { M-H,Random,95\% Cl }\end{array}$} & \multirow{2}{*}{$\begin{array}{c}\text { (... Continued) } \\
\text { Risk Ratio }\end{array}$} \\
\hline & & & & \\
\hline & & & & M-H,Random, $95 \% \mathrm{Cl}$ \\
\hline Niskanen 2005 & $5 / 19$ & $8 / 20$ & & $0.66[0.26,1.66]$ \\
\hline Orpen 2006 & $1 / 15$ & $3 / 14$ & & $0.31[0.04,2.65]$ \\
\hline Sadeghi 2007 & $12 / 32$ & 20/35 & $\longrightarrow$ & $0.66[0.39,1.12]$ \\
\hline Sorin 1999 & $2 / 21$ & $13 / 21$ & - & $0.15[0.04,0.60]$ \\
\hline Tanaka 200I & $47 / 73$ & $26 / 26$ & + & $0.65[0.55,0.78]$ \\
\hline Veien 2002 & $0 / 15$ & $2 / 15$ & & $0.20[0.01,3.85]$ \\
\hline Wong 2008 & $23 / 73$ & $30 / 74$ & $一$ & $0.78[0.50,1.20]$ \\
\hline Yamasaki 2004 & $0 / 20$ & 0/20 & & $0.0[0.0,0.0]$ \\
\hline Zohar 2004 & $3 / 20$ & $12 / 20$ & - & $0.25[0.08,0.75]$ \\
\hline Subtotal (95\% CI) & 722 & 659 & $\bullet$ & $0.49[0.39,0.62]$ \\
\hline \multicolumn{5}{|c|}{ Total events: 193 (TXA), 328 (Control) } \\
\hline \multicolumn{5}{|c|}{ Heterogeneity: Tau $^{2}=0.12 ; \mathrm{Chi}^{2}=53.86, \mathrm{df}=25(\mathrm{P}=0.00069) ; \mathrm{I}^{2}=54 \%$} \\
\hline \multicolumn{5}{|c|}{ Test for overall effect: $Z=6.20(P<0.0000 I)$} \\
\hline \multicolumn{5}{|l|}{3 Liver surgery } \\
\hline Dalmau 2000 & $29 / 42$ & $37 / 40$ & + & $0.75[0.60,0.93]$ \\
\hline Wu 2006 & 0/106 & $17 / 108$ & & $0.03[0.00,0.48]$ \\
\hline Subtotal $(95 \% \mathrm{CI})$ & 148 & 148 & & $0.16[0.00,32.47]$ \\
\hline \multicolumn{5}{|c|}{ Total events: 29 (TXA), 54 (Control) } \\
\hline \multicolumn{5}{|c|}{ Heterogeneity: Tau $^{2}=13.57 ; \mathrm{Chi}^{2}=14.23, \mathrm{df}=|(P=0.00016) ;|^{2}=93 \%$} \\
\hline \multicolumn{5}{|c|}{ Test for overall effect: $Z=0.67(P=0.50)$} \\
\hline \multicolumn{5}{|l|}{4 Vascular surgery } \\
\hline Casati 2002 & $11 / 30$ & 19/29 & & $0.56[0.33,0.96]$ \\
\hline Subtotal $(95 \% \mathrm{CI})$ & 30 & 29 & & $0.56[0.33,0.96]$ \\
\hline \multicolumn{5}{|c|}{ Total events: II (TXA), 19 (Control) } \\
\hline \multicolumn{5}{|c|}{ Heterogeneity: not applicable } \\
\hline \multicolumn{5}{|c|}{ Test for overall effect: $Z=2.11(P=0.035)$} \\
\hline \multicolumn{5}{|l|}{5 Gynaecological surgery } \\
\hline Caglar 2008 & $15 / 50$ & $10 / 50$ & & $1.50[0.75,3.01]$ \\
\hline Subtotal $(95 \% \mathrm{CI})$ & 50 & 50 & & $1.50[0.75,3.01]$ \\
\hline \multicolumn{5}{|c|}{ Total events: 15 (TXA), 10 (Control) } \\
\hline \multicolumn{5}{|c|}{ Heterogeneity: not applicable } \\
\hline \multicolumn{5}{|c|}{ Test for overall effect: $Z=1.14(P=0.25)$} \\
\hline Total $(95 \% \mathrm{CI})$ & 2528 & 2314 & $\bullet$ & $0.61[0.53,0.70]$ \\
\hline \multicolumn{5}{|c|}{ Total events: 707 (TXA), 1028 (Control) } \\
\hline \multicolumn{5}{|c|}{ Heterogeneity: $\mathrm{Tau}^{2}=0.16 ; \mathrm{Chi}^{2}=249.33, \mathrm{df}=63(\mathrm{P}<0.0000 \mathrm{I}) ; \mathrm{I}^{2}=75 \%$} \\
\hline Test for overall effect: $Z=$ & & & & \\
\hline
\end{tabular}


Analysis 2.3. Comparison 2 Tranexamic Acid versus Control (Blood Transfusion \& Blood Loss), Outcome 3 No. Exposed to Allogeneic Blood - Transfusion Protocol.

Review: Anti-fibrinolytic use for minimising perioperative allogeneic blood transfusion

Comparison: 2 Tranexamic Acid versus Control (Blood Transfusion \% Blood Loss)

Outcome: 3 No. Exposed to Allogeneic Blood - Transfusion Protocol

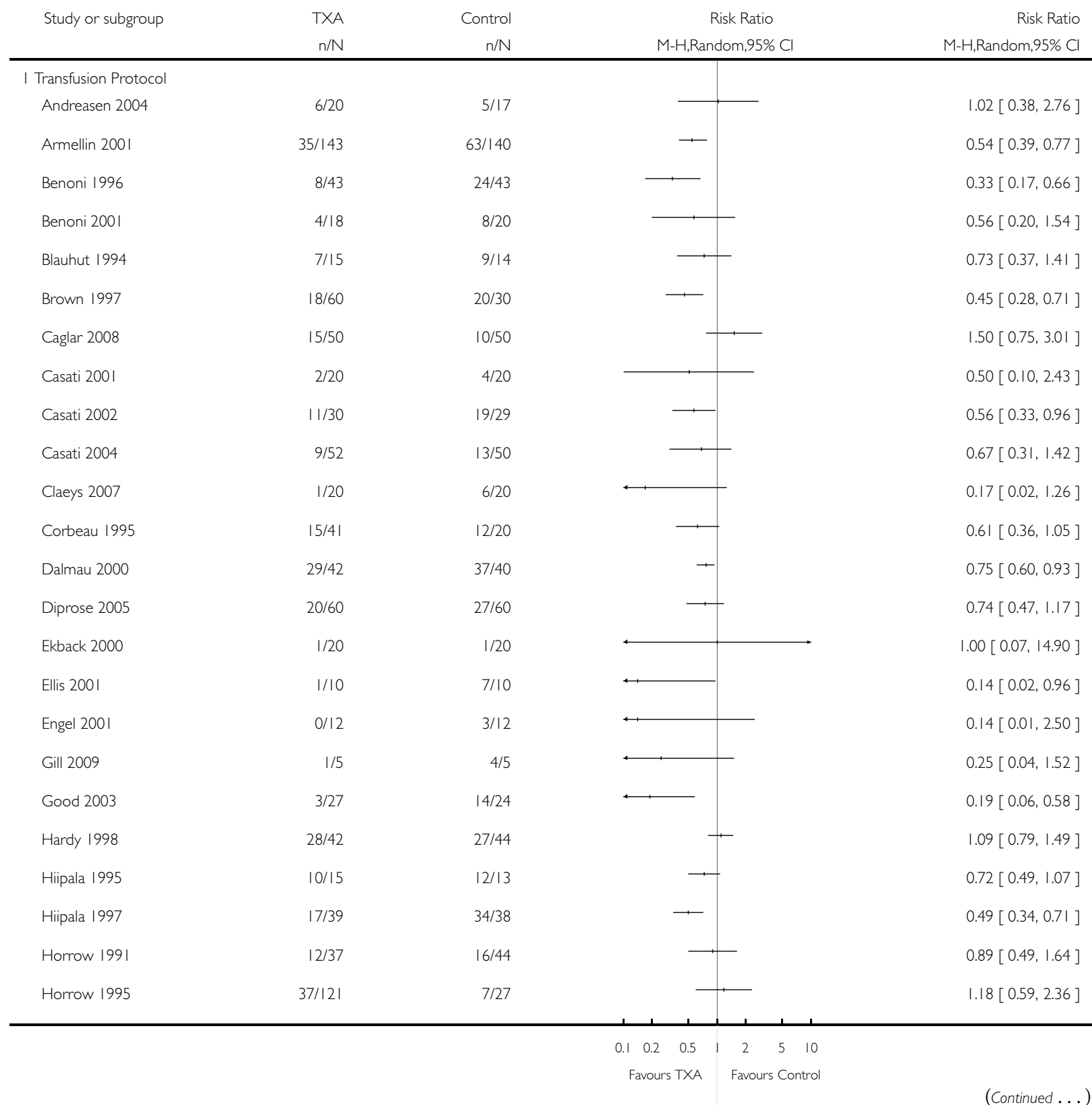




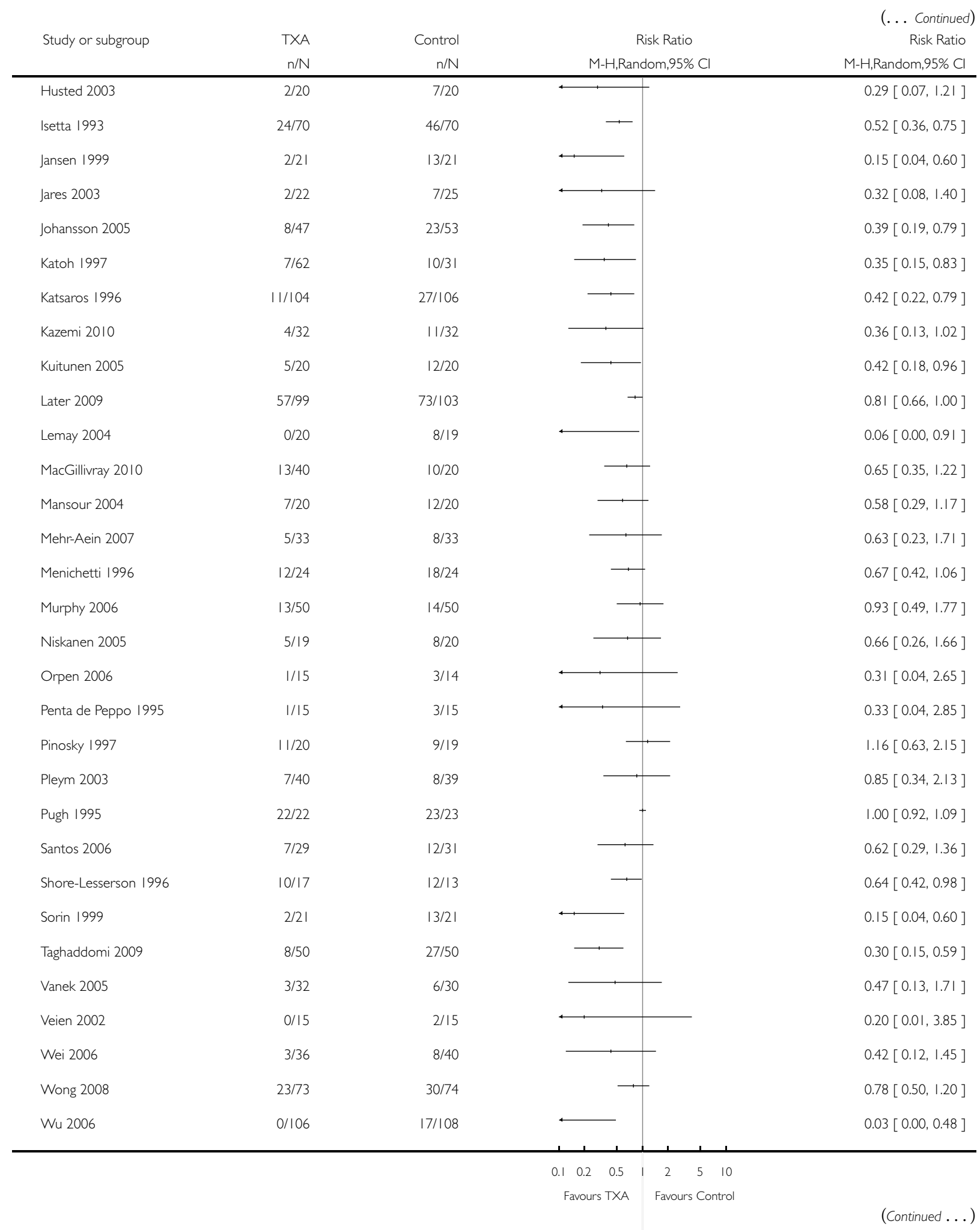

Anti-fibrinolytic use for minimising perioperative allogeneic blood transfusion (Review) 


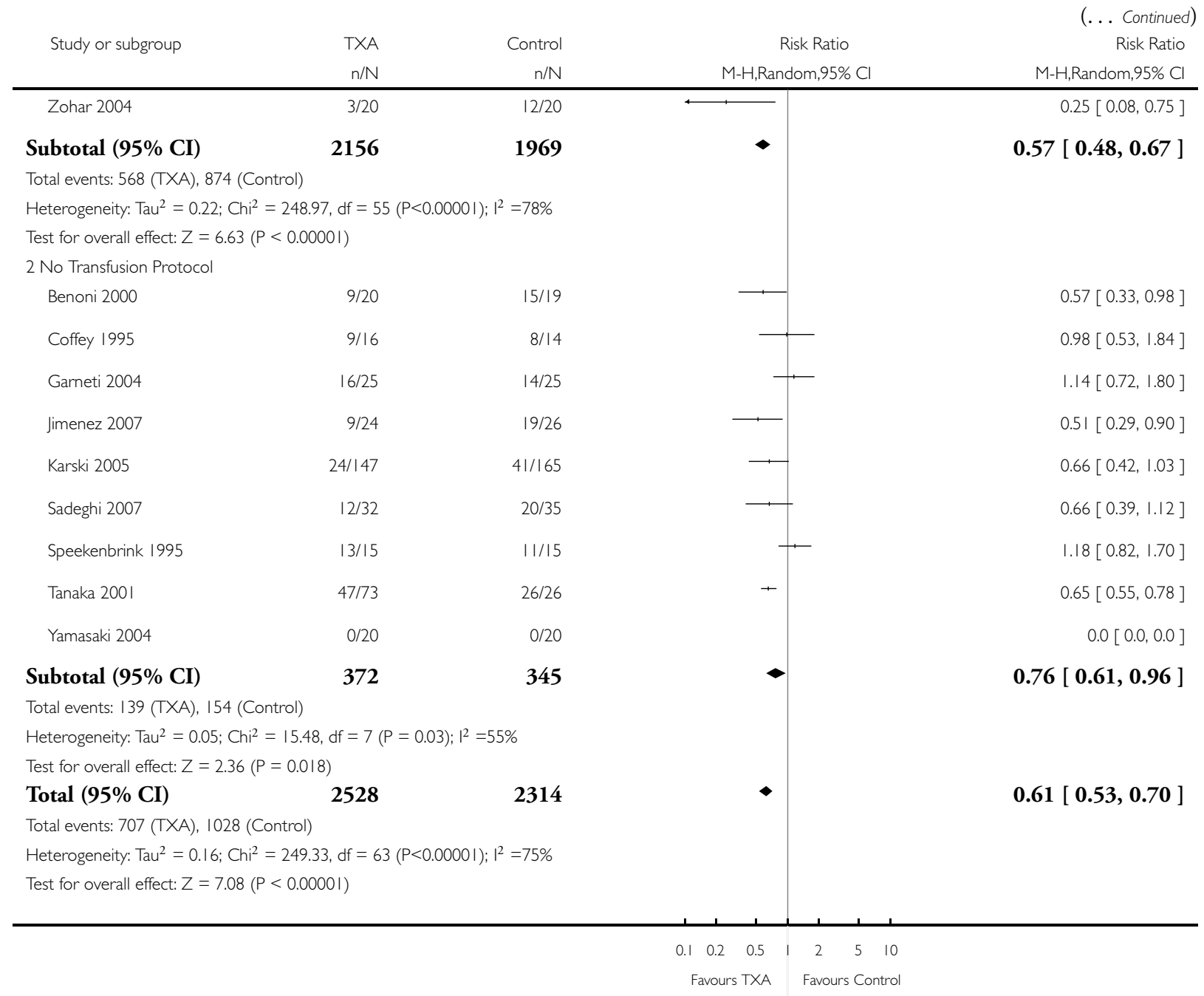


Analysis 2.4. Comparison 2 Tranexamic Acid versus Control (Blood Transfusion \& Blood Loss), Outcome 4 No. Exposed to Allogeneic Blood - Dose (Cardiac Surgery).

Review: Anti-fibrinolytic use for minimising perioperative allogeneic blood transfusion

Comparison: 2 Tranexamic Acid versus Control (Blood Transfusion \% Blood Loss)

Outcome: 4 No. Exposed to Allogeneic Blood - Dose (Cardiac Surgery)

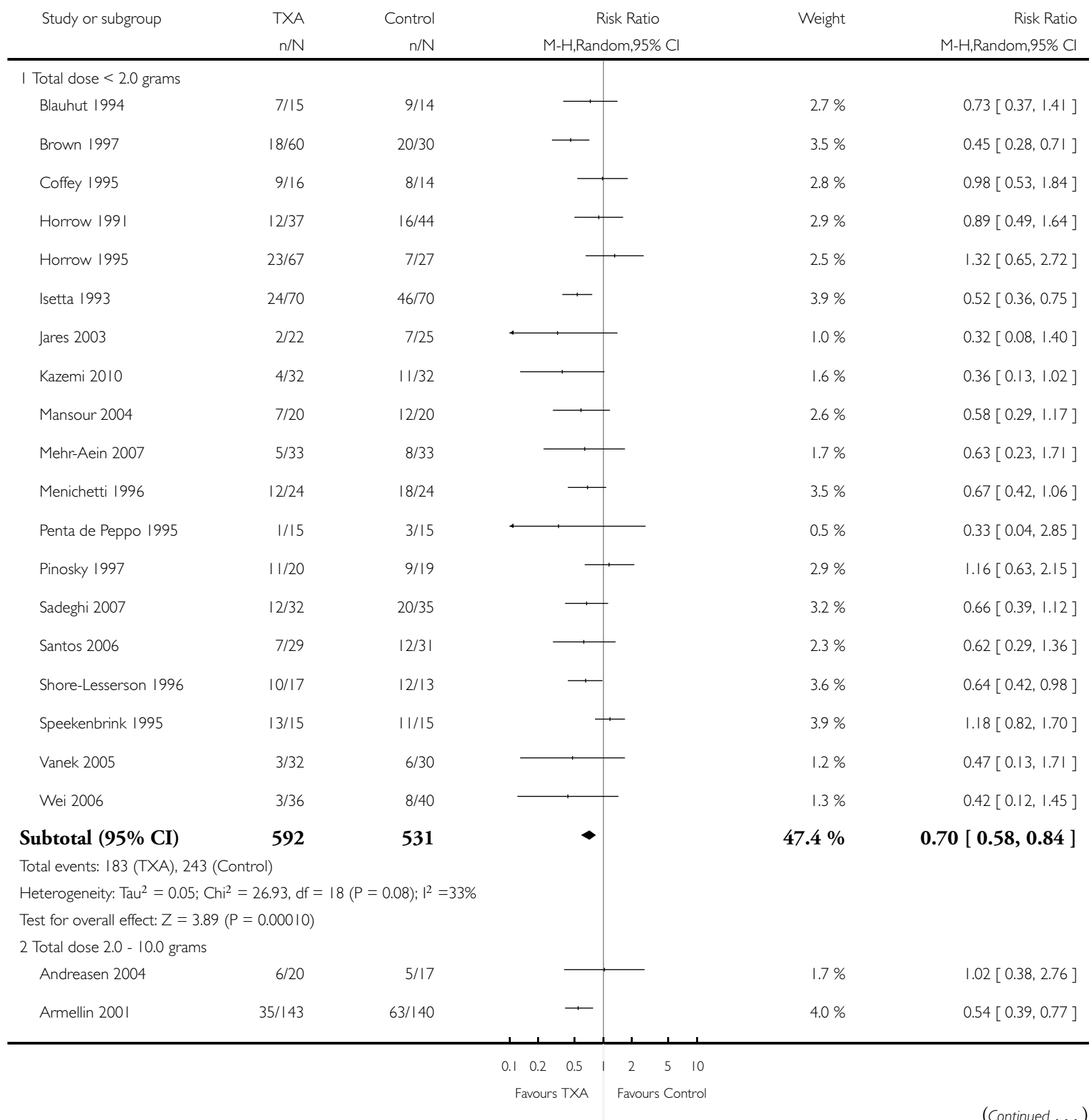




\begin{tabular}{|c|c|c|c|c|c|}
\hline \multirow[t]{2}{*}{ Study or subgroup } & TXA & Control & Risk Ratio & \multirow[t]{2}{*}{ Weight } & $\begin{array}{c}(\ldots \text { Continued }) \\
\text { Risk Ratio }\end{array}$ \\
\hline & $n / N$ & $\mathrm{n} / \mathrm{N}$ & $\mathrm{M}-\mathrm{H}$, Random, $95 \% \mathrm{Cl}$ & & $\mathrm{M}-\mathrm{H}$, Random, $95 \% \mathrm{Cl}$ \\
\hline Casati 2001 & $2 / 20$ & $4 / 20$ & & $0.9 \%$ & $0.50[0.10,2.43]$ \\
\hline Casati 2004 & $9 / 52$ & $13 / 50$ & - & $2.4 \%$ & $0.67[0.31,1.42]$ \\
\hline Corbeau 1995 & $|5 / 4|$ & $12 / 20$ & 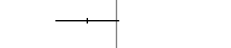 & $3.2 \%$ & $0.61[0.36,1.05]$ \\
\hline Diprose 2005 & $20 / 60$ & $27 / 60$ & $\longrightarrow$ & $3.5 \%$ & $0.74[0.47,1.17]$ \\
\hline Hardy 1998 & $28 / 42$ & $27 / 44$ & + & $4.1 \%$ & $1.09[0.79,1.49]$ \\
\hline Horrow 1995 & $14 / 54$ & $14 / 54$ & - & $2.8 \%$ & $1.00[0.53,1.89]$ \\
\hline Jimenez 2007 & $9 / 24$ & 19/26 & + & $3.1 \%$ & $0.51[0.29,0.90]$ \\
\hline Karski 2005 & $24 / 147$ & $41 / 165$ & & $3.5 \%$ & $0.66[0.42,1.03]$ \\
\hline Katoh 1997 & $7 / 62$ & $|0 / 3|$ & & $2.0 \%$ & $0.35[0.15,0.83]$ \\
\hline Katsaros 1996 & $11 / 104$ & $27 / 106$ & — & $2.7 \%$ & $0.42[0.22,0.79]$ \\
\hline Kuitunen 2005 & $5 / 20$ & $12 / 20$ & & $2.1 \%$ & $0.42[0.18,0.96]$ \\
\hline Later 2009 & $57 / 99$ & $73 / 103$ & + & $4.5 \%$ & $0.81[0.66,1.00]$ \\
\hline Murphy 2006 & $13 / 50$ & $14 / 50$ & - & $2.8 \%$ & $0.93[0.49,1.77]$ \\
\hline Pleym 2003 & $7 / 40$ & $8 / 39$ & - & $1.9 \%$ & $0.85[0.34,2.13]$ \\
\hline Pugh 1995 & $22 / 22$ & $23 / 23$ & - & $4.8 \%$ & $1.00[0.92,1.09]$ \\
\hline Taghaddomi 2009 & $8 / 50$ & $27 / 50$ & - & $2.6 \%$ & $0.30[0.15,0.59]$ \\
\hline Subtotal $(95 \%$ CI $)$ & 1050 & 1018 & - & $52.6 \%$ & $0.67[0.52,0.86]$ \\
\hline \multicolumn{6}{|c|}{ Total events: 292 (TXA), 419 (Control) } \\
\hline \multicolumn{6}{|c|}{ Heterogeneity: $\mathrm{Tau}^{2}=0.20 ; \mathrm{Chi}^{2}=107.97, \mathrm{df}=17(\mathrm{P}<0.0000 \mathrm{I}) ; \mathrm{I}^{2}=84 \%$} \\
\hline \multicolumn{6}{|c|}{ Test for overall effect: $Z=3.15(P=0.0016)$} \\
\hline Total (95\% CI) & 1642 & 1549 & $\bullet$ & $100.0 \%$ & $0.68[0.58,0.80]$ \\
\hline \multicolumn{6}{|c|}{ Total events: 475 (TXA), 662 (Control) } \\
\hline \multicolumn{6}{|c|}{ Heterogeneity: Tau $^{2}=0.14 ; \mathrm{Chi}^{2}=139.40, \mathrm{df}=36(\mathrm{P}<0.0000 \mathrm{I}) ; \mathrm{I}^{2}=74 \%$} \\
\hline Test for overall effect: $Z=$ & $0.00001)$ & & & & \\
\hline
\end{tabular}


Analysis 2.5. Comparison 2 Tranexamic Acid versus Control (Blood Transfusion \& Blood Loss), Outcome 5 Trial Methodological Quality - Allocation Concealment.

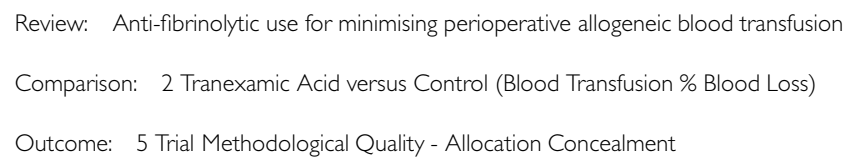

$\mathrm{n} / \mathrm{N} \quad \mathrm{n} / \mathrm{N} \quad \mathrm{M}-\mathrm{H}$, Random,95\% Cl M-H,Random,95\% Cl

$8 / 43 \quad 24 / 43$

$\begin{array}{lll}\text { Benoni } 2000 & 9 / 20 \quad 15 / 19\end{array}$

$\begin{array}{lll}\text { Benoni } 2001 & 4 / 18 & 8 / 20\end{array}$

$\begin{array}{lll}\text { Casati 2001 } & \text { 2/20 }\end{array}$

$\begin{array}{lll}\text { Casati } 2002 & \text { 11/30 }\end{array}$

$\begin{array}{lll}\text { Casati } 2004 & 9 / 52 & 13 / 50\end{array}$

Coffey $1995 \quad 9 / 16 \quad 8 / 14$

Gill $2009 \quad$ |/5 4/5

Good $2003 \quad 3 / 27 \quad$ 14/24

$\begin{array}{lll}\text { Hardy } 1998 & 28 / 42 & 27 / 44\end{array}$

$\begin{array}{lll}\text { Husted } 2003 & \text { 2/20 }\end{array}$

$\begin{array}{lrl}\text { Jimenez } 2007 & 9 / 24 & 19 / 26\end{array}$

$\begin{array}{lll}\text { Johansson } 2005 & \text { 23/57 }\end{array}$

$\begin{array}{lll}\text { Karski } 2005 & \text { 24/147 4I/I65 }\end{array}$

$\begin{array}{lll}\text { Katsaros } 1996 & \text { II/104 27/106 }\end{array}$

Lemay $2004 \quad 0 / 20 \quad 8 / 19$

MacGillivray $2010 \quad 13 / 40 \quad 10 / 20$

$\begin{array}{lll}\text { Mansour } 2004 & 7 / 20 & 12 / 20\end{array}$

Mehr-Aein $2007 \quad 5 / 33 \quad 8 / 33$

$\begin{array}{lll}\text { Niskanen } 2005 & 5 / 19 & 8 / 20\end{array}$

Orpen $2006 \quad$ I/15 3/14

Pleym $2003 \quad 7 / 40 \quad 8 / 39$

\begin{tabular}{l|l} 
Sadeghi 2007 & 12/32
\end{tabular}

$\begin{array}{lll}\text { Shore-Lesserson } 1996 & 10 / 17 & 12 / 13\end{array}$

$0.33[0.17,0.66]$

$0.57[0.33,0.98]$

$0.56[0.20,1.54]$

$0.50[0.10,2.43]$

$0.56[0.33,0.96]$

$0.67[0.31,1.42]$

$0.98[0.53,1.84]$

$0.25[0.04,1.52]$

$0.19[0.06,0.58]$

$1.09[0.79,1.49]$

$0.29[0.07,1.21]$

$0.51[0.29,0.90]$

$0.39[0.19,0.79]$

$0.66[0.42,1.03]$

$0.42[0.22,0.79]$

$0.06[0.00,0.91]$

$0.65[0.35,1.22]$

$0.58[0.29,1.17]$

$0.63[0.23,1.71]$

$0.66[0.26,1.66]$

$0.31[0.04,2.65]$

$0.85[0.34,2.13]$

$0.66[0.39,1.12]$

$0.64[0.42,0.98]$

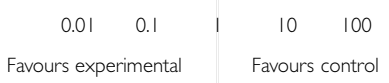

(Continued....) 


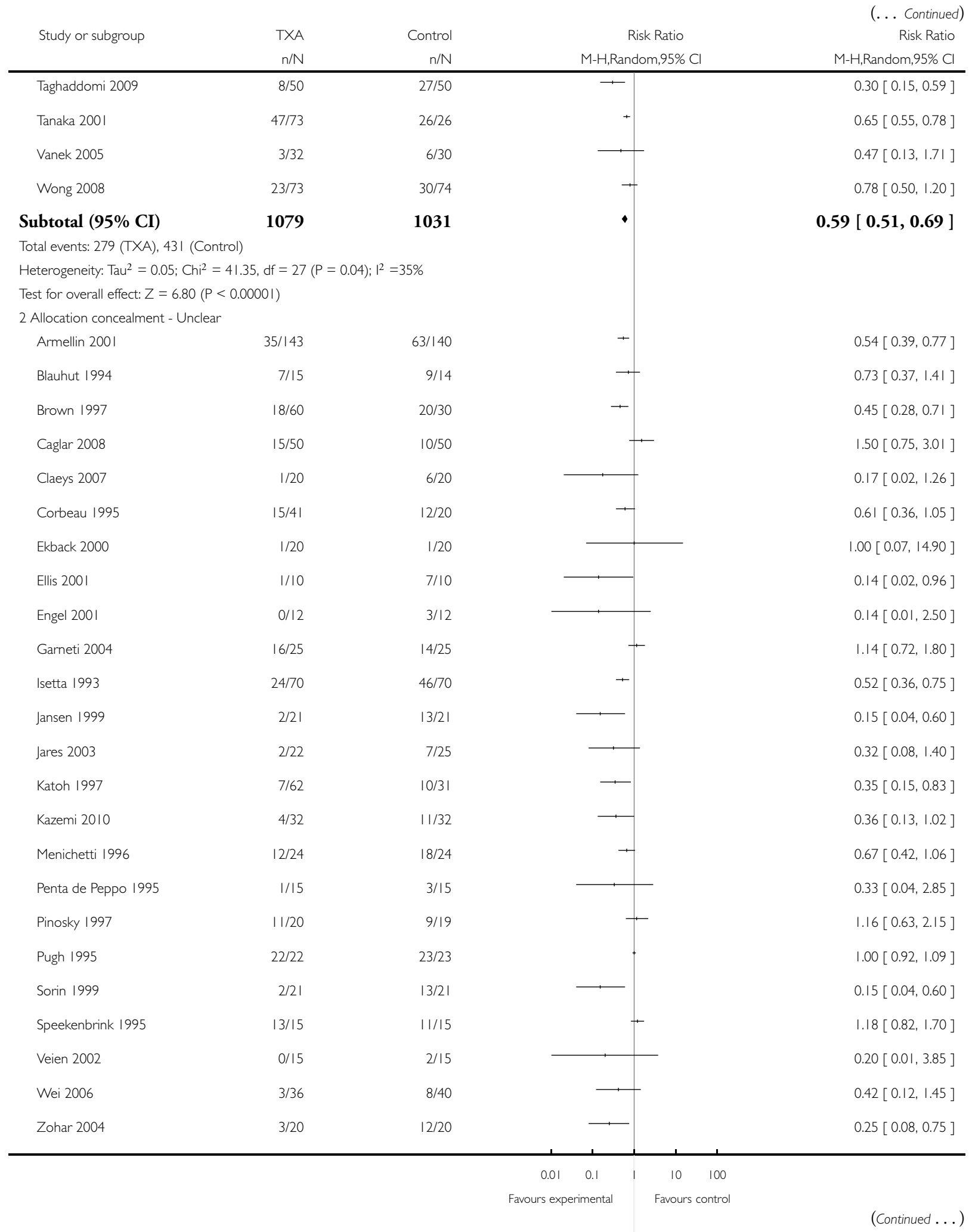

Anti-fibrinolytic use for minimising perioperative allogeneic blood transfusion (Review)

Copyright (C) 201 I The Cochrane Collaboration. Published by John Wiley \& Sons, Ltd. 


\begin{tabular}{|c|c|c|c|c|}
\hline \multirow[t]{2}{*}{ Study or subgroup } & TXA & Control & Risk Ratio & $\begin{array}{r}\text { (... Continued) } \\
\text { Risk Ratio } \\
\text { M-H.Random. } 95 \% \text { C }\end{array}$ \\
\hline & $\mathrm{n} / \mathrm{N}$ & $n / N$ & M-H,Random, $95 \% \mathrm{Cl}$ & $\mathrm{M}-\mathrm{H}$, Random, $95 \% \mathrm{Cl}$ \\
\hline Subtotal $(95 \% \mathrm{CI})$ & 791 & 712 & $\bullet$ & $0.53[0.37,0.76]$ \\
\hline \multicolumn{5}{|c|}{ Total events: 215 (TXA), 33I (Control) } \\
\hline \multicolumn{5}{|c|}{ Heterogeneity: $\mathrm{Tau}^{2}=0.53 ; \mathrm{Ch}^{2}=209.62, \mathrm{df}=23(\mathrm{P}<0.0000 \mathrm{I}) ; \mathrm{I}^{2}=89 \%$} \\
\hline \multicolumn{5}{|c|}{ Test for overall effect: $Z=3.44(P=0.00059)$} \\
\hline \multicolumn{5}{|c|}{3 Allocation concealment - No } \\
\hline Andreasen 2004 & $6 / 20$ & $5 / 17$ & $\longrightarrow$ & $1.02[0.38,2.76]$ \\
\hline Dalmau 2000 & $29 / 42$ & $37 / 40$ & + & $0.75[0.60,0.93]$ \\
\hline Diprose 2005 & $20 / 60$ & $27 / 60$ & + & $0.74[0.47,1.17]$ \\
\hline Hiipala 1995 & $10 / 15$ & $12 / 13$ & + & $0.72[0.49,1.07]$ \\
\hline Hiipala 1997 & $17 / 39$ & $34 / 38$ & + & $0.49[0.34,0.71]$ \\
\hline Horrow 1991 & $12 / 37$ & $16 / 44$ & + & $0.89[0.49,1.64]$ \\
\hline Horrow 1995 & $37 / 121$ & $7 / 27$ & $\rightarrow$ & $1.18[0.59,2.36]$ \\
\hline Kuitunen 2005 & $5 / 20$ & $12 / 20$ & 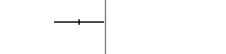 & $0.42[0.18,0.96]$ \\
\hline Later 2009 & $57 / 99$ & $73 / 103$ & + & $0.81[0.66,1.00]$ \\
\hline Murphy 2006 & $13 / 50$ & $14 / 50$ & $\rightarrow$ & $0.93[0.49,1.77]$ \\
\hline Santos 2006 & 7/29 & $|2 / 3|$ & 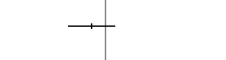 & $0.62[0.29,1.36]$ \\
\hline Wu 2006 & $0 / 106$ & $17 / 108$ & - & $0.03[0.00,0.48]$ \\
\hline Yamasaki 2004 & $0 / 20$ & $0 / 20$ & & $0.0[0.0,0.0]$ \\
\hline Subtotal $(95 \% \mathrm{CI})$ & 658 & 571 & - & $0.73[0.62,0.86]$ \\
\hline \multicolumn{5}{|c|}{ Total events: 213 (TXA), 266 (Control) } \\
\hline \multicolumn{5}{|c|}{ Heterogeneity: $\operatorname{Tau}^{2}=0.02 ; \mathrm{Chi}^{2}=16.38, \mathrm{df}=11(P=0.13) ;\left.\right|^{2}=33 \%$} \\
\hline \multicolumn{5}{|c|}{ Test for overall effect: $Z=3.72(P=0.00020)$} \\
\hline Total $(95 \% \mathrm{CI})$ & 2528 & 2314 & $\cdot$ & $0.61[0.53,0.70]$ \\
\hline \multicolumn{5}{|c|}{ Total events: 707 (TXA), 1028 (Control) } \\
\hline \multicolumn{5}{|c|}{ Heterogeneity: $\mathrm{Tau}^{2}=0.16 ; \mathrm{Chi}^{2}=249.33, \mathrm{df}=63(\mathrm{P}<0.0000 \mathrm{I}) ; \mathrm{I}^{2}=75 \%$} \\
\hline \multicolumn{5}{|c|}{ Test for overall effect: $Z=7.08(P<0.0000 I)$} \\
\hline
\end{tabular}


Analysis 2.6. Comparison 2 Tranexamic Acid versus Control (Blood Transfusion \& Blood Loss), Outcome 6 Units Allogeneic Blood Transfused - Transfused Patients.

Review: Anti-fibrinolytic use for minimising perioperative allogeneic blood transfusion

Comparison: 2 Tranexamic Acid versus Control (Blood Transfusion \% Blood Loss)

Outcome: 6 Units Allogeneic Blood Transfused - Transfused Patients

\begin{tabular}{|c|c|c|c|c|c|c|c|}
\hline \multirow[t]{2}{*}{ Study or subgroup } & \multirow{2}{*}{$\begin{array}{r}\text { TXA } \\
\mathrm{N} \\
\end{array}$} & \multicolumn{3}{|c|}{ Control } & \multirow{2}{*}{$\begin{array}{l}\text { Mean Difference } \\
\text { IV,Random,95\% Cl }\end{array}$} & \multirow[t]{2}{*}{ Weight } & \multirow{2}{*}{$\begin{array}{l}\text { Mean Difference } \\
\text { IV,Random,95\% C }\end{array}$} \\
\hline & & Mean(SD) & $\mathrm{N}$ & Mean(SD) & & & \\
\hline Armellin 200I & 35 & $1.68(1.2)$ & 63 & $1.93(1.3)$ & + & $10.8 \%$ & $-0.25[-0.76,0.26]$ \\
\hline Blauhut 1994 & 7 & ।.7| (0.95) & 9 & $2.44(1.13)$ & $\rightarrow$ & $7.7 \%$ & $-0.73[-1.75,0.29]$ \\
\hline Caglar 2008 & 15 & $1.8(0.54)$ & 10 & $1.6(0.66)$ & F & $10.9 \%$ & $0.20[-0.29,0.69]$ \\
\hline Corbeau 1995 & 15 & $2.19(0.46)$ & 12 & $2.83(1.45)$ & $\rightarrow$ & $8.7 \%$ & $-0.64[-1.49,0.21]$ \\
\hline Dalmau 2000 & 29 & $7.72(5.44)$ & 37 & $8.38(6.13)$ & +1 & $2.2 \%$ & $-0.66[-3.46,2.14]$ \\
\hline Diprose 2005 & 20 & $2.61(1.55)$ & 27 & $3.73(4.47)$ & $\longrightarrow$ & $4.2 \%$ & $-1.12[-2.94,0.70]$ \\
\hline Garneti 2004 & 16 & $2.31(0.87)$ & 14 & $1.29(0.73)$ & \# & $10.4 \%$ & $1.02[0.45,1.59]$ \\
\hline Good 2003 & 3 & $2.33(1.53)$ & 14 & $2.5(1.02)$ & $\rightarrow$ & $4.2 \%$ & $-0.17[-1.98,1.64]$ \\
\hline Hiipala 1995 & 10 & $2.25(0.87)$ & 12 & $3.58(1.57)$ & $*$ & $7.6 \%$ & $-1.33[-2.37,-0.29]$ \\
\hline Hiipala 1997 & 17 & $2.29(0.52)$ & 34 & $3.46(1.25)$ & 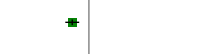 & $10.9 \%$ & $-1.17[-1.66,-0.68]$ \\
\hline Johansson 2005 & 8 & $2.13(0.99)$ & 23 & $2.48(1.31)$ & 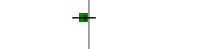 & $8.6 \%$ & $-0.35[-1.22,0.52]$ \\
\hline Murphy 2006 & 13 & $1.46(0.97)$ & 14 & $1.21(0.43)$ & $\rightleftarrows$ & $10.4 \%$ & $0.25[-0.32,0.82]$ \\
\hline Speekenbrink 1995 & 13 & $3.31(1.62)$ & 11 & $4.27(3.15)$ & 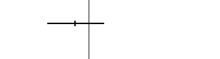 & $3.5 \%$ & $-0.96[-3.02,1.10]$ \\
\hline Total (95\% CI) & 201 & & 280 & & 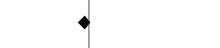 & $100.0 \%$ & $-0.34[-0.80,0.11]$ \\
\hline \multicolumn{8}{|c|}{ Heterogeneity: Tau $^{2}=0.43 ;$ Chi $^{2}=45.89, d f=12(P<0.0000 I) ; I^{2}=74 \%$} \\
\hline \multicolumn{8}{|c|}{ Test for overall effect: $Z=1.49(P=0.14)$} \\
\hline
\end{tabular}

$\begin{array}{ccccc}-10 & -5 & 0 & 5 & 10 \\ \text { Favours TXA } & & \text { Favours Contro }\end{array}$ 
Analysis 2.7. Comparison 2 Tranexamic Acid versus Control (Blood Transfusion \& Blood Loss), Outcome 7 Units of Allogeneic Blood Transfused - All Patients.

Review: Anti-fibrinolytic use for minimising perioperative allogeneic blood transfusion

Comparison: 2 Tranexamic Acid versus Control (Blood Transfusion \% Blood Loss)

Outcome: 7 Units of Allogeneic Blood Transfused - All Patients

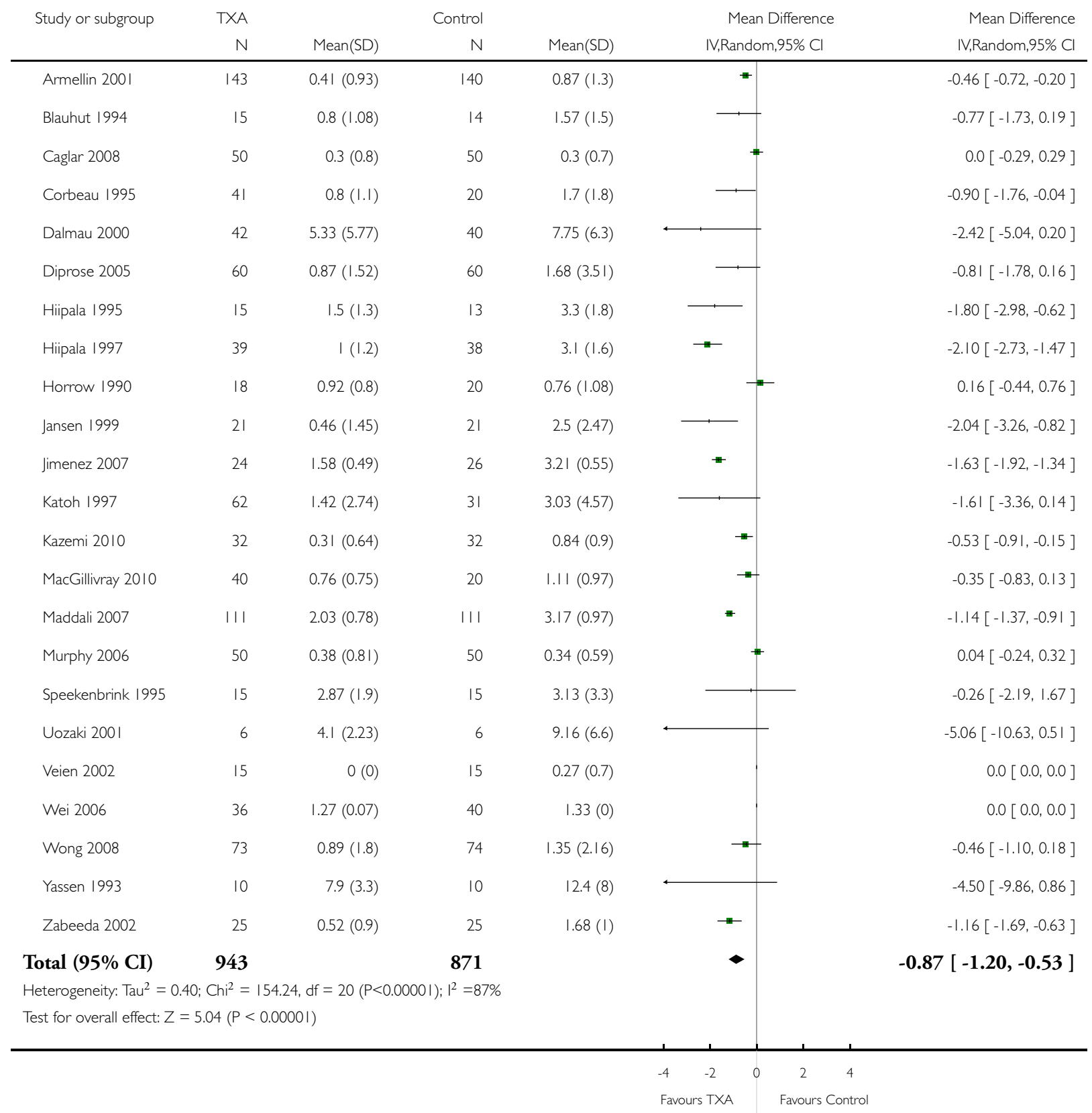


Analysis 2.8. Comparison 2 Tranexamic Acid versus Control (Blood Transfusion \& Blood Loss), Outcome 8 Blood loss - Intra-operative.

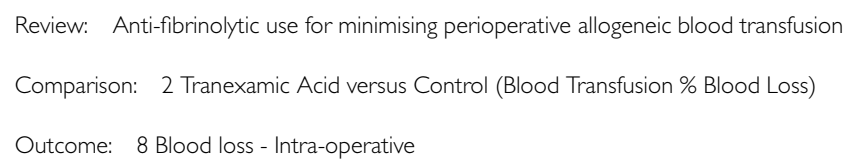

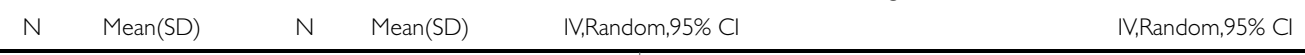

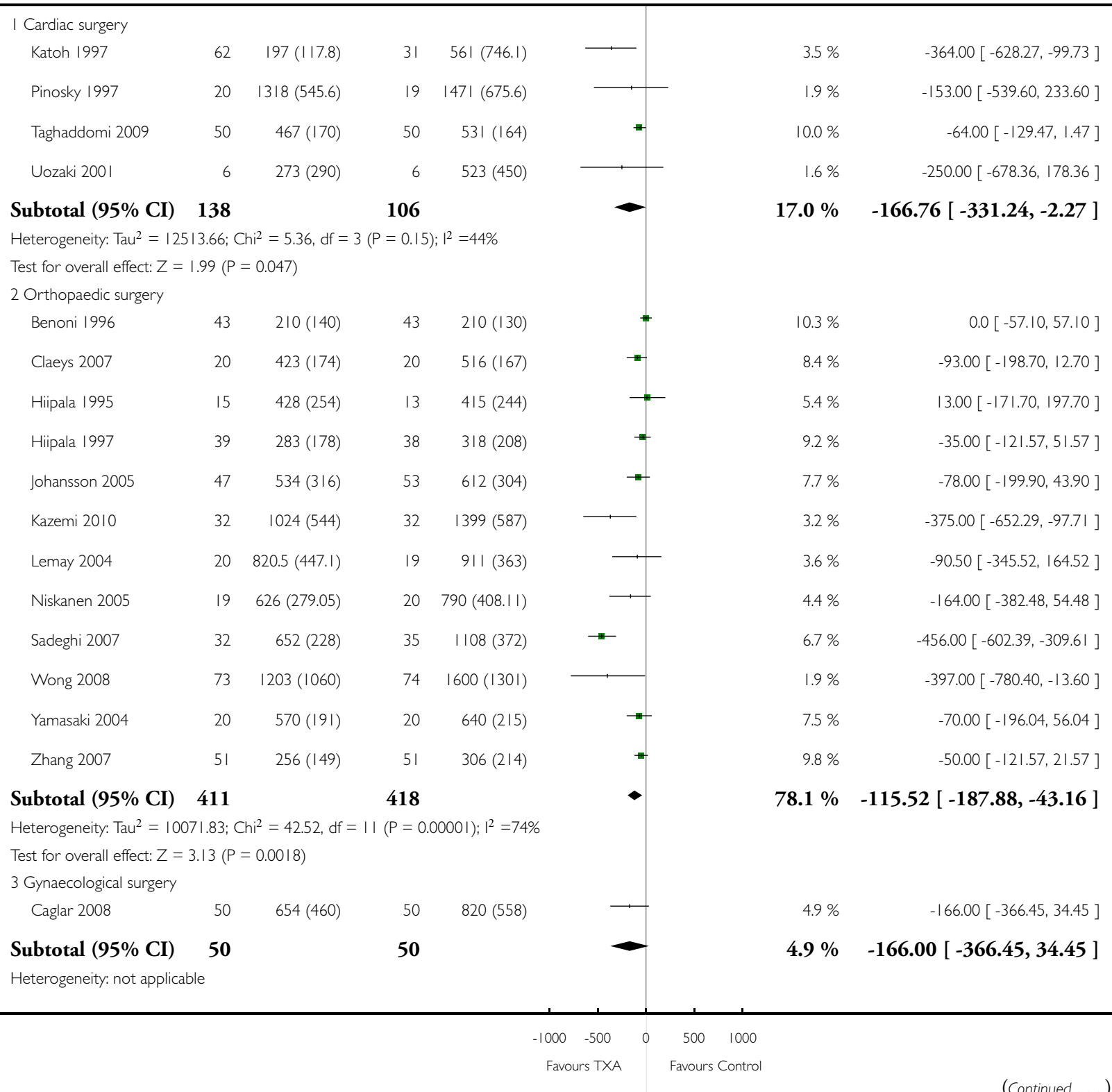


Test for overall effect: $Z=1.62(P=0.10)$

4 Head \% neck surgery

Subtotal (95\% CI)

\section{0}

$\mathbf{0}$

Mean(SD)

IV,Random,95\% Cl

Heterogeneity: not applicable

Test for overall effect: not applicable

Total (95\% CI) $\mathbf{5 9 9}$

$0.0 \%$

$0.0[0.0,0.0]$

Heterogeneity: Tau $^{2}=7861.99 ; \mathrm{Chi}^{2}=49.05, \mathrm{df}=16(\mathrm{P}=0.00003) ; \mathrm{I}^{2}=67 \%$

Test for overall effect: $Z=4.05(P=0.000052)$

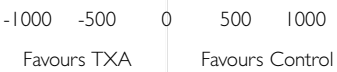

Analysis 2.9. Comparison 2 Tranexamic Acid versus Control (Blood Transfusion \& Blood Loss), Outcome 9 Blood loss - Post-operative.

Review: Anti-fibrinolytic use for minimising perioperative allogeneic blood transfusion

Comparison: 2 Tranexamic Acid versus Control (Blood Transfusion \% Blood Loss)

Outcome: 9 Blood loss - Post-operative

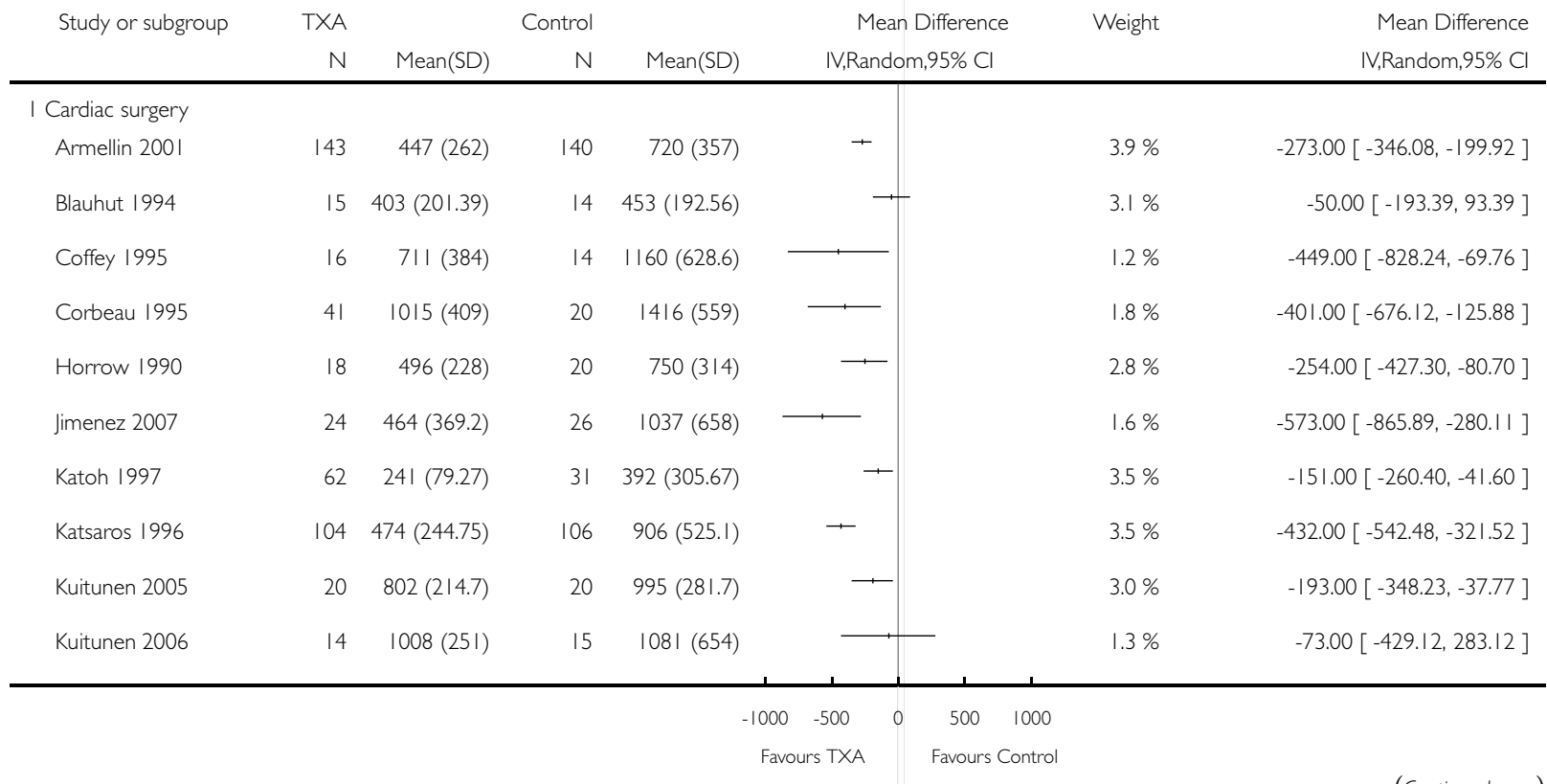




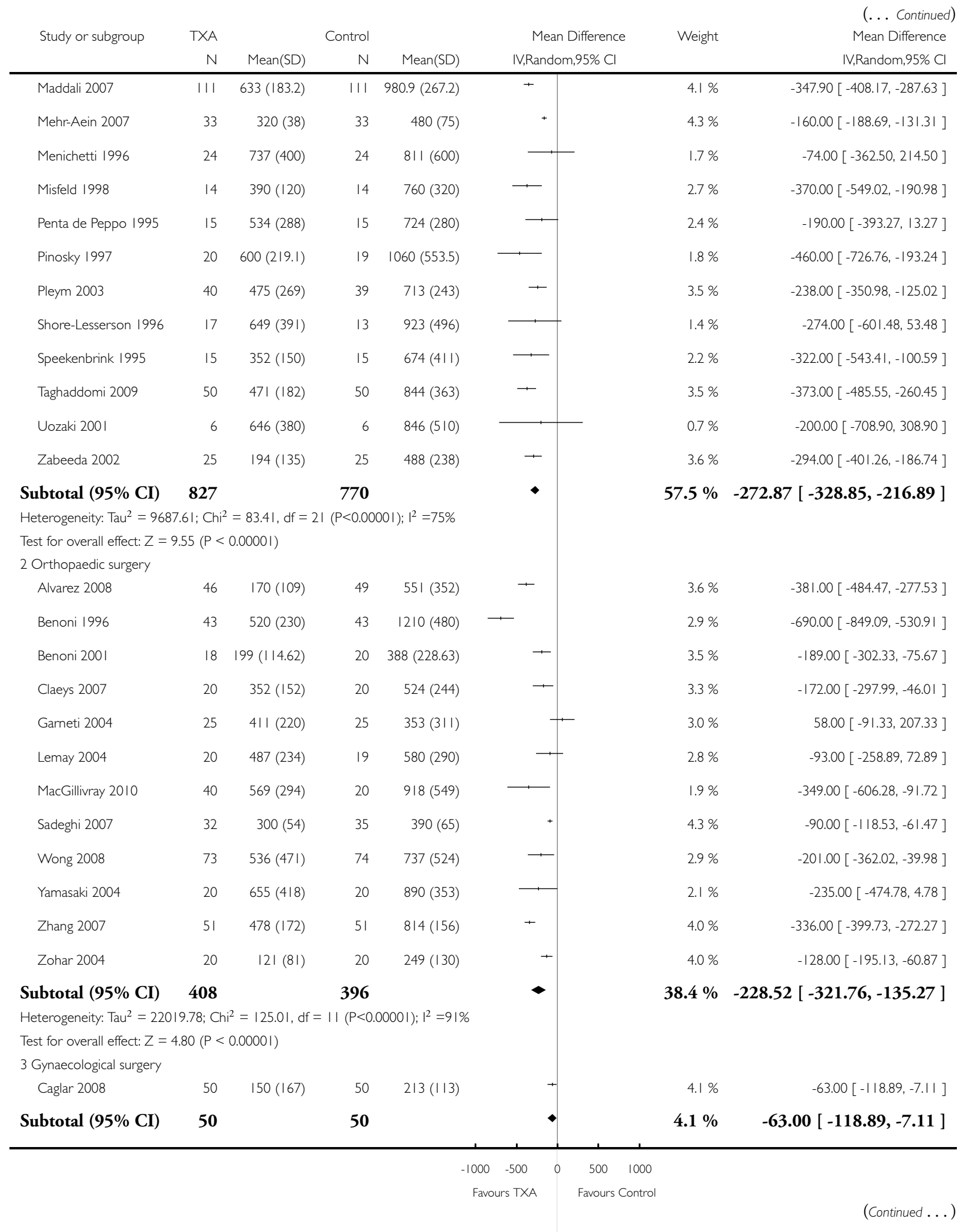

Anti-fibrinolytic use for minimising perioperative allogeneic blood transfusion (Review)

Copyright (@) 20II The Cochrane Collaboration. Published by John Wiley \& Sons, Ltd. 
Heterogeneity: not applicable

Test for overall effect: $Z=2.21(P=0.027)$

4 Head \% neck surgery

Subtotal (95\% CI)

$\mathbf{0}$

0

0

$0.0 \%$

$0.0[0.0,0.0]$

Heterogeneity: not applicable

Test for overall effect: not applicable

Total (95\% CI) 1285

Heterogeneity: $\mathrm{Tau}^{2}=$ I3588.50; $\mathrm{Chi}^{2}=248.36, \mathrm{df}=34(\mathrm{P}<0.0000 \mathrm{I}) ; \mathrm{I}^{2}=86 \%$

Test for overall effect: $Z=10.18(P<0.00001)$

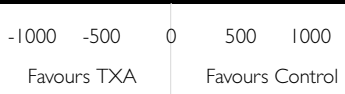

Analysis 2.10. Comparison 2 Tranexamic Acid versus Control (Blood Transfusion \& Blood Loss), Outcome 10 Blood loss - Post-operative - Dose (Cardiac Surgery).

Review: Anti-fibrinolytic use for minimising perioperative allogeneic blood transfusion

Comparison: 2 Tranexamic Acid versus Control (Blood Transfusion \% Blood Loss)

Outcome: 10 Blood loss - Post-operative - Dose (Cardiac Surgery)

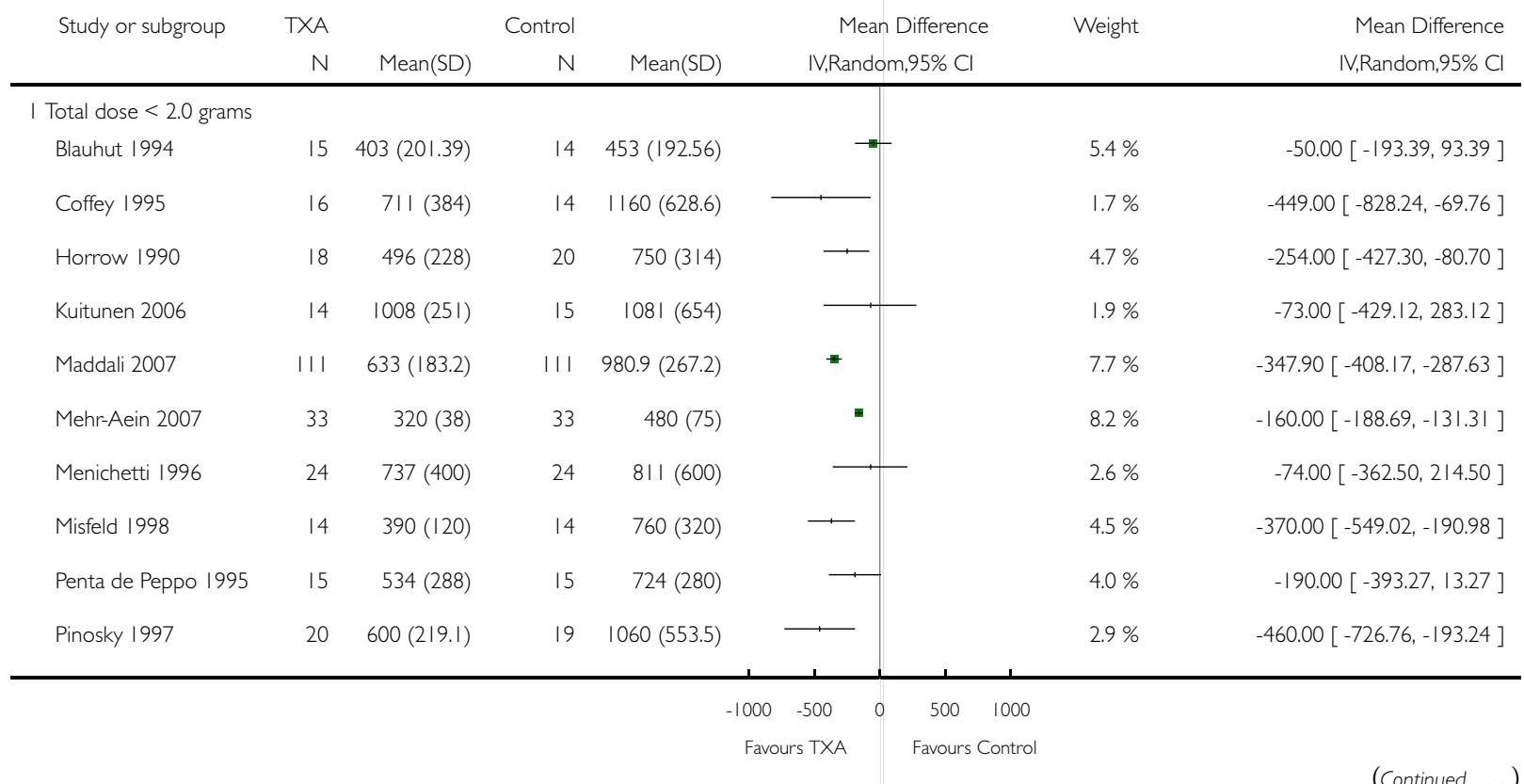




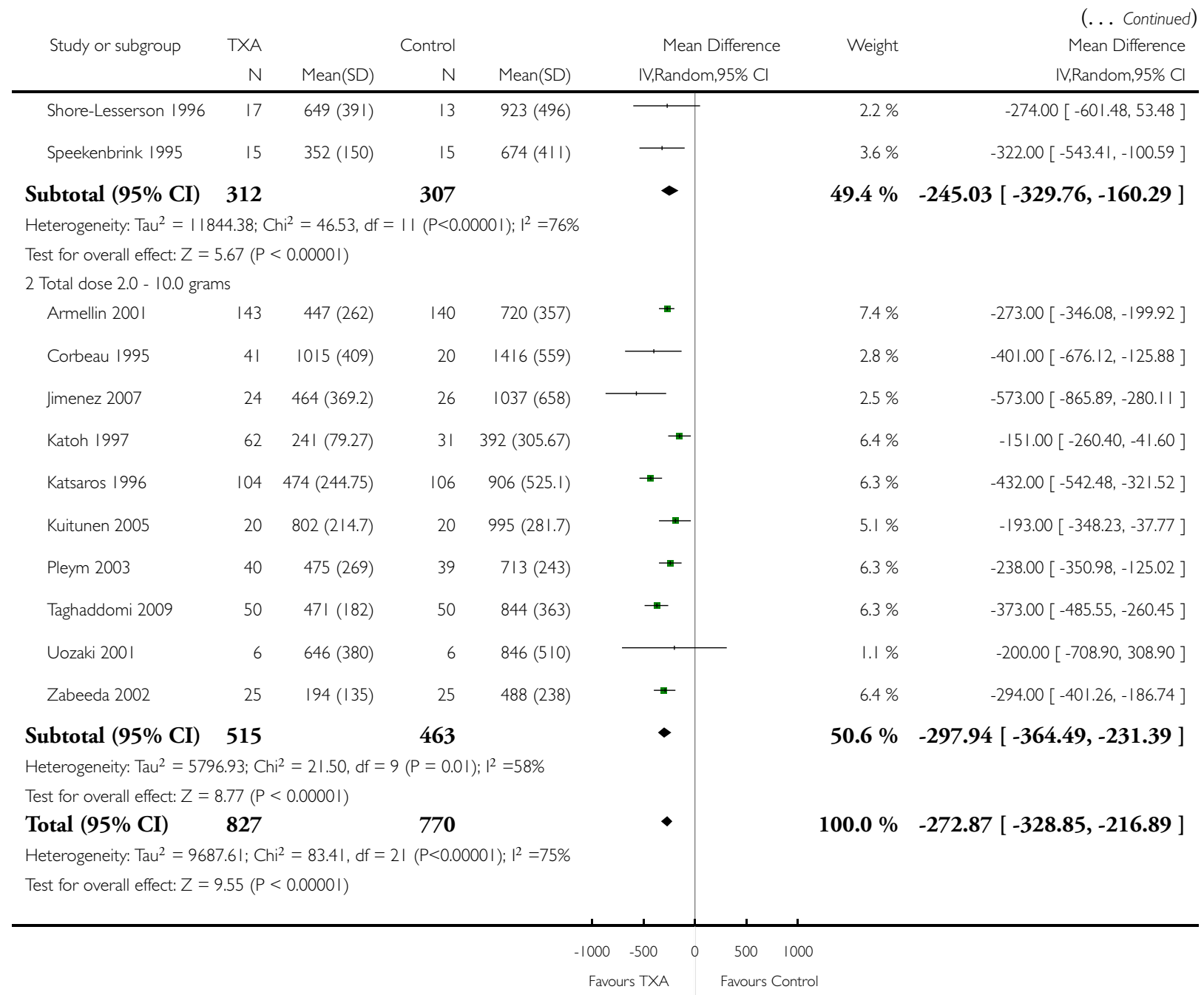




\section{Analysis 2.1 I. Comparison 2 Tranexamic Acid versus Control (Blood Transfusion \& Blood Loss), Outcome I I Blood loss - Total.}

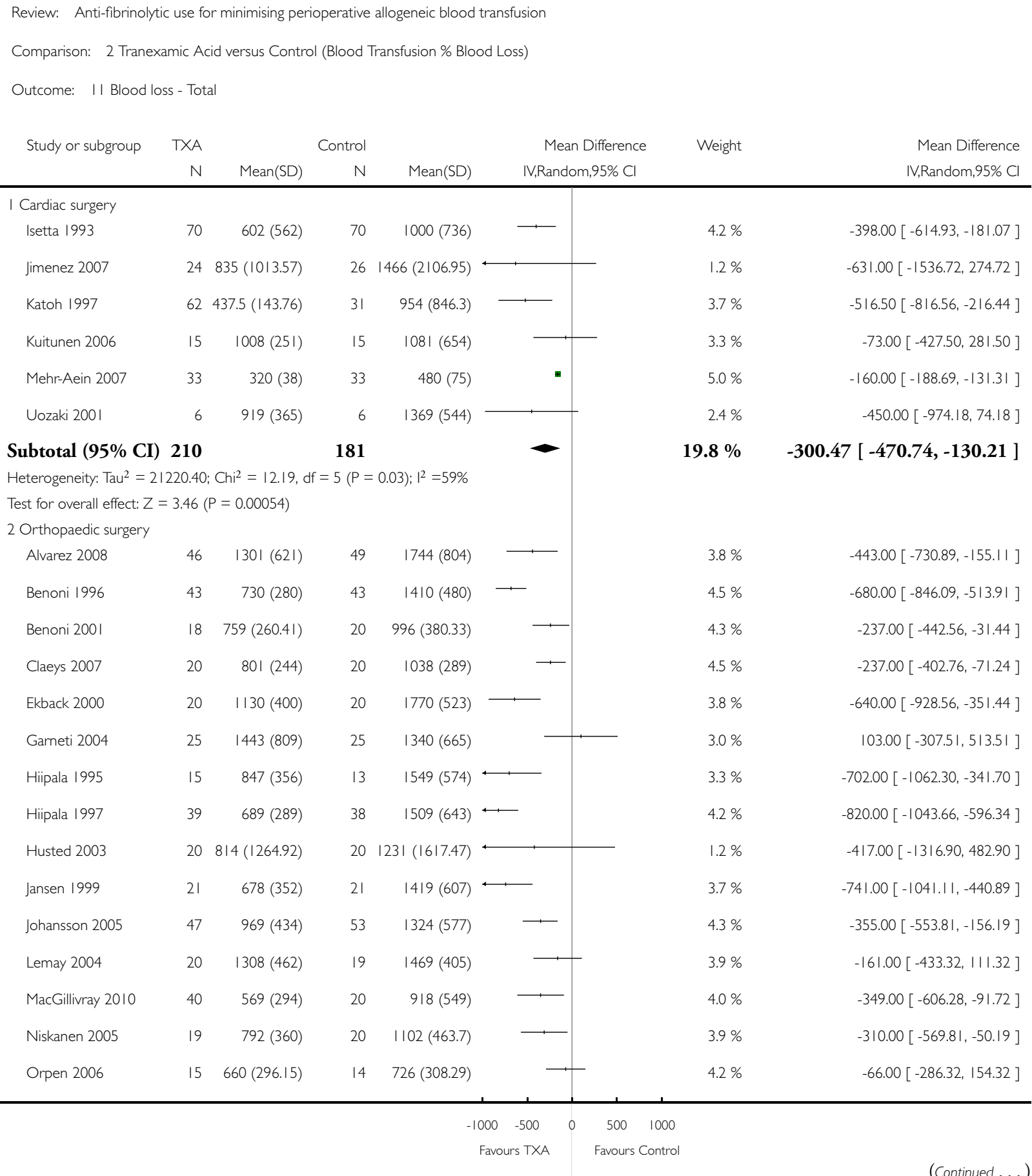




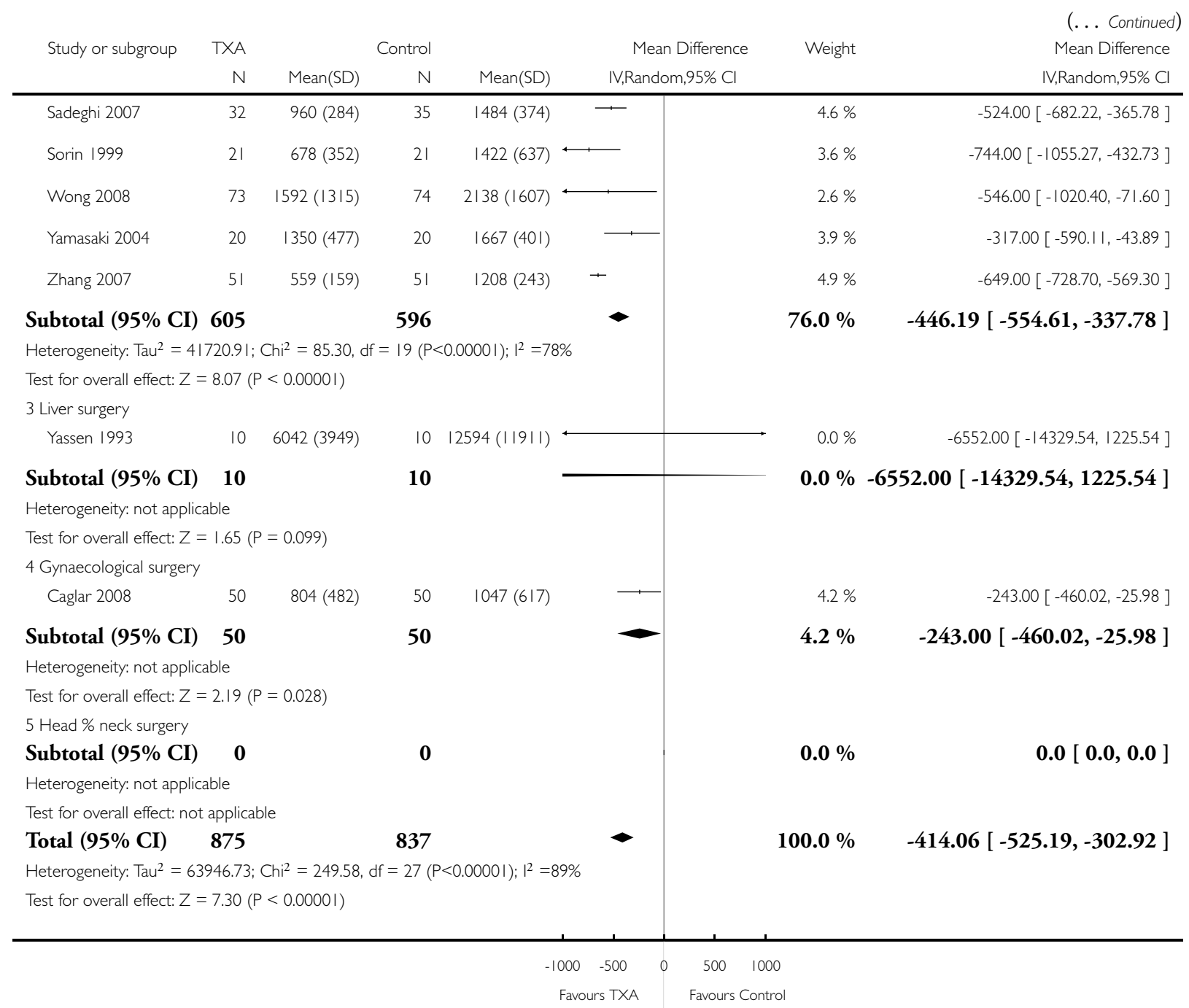


Analysis 3.I. Comparison 3 Epsilon Aminocaproic Acid versus Control (Blood Transfusion \& Blood Loss), Outcome I No. Exposed to Allogeneic Blood.

Review: Anti-fibrinolytic use for minimising perioperative allogeneic blood transfusion

Comparison: 3 Epsilon Aminocaproic Acid versus Control (Blood Transfusion \% Blood Loss)

Outcome: I No. Exposed to Allogeneic Blood

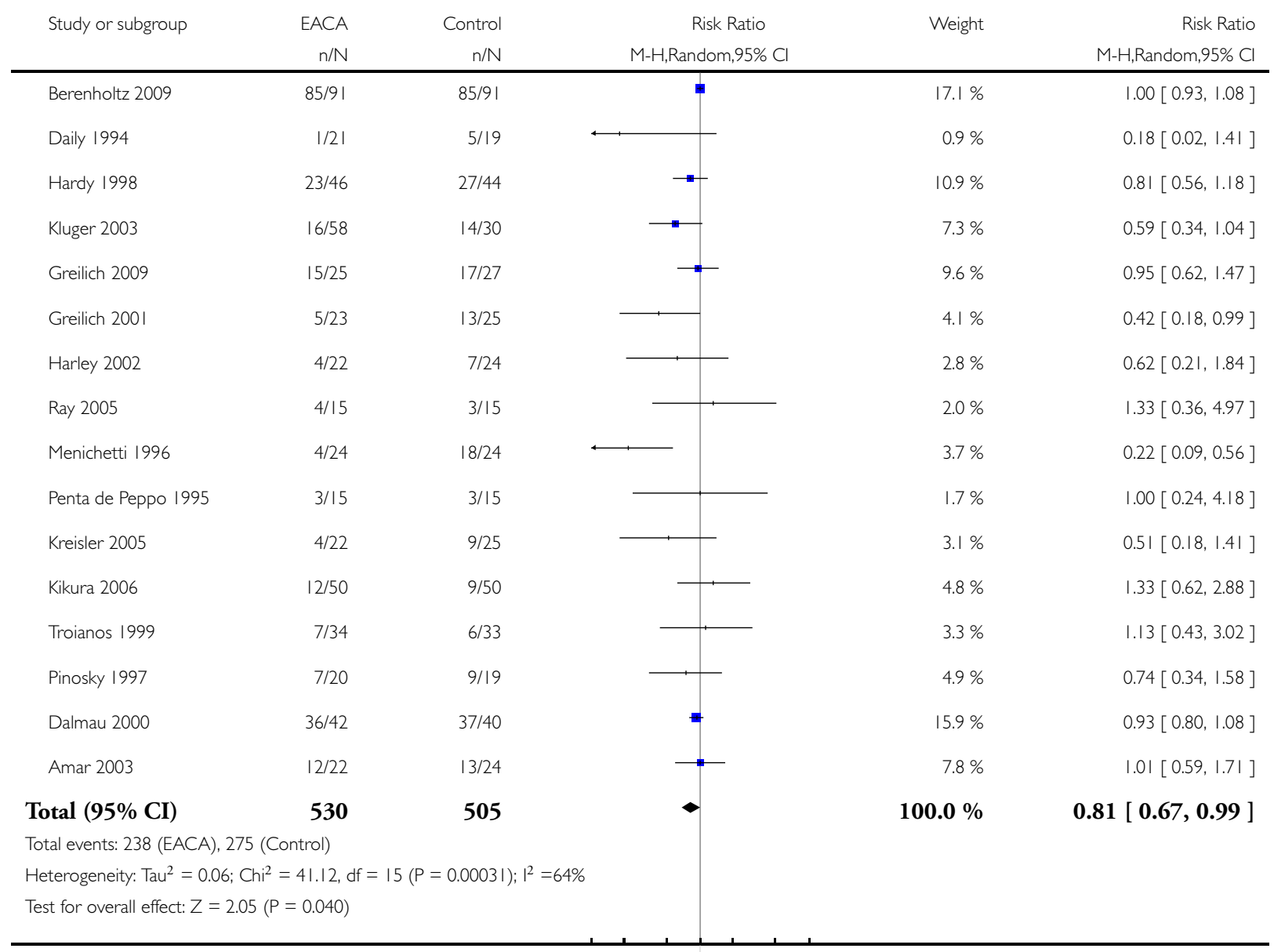

$\begin{array}{lllllll}0.1 & 0.2 & 0.5 & 1 & 2 & 5 & 10\end{array}$

Favours EACA Favours Control 
Analysis 3.2. Comparison 3 Epsilon Aminocaproic Acid versus Control (Blood Transfusion \& Blood Loss), Outcome 2 No. Exposed to Allogeneic Blood - Type of Surgery.

Review: Anti-fibrinolytic use for minimising perioperative allogeneic blood transfusion

Comparison: 3 Epsilon Aminocaproic Acid versus Control (Blood Transfusion \% Blood Loss)

Outcome: 2 No. Exposed to Allogeneic Blood - Type of Surgery

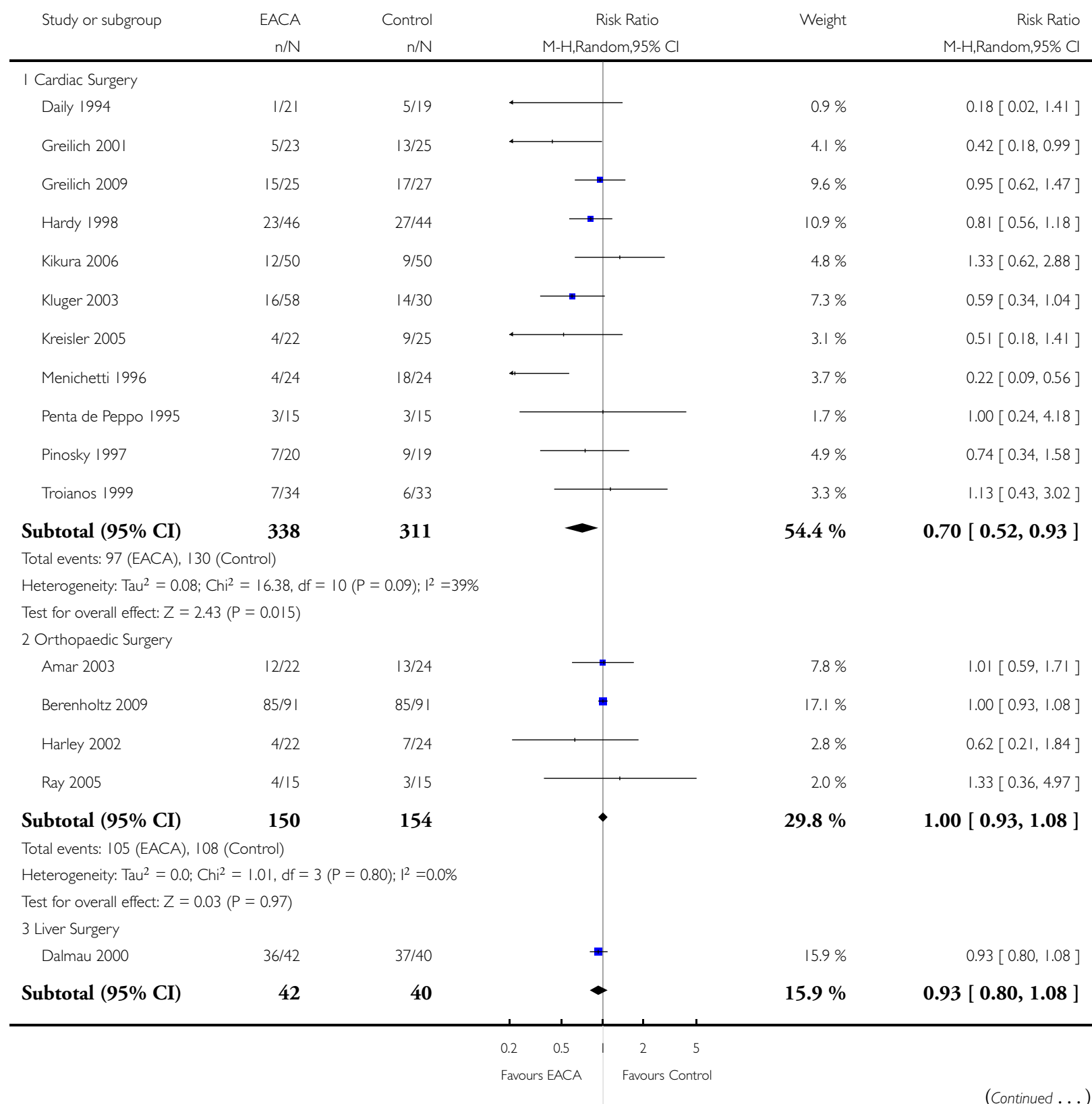




\begin{tabular}{|c|c|c|c|c|c|}
\hline Study or subgroup & $\begin{array}{r}\text { EACA } \\
n / N\end{array}$ & $\begin{array}{r}\text { Control } \\
\mathrm{n} / \mathrm{N}\end{array}$ & $\begin{array}{c}\text { Risk Ratio } \\
\text { M-H,Random,95\% Cl}\end{array}$ & Weight & $\begin{array}{r}\text { (. . Continued } \\
\text { Risk Ratio } \\
\text { M-H,Random, } 95 \% \text { Cl }\end{array}$ \\
\hline \multicolumn{6}{|c|}{ Total events: 36 (EACA), 37 (Control) } \\
\hline \multicolumn{6}{|c|}{ Heterogeneity: not applicable } \\
\hline \multicolumn{6}{|c|}{ Test for overall effect: $Z=0.98(P=0.33)$} \\
\hline Total $(95 \% \mathrm{CI})$ & 530 & 505 & - & $100.0 \%$ & $0.81[0.67,0.99]$ \\
\hline \multicolumn{6}{|c|}{ Total events: 238 (EACA), 275 (Control) } \\
\hline \multicolumn{6}{|c|}{ Heterogeneity: $\mathrm{Tau}^{2}=0.06 ; \mathrm{Ch}^{2}=41.12, \mathrm{df}=15(P=0.0003 \mathrm{I}) ; \mathrm{I}^{2}=64 \%$} \\
\hline \multicolumn{6}{|c|}{ Test for overall effect: $Z=2.05(P=0.040)$} \\
\hline
\end{tabular}

Analysis 3.3. Comparison 3 Epsilon Aminocaproic Acid versus Control (Blood Transfusion \& Blood Loss), Outcome 3 No. Exposed to Allogeneic Blood - Transfusion Protocol.

Review: Anti-fibrinolytic use for minimising perioperative allogeneic blood transfusion

Comparison: 3 Epsilon Aminocaproic Acid versus Control (Blood Transfusion \% Blood Loss)

Outcome: 3 No. Exposed to Allogeneic Blood - Transfusion Protocol

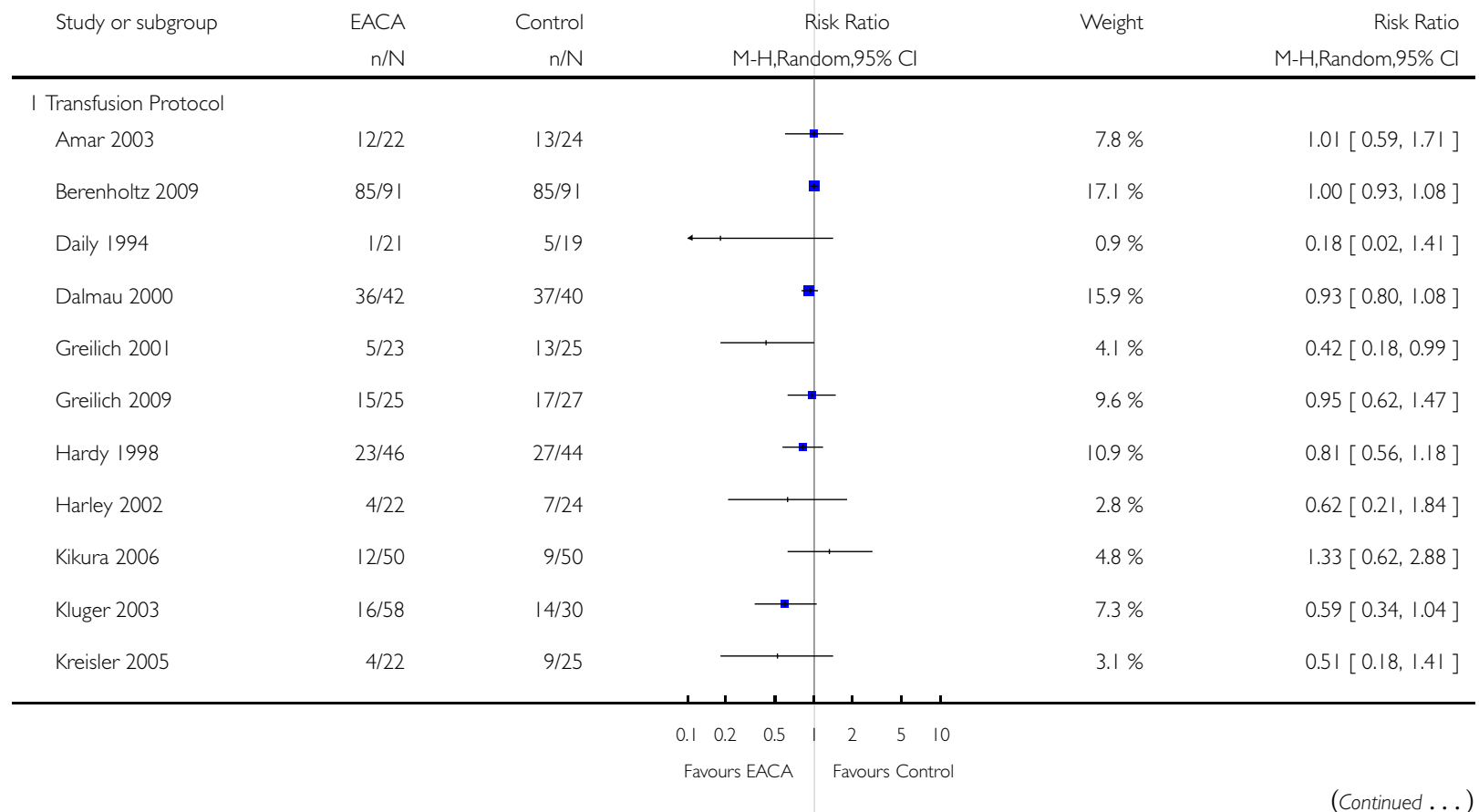




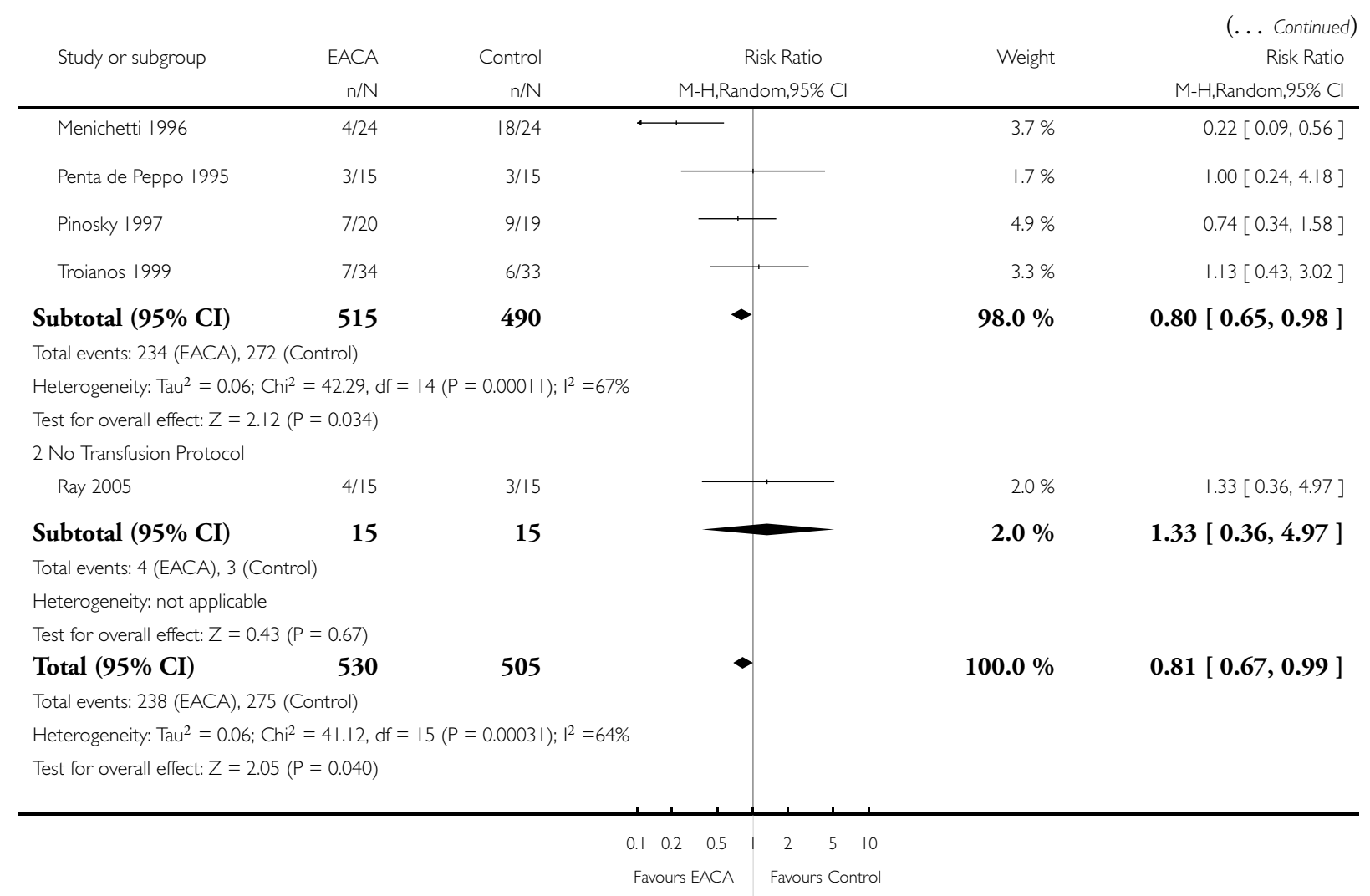


Analysis 3.4. Comparison 3 Epsilon Aminocaproic Acid versus Control (Blood Transfusion \& Blood Loss), Outcome 4 Trial Methodological Quality - Allocation Concealment.

Review: Anti-fibrinolytic use for minimising perioperative allogeneic blood transfusion

Comparison: 3 Epsilon Aminocaproic Acid versus Control (Blood Transfusion \% Blood Loss)

Outcome: 4 Trial Methodological Quality - Allocation Concealment

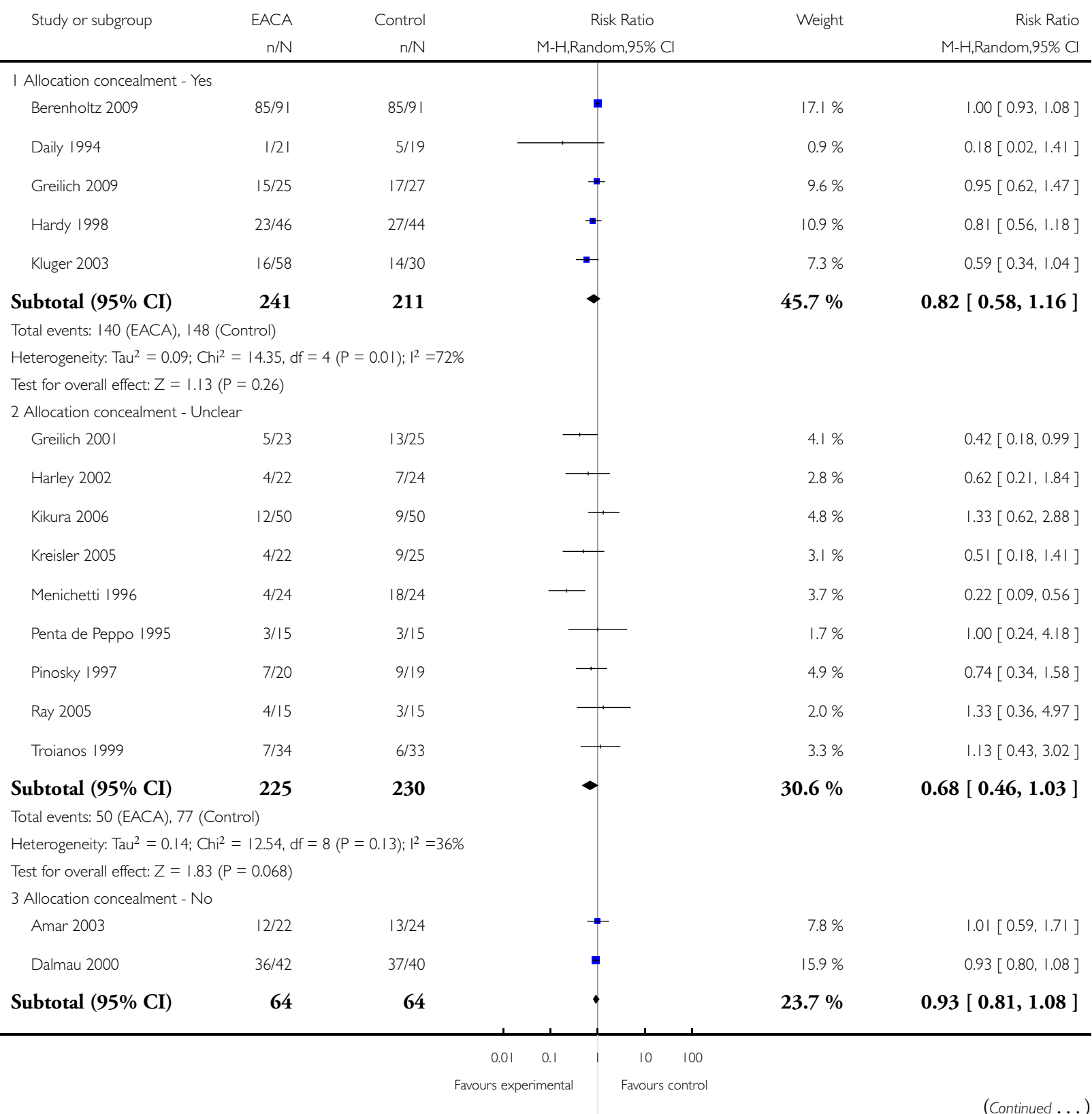




\begin{tabular}{|c|c|c|c|c|c|c|}
\hline Study or subgroup & $\begin{array}{r}\text { EACA } \\
n / N\end{array}$ & $\begin{array}{r}\text { Control } \\
n / N\end{array}$ & \multicolumn{2}{|c|}{ Risk Ratio } & Weight & $\begin{array}{r}\text { (... Continued }) \\
\text { Risk Ratio } \\
\text { M-H,Random,95\% Cl }\end{array}$ \\
\hline \multicolumn{7}{|c|}{ Total events: 48 (EACA), 50 (Control) } \\
\hline \multicolumn{7}{|c|}{ Heterogeneity: $\operatorname{Tau}^{2}=0.0 ; \mathrm{Chi}^{2}=0.13, \mathrm{df}=1(\mathrm{P}=0.72) ; \mathrm{I}^{2}=0.0 \%$} \\
\hline \multicolumn{7}{|c|}{ Test for overall effect: $Z=0.94(P=0.35)$} \\
\hline Total $(95 \% \mathrm{CI})$ & 530 & 505 & $\cdot$ & & $100.0 \%$ & $0.81[0.67,0.99]$ \\
\hline \multicolumn{7}{|c|}{ Total events: 238 (EACA), 275 (Control) } \\
\hline \multicolumn{7}{|c|}{ Heterogeneity: $\mathrm{Tau}^{2}=0.06 ; \mathrm{Ch}^{2}=41.12, \mathrm{df}=15(P=0.0003 \mathrm{I}) ; \mathrm{I}^{2}=64 \%$} \\
\hline \multicolumn{7}{|c|}{ Test for overall effect: $Z=2.05(P=0.040)$} \\
\hline
\end{tabular}

\section{Analysis 3.5. Comparison 3 Epsilon Aminocaproic Acid versus Control (Blood Transfusion \& Blood Loss),} Outcome 5 Units of Allogeneic Blood Transfused - Transfused Patients.

Review: Anti-fibrinolytic use for minimising perioperative allogeneic blood transfusion

Comparison: 3 Epsilon Aminocaproic Acid versus Control (Blood Transfusion \% Blood Loss)

Outcome: 5 Units of Allogeneic Blood Transfused - Transfused Patients

\begin{tabular}{|c|c|c|c|c|c|c|c|}
\hline \multirow[t]{2}{*}{ Study or subgroup } & \multirow{2}{*}{$\begin{array}{r}\text { EACA } \\
N\end{array}$} & \multicolumn{3}{|c|}{ Control } & \multirow{2}{*}{$\begin{array}{l}\text { Mean Difference } \\
\text { IV,Random,95\% Cl }\end{array}$} & \multirow[t]{2}{*}{ Weight } & \multirow{2}{*}{$\begin{array}{l}\text { Mean Difference } \\
\text { IV,Random,95\% Cl }\end{array}$} \\
\hline & & Mean(SD) & $N$ & Mean(SD) & & & \\
\hline Amar 2003 & 12 & $3.12(2.67)$ & 13 & $3.32(2.55)$ & $\longrightarrow$ & $7.7 \%$ & $-0.20[-2.25,1.85]$ \\
\hline Dalmau 2000 & 36 & $7.81(5.67)$ & 37 & $8.38(6.13)$ & 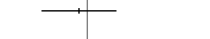 & $4.4 \%$ & $-0.57[-3.28,2.14]$ \\
\hline Kikura 2006 & 12 & $2.2(0.7)$ & 9 & $1.9(0.7)$ & & $88.0 \%$ & $0.30[-0.30,0.90]$ \\
\hline
\end{tabular}

Total (95\% CI)

\section{0}

59

$100.0 \% \quad 0.22[-0.34,0.79]$

Heterogeneity: $\mathrm{Tau}^{2}=0.0 ; \mathrm{Chi}^{2}=0.56, \mathrm{df}=2(\mathrm{P}=0.76) ; \mathrm{I}^{2}=0.0 \%$

Test for overall effect: $Z=0.77(P=0.44)$

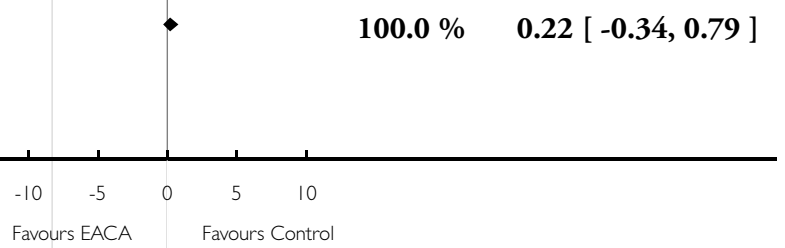


Analysis 3.6. Comparison 3 Epsilon Aminocaproic Acid versus Control (Blood Transfusion \& Blood Loss), Outcome 6 Units of Allogeneic Blood Transfused - All Patients.

Review: Anti-fibrinolytic use for minimising perioperative allogeneic blood transfusion

Comparison: 3 Epsilon Aminocaproic Acid versus Control (Blood Transfusion \% Blood Loss)

Outcome: 6 Units of Allogeneic Blood Transfused - All Patients

\begin{tabular}{|c|c|c|c|c|c|c|c|c|}
\hline \multirow[t]{2}{*}{ Study or subgroup } & \multirow{2}{*}{$\begin{array}{r}\text { EACA } \\
N\end{array}$} & \multicolumn{3}{|c|}{ Control } & & \multirow{2}{*}{$\begin{array}{l}\text { Mean Difference } \\
\text { IV,Random,95\% Cl }\end{array}$} & \multirow[t]{2}{*}{ Weight } & \multirow{2}{*}{$\begin{array}{l}\text { Mean Difference } \\
\text { IV,Random,95\% Cl }\end{array}$} \\
\hline & & Mean(SD) & $N$ & Mean(SD) & & & & \\
\hline Amar 2003 & 22 & $1.7(2.5)$ & 24 & $1.8(2.5)$ & & $\rightarrow$ & $14.5 \%$ & $-0.10[-1.55,1.35]$ \\
\hline Berenholtz 2009 & 91 & $5.9(4.7)$ & 91 & $6.9(5.4)$ & & $\rightarrow$ & $14.3 \%$ & $-1.00[-2.47,0.47]$ \\
\hline Dalmau 2000 & 42 & $6.69(5.92)$ & 40 & $7.75(6.3)$ & & $\longrightarrow$ & $7.2 \%$ & $-1.06[-3.71,1.59]$ \\
\hline Greilich 2009 & 25 & $1.7(1.8)$ & 27 & $1.8(2)$ & & 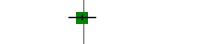 & $18.3 \%$ & $-0.10[-1.13,0.93]$ \\
\hline Liu 1998 & 20 & $6.8(1.2)$ & 20 & $9.4(1.1)$ & & \# & $21.4 \%$ & $-2.60[-3.31,-1.89]$ \\
\hline Rao 1999 & 15 & $1.2(0.4)$ & 15 & $3.2(0.45)$ & & $\mathbf{m}$ & $24.4 \%$ & $-2.00[-2.30,-1.70]$ \\
\hline Total (95\% CI) & 215 & & 217 & & & - & $100.0 \%$ & $-1.30[-2.14,-0.45]$ \\
\hline \multicolumn{9}{|c|}{ Heterogeneity: $\operatorname{Tau}^{2}=0.73 ; \mathrm{Chi}^{2}=23.45, \mathrm{df}=5(\mathrm{P}=0.00028) ; \mathrm{I}^{2}=79 \%$} \\
\hline & & & & & -10 & -5 & 10 & \\
\hline & & & & & Favou & Favours $\mathrm{Co}$ & ontrol & \\
\hline
\end{tabular}


Analysis 3.7. Comparison 3 Epsilon Aminocaproic Acid versus Control (Blood Transfusion \& Blood Loss), Outcome 7 Blood loss - Intra-operative.

Review: Anti-fibrinolytic use for minimising perioperative allogeneic blood transfusion

Comparison: 3 Epsilon Aminocaproic Acid versus Control (Blood Transfusion \% Blood Loss)

Outcome: 7 Blood loss - Intra-operative

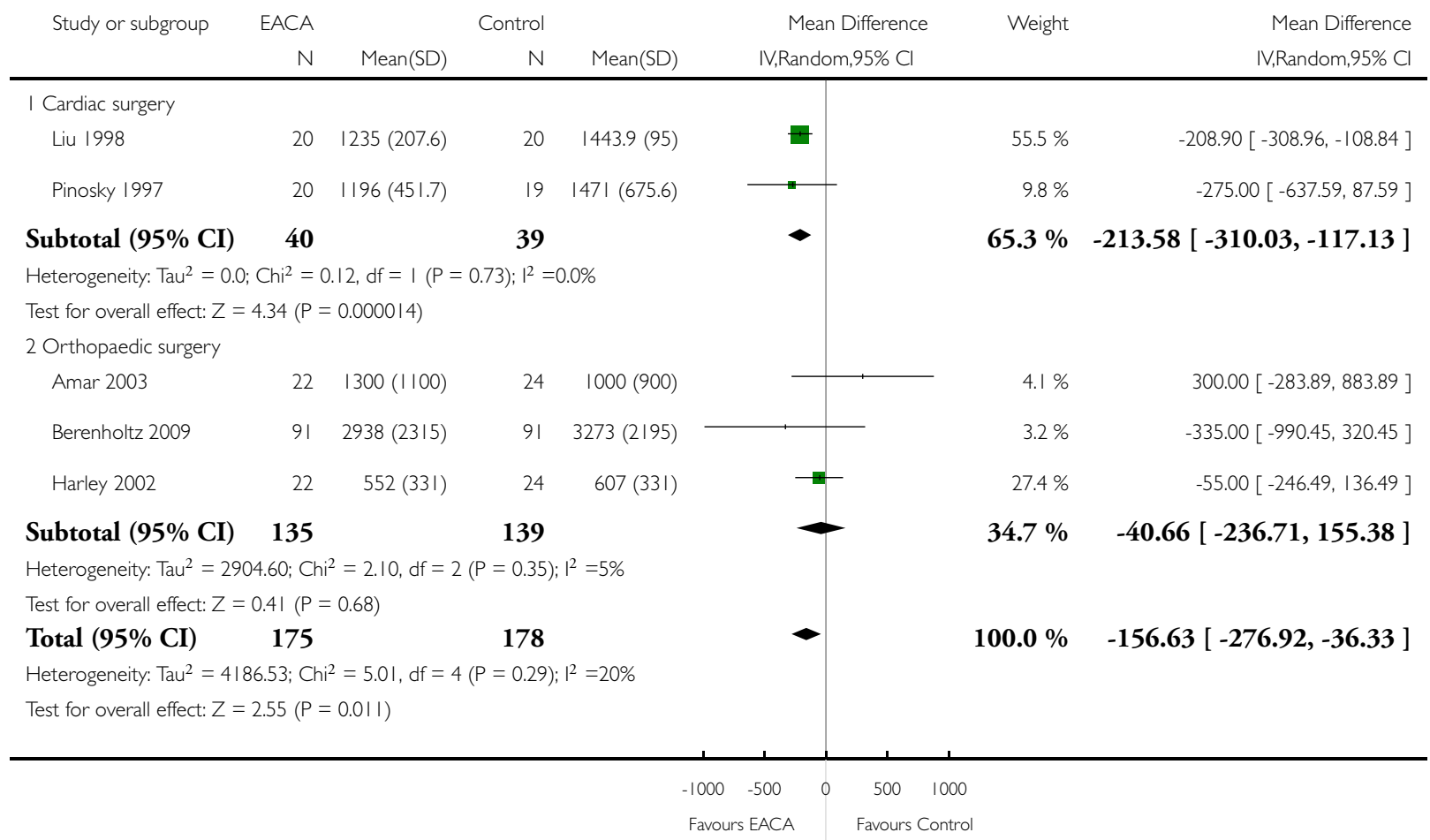


Analysis 3.8. Comparison 3 Epsilon Aminocaproic Acid versus Control (Blood Transfusion \& Blood Loss), Outcome 8 Blood loss - Post-operative.

Review: Anti-fibrinolytic use for minimising perioperative allogeneic blood transfusion

Comparison: 3 Epsilon Aminocaproic Acid versus Control (Blood Transfusion \% Blood Loss)

Outcome: 8 Blood loss - Post-operative

\begin{tabular}{|c|c|c|c|c|c|c|c|}
\hline \multirow[t]{2}{*}{ Study or subgroup } & \multirow{2}{*}{$\begin{array}{r}\text { EACA } \\
N\end{array}$} & \multicolumn{3}{|c|}{ Control } & \multirow{2}{*}{$\begin{array}{l}\text { Mean Difference } \\
\text { IV,Random,95\% Cl }\end{array}$} & \multirow[t]{2}{*}{ Weight } & \multirow{2}{*}{$\begin{array}{l}\text { Mean Difference } \\
\text { IV,Random,95\% Cl }\end{array}$} \\
\hline & & Mean(SD) & N & Mean(SD) & & & \\
\hline \multicolumn{8}{|l|}{ I Cardiac surgery } \\
\hline Daily 1994 & 21 & $623(380)$ & 19 & 845 (339) & $\longrightarrow-$ & $5.5 \%$ & $-222.00[-444.82,0.82]$ \\
\hline Del Rossi 1989 & 170 & $617.2(43.9)$ & 180 & $883.2(29.3)$ & m & $12.7 \%$ & $-266.00[-273.87,-258.13]$ \\
\hline Greilich 2009 & 25 & $715(394)$ & 27 & $1002(627)$ & 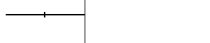 & $4.1 \%$ & $-287.00[-569.46,-4.54]$ \\
\hline Kikura 2006 & 50 & $650(261)$ & 50 & $940(627)$ & 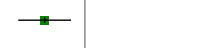 & $6.5 \%$ & $-290.00[-478.25,-101.75]$ \\
\hline Kreisler 2005 & 22 & $269(113)$ & 25 & $284(128)$ & 7 & $11.2 \%$ & $-15.00[-83.90,53.90]$ \\
\hline Liu 1998 & 20 & $987.2(103.7)$ & 20 & $1298.3(143.2)$ & $\varpi$ & $10.9 \%$ & $-311.10[-388.59,-233.61]$ \\
\hline Menichetti 1996 & 24 & $512(250)$ & 24 & $811(600)$ & 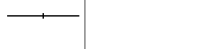 & $4.5 \%$ & $-299.00[-559.05,-38.95]$ \\
\hline Penta de Peppo 1995 & 15 & $509(148)$ & 15 & $724(280)$ & $\rightarrow$ & $7.5 \%$ & $-215.00[-375.27,-54.73]$ \\
\hline Pinosky 1997 & 20 & $961(661.9)$ & 19 & $1060(553.5)$ & & $2.6 \%$ & $-99.00[-481.22,283.22]$ \\
\hline Rao 1999 & 15 & $372(55.5)$ & 15 & $494(62.4)$ & $\#$ & $12.1 \%$ & $-122.00[-164.26,-79.74]$ \\
\hline Troianos 1999 & 34 & $529(24 I)$ & 33 & $691(286)$ & 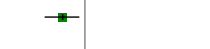 & $8.9 \%$ & $-162.00[-288.82,-35.18]$ \\
\hline Vander-Salm 1996 & 51 & $647(488)$ & 52 & $839(634)$ & $\longrightarrow$ & $5.6 \%$ & $-192.00[-410.25,26.25]$ \\
\hline Subtotal (95\% CI) & 467 & & 479 & & $\bullet$ & $92.0 \%$ & $-200.27[-273.44,-127.09]$ \\
\hline \multicolumn{8}{|c|}{ Heterogeneity: $\mathrm{Tau}^{2}=10265.78 ; \mathrm{Chi}^{2}=97.18, \mathrm{df}=||(\mathrm{P}<0.0000 \mathrm{I}) ;\left.\right|^{2}=89 \%$} \\
\hline \multicolumn{8}{|c|}{ Test for overall effect: $Z=5.36(P<0.0000 I)$} \\
\hline \multicolumn{8}{|l|}{2 Orthopaedic surgery } \\
\hline Berenholtz 2009 & 91 & $3265(2416)$ & 91 & $3695(234 I)$ & & $0.9 \%$ & $-430.00[-1121.19,261.19]$ \\
\hline Harley 2002 & 22 & $315(207)$ & 24 & $591(374)$ & 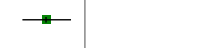 & $7.1 \%$ & $-276.00[-448.83,-103.17]$ \\
\hline Subtotal (95\% CI) & 113 & & 115 & & - & $8.0 \%$ & $-285.06[-452.73,-117.39]$ \\
\hline \multicolumn{8}{|c|}{ Heterogeneity: $\operatorname{Tau}^{2}=0.0 ; \mathrm{Chi}^{2}=0.18, \mathrm{df}=\mathrm{I}(\mathrm{P}=0.67) ; \mathrm{I}^{2}=0.0 \%$} \\
\hline \multicolumn{8}{|c|}{ Test for overall effect: $Z=3.33(P=0.00086)$} \\
\hline Total (95\% CI) & 580 & & 594 & & $\bullet$ & $100.0 \%$ & $-207.49[-276.43,-138.54]$ \\
\hline \multicolumn{8}{|c|}{ Heterogeneity: $\mathrm{Tau}^{2}=9763.54 ; \mathrm{Chi}^{2}=97.46, \mathrm{df}=13(\mathrm{P}<0.0000 \mathrm{I}) ; \mathrm{I}^{2}=87 \%$} \\
\hline Test for overall effect: $Z=$ & $5.90(\mathrm{P}<$ & $=0.00001)$ & & & & & \\
\hline
\end{tabular}

$\begin{array}{rrrrr}-1000 & -500 \quad 0 & 500 \quad 1000\end{array}$

Favours EACA Favours Control 
Analysis 3.9. Comparison 3 Epsilon Aminocaproic Acid versus Control (Blood Transfusion \& Blood Loss), Outcome 9 Blood loss - Total.

Review: Anti-fibrinolytic use for minimising perioperative allogeneic blood transfusion

Comparison: 3 Epsilon Aminocaproic Acid versus Control (Blood Transfusion \% Blood Loss)

Outcome: 9 Blood loss - Total

\begin{tabular}{|c|c|c|c|c|c|c|c|}
\hline \multirow[t]{2}{*}{ Study or subgroup } & \multirow{2}{*}{$\begin{array}{r}\text { EACA } \\
N\end{array}$} & \multicolumn{3}{|c|}{ Control } & \multirow{2}{*}{$\begin{array}{l}\text { Mean Difference } \\
\text { IV,Random,95\% Cl }\end{array}$} & \multirow[t]{2}{*}{ Weight } & \multirow{2}{*}{$\begin{array}{l}\text { Mean Difference } \\
\text { IV,Random,95\% Cl }\end{array}$} \\
\hline & & Mean(SD) & $N$ & Mean(SD) & & & \\
\hline \multicolumn{8}{|c|}{ I Orthopaedic surgery } \\
\hline Amar 2003 & 22 & $1600(1100)$ & 24 & $1600(1400)$ & & $9.5 \%$ & $0.0[-724.57,724.57]$ \\
\hline Harley 2002 & 22 & $867(207)$ & 24 & I 198 (544) & - & $90.5 \%$ & $-331.00[-565.20,-96.80]$ \\
\hline
\end{tabular}

Total (95\% CI) 44

Heterogeneity: $\mathrm{Tau}^{2}=0.0 ; \mathrm{Chi}^{2}=0.73, \mathrm{df}=\mathrm{I}(\mathrm{P}=0.39) ; \mathrm{I}^{2}=0.0 \%$

Test for overall effect: $Z=2.64(P=0.0084)$

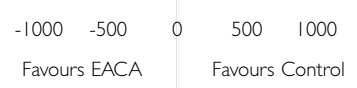

Analysis 4.I. Comparison 4 Aprotinin versus Tranexamic Acid (Blood Transfusion \& Blood Loss), Outcome I No. Exposed to Allogeneic Blood.

Review: Anti-fibrinolytic use for minimising perioperative allogeneic blood transfusion

Comparison: 4 Aprotinin versus Tranexamic Acid (Blood Transfusion \% Blood Loss)

Outcome: I No. Exposed to Allogeneic Blood

\begin{tabular}{|c|c|c|c|c|c|}
\hline \multirow[t]{2}{*}{ Study or subgroup } & Aprotinin & TXA & Risk Ratio & Weight & Risk Ratio \\
\hline & $\mathrm{n} / \mathrm{N}$ & $\mathrm{n} / \mathrm{N}$ & M-H,Random,95\% Cl & & $\mathrm{M}-\mathrm{H}$, Random,95\% Cl \\
\hline Dietrich 2008 & $52 / 110$ & $67 / 110$ & - & $8.0 \%$ & $0.78[0.61,0.99]$ \\
\hline Mansour 2004 & $1 / 20$ & $7 / 20$ & $\leftrightarrow$ & $0.3 \%$ & $0.14[0.02,1.06]$ \\
\hline Fergusson 2008 & $419 / 780$ & $506 / 770$ & - & $12.4 \%$ & $0.82[0.75,0.89]$ \\
\hline Vanek 2005 & $1 / 29$ & $3 / 32$ & & $0.2 \%$ & $0.37[0.04,3.34]$ \\
\hline Mengistu 2008 & $10 / 25$ & $17 / 25$ & & $3.1 \%$ & $0.59[0.34,1.02]$ \\
\hline Menichetti 1996 & $2 / 24$ & $12 / 24$ & & $0.6 \%$ & $0.17[0.04,0.67]$ \\
\hline Corbeau 1995 & $15 / 43$ & $|5 / 4|$ & . & $2.9 \%$ & $0.95[0.54,1.69]$ \\
\hline Blauhut 1994 & $3 / 14$ & $7 / 15$ & - & $0.9 \%$ & $0.46[0.15,1.44]$ \\
\hline
\end{tabular}

$\begin{array}{lllllll}0.1 & 0.2 & 0.5 & 1 & 2 & 5 & 10\end{array}$

Favours Aprotinin Favours TXA

(Continued ... ) 


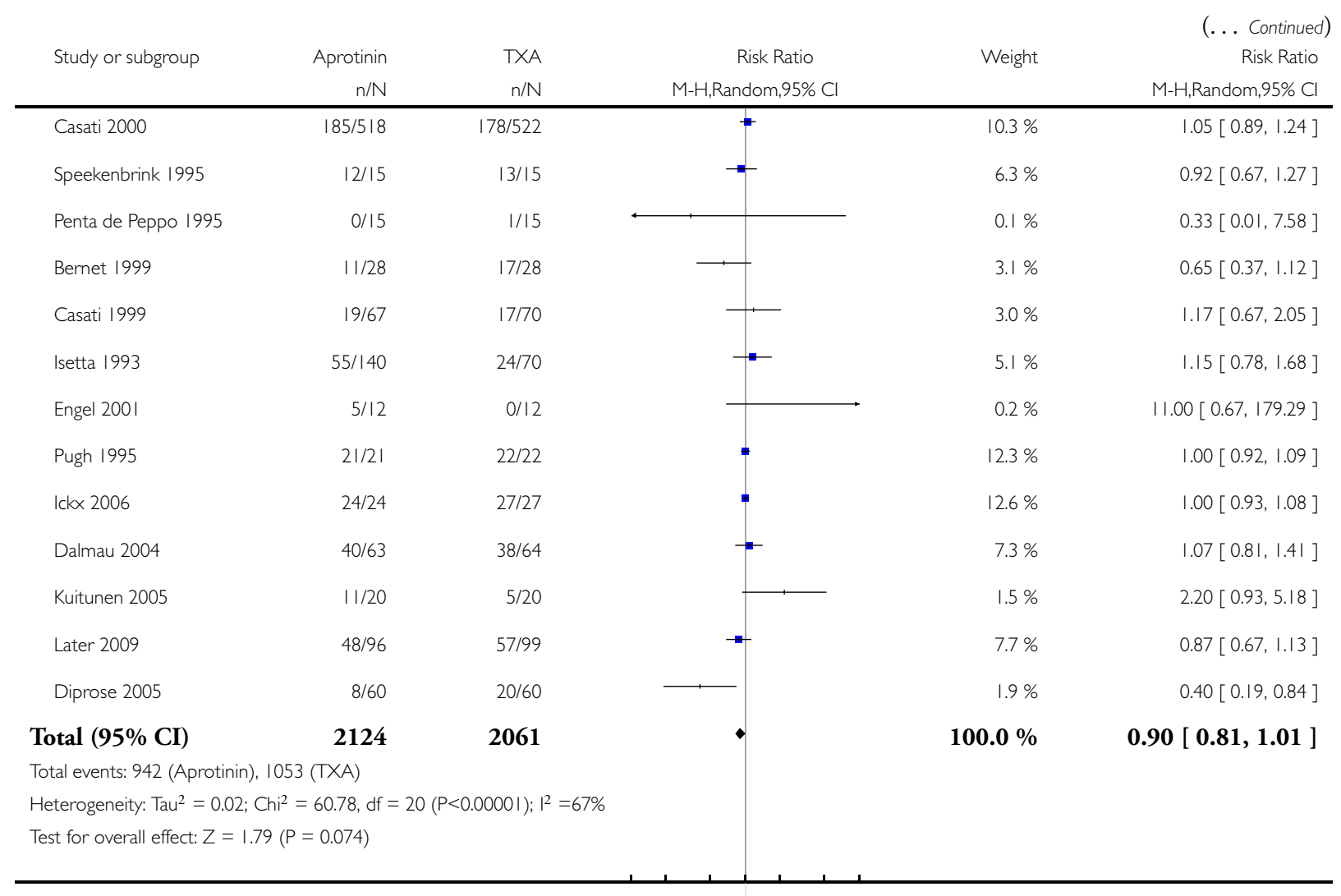

$\begin{array}{lllllll}0.1 & 0.2 & 0.5 & 1 & 2 & 5 & 10\end{array}$

Favours Aprotinin Favours TXA 
Analysis 4.2. Comparison 4 Aprotinin versus Tranexamic Acid (Blood Transfusion \& Blood Loss), Outcome 2 No. Exposed to Allogeneic Blood - Type of Surgery.

Review: Anti-fibrinolytic use for minimising perioperative allogeneic blood transfusion

Comparison: 4 Aprotinin versus Tranexamic Acid (Blood Transfusion \% Blood Loss)

Outcome: 2 No. Exposed to Allogeneic Blood - Type of Surgery

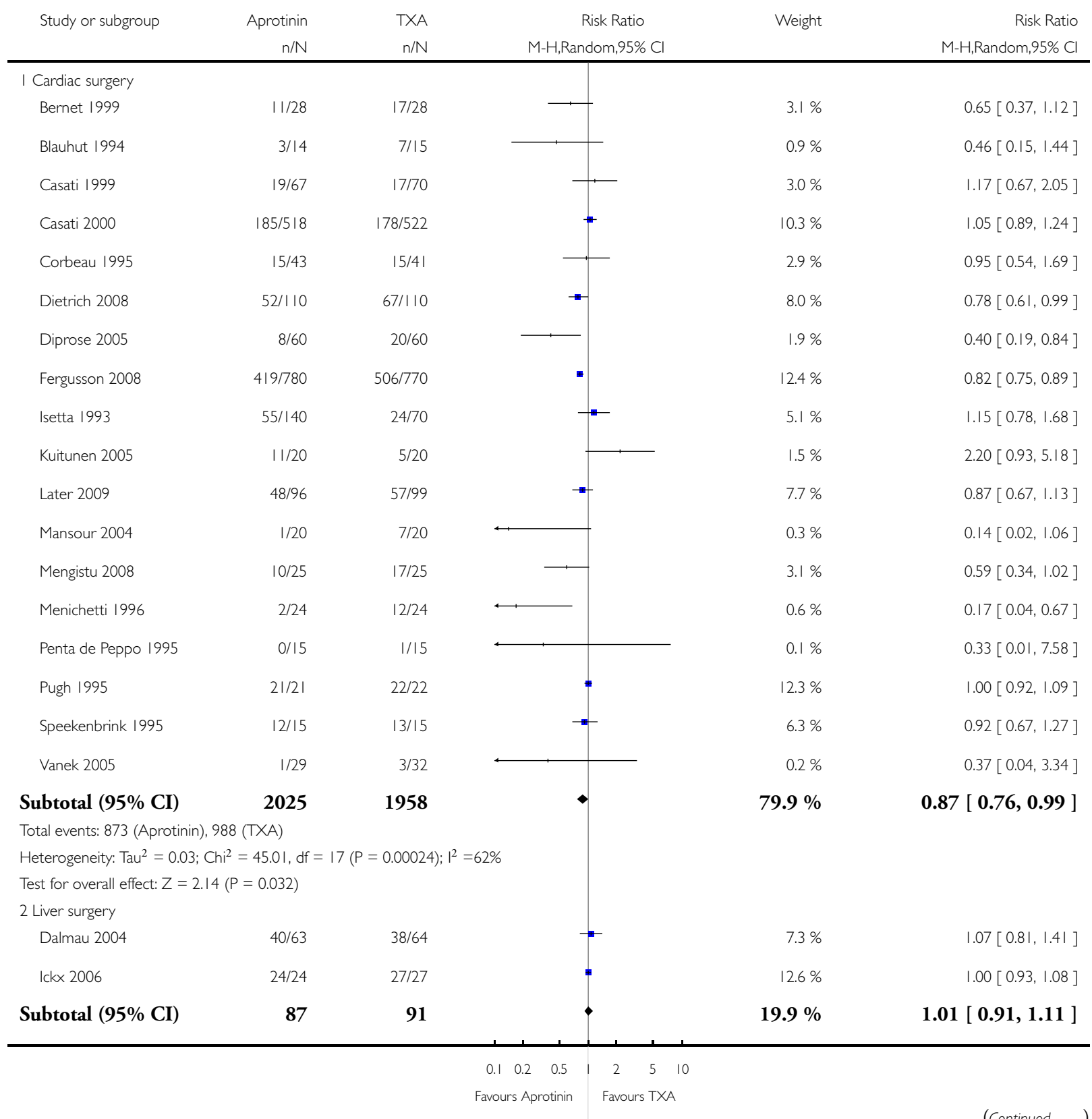




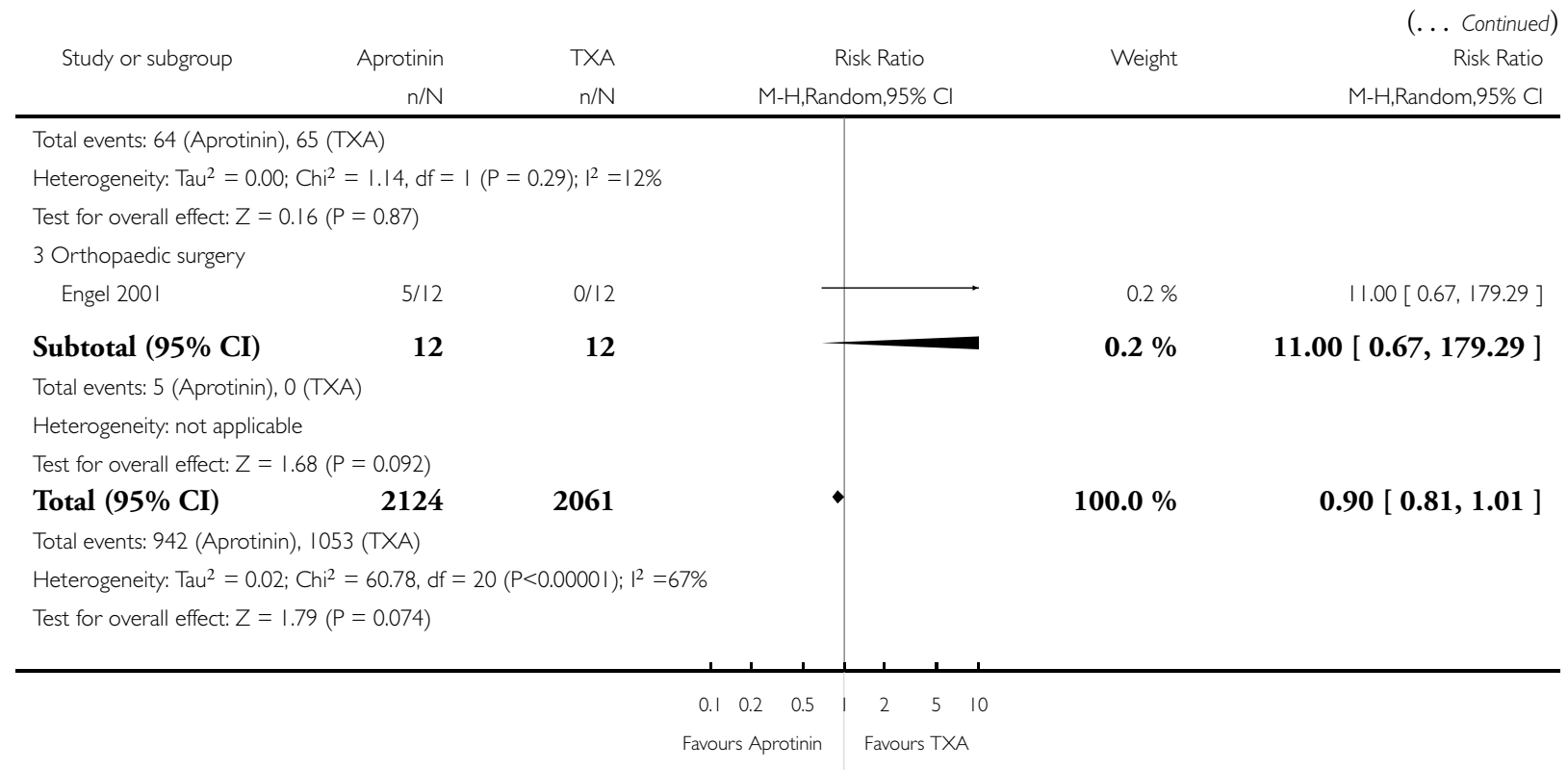

Analysis 4.3. Comparison 4 Aprotinin versus Tranexamic Acid (Blood Transfusion \& Blood Loss), Outcome 3 No. Exposed to Allogeneic Blood - Transfusion Protocol.

Review: Anti-fibrinolytic use for minimising perioperative allogeneic blood transfusion

Comparison: 4 Aprotinin versus Tranexamic Acid (Blood Transfusion \% Blood Loss)

Outcome: 3 No. Exposed to Allogeneic Blood - Transfusion Protocol

\begin{tabular}{|c|c|c|c|c|c|}
\hline \multirow[t]{2}{*}{ Study or subgroup } & Aprotinin & TXA & Risk Ratio & \multirow[t]{2}{*}{ Weight } & \multirow{2}{*}{$\begin{array}{r}\text { Risk Ratio } \\
\mathrm{M}-\mathrm{H}, \text { Random,95\% Cl }\end{array}$} \\
\hline & $\mathrm{n} / \mathrm{N}$ & $\mathrm{n} / \mathrm{N}$ & $\mathrm{M}-\mathrm{H}$, Random,95\% Cl & & \\
\hline \multicolumn{6}{|l|}{ I Transfusion Protocol } \\
\hline Bernet 1999 & $1 \mid / 28$ & $17 / 28$ & & $3.1 \%$ & $0.65[0.37,1.12]$ \\
\hline Blauhut 1994 & $3 / 14$ & $7 / 15$ & - & $0.9 \%$ & $0.46[0.15,1.44]$ \\
\hline Casati 1999 & $19 / 67$ & $17 / 70$ & +1 & $3.0 \%$ & $1.17[0.67,2.05]$ \\
\hline Casati 2000 & $|85 / 5| 8$ & $178 / 522$ & + & $10.3 \%$ & $1.05[0.89,1.24]$ \\
\hline Corbeau 1995 & $15 / 43$ & $|5 / 4|$ & +1 & $2.9 \%$ & $0.95[0.54,1.69]$ \\
\hline Dalmau 2004 & $40 / 63$ & $38 / 64$ & $\rightarrow$ & $7.3 \%$ & $1.07[0.81,1.41]$ \\
\hline Dietrich 2008 & $52 / 110$ & $67 / 110$ & $\rightarrow$ & $8.0 \%$ & $0.78[0.61,0.99]$ \\
\hline
\end{tabular}

Favours Aprotinin Favours TXA 


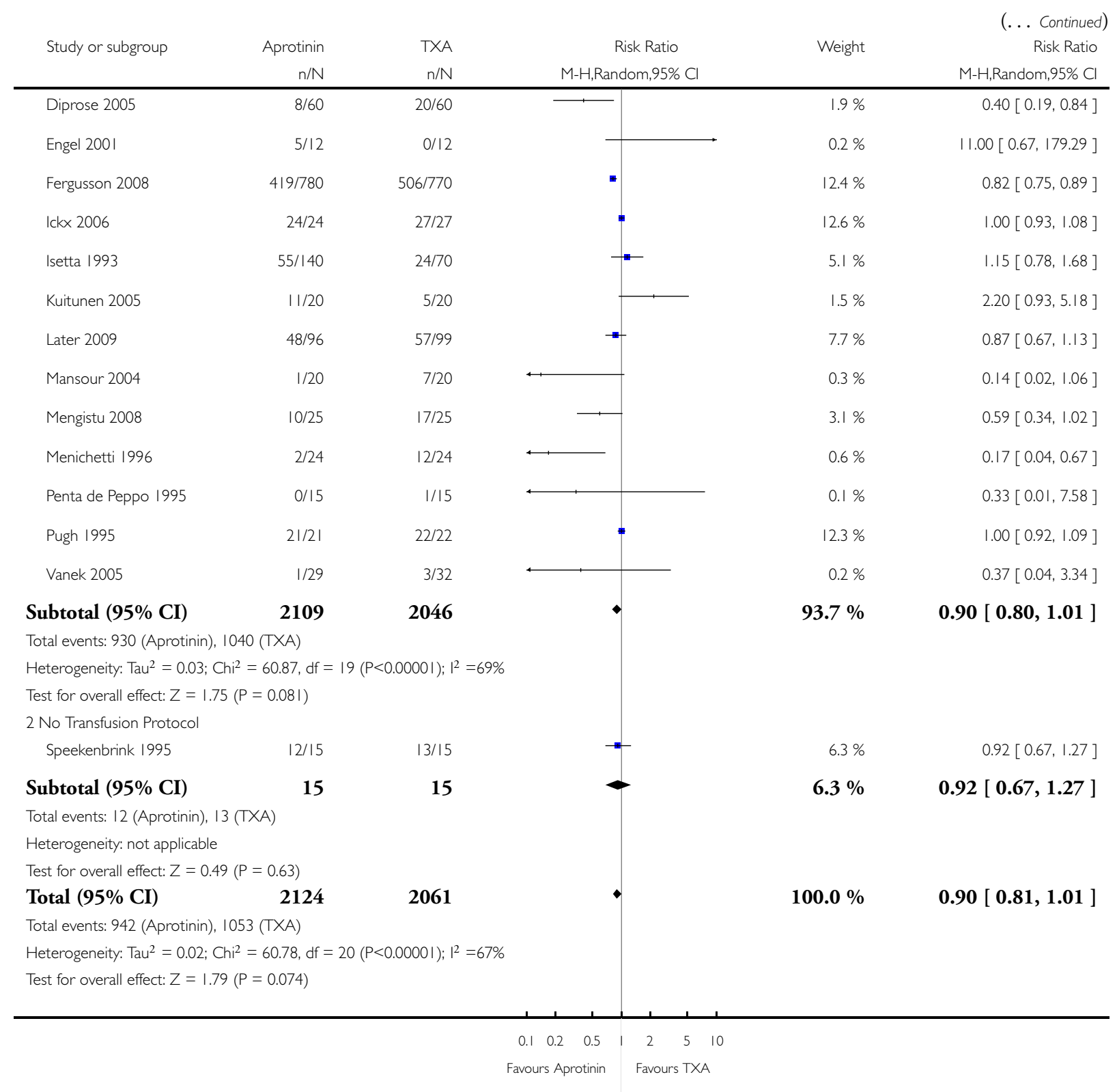


Analysis 4.4. Comparison 4 Aprotinin versus Tranexamic Acid (Blood Transfusion \& Blood Loss), Outcome 4 Trial Methodological Quality - Allocation Concealment.

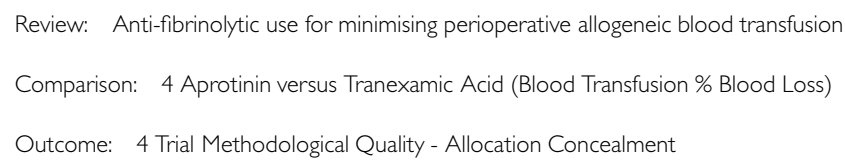

Total events: 473 (Aprotinin), 583 (TXA)

Heterogeneity: Tau $^{2}=0.01 ; \mathrm{Chi}^{2}=3.60, \mathrm{df}=3(\mathrm{P}=0.3 \mathrm{I}) ; \mathrm{I}^{2}=17 \%$

Test for overall effect: $Z=3.14(P=0.0017)$

2 Allocation concealment - Unclear

$\begin{array}{lrr}\text { Bernet 1999 } & 11 / 28 & 17 / 28 \\ \text { Blauhut 1994 } & 3 / 14 & 7 / 15 \\ \text { Casati 1999 } & 19 / 67 & 17 / 70 \\ \text { Casati 2000 } & 185 / 518 & 178 / 522 \\ \text { Corbeau 1995 } & 15 / 43 & 15 / 4 \mid \\ \text { Engel 200| } & 5 / 12 & 0 / 12 \\ \text { Ickx 2006 } & 24 / 24 & 27 / 27 \\ \text { Isetta 1993 } & 55 / 140 & 24 / 70 \\ \text { Mengistu 2008 } & 10 / 25 & 17 / 25 \\ \text { Menichetti 1996 } & 2 / 24 & 12 / 24 \\ \text { Penta de Peppo 1995 } & 0 / 15 & 1 / 15 \\ \text { Pugh 1995 } & 21 / 21 & 22 / 22 \\ \text { Speekenbrink 1995 } & 12 / 15 & 13 / 15\end{array}$

Subtotal (95\% CI) 


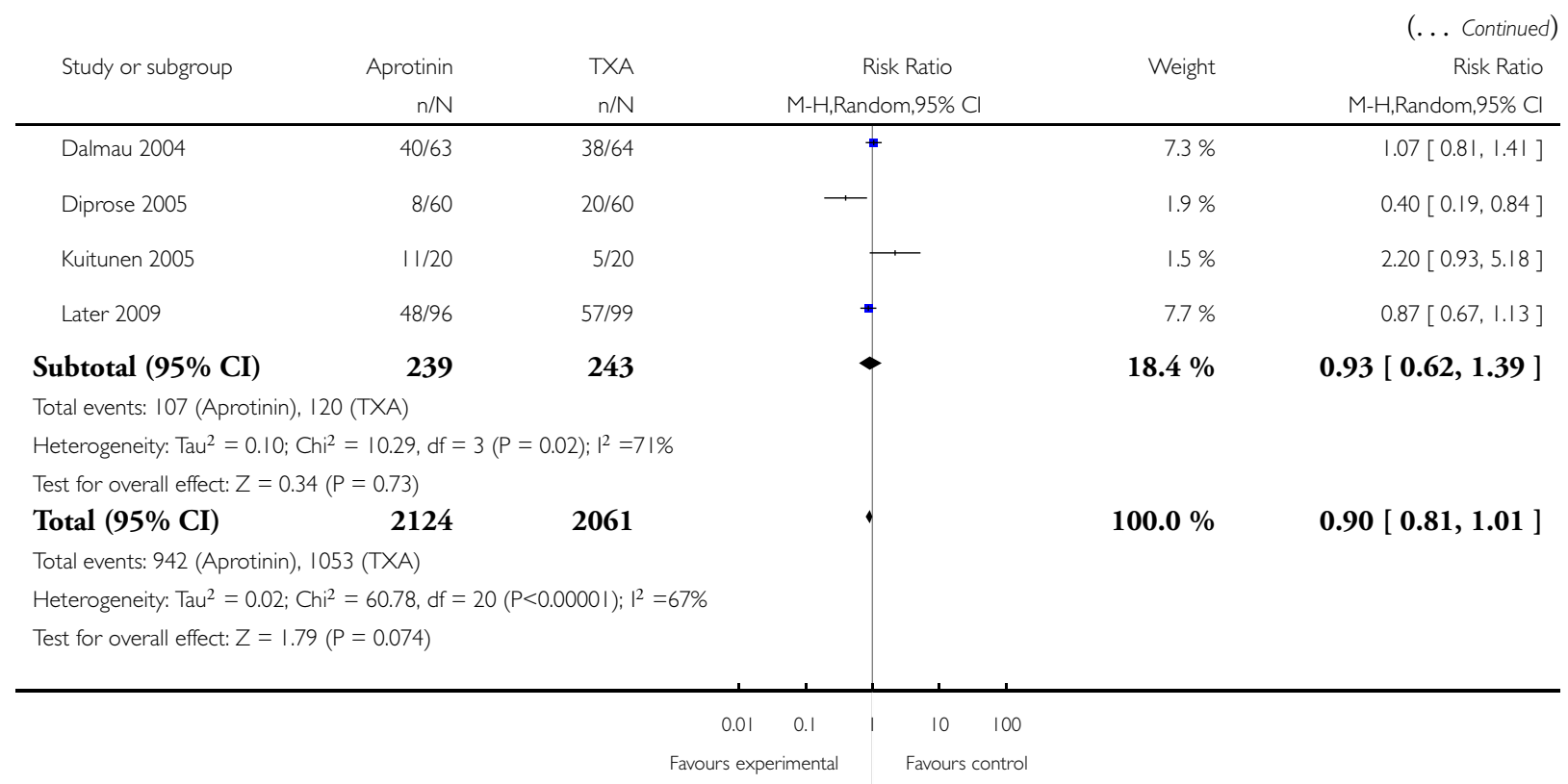

\section{Analysis 4.5. Comparison 4 Aprotinin versus Tranexamic Acid (Blood Transfusion \& Blood Loss), Outcome} 5 Units Allogeneic Blood Transfused - Transfused Patients.

Review: Anti-fibrinolytic use for minimising perioperative allogeneic blood transfusion

Comparison: 4 Aprotinin versus Tranexamic Acid (Blood Transfusion \% Blood Loss)

Outcome: 5 Units Allogeneic Blood Transfused - Transfused Patients

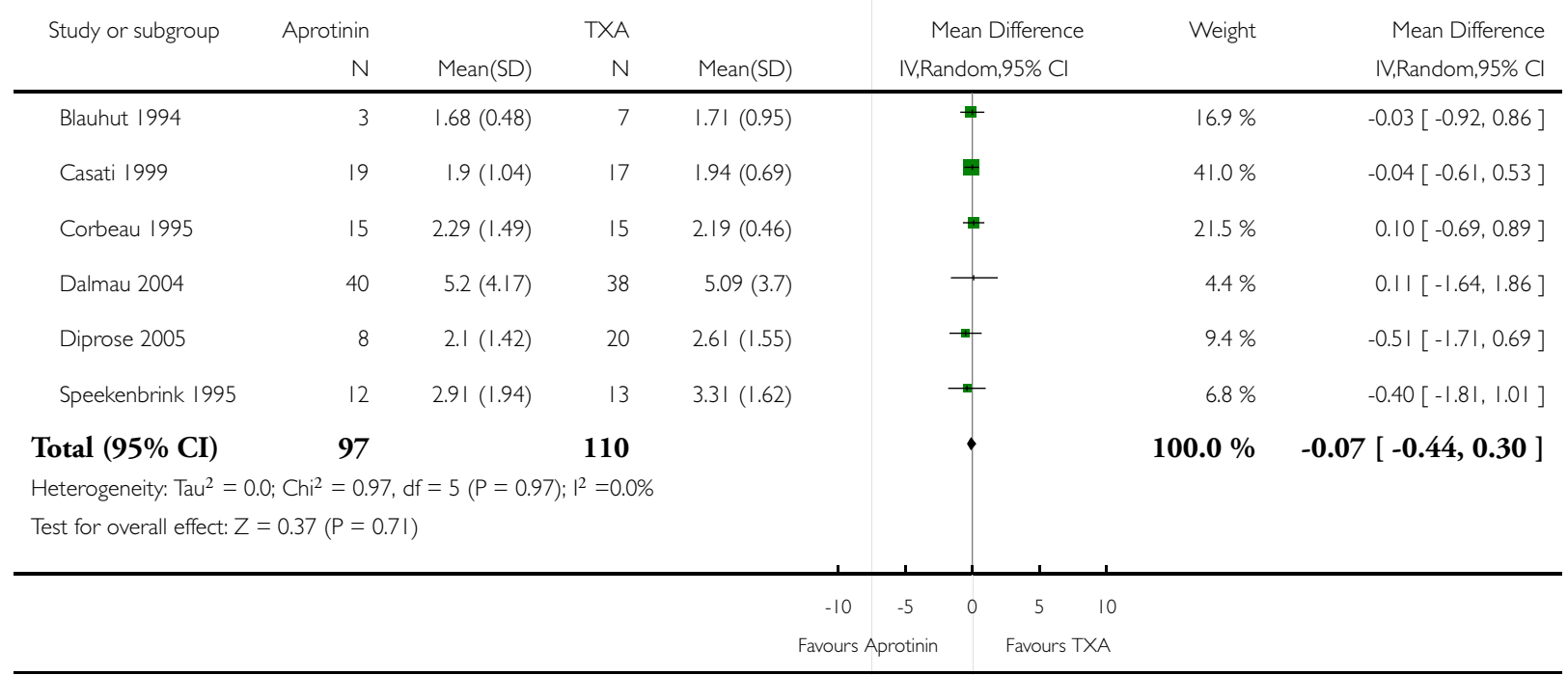


Analysis 4.6. Comparison 4 Aprotinin versus Tranexamic Acid (Blood Transfusion \& Blood Loss), Outcome 6 Units Allogeneic Blood Transfused - All Patients.

Review: Anti-fibrinolytic use for minimising perioperative allogeneic blood transfusion

Comparison: 4 Aprotinin versus Tranexamic Acid (Blood Transfusion \% Blood Loss)

Outcome: 6 Units Allogeneic Blood Transfused - All Patients

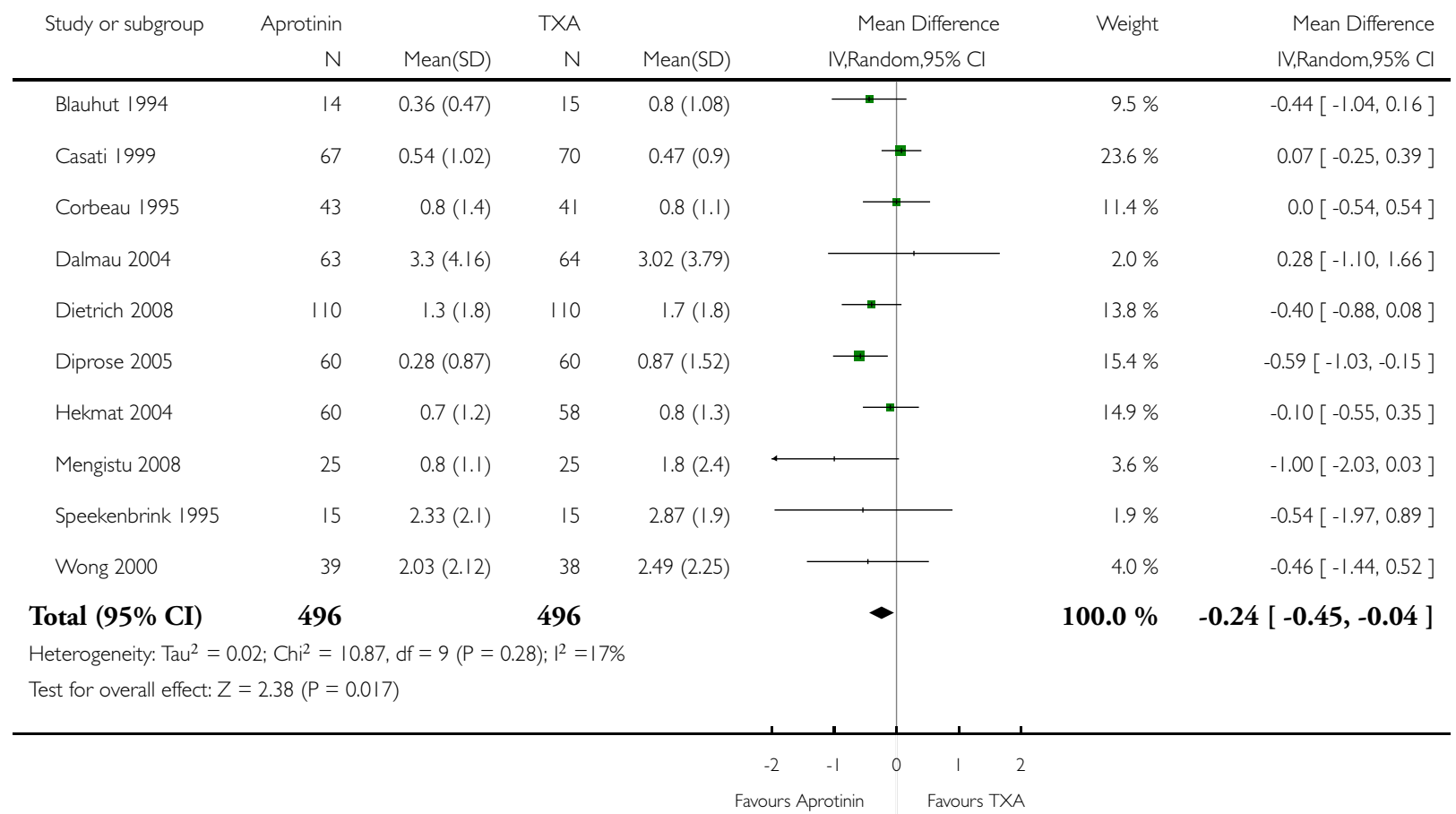




\section{Analysis 4.7. Comparison 4 Aprotinin versus Tranexamic Acid (Blood Transfusion \& Blood Loss), Outcome 7 Blood loss.}

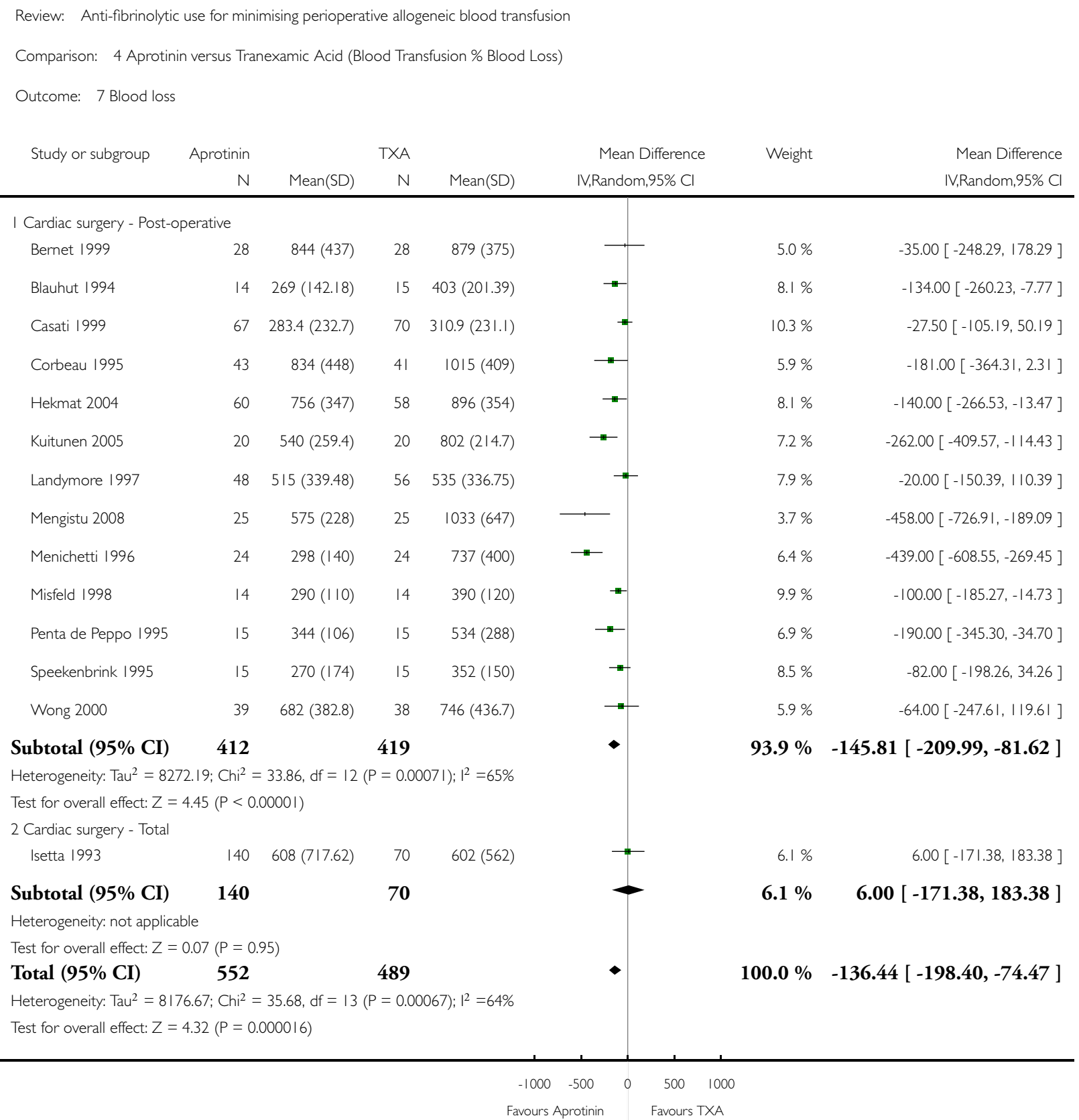


Analysis 5.1. Comparison 5 Aprotinin versus Epsilon Aminocaproic Acid (Blood Transfusion \& Blood Loss), Outcome I No. Exposed to Allogeneic Blood.

Review: Anti-fibrinolytic use for minimising perioperative allogeneic blood transfusion

Comparison: 5 Aprotinin versus Epsilon Aminocaproic Acid (Blood Transfusion \% Blood Loss)

Outcome: I No. Exposed to Allogeneic Blood

\begin{tabular}{|c|c|c|c|c|c|}
\hline \multirow[t]{2}{*}{ Study or subgroup } & Aprotinin & EACA & Risk Ratio & \multirow[t]{2}{*}{ Weight } & \multirow{2}{*}{$\begin{array}{r}\text { Risk Ratio } \\
\text { M-H,Random,95\% Cl }\end{array}$} \\
\hline & $n / N$ & $\mathrm{n} / \mathrm{N}$ & $\mathrm{M}-\mathrm{H}$, Random, $95 \% \mathrm{Cl}$ & & \\
\hline Eberle 1998 & $9 / 20$ & $12 / 20$ & - & $1.6 \%$ & $0.75[0.41,1.37]$ \\
\hline Greilich 2009 & $18 / 26$ & $15 / 25$ & + & $3.4 \%$ & $1.15[0.77,1.74]$ \\
\hline Fergusson 2008 & $419 / 780$ & $514 / 780$ & & $84.9 \%$ & $0.82[0.75,0.89]$ \\
\hline Ray 200I & $14 / 49$ & $|2 / 5|$ & $\longrightarrow$ & $1.3 \%$ & $1.21[0.63,2.36]$ \\
\hline Casati 1999 & $19 / 67$ & $23 / 66$ & - & $2.3 \%$ & $0.81[0.49,1.35]$ \\
\hline Trinh-Duc 1992 & $12 / 29$ & $18 / 27$ & 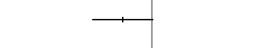 & $2.2 \%$ & $0.62[0.37,1.03]$ \\
\hline Penta de Peppo 1995 & $0 / 15$ & $3 / 15$ & $\leftarrow$ & $0.1 \%$ & $0.14[0.01,2.55]$ \\
\hline Greilich 200I & $7 / 24$ & $5 / 23$ & & $0.6 \%$ & $1.34[0.50,3.63]$ \\
\hline Dorman 2008 & $9 / 30$ & $15 / 30$ & & $1.4 \%$ & $0.60[0.31,1.15]$ \\
\hline Ray 2005 & $2 / 15$ & $4 / 15$ & & $0.2 \%$ & $0.50[0.11,2.33]$ \\
\hline Menichetti 1996 & $2 / 24$ & $4 / 24$ & & $0.2 \%$ & $0.50[0.10,2.48]$ \\
\hline Amar 2003 & $11 / 23$ & $12 / 22$ & 1 & $1.8 \%$ & $0.88[0.49,1.55]$ \\
\hline Total (95\% CI) & 1102 & 1098 & - & $100.0 \%$ & $0.82[0.76,0.89]$ \\
\hline \multicolumn{6}{|c|}{ Total events: 522 (Aprotinin), 637 (EACA) } \\
\hline \multicolumn{6}{|c|}{ Heterogeneity: $\mathrm{Tau}^{2}=0.0 ; \mathrm{Chi}^{2}=9.33, \mathrm{df}=\mathrm{II}(\mathrm{P}=0.59) ; \mathrm{I}^{2}=0.0 \%$} \\
\hline \multicolumn{6}{|c|}{ Test for overall effect: $Z=5.11(P<0.0000 I)$} \\
\hline
\end{tabular}

$\begin{array}{lllllll}0.1 & 0.2 & 0.5 & 1 & 2 & 5 & 10\end{array}$

Favours Aprotinin Favours EACA 
Analysis 5.2. Comparison 5 Aprotinin versus Epsilon Aminocaproic Acid (Blood Transfusion \& Blood Loss), Outcome 2 No. Exposed to Allogeneic Blood - Type of Surgery.

Review: Anti-fibrinolytic use for minimising perioperative allogeneic blood transfusion

Comparison: 5 Aprotinin versus Epsilon Aminocaproic Acid (Blood Transfusion \% Blood Loss)

Outcome: 2 No. Exposed to Allogeneic Blood - Type of Surgery

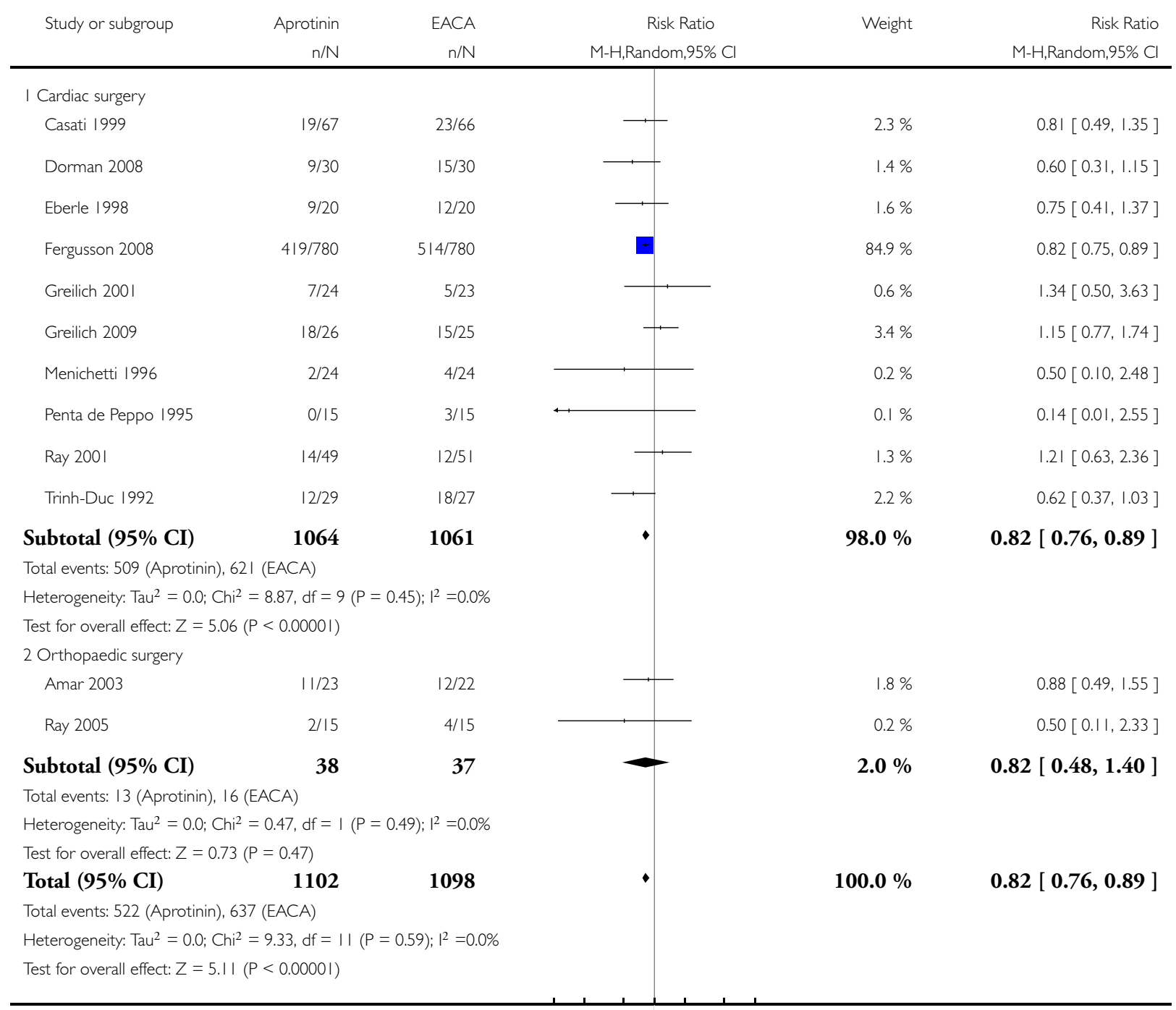

$\begin{array}{lllllll}0.1 & 0.2 & 0.5 & 1 & 2 & 5 & 10\end{array}$

Favours Aprotinin Favours EACA 
Analysis 5.3. Comparison 5 Aprotinin versus Epsilon Aminocaproic Acid (Blood Transfusion \& Blood Loss), Outcome 3 No. Exposed to Allogeneic Blood - Transfusion Protocol.

Review: Anti-fibrinolytic use for minimising perioperative allogeneic blood transfusion

Comparison: 5 Aprotinin versus Epsilon Aminocaproic Acid (Blood Transfusion \% Blood Loss)

Outcome: 3 No. Exposed to Allogeneic Blood - Transfusion Protocol

\begin{tabular}{|c|c|c|c|c|c|}
\hline \multirow[t]{2}{*}{ Study or subgroup } & Aprotinin & EACA & Risk Ratio & \multirow[t]{2}{*}{ Weight } & \multirow{2}{*}{$\begin{array}{r}\text { Risk Ratio } \\
\text { M-H,Random,95\% Cl}\end{array}$} \\
\hline & $n / N$ & $\mathrm{n} / \mathrm{N}$ & $\mathrm{M}-\mathrm{H}, \mathrm{Random}, 95 \% \mathrm{Cl}$ & & \\
\hline \multicolumn{6}{|l|}{ I Transfusion Protocol } \\
\hline Amar 2003 & $11 / 23$ & $12 / 22$ & - & $1.8 \%$ & $0.88[0.49,1.55]$ \\
\hline Casati 1999 & $19 / 67$ & $23 / 66$ & $\cdot$ & $2.3 \%$ & $0.81[0.49,1.35]$ \\
\hline Dorman 2008 & $9 / 30$ & $15 / 30$ & - & $1.4 \%$ & $0.60[0.31,1.15]$ \\
\hline Eberle 1998 & 9/20 & $12 / 20$ & 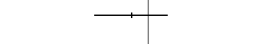 & $1.6 \%$ & $0.75[0.41,1.37]$ \\
\hline Fergusson 2008 & $419 / 780$ & $514 / 780$ & & $84.9 \%$ & $0.82[0.75,0.89]$ \\
\hline Greilich 200I & $7 / 24$ & $5 / 23$ & - & $0.6 \%$ & $1.34[0.50,3.63]$ \\
\hline Greilich 2009 & $18 / 26$ & $15 / 25$ & 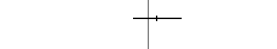 & $3.4 \%$ & $1.15[0.77,1.74]$ \\
\hline Menichetti 1996 & $2 / 24$ & $4 / 24$ & & $0.2 \%$ & $0.50[0.10,2.48]$ \\
\hline Penta de Peppo 1995 & $0 / 15$ & $3 / 15$ & & $0.1 \%$ & $0.14[0.01,2.55]$ \\
\hline Subtotal (95\% CI) & 1009 & 1005 & - & $96.2 \%$ & $0.82[0.76,0.89]$ \\
\hline \multicolumn{6}{|c|}{ Total events: 494 (Aprotinin), 603 (EACA) } \\
\hline \multicolumn{6}{|c|}{ Heterogeneity: $\operatorname{Tau}^{2}=0.0 ; \mathrm{Chi}^{2}=6.45, \mathrm{df}=8(\mathrm{P}=0.60) ; \mathrm{I}^{2}=0.0 \%$} \\
\hline \multicolumn{6}{|c|}{ Test for overall effect: $Z=4.95(P<0.0000 I)$} \\
\hline \multicolumn{6}{|l|}{2 No Transfusion Protocol } \\
\hline Ray 200। & $14 / 49$ & $|2 / 5|$ & 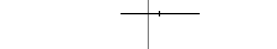 & $1.3 \%$ & $1.21[0.63,2.36]$ \\
\hline Ray 2005 & $2 / 15$ & $4 / 15$ & & $0.2 \%$ & $0.50[0.11,2.33]$ \\
\hline Trinh-Duc 1992 & $12 / 29$ & $18 / 27$ & 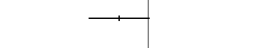 & $2.2 \%$ & $0.62[0.37,1.03]$ \\
\hline Subtotal (95\% CI) & 93 & 93 & & $3.8 \%$ & $0.78[0.47,1.31]$ \\
\hline \multicolumn{6}{|c|}{ Total events: 28 (Aprotinin), 34 (EACA) } \\
\hline \multicolumn{6}{|c|}{ Heterogeneity: $\operatorname{Tau}^{2}=0.06 ; \mathrm{Chi}^{2}=2.86, \mathrm{df}=2(P=0.24) ; \mathrm{I}^{2}=30 \%$} \\
\hline \multicolumn{6}{|c|}{ Test for overall effect: $Z=0.93(P=0.35)$} \\
\hline Total (95\% CI) & 1102 & 1098 & - & $100.0 \%$ & $0.82[0.76,0.89]$ \\
\hline \multicolumn{6}{|c|}{ Total events: 522 (Aprotinin), 637 (EACA) } \\
\hline \multicolumn{6}{|c|}{ Heterogeneity: $\operatorname{Tau}^{2}=0.0 ; \mathrm{Chi}^{2}=9.33, \mathrm{df}=\mathrm{II}(\mathrm{P}=0.59) ; \mathrm{I}^{2}=0.0 \%$} \\
\hline Test for overall effect: $Z=$ & $<0.00001)$ & & & & \\
\hline
\end{tabular}

$\begin{array}{lllllll}0.1 & 0.2 & 0.5 & 1 & 2 & 5 & 10\end{array}$

Favours Aprotinin Favours EACA 
Analysis 5.4. Comparison 5 Aprotinin versus Epsilon Aminocaproic Acid (Blood Transfusion \& Blood Loss), Outcome 4 Trial Methodological Quality - Allocation Concealment.

Review: Anti-fibrinolytic use for minimising perioperative allogeneic blood transfusion

Comparison: 5 Aprotinin versus Epsilon Aminocaproic Acid (Blood Transfusion \% Blood Loss)

Outcome: 4 Trial Methodological Quality - Allocation Concealment

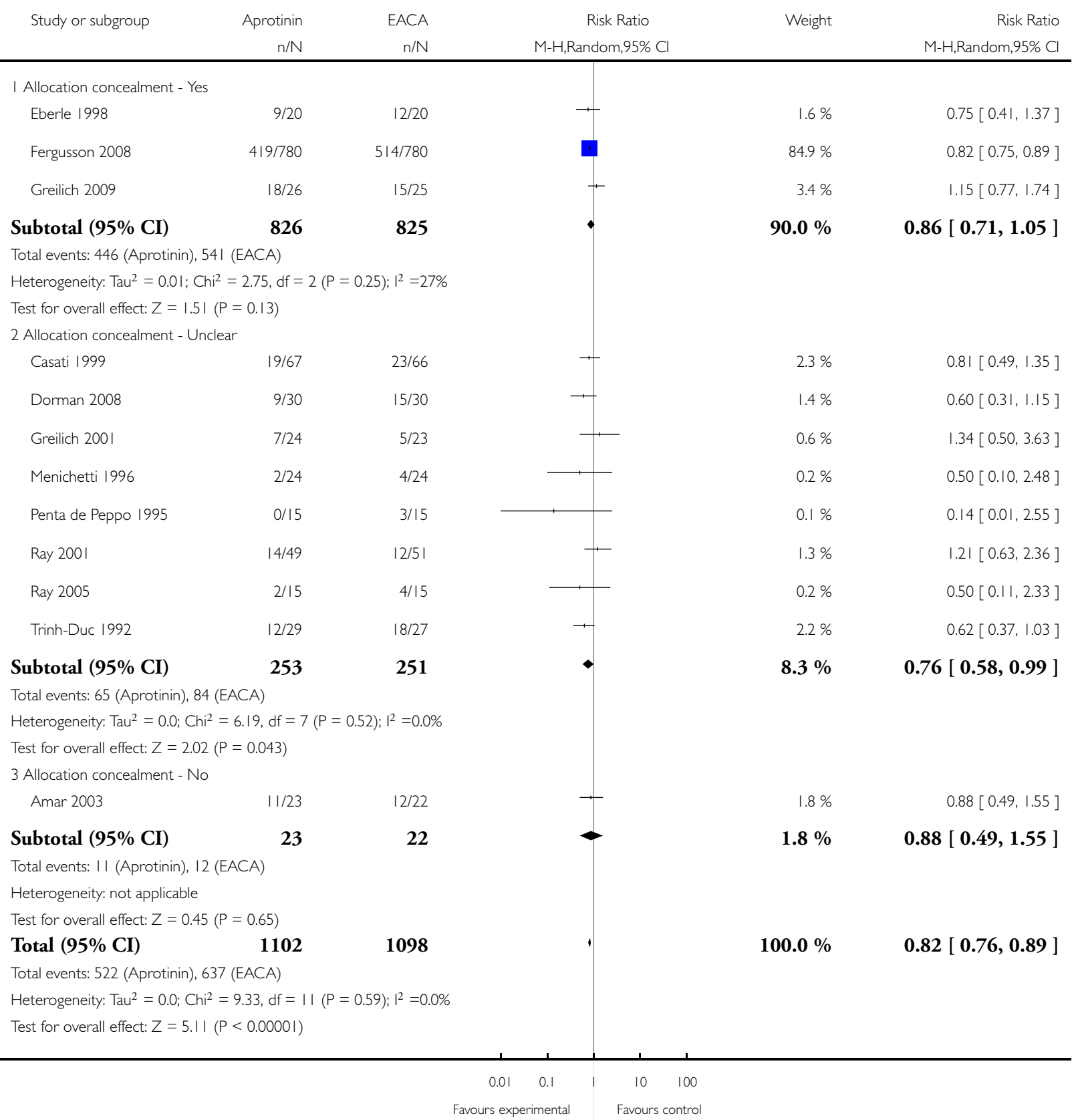


Analysis 5.5. Comparison 5 Aprotinin versus Epsilon Aminocaproic Acid (Blood Transfusion \& Blood Loss), Outcome 5 Units of Allogeneic Blood Transfused - Transfused Patients.

Review: Anti-fibrinolytic use for minimising perioperative allogeneic blood transfusion

Comparison: 5 Aprotinin versus Epsilon Aminocaproic Acid (Blood Transfusion \% Blood Loss)

Outcome: 5 Units of Allogeneic Blood Transfused - Transfused Patients

\begin{tabular}{ccccccr} 
Study or subgroup & Aprotinin & EACA & Mean(SD) & Mean Difference & $\begin{array}{r}\text { Weight } \\
\text { IV,Random,95\% Cl }\end{array}$ & $\begin{array}{r}\text { Mean Difference } \\
\text { IV,Random,95\% Cl }\end{array}$ \\
\hline Casati 1999 & 19 & $1.9(1.04)$ & 23 & $2.24(0.92)$ & $-0.34[-0.94,0.26]$ \\
Eberle 1998 & 9 & $1.29(0.88)$ & 12 & $1.25(0.67)$ & $0.04[-0.65,0.73]$
\end{tabular}

Total (95\% CI) $28 \quad 35$

Heterogeneity: $\mathrm{Tau}^{2}=0.0 ; \mathrm{Chi}^{2}=0.66, \mathrm{df}=\mathrm{I}(\mathrm{P}=0.4 \mathrm{I}) ; \mathrm{I}^{2}=0.0 \%$

Test for overall effect: $Z=0.76(P=0.45)$

Analysis 5.6. Comparison 5 Aprotinin versus Epsilon Aminocaproic Acid (Blood Transfusion \& Blood Loss), Outcome 6 Units of Allogeneic Blood Transfused - All Patients.

Review: Anti-fibrinolytic use for minimising perioperative allogeneic blood transfusion

Comparison: 5 Aprotinin versus Epsilon Aminocaproic Acid (Blood Transfusion \% Blood Loss)

Outcome: 6 Units of Allogeneic Blood Transfused - All Patients

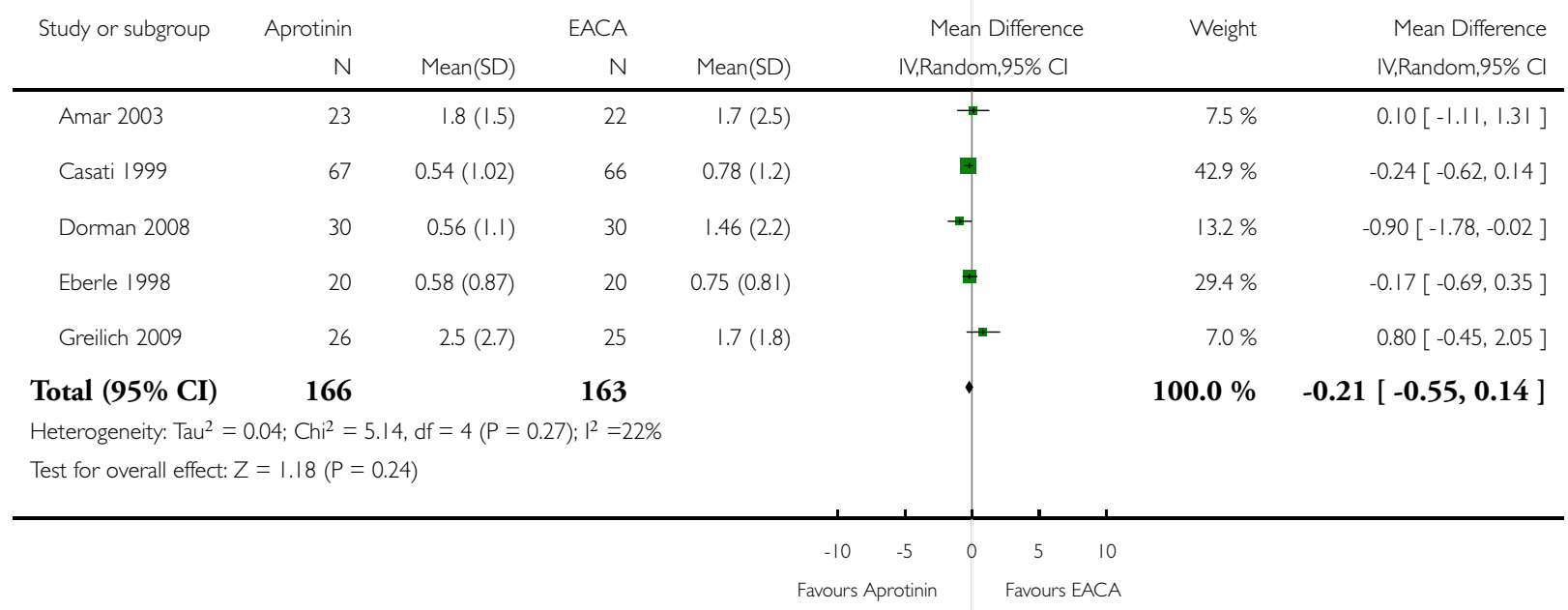


Analysis 5.7. Comparison 5 Aprotinin versus Epsilon Aminocaproic Acid (Blood Transfusion \& Blood Loss), Outcome 7 Blood loss.

Review: Anti-fibrinolytic use for minimising perioperative allogeneic blood transfusion

Comparison: 5 Aprotinin versus Epsilon Aminocaproic Acid (Blood Transfusion \% Blood Loss)

Outcome: 7 Blood loss

\begin{tabular}{|c|c|c|c|c|c|c|c|c|}
\hline \multirow[t]{2}{*}{ Study or subgroup } & \multirow{2}{*}{$\begin{array}{r}\text { Aprotinin } \\
\mathrm{N}\end{array}$} & \multicolumn{3}{|c|}{ EACA } & \multirow{2}{*}{\multicolumn{2}{|c|}{$\begin{array}{l}\text { Mean Difference } \\
\text { IV,Random,95\% Cl }\end{array}$}} & \multirow[t]{2}{*}{ Weight } & \multirow{2}{*}{$\begin{array}{r}\text { Mean Difference } \\
\text { IV,Random,95\% Cl }\end{array}$} \\
\hline & & Mean(SD) & $\mathrm{N}$ & Mean(SD) & & & & \\
\hline \multicolumn{9}{|c|}{ I Cardiac surgery - Post-operative } \\
\hline Casati 1999 & 67 & $283.4(232.7)$ & 66 & $466.9(234.2)$ & $\#$ & & $19.2 \%$ & $-183.50[-262.85,-104.15]$ \\
\hline Dorman 2008 & 30 & $423(1363.83)$ & 30 & $596(2026.57)$ & & & $1.4 \%$ & $-173.00[-1047.11,701.11]$ \\
\hline Eberle 1998 & 20 & $391(220)$ & 20 & $582(274)$ & $\rightarrow$ & & $14.8 \%$ & $-191.00[-345.00,-37.00]$ \\
\hline Greilich 2009 & 26 & $685(505)$ & 25 & $715(394)$ & $\longrightarrow$ & & $9.9 \%$ & $-30.00[-278.06,218.06]$ \\
\hline Landymore 1997 & 48 & $515(339.48)$ & 44 & $343(305.13)$ & & $\rightarrow$ & $16.2 \%$ & $172.00[40.27,303.73]$ \\
\hline Menichetti 1996 & 24 & $298(140)$ & 24 & $512(250)$ & $\rightleftarrows$ & & $17.2 \%$ & $-214.00[-328.63,-99.37]$ \\
\hline Penta de Peppo 1995 & 15 & $344(106)$ & 15 & $509(148)$ & 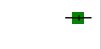 & & $18.5 \%$ & $-165.00[-257.13,-72.87]$ \\
\hline Subtotal (95\% CI) & 230 & & 224 & & - & & $97.4 \%$ & $-111.43[-220.64,-2.21]$ \\
\hline \multicolumn{9}{|c|}{ Heterogeneity: $\mathrm{Tau}^{2}=|4| 45.97 ; \mathrm{Chi}^{2}=25.74, \mathrm{df}=6(P=0.00025) ;\left.\right|^{2}=77 \%$} \\
\hline \multicolumn{9}{|c|}{ Test for overall effect: $Z=2.00(P=0.046)$} \\
\hline \multicolumn{9}{|c|}{2 Orthopaedic surgery - Total } \\
\hline Amar 2003 & 23 & $1700(1000)$ & 22 & $1600(1100)$ & & & $2.6 \%$ & $100.00[-515.06,715.06]$ \\
\hline Subtotal (95\% CI) & 23 & & 22 & & & & $2.6 \%$ & $100.00[-515.06,715.06]$ \\
\hline \multicolumn{9}{|c|}{ Heterogeneity: not applicable } \\
\hline \multicolumn{9}{|c|}{ Test for overall effect: $Z=0.32(P=0.75)$} \\
\hline Total $(95 \% \mathrm{CI})$ & 253 & & 246 & & 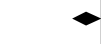 & & $100.0 \%$ & $-106.01[-212.50,0.47]$ \\
\hline \multicolumn{9}{|c|}{ Heterogeneity: $\operatorname{Tau}^{2}=13708.99 ; \mathrm{Chi}^{2}=26.30, \mathrm{df}=7(\mathrm{P}=0.00045) ; \mathrm{I}^{2}=73 \%$} \\
\hline \multicolumn{9}{|c|}{ Test for overall effect: $Z=1.95(P=0.05 \mathrm{I})$} \\
\hline & & & & -1000 & -500 & 500 & 1000 & \\
\hline & & & & Favour & protinin & Favours Ef & EACA & \\
\hline
\end{tabular}


Analysis 6.I. Comparison 6 Tranexamic Acid versus Epsilon Aminocaproic Acid (Blood Transfusion \& Blood Loss), Outcome I No. Exposed to Allogeneic Blood.

Review: Anti-fibrinolytic use for minimising perioperative allogeneic blood transfusion

Comparison: 6 Tranexamic Acid versus Epsilon Aminocaproic Acid (Blood Transfusion \% Blood Loss)

Outcome: I No. Exposed to Allogeneic Blood

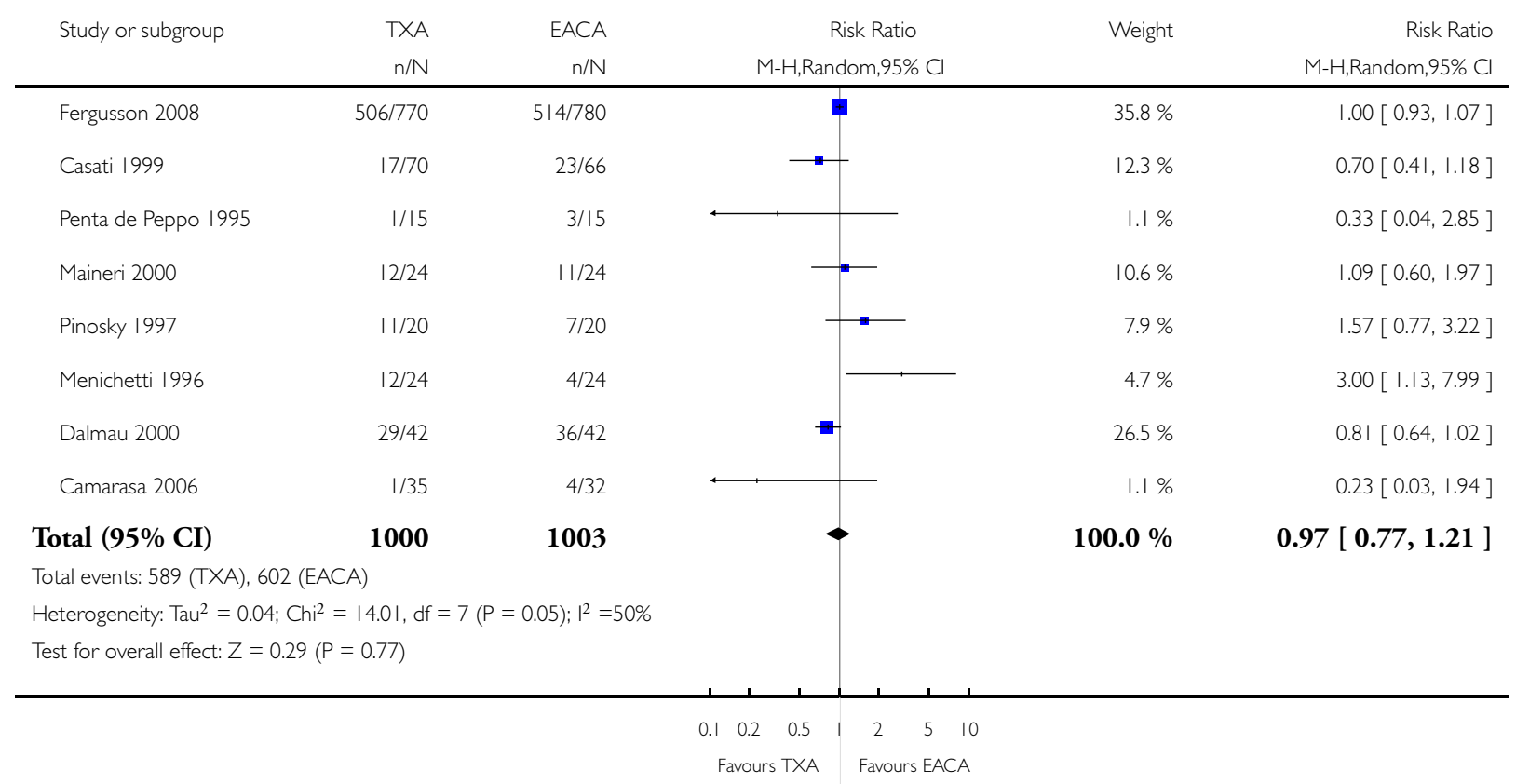


Analysis 6.2. Comparison 6 Tranexamic Acid versus Epsilon Aminocaproic Acid (Blood Transfusion \& Blood Loss), Outcome 2 No. Exposed to Allogeneic Blood - Type of Surgery.

Review: Anti-fibrinolytic use for minimising perioperative allogeneic blood transfusion

Comparison: 6 Tranexamic Acid versus Epsilon Aminocaproic Acid (Blood Transfusion \% Blood Loss)

Outcome: 2 No. Exposed to Allogeneic Blood - Type of Surgery

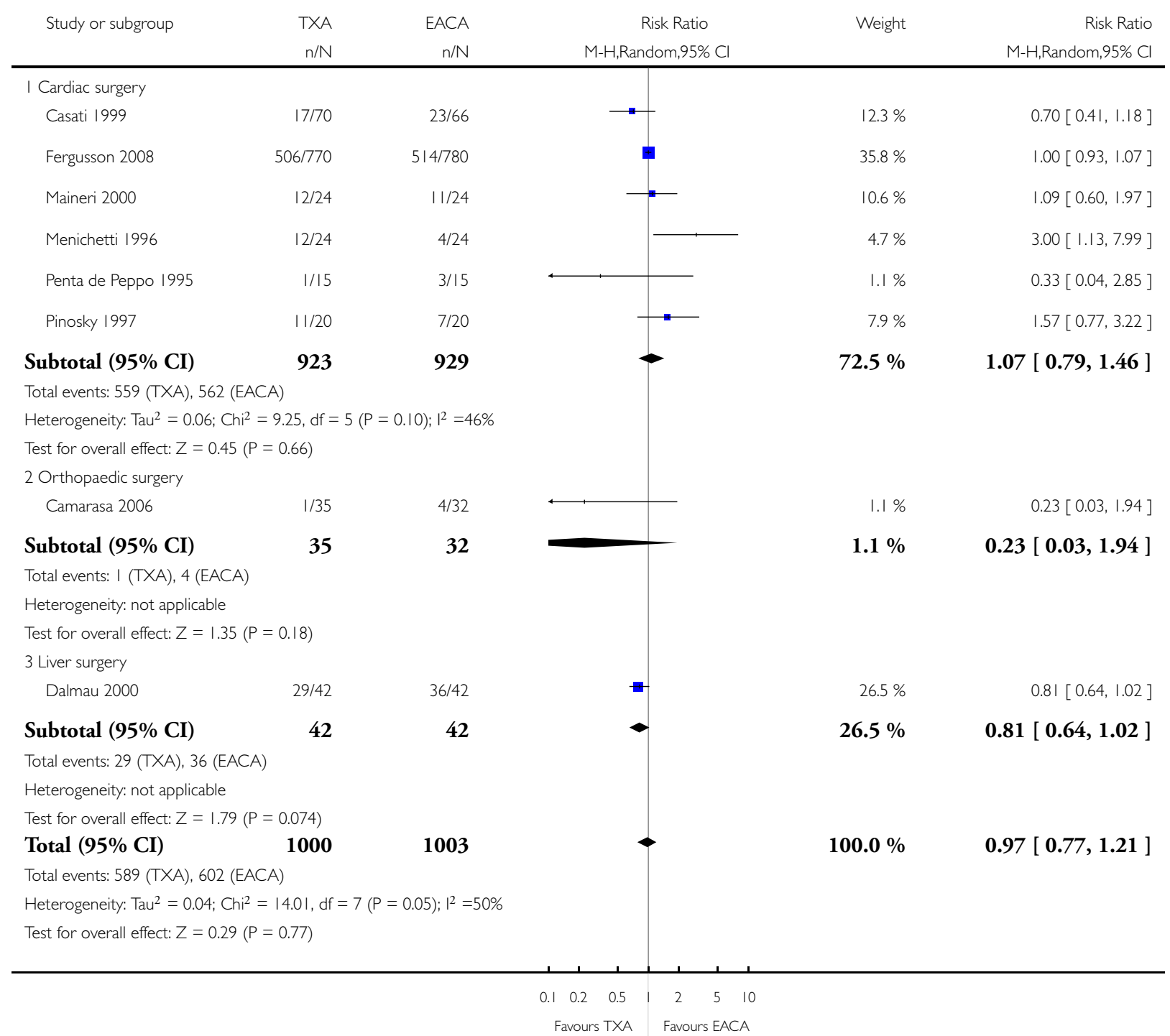


Analysis 6.3. Comparison 6 Tranexamic Acid versus Epsilon Aminocaproic Acid (Blood Transfusion \& Blood Loss), Outcome 3 No. Exposed to Allogeneic Blood - Transfusion Protocol.

Review: Anti-fibrinolytic use for minimising perioperative allogeneic blood transfusion

Comparison: 6 Tranexamic Acid versus Epsilon Aminocaproic Acid (Blood Transfusion \% Blood Loss)

Outcome: 3 No. Exposed to Allogeneic Blood - Transfusion Protocol

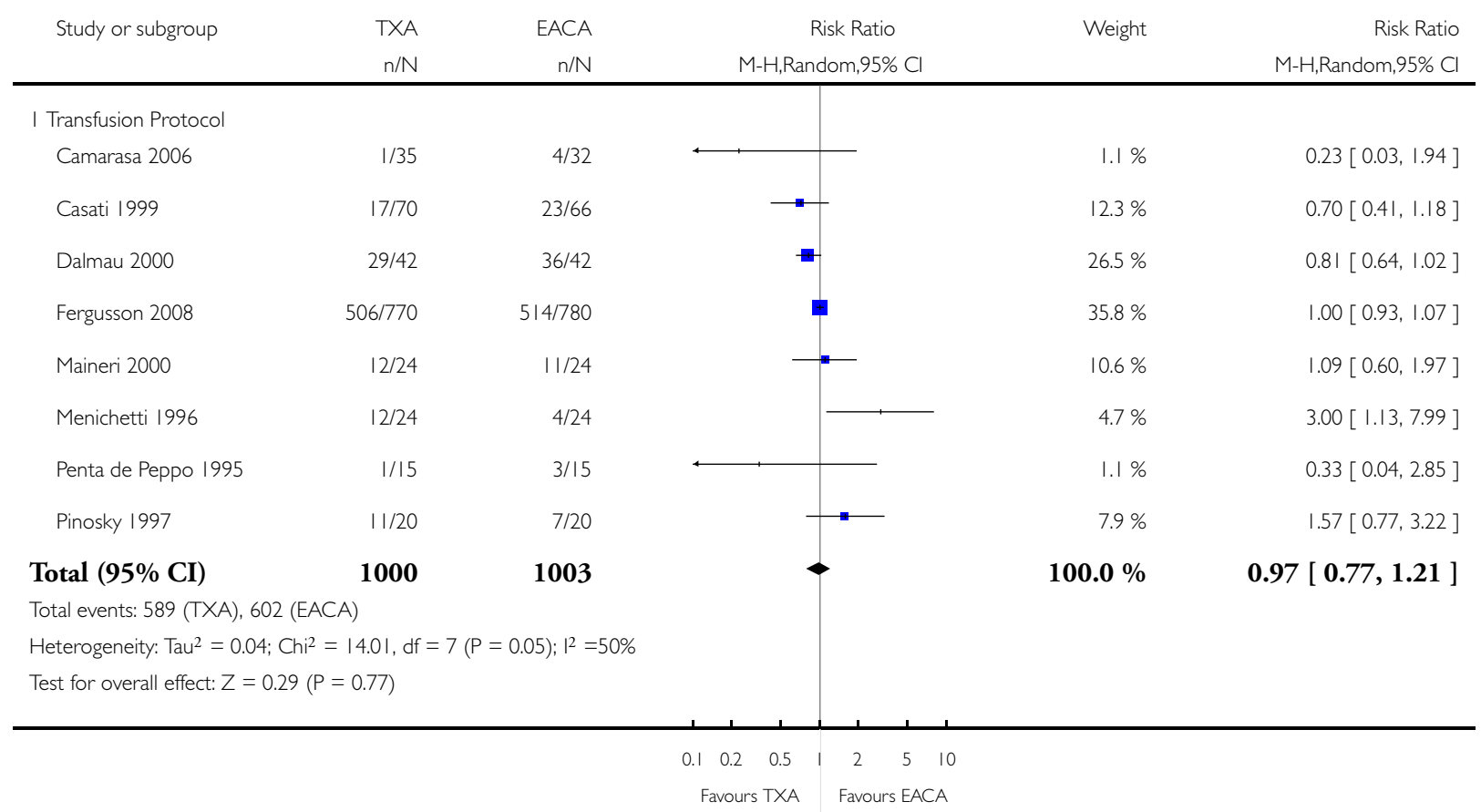




\section{Analysis 6.4. Comparison 6 Tranexamic Acid versus Epsilon Aminocaproic Acid (Blood Transfusion \& Blood Loss), Outcome 4 Trial Methodological Quality - Allocation Concealment.}

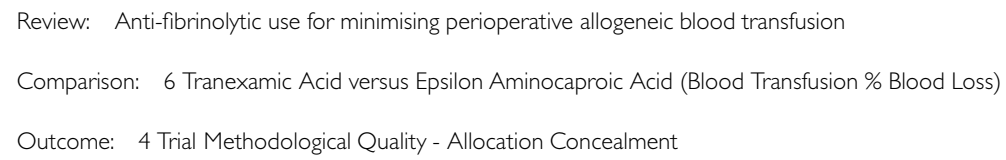

$\mathrm{n} / \mathrm{N} \quad \mathrm{n} / \mathrm{N}-\mathrm{H}$, Random,95\% Cl M-H,Random,95\% Cl

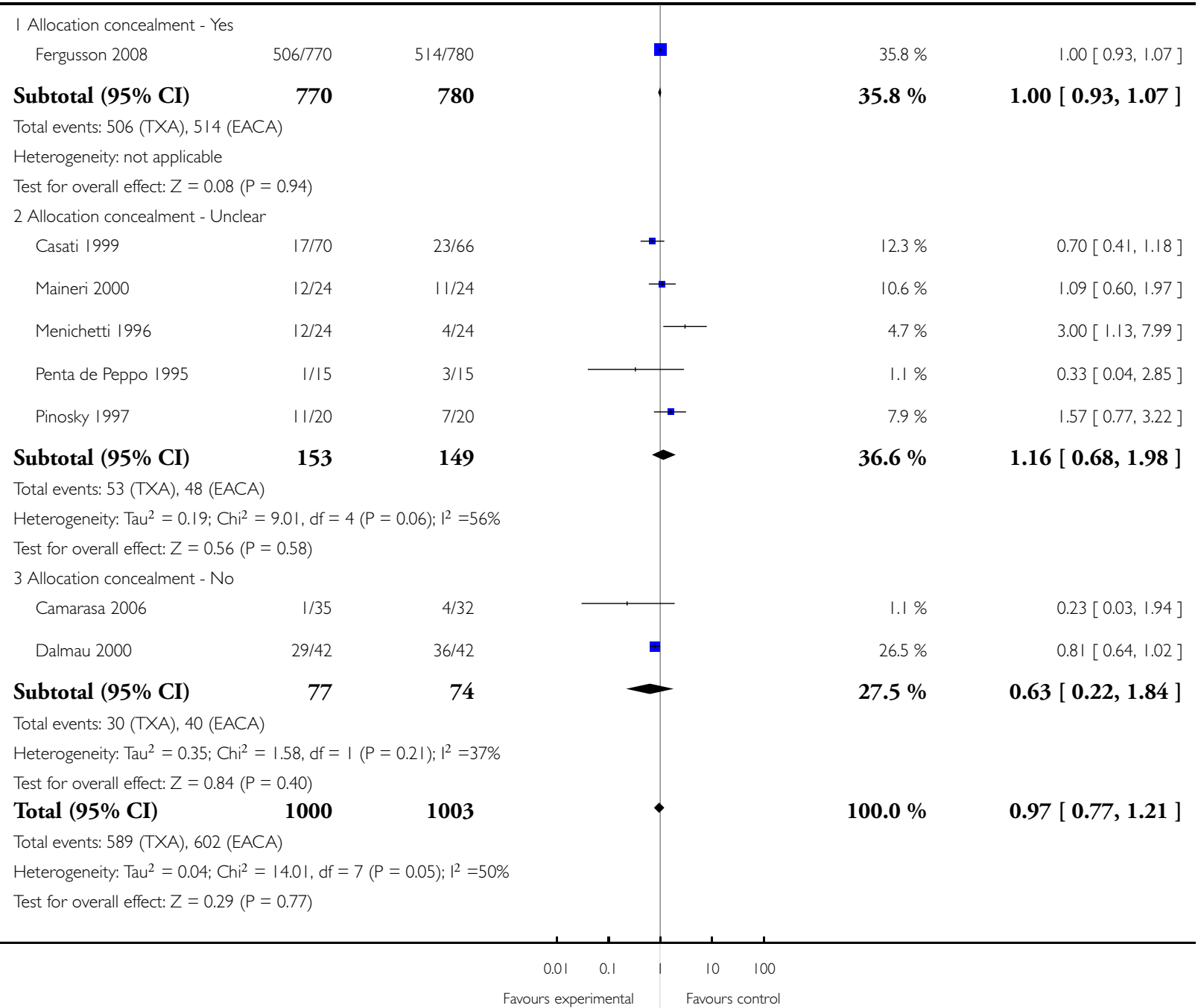




\section{Analysis 6.5. Comparison 6 Tranexamic Acid versus Epsilon Aminocaproic Acid (Blood Transfusion \& Blood Loss), Outcome 5 Units of Allogeneic Blood Transfused - Transfused Patients.}

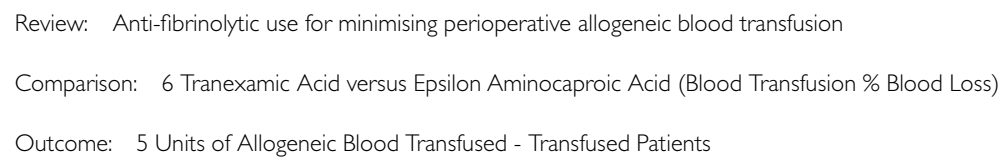

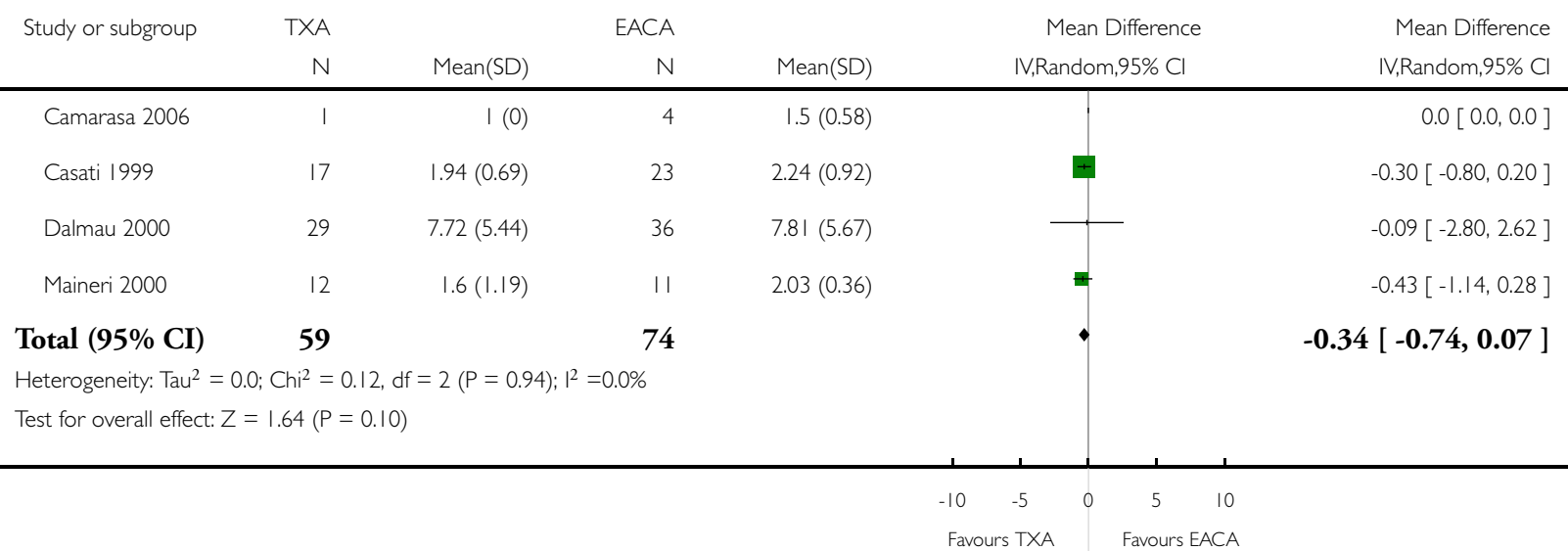

Analysis 6.6. Comparison 6 Tranexamic Acid versus Epsilon Aminocaproic Acid (Blood Transfusion \& Blood Loss), Outcome 6 Units of Allogeneic Blood Transfused - All Patients.

Review: Anti-fibrinolytic use for minimising perioperative allogeneic blood transfusion

Comparison: 6 Tranexamic Acid versus Epsilon Aminocaproic Acid (Blood Transfusion \% Blood Loss)

Outcome: 6 Units of Allogeneic Blood Transfused - All Patients

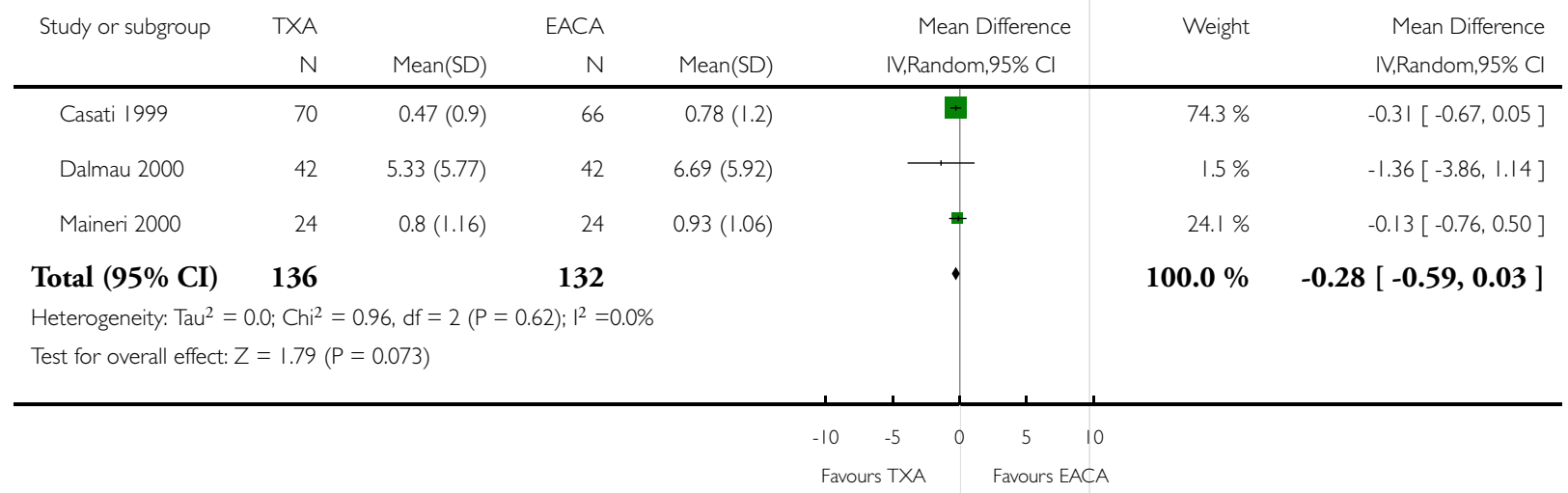


Analysis 6.7. Comparison 6 Tranexamic Acid versus Epsilon Aminocaproic Acid (Blood Transfusion \& Blood Loss), Outcome 7 Blood loss.

Review: Anti-fibrinolytic use for minimising perioperative allogeneic blood transfusion

Comparison: 6 Tranexamic Acid versus Epsilon Aminocaproic Acid (Blood Transfusion \% Blood Loss)

Outcome: 7 Blood loss

\begin{tabular}{|c|c|c|c|c|c|c|c|c|c|}
\hline \multirow[t]{2}{*}{ Study or subgroup } & \multirow{2}{*}{$\begin{array}{r}\text { TXA } \\
N\end{array}$} & \multicolumn{3}{|c|}{ EACA } & \multirow{2}{*}{\multicolumn{3}{|c|}{$\begin{array}{l}\text { Mean Difference } \\
\text { IV,Random,95\% Cl }\end{array}$}} & \multirow[t]{2}{*}{ Weight } & \multirow{2}{*}{$\begin{array}{l}\text { Mean Difference } \\
\text { IV,Random,95\% Cl }\end{array}$} \\
\hline & & Mean(SD) & $\mathrm{N}$ & Mean(SD) & & & & & \\
\hline \multicolumn{10}{|c|}{ I Cardiac surgery - Post-operative } \\
\hline Casati 1999 & 70 & $310.9(231.1)$ & 66 & $466.9(234.2)$ & $\hookrightarrow$ & - & & $17.8 \%$ & $-156.00[-234.25,-77.75]$ \\
\hline Landymore 1997 & 56 & $535(336.75)$ & 44 & $343(305.13)$ & & & 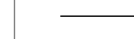 & $16.4 \%$ & $192.00[65.87,318.13]$ \\
\hline Maineri 2000 & 24 & $750(314)$ & 24 & $790(274)$ & & & & $15.0 \%$ & $-40.00[-206.73,126.73]$ \\
\hline Menichetti 1996 & 24 & $737(400)$ & 24 & $512(250)$ & & & & $14.2 \%$ & $225.00[36.28,413.72]$ \\
\hline Penta de Peppo 1995 & 15 & $534(288)$ & 15 & $509(148)$ & & & 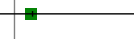 & $15.1 \%$ & $25.00[-138.86,188.86]$ \\
\hline Pinosky 1997 & 20 & $600(219.1)$ & 20 & $961(661.9)$ & $\longleftarrow$ & & & $10.1 \%$ & $-361.00[-666.56,-55.44]$ \\
\hline Subtotal (95\% CI) & 209 & & 193 & & & & & $88.4 \%$ & $-4.36[-163.35,154.63]$ \\
\hline \multicolumn{10}{|c|}{ Heterogeneity: $\operatorname{Tau}^{2}=31640.08 ; \mathrm{Chi}^{2}=33.8 \mathrm{I}, \mathrm{df}=5(\mathrm{P}<0.0000 \mathrm{I}) ; \mathrm{I}^{2}=85 \%$} \\
\hline \multicolumn{10}{|c|}{ Test for overall effect: $Z=0.05(P=0.96)$} \\
\hline \multicolumn{10}{|c|}{2 Orthopaedic surgery - Total } \\
\hline Camarasa 2006 & 35 & $1095(473)$ & 32 & $1104(603)$ & & & & $11.6 \%$ & $-9.00[-270.16,252.16]$ \\
\hline Subtotal (95\% CI) & 35 & & 32 & & & & & $11.6 \%$ & $-9.00[-270.16,252.16]$ \\
\hline \multicolumn{10}{|c|}{ Heterogeneity: not applicable } \\
\hline \multicolumn{10}{|c|}{ Test for overall effect: $Z=0.07(P=0.95)$} \\
\hline \multicolumn{10}{|c|}{3 Gynaecological surgery - Total } \\
\hline Subtotal (95\% CI) & $\mathbf{0}$ & & $\mathbf{0}$ & & & & & $0.0 \%$ & $0.0[0.0,0.0]$ \\
\hline \multicolumn{10}{|c|}{ Heterogeneity: not applicable } \\
\hline \multicolumn{10}{|c|}{ Test for overall effect: not applicable } \\
\hline Total (95\% CI) & 244 & & 225 & & & & & $100.0 \%$ & $-4.20[-147.29,138.89]$ \\
\hline \multicolumn{10}{|c|}{ Heterogeneity: $\mathrm{Tau}^{2}=28391.91 ; \mathrm{Chi}^{2}=33.84, \mathrm{df}=6(\mathrm{P}<0.0000 \mathrm{I}) ; \mathrm{I}^{2}=82 \%$} \\
\hline \multicolumn{10}{|c|}{ Test for overall effect: $Z=0.06(P=0.95)$} \\
\hline & & & & & -200 & -100 & 100 & 200 & \\
\hline & & & & & & rs TXA & Favours & & \\
\hline
\end{tabular}


Analysis 7.I. Comparison 7 Aprotinin versus Lysine Analogues (Blood Transfusion), Outcome I No. Exposed to Allogeneic Blood.

Review: Anti-fibrinolytic use for minimising perioperative allogeneic blood transfusion

Comparison: 7 Aprotinin versus Lysine Analogues (Blood Transfusion)

Outcome: I No. Exposed to Allogeneic Blood

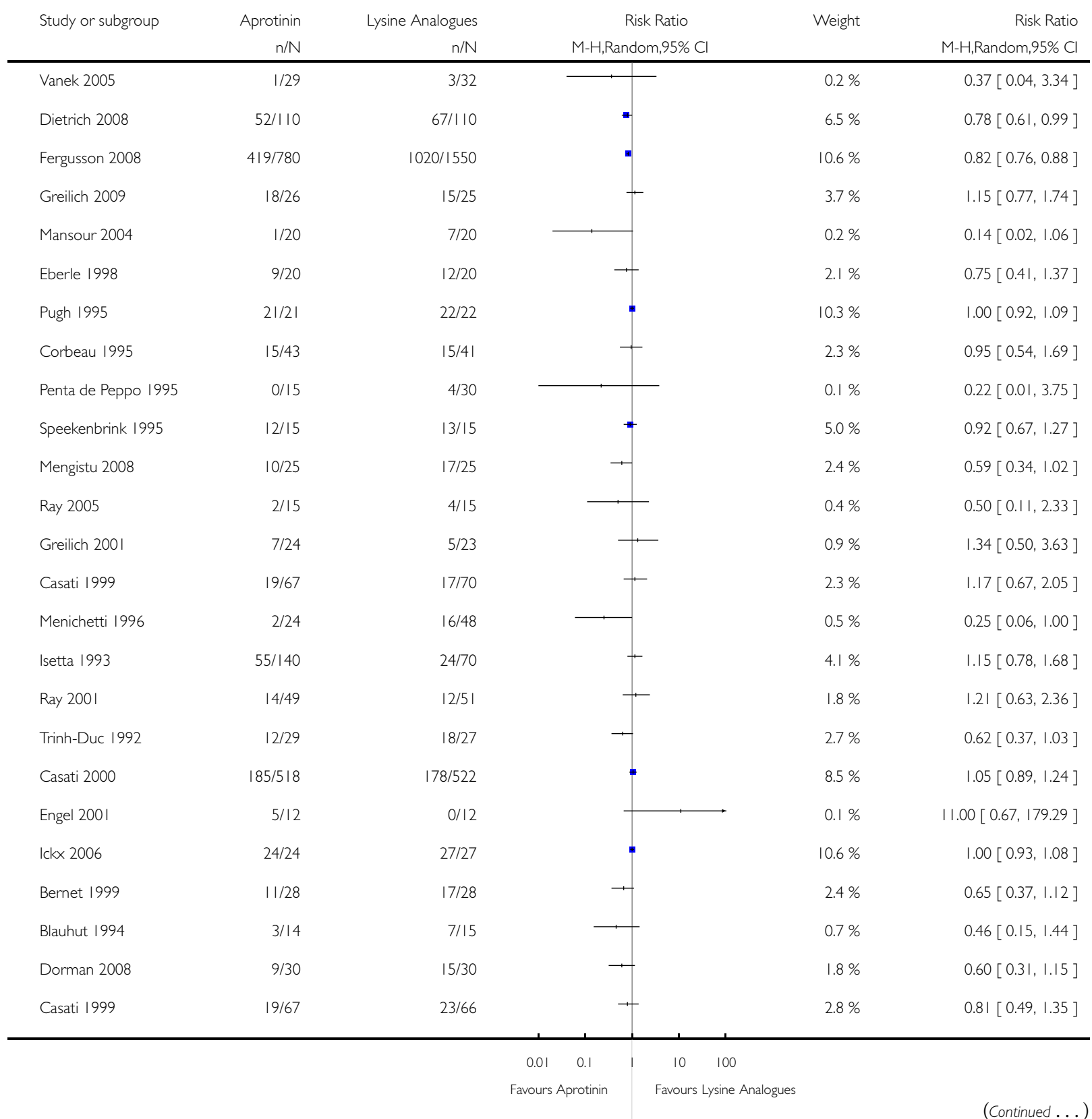




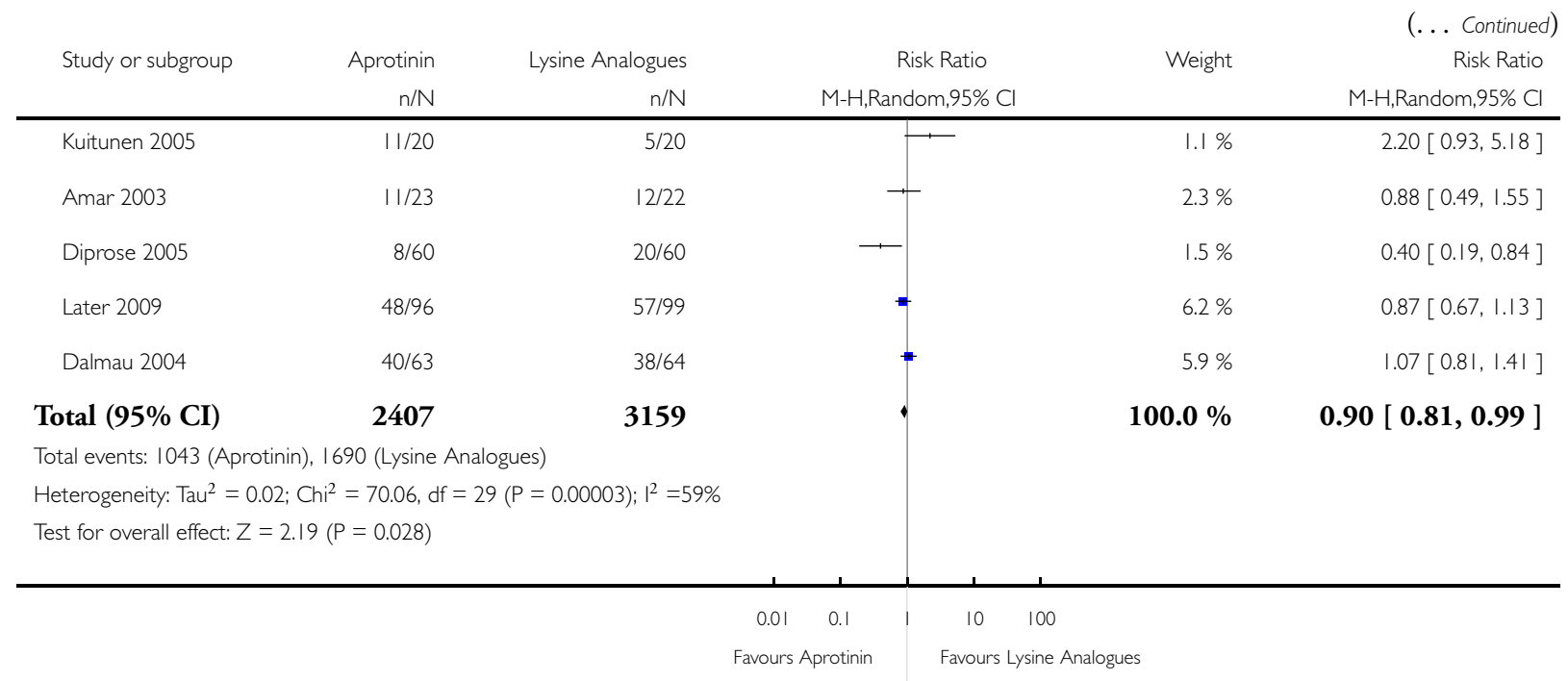

Analysis 7.2. Comparison 7 Aprotinin versus Lysine Analogues (Blood Transfusion), Outcome 2 No. Exposed to Allogeneic Blood - Type of Surgery.

Review: Anti-fibrinolytic use for minimising perioperative allogeneic blood transfusion

Comparison: 7 Aprotinin versus Lysine Analogues (Blood Transfusion)

Outcome: 2 No. Exposed to Allogeneic Blood - Type of Surgery

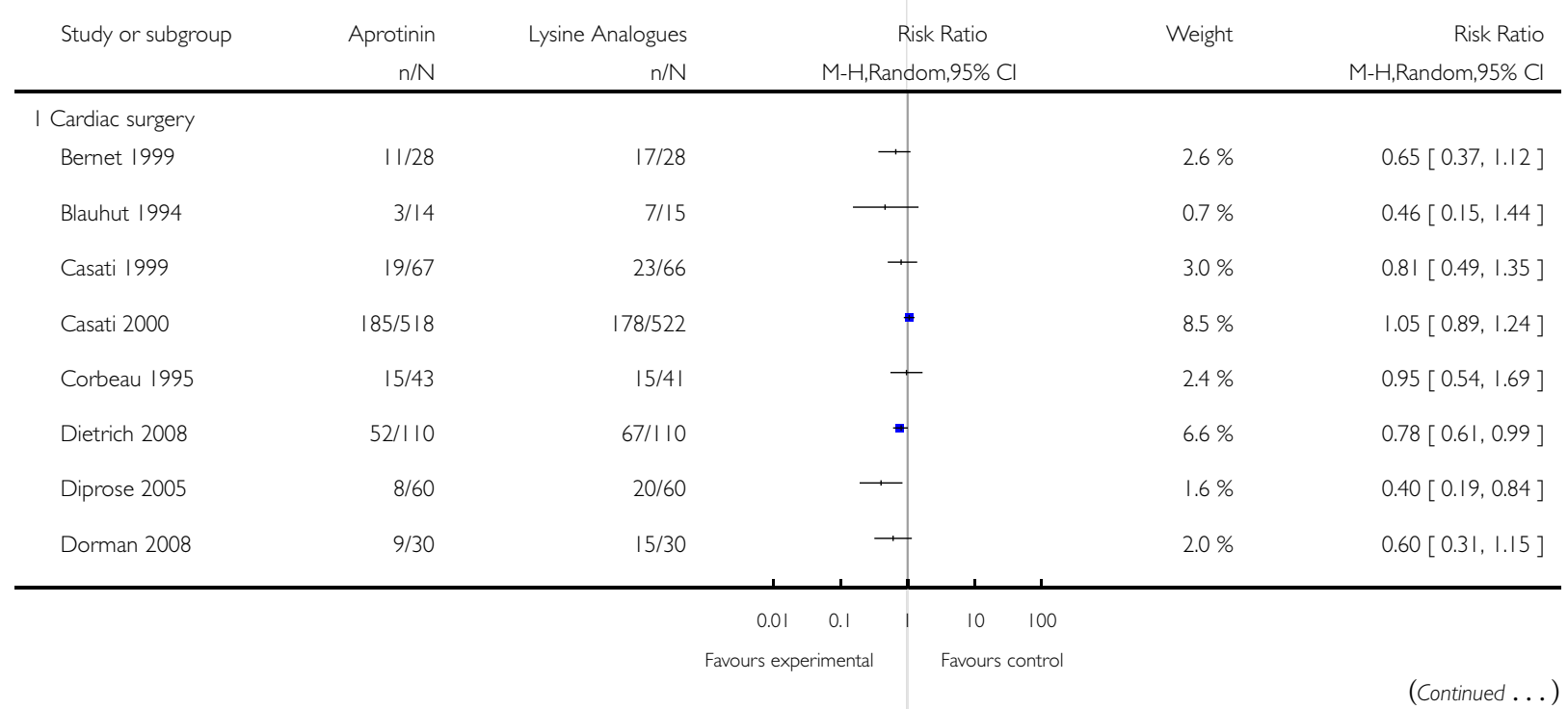




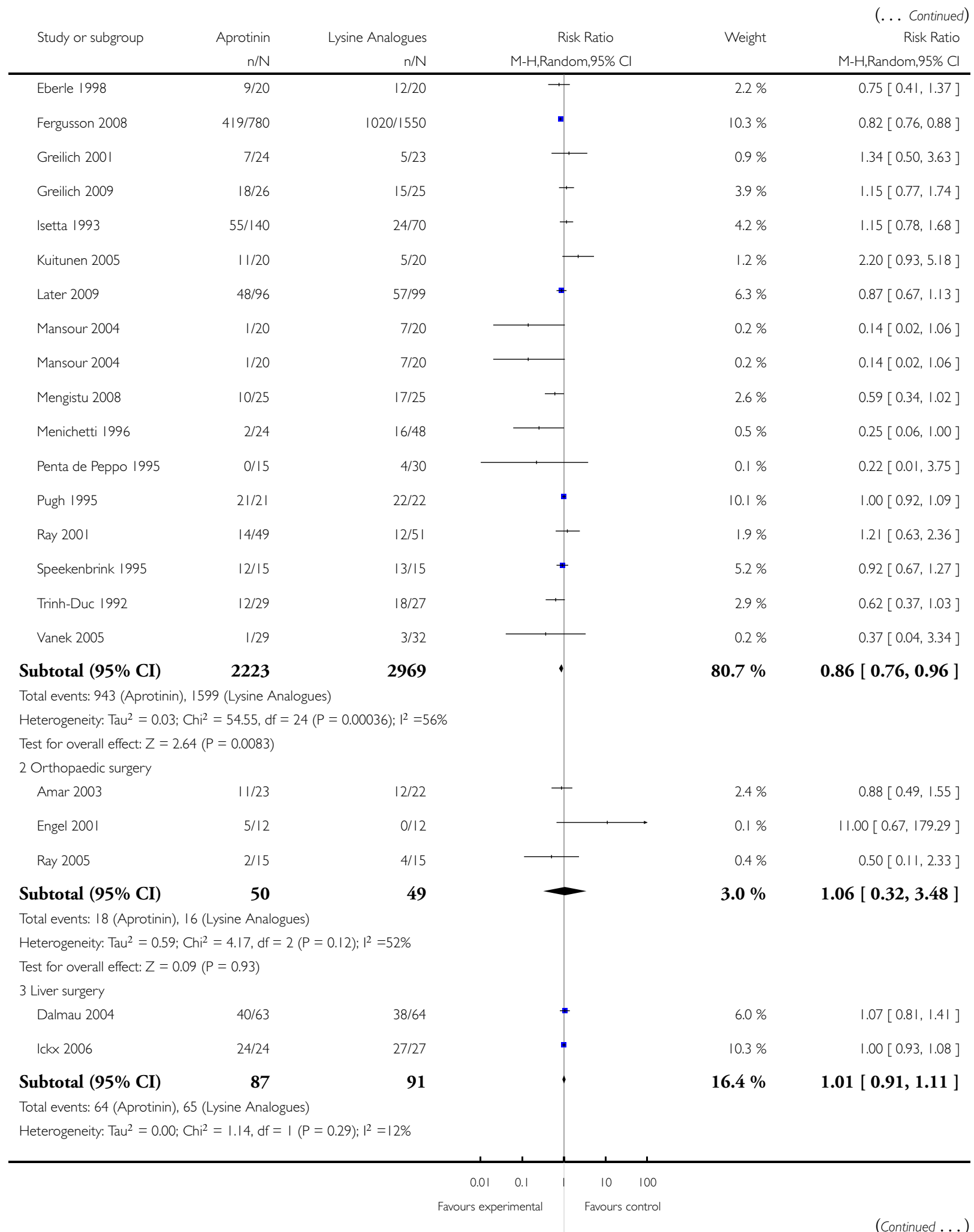

Anti-fibrinolytic use for minimising perioperative allogeneic blood transfusion (Review)

Copyright (C) 201 I The Cochrane Collaboration. Published by John Wiley \& Sons, Ltd. 


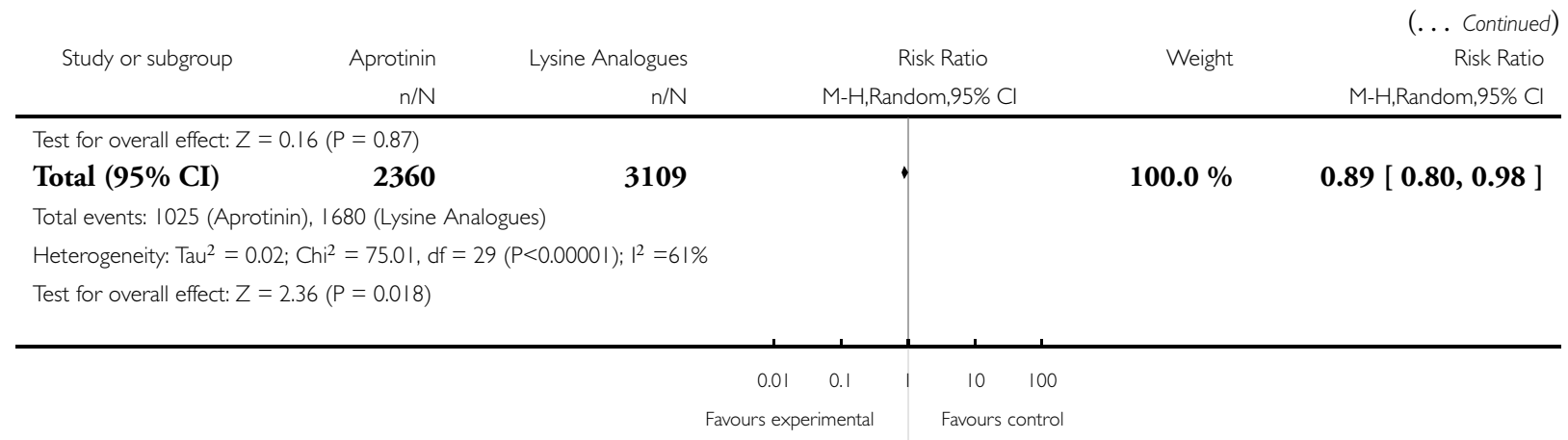

Analysis 7.3. Comparison 7 Aprotinin versus Lysine Analogues (Blood Transfusion), Outcome 3 No. Exposed to Allogeneic Blood - Transfusion Protocol.

Review: Anti-fibrinolytic use for minimising perioperative allogeneic blood transfusion

Comparison: 7 Aprotinin versus Lysine Analogues (Blood Transfusion)

Outcome: 3 No. Exposed to Allogeneic Blood - Transfusion Protocol

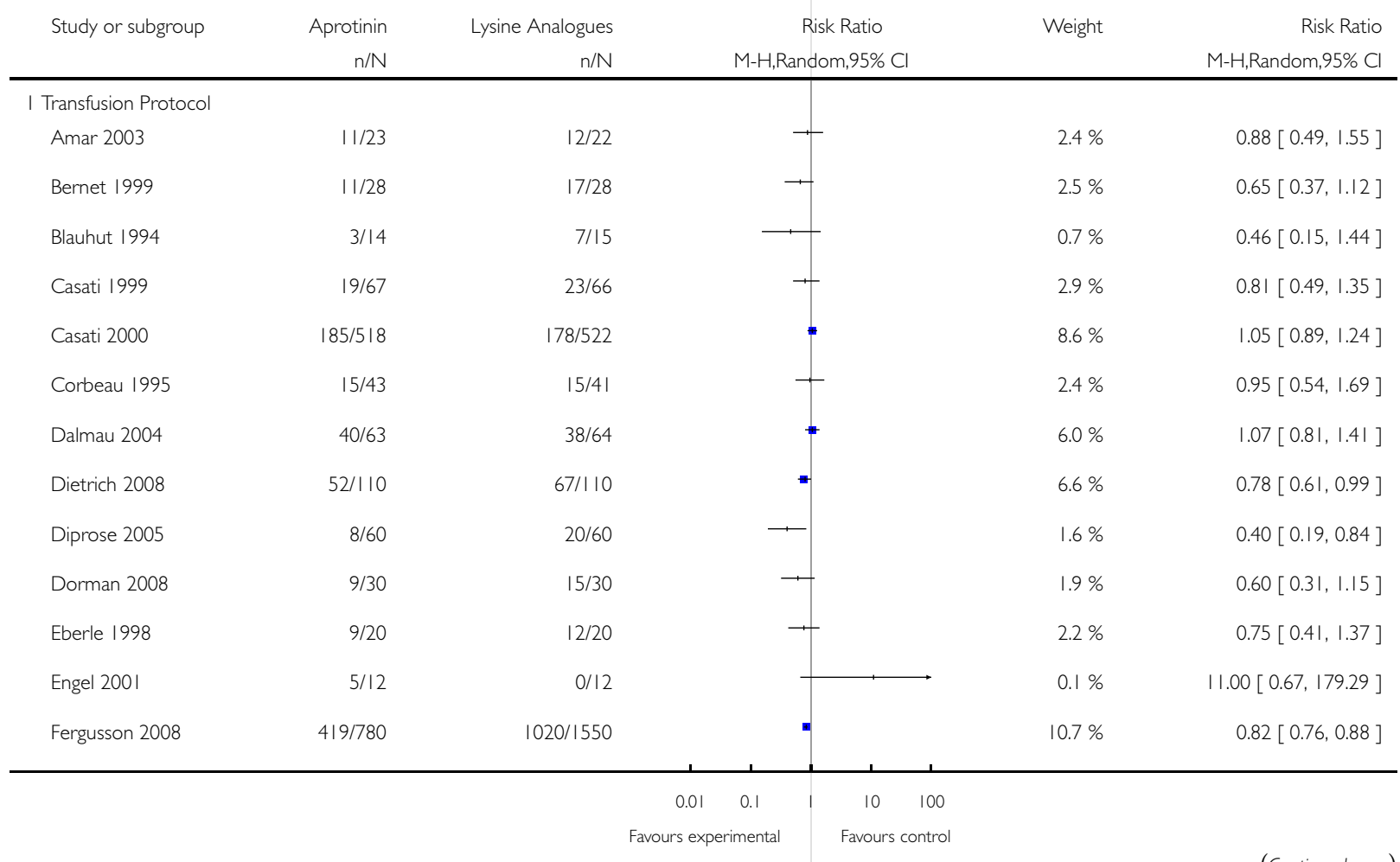

(Continued ... ) 


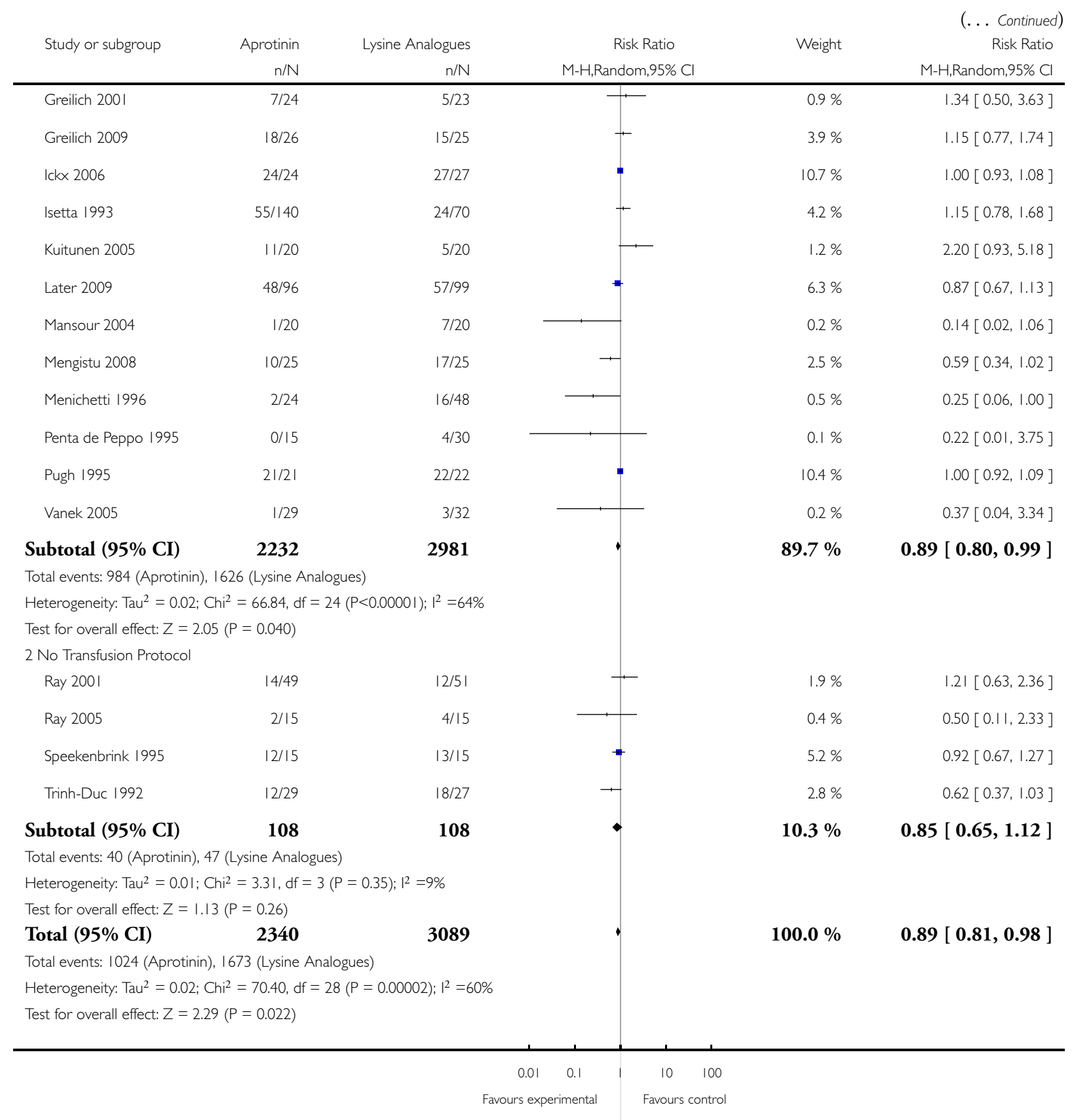


Analysis 7.4. Comparison 7 Aprotinin versus Lysine Analogues (Blood Transfusion), Outcome 4 Units of Allogeneic Blood Transfused - Transfused Patients.

Review: Anti-fibrinolytic use for minimising perioperative allogeneic blood transfusion

Comparison: 7 Aprotinin versus Lysine Analogues (Blood Transfusion)

Outcome: 4 Units of Allogeneic Blood Transfused - Transfused Patients

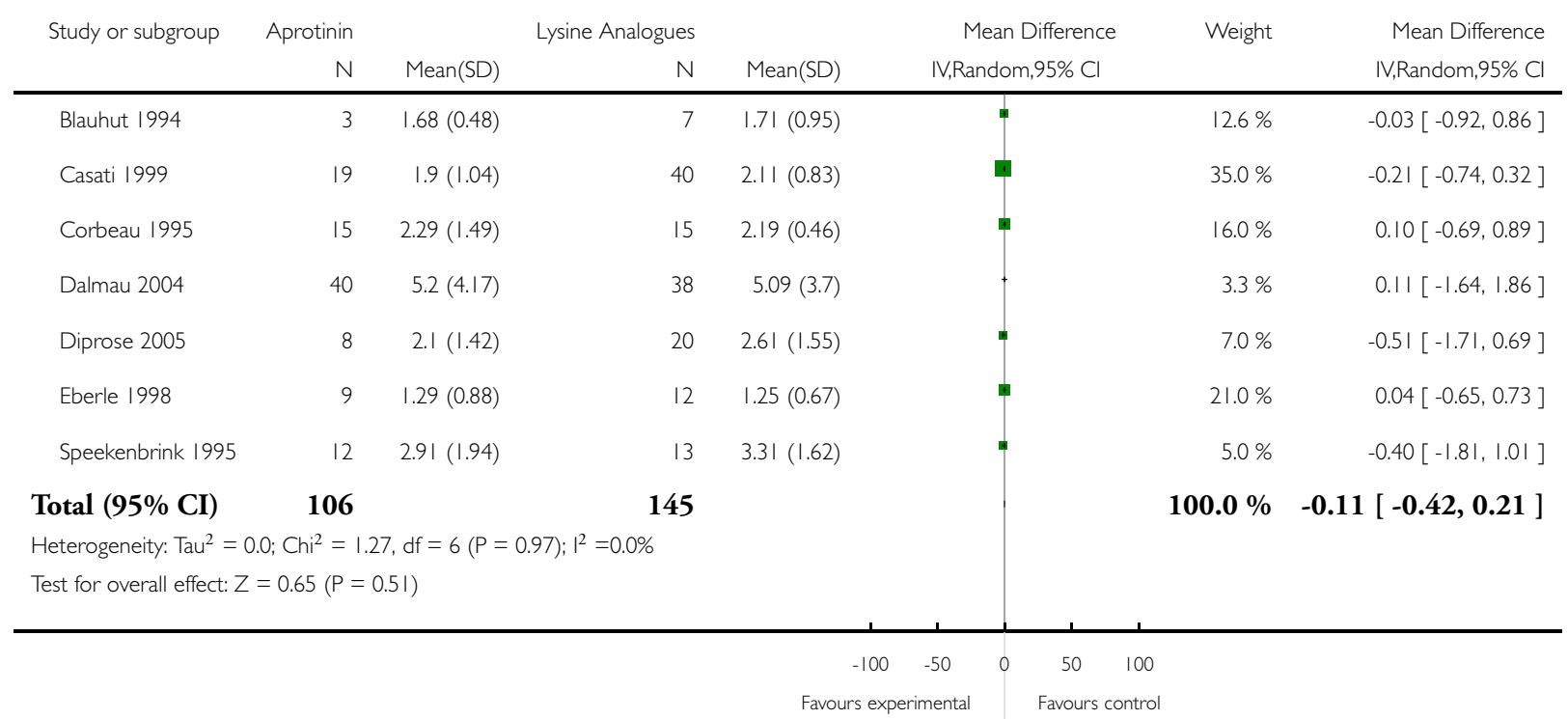


Analysis 7.5. Comparison 7 Aprotinin versus Lysine Analogues (Blood Transfusion), Outcome 5 Units of Allogeneic Blood Transfused - All Patients.

Review: Anti-fibrinolytic use for minimising perioperative allogeneic blood transfusion

Comparison: 7 Aprotinin versus Lysine Analogues (Blood Transfusion)

Outcome: 5 Units of Allogeneic Blood Transfused - All Patients

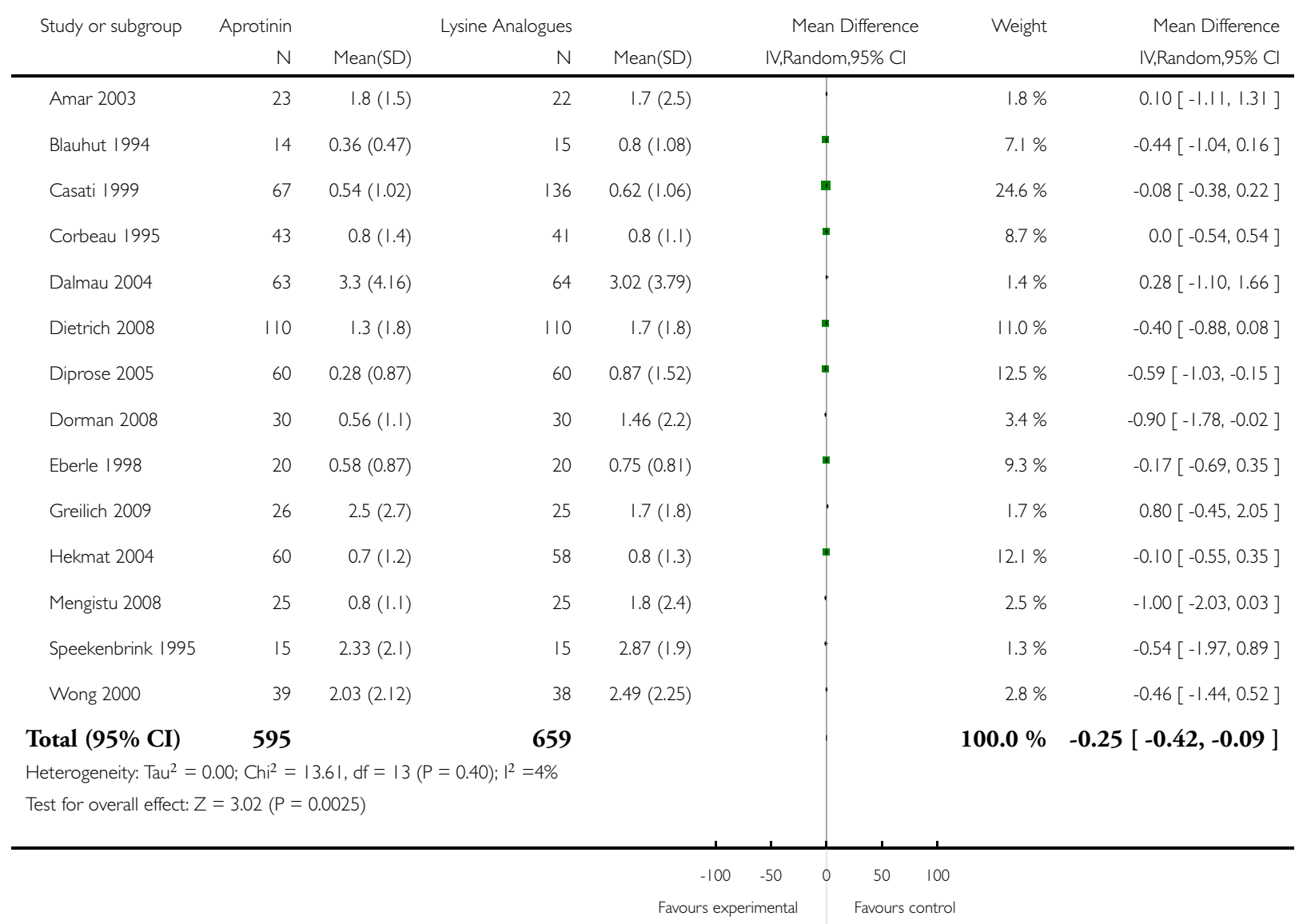


Analysis 7.6. Comparison 7 Aprotinin versus Lysine Analogues (Blood Transfusion), Outcome 6 Trial Methodological Quality - Allocation Concealment.

Review: Anti-fibrinolytic use for minimising perioperative allogeneic blood transfusion

Comparison: 7 Aprotinin versus Lysine Analogues (Blood Transfusion)

Outcome: 6 Trial Methodological Quality - Allocation Concealment

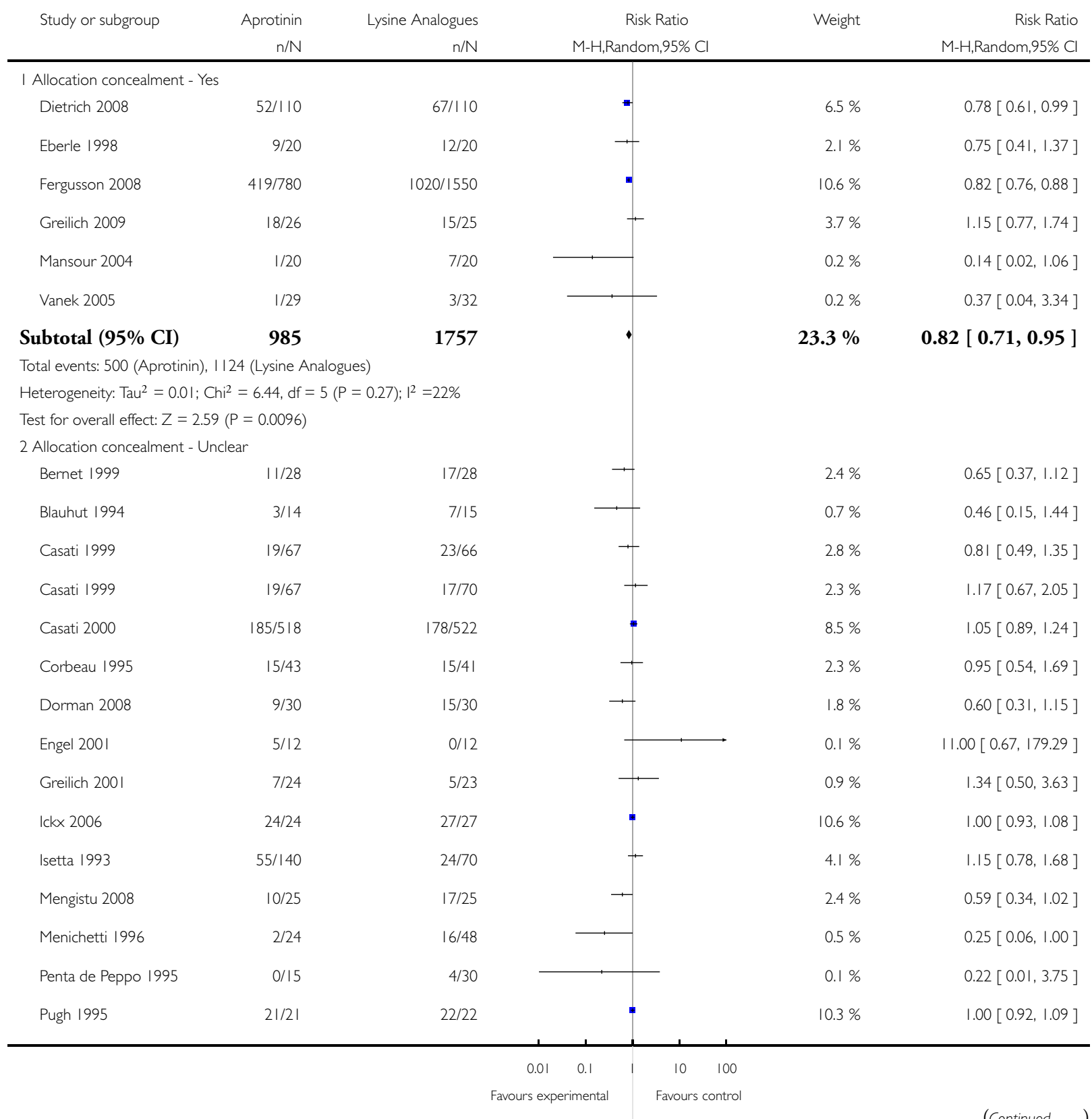




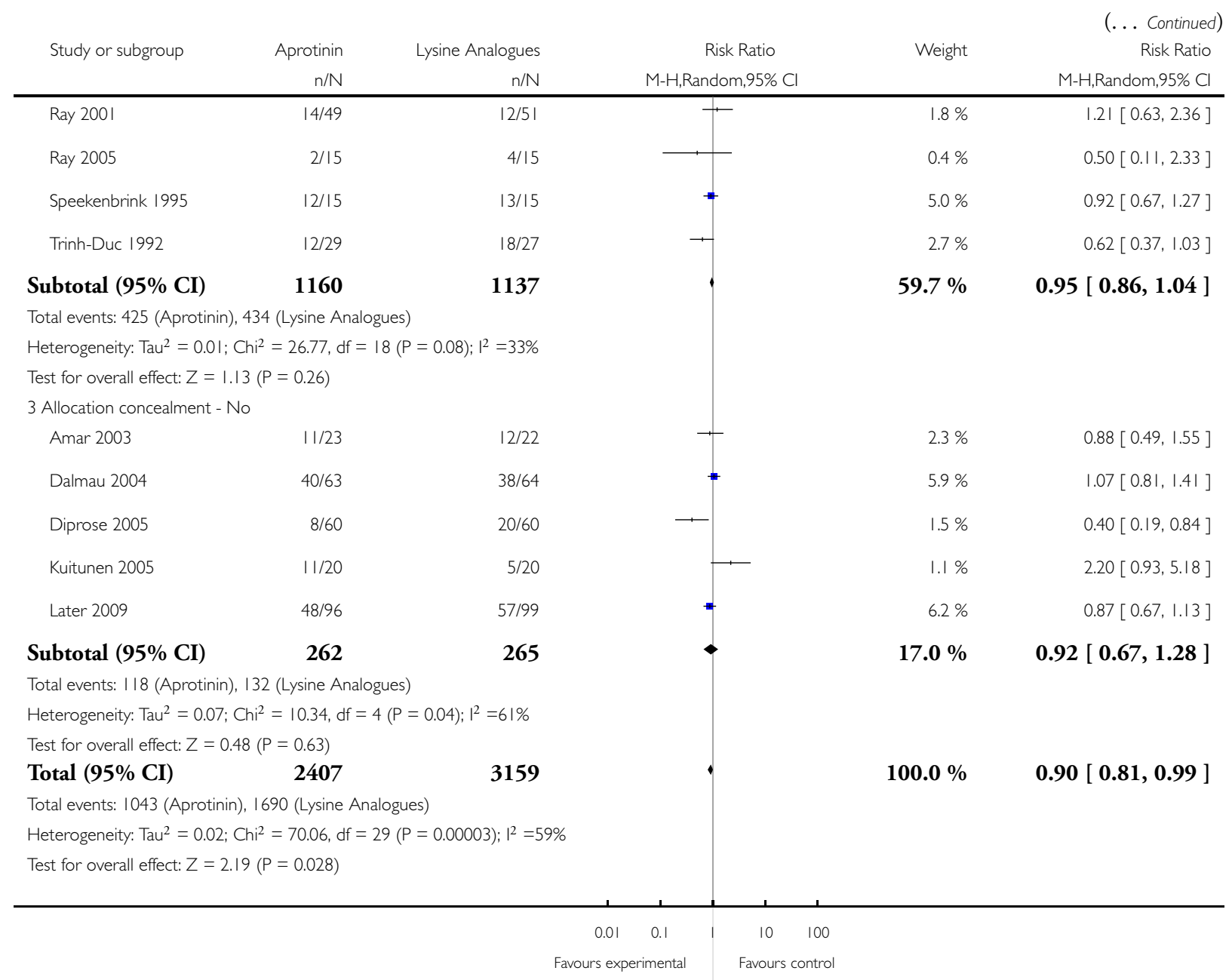




\section{Analysis 8.I. Comparison 8 Adverse Events and Other Outcomes (Active versus Control), Outcome I Re- operation for bleeding.}

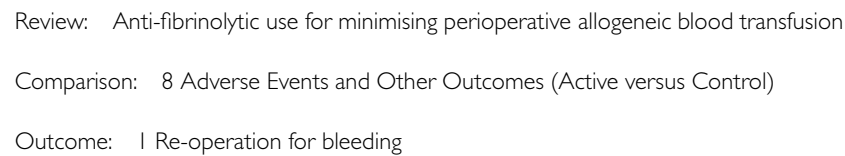

\begin{tabular}{|c|c|c|c|c|}
\hline & $\mathrm{n} / \mathrm{N}$ & $\mathrm{n} / \mathrm{N}$ & M-H,Random,95\% Cl & M-H,Random,95\% Cl \\
\hline \multicolumn{5}{|l|}{ I Aprotinin versus Control } \\
\hline Alderman 1998 & $11 / 436$ & $19 / 434$ & - & $0.58[0.28,1.20]$ \\
\hline Alvarez 1995 & $6 / 49$ & $2 / 51$ & & $3.12[0.66,14.73]$ \\
\hline Alvarez 2001 & $0 / 26$ & $0 / 29$ & & $0.0[0.0,0.0]$ \\
\hline Apostolakis 2008 & $0 / 29$ & $0 / 30$ & & $0.0[0.0,0.0]$ \\
\hline Ashraf 1997 & $0 / 19$ & $0 / 19$ & & $0.0[0.0,0.0]$ \\
\hline Asimakopoulos 2000 & $0 / 8$ & $0 / 10$ & & $0.0[0.0,0.0]$ \\
\hline Bert 2008 & $0 / 25$ & $1 / 25$ & & $0.33[0.01,7.81]$ \\
\hline Bidstrup 1989 & $0 / 40$ & $2 / 40$ & - & $0.20[0.01,4.04]$ \\
\hline Bidstrup 1990 & $1 / 26$ & $4 / 18$ & & $0.17[0.02,1.42]$ \\
\hline Bidstrup 1993 & $0 / 43$ & $3 / 47$ & - & $0.16[0.01,2.93]$ \\
\hline Bidstrup 2000 & $1 / 30$ & $1 / 30$ & & $1.00[0.07,15.26]$ \\
\hline Boldt 1994 & $0 / 20$ & $0 / 20$ & & $0.0[0.0,0.0]$ \\
\hline Casas 1995 & $1 / 47$ & $0 / 51$ & & $3.25[0.14,77.88]$ \\
\hline Cicekcioglu 2006 & $0 / 24$ & $1 / 20$ & \begin{tabular}{l|l}
1 & \\
\end{tabular} & $0.28[0.01,6.52]$ \\
\hline Cosgrove 1992 & $4 / 113$ & $0 / 56$ & & $4.50[0.25,82.14]$ \\
\hline D'Ambra 1996 & $2 / 141$ & $5 / 71$ & $\square$ & $0.20[0.04,1.01]$ \\
\hline Deleuze 1991 & $0 / 30$ & $4 / 30$ & - & $0.11[0.01,1.98]$ \\
\hline Demeyere 2006 & $0 / 20$ & $0 / 20$ & & $0.0[0.0,0.0]$ \\
\hline Desai 2009 & $1 / 38$ & $2 / 37$ & - & $0.49[0.05,5.14]$ \\
\hline Dietrich 1990 & $0 / 19$ & $0 / 20$ & & $0.0[0.0,0.0]$ \\
\hline Dietrich 1995 & $1 / 15$ & $4 / 15$ & - & $0.25[0.03,1.98]$ \\
\hline Dignan 200I & $2 / 101$ & 2/99 & & $0.98[0.14,6.82]$ \\
\hline Diprose 2005 & $2 / 60$ & $7 / 60$ & t & $0.29[0.06,1.32]$ \\
\hline Ehrlich 1998 & $0 / 25$ & $0 / 25$ & & $0.0[0.0,0.0]$ \\
\hline
\end{tabular}

$0.0010 .010 .1 \quad 1 \quad 10 \quad 1001000$

Favours Treatment Favours Control 


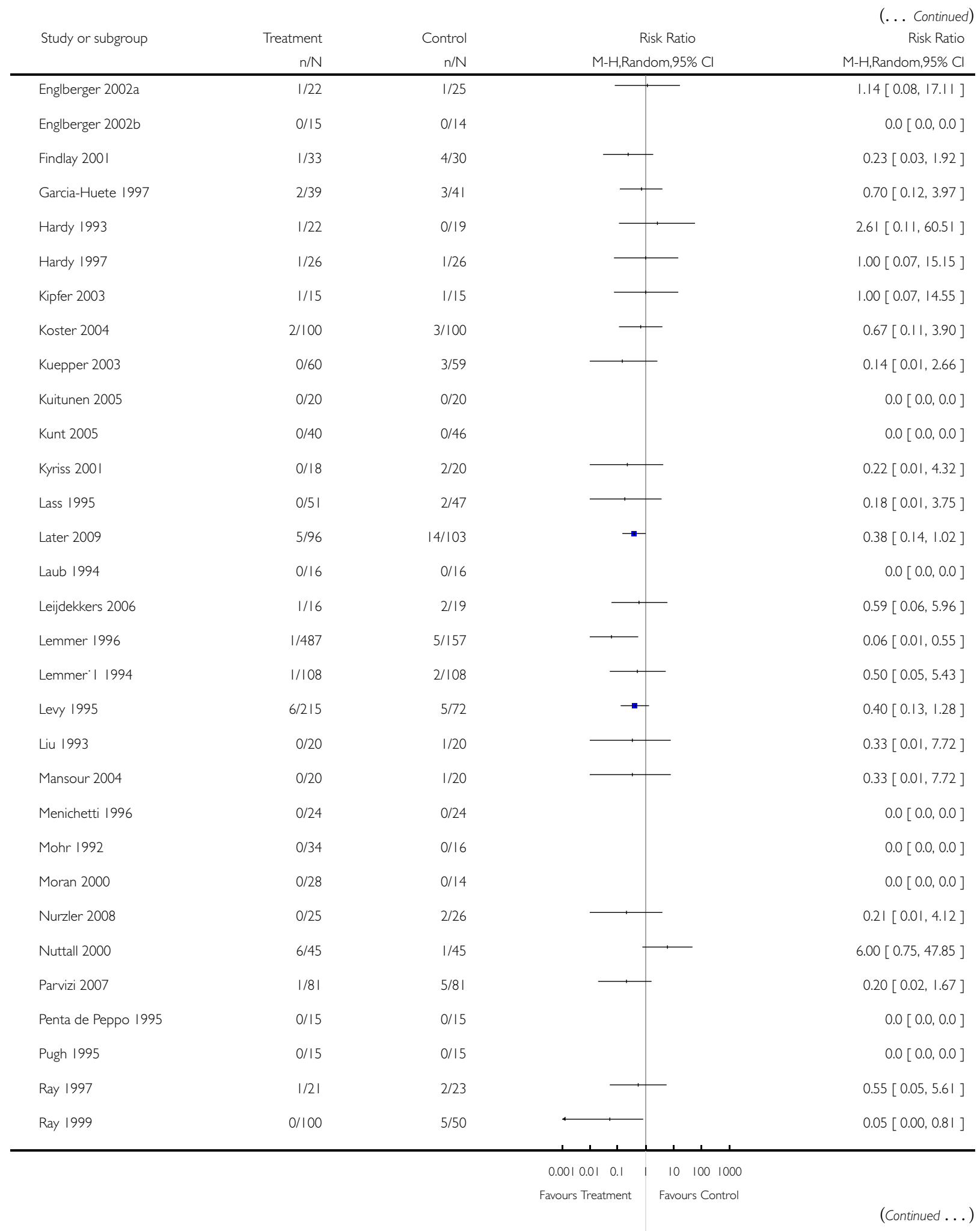

Anti-fibrinolytic use for minimising perioperative allogeneic blood transfusion (Review)

Copyright (@) 20II The Cochrane Collaboration. Published by John Wiley \& Sons, Ltd. 


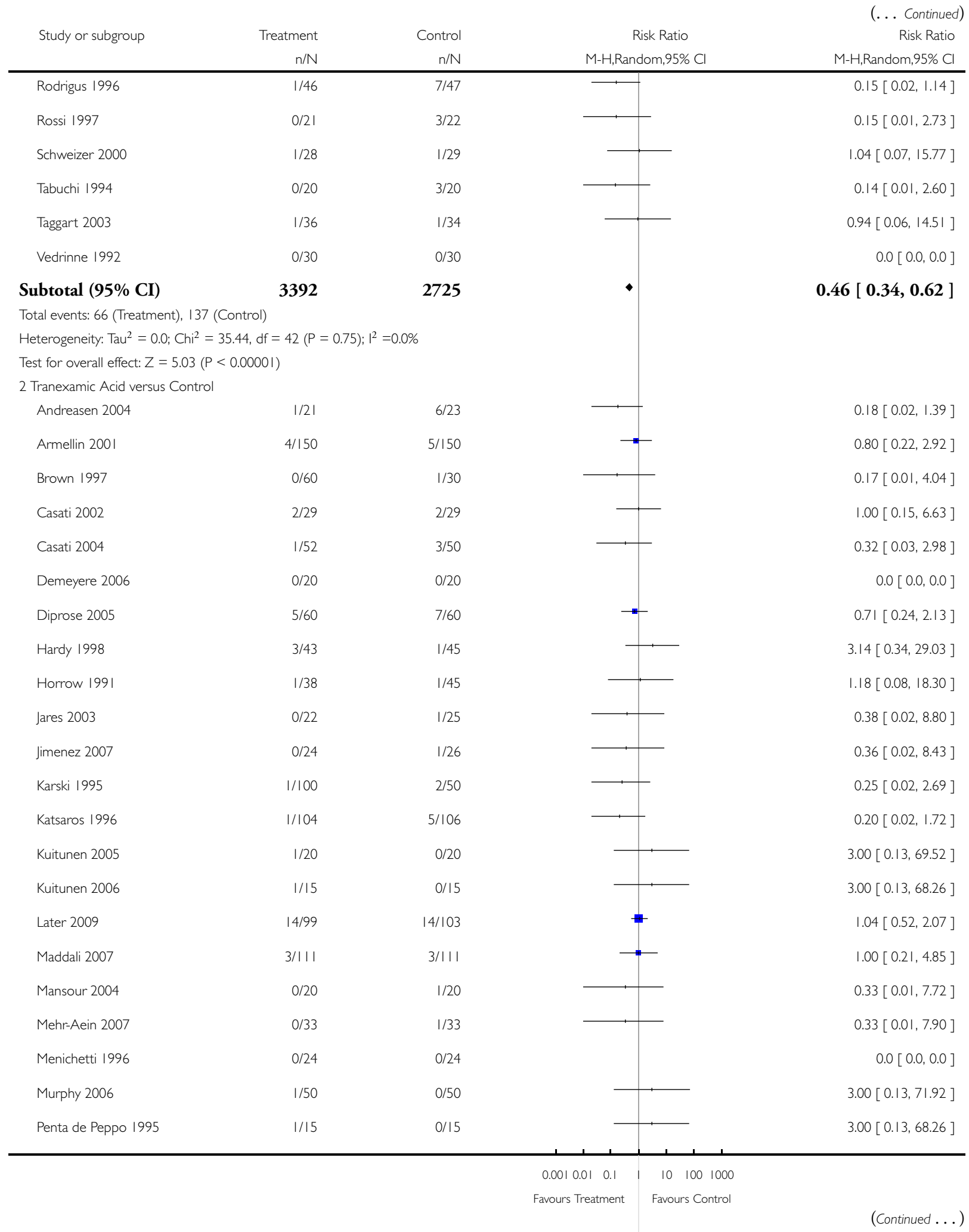

Anti-fibrinolytic use for minimising perioperative allogeneic blood transfusion (Review) 


\begin{tabular}{|c|c|c|c|c|}
\hline \multirow[t]{2}{*}{ Study or subgroup } & Treatment & Control & Risk Ratio & $\begin{array}{r}\text { (. . . Continued) } \\
\text { Risk Ratio } \\
\text { M-H.Random, } 95 \% \text { Cl }\end{array}$ \\
\hline & $n / N$ & $n / N$ & M-H,Random,95\% Cl & M-H,Random,, $95 \% \mathrm{Cl}$ \\
\hline Pleym 2003 & $0 / 40$ & $1 / 39$ & 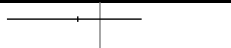 & $0.33[0.01,7.75]$ \\
\hline Pugh 1995 & $2 / 22$ & $2 / 23$ & + & $1.05[0.16,6.79]$ \\
\hline Santos 2006 & 1/29 & $|/ 3|$ & & $1.07[0.07,16.31]$ \\
\hline Shore-Lesserson 1996 & 0/17 & $0 / 13$ & & $0.0[0.0,0.0]$ \\
\hline Uozaki 2001 & 1/6 & $1 / 6$ & & $1.00[0.08,12.56]$ \\
\hline Subtotal (95\% CI) & 1224 & 1162 & $\bullet$ & $0.80[0.55,1.17]$ \\
\hline \multicolumn{5}{|c|}{ Total events: 44 (Treatment), 59 (Control) } \\
\hline \multicolumn{5}{|c|}{ Heterogeneity: Tau $^{2}=0.0 ; \mathrm{Chi}^{2}=12.66, \mathrm{df}=23(\mathrm{P}=0.96) ; \mathrm{I}^{2}=0.0 \%$} \\
\hline \multicolumn{5}{|c|}{ Test for overall effect: $Z=1.16(P=0.25)$} \\
\hline \multicolumn{5}{|c|}{3 Epsilon Aminocaproic Acid versus Control } \\
\hline Berenholtz 2009 & $0 / 91$ & 2/91 & .7 & $0.20[0.01,4.11]$ \\
\hline Del Rossi 1989 & $0 / 170$ & $6 / 180$ & & $0.08[0.00,1.43]$ \\
\hline Hardy 1998 & 1/46 & $1 / 45$ & & $0.98[0.06,15.17]$ \\
\hline Kluger 2003 & 1/58 & $1 / 30$ & - & $0.52[0.03,7.98]$ \\
\hline Menichetti 1996 & $0 / 24$ & $0 / 24$ & & $0.0[0.0,0.0]$ \\
\hline Penta de Peppo 1995 & $0 / 15$ & $0 / 15$ & & $0.0[0.0,0.0]$ \\
\hline Rao 1999 & $0 / 15$ & $1 / 15$ & $\rightarrow-$ & $0.33[0.01,7.58]$ \\
\hline Vander-Salm 1996 & $|/ 5|$ & $3 / 52$ & - & $0.34[0.04,3.16]$ \\
\hline Subtotal (95\% CI) & 470 & 452 & - & $0.32[0.11,0.99]$ \\
\hline \multicolumn{5}{|c|}{ Total events: 3 (Treatment), 14 (Control) } \\
\hline \multicolumn{5}{|c|}{ Heterogeneity: $\operatorname{Tau}^{2}=0.0 ; \mathrm{Chi}^{2}=1.84, \mathrm{df}=5(\mathrm{P}=0.87) ; \mathrm{I}^{2}=0.0 \%$} \\
\hline \multicolumn{5}{|c|}{ Test for overall effect: $Z=1.97(P=0.048)$} \\
\hline
\end{tabular}


Analysis 8.2. Comparison 8 Adverse Events and Other Outcomes (Active versus Control), Outcome 2 Mortality.

Review: Anti-fibrinolytic use for minimising perioperative allogeneic blood transfusion

Comparison: 8 Adverse Events and Other Outcomes (Active versus Control)

Outcome: 2 Mortality

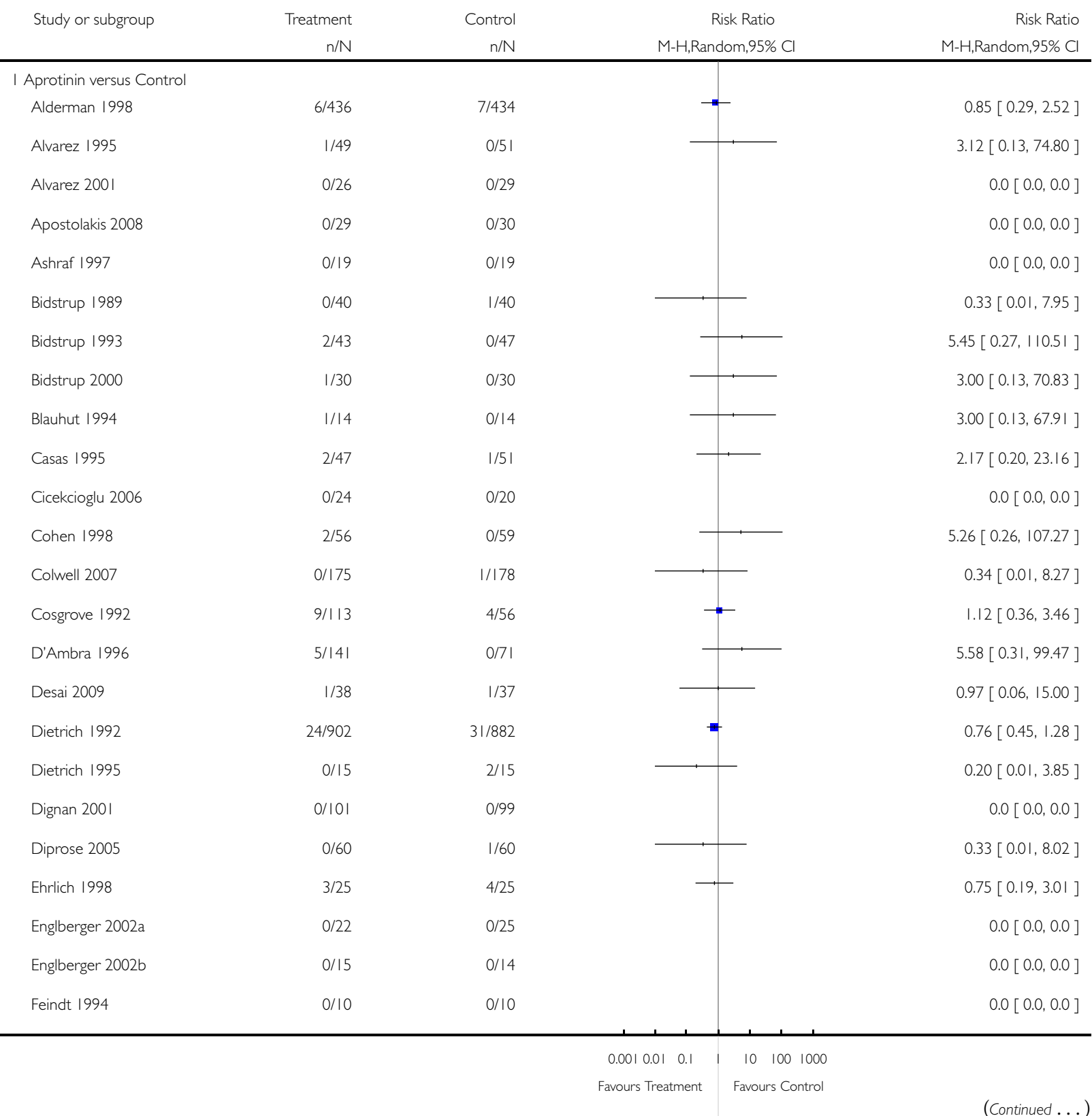




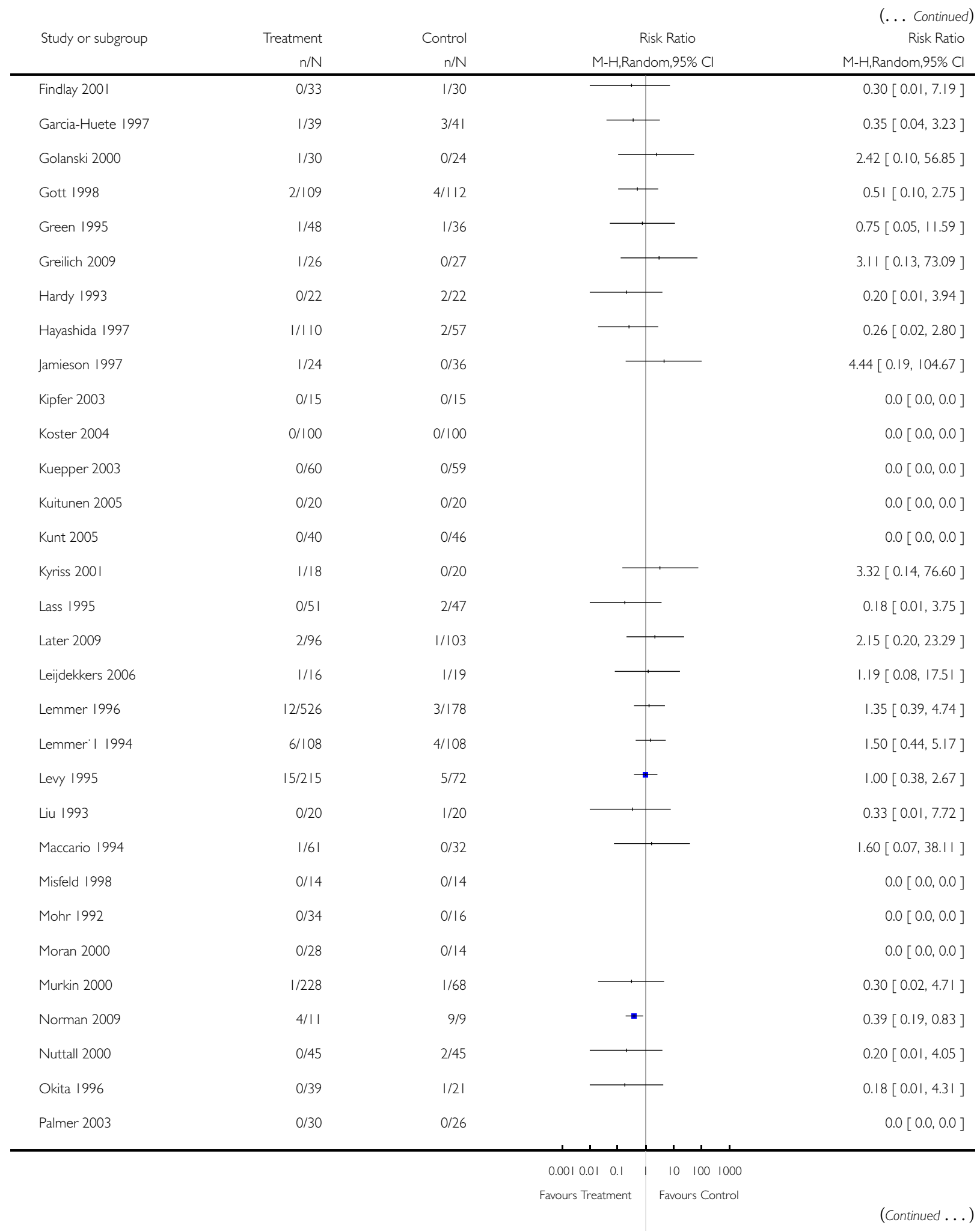

Anti-fibrinolytic use for minimising perioperative allogeneic blood transfusion (Review) 


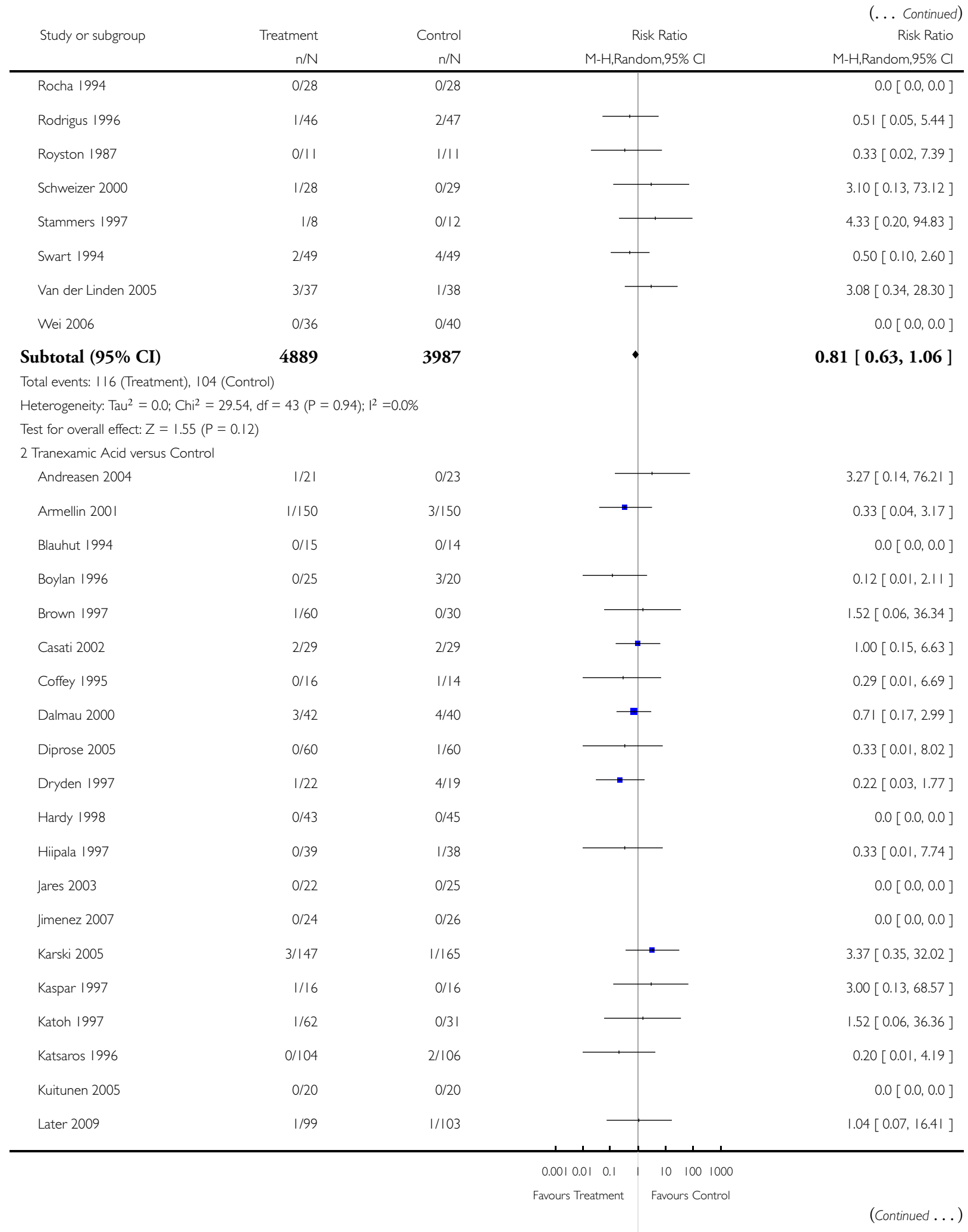

Anti-fibrinolytic use for minimising perioperative allogeneic blood transfusion (Review)

Copyright (C) 201 I The Cochrane Collaboration. Published by John Wiley \& Sons, Ltd. 


\begin{tabular}{|c|c|c|c|c|}
\hline \multirow[t]{2}{*}{ Study or subgroup } & Treatment & Control & \multirow{2}{*}{$\begin{array}{c}\text { Risk Ratio } \\
\text { M-H,Random,95\% Cl }\end{array}$} & \multirow{2}{*}{$\begin{array}{r}(\ldots \text { Continued }) \\
\text { Risk Ratio } \\
\mathrm{M}-\mathrm{H}, \text { Random,95\% Cl } \\
\end{array}$} \\
\hline & $n / N$ & $n / N$ & & \\
\hline Maddali 2007 & $0 / 111$ & $0 / 111$ & & $0.0[0.0,0.0]$ \\
\hline Mehr-Aein 2007 & 0/33 & $0 / 33$ & & $0.0[0.0,0.0]$ \\
\hline Misfeld 1998 & $0 / 14$ & $0 / 14$ & & $0.0[0.0,0.0]$ \\
\hline Murphy 2006 & $0 / 50$ & $0 / 50$ & & $0.0[0.0,0.0]$ \\
\hline Nuttall 2000 & $0 / 45$ & $2 / 45$ & 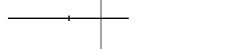 & $0.20[0.01,4.05]$ \\
\hline Sadeghi 2007 & $0 / 32$ & $1 / 35$ & - & $0.36[0.02,8.62]$ \\
\hline Santos 2006 & $0 / 29$ & $2 / 31$ & - & $0.21[0.01,4.26]$ \\
\hline Shore-Lesserson 1996 & 0/17 & $0 / 13$ & & $0.0[0.0,0.0]$ \\
\hline Wu 2006 & 0/106 & 0/108 & & $0.0[0.0,0.0]$ \\
\hline Zabeeda 2002 & $0 / 25$ & $0 / 25$ & & $0.0[0.0,0.0]$ \\
\hline Subtotal (95\% CI) & 1478 & 1439 & - & $0.60[0.33,1.10]$ \\
\hline \multicolumn{5}{|c|}{ Total events: 15 (Treatment), 28 (Control) } \\
\hline \multicolumn{5}{|c|}{ Heterogeneity: Tau $^{2}=0.0 ; \mathrm{Chi}^{2}=10.00, \mathrm{df}=17(P=0.90) ; 1^{2}=0.0 \%$} \\
\hline \multicolumn{5}{|c|}{ Test for overall effect: $Z=1.64(P=0.10)$} \\
\hline \multicolumn{5}{|c|}{3 Epsilon Aminocaproic Acid versus Control } \\
\hline Berenholtz 2009 & $0 / 91$ & $|/ 9|$ & 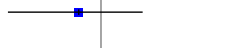 & $0.33[0.01,8.08]$ \\
\hline Daily 1994 & $0 / 21$ & $0 / 19$ & & $0.0[0.0,0.0]$ \\
\hline Dalmau 2000 & $3 / 42$ & $4 / 40$ & - & $0.71[0.17,2.99]$ \\
\hline Del Rossi 1989 & $3 / 170$ & $3 / 180$ & & $1.06[0.22,5.17]$ \\
\hline Greilich 2009 & 0/25 & $0 / 27$ & & $0.0[0.0,0.0]$ \\
\hline Hardy 1998 & 2/46 & 0/45 & - & $4.89[0.24,99.18]$ \\
\hline Kluger 2003 & 1/58 & $0 / 30$ & - & $1.58[0.07,37.56]$ \\
\hline Vander-Salm 1996 & $|/ 5|$ & $0 / 52$ & - & $3.06[0.13,73.36]$ \\
\hline Subtotal (95\% CI) & 504 & 484 & 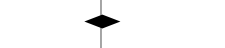 & $1.07[0.44,2.57]$ \\
\hline \multicolumn{5}{|c|}{ Total events: 10 (Treatment), 8 (Control) } \\
\hline \multicolumn{5}{|c|}{ Heterogeneity: $\operatorname{Tau}^{2}=0.0 ; \mathrm{Chi}^{2}=2.30, \mathrm{df}=5(\mathrm{P}=0.81) ; 1^{2}=0.0 \%$} \\
\hline Test for overall effect: $Z=$ & & & & \\
\hline
\end{tabular}


Analysis 8.3. Comparison 8 Adverse Events and Other Outcomes (Active versus Control), Outcome 3 Myocardial Infarction (MI).

Review: Anti-fibrinolytic use for minimising perioperative allogeneic blood transfusion

Comparison: 8 Adverse Events and Other Outcomes (Active versus Control)

Outcome: 3 Myocardial Infarction (MI)

\begin{tabular}{|c|c|c|c|c|}
\hline Study or subgroup & Treatment & Control & Risk Ratio & Risk Ratio \\
\hline & $\mathrm{n} / \mathrm{N}$ & $\mathrm{n} / \mathrm{N}$ & M-H,Random,95\% Cl & M-H,Random,95\% Cl \\
\hline \multicolumn{5}{|l|}{ I Aprotinin versus Control } \\
\hline Alderman 1998 & $12 / 410$ & $|6 / 42|$ & & $0.77[0.37,1.61]$ \\
\hline Alvarez 1995 & $2 / 49$ & $0 / 51$ & - & $5.20[0.26,105.65]$ \\
\hline Alvarez 200I & $0 / 26$ & 0/29 & & $0.0[0.0,0.0]$ \\
\hline Asimakopoulos 2000 & $0 / 8$ & $0 / 10$ & & $0.0[0.0,0.0]$ \\
\hline Baele 1992 & $4 / 58$ & $8 / 57$ & $\because$ & $0.49[0.16,1.54]$ \\
\hline Bidstrup 1993 & $12 / 410$ & $|6 / 42|$ & - & $0.77[0.37,1.61]$ \\
\hline Bidstrup 2000 & $2 / 30$ & $2 / 30$ & \begin{tabular}{l|l} 
\\
\end{tabular} & $1.00[0.15,6.64]$ \\
\hline Carrera 1994 & $3 / 51$ & $2 / 51$ & $\longrightarrow$ & $1.50[0.26,8.60]$ \\
\hline Cicek 1996a & $0 / 50$ & $0 / 25$ & & $0.0[0.0,0.0]$ \\
\hline Cicek 1996b & $0 / 29$ & $0 / 28$ & & $0.0[0.0,0.0]$ \\
\hline Cicekcioglu 2006 & $0 / 24$ & $0 / 20$ & & $0.0[0.0,0.0]$ \\
\hline Cohen 1998 & $2 / 56$ & 0/59 & & $5.26[0.26,107.27]$ \\
\hline Colwell 2007 & $1 / 175$ & I/I77 & & $1.01[0.06,16.04]$ \\
\hline Cosgrove 1992 & $14 / 113$ & $4 / 56$ & -6 & $1.73[0.60,5.03]$ \\
\hline D'Ambra 1996 & $5 /|4|$ & $3 / 71$ & - & $0.84[0.21,3.41]$ \\
\hline Desai 2009 & $1 / 38$ & $4 / 37$ & - & $0.24[0.03,2.08]$ \\
\hline Dignan 200। & $3 / 101$ & $5 / 99$ & - & $0.59[0.14,2.40]$ \\
\hline Diprose 2005 & $3 / 60$ & $4 / 60$ & & $0.75[0.18,3.21]$ \\
\hline Ehrlich 1998 & $0 / 25$ & $1 / 25$ & & $0.33[0.01,7.81]$ \\
\hline Englberger 2002a & $1 / 22$ & $1 / 25$ & & $1.14[0.08,17.11]$ \\
\hline Englberger $2002 b$ & $1 / 15$ & $1 / 14$ & & $0.93[0.06,13.54]$ \\
\hline Golanski 2000 & $6 / 30$ & $2 / 24$ & & $2.40[0.53,10.84]$ \\
\hline Greilich 2009 & $6 / 26$ & $7 / 27$ & & $0.89[0.34,2.30]$ \\
\hline Harder 199| & $0 / 40$ & $0 / 40$ & & $0.0[0.0,0.0]$ \\
\hline
\end{tabular}

$0.0010 .010 .1 \quad 1 \quad 10 \quad 100 \quad 1000$

Favours Treatment Favours Control

(Continued ....) 


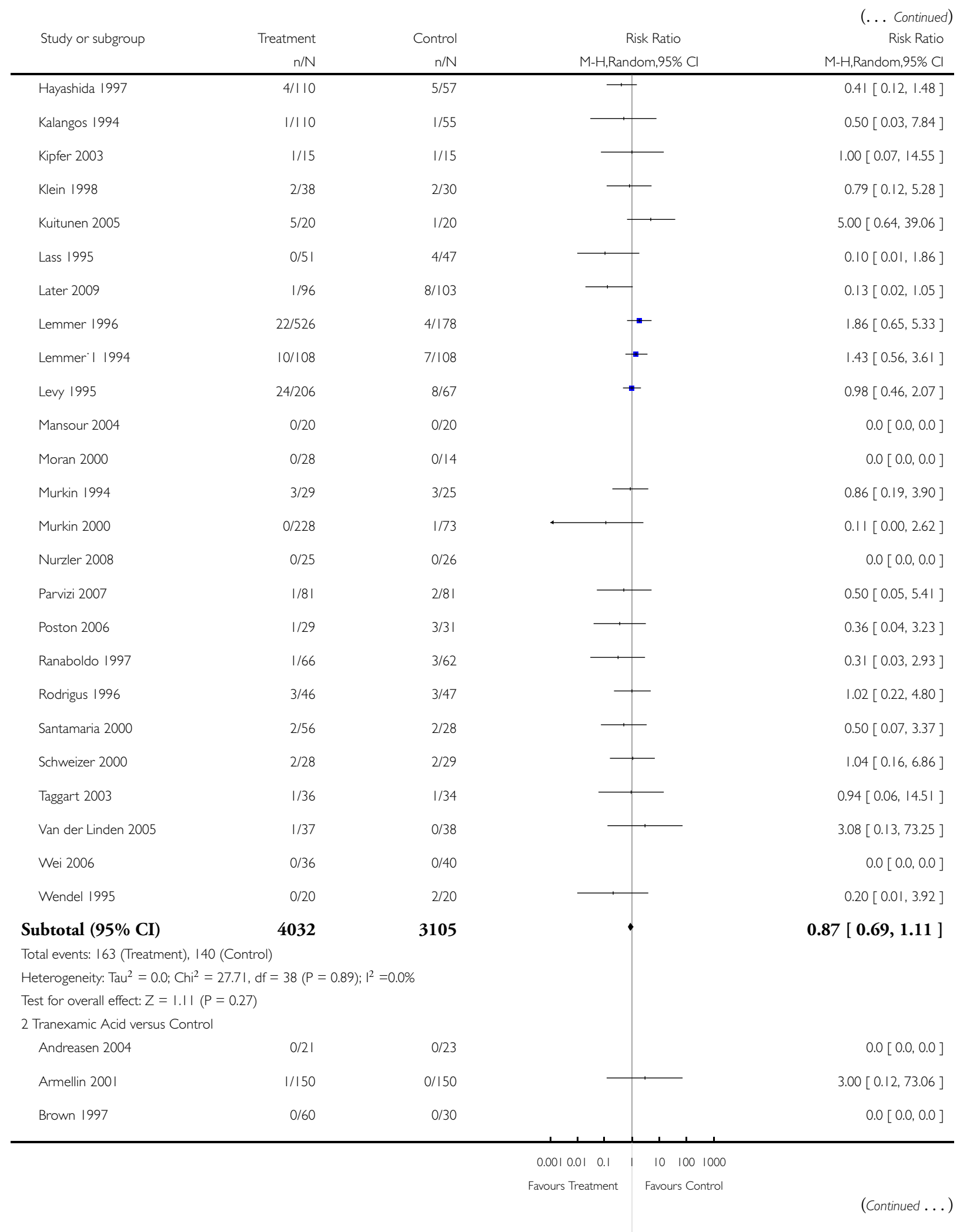

Anti-fibrinolytic use for minimising perioperative allogeneic blood transfusion (Review)

Copyright (C) 201 I The Cochrane Collaboration. Published by John Wiley \& Sons, Ltd. 


\begin{tabular}{|c|c|c|c|c|}
\hline \multirow[t]{2}{*}{ Study or subgroup } & Treatment & Control & Risk Ratio & $\begin{array}{r}\text { Risk Ratio } \\
\end{array}$ \\
\hline & $\mathrm{n} / \mathrm{N}$ & $\mathrm{n} / \mathrm{N}$ & M-H,Random,95\% Cl & M-H,Random,95\% Cl \\
\hline Casati 2002 & 1/29 & $1 / 29$ & $\vec{\longrightarrow}$ & $1.00[0.07,15.24]$ \\
\hline Diprose 2005 & $5 / 60$ & $4 / 60$ & $\rightarrow$ & $1.25[0.35,4.43]$ \\
\hline Hardy 1998 & |/43 & $2 / 45$ & $\rightarrow$ & $0.52[0.05,5.56]$ \\
\hline Hiipala 1995 & $1 / 15$ & $0 / 13$ & - & $2.63[0.12,59.40]$ \\
\hline Horrow 1991 & 1/37 & $0 / 44$ & & $3.55[0.15,84.69]$ \\
\hline Jares 2003 & $1 / 22$ & $1 / 25$ & & $1.14[0.08,17.11]$ \\
\hline Karski 1995 & $0 / 100$ & $0 / 50$ & & $0.0[0.0,0.0]$ \\
\hline Karski 2005 & $2 / 147$ & $3 / 165$ & & $0.75[0.13,4.42]$ \\
\hline Katsaros 1996 & $0 / 104$ & $0 / 106$ & & $0.0[0.0,0.0]$ \\
\hline Kuitunen 2005 & $1 / 20$ & $1 / 20$ & & $1.00[0.07,14.90]$ \\
\hline Later 2009 & $0 / 99$ & $8 / 103$ & & $0.06[0.00,1.05]$ \\
\hline Mansour 2004 & $0 / 20$ & $0 / 20$ & & $0.0[0.0,0.0]$ \\
\hline Mehr-Aein 2007 & 0/33 & 0/33 & & $0.0[0.0,0.0]$ \\
\hline Murphy 2006 & $0 / 50$ & $1 / 50$ & & $0.33[0.01,7.99]$ \\
\hline Shore-Lesserson 1996 & $1 / 17$ & $2 / 13$ & & $0.38[0.04,3.77]$ \\
\hline Speekenbrink 1995 & $0 / 15$ & $2 / 15$ & & $0.20[0.01,3.85]$ \\
\hline Taghaddomi 2009 & $0 / 50$ & $0 / 50$ & & $0.0[0.0,0.0]$ \\
\hline Zabeeda 2002 & $0 / 25$ & $0 / 25$ & & $0.0[0.0,0.0]$ \\
\hline Subtotal (95\% CI) & 1117 & 1069 & & $0.79[0.41,1.52]$ \\
\hline \multicolumn{5}{|c|}{ Total events: I5 (Treatment), 25 (Control) } \\
\hline \multicolumn{5}{|c|}{ Heterogeneity: $\operatorname{Tau}^{2}=0.0 ; \mathrm{Chi}^{2}=7.84, \mathrm{df}=12(\mathrm{P}=0.80) ;\left.\right|^{2}=0.0 \%$} \\
\hline \multicolumn{5}{|c|}{ Test for overall effect: $Z=0.69(P=0.49)$} \\
\hline \multicolumn{5}{|c|}{3 Epsilon aminocaproic Acid versus Control } \\
\hline Berenholtz 2009 & $0 / 91$ & $0 / 91$ & & $0.0[0.0,0.0]$ \\
\hline Del Rossi 1989 & $4 / 170$ & $10 / 180$ & $\rightarrow$ & $0.42[0.14,1.32]$ \\
\hline Greilich 2009 & $6 / 25$ & $7 / 27$ & & $0.93[0.36,2.38]$ \\
\hline Hardy 1998 & $2 / 46$ & $2 / 45$ & & $0.98[0.14,6.65]$ \\
\hline Kluger 2003 & $1 / 58$ & $0 / 30$ & & $1.58[0.07,37.56]$ \\
\hline Rao 1999 & $0 / 15$ & $0 / 15$ & & $0.0[0.0,0.0]$ \\
\hline Vander-Salm 1996 & $5 / 51$ & $2 / 52$ & $=$ & $2.55[0.52,12.55]$ \\
\hline Subtotal (95\% CI) & 456 & 440 & 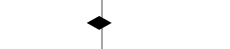 & $0.88[0.48,1.63]$ \\
\hline \multicolumn{5}{|c|}{ Total events: I8 (Treatment), 2 I (Control) } \\
\hline \multicolumn{5}{|c|}{ Heterogeneity: $\operatorname{Tau}^{2}=0.0 ; \mathrm{Chi}^{2}=3.44, \mathrm{df}=4(\mathrm{P}=0.49) ; \mathrm{I}^{2}=0.0 \%$} \\
\hline Test for overall effect: $Z=$ & & & & \\
\hline
\end{tabular}


Analysis 8.4. Comparison 8 Adverse Events and Other Outcomes (Active versus Control), Outcome 4 Stroke (CVA).

Review: Anti-fibrinolytic use for minimising perioperative allogeneic blood transfusion

Comparison: 8 Adverse Events and Other Outcomes (Active versus Control)

Outcome: 4 Stroke (CVA)

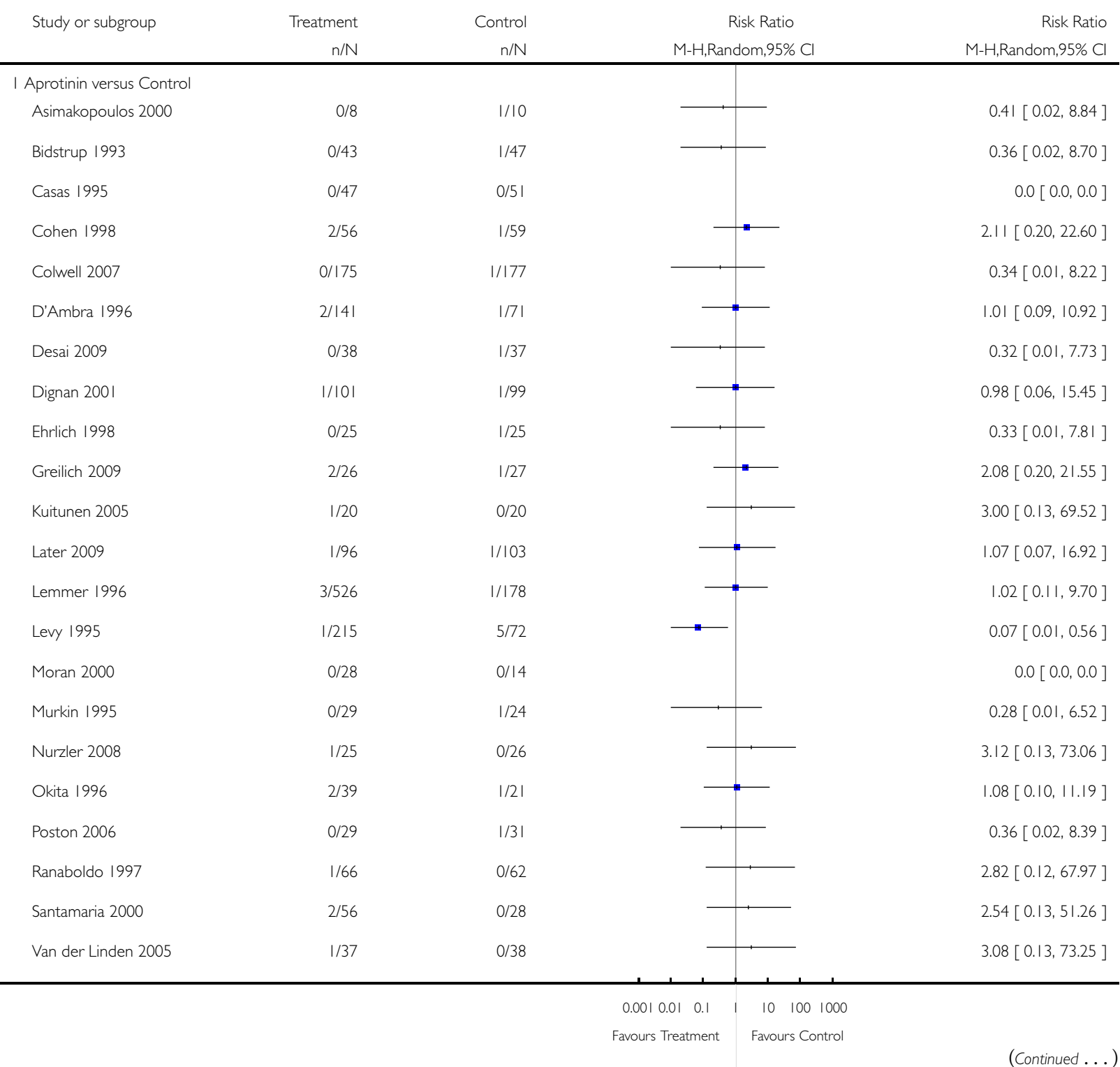

Anti-fibrinolytic use for minimising perioperative allogeneic blood transfusion (Review) 


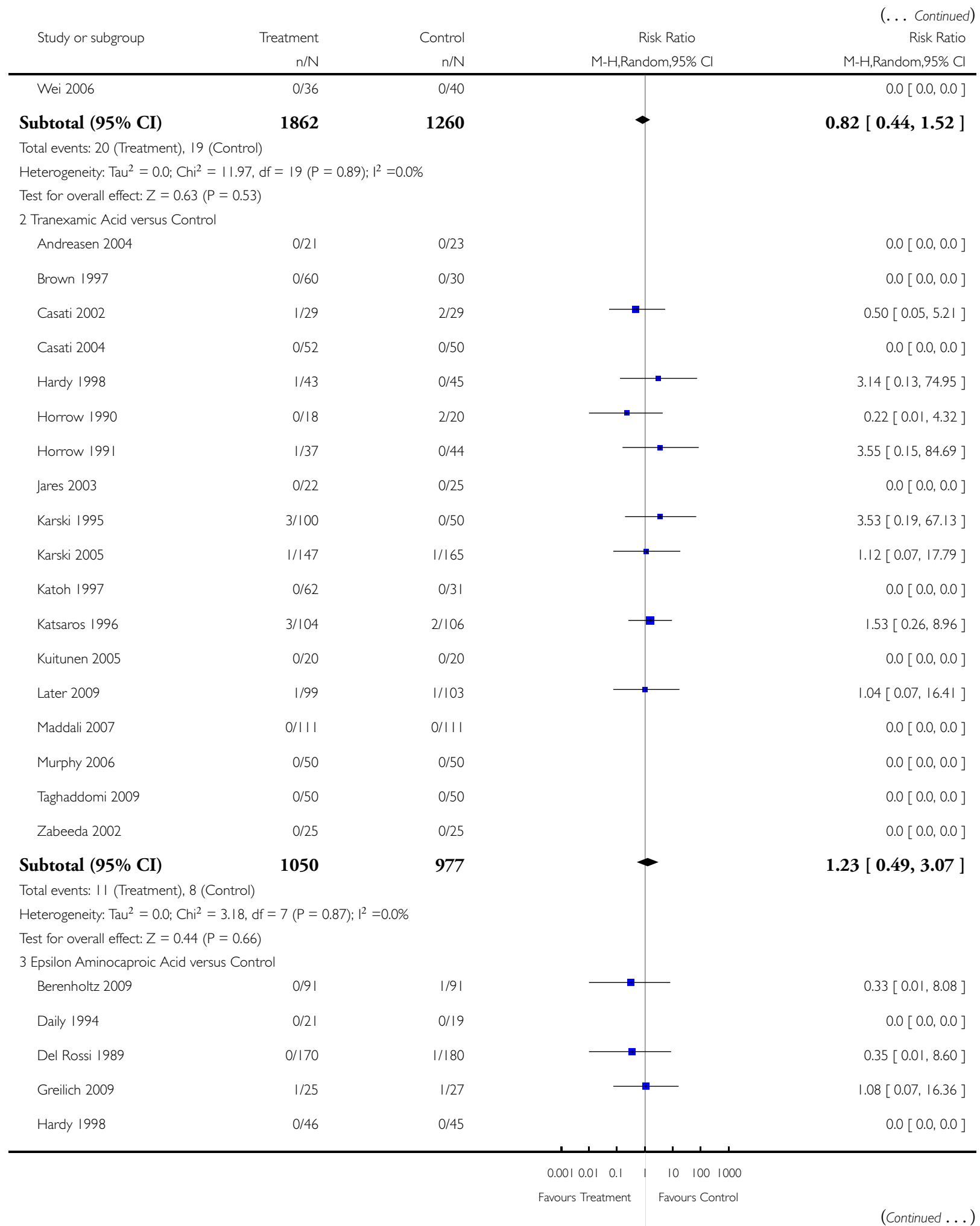

Anti-fibrinolytic use for minimising perioperative allogeneic blood transfusion (Review) 


\begin{tabular}{|c|c|c|c|c|}
\hline \multirow[t]{2}{*}{ Study or subgroup } & Treatment & Control & Risk Ratio & $\begin{array}{c}\text { (... Continued) } \\
\text { Risk Ratio }\end{array}$ \\
\hline & $n / N$ & $n / N$ & M-H,Random, $95 \% \mathrm{Cl}$ & M-H,Random, $95 \% \mathrm{Cl}$ \\
\hline Kluger 2003 & $2 / 58$ & $0 / 30$ & $\longrightarrow$ & $2.63[0.13,53.04]$ \\
\hline Rao 1999 & $0 / 15$ & $0 / 15$ & & $0.0[0.0,0.0]$ \\
\hline Vander-Salm 1996 & $0 / 51$ & $2 / 52$ & 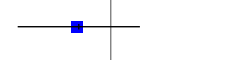 & $0.20[0.01,4.14]$ \\
\hline Subtotal $(95 \% \mathrm{CI})$ & 477 & 459 & $\sim$ & $0.62[0.16,2.36]$ \\
\hline \multicolumn{5}{|c|}{ Total events: 3 (Treatment), 5 (Control) } \\
\hline \multicolumn{5}{|c|}{ Heterogeneity: $\mathrm{Tau}^{2}=0.0 ; \mathrm{Chi}^{2}=1.84, \mathrm{df}=4(\mathrm{P}=0.77) ; \mathrm{I}^{2}=0.0 \%$} \\
\hline \multicolumn{5}{|c|}{ Test for overall effect: $Z=0.7 \mid(P=0.48)$} \\
\hline
\end{tabular}

$0.0010 .010 .1 \quad 1 \quad 10 \quad 1001000$

Favours Treatment Favours Control

\section{Analysis 8.5. Comparison 8 Adverse Events and Other Outcomes (Active versus Control), Outcome 5} Deep Vein Thrombosis (DVT).

Review: Anti-fibrinolytic use for minimising perioperative allogeneic blood transfusion

Comparison: 8 Adverse Events and Other Outcomes (Active versus Control)

Outcome: 5 Deep Vein Thrombosis (DVT)

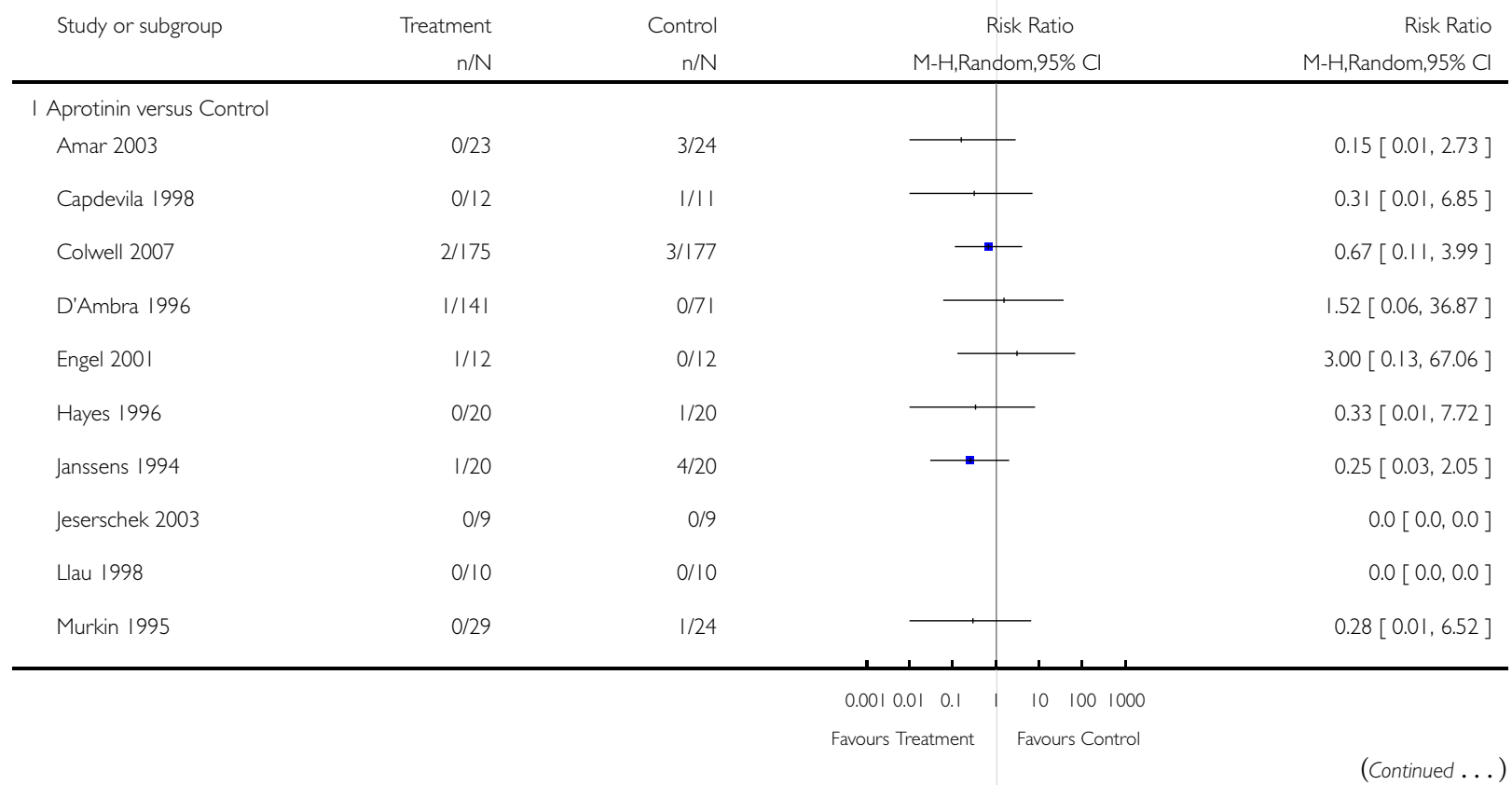




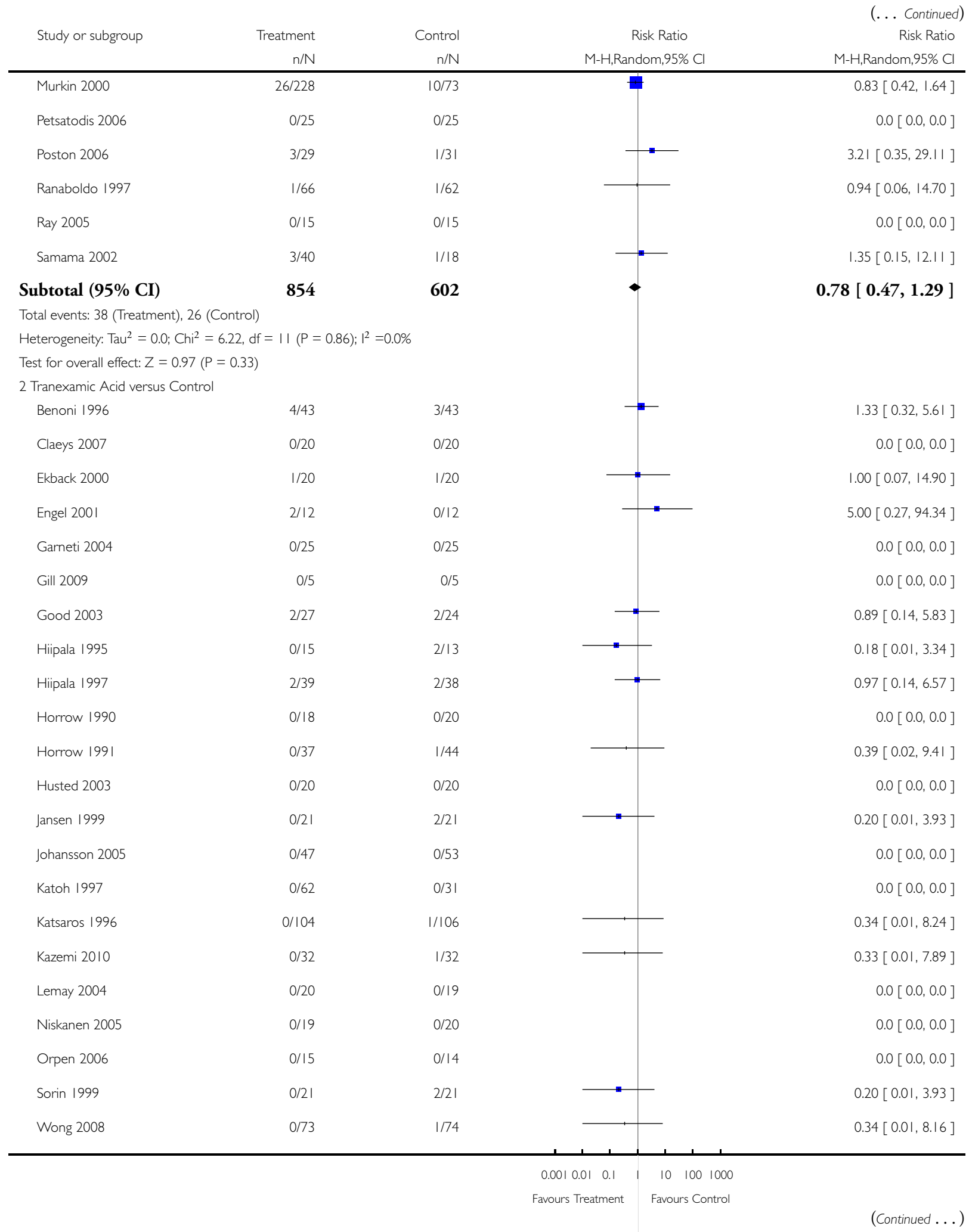

Anti-fibrinolytic use for minimising perioperative allogeneic blood transfusion (Review) 


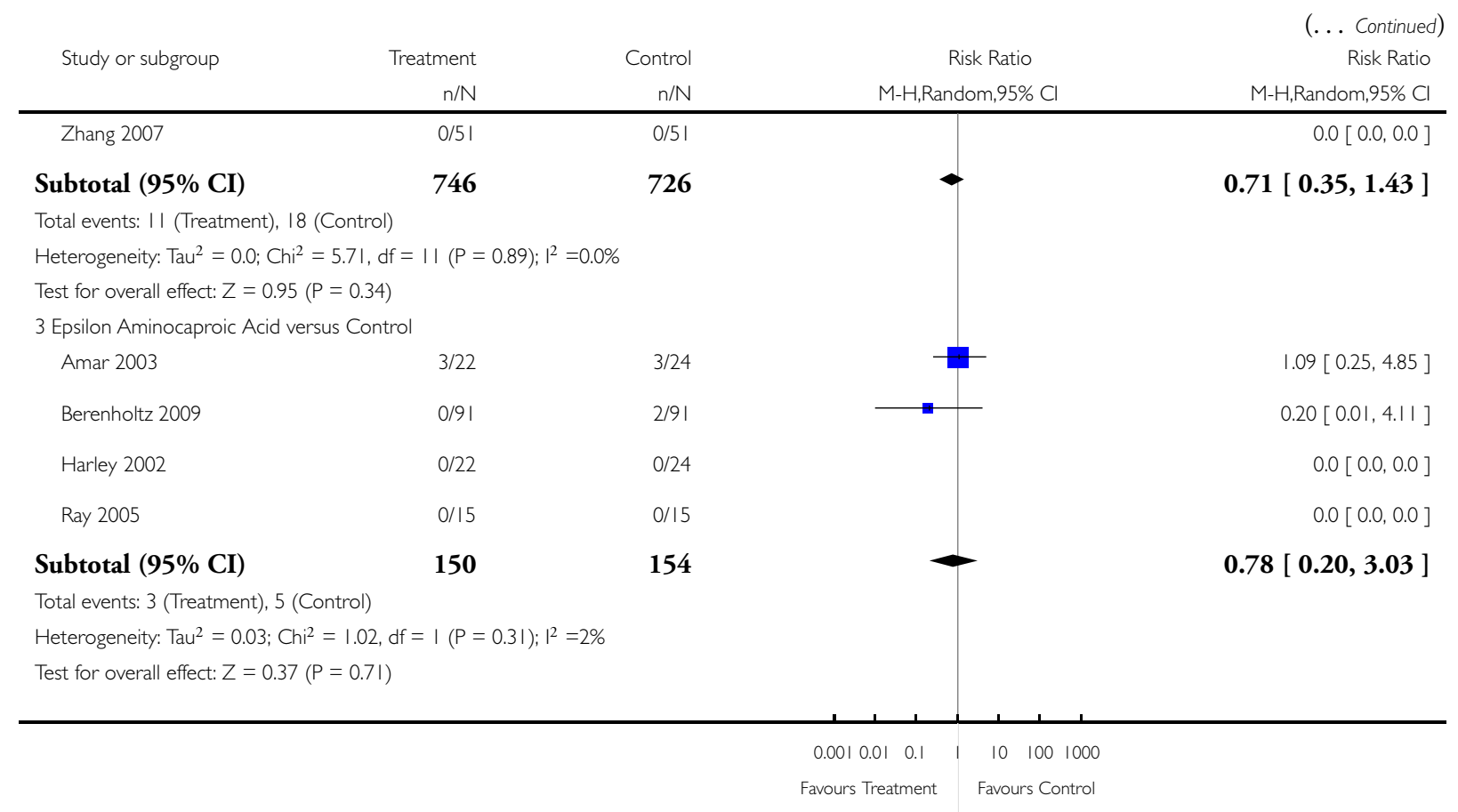


Analysis 8.6. Comparison 8 Adverse Events and Other Outcomes (Active versus Control), Outcome 6 Pulmonary Embolism (PE).

Review: Anti-fibrinolytic use for minimising perioperative allogeneic blood transfusion

Comparison: 8 Adverse Events and Other Outcomes (Active versus Control)

Outcome: 6 Pulmonary Embolism (PE)

\begin{tabular}{|c|c|c|c|c|}
\hline Study or subgroup & $\begin{array}{r}\text { Treatment } \\
\mathrm{n} / \mathrm{N} \\
\end{array}$ & $\begin{array}{r}\text { Control } \\
\mathrm{n} / \mathrm{N} \\
\end{array}$ & $\begin{array}{c}\text { Risk Ratio } \\
\text { M-H,Random,95\% Cl }\end{array}$ & $\begin{array}{r}\text { Risk Ratio } \\
\text { M-H,Random,95\% Cl }\end{array}$ \\
\hline \multicolumn{5}{|l|}{ I Aprotinin versus Control } \\
\hline Amar 2003 & $2 / 23$ & $1 / 24$ & 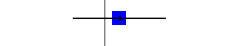 & $2.09[0.20,21.48]$ \\
\hline Colwell 2007 & $2 / 175$ & $2 / 177$ & + & $1.01[0.14,7.10]$ \\
\hline Ranaboldo 1997 & $2 / 66$ & $1 / 62$ & $=$ & $1.88[0.17,20.20]$ \\
\hline Samama 2002 & $0 / 40$ & $0 / 18$ & & $0.0[0.0,0.0]$ \\
\hline Subtotal (95\% CI) & 304 & 281 & & $1.49[0.42,5.29]$ \\
\hline \multicolumn{5}{|c|}{ Total events: 6 (Treatment), 4 (Control) } \\
\hline \multicolumn{5}{|c|}{ Heterogeneity: $\operatorname{Tau}^{2}=0.0 ; \mathrm{Chi}^{2}=0.27, \mathrm{df}=2(P=0.87) ;\left.\right|^{2}=0.0 \%$} \\
\hline \multicolumn{5}{|c|}{ Test for overall effect: $Z=0.62(P=0.54)$} \\
\hline \multicolumn{5}{|c|}{2 Tranexamic Acid versus Control } \\
\hline Benoni 1996 & $0 / 43$ & $1 / 43$ & & $0.33[0.01,7.96]$ \\
\hline Benoni 200I & $1 / 18$ & $1 / 20$ & & I.II $[0.07,16.49]$ \\
\hline Casati 2002 & $0 / 29$ & $0 / 29$ & & $0.0[0.0,0.0]$ \\
\hline Casati 2004 & $0 / 52$ & $0 / 50$ & & $0.0[0.0,0.0]$ \\
\hline Garneti 2004 & $1 / 25$ & $0 / 25$ & & $3.00[0.13,70.30]$ \\
\hline Hiipala 1995 & $0 / 15$ & $1 / 13$ & - & $0.29[0.01,6.60]$ \\
\hline Hiipala 1997 & $0 / 39$ & $1 / 38$ & - & $0.33[0.01,7.74]$ \\
\hline Horrow 1990 & $0 / 18$ & $0 / 20$ & & $0.0[0.0,0.0]$ \\
\hline Husted 2003 & $0 / 20$ & $0 / 20$ & & $0.0[0.0,0.0]$ \\
\hline Jares 2003 & $0 / 22$ & $0 / 25$ & & $0.0[0.0,0.0]$ \\
\hline Katoh 1997 & $0 / 62$ & $0 / 31$ & & $0.0[0.0,0.0]$ \\
\hline Katsaros 1996 & $0 / 104$ & $1 / 106$ & 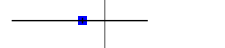 & $0.34[0.01,8.24]$ \\
\hline MacGillivray 2010 & $2 / 40$ & $0 / 20$ & $\square$ & $2.56[0.13,50.95]$ \\
\hline Pleym 2003 & $0 / 40$ & $1 / 39$ & $\rightarrow$ & $0.33[0.01,7.75]$ \\
\hline Subtotal (95\% CI) & 527 & 479 & - & $0.67[0.23,1.99]$ \\
\hline \multicolumn{5}{|c|}{ Total events: 4 (Treatment), 6 (Control) } \\
\hline \multicolumn{5}{|c|}{ Heterogeneity: $\operatorname{Tau}^{2}=0.0 ; \mathrm{Chi}^{2}=2.8 \mathrm{I}, \mathrm{df}=7(\mathrm{P}=0.90) ; \mathrm{I}^{2}=0.0 \%$} \\
\hline
\end{tabular}

$0.0010 .01 \quad 0.1 \quad 1 \quad 10 \quad 100 \quad 1000$

Favours Treatment Favours Control

(Continued ... ) 


\begin{tabular}{|c|c|c|c|c|}
\hline Study or subgroup & $\begin{array}{r}\text { Treatment } \\
\mathrm{n} / \mathrm{N}\end{array}$ & $\begin{array}{r}\text { Control } \\
n / N\end{array}$ & $\begin{array}{c}\text { Risk Ratio } \\
\text { M-H,Random,95\% Cl }\end{array}$ & $\begin{array}{r}\text { (. . . Continued }) \\
\text { Risk Ratio } \\
\text { M-H,Random,95\% Cl }\end{array}$ \\
\hline \multicolumn{5}{|c|}{ Test for overall effect: $Z=0.7 \mathrm{I}(P=0.48)$} \\
\hline \multicolumn{5}{|c|}{3 Epsilon Aminocaproic Acid versus Control } \\
\hline Amar 2003 & 0/22 & 1/24 & $\longrightarrow$ & $0.36[0.02,8.46]$ \\
\hline Berenholtz 2009 & |/9| & 3/91 & (1) & $0.33[0.04,3.15]$ \\
\hline Harley 2002 & $0 / 22$ & $0 / 24$ & & $0.0[0.0,0.0]$ \\
\hline Subtotal (95\% CI) & 135 & 139 & 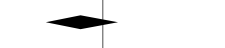 & $0.34[0.06,2.13]$ \\
\hline \multicolumn{5}{|c|}{ Total events: I (Treatment), 4 (Control) } \\
\hline \multicolumn{5}{|c|}{ Heterogeneity: $\operatorname{Tau}^{2}=0.0 ; \mathrm{Chi}^{2}=0.00, \mathrm{df}=\mathrm{I}(\mathrm{P}=0.97) ; \mathrm{I}^{2}=0.0 \%$} \\
\hline \multicolumn{5}{|c|}{ Test for overall effect: $Z=1.15(P=0.25)$} \\
\hline
\end{tabular}

\section{Analysis 8.7. Comparison 8 Adverse Events and Other Outcomes (Active versus Control), Outcome 7} Other Thrombosis.

Review: Anti-fibrinolytic use for minimising perioperative allogeneic blood transfusion

Comparison: 8 Adverse Events and Other Outcomes (Active versus Control)

Outcome: 7 Other Thrombosis

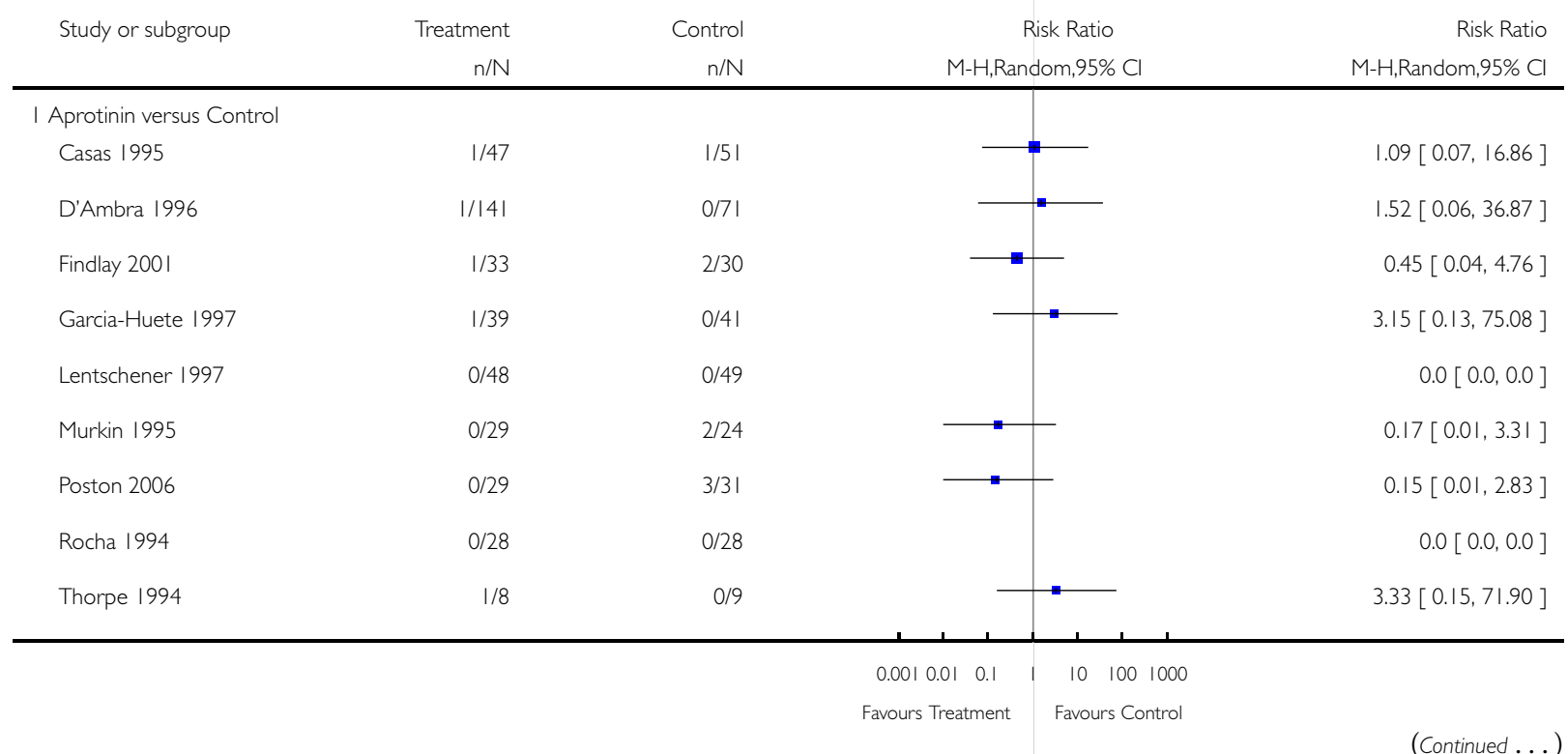




\begin{tabular}{|c|c|c|c|c|}
\hline \multirow[t]{2}{*}{ Study or subgroup } & Treatment & Control & Risk Ratio & $\begin{array}{c}\text { (... Continued) } \\
\text { Risk Ratio }\end{array}$ \\
\hline & $\mathrm{n} / \mathrm{N}$ & $\mathrm{n} / \mathrm{N}$ & M-H,Random, $95 \% \mathrm{Cl}$ & M-H,Random, $95 \% \mathrm{Cl}$ \\
\hline Subtotal $(95 \% \mathrm{CI})$ & 402 & 334 & - & $0.73[0.25,2.15]$ \\
\hline \multicolumn{5}{|c|}{ Total events: 5 (Treatment), 8 (Control) } \\
\hline \multicolumn{5}{|c|}{ Heterogeneity: $\mathrm{Tau}^{2}=0.0 ; \mathrm{Chi}^{2}=4.27, \mathrm{df}=6(\mathrm{P}=0.64) ; \mathrm{I}^{2}=0.0 \%$} \\
\hline \multicolumn{5}{|c|}{ Test for overall effect: $Z=0.58(P=0.57)$} \\
\hline \multicolumn{5}{|c|}{2 Tranexamic Acid versus Control } \\
\hline Alvarez 2008 & $0 / 46$ & $0 / 49$ & & $0.0[0.0,0.0]$ \\
\hline Boylan 1996 & 0/25 & $0 / 20$ & & $0.0[0.0,0.0]$ \\
\hline Dalmau 2000 & $4 / 42$ & $2 / 40$ & & $1.90[0.37,9.83]$ \\
\hline Kaspar 1997 & $1 / 16$ & $0 / 16$ & - & $3.00[0.13,68.57]$ \\
\hline Taghaddomi 2009 & 0/50 & $0 / 50$ & & $0.0[0.0,0.0]$ \\
\hline Veien 2002 & $0 / 15$ & $0 / 15$ & & $0.0[0.0,0.0]$ \\
\hline Yamasaki 2004 & 0/20 & $0 / 20$ & & $0.0[0.0,0.0]$ \\
\hline Yassen 1993 & $0 / 10$ & $0 / 10$ & & $0.0[0.0,0.0]$ \\
\hline Zohar 2004 & 0/20 & $0 / 20$ & & $0.0[0.0,0.0]$ \\
\hline Subtotal $(95 \% \mathrm{CI})$ & 244 & 240 & - & $2.10[0.49,8.99]$ \\
\hline \multicolumn{5}{|c|}{ Total events: 5 (Treatment), 2 (Control) } \\
\hline \multicolumn{5}{|c|}{ Heterogeneity: $\mathrm{Tau}^{2}=0.0 ; \mathrm{Chi}^{2}=0.06, \mathrm{df}=1(\mathrm{P}=0.80) ; \mathrm{I}^{2}=0.0 \%$} \\
\hline \multicolumn{5}{|c|}{ Test for overall effect: $Z=1.00(P=0.32)$} \\
\hline \multicolumn{5}{|c|}{3 Epsilon Aminocaproic Acid versus Control } \\
\hline Berenholtz 2009 & 2/91 & $6 / 91$ & - & $0.33[0.07,1.61]$ \\
\hline Dalmau 2000 & $2 / 42$ & $2 / 40$ & 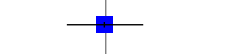 & $0.95[0.14,6.44]$ \\
\hline Subtotal $(95 \% \mathrm{CI})$ & 133 & 131 & & $0.51[0.15,1.72]$ \\
\hline \multicolumn{5}{|c|}{ Total events: 4 (Treatment), 8 (Control) } \\
\hline \multicolumn{5}{|c|}{ Heterogeneity: $\operatorname{Tau}^{2}=0.0 ; \mathrm{Chi}^{2}=0.69, \mathrm{df}=\mathrm{I}(\mathrm{P}=0.40) ; \mathrm{I}^{2}=0.0 \%$} \\
\hline Test for overall effect: $Z=$ & & & & \\
\hline
\end{tabular}


Analysis 8.8. Comparison 8 Adverse Events and Other Outcomes (Active versus Control), Outcome 8 Coronary artery graft occlusion.

Review: Anti-fibrinolytic use for minimising perioperative allogeneic blood transfusion

Comparison: 8 Adverse Events and Other Outcomes (Active versus Control)

Outcome: 8 Coronary artery graft occlusion

\begin{tabular}{|c|c|c|c|c|c|}
\hline \multirow[t]{2}{*}{ Study or subgroup } & Treatment & Control & Risk Ratio & \multirow[t]{2}{*}{ Weight } & \multirow{2}{*}{$\begin{array}{r}\text { Risk Ratio } \\
\mathrm{M}-\mathrm{H}, \text { Random,95\% Cl}\end{array}$} \\
\hline & $\mathrm{n} / \mathrm{N}$ & $\mathrm{n} / \mathrm{N}$ & M-H,Random,95\% Cl & & \\
\hline \multicolumn{6}{|c|}{ I Aprotinin versus Control } \\
\hline Alderman 1998 & $54 / 340$ & $36 / 328$ & & $71.2 \%$ & $1.45[0.98,2.14]$ \\
\hline Poston 2006 & $0 / 29$ & $3 / 31$ & $\longrightarrow$ & $28.8 \%$ & $0.15[0.01,2.83]$ \\
\hline Total (95\% CI) & 369 & 359 & & $100.0 \%$ & $0.76[0.10,5.67]$ \\
\hline \multicolumn{6}{|c|}{ Total events: 54 (Treatment), 39 (Control) } \\
\hline \multicolumn{6}{|c|}{ Heterogeneity: $\mathrm{Tau}^{2}=1.45 ; \mathrm{Chi}^{2}=2.28, \mathrm{df}=\mathrm{I}(\mathrm{P}=0.13) ; \mathrm{I}^{2}=56 \%$} \\
\hline \multicolumn{6}{|c|}{ Test for overall effect: $Z=0.27(P=0.79)$} \\
\hline
\end{tabular}


Analysis 8.9. Comparison 8 Adverse Events and Other Outcomes (Active versus Control), Outcome 9 Renal Failure / Dysfunction.

Review: Anti-fibrinolytic use for minimising perioperative allogeneic blood transfusion

Comparison: 8 Adverse Events and Other Outcomes (Active versus Control)

Outcome: 9 Renal Failure / Dysfunction

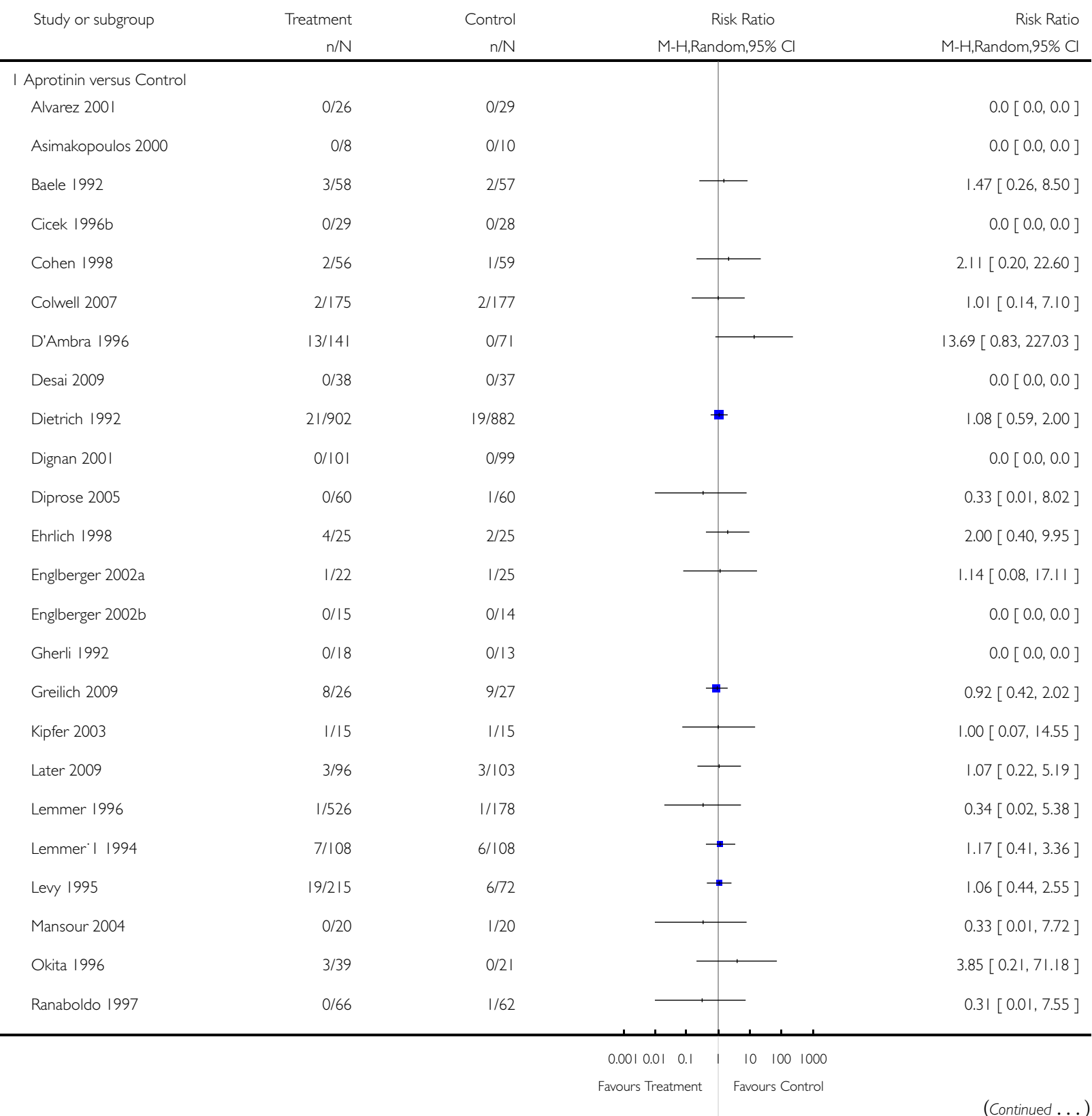




\begin{tabular}{|c|c|c|c|c|}
\hline Study or subgroup & $\begin{array}{r}\text { Treatment } \\
n / N\end{array}$ & $\begin{array}{r}\text { Control } \\
n / N\end{array}$ & $\begin{array}{c}\text { Risk Ratio } \\
\text { M-H,Random,95\% Cl } \\
\end{array}$ & $\begin{array}{r}(\ldots \text { Continued }) \\
\text { Risk Ratio } \\
\mathrm{M}-\mathrm{H}, \text { Random,95\% Cl } \\
\end{array}$ \\
\hline Speekenbrink 1996 & 0/75 & 0/37 & & $0.0[0.0,0.0]$ \\
\hline Stammers 1997 & $0 / 8$ & $0 / 12$ & & $0.0[0.0,0.0]$ \\
\hline Wei 2006 & 0/36 & 0/40 & & $0.0[0.0,0.0]$ \\
\hline Subtotal (95\% CI) & 2904 & 2281 & - & $1.10[0.79,1.54]$ \\
\hline \multicolumn{5}{|c|}{ Total events: 88 (Treatment), 56 (Control) } \\
\hline \multicolumn{5}{|c|}{ Heterogeneity: $\mathrm{Tau}^{2}=0.0 ; \mathrm{Chi}^{2}=7.64, \mathrm{df}=16(\mathrm{P}=0.96) ; \mathrm{I}^{2}=0.0 \%$} \\
\hline \multicolumn{5}{|c|}{ Test for overall effect: $Z=0.58(P=0.56)$} \\
\hline \multicolumn{5}{|c|}{2 Tranexamic Acid versus Control } \\
\hline Andreasen 2004 & $0 / 21$ & 0/23 & & $0.0[0.0,0.0]$ \\
\hline Diprose 2005 & $0 / 60$ & 1/60 & - & $0.33[0.01,8.02]$ \\
\hline Katsaros 1996 & 1/104 & 0/106 & - & $3.06[0.13,74.20]$ \\
\hline Later 2009 & 3/99 & $3 / 103$ & - & $1.04[0.22,5.03]$ \\
\hline Mansour 2004 & $1 / 20$ & $1 / 20$ & & $1.00[0.07,14.90]$ \\
\hline Mehr-Aein 2007 & 0/33 & $1 / 33$ & $\cdot-$ & $0.33[0.01,7.90]$ \\
\hline Murphy 2006 & 1/50 & 0/50 & 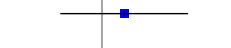 & $3.00[0.13,71.92]$ \\
\hline Shore-Lesserson 1996 & $0 / 17$ & $1 / 13$ & - & $0.26[0.01,5.89]$ \\
\hline Taghaddomi 2009 & 0/50 & 0/50 & & $0.0[0.0,0.0]$ \\
\hline Subtotal $(95 \% \mathrm{CI})$ & 454 & 458 & 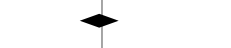 & $0.89[0.33,2.37]$ \\
\hline \multicolumn{5}{|c|}{ Total events: 6 (Treatment), 7 (Control) } \\
\hline \multicolumn{5}{|c|}{ Heterogeneity: $\operatorname{Tau}^{2}=0.0 ; \mathrm{Chi}^{2}=2.52, \mathrm{df}=6(\mathrm{P}=0.87) ; \mathrm{I}^{2}=0.0 \%$} \\
\hline \multicolumn{5}{|c|}{ Test for overall effect: $Z=0.23(P=0.8 \mathrm{I})$} \\
\hline \multicolumn{5}{|c|}{3 Epsilon Aminocaproic Acid versus control } \\
\hline Berenholtz 2009 & $|/ 9|$ & $|/ 9|$ & & $1.00[0.06,15.75]$ \\
\hline Greilich 2009 & $3 / 26$ & 9/27 & - & $0.35[0.11,1.14]$ \\
\hline Subtotal $(95 \% \mathrm{CI})$ & 117 & 118 & 0 & $0.41[0.14,1.22]$ \\
\hline \multicolumn{5}{|c|}{ Total events: 4 (Treatment), 10 (Control) } \\
\hline \multicolumn{5}{|c|}{ Heterogeneity: $\mathrm{Tau}^{2}=0.0 ; \mathrm{Chi}^{2}=0.48, \mathrm{df}=\mathrm{I}(\mathrm{P}=0.49) ; \mathrm{I}^{2}=0.0 \%$} \\
\hline Test for overall effect: $Z=$ & & & & \\
\hline
\end{tabular}


Analysis 8.10. Comparison 8 Adverse Events and Other Outcomes (Active versus Control), Outcome 10 Hospital Length of Stay.

Review: Anti-fibrinolytic use for minimising perioperative allogeneic blood transfusion

Comparison: 8 Adverse Events and Other Outcomes (Active versus Control)

Outcome: 10 Hospital Length of Stay

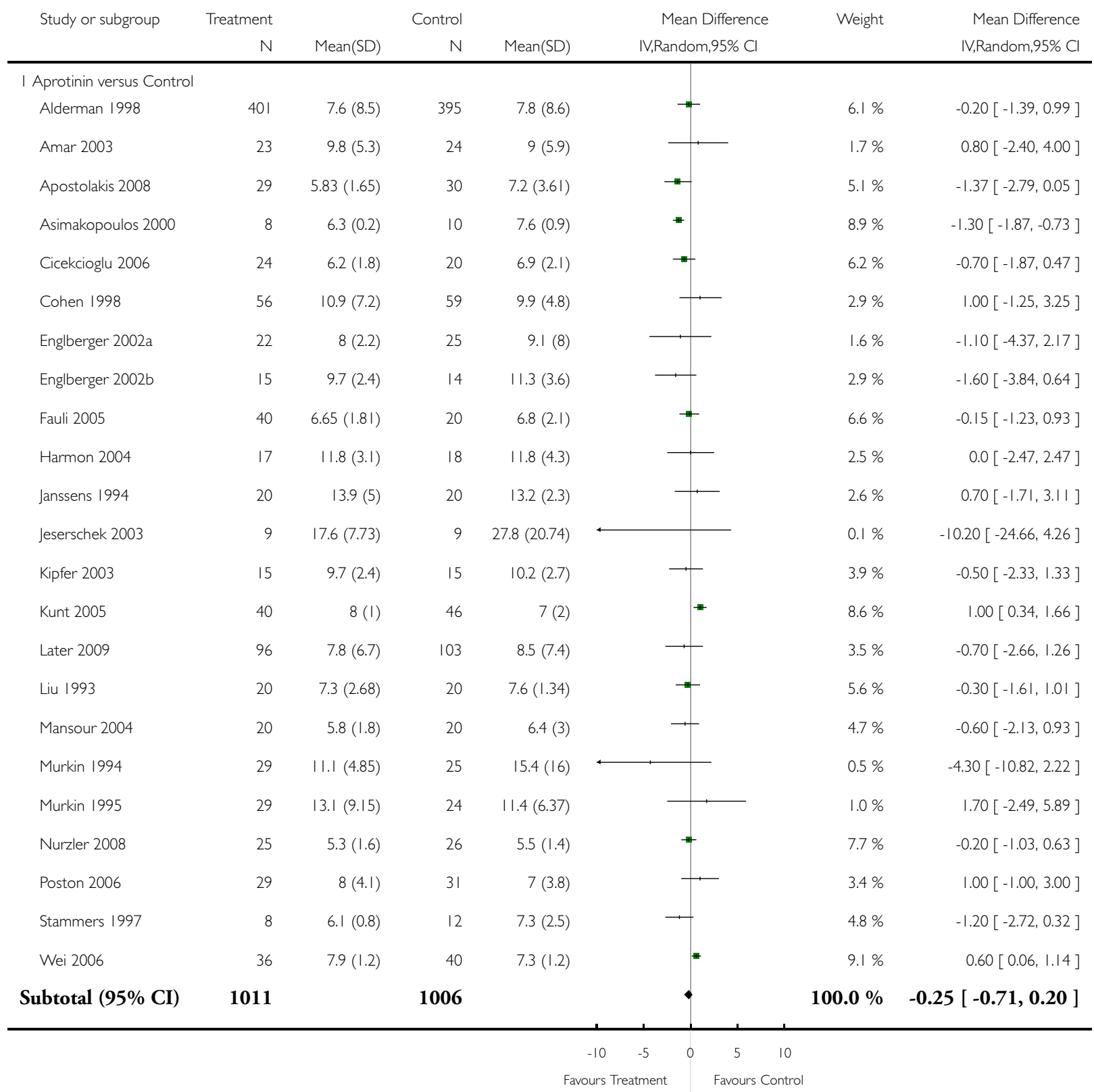

(Continued ....) 


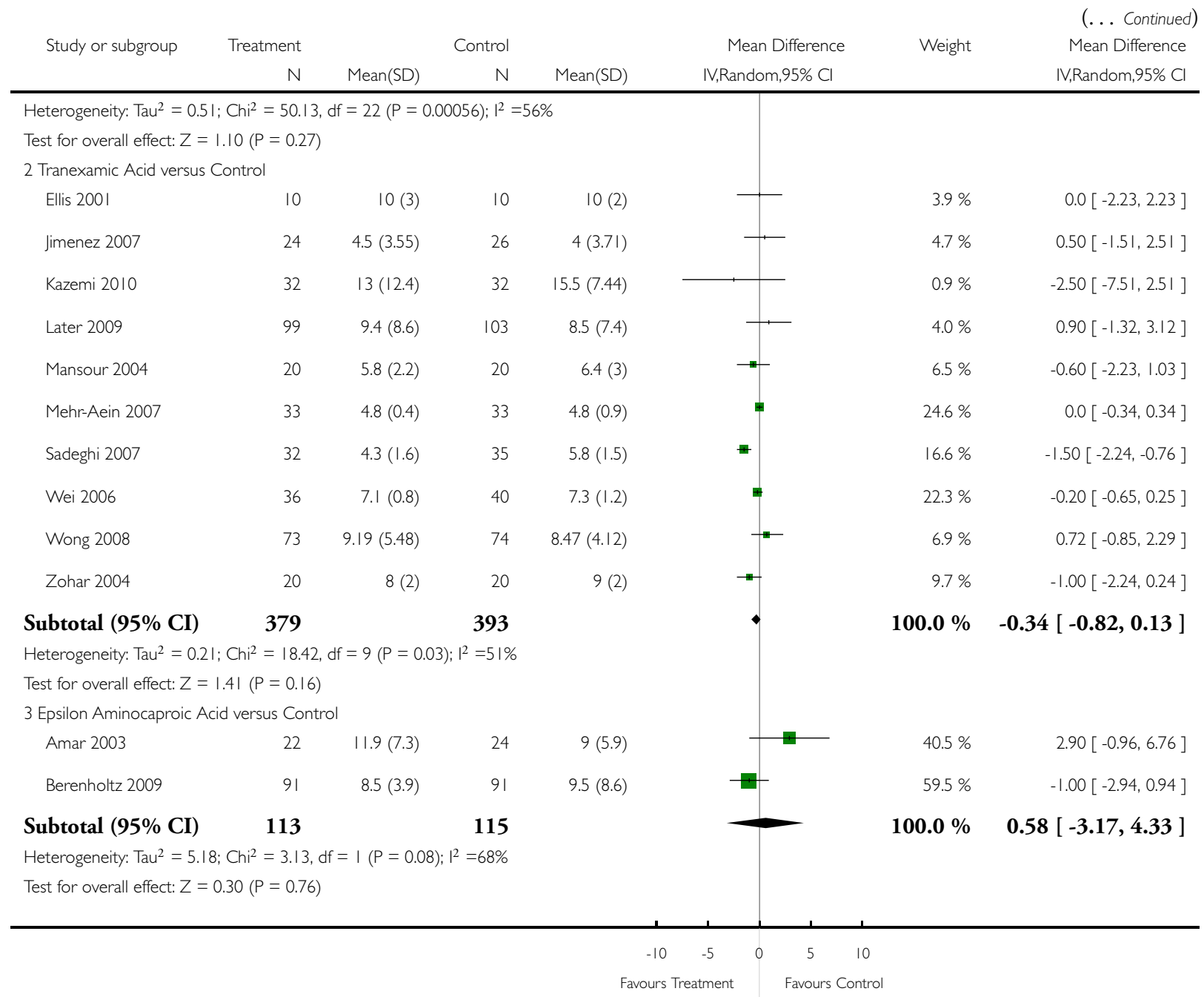


Analysis 9.1. Comparison 9 Adverse Events and Other Outcomes (Active versus Active), Outcome I Reoperation for bleeding - Aprotinin versus Tranexamic Acid.

Review: Anti-fibrinolytic use for minimising perioperative allogeneic blood transfusion

Comparison: 9 Adverse Events and Other Outcomes (Active versus Active)

Outcome: I Re-operation for bleeding - Aprotinin versus Tranexamic Acid

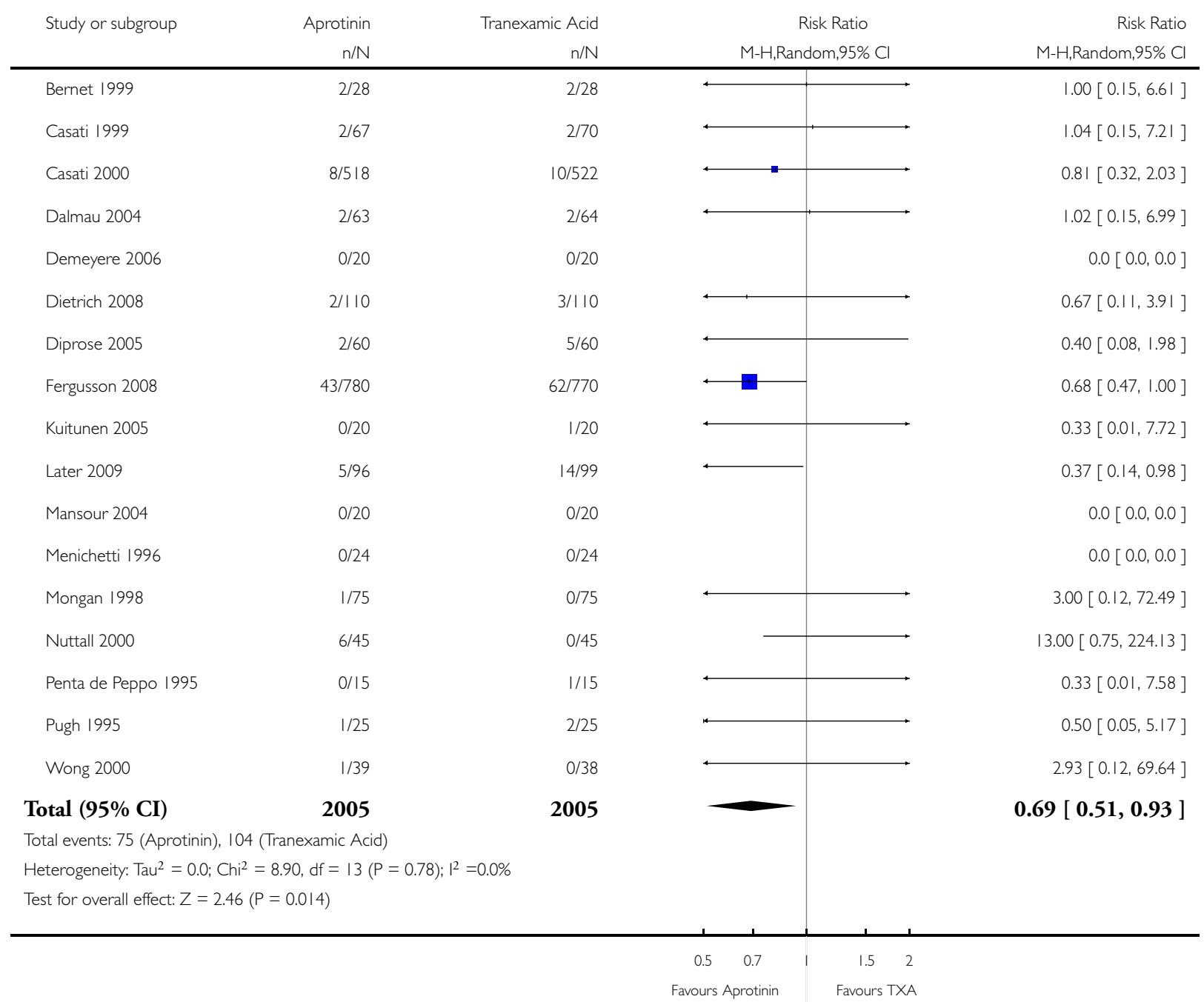


Analysis 9.2. Comparison 9 Adverse Events and Other Outcomes (Active versus Active), Outcome 2 Reoperation for bleeding - Aprotinin versus Epsilon Aminocaproic Acid.

Review: Anti-fibrinolytic use for minimising perioperative allogeneic blood transfusion

Comparison: 9 Adverse Events and Other Outcomes (Active versus Active)

Outcome: 2 Re-operation for bleeding - Aprotinin versus Epsilon Aminocaproic Acid

\begin{tabular}{|c|c|c|c|c|}
\hline \multirow[t]{2}{*}{ Study or subgroup } & Aprotinin & EACA & Risk Ratio & Risk Ratio \\
\hline & $\mathrm{n} / \mathrm{N}$ & $n / N$ & M-H,Random,95\% Cl & M-H,Random,95\% Cl \\
\hline Bennett-Guerrero 1997 & $4 / 99$ & $3 / 105$ & - & $1.41[0.32,6.16]$ \\
\hline Casati 1999 & $2 / 67$ & $3 / 66$ & 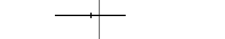 & $0.66[0.11,3.80]$ \\
\hline Fergusson 2008 & $43 / 780$ & $64 / 780$ & 1 & $0.67[0.46,0.98]$ \\
\hline Menichetti 1996 & $0 / 24$ & $0 / 24$ & & $0.0[0.0,0.0]$ \\
\hline Penta de Peppo 1995 & $0 / 15$ & $0 / 15$ & & $0.0[0.0,0.0]$ \\
\hline Ray 2001 & 0/49 & $0 / 51$ & & $0.0[0.0,0.0]$ \\
\hline Total $(95 \% \mathrm{CI})$ & 1034 & 1041 & $\bullet$ & $0.70[0.49,1.00]$ \\
\hline \multicolumn{5}{|c|}{ Total events: 49 (Aprotinin), 70 (EACA) } \\
\hline \multicolumn{5}{|c|}{ Heterogeneity: $\operatorname{Tau}^{2}=0.0 ; \mathrm{Chi}^{2}=0.93, \mathrm{df}=2(\mathrm{P}=0.63) ;\left.\right|^{2}=0.0 \%$} \\
\hline \multicolumn{5}{|c|}{ Test for overall effect: $Z=1.96(P=0.049)$} \\
\hline
\end{tabular}

$0.0010 .010 .1 \quad 1 \quad 10 \quad 1001000$

Favours Aprotinin Favours EACA 
Analysis 9.3. Comparison 9 Adverse Events and Other Outcomes (Active versus Active), Outcome 3 Reoperation for bleeding - Tranexamic Acid versus Epsilon Aminocaproic Acid.

Review: Anti-fibrinolytic use for minimising perioperative allogeneic blood transfusion

Comparison: 9 Adverse Events and Other Outcomes (Active versus Active)

Outcome: 3 Re-operation for bleeding - Tranexamic Acid versus Epsilon Aminocaproic Acid

\begin{tabular}{|c|c|c|c|c|}
\hline \multirow[t]{2}{*}{ Study or subgroup } & \multirow{2}{*}{$\begin{array}{r}\text { Tranexamic Acid } \\
n / N\end{array}$} & \multirow{2}{*}{$\begin{array}{r}\text { EACA } \\
\mathrm{n} / \mathrm{N}\end{array}$} & \multirow{2}{*}{$\begin{array}{c}\text { Risk Ratio } \\
\mathrm{M}-\mathrm{H}, \text { Random,95\% Cl }\end{array}$} & \multirow{2}{*}{$\begin{array}{r}\text { Risk Ratio } \\
\mathrm{M}-\mathrm{H}, \text { Random,95\% Cl}\end{array}$} \\
\hline & & & & \\
\hline Casati 1999 & $2 / 70$ & $3 / 66$ & - & $0.63[0.11,3.64]$ \\
\hline Fergusson 2008 & $62 / 770$ & $64 / 780$ & & $0.98[0.70,1.37]$ \\
\hline Hardy 1998 & $3 / 43$ & $1 / 46$ & & $3.21[0.35,29.69]$ \\
\hline Menichetti 1996 & $0 / 24$ & $0 / 24$ & & $0.0[0.0,0.0]$ \\
\hline Penta de Peppo 1995 & $1 / 15$ & $0 / 15$ & & $3.00[0.13,68.26]$ \\
\hline Total (95\% CI) & 922 & 931 & & $1.00[0.73,1.39]$ \\
\hline \multicolumn{5}{|c|}{ Total events: 68 (Tranexamic Acid), 68 (EACA) } \\
\hline \multicolumn{5}{|c|}{ Heterogeneity: $\operatorname{Tau}^{2}=0.0 ; \mathrm{Chi}^{2}=1.8 \mathrm{I}, \mathrm{df}=3(\mathrm{P}=0.6 \mathrm{I}) ; \mathrm{I}^{2}=0.0 \%$} \\
\hline Test for overall effect: $Z=$ & & & & \\
\hline
\end{tabular}

$\begin{array}{lllllll}0.1 & 0.2 & 0.5 & 1 & 2 & 5 & 10\end{array}$

Favours TXA Favours EACA 
Analysis 9.4. Comparison 9 Adverse Events and Other Outcomes (Active versus Active), Outcome 4 Mortality - Aprotinin versus Tranexamic Acid.

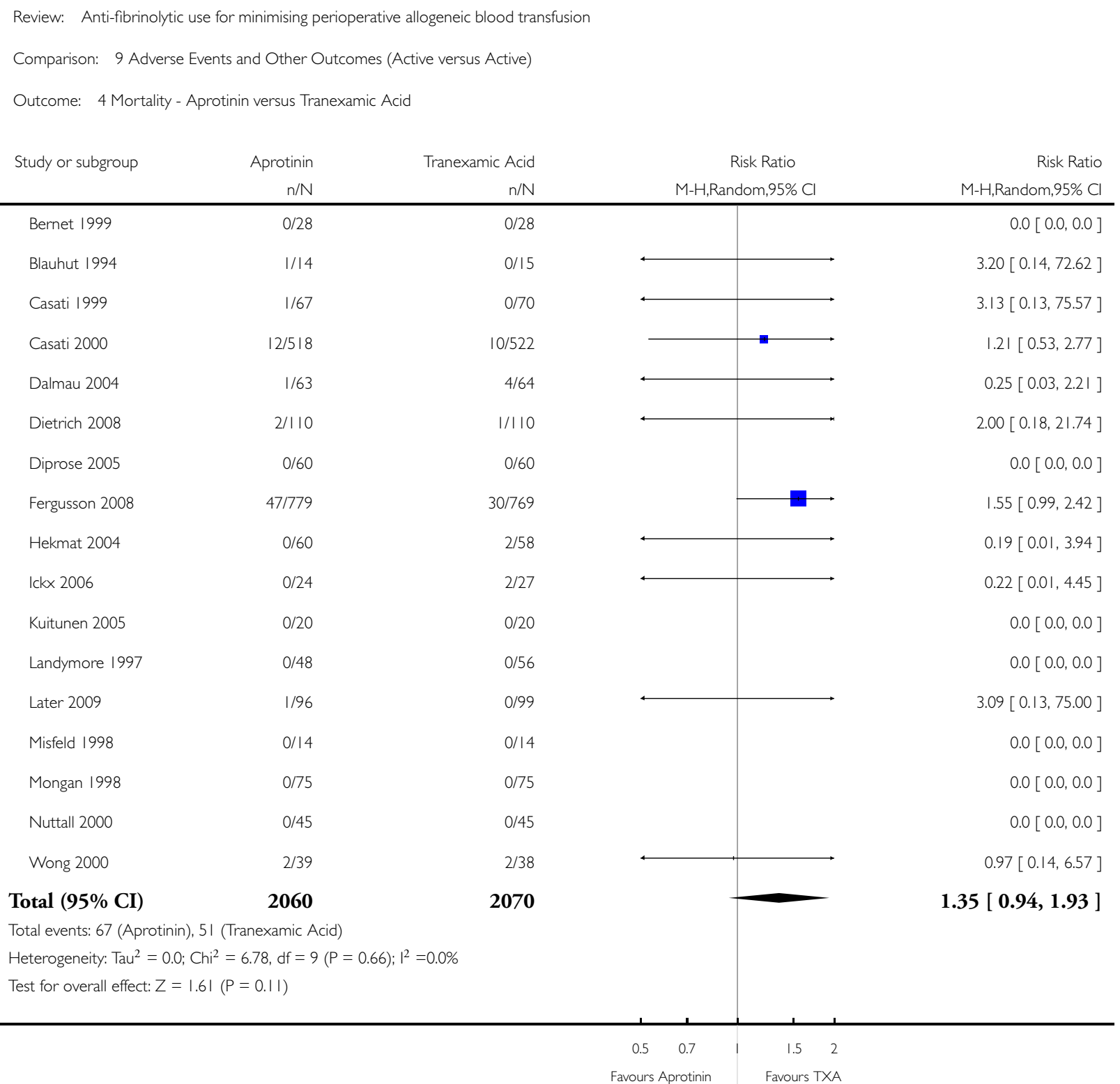


Analysis 9.5. Comparison 9 Adverse Events and Other Outcomes (Active versus Active), Outcome 5 Mortality - Aprotinin versus Epsilon Aminocaproic Acid.

Review: Anti-fibrinolytic use for minimising perioperative allogeneic blood transfusion

Comparison: 9 Adverse Events and Other Outcomes (Active versus Active)

Outcome: 5 Mortality - Aprotinin versus Epsilon Aminocaproic Acid

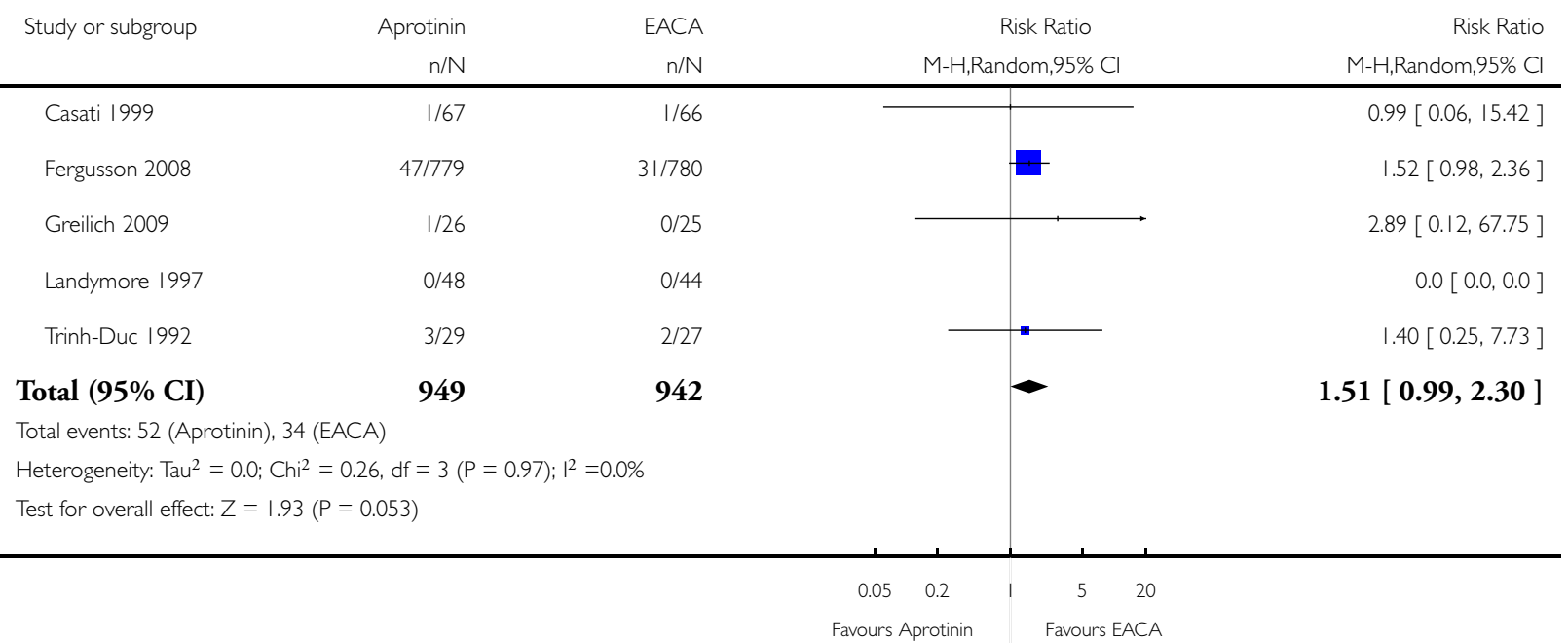


Analysis 9.6. Comparison 9 Adverse Events and Other Outcomes (Active versus Active), Outcome 6 Mortality - Tranexamic Acid versus Epsilon Aminocaproic Acid.

Review: Anti-fibrinolytic use for minimising perioperative allogeneic blood transfusion

Comparison: 9 Adverse Events and Other Outcomes (Active versus Active)

Outcome: 6 Mortality - Tranexamic Acid versus Epsilon Aminocaproic Acid

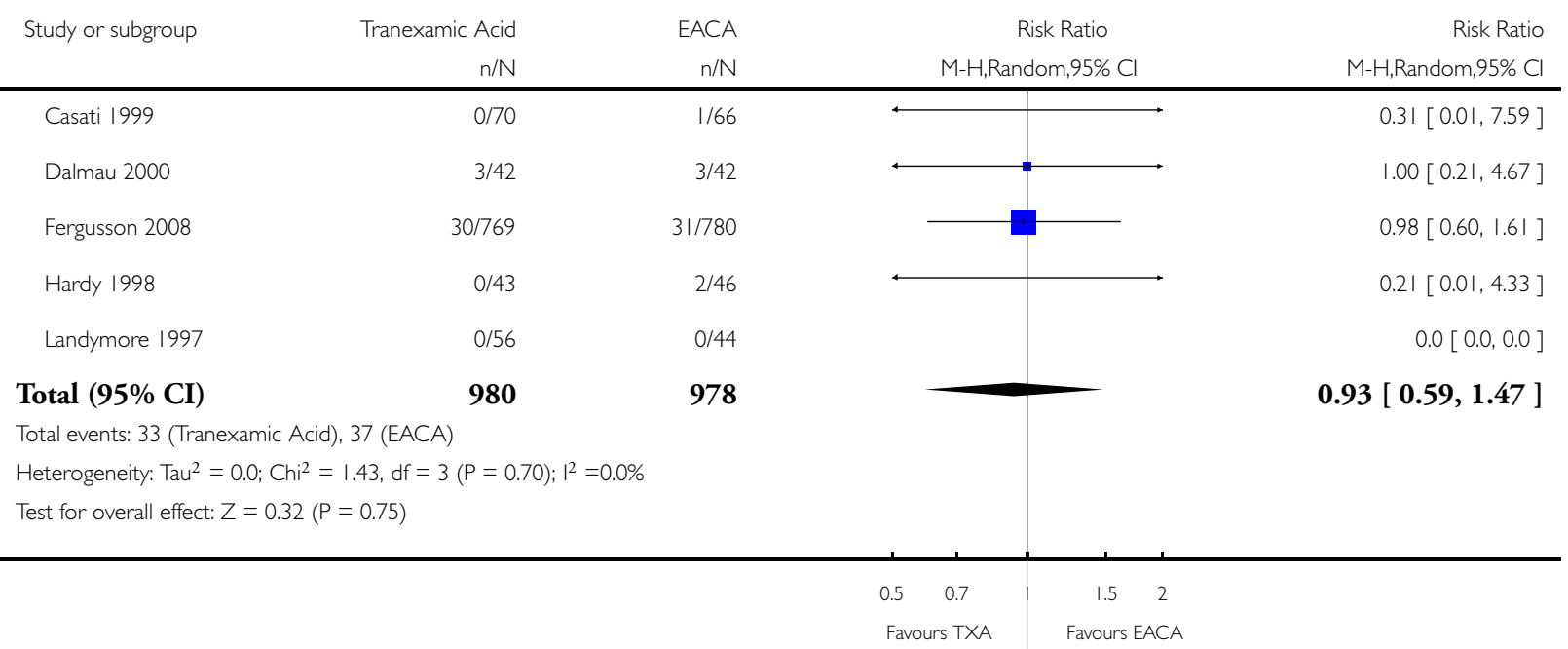


Analysis 9.7. Comparison 9 Adverse Events and Other Outcomes (Active versus Active), Outcome 7 Mortality - Aprotinin versus Lysine Analogues.

Review: Anti-fibrinolytic use for minimising perioperative allogeneic blood transfusion

Comparison: 9 Adverse Events and Other Outcomes (Active versus Active)

Outcome: 7 Mortality - Aprotinin versus Lysine Analogues

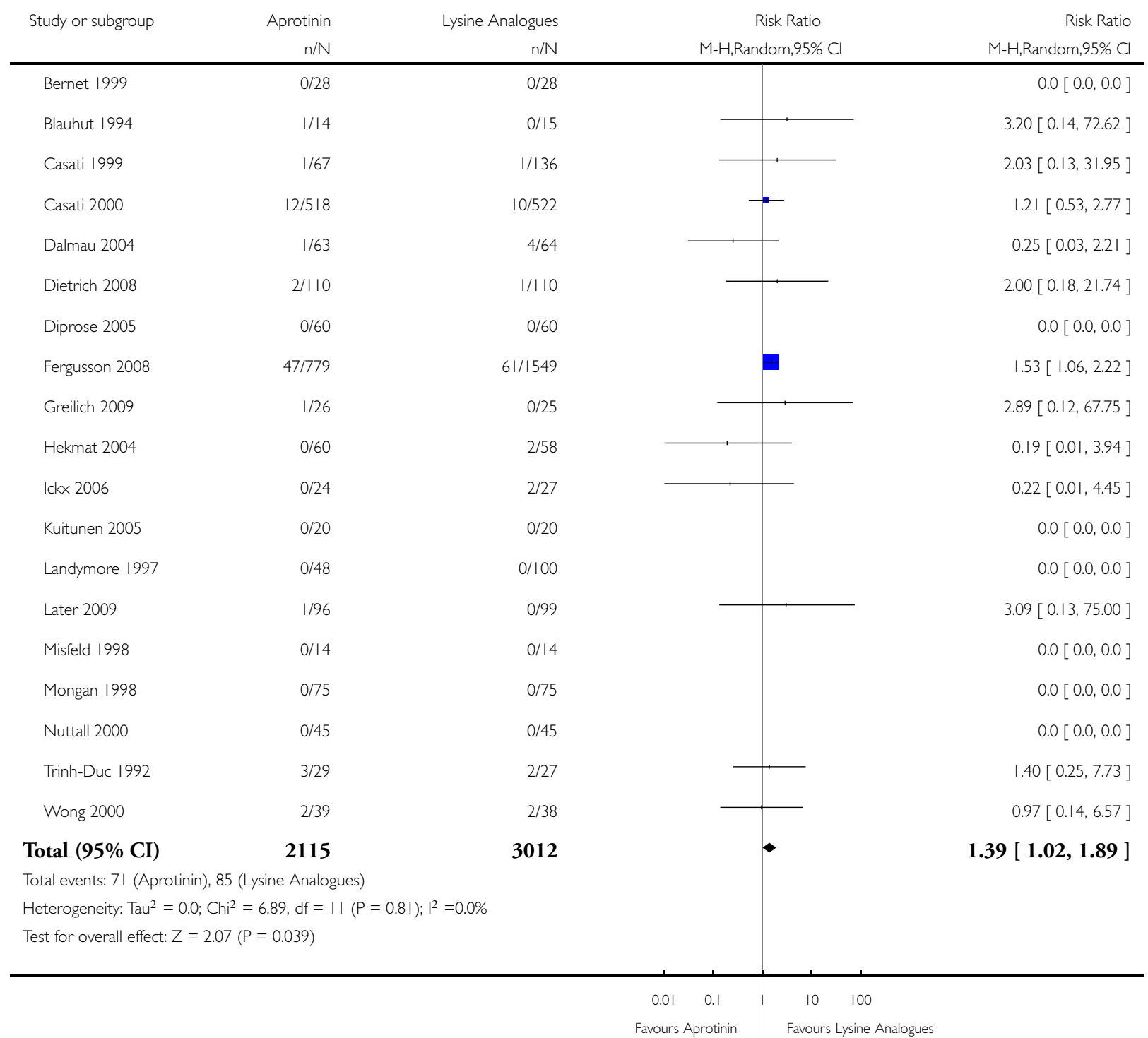


Analysis 9.8. Comparison 9 Adverse Events and Other Outcomes (Active versus Active), Outcome 8 Myocardial Infarction - Aprotinin versus Tranexamic Acid.

Review: Anti-fibrinolytic use for minimising perioperative allogeneic blood transfusion

Comparison: 9 Adverse Events and Other Outcomes (Active versus Active)

Outcome: 8 Myocardial Infarction - Aprotinin versus Tranexamic Acid

\begin{tabular}{|c|c|c|c|c|}
\hline \multirow[t]{2}{*}{ Study or subgroup } & Aprotinin & \multirow{2}{*}{$\begin{array}{r}\text { Tranexamic Acid } \\
n / N\end{array}$} & \multirow{2}{*}{$\begin{array}{c}\text { Risk Ratio } \\
\mathrm{M}-\mathrm{H}, \text { Random,95\% Cl }\end{array}$} & \multirow{2}{*}{$\begin{array}{r}\text { Risk Ratio } \\
\text { M-H,Random,95\% Cl }\end{array}$} \\
\hline & $\mathrm{n} / \mathrm{N}$ & & & \\
\hline Bernet 1999 & $0 / 28$ & $0 / 28$ & & $0.0[0.0,0.0]$ \\
\hline Casati 1999 & $3 / 67$ & $4 / 70$ & $\longrightarrow$ & $0.78[0.18,3.37]$ \\
\hline Casati 2000 & $9 / 518$ & $11 / 522$ & & $0.82[0.34,1.97]$ \\
\hline Dalmau 2004 & $0 / 63$ & $1 / 64$ & & $0.34[0.01,8.16]$ \\
\hline Diprose 2005 & $3 / 60$ & $5 / 60$ & & $0.60[0.15,2.40]$ \\
\hline Fergusson 2008 & $33 / 717$ & $28 / 727$ & 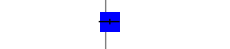 & $1.20[0.73,1.96]$ \\
\hline Hekmat 2004 & $2 / 60$ & $4 / 58$ & & $0.48[0.09,2.54]$ \\
\hline Kuitunen 2005 & $5 / 20$ & $1 / 20$ & & $5.00[0.64,39.06]$ \\
\hline Later 2009 & 1/96 & 0/99 & & $3.09[0.13,75.00]$ \\
\hline Mansour 2004 & $0 / 20$ & $0 / 20$ & & $0.0[0.0,0.0]$ \\
\hline Mongan 1998 & $2 / 75$ & $3 / 75$ & & $0.67[0.11,3.88]$ \\
\hline Speekenbrink 1995 & $1 / 15$ & $0 / 15$ & & $3.00[0.13,68.26]$ \\
\hline Wong 2000 & $4 / 39$ & $5 / 38$ & 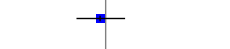 & $0.78[0.23,2.68]$ \\
\hline Total (95\% CI) & 1778 & 1796 & $\bullet$ & $1.00[0.71,1.42]$ \\
\hline \multicolumn{5}{|c|}{ Total events: 63 (Aprotinin), 62 (Tranexamic Acid) } \\
\hline \multicolumn{5}{|c|}{ Heterogeneity: $\mathrm{Tau}^{2}=0.0 ; \mathrm{Chi}^{2}=6.18, \mathrm{df}=10(\mathrm{P}=0.80) ;\left.\right|^{2}=0.0 \%$} \\
\hline \multicolumn{5}{|c|}{ Test for overall effect: $Z=0.03(P=0.98)$} \\
\hline
\end{tabular}

$0.0010 .01 \quad 0.1 \quad 1 \quad 10 \quad 100 \quad 1000$

Favours Aprotinin Favours TXA 
Analysis 9.9. Comparison 9 Adverse Events and Other Outcomes (Active versus Active), Outcome 9 Myocardial Infarction - Aprotinin versus Epsilon Aminocaproic Acid.

Review: Anti-fibrinolytic use for minimising perioperative allogeneic blood transfusion

Comparison: 9 Adverse Events and Other Outcomes (Active versus Active)

Outcome: 9 Myocardial Infarction - Aprotinin versus Epsilon Aminocaproic Acid

\begin{tabular}{|c|c|c|c|c|c|}
\hline \multirow[t]{2}{*}{ Study or subgroup } & Aprotinin & EACA & Risk Ratio & Weight & Risk Ratio \\
\hline & $\mathrm{n} / \mathrm{N}$ & $\mathrm{n} / \mathrm{N}$ & $\mathrm{M}-\mathrm{H}$, Random,95\% Cl & & $\mathrm{M}-\mathrm{H}$, Random,95\% Cl \\
\hline Casati 1999 & $3 / 67$ & $3 / 66$ & $\longrightarrow$ & $8.3 \%$ & $0.99[0.21,4.71]$ \\
\hline Eberle 1998 & $1 / 20$ & $1 / 20$ & & $2.8 \%$ & $1.00[0.07,14.90]$ \\
\hline Fergusson 2008 & $33 / 717$ & $20 / 735$ & - & $68.2 \%$ & $1.69[0.98,2.92]$ \\
\hline Greilich 2009 & $6 / 26$ & $6 / 25$ & $\rightarrow$ & $20.7 \%$ & $0.96[0.36,2.59]$ \\
\hline
\end{tabular}

Total (95\% CI)

830

846

$100.0 \%$

$1.42[0.90,2.22]$

Total events: 43 (Aprotinin), 30 (EACA)

Heterogeneity: $\mathrm{Tau}^{2}=0.0 ; \mathrm{Chi}^{2}=1.27, \mathrm{df}=3(\mathrm{P}=0.74) ;\left.\right|^{2}=0.0 \%$

Test for overall effect: $Z=1.52(P=0.13)$

$0.0010 .010 .1 \quad 1 \quad 10 \quad 1001000$

Favours Aprotinin Favours EACA

Analysis 9.10. Comparison 9 Adverse Events and Other Outcomes (Active versus Active), Outcome 10 Myocardial Infarction - Tranexamic Acid versus Epsilon Aminocaproic Acid.

Review: Anti-fibrinolytic use for minimising perioperative allogeneic blood transfusion

Comparison: 9 Adverse Events and Other Outcomes (Active versus Active)

Outcome: 10 Myocardial Infarction - Tranexamic Acid versus Epsilon Aminocaproic Acid

\begin{tabular}{|c|c|c|c|c|c|}
\hline \multirow[t]{2}{*}{ Study or subgroup } & Tranexamic Acid & EACA & Risk Ratio & Weight & Risk Ratio \\
\hline & $n / N$ & $\mathrm{n} / \mathrm{N}$ & M-H,Random,95\% C & & M-H,Random,95\% Cl \\
\hline Casati 1999 & $4 / 70$ & $3 / 66$ & $\longrightarrow$ & $12.4 \%$ & $1.26[0.29,5.41]$ \\
\hline Fergusson 2008 & $28 / 727$ & 20/735 & & $82.9 \%$ & $1.42[0.80,2.49]$ \\
\hline Hardy 1998 & $1 / 43$ & 2/46 & '. & $4.7 \%$ & $0.53[0.05,5.69]$ \\
\hline Total (95\% CI) & 840 & 847 & - & $100.0 \%$ & $1.33[0.80,2.23]$ \\
\hline \multicolumn{6}{|c|}{ Total events: 33 (Tranexamic Acid), 25 (EACA) } \\
\hline \multicolumn{6}{|c|}{ Heterogeneity: $\operatorname{Tau}^{2}=0.0 ; \mathrm{Chi}^{2}=0.62, \mathrm{df}=2(\mathrm{P}=0.73) ;\left.\right|^{2}=0.0 \%$} \\
\hline \multicolumn{6}{|c|}{ Test for overall effect: $Z=1.09(P=0.27)$} \\
\hline
\end{tabular}


Analysis 9.1 I. Comparison 9 Adverse Events and Other Outcomes (Active versus Active), Outcome II Myocardial infarction - Aprotinin versus Lysine Analogues.

Review: Anti-fibrinolytic use for minimising perioperative allogeneic blood transfusion

Comparison: 9 Adverse Events and Other Outcomes (Active versus Active)

Outcome: II Myocardial infarction - Aprotinin versus Lysine Analogues

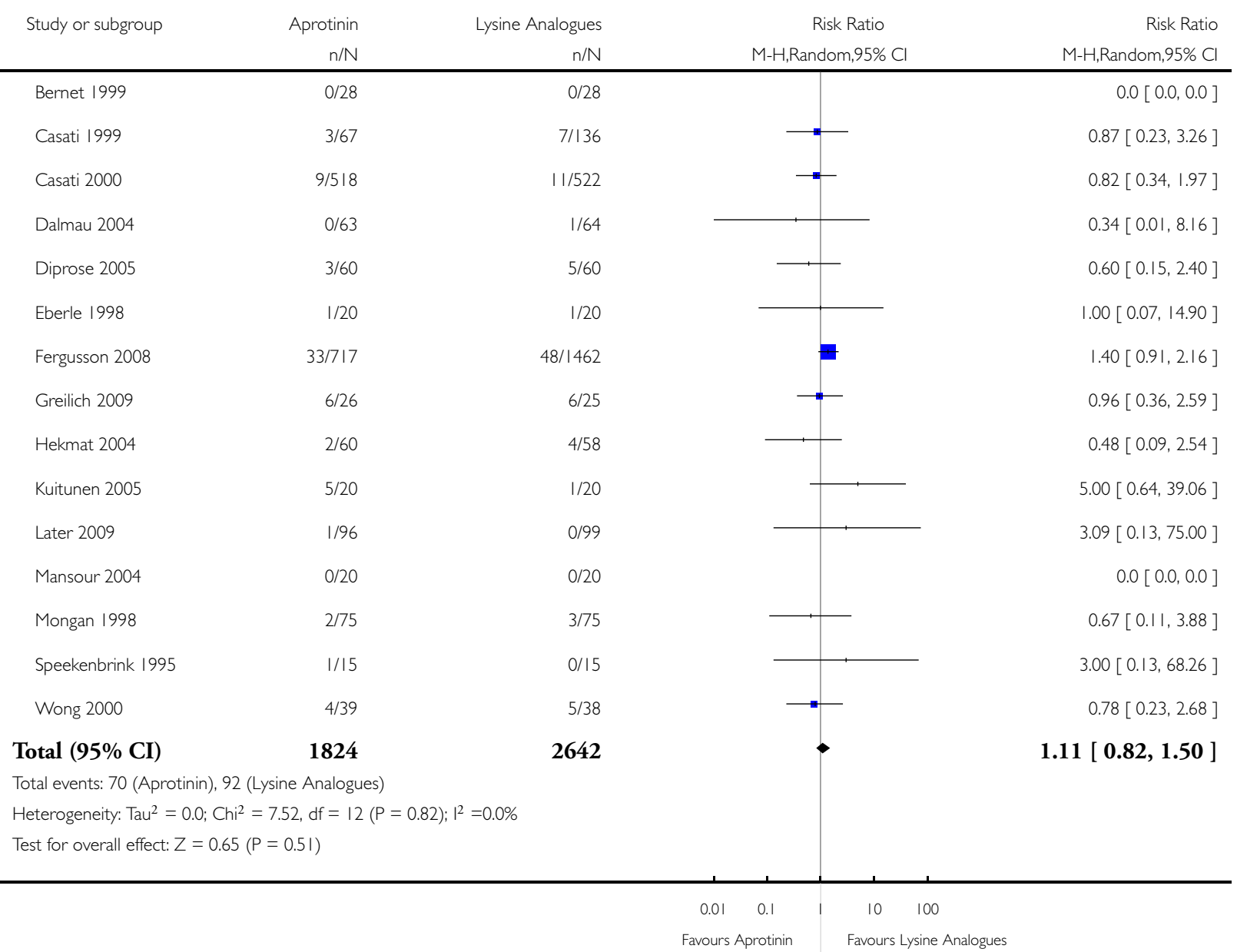


Analysis 9.12. Comparison 9 Adverse Events and Other Outcomes (Active versus Active), Outcome 12 Stroke (CVA) - Aprotinin versus Tranexamic Acid.

Review: Anti-fibrinolytic use for minimising perioperative allogeneic blood transfusion

Comparison: 9 Adverse Events and Other Outcomes (Active versus Active)

Outcome: 12 Stroke (CVA) - Aprotinin versus Tranexamic Acid

\begin{tabular}{|c|c|c|c|c|}
\hline \multirow[t]{2}{*}{ Study or subgroup } & Aprotinin & Tranexamic Acid & Risk Ratio & Risk Ratio \\
\hline & $\mathrm{n} / \mathrm{N}$ & $\mathrm{n} / \mathrm{N}$ & M-H,Random,95\% Cl & M-H,Random,95\% Cl \\
\hline Bernet 1999 & $0 / 28$ & $0 / 28$ & & $0.0[0.0,0.0]$ \\
\hline Fergusson 2008 & $22 / 759$ & $28 / 753$ & & $0.78[0.45,1.35]$ \\
\hline Kuitunen 2005 & $1 / 20$ & $0 / 20$ & & $3.00[0.13,69.52]$ \\
\hline Later 2009 & 1/96 & $1 / 99$ & & $1.03[0.07,16.25]$ \\
\hline Mongan 1998 & $1 / 75$ & 0/75 & & $3.00[0.12,72.49]$ \\
\hline Wong 2000 & $1 / 39$ & $0 / 38$ & & $2.93[0.12,69.64]$ \\
\hline Total (95\% CI) & 1017 & 1013 & 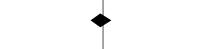 & $0.88[0.52,1.47]$ \\
\hline \multicolumn{5}{|c|}{ Total events: 26 (Aprotinin), 29 (Tranexamic Acid) } \\
\hline \multicolumn{5}{|c|}{ Heterogeneity: $\operatorname{Tau}^{2}=0.0 ; \mathrm{Chi}^{2}=1.91, \mathrm{df}=4(\mathrm{P}=0.75) ; \mathbf{1}^{2}=0.0 \%$} \\
\hline \multicolumn{5}{|c|}{ Test for overall effect: $Z=0.50(P=0.62)$} \\
\hline
\end{tabular}

$0.0010 .010 .1 \quad 1 \quad 10 \quad 1001000$

Favours Aprotinin Favours TXA

Analysis 9.13. Comparison 9 Adverse Events and Other Outcomes (Active versus Active), Outcome 13 Stroke (CVA) - Aprotinin versus Epsilon Aminocaproic Acid.

Review: Anti-fibrinolytic use for minimising perioperative allogeneic blood transfusion

Comparison: 9 Adverse Events and Other Outcomes (Active versus Active)

Outcome: 13 Stroke (CVA) - Aprotinin versus Epsilon Aminocaproic Acid

\begin{tabular}{|c|c|c|c|c|c|}
\hline \multirow[t]{2}{*}{ Study or subgroup } & Aprotinin & Epsilon Aminocaproic Acid & Risk Ratio & Weight & Risk Ratio \\
\hline & $\mathrm{n} / \mathrm{N}$ & $\mathrm{n} / \mathrm{N}$ & $\mathrm{M}-\mathrm{H}$, Random,95\% Cl & & $\mathrm{M}-\mathrm{H}$, Random,95\% Cl \\
\hline Fergusson 2008 & $22 / 759$ & $22 / 768$ & & $94.2 \%$ & $1.01[0.57,1.81]$ \\
\hline Greilich 2009 & $2 / 26$ & $1 / 25$ & & $5.8 \%$ & $1.92[0.19,19.90]$ \\
\hline
\end{tabular}

Total (95\% CI)

785

793

$100.0 \%$

$1.05[0.60,1.85]$

Total events: 24 (Aprotinin), 23 (Epsilon Aminocaproic Acid)

Heterogeneity: $\mathrm{Tau}^{2}=0.0 ; \mathrm{Chi}^{2}=0.27, \mathrm{df}=\mathrm{I}(\mathrm{P}=0.60) ; \mathrm{I}^{2}=0.0 \%$

Test for overall effect: $Z=0.17(P=0.86)$ 
Analysis 9.14. Comparison 9 Adverse Events and Other Outcomes (Active versus Active), Outcome 14 Stroke (CVA) - Tranexamic Acid versus Epsilon Aminocaproic Acid.

Review: Anti-fibrinolytic use for minimising perioperative allogeneic blood transfusion

Comparison: 9 Adverse Events and Other Outcomes (Active versus Active)

Outcome: 14 Stroke (CVA) - Tranexamic Acid versus Epsilon Aminocaproic Acid

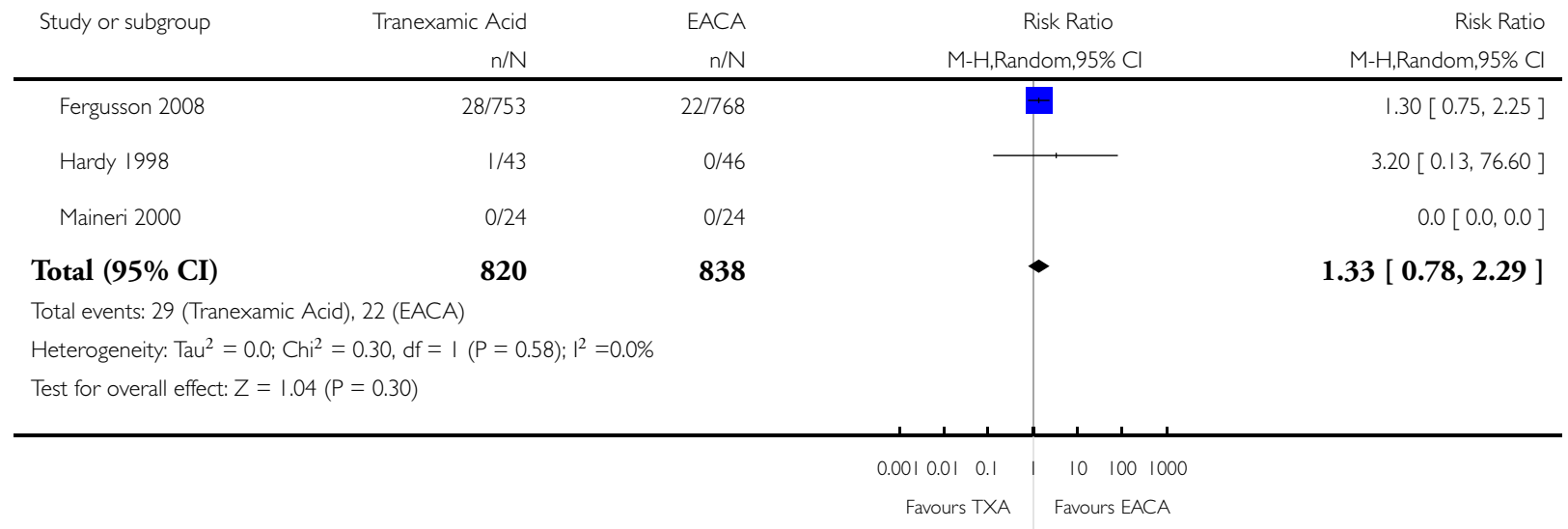




\section{Analysis 9.15. Comparison 9 Adverse Events and Other Outcomes (Active versus Active), Outcome I5 Deep Vein Thrombosis (DVT) - Aprotinin versus Tranexamic Acid.}

Review: Anti-fibrinolytic use for minimising perioperative allogeneic blood transfusion

Comparison: 9 Adverse Events and Other Outcomes (Active versus Active)

Outcome: 15 Deep Vein Thrombosis (DVT) - Aprotinin versus Tranexamic Acid

\begin{tabular}{|c|c|c|c|c|}
\hline \multirow[t]{2}{*}{ Study or subgroup } & Aprotinin & Tranexamic Acid & \multirow{2}{*}{$\begin{array}{c}\text { Risk Ratio } \\
\text { M-H,Random,95\% Cl }\end{array}$} & \multirow{2}{*}{$\begin{array}{r}\text { Risk Ratio } \\
\text { M-H,Random,95\% Cl }\end{array}$} \\
\hline & $\mathrm{n} / \mathrm{N}$ & $\mathrm{n} / \mathrm{N}$ & & \\
\hline Casati 1999 & $0 / 67$ & $0 / 70$ & & $0.0[0.0,0.0]$ \\
\hline Engel 2001 & $1 / 12$ & $2 / 12$ & & $0.50[0.05,4.81]$ \\
\hline Landymore 1997 & $0 / 48$ & $0 / 56$ & & $0.0[0.0,0.0]$ \\
\hline Total (95\% CI) & 127 & 138 & & $0.50[0.05,4.81]$ \\
\hline \multicolumn{5}{|c|}{ Total events: I (Aprotinin), 2 (Tranexamic Acid) } \\
\hline \multicolumn{5}{|c|}{ Heterogeneity: Tau $^{2}=0.0 ; \mathrm{Chi}^{2}=0.0, \mathrm{df}=0(P=1.00) ;\left.\right|^{2}=0.0 \%$} \\
\hline \multicolumn{5}{|c|}{ Test for overall effect: $Z=0.60(P=0.55)$} \\
\hline
\end{tabular}

Analysis 9.16. Comparison 9 Adverse Events and Other Outcomes (Active versus Active), Outcome 16 Deep Vein Thrombosis (DVT) - Aprotinin versus Epsilon Aminocaproic Acid.

Review: Anti-fibrinolytic use for minimising perioperative allogeneic blood transfusion

Comparison: 9 Adverse Events and Other Outcomes (Active versus Active)

Outcome: 16 Deep Vein Thrombosis (DVT) - Aprotinin versus Epsilon Aminocaproic Acid

\begin{tabular}{|c|c|c|c|c|}
\hline Study or subgroup & $\begin{array}{r}\text { Aprotinin } \\
\mathrm{n} / \mathrm{N}\end{array}$ & $\begin{array}{r}\text { EACA } \\
\mathrm{n} / \mathrm{N}\end{array}$ & $\begin{array}{c}\text { Risk Ratio } \\
M-H, \text { Random,95\% Cl}\end{array}$ & 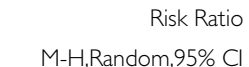 \\
\hline Amar 2003 & $0 / 23$ & $3 / 22$ & $\square$ & $0.14[0.01,2.51]$ \\
\hline Casati 1999 & $0 / 67$ & $0 / 66$ & & $0.0[0.0,0.0]$ \\
\hline Landymore 1997 & $0 / 48$ & $0 / 44$ & & $0.0[0.0,0.0]$ \\
\hline Ray 2005 & $0 / 15$ & $0 / 15$ & & $0.0[0.0,0.0]$ \\
\hline Total (95\% CI) & 153 & 147 & & $0.14[0.01,2.51]$ \\
\hline \multicolumn{5}{|c|}{ Total events: 0 (Aprotinin), 3 (EACA) } \\
\hline \multicolumn{5}{|c|}{ Heterogeneity: $\operatorname{Tau}^{2}=0.0 ; \mathrm{Chi}^{2}=0.0, \mathrm{df}=0(P=1.00) ;\left.\right|^{2}=0.0 \%$} \\
\hline \multicolumn{5}{|c|}{ Test for overall effect: $Z=1.34(P=0.18)$} \\
\hline
\end{tabular}


Analysis 9.17. Comparison 9 Adverse Events and Other Outcomes (Active versus Active), Outcome 17 Deep Vein Thrombosis (DVT) - Tranexamic Acid versus Epsilon Aminocaproic Acid.

Review: Anti-fibrinolytic use for minimising perioperative allogeneic blood transfusion

Comparison: 9 Adverse Events and Other Outcomes (Active versus Active)

Outcome: 17 Deep Vein Thrombosis (DVT) - Tranexamic Acid versus Epsilon Aminocaproic Acid

\begin{tabular}{|c|c|c|c|c|}
\hline \multirow[t]{2}{*}{ Study or subgroup } & \multirow{2}{*}{$\begin{array}{r}\text { Tranexamic Acid } \\
n / N\end{array}$} & \multirow{2}{*}{$\begin{array}{r}\text { EACA } \\
n / N\end{array}$} & \multirow{2}{*}{$\begin{array}{c}\text { Risk Ratio } \\
\mathrm{M}-\mathrm{H}, \text { Random,95\% Cl }\end{array}$} & \multirow{2}{*}{$\begin{array}{r}\text { Risk Ratio } \\
\mathrm{M}-\mathrm{H}, \text { Random,95\% Cl}\end{array}$} \\
\hline & & & & \\
\hline Camarasa 2006 & $0 / 35$ & $0 / 32$ & & $0.0[0.0,0.0]$ \\
\hline Casati 1999 & $0 / 70$ & $0 / 66$ & & $0.0[0.0,0.0]$ \\
\hline Landymore 1997 & $0 / 56$ & $0 / 44$ & & $0.0[0.0,0.0]$ \\
\hline Total $(95 \% \mathrm{CI})$ & 161 & 142 & & $0.0[0.0,0.0]$ \\
\hline \multicolumn{5}{|c|}{ Total events: 0 (Tranexamic Acid), 0 (EACA) } \\
\hline \multicolumn{5}{|c|}{ Heterogeneity: $\operatorname{Tau}^{2}=\mathrm{iI}^{1} / 2 ; \mathrm{Chi}^{2}=0.0, \mathrm{df}=0(\mathrm{P}<0.0000 \mathrm{I}) ; \mathrm{I}^{2}=0.0 \%$} \\
\hline \multicolumn{5}{|c|}{ Test for overall effect: $Z=0.0(P<0.0000 I)$} \\
\hline
\end{tabular}

Analysis 9.18. Comparison 9 Adverse Events and Other Outcomes (Active versus Active), Outcome 18 Pulmonary Embolism (PE) - Aprotinin versus Tranexamic Acid.

Review: Anti-fibrinolytic use for minimising perioperative allogeneic blood transfusion

Comparison: 9 Adverse Events and Other Outcomes (Active versus Active)

Outcome: 18 Pulmonary Embolism (PE) - Aprotinin versus Tranexamic Acid

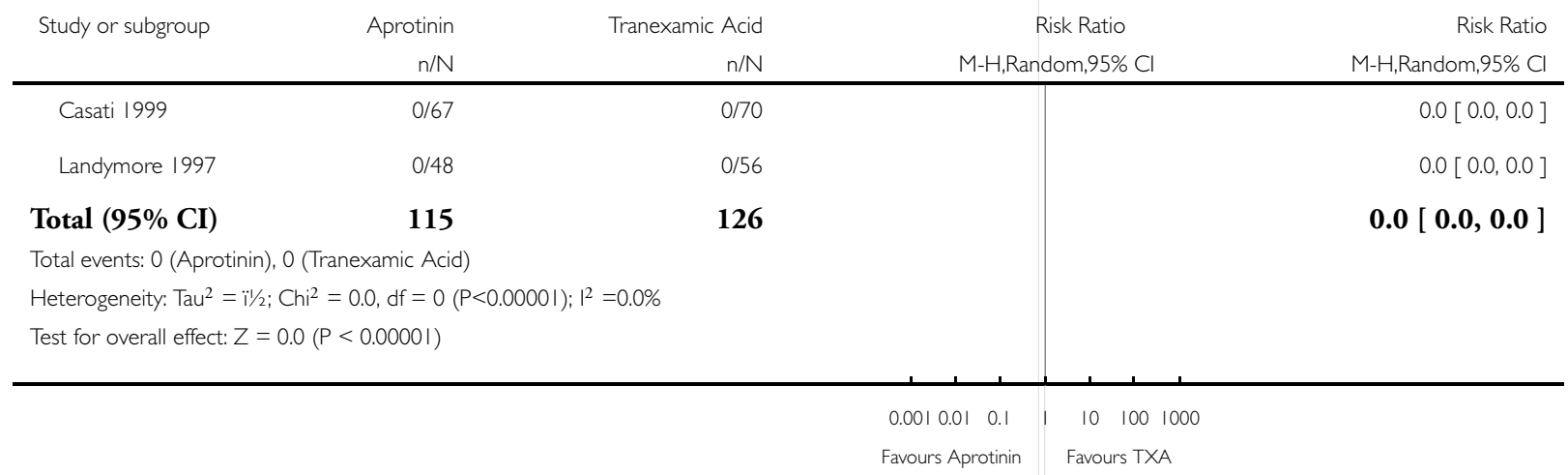


Analysis 9.19. Comparison 9 Adverse Events and Other Outcomes (Active versus Active), Outcome 19 Pulmonary Embolism (PE) - Aprotinin versus Epsilon Aminocaproic Acid.

Review: Anti-fibrinolytic use for minimising perioperative allogeneic blood transfusion

Comparison: 9 Adverse Events and Other Outcomes (Active versus Active)

Outcome: 19 Pulmonary Embolism (PE) - Aprotinin versus Epsilon Aminocaproic Acid

\begin{tabular}{|c|c|c|c|c|}
\hline \multirow[t]{2}{*}{ Study or subgroup } & Aprotinin & EACA & Risk Ratio & Risk Ratio \\
\hline & $\mathrm{n} / \mathrm{N}$ & $\mathrm{n} / \mathrm{N}$ & M-H,Random,95\% Cl & $\mathrm{M}-\mathrm{H}$, Random, $95 \% \mathrm{Cl}$ \\
\hline Amar 2003 & $2 / 23$ & $0 / 22$ & $+\quad$ & $4.79[0.24,94.53]$ \\
\hline Casati 1999 & $0 / 67$ & $1 / 66$ & $\rightarrow$ & $0.33[0.01,7.92]$ \\
\hline Landymore 1997 & $0 / 48$ & $0 / 44$ & & $0.0[0.0,0.0]$ \\
\hline
\end{tabular}

Total $(95 \% \mathrm{CI})$

138

132

$1.33[0.10,18.42]$

Total events: 2 (Aprotinin), I (EACA)

Heterogeneity: $\mathrm{Tau}^{2}=1.12 ; \mathrm{Chi}^{2}=1.45, \mathrm{df}=1(\mathrm{P}=0.23) ; \mathrm{I}^{2}=31 \%$

Test for overall effect: $Z=0.21(P=0.83)$

$0.0010 .01 \quad 0.1 \quad 1 \quad 10 \quad 100 \quad 1000$

Favours Aprotinin Favours EACA

Analysis 9.20. Comparison 9 Adverse Events and Other Outcomes (Active versus Active), Outcome 20 Pulmonary Embolism (PE) - Tranexamic Acid versus Epsilon Aminocaproic Acid.

Review: Anti-fibrinolytic use for minimising perioperative allogeneic blood transfusion

Comparison: 9 Adverse Events and Other Outcomes (Active versus Active)

Outcome: 20 Pulmonary Embolism (PE) - Tranexamic Acid versus Epsilon Aminocaproic Acid

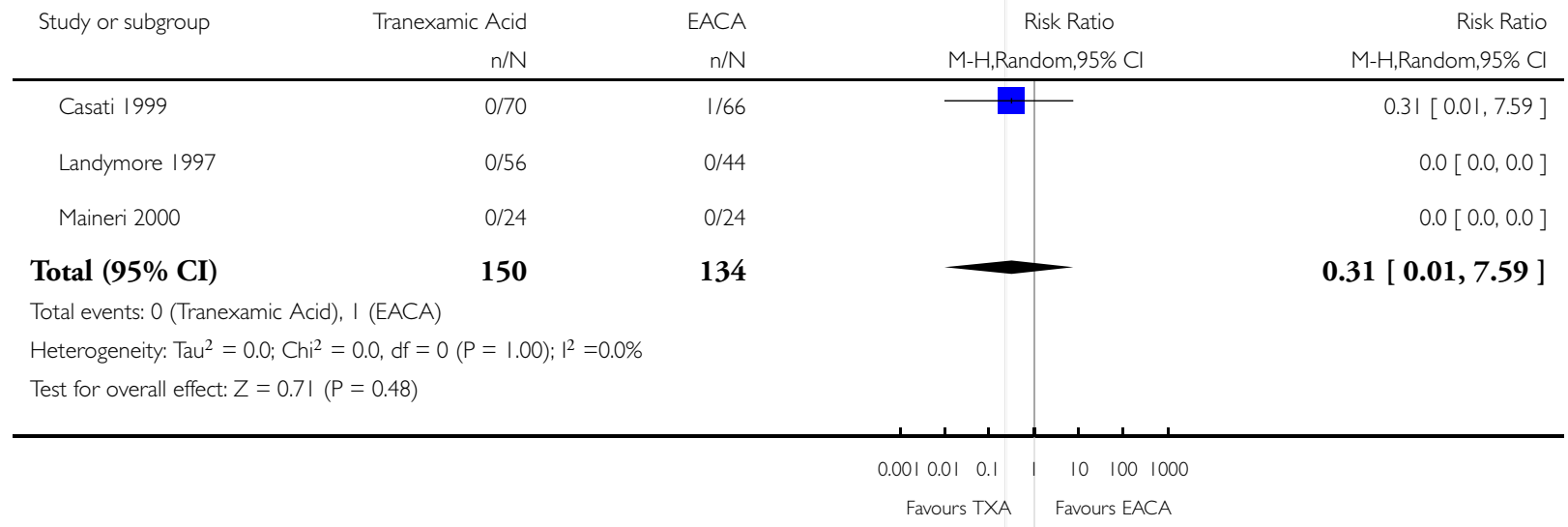


Analysis 9.21. Comparison 9 Adverse Events and Other Outcomes (Active versus Active), Outcome 21 Other Thrombosis - Aprotinin versus Tranexamic Acid.

Review: Anti-fibrinolytic use for minimising perioperative allogeneic blood transfusion

Comparison: 9 Adverse Events and Other Outcomes (Active versus Active)

Outcome: 21 Other Thrombosis - Aprotinin versus Tranexamic Acid

\begin{tabular}{|c|c|c|c|c|}
\hline \multirow[t]{2}{*}{ Study or subgroup } & Aprotinin & \multirow{2}{*}{$\begin{array}{r}\text { Tranexamic Acid } \\
\mathrm{n} / \mathrm{N}\end{array}$} & \multirow{2}{*}{$\begin{array}{c}\text { Risk Ratio } \\
\mathrm{M}-\mathrm{H}, \text { Random,95\% Cl }\end{array}$} & \multirow{2}{*}{$\begin{array}{r}\text { Risk Ratio } \\
\text { M-H,Random,95\% Cl }\end{array}$} \\
\hline & $\mathrm{n} / \mathrm{N}$ & & & \\
\hline Bernet 1999 & $0 / 28$ & $0 / 28$ & & $0.0[0.0,0.0]$ \\
\hline Dalmau 2004 & $2 / 63$ & $4 / 64$ & & $0.51[0.10,2.68]$ \\
\hline Landymore 1997 & $0 / 48$ & $0 / 56$ & & $0.0[0.0,0.0]$ \\
\hline Total (95\% CI) & 139 & 148 & & $0.51[0.10,2.68]$ \\
\hline \multicolumn{5}{|c|}{ Total events: 2 (Aprotinin), 4 (Tranexamic Acid) } \\
\hline \multicolumn{5}{|c|}{ Heterogeneity: $\operatorname{Tau}^{2}=0.0 ; \mathrm{Chi}^{2}=0.0, \mathrm{df}=0(P=1.00) ;\left.\right|^{2}=0.0 \%$} \\
\hline Test for overall effect: & $=0.42)$ & & & \\
\hline
\end{tabular}

$0.0010 .01 \quad 0.1 \quad 1 \quad 10 \quad 100 \quad 1000$

Favours Aprotinin Favours TXA

Analysis 9.22. Comparison 9 Adverse Events and Other Outcomes (Active versus Active), Outcome 22 Other Thrombosis - Aprotinin versus Epsilon Aminocaproic Acid.

Review: Anti-fibrinolytic use for minimising perioperative allogeneic blood transfusion

Comparison: 9 Adverse Events and Other Outcomes (Active versus Active)

Outcome: 22 Other Thrombosis - Aprotinin versus Epsilon Aminocaproic Acid

\begin{tabular}{|c|c|c|c|c|}
\hline \multirow{2}{*}{ Study or subgroup } & \multirow{2}{*}{$\begin{array}{r}\text { Aproinin } \\
\mathrm{n} / \mathrm{N}\end{array}$} & \multirow{2}{*}{$\begin{array}{r}\text { EACA } \\
\mathrm{n} / \mathrm{N}\end{array}$} & \multirow{2}{*}{$\begin{array}{c}\text { Risk Ratio } \\
\mathrm{M}-\mathrm{H}, \text { Random,95\% Cl }\end{array}$} & Risk Ratio \\
\hline & & & & M-H,Random,95\% Cl \\
\hline Landymore 1997 & $0 / 48$ & $0 / 44$ & & $0.0[0.0,0.0]$ \\
\hline Total $(95 \% \mathrm{CI})$ & 48 & 44 & & $0.0[0.0,0.0]$ \\
\hline \multicolumn{5}{|c|}{ Total events: 0 (Aproinin), 0 (EACA) } \\
\hline \multicolumn{5}{|c|}{ Heterogeneity: not applicable } \\
\hline \multicolumn{5}{|c|}{ Test for overall effect: $Z=0.0(P<0.0000 I)$} \\
\hline
\end{tabular}


Analysis 9.23. Comparison 9 Adverse Events and Other Outcomes (Active versus Active), Outcome 23 Other Thrombosis - Tranexamic Acid versus Epsilon Aminocaproic Acid.

Review: Anti-fibrinolytic use for minimising perioperative allogeneic blood transfusion

Comparison: 9 Adverse Events and Other Outcomes (Active versus Active)

Outcome: 23 Other Thrombosis - Tranexamic Acid versus Epsilon Aminocaproic Acid

\begin{tabular}{|c|c|c|c|c|}
\hline Study or subgroup & Tranexamic Acid & EACA & Risk Ratio & Risk Ratio \\
\hline & $\mathrm{n} / \mathrm{N}$ & $\mathrm{n} / \mathrm{N}$ & M-H,Random,95\% Cl & M-H,Random,95\% Cl \\
\hline Dalmau 2000 & $4 / 42$ & $2 / 42$ & & $2.00[0.39,10.34]$ \\
\hline Landymore 1997 & $0 / 56$ & $0 / 44$ & & $0.0[0.0,0.0]$ \\
\hline
\end{tabular}

Total (95\% CI)

98

86

$2.00[0.39,10.34]$

Total events: 4 (Tranexamic Acid), 2 (EACA)

Heterogeneity: $\mathrm{Tau}^{2}=0.0 ; \mathrm{Chi}^{2}=0.0, \mathrm{df}=0(\mathrm{P}=1.00) ; \mathrm{I}^{2}=0.0 \%$

Test for overall effect: $Z=0.83(P=0.41)$

$0.0010 .01 \quad 0.1 \quad 1 \quad 10 \quad 100 \quad 1000$

Favours TXA Favours EACA

Analysis 9.24. Comparison 9 Adverse Events and Other Outcomes (Active versus Active), Outcome 24 Renal Failure / Dysfunction - Aprotinin versus Tranexamic Acid.

Review: Anti-fibrinolytic use for minimising perioperative allogeneic blood transfusion

Comparison: 9 Adverse Events and Other Outcomes (Active versus Active)

Outcome: 24 Renal Failure / Dysfunction - Aprotinin versus Tranexamic Acid

\begin{tabular}{|c|c|c|c|c|}
\hline Study or subgroup & $\begin{array}{r}\text { Aprotinin } \\
\mathrm{n} / \mathrm{N}\end{array}$ & $\begin{array}{r}\text { Tranexamic Acid } \\
\mathrm{n} / \mathrm{N}\end{array}$ & $\begin{array}{c}\text { Risk Ratio } \\
\text { M-H,Random,95\% Cl }\end{array}$ & $\begin{array}{r}\text { Risk Ratio } \\
\text { M-H,Random,95\% Cl}\end{array}$ \\
\hline Dalmau 2004 & $2 / 63$ & $4 / 64$ & $\longrightarrow$ & $0.51[0.10,2.68]$ \\
\hline Dietrich 2008 & $0 / 110$ & $0 / 110$ & & $0.0[0.0,0.0]$ \\
\hline Diprose 2005 & $0 / 60$ & $0 / 60$ & & $0.0[0.0,0.0]$ \\
\hline Fergusson 2008 & $102 / 770$ & $97 / 766$ & & $1.05[0.81,1.36]$ \\
\hline Later 2009 & $3 / 96$ & $3 / 99$ & \begin{tabular}{l|l}
1 \\
\end{tabular} & $1.03[0.21,4.98]$ \\
\hline Mansour 2004 & $0 / 20$ & $1 / 20$ & & $0.33[0.01,7.72]$ \\
\hline Total (95\% CI) & 1119 & 1119 & 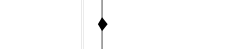 & $1.02[0.79,1.31]$ \\
\hline \multicolumn{5}{|c|}{ Total events: 107 (Aprotinin), 105 (Tranexamic Acid) } \\
\hline \multicolumn{5}{|c|}{ Heterogeneity: $\operatorname{Tau}^{2}=0.0 ; \mathrm{Chi}^{2}=1.20, \mathrm{df}=3(\mathrm{P}=0.75) ; \mathrm{I}^{2}=0.0 \%$} \\
\hline \multicolumn{5}{|c|}{ Test for overall effect: $Z=0.16(P=0.87)$} \\
\hline
\end{tabular}

$0.0010 .010 .1 \quad 1 \quad 10 \quad 1001000$

Favours Aprotinin Favours TXA 
Analysis 9.25. Comparison 9 Adverse Events and Other Outcomes (Active versus Active), Outcome 25 Renal Failure / Dysfunction - Aprotinin versus Epsilon Aminocaproic Acid.

Review: Anti-fibrinolytic use for minimising perioperative allogeneic blood transfusion

Comparison: 9 Adverse Events and Other Outcomes (Active versus Active)

Outcome: 25 Renal Failure / Dysfunction - Aprotinin versus Epsilon Aminocaproic Acid

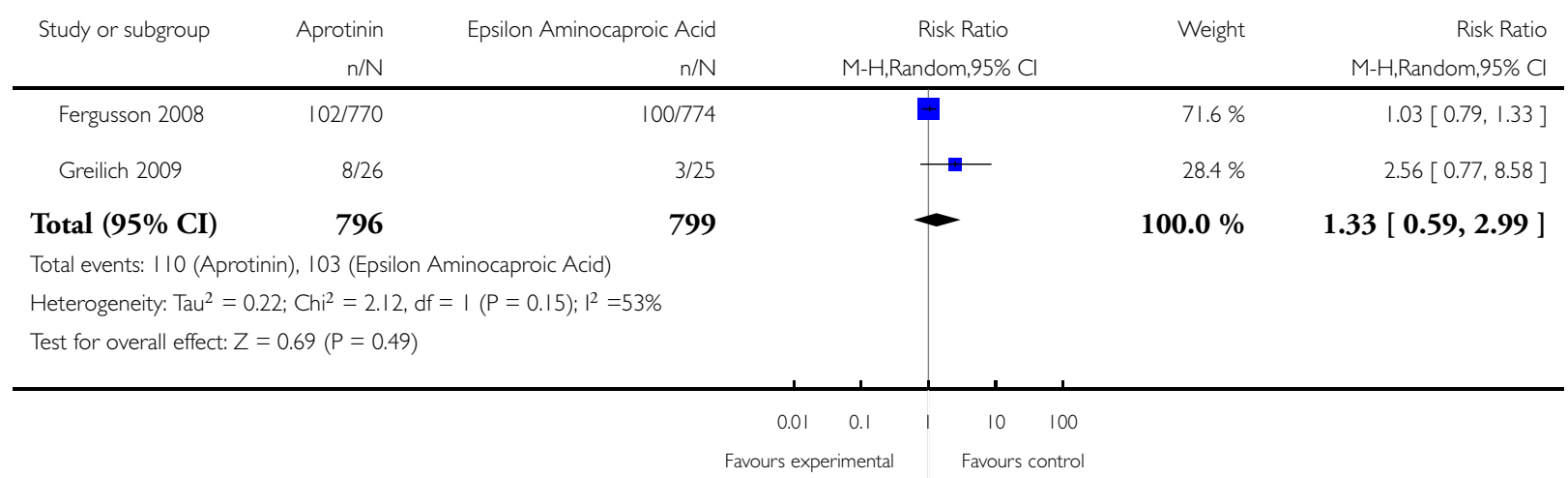

Analysis 9.26. Comparison 9 Adverse Events and Other Outcomes (Active versus Active), Outcome 26 Renal Failure / Dysfunction - Tranexamic Acid versus Epsilon Aminocaproic Acid.

Review: Anti-fibrinolytic use for minimising perioperative allogeneic blood transfusion

Comparison: 9 Adverse Events and Other Outcomes (Active versus Active)

Outcome: 26 Renal Failure / Dysfunction - Tranexamic Acid versus Epsilon Aminocaproic Acid

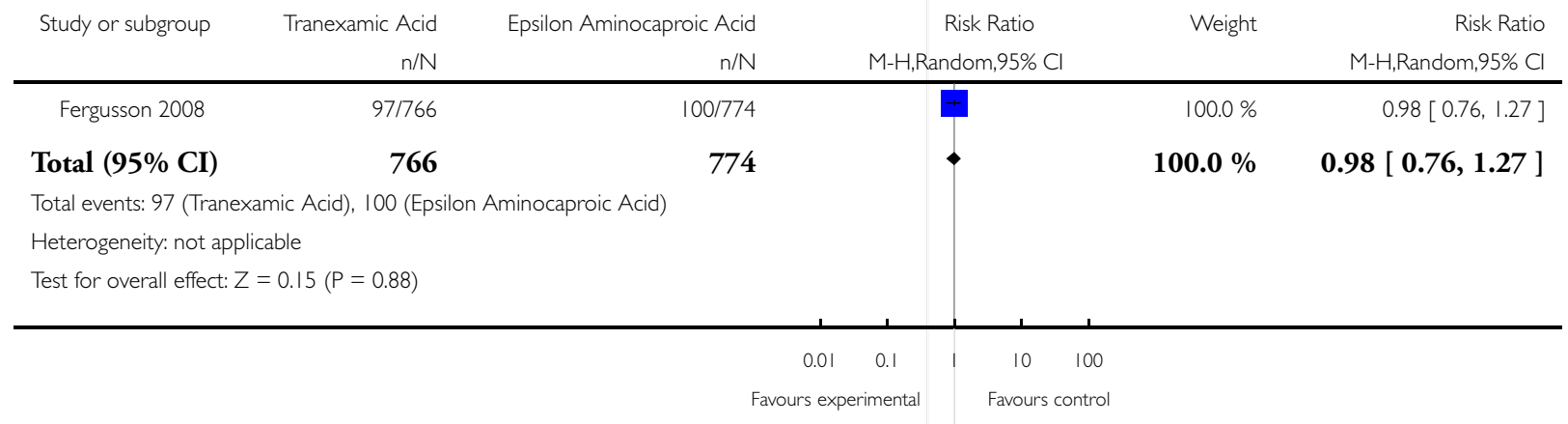




\section{Analysis 9.27. Comparison 9 Adverse Events and Other Outcomes (Active versus Active), Outcome 27 Hospital Length of Stay - Aprotinin versus Tranexamic Acid.}

Review: Anti-fibrinolytic use for minimising perioperative allogeneic blood transfusion

Comparison: 9 Adverse Events and Other Outcomes (Active versus Active)

Outcome: 27 Hospital Length of Stay - Aprotinin versus Tranexamic Acid

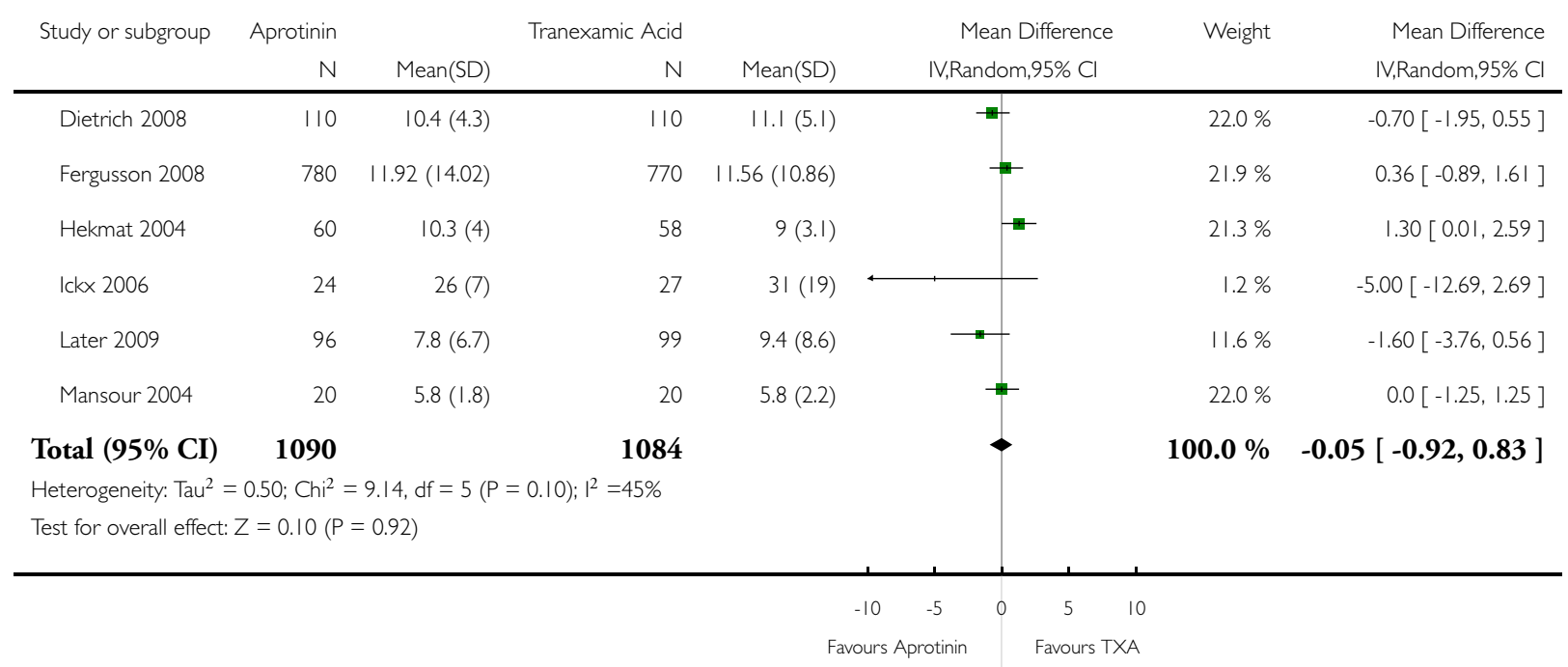


Analysis 9.28. Comparison 9 Adverse Events and Other Outcomes (Active versus Active), Outcome 28 Hospital Length of Stay - Aprotinin versus Epsilon Aminocaproic Acid.

Review: Anti-fibrinolytic use for minimising perioperative allogeneic blood transfusion

Comparison: 9 Adverse Events and Other Outcomes (Active versus Active)

Outcome: 28 Hospital Length of Stay - Aprotinin versus Epsilon Aminocaproic Acid

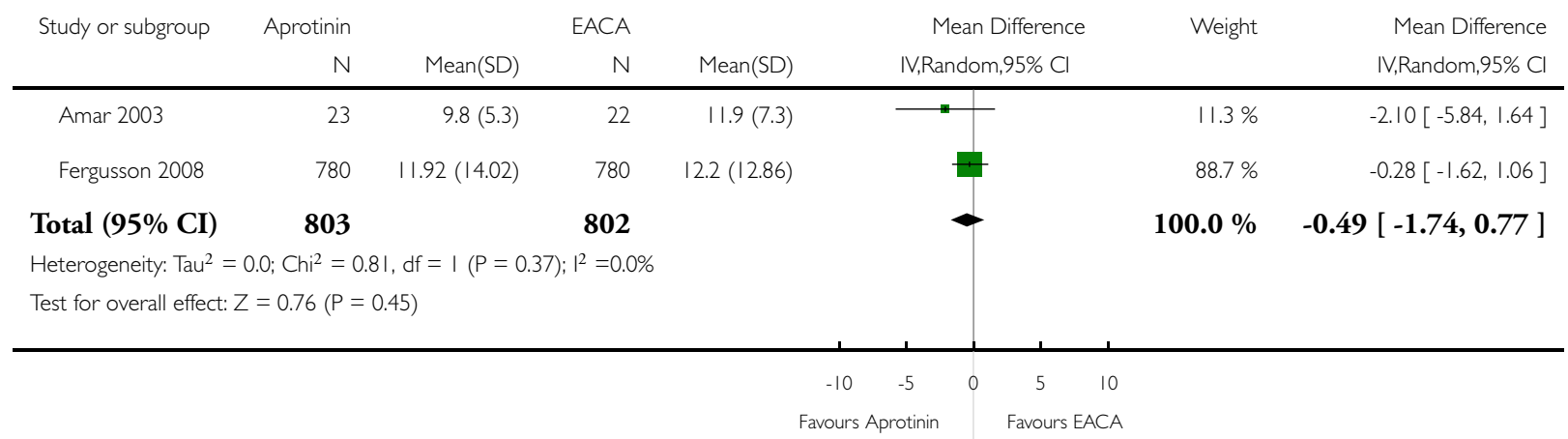

Analysis 9.29. Comparison 9 Adverse Events and Other Outcomes (Active versus Active), Outcome 29 Hospital Length of Stay - Tranexamic Acid versus Epsilon Aminocaproic Acid.

Review: Anti-fibrinolytic use for minimising perioperative allogeneic blood transfusion

Comparison: 9 Adverse Events and Other Outcomes (Active versus Active)

Outcome: 29 Hospital Length of Stay - Tranexamic Acid versus Epsilon Aminocaproic Acid

\begin{tabular}{|c|c|c|c|c|c|c|c|c|}
\hline \multirow[t]{2}{*}{ Study or subgroup } & \multirow{2}{*}{$\begin{array}{r}\text { TXA } \\
N\end{array}$} & \multicolumn{3}{|c|}{ EACA } & & \multirow{2}{*}{$\begin{array}{l}\text { Mean Difference } \\
\text { IV,Random,95\% Cl }\end{array}$} & \multirow[t]{2}{*}{ Weight } & \multirow{2}{*}{$\begin{array}{l}\text { Mean Difference } \\
\text { IV,Random,95\% Cl }\end{array}$} \\
\hline & & Mean(SD) & N & Mean(SD) & & & & \\
\hline Fergusson 2008 & 770 & I I.56 (I0.86) & 780 & $12.2(12.86)$ & & & $100.0 \%$ & $-0.64[-1.82,0.54]$ \\
\hline Total $(95 \% \mathrm{CI})$ & 770 & & 780 & & & & $100.0 \%$ & $-0.64[-1.82,0.54]$ \\
\hline \multicolumn{9}{|c|}{ Heterogeneity: not applicable } \\
\hline \multicolumn{9}{|c|}{ Test for overall effect: $Z=1.06(P=0.29)$} \\
\hline & & & & & -4 & -2 & 4 & \\
\hline & & & & & Fav & Favours EA & CA & \\
\hline
\end{tabular}


Analysis 10.1. Comparison 10 Adverse Events and Other Outcomes (Active versus Control) - Cardiac Surgery, Outcome I Re-operation for bleeding.

Review: Anti-fibrinolytic use for minimising perioperative allogeneic blood transfusion

Comparison: 10 Adverse Events and Other Outcomes (Active versus Control) - Cardiac Surgery

Outcome: I Re-operation for bleeding

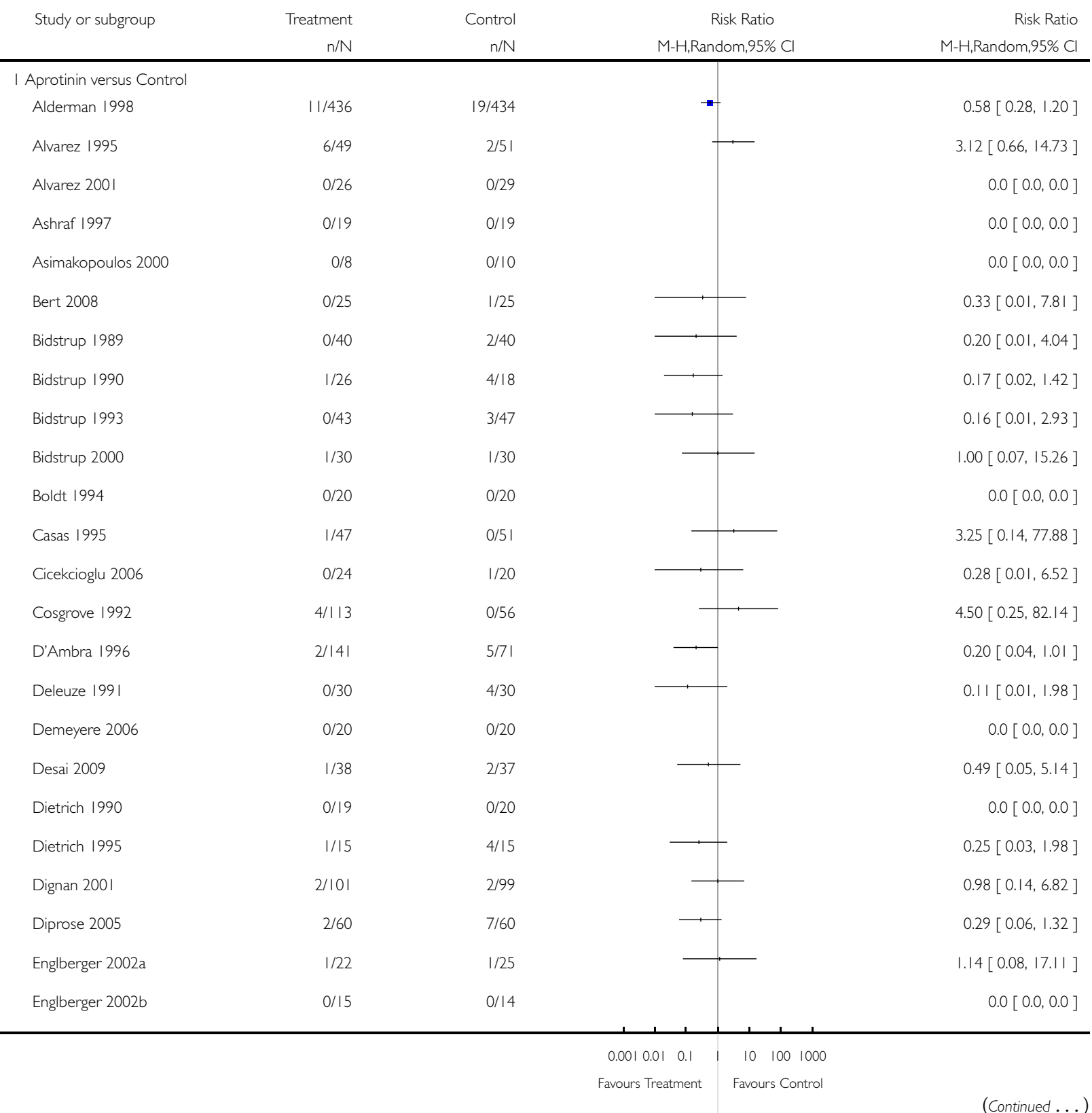




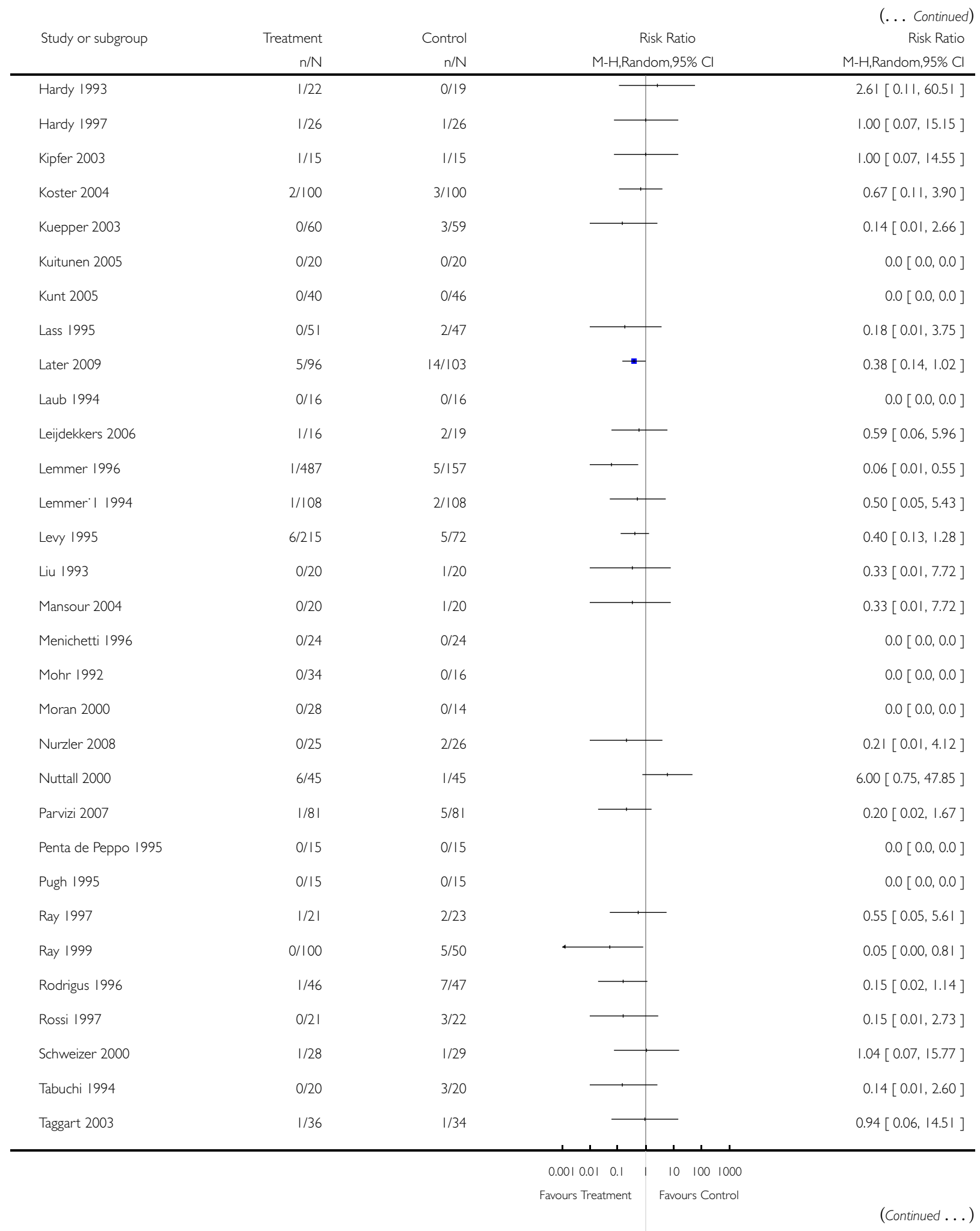

Anti-fibrinolytic use for minimising perioperative allogeneic blood transfusion (Review) 


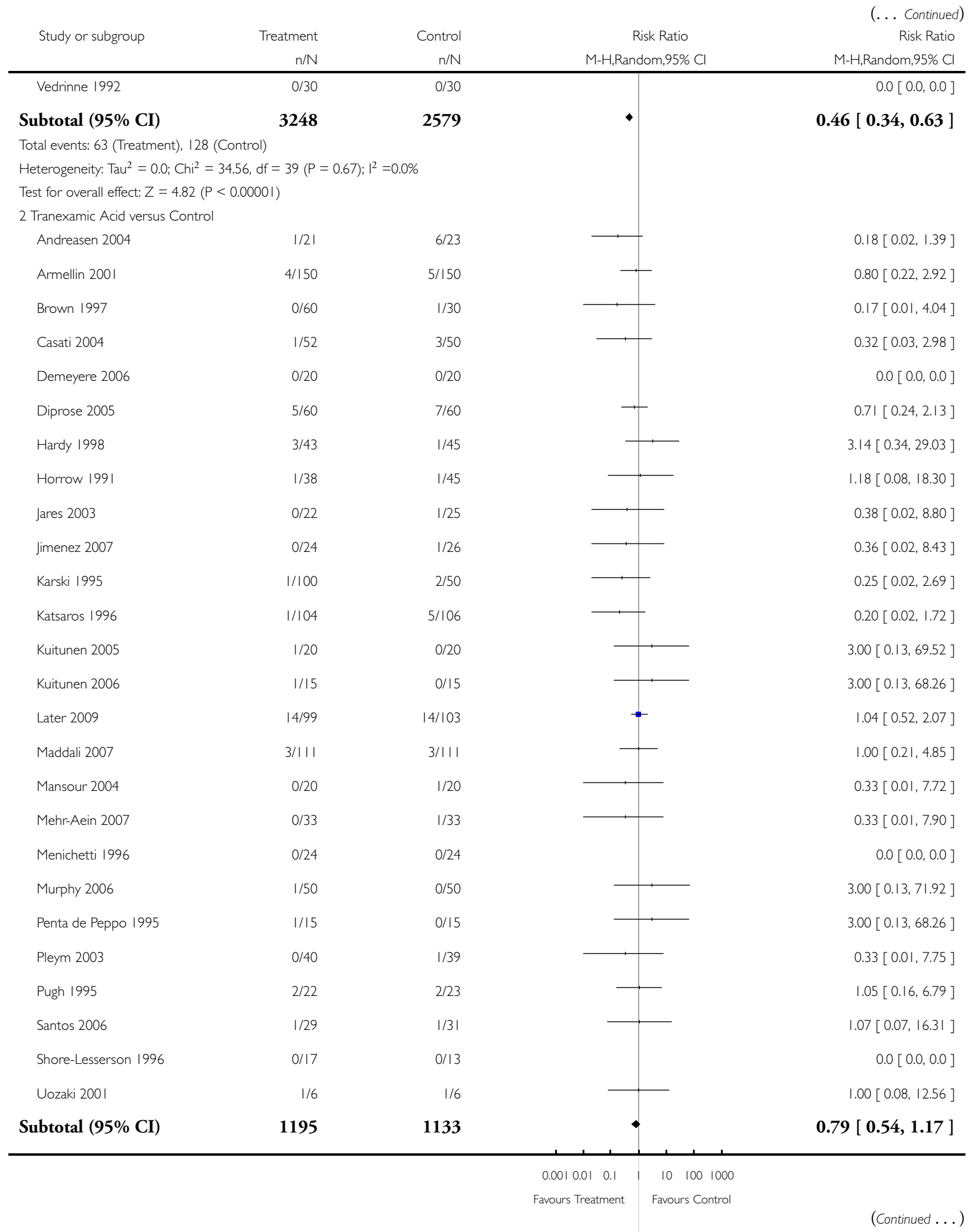

Anti-fibrinolytic use for minimising perioperative allogeneic blood transfusion (Review) 


\begin{tabular}{|c|c|c|c|c|}
\hline Study or subgroup & $\begin{array}{r}\text { Treatment } \\
n / N\end{array}$ & $\begin{array}{r}\text { Control } \\
n / N\end{array}$ & Risk Ratio & $\begin{array}{r}\text { (... Continued) } \\
\text { Risk Ratio } \\
\mathrm{M}-\mathrm{H}, \text { Random, } 95 \% \mathrm{Cl}\end{array}$ \\
\hline \multicolumn{5}{|c|}{ Total events: 42 (Treatment), 57 (Control) } \\
\hline \multicolumn{5}{|c|}{ Heterogeneity: Tau $^{2}=0.0 ; \mathrm{Chi}^{2}=12.60, \mathrm{df}=22(P=0.94) ;\left.\right|^{2}=0.0 \%$} \\
\hline \multicolumn{5}{|c|}{ Test for overall effect: $Z=1.18(P=0.24)$} \\
\hline \multicolumn{5}{|c|}{3 Epsilon Aminocaproic Acid versus Control } \\
\hline Del Rossi 1989 & $0 / 170$ & $6 / 180$ & & $0.08[0.00,1.43]$ \\
\hline Hardy 1998 & 1/46 & $1 / 45$ & & $0.98[0.06,15.17]$ \\
\hline Kluger 2003 & 1/58 & $1 / 30$ & & $0.52[0.03,7.98]$ \\
\hline Menichetti 1996 & 0/24 & $0 / 24$ & & $0.0[0.0,0.0]$ \\
\hline Penta de Peppo 1995 & $0 / 15$ & $0 / 15$ & & $0.0[0.0,0.0]$ \\
\hline Rao 1999 & $0 / 15$ & $1 / 15$ & - & $0.33[0.01,7.58]$ \\
\hline Vander-Salm 1996 & $|/ 5|$ & $3 / 52$ & + & $0.34[0.04,3.16]$ \\
\hline Subtotal $(95 \% \mathrm{CI})$ & 379 & 361 & - & $0.35[0.11,1.17]$ \\
\hline \multicolumn{5}{|c|}{ Total events: 3 (Treatment), 12 (Control) } \\
\hline \multicolumn{5}{|c|}{ Heterogeneity: $\operatorname{Tau}^{2}=0.0 ; \mathrm{Chi}^{2}=1.74, \mathrm{df}=4(\mathrm{P}=0.78) ; \mathrm{I}^{2}=0.0 \%$} \\
\hline \multicolumn{5}{|c|}{ Test for overall effect: $Z=1.71(P=0.087)$} \\
\hline Total $(95 \% \mathrm{CI})$ & 4822 & 4073 & $\bullet$ & $0.56[0.44,0.71]$ \\
\hline \multicolumn{5}{|c|}{ Total events: 108 (Treatment), 197 (Control) } \\
\hline \multicolumn{5}{|c|}{ Heterogeneity: $\mathrm{Tau}^{2}=0.0 ; \mathrm{Chi}^{2}=54.09, \mathrm{df}=67(\mathrm{P}=0.87) ;\left.\right|^{2}=0.0 \%$} \\
\hline \multicolumn{5}{|c|}{ Test for overall effect: $Z=4.73(P<0.00001)$} \\
\hline
\end{tabular}




\section{Analysis 10.2. Comparison 10 Adverse Events and Other Outcomes (Active versus Control) - Cardiac Surgery, Outcome 2 Mortality.}

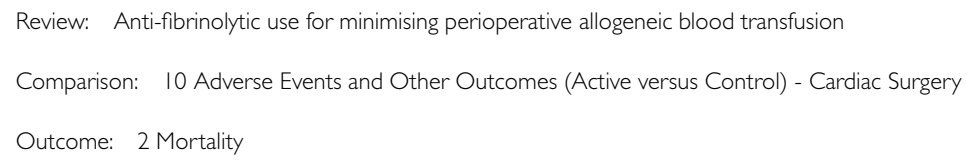

$0 / 51$
$0.85[0.29,2.52]$

$3.12[0.13,74.80]$

$0.0[0.0,0.0]$

$0.0[0.0,0.0]$

0.33 [0.01, 7.95 ]

$5.45[0.27,|10.5|]$

$3.00[0.13,70.83]$

$3.00[0.13,67.91]$

$2.17[0.20,23.16]$

$0.0[0.0,0.0]$

$5.26[0.26,107.27]$

0.34 [0.01, 8.27]

I. $12[0.36,3.46]$

$5.58[0.31,99.47]$

$0.97[0.06,15.00]$

$0.76[0.45,1.28]$

$0.20[0.01,3.85]$

$0.0[0.0,0.0]$

$0.33[0.01,8.02]$

$0.0[0.0,0.0]$

$0.0[0.0,0.0]$

$0.0[0.0,0.0]$

$2.42[0.10,56.85]$

$0.51[0.10,2.75]$ 


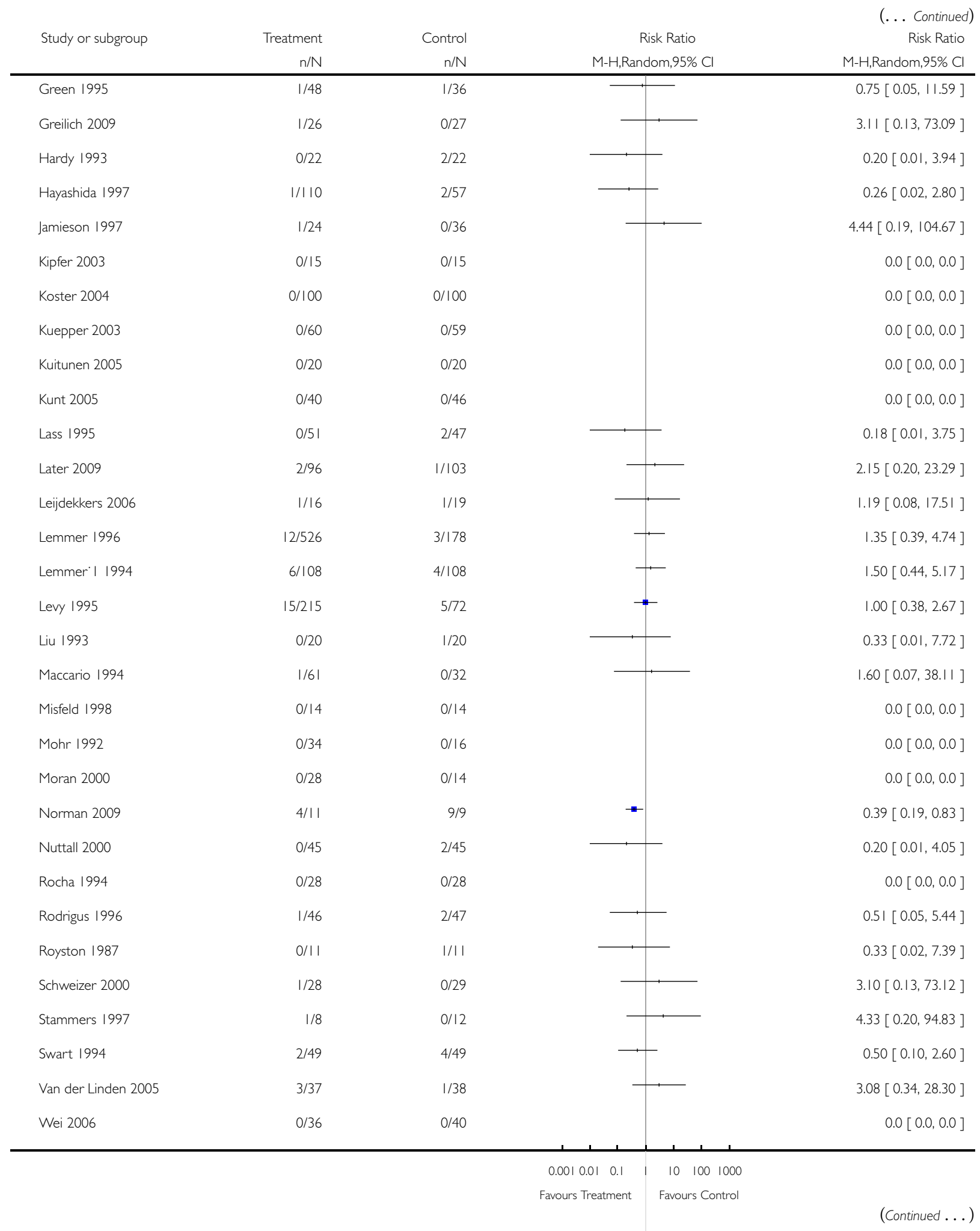

Anti-fibrinolytic use for minimising perioperative allogeneic blood transfusion (Review)

Copyright (? 20II The Cochrane Collaboration. Published by John Wiley \& Sons, Ltd. 


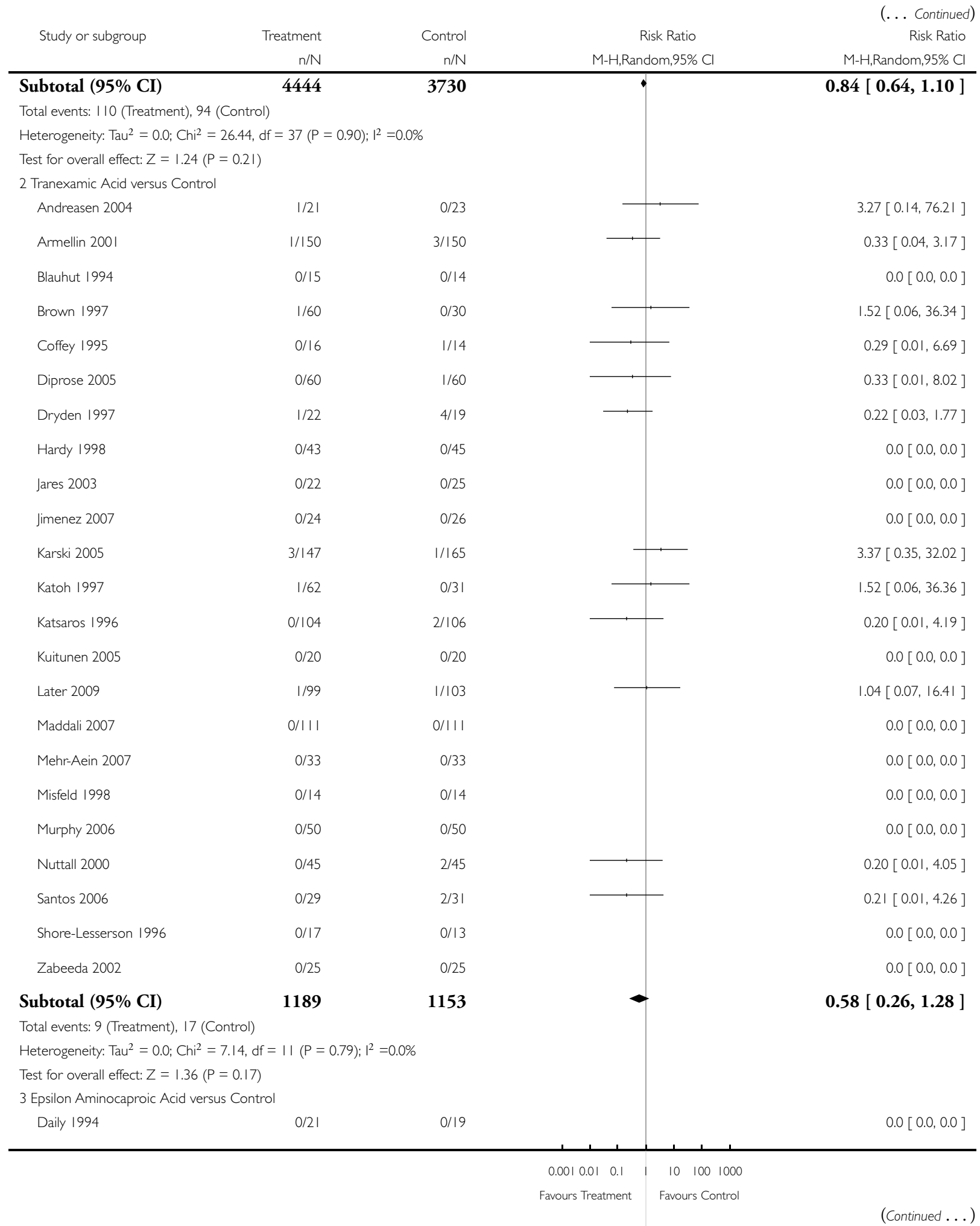

Anti-fibrinolytic use for minimising perioperative allogeneic blood transfusion (Review) 


\begin{tabular}{|c|c|c|c|c|}
\hline Study or subgroup & $\begin{array}{r}\text { Treatment } \\
n / N\end{array}$ & $\begin{array}{r}\text { Control } \\
n / N\end{array}$ & $\begin{array}{c}\text { Risk Ratio } \\
\text { M-H,Random,95\% Cl }\end{array}$ & $\begin{array}{r}\text { ( . . . Continued) } \\
\text { Risk Ratio } \\
\text { M-H,Random,95\% Cl}\end{array}$ \\
\hline Del Rossi 1989 & $3 / 170$ & $3 / 180$ & 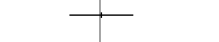 & $1.06[0.22,5.17]$ \\
\hline Greilich 2009 & 0/25 & $0 / 27$ & & $0.0[0.0,0.0]$ \\
\hline Hardy 1998 & $2 / 46$ & $0 / 45$ & 7 & $4.89[0.24,99.18]$ \\
\hline Kluger 2003 & 1/58 & $0 / 30$ & 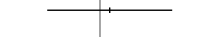 & $1.58[0.07,37.56]$ \\
\hline Vander-Salm 1996 & $|/ 5|$ & 0/52 & 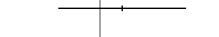 & $3.06[0.13,73.36]$ \\
\hline Subtotal $(95 \% \mathrm{CI})$ & 371 & 353 & - & $1.65[0.50,5.43]$ \\
\hline \multicolumn{5}{|c|}{ Total events: 7 (Treatment), 3 (Control) } \\
\hline \multicolumn{5}{|c|}{ Heterogeneity: $\operatorname{Tau}^{2}=0.0 ; \mathrm{Chi}^{2}=0.96, \mathrm{df}=3(\mathrm{P}=0.81) ; \mathrm{I}^{2}=0.0 \%$} \\
\hline \multicolumn{5}{|c|}{ Test for overall effect: $Z=0.83(P=0.4 \mathrm{I})$} \\
\hline Total $(95 \% \mathrm{CI})$ & 6004 & 5236 & - & $0.84[0.65,1.07]$ \\
\hline \multicolumn{5}{|c|}{ Total events: 126 (Treatment), I I 4 (Control) } \\
\hline \multicolumn{5}{|c|}{ Heterogeneity: Tau $^{2}=0.0 ; \mathrm{Chi}^{2}=36.5 \mathrm{I}, \mathrm{df}=53(\mathrm{P}=0.96) ; \mathrm{I}^{2}=0.0 \%$} \\
\hline \multicolumn{5}{|c|}{ Test for overall effect: $Z=1.41(P=0.16)$} \\
\hline
\end{tabular}




\section{Analysis 10.3. Comparison 10 Adverse Events and Other Outcomes (Active versus Control) - Cardiac Surgery, Outcome 3 Myocardial Infarction.}

Review: Anti-fibrinolytic use for minimising perioperative allogeneic blood transfusion

Comparison: 10 Adverse Events and Other Outcomes (Active versus Control) - Cardiac Surgery

Outcome: 3 Myocardial Infarction

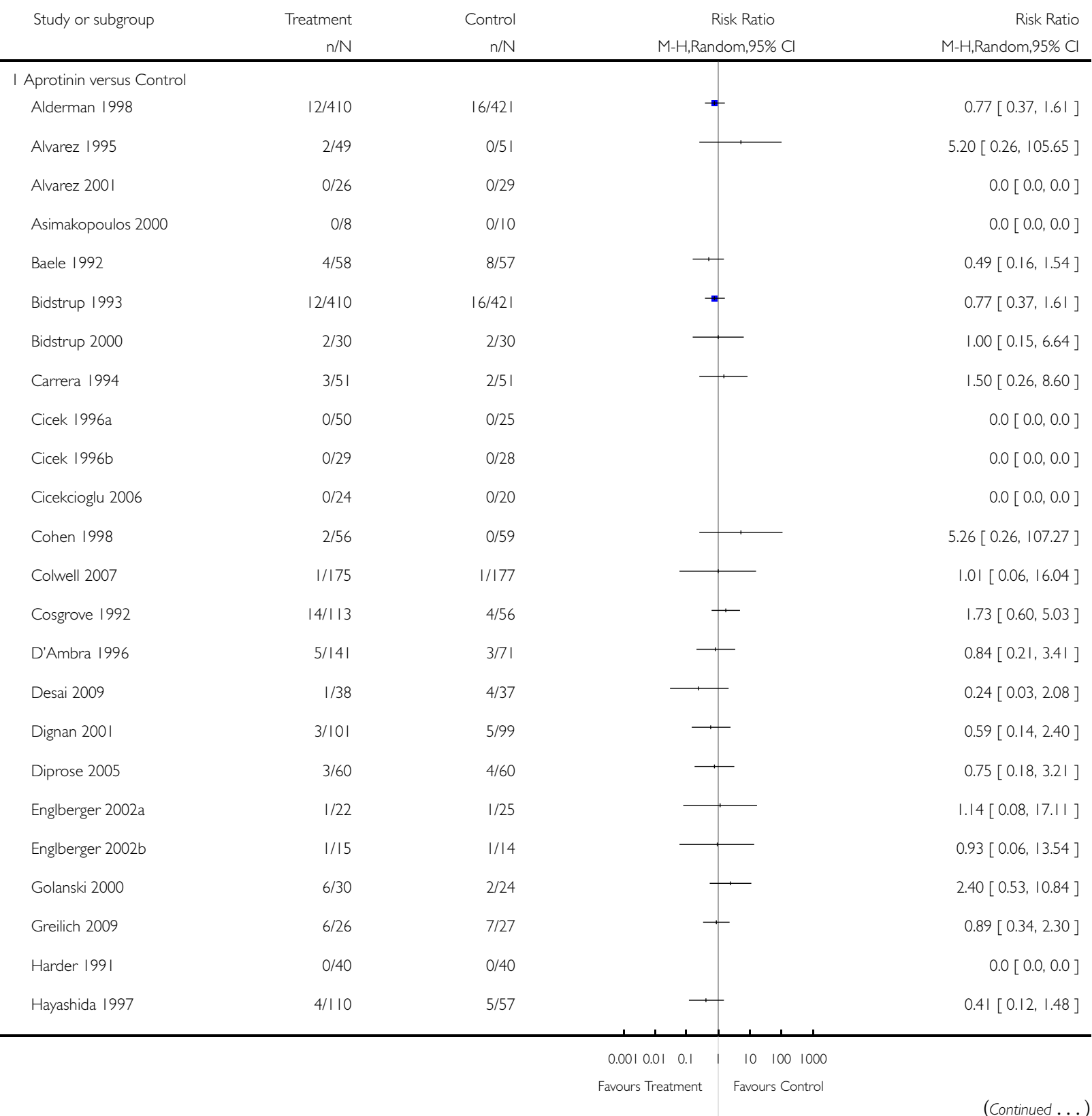




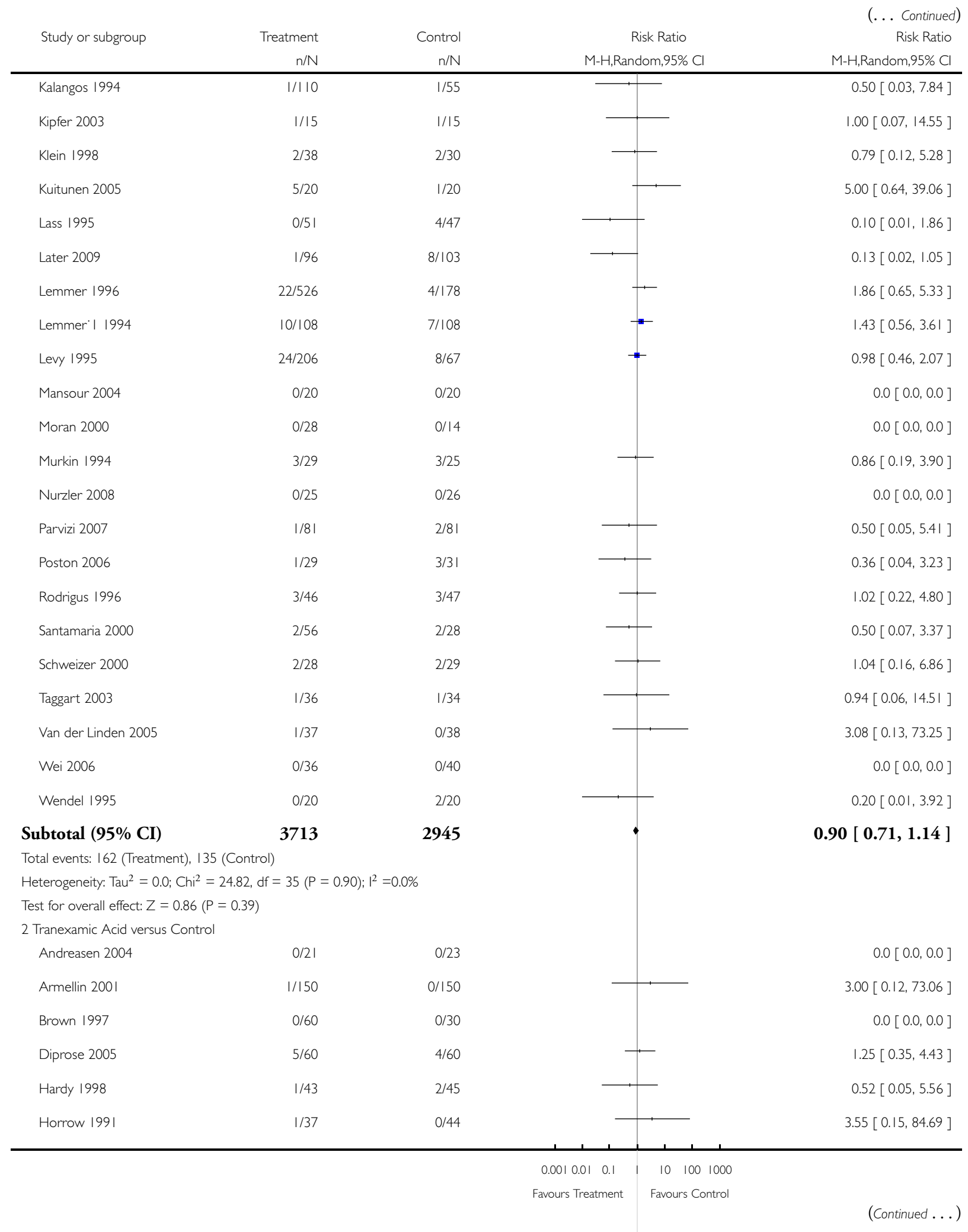

Anti-fibrinolytic use for minimising perioperative allogeneic blood transfusion (Review)

Copyright @ 201 I The Cochrane Collaboration. Published by John Wiley \& Sons, Ltd. 


\begin{tabular}{|c|c|c|c|c|}
\hline \multirow[t]{2}{*}{ Study or subgroup } & Treatment & Control & Risk Ratio & $\begin{array}{c}\text { (... Continued) } \\
\text { Risk Ratio }\end{array}$ \\
\hline & $\mathrm{n} / \mathrm{N}$ & $\mathrm{n} / \mathrm{N}$ & M-H,Random, $95 \% \mathrm{Cl}$ & M-H,Random, $95 \% \mathrm{Cl}$ \\
\hline Jares 2003 & $1 / 22$ & $1 / 25$ & \begin{tabular}{l|l} 
&
\end{tabular} & $1.14[0.08,17.11]$ \\
\hline Karski 1995 & $0 / 100$ & $0 / 50$ & & $0.0[0.0,0.0]$ \\
\hline Karski 2005 & $2 / 147$ & $3 / 165$ & - & $0.75[0.13,4.42]$ \\
\hline Katsaros 1996 & 0/104 & $0 / 106$ & & $0.0[0.0,0.0]$ \\
\hline Kuitunen 2005 & $1 / 20$ & $1 / 20$ & \begin{tabular}{l|l}
\end{tabular} & $1.00[0.07,14.90]$ \\
\hline Later 2009 & 0/99 & $8 / 103$ & & $0.06[0.00,1.05]$ \\
\hline Mansour 2004 & $0 / 20$ & $0 / 20$ & & $0.0[0.0,0.0]$ \\
\hline Mehr-Aein 2007 & 0/33 & $0 / 33$ & & $0.0[0.0,0.0]$ \\
\hline Murphy 2006 & $0 / 50$ & $1 / 50$ & - & $0.33[0.01,7.99]$ \\
\hline Shore-Lesserson 1996 & $1 / 17$ & $2 / 13$ & - & $0.38[0.04,3.77]$ \\
\hline Speekenbrink 1995 & $0 / 15$ & $2 / 15$ & - & $0.20[0.01,3.85]$ \\
\hline Taghaddomi 2009 & $0 / 50$ & $0 / 50$ & & $0.0[0.0,0.0]$ \\
\hline Zabeeda 2002 & $0 / 25$ & $0 / 25$ & & $0.0[0.0,0.0]$ \\
\hline Subtotal (95\% CI) & 1073 & 1027 & - & $0.74[0.37,1.47]$ \\
\hline \multicolumn{5}{|c|}{ Total events: I3 (Treatment), 24 (Control) } \\
\hline \multicolumn{5}{|c|}{ Heterogeneity: $\mathrm{Tau}^{2}=0.0 ; \mathrm{Chi}^{2}=7.21, \mathrm{df}=10(\mathrm{P}=0.71) ; 1^{2}=0.0 \%$} \\
\hline \multicolumn{5}{|c|}{ Test for overall effect: $Z=0.86(P=0.39)$} \\
\hline \multicolumn{5}{|c|}{3 Epsilon Aminocaproic Acid versus Control } \\
\hline Del Rossi 1989 & $4 / 170$ & $10 / 180$ & 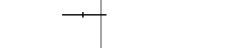 & $0.42[0.14,1.32]$ \\
\hline Greilich 2009 & $6 / 25$ & $7 / 27$ & - & $0.93[0.36,2.38]$ \\
\hline Hardy 1998 & 2/46 & $2 / 45$ & & $0.98[0.14,6.65]$ \\
\hline Kluger 2003 & 1/58 & $0 / 30$ & & $1.58[0.07,37.56]$ \\
\hline Rao 1999 & $0 / 15$ & $0 / 15$ & & $0.0[0.0,0.0]$ \\
\hline Vander-Salm 1996 & $5 / 51$ & 2/52 & 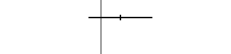 & $2.55[0.52,12.55]$ \\
\hline Subtotal $(95 \% \mathrm{CI})$ & 365 & 349 & - & $0.88[0.48,1.63]$ \\
\hline \multicolumn{5}{|c|}{ Total events: 18 (Treatment), 21 (Control) } \\
\hline \multicolumn{5}{|c|}{ Heterogeneity: Tau $^{2}=0.0 ; \mathrm{Chi}^{2}=3.44, \mathrm{df}=4(\mathrm{P}=0.49) ; \mathrm{I}^{2}=0.0 \%$} \\
\hline \multicolumn{5}{|c|}{ Test for overall effect: $Z=0.41 \quad(P=0.68)$} \\
\hline Total $(95 \% \mathrm{CI})$ & 5151 & 4321 & $\bullet$ & $0.88[0.71,1.09]$ \\
\hline \multicolumn{5}{|c|}{ Total events: 193 (Treatment), 180 (Control) } \\
\hline \multicolumn{5}{|c|}{ Heterogeneity: $\mathrm{Tau}^{2}=0.0 ; \mathrm{Ch}^{2}=35.49, \mathrm{df}=5 \mathrm{I}(\mathrm{P}=0.95) ; \mathrm{I}^{2}=0.0 \%$} \\
\hline Test for overall effect: $Z=$ & & & & \\
\hline
\end{tabular}




\section{Analysis 10.4. Comparison 10 Adverse Events and Other Outcomes (Active versus Control) - Cardiac Surgery, Outcome 4 Stroke.}

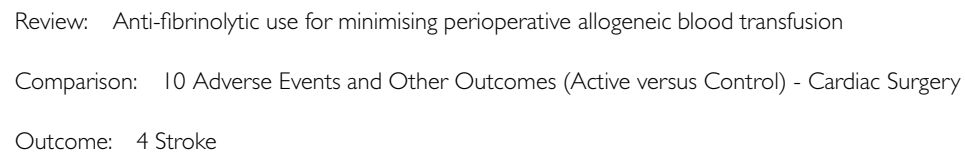

\section{Aprotinin versus Control}

Asimakopoulos 2000

$0 / 8$

Bidstrup 1993

Casas 1995

Cohen 1998

Colwell 2007

D'Ambra 1996

Desai 2009

Dignan 200।

Greilich 2009

Levy 1995

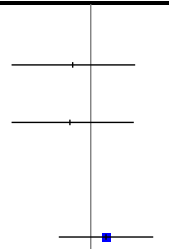

$0.41[0.02,8.84]$

$0.36[0.02,8.70]$

$0.0[0.0,0.0]$

$2.11[0.20,22.60]$

$0.34[0.01,8.22]$

I.01 $[0.09,10.92$ ]

$0.32[0.01,7.73]$

$0.98[0.06,15.45]$

$2.08[0.20,21.55]$

$3.00[0.13,69.52]$

$1.07[0.07,16.92]$

$0.07[0.01,0.56]$

$0.0[0.0,0.0]$

$3.12[0.13,73.06]$

$0.36[0.02,8.39]$

$2.54[0.13,51.26]$

$3.08[0.13,73.25]$

$0.0[0.0,0.0]$

Total events: 14 (Treatment), 15 (Control)

Heterogeneity: $\operatorname{Tau}^{2}=0.0 ; \mathrm{Chi}^{2}=10.55, \mathrm{df}=14(\mathrm{P}=0.72) ; \mathrm{I}^{2}=0.0 \%$

Test for overall effect: $Z=0.56(P=0.57)$

2 Tranexamic Acid versus Control 


\begin{tabular}{|c|c|c|c|c|}
\hline Hardy 1998 & $1 / 43$ & $0 / 45$ & & $\frac{3.14[0.13,74.95]}{3.13}$ \\
\hline Horrow 1990 & $0 / 18$ & $2 / 20$ & - & $0.22[0.01,4.32]$ \\
\hline Horrow 1991 & 1/37 & $0 / 44$ & & $3.55[0.15,84.69]$ \\
\hline Jares 2003 & $0 / 22$ & $0 / 25$ & & $0.0[0.0,0.0]$ \\
\hline Karski 1995 & $3 / 100$ & $0 / 50$ & - & $3.53[0.19,67.13]$ \\
\hline Karski 2005 & $1 / 147$ & $1 / 165$ & - & $1.12[0.07,17.79]$ \\
\hline Katoh 1997 & $0 / 62$ & $0 / 31$ & & $0.0[0.0,0.0]$ \\
\hline Katsaros 1996 & $3 / 104$ & $2 / 106$ & 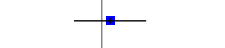 & $1.53[0.26,8.96]$ \\
\hline Kuitunen 2005 & $0 / 20$ & $0 / 20$ & & $0.0[0.0,0.0]$ \\
\hline Later 2009 & 1/99 & $1 / 103$ & & $1.04[0.07,16.41]$ \\
\hline Maddali 2007 & $0 / 111$ & $0 / 111$ & & $0.0[0.0,0.0]$ \\
\hline Murphy 2006 & $0 / 50$ & $0 / 50$ & & $0.0[0.0,0.0]$ \\
\hline Taghaddomi 2009 & $0 / 50$ & $0 / 50$ & & $0.0[0.0,0.0]$ \\
\hline Zabeeda 2002 & $0 / 25$ & $0 / 25$ & & $0.0[0.0,0.0]$ \\
\hline Subtotal $(95 \%$ CI) & 1021 & 948 & - & $1.44[0.53,3.91]$ \\
\hline \multicolumn{5}{|c|}{ Total events: I0 (Treatment), 6 (Control) } \\
\hline \multicolumn{5}{|c|}{ Heterogeneity: $\operatorname{Tau}^{2}=0.0 ; \mathrm{Chi}^{2}=2.52, \mathrm{df}=6(P=0.87) ; 1^{2}=0.0 \%$} \\
\hline \multicolumn{5}{|c|}{ Test for overall effect: $Z=0.72(P=0.47)$} \\
\hline \multicolumn{5}{|c|}{3 Epsilon Aminocaproic Acid versus Control } \\
\hline Daily 1994 & $0 / 21$ & $0 / 19$ & & $0.0[0.0,0.0]$ \\
\hline Del Rossi 1989 & 0/170 & $1 / 180$ & - & $0.35[0.01,8.60]$ \\
\hline Greilich 2009 & $1 / 25$ & $1 / 27$ & & $1.08[0.07,16.36]$ \\
\hline Hardy 1998 & $0 / 46$ & $0 / 45$ & & $0.0[0.0,0.0]$ \\
\hline Kluger 2003 & 2/58 & 0/30 & & $2.63[0.13,53.04]$ \\
\hline Rao 1999 & $0 / 15$ & $0 / 15$ & & $0.0[0.0,0.0]$ \\
\hline Vander-Salm 1996 & $0 / 51$ & $2 / 52$ & - & $0.20[0.01,4.14]$ \\
\hline Subtotal $(95 \% \mathrm{CI})$ & 386 & 368 & & $0.70[0.16,3.10]$ \\
\hline \multicolumn{5}{|c|}{ Total events: 3 (Treatment), 4 (Control) } \\
\hline \multicolumn{5}{|c|}{ Heterogeneity: $\operatorname{Tau}^{2}=0.0 ; \mathrm{Chi}^{2}=1.66, \mathrm{df}=3(\mathrm{P}=0.64) ; \mathrm{I}^{2}=0.0 \%$} \\
\hline \multicolumn{5}{|c|}{ Test for overall effect: $Z=0.47(P=0.64)$} \\
\hline Total $(95 \% \mathrm{CI})$ & 2584 & 2266 & 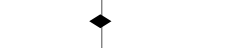 & $0.95[0.55,1.63]$ \\
\hline \multicolumn{5}{|c|}{ Total events: 27 (Treatment), 25 (Control) } \\
\hline \multicolumn{5}{|c|}{ Heterogeneity: Tau $^{2}=0.0 ; \mathrm{Chi}^{2}=15.75, \mathrm{df}=25(\mathrm{P}=0.92) ; 1^{2}=0.0 \%$} \\
\hline Test for overall effect: $Z=$ & & & & \\
\hline
\end{tabular}




\section{Analysis 10.5. Comparison 10 Adverse Events and Other Outcomes (Active versus Control) - Cardiac Surgery, Outcome 5 Deep Vein Thrombosis (DVT).}

Review: Anti-fibrinolytic use for minimising perioperative allogeneic blood transfusion

Comparison: 10 Adverse Events and Other Outcomes (Active versus Control) - Cardiac Surgery

Outcome: 5 Deep Vein Thrombosis (DVT)

\begin{tabular}{|c|c|c|c|c|}
\hline \multirow[t]{2}{*}{ Study or subgroup } & Treatment & Control & Risk Ratio & Risk Ratio \\
\hline & $\mathrm{n} / \mathrm{N}$ & $\mathrm{n} / \mathrm{N}$ & $\mathrm{M}-\mathrm{H}$, Random,95\% Cl & $\mathrm{M}-\mathrm{H}$, Random,95\% Cl \\
\hline \multicolumn{5}{|l|}{ I Aprotinin versus Control } \\
\hline Colwell 2007 & $2 / 175$ & $3 / 177$ & - & $0.67[0.11,3.99]$ \\
\hline D'Ambra 1996 & $|/| 4 \mid$ & $0 / 71$ & 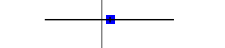 & $1.52[0.06,36.87]$ \\
\hline Poston 2006 & $3 / 29$ & $|/ 3|$ & - & $3.21[0.35,29.11]$ \\
\hline Subtotal (95\% CI) & 345 & 279 & & $1.29[0.36,4.58]$ \\
\hline \multicolumn{5}{|c|}{ Total events: 6 (Treatment), 4 (Control) } \\
\hline \multicolumn{5}{|c|}{ Heterogeneity: $\operatorname{Tau}^{2}=0.0 ; \mathrm{Chi}^{2}=1.18, \mathrm{df}=2(\mathrm{P}=0.55) ;\left.\right|^{2}=0.0 \%$} \\
\hline \multicolumn{5}{|c|}{ Test for overall effect: $Z=0.39(P=0.70)$} \\
\hline \multicolumn{5}{|c|}{2 Tranexamic Acid versus Control } \\
\hline Horrow 1990 & $0 / 18$ & $0 / 20$ & & $0.0[0.0,0.0]$ \\
\hline Horrow 1991 & $0 / 37$ & $1 / 44$ & - & $0.39[0.02,9.41]$ \\
\hline Katoh 1997 & $0 / 62$ & $0 / 31$ & & $0.0[0.0,0.0]$ \\
\hline Katsaros 1996 & $0 / 104$ & $1 / 106$ & \pm & $0.34[0.01,8.24]$ \\
\hline Subtotal (95\% CI) & 221 & 201 & & $0.37[0.04,3.47]$ \\
\hline \multicolumn{5}{|c|}{ Total events: 0 (Treatment), 2 (Control) } \\
\hline \multicolumn{5}{|c|}{ Heterogeneity: Tau $^{2}=0.0 ; \mathrm{Chi}^{2}=0.00, \mathrm{df}=\mathrm{I}(\mathrm{P}=0.95) ; \mathrm{I}^{2}=0.0 \%$} \\
\hline \multicolumn{5}{|c|}{ Test for overall effect: $Z=0.88(P=0.38)$} \\
\hline Total (95\% CI) & 566 & 480 & & $0.95[0.31,2.87]$ \\
\hline \multicolumn{5}{|c|}{ Total events: 6 (Treatment), 6 (Control) } \\
\hline \multicolumn{5}{|c|}{ Heterogeneity: $\operatorname{Tau}^{2}=0.0 ; \mathrm{Chi}^{2}=2.09, \mathrm{df}=4(\mathrm{P}=0.72) ; \mathrm{I}^{2}=0.0 \%$} \\
\hline Test for overall effect: $Z=$ & & & & \\
\hline
\end{tabular}

$0.0010 .01 \quad 0.1 \quad 1 \quad 10 \quad 100 \quad 1000$

Favours Treatment Favours Control 
Analysis 10.6. Comparison 10 Adverse Events and Other Outcomes (Active versus Control) - Cardiac Surgery, Outcome 6 Pulmonary Embolism.

Review: Anti-fibrinolytic use for minimising perioperative allogeneic blood transfusion

Comparison: 10 Adverse Events and Other Outcomes (Active versus Control) - Cardiac Surgery

Outcome: 6 Pulmonary Embolism

\begin{tabular}{|c|c|c|c|c|}
\hline \multirow[t]{2}{*}{ Study or subgroup } & Treatment & Control & Risk Ratio & Risk Ratio \\
\hline & $\mathrm{n} / \mathrm{N}$ & $n / N$ & M-H,Random,95\% Cl & $\mathrm{M}-\mathrm{H}$, Random,95\% Cl \\
\hline \multicolumn{5}{|c|}{ I Tranexamic Acid versus Control } \\
\hline Casati 2004 & $0 / 52$ & $0 / 50$ & & $0.0[0.0,0.0]$ \\
\hline Horrow 1990 & $0 / 18$ & $0 / 20$ & & $0.0[0.0,0.0]$ \\
\hline Jares 2003 & $0 / 22$ & $0 / 25$ & & $0.0[0.0,0.0]$ \\
\hline Katoh 1997 & $0 / 62$ & $0 / 31$ & & $0.0[0.0,0.0]$ \\
\hline Katsaros 1996 & $0 / 104$ & $1 / 106$ & $\div$ & $0.34[0.01,8.24]$ \\
\hline Pleym 2003 & $0 / 40$ & $1 / 39$ & ? & $0.33[0.01,7.75]$ \\
\hline Subtotal (95\% CI) & 298 & 271 & & $0.33[0.04,3.15]$ \\
\hline \multicolumn{5}{|c|}{ Total events: 0 (Treatment), 2 (Control) } \\
\hline \multicolumn{5}{|c|}{ Heterogeneity: $\operatorname{Tau}^{2}=0.0 ; \mathrm{Chi}^{2}=0.00, \mathrm{df}=\mathrm{I}(\mathrm{P}=0.98) ; \mathrm{I}^{2}=0.0 \%$} \\
\hline \multicolumn{5}{|c|}{ Test for overall effect: $Z=0.96(P=0.34)$} \\
\hline \multicolumn{5}{|l|}{2 Aprotinin versus Control } \\
\hline Colwell 2007 & $2 / 175$ & $2 / 177$ & & $1.01[0.14,7.10]$ \\
\hline Subtotal (95\% CI) & 175 & 177 & & $1.01[0.14,7.10]$ \\
\hline \multicolumn{5}{|c|}{ Total events: 2 (Treatment), 2 (Control) } \\
\hline \multicolumn{5}{|c|}{ Heterogeneity: not applicable } \\
\hline \multicolumn{5}{|c|}{ Test for overall effect: $Z=0.01(P=0.99)$} \\
\hline Total (95\% CI) & 473 & 448 & & $0.63[0.14,2.74]$ \\
\hline \multicolumn{5}{|c|}{ Total events: 2 (Treatment), 4 (Control) } \\
\hline \multicolumn{5}{|c|}{ Heterogeneity: $\operatorname{Tau}^{2}=0.0 ; \mathrm{Chi}^{2}=0.54, \mathrm{df}=2(P=0.76) ; 1^{2}=0.0 \%$} \\
\hline Test for overall effect: $Z=$ & & & & \\
\hline
\end{tabular}

$\begin{array}{lllllll}0.001 & 0.01 & 0.1 & 1 & 10 & 100 & 1000\end{array}$

Favours Treatment Favours Control 
Analysis 10.7. Comparison 10 Adverse Events and Other Outcomes (Active versus Control) - Cardiac Surgery, Outcome 7 Other Thrombosis.

Review: Anti-fibrinolytic use for minimising perioperative allogeneic blood transfusion

Comparison: 10 Adverse Events and Other Outcomes (Active versus Control) - Cardiac Surgery

Outcome: 7 Other Thrombosis

Study or subgroup

Treatment

$n / N$

$\mathrm{n} / \mathrm{N}$

| Aprotinin versus Control

Casas 1995

D'Ambra 1996

Poston 2006

Rocha 1994

Subtotal (95\% CI)

Total events: 2 (Treatment), 4 (Control)

Heterogeneity: $\operatorname{Tau}^{2}=0.0 ; \mathrm{Chi}^{2}=1.40, \mathrm{df}=2(\mathrm{P}=0.50) ; \mathrm{I}^{2}=0.0 \%$

Test for overall effect: $Z=0.56(P=0.58)$

2 Tranexamic Acid versus Control

Taghaddomi 2009

50

$0 / 50$

$|/ 5|$

$0 / 71$

$3 / 31$

$0 / 28$

181

Risk Ratio

Risk Ratio

M-H,Random,95\% Cl

$\mathrm{M}-\mathrm{H}$, Random, $95 \% \mathrm{Cl}$

Subtotal (95\% CI)

Total events: 0 (Treatment), 0 (Control)

Heterogeneity: not applicable

Test for overall effect: $Z=0.0(P<0.0000 I)$

$1.09[0.07,16.86]$
$1.52[0.06,36.87]$
$0.15[0.01,2.83]$
$0.0[0.0,0.0]$

$0.62[0.11,3.36]$

$0.0[0.0,0.0]$

$0.0[0.0,0.0]$ 
Analysis 10.8. Comparison 10 Adverse Events and Other Outcomes (Active versus Control) - Cardiac Surgery, Outcome 8 Renal Failure / Dysfunction.

Review: Anti-fibrinolytic use for minimising perioperative allogeneic blood transfusion

Comparison: 10 Adverse Events and Other Outcomes (Active versus Control) - Cardiac Surgery

Outcome: 8 Renal Failure / Dysfunction

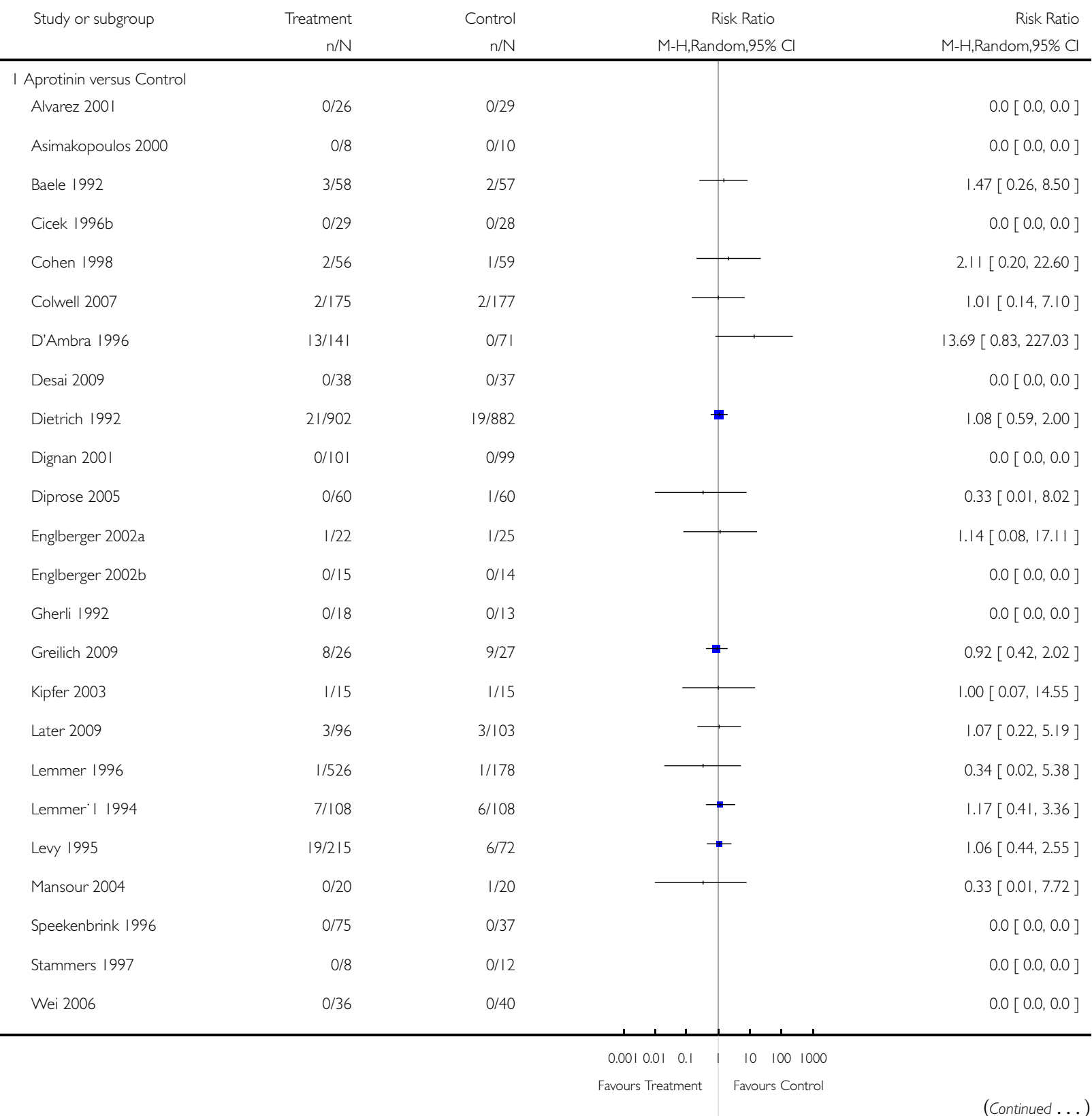




\begin{tabular}{|c|c|c|c|c|}
\hline Study or subgroup & $\begin{array}{r}\text { Treatment } \\
\mathrm{n} / \mathrm{N}\end{array}$ & $\begin{array}{r}\text { Control } \\
n / N\end{array}$ & $\begin{array}{c}\text { Risk Ratio } \\
\text { M-H,Random,95\% Cl }\end{array}$ & $\begin{array}{r}\text { (... Continued }) \\
\text { Risk Ratio } \\
\text { M-H,Random, } 95 \% \mathrm{Cl}\end{array}$ \\
\hline Subtotal $(95 \% \mathrm{CI})$ & 2774 & 2173 & 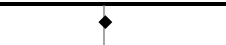 & $1.07[0.76,1.51]$ \\
\hline \multicolumn{5}{|c|}{ Total events: 81 (Treatment), 53 (Control) } \\
\hline \multicolumn{5}{|c|}{ Heterogeneity: $\mathrm{Tau}^{2}=0.0 ; \mathrm{Ch}^{2}=5.78, \mathrm{df}=13(\mathrm{P}=0.95) ; \mathrm{I}^{2}=0.0 \%$} \\
\hline \multicolumn{5}{|c|}{ Test for overall effect: $Z=0.39(P=0.70)$} \\
\hline \multicolumn{5}{|c|}{2 Tranexamic Acid versus Control } \\
\hline Andreasen 2004 & $0 / 21$ & $0 / 23$ & & $0.0[0.0,0.0]$ \\
\hline Diprose 2005 & $0 / 60$ & $1 / 60$ & & $0.33[0.01,8.02]$ \\
\hline Katsaros 1996 & $1 / 104$ & $0 / 106$ & & $3.06[0.13,74.20]$ \\
\hline Later 2009 & 3/99 & $3 / 103$ & - & $1.04[0.22,5.03]$ \\
\hline Mansour 2004 & $1 / 20$ & $1 / 20$ & & $1.00[0.07,14.90]$ \\
\hline Mehr-Aein 2007 & 0/33 & $1 / 33$ & - & $0.33[0.01,7.90]$ \\
\hline Murphy 2006 & $1 / 50$ & $0 / 50$ & & $3.00[0.13,71.92]$ \\
\hline Shore-Lesserson 1996 & $0 / 17$ & $1 / 13$ & - & $0.26[0.01,5.89]$ \\
\hline Taghaddomi 2009 & $0 / 50$ & $0 / 50$ & & $0.0[0.0,0.0]$ \\
\hline Subtotal $(95 \% \mathrm{CI})$ & 454 & 458 & - & $0.89[0.33,2.37]$ \\
\hline \multicolumn{5}{|c|}{ Total events: 6 (Treatment), 7 (Control) } \\
\hline \multicolumn{5}{|c|}{ Heterogeneity: $\mathrm{Tau}^{2}=0.0 ; \mathrm{Chi}^{2}=2.52, \mathrm{df}=6(\mathrm{P}=0.87) ; \mathrm{I}^{2}=0.0 \%$} \\
\hline \multicolumn{5}{|c|}{ Test for overall effect: $Z=0.23(P=0.81)$} \\
\hline \multicolumn{5}{|c|}{3 Epsilon Aminocaproic Acid versus control } \\
\hline Greilich 2009 & $3 / 26$ & 9/27 & $\rightarrow$ & $0.35[0.11,1.14]$ \\
\hline Subtotal $(95 \% \mathrm{CI})$ & 26 & 27 & 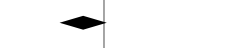 & $0.35[0.11,1.14]$ \\
\hline \multicolumn{5}{|c|}{ Total events: 3 (Treatment), 9 (Control) } \\
\hline \multicolumn{5}{|c|}{ Heterogeneity: not applicable } \\
\hline \multicolumn{5}{|c|}{ Test for overall effect: $Z=1.75(P=0.081)$} \\
\hline Total $(95 \% \mathrm{CI})$ & 3254 & 2658 & $\cdot$ & $0.97[0.71,1.33]$ \\
\hline \multicolumn{5}{|c|}{ Total events: 90 (Treatment), 69 (Control) } \\
\hline \multicolumn{5}{|c|}{ Heterogeneity: $\mathrm{Tau}^{2}=0.0 ; \mathrm{Chi}^{2}=11.43, \mathrm{df}=21 \quad(P=0.95) ; \mathrm{I}^{2}=0.0 \%$} \\
\hline \multicolumn{5}{|c|}{ Test for overall effect: $Z=0.19(P=0.85)$} \\
\hline
\end{tabular}




\section{Analysis 10.9. Comparison 10 Adverse Events and Other Outcomes (Active versus Control) - Cardiac Surgery, Outcome 9 Hospital Length of Stay.}

Review: Anti-fibrinolytic use for minimising perioperative allogeneic blood transfusion

Comparison: 10 Adverse Events and Other Outcomes (Active versus Control) - Cardiac Surgery

Outcome: 9 Hospital Length of Stay

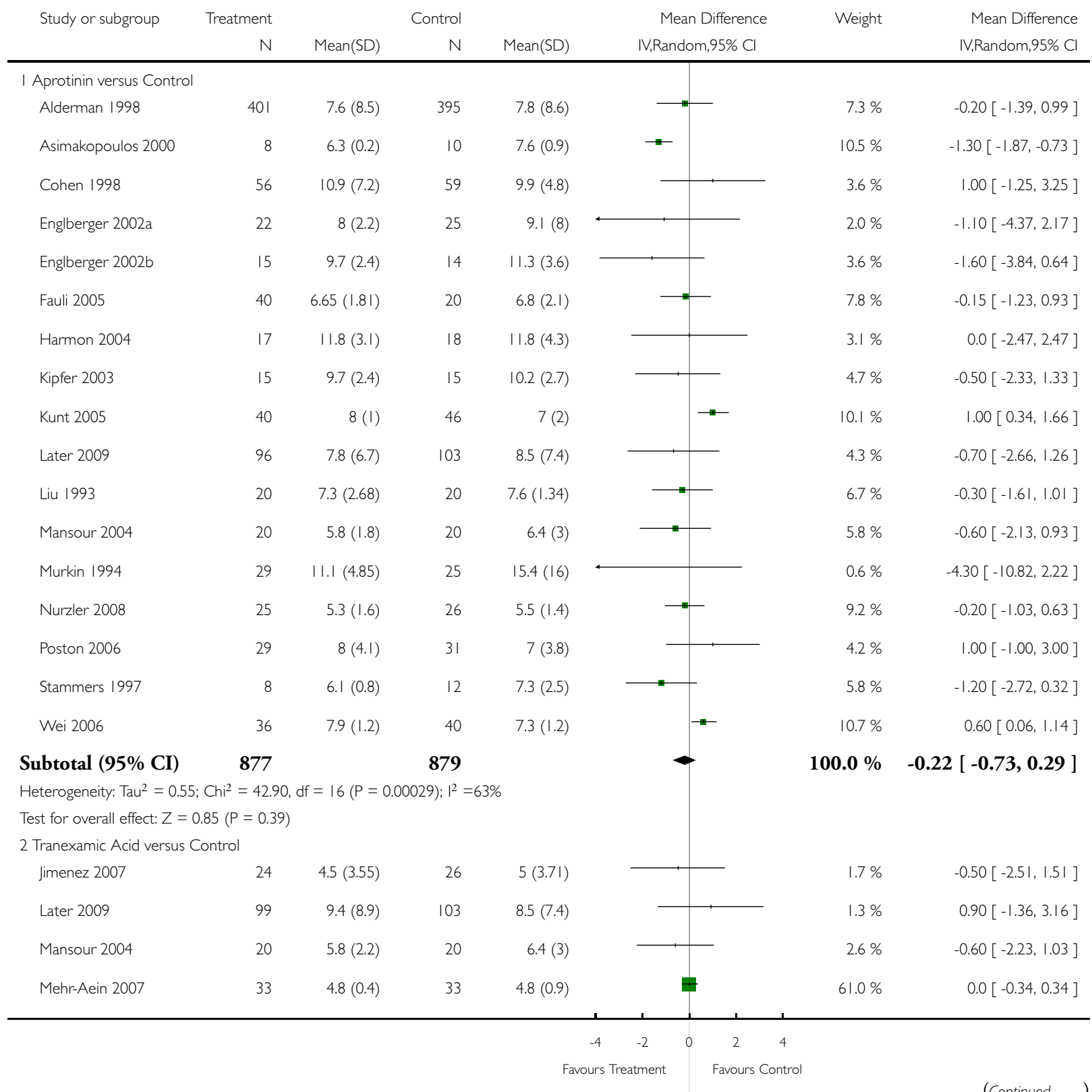

(Continued ....) 


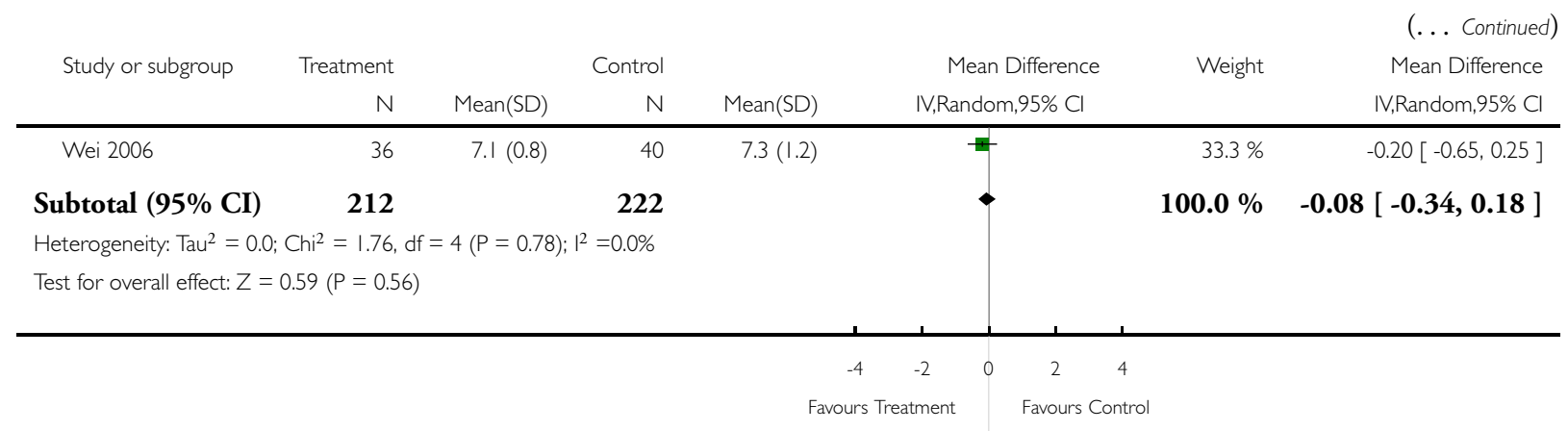

\section{A P PEN DICES}

\section{Appendix I. Search strategy}

The original search strategy at the outset of the review included the following terms;

Exploded MeSH terms: 'aprotinin' 'tranexamic acid' 'Aminocaproic acids' 'Blood transfusion' 'Hemorrhage' 'Anesthesia'.

Text-word terms:aprotinin, antilysin, contrical, kallikrein-trypsin, bovine pancreatic trypsin, tranexamic, cyklokapron, pharmacia, $\mathrm{t}$ amcha, amcha, ugurol, transamin, kabi, epsilon-aminocaproic acid, aminocaproic, lederle, amicar, transfusion\$, bleed\$, blood loss\$, hemorrhag\$.

\section{Appendix 2. Search strategy: 2010 update}

Cochrane Injuries Group Specialised Register (searched July 2010)

(Aprotinin* or kallikrein-trypsin inactivator* or bovine kunitz pancreatic trypsin inhibitor* or bovine pancreatic trypsin inhibitor* or basic pancreatic trypsin inhibitor* or BPTI or contrykal or kontrykal or kontrikal or contrical or dilmintal or iniprol or zymofren or traskolan or antilysin or pulmin or amicar or caprocid or epsamon or epsikapron or antilysin or iniprol or kontrikal or kontrykal or pulmin* or Trasylol or Antilysin Spofa or rp?9921 or antagosan or antilysin or antilysine or apronitin* or apronitrine or bayer a? 128 or bovine pancreatic secretory trypsin inhibitor* or contrycal or frey inhibitor* or gordox or kallikrein trypsin inhibitor* or kazal type trypsin inhibitor* or (Kunitz adj3 inhibitor*) or midran or (pancrea* adj2 antitrypsin) or (pancrea* adj2 trypsin inhibitor*) or riker?52g or rp?9921 or tracylol or trascolan or trasilol or traskolan or trazylol or zymofren or zymophren) or (tranexamic or Cyclohexanecarboxylic Acid* or Methylamine* or amcha or trans-4-aminomethyl-cyclohexanecarboxylic acid* or t-amcha or amca or kabi 2161 or transamin* or exacyl or amchafibrin or anvitoff or spotof or cyklokapron or ugurol oramino methylcyclohexane carboxylate or aminomethylcyclohexanecarbonic acid or aminomethylcyclohexanecarboxylic acid or AMCHA or amchafibrin or amikapron or aminomethyl cyclohexane carboxylic acid or aminomethyl cyclohexanecarboxylic acid or aminomethylcyclohexane carbonic acid or aminomethylcyclohexane carboxylic acid or aminomethylcyclohexanecarbonic acid or aminomethylcyclohexanecarboxylic acid or aminomethylcyclohexanocarboxylic acid or aminomethylcyclohexanoic acid or amstat or anvitoff or cl?65336 or cl65336 or cyclocapron or cyclokapron or cyklocapron or exacyl or frenolyse or hexacapron or hexakapron or tranex or TXA) or (aminocaproic or amino?caproic or aminohexanoic or amino?hexanoic or epsilon-aminocaproic or E-aminocaproic) adj2 acid*) or epsikapron or cy-116 or cy116 or epsamon or amicar or caprocid or lederle or Aminocaproic or aminohexanoic or amino caproic or amino n hexanoic or acikaprin or afibrin or capracid or capramol or caprogel or caprolest or caprolisine or caprolysin or capromol or cl 10304 or EACA or eaca roche or ecapron or ekaprol or epsamon or epsicapron or epsilcapramin or epsilon amino caproate or epsilon aminocaproate or epsilonaminocaproic or etha?aminocaproic or ethaaminocaproich or emocaprol or hepin or ipsilon or jd?177or neocaprol or nsc? 26154 or tachostyptan)

MEDLINE(Ovid) 1950 to July Week 22010

1. exp Antifibrinolytic Agents/ 
2. (anti-fibrinolytic* or antifibrinolytic* or antifibrinolysin* or anti-fibrinolysin* or antiplasmin* or anti-plasmin* or ((plasmin or fibrinolysis) adj3 inhibitor*)).ab,ti.

3. exp Aprotinin/

4. (Aprotinin* or kallikrein-trypsin inactivator* or bovine kunitz pancreatic trypsin inhibitor* or bovine pancreatic trypsin inhibitor* or basic pancreatic trypsin inhibitor* or BPTI or contrykal or kontrykal or kontrikal or contrical or dilmintal or iniprol or zymofren or traskolan or antilysin or pulmin or amicar or caprocid or epsamon or epsikapron or antilysin or iniprol or kontrikal or kontrykal or pulmin* or Trasylol or Antilysin Spofa or rp?9921 or antagosan or antilysin or antilysine or apronitin* or apronitrine or bayer a? 128 or bovine pancreatic secretory trypsin inhibitor* or contrycal or frey inhibitor* or gordox or kallikrein trypsin inhibitor* or kazal type trypsin inhibitor* or (Kunitz adj3 inhibitor*) or midran or (pancrea* adj2 antitrypsin) or (pancrea* adj2 trypsin inhibitor*) or riker? $52 \mathrm{~g}$ or rp?9921 or tracylol or trascolan or trasilol or traskolan or trazylol or zymofren or zymophren).ab,ti.

5. exp Tranexamic Acid/

6. (tranexamic or Cyclohexanecarboxylic Acid* or Methylamine* or amcha or trans-4-aminomethyl-cyclohexanecarboxylic acid* or tamcha or amca or kabi 2161 or transamin* or exacyl or amchafibrin or anvitoff or spotof or cyklokapron or ugurol oramino methylcyclohexane carboxylate or aminomethylcyclohexanecarbonic acid or aminomethylcyclohexanecarboxylic acid or AMCHA or amchafibrin or amikapron or aminomethyl cyclohexane carboxylic acid or aminomethyl cyclohexanecarboxylic acid or aminomethylcyclohexane carbonic acid or aminomethylcyclohexane carboxylic acid or aminomethylcyclohexanecarbonic acid or aminomethylcyclohexanecarboxylic acid or aminomethylcyclohexanocarboxylic acid or aminomethylcyclohexanoic acid or amstat or anvitoff or cl?65336 or cl65336 or cyclocapron or cyclokapron or cyklocapron or exacyl or frenolyse or hexacapron or hexakapron or tranex or TXA).ab,ti.

7. exp Aminocaproic Acids/ or exp 6-Aminocaproic Acid/

8. (((aminocaproic or amino?caproic or aminohexanoic or amino?hexanoic or epsilon-aminocaproic or E-aminocaproic) adj2 acid*) or epsikapron or cy-116 or cy116 or epsamon or amicar or caprocid or lederle or Aminocaproic or aminohexanoic or amino caproic or amino $\mathrm{n}$ hexanoic or acikaprin or afibrin or capracid or capramol or caprogel or caprolest or caprolisine or caprolysin or capromol or cl 10304 or EACA or eaca roche or ecapron or ekaprol or epsamon or epsicapron or epsilcapramin or epsilon amino caproate or epsilon aminocaproate or epsilonaminocaproic or etha?aminocaproic or ethaaminocaproich or emocaprol or hepin or ipsilon or jd? 177or neocaprol or nsc?26154 or tachostyptan).ab,ti.

9. 1 or 2 or 3 or 4 or 5 or 6 or 7 or 8

10. randomi?ed.ab,ti.

11. randomized controlled trial.pt.

12. controlled clinical trial.pt.

13. placebo.ab.

14. clinical trials as topic.sh.

15. randomly.ab.

16. trial.ti.

17. 10 or 11 or 12 or 13 or 14 or 15 or 16

18. (animals not (humans and animals)).sh.

19.17 not 18

20. 9 and 19

\section{EMBASE (Ovid) 1980 to 2010 Week 28}

1. exp Antifibrinolytic Agent/

2. (anti-fibrinolytic* or antifibrinolytic* or antifibrinolysin* or anti-fibrinolysin* or antiplasmin* or anti-plasmin* or ((plasmin or fibrinolysis) adj3 inhibitor*)).ab,ti.

3. exp Aprotinin/

4. (Aprotinin* or kallikrein-trypsin inactivator* or bovine kunitz pancreatic trypsin inhibitor* or bovine pancreatic trypsin inhibitor* or basic pancreatic trypsin inhibitor* or BPTI or contrykal or kontrykal or kontrikal or contrical or dilmintal or iniprol or zymofren or traskolan or antilysin or pulmin or amicar or caprocid or epsamon or epsikapron or antilysin or iniprol or kontrikal or kontrykal or pulmin* or Trasylol or Antilysin Spofa or rp?9921 or antagosan or antilysin or antilysine or apronitin* or apronitrine or bayer a?128 or bovine pancreatic secretory trypsin inhibitor* or contrycal or frey inhibitor* or gordox or kallikrein trypsin inhibitor* or kazal type trypsin inhibitor* or (Kunitz adj3 inhibitor*) or midran or (pancrea* adj2 antitrypsin) or (pancrea* adj2 trypsin inhibitor*) or riker? $52 \mathrm{~g}$ or rp?9921or tracylol or trascolan or trasilol or traskolan or trazylol or zymofren or zymophren).ab,ti.

5. exp Tranexamic Acid/

6. (tranexamic or Cyclohexanecarboxylic Acid* or Methylamine* or amcha or trans-4-aminomethyl-cyclohexanecarboxylic acid* or tamcha or amca or kabi 2161 or transamin* or exacyl or amchafibrin or anvitoff or spotof or cyklokapron or ugurol oramino methylcyclo- 
hexane carboxylate or aminomethylcyclohexanecarbonic acid or aminomethylcyclohexanecarboxylic acid or AMCHA or amchafibrin or amikapron or aminomethyl cyclohexane carboxylic acid or aminomethyl cyclohexanecarboxylic acid or aminomethylcyclohexane carbonic acid or aminomethylcyclohexane carboxylic acid or aminomethylcyclohexanecarbonic acid or aminomethylcyclohexanecarboxylic acid or aminomethylcyclohexanocarboxylic acid or aminomethylcyclohexanoic acid or amstat or anvitoff or cl?65336 or cl65336 or cyclocapron or cyclokapron or cyklocapron or exacyl or frenolyse or hexacapron or hexakapron or tranex or TXA).ab,ti.

7. exp Aminocaproic Acid/

8. (((aminocaproic or amino?caproic or aminohexanoic or amino?hexanoic or epsilon-aminocaproic or E-aminocaproic) adj2 acid*) or epsikapron or cy-116 or cy116 or epsamon or amicar or caprocid or lederle or Aminocaproic or aminohexanoic or amino caproic or amino $\mathrm{n}$ hexanoic or acikaprin or afibrin or capracid or capramol or caprogel or caprolest or caprolisine or caprolysin or capromol or cl 10304 or EACA or eaca roche or ecapron or ekaprol or epsamon or epsicapron or epsilcapramin or epsilon amino caproate or epsilon aminocaproate or epsilonaminocaproic or etha?aminocaproic or ethaaminocaproich or emocaprol or hepin or ipsilon or jd? 177or neocaprol or nsc?26154 or tachostyptan).ab,ti.

9. 1 or 2 or 3 or 4 or 5 or 6 or 7 or 8

10. exp Randomized Controlled Trial/

11. exp controlled clinical trial/

12. randomi?ed.ab,ti.

13. placebo.ab.

14. * Clinical Trial/

15. randomly.ab.

16. trial.ti.

17. 10 or 11 or 12 or 13 or 14 or 15 or 16

18. exp animal/ not (exp human/ and exp animal/)

19. 17 not 18

20. 9 and 19

Cochrane Central Register of Controlled Trials (The Cochrane Library 2010, Issue 3)

\#1 MeSH descriptor Antifibrinolytic Agents explode all trees

\#2 (anti-fibrinolytic* or antifibrinolytic* or antifibrinolysin* or anti-fibrinolysin* or antiplasmin* or anti-plasmin*):ab,ti or ((plasmin or fibrinolysis) near3 inhibitor*):ab,ti

\#3 MeSH descriptor Aprotinin explode all trees

\#4 (Aprotinin* or kallikrein-trypsin inactivator* or bovine kunitz pancreatic trypsin inhibitor* or bovine pancreatic trypsin inhibitor* or basic pancreatic trypsin inhibitor* or BPTI or contrykal or kontrykal or kontrikal or contrical or dilmintal or iniprol or zymofren or traskolan or antilysin or pulmin or amicar or caprocid or epsamon or epsikapron or antilysin or iniprol or kontrikal or kontrykal or pulmin* or Trasylol or Antilysin Spofa or rp?9921 or antagosan or antilysin or antilysine or apronitin* or apronitrine or bayer a? 128 or bovine pancreatic secretory trypsin inhibitor* or contrycal or frey inhibitor* or gordox or kallikrein trypsin inhibitor* or kazal type trypsin inhibitor or riker?52g or rp?9921or tracylol or trascolan or trasilol or traskolan or trazylol or zymofren or zymophren or midran):ab,ti or ((Kunitz near3 inhibitor*) or (pancrea* near3 antitrypsin) or (pancrea* near3 trypsin next inhibitor*)):ab,ti

\#5 MeSH descriptor Tranexamic Acid explode all trees

\#6 (tranexamic or Cyclohexanecarboxylic Acid* or Methylamine* or amcha or trans-4-aminomethyl-cyclohexanecarboxylic acid* or tamcha or amca or kabi 2161 or transamin* or exacyl or amchafibrin or anvitoff or spotof or cyklokapron or ugurol oramino methylcyclohexane carboxylate or aminomethylcyclohexanecarbonic acid or aminomethylcyclohexanecarboxylic acid or AMCHA or amchafibrin or amikapron or aminomethyl cyclohexane carboxylic acid or aminomethyl cyclohexanecarboxylic acid or aminomethylcyclohexane carbonic acid or aminomethylcyclohexane carboxylic acid or aminomethylcyclohexanecarbonic acid or aminomethylcyclohexanecarboxylic acid or aminomethylcyclohexanocarboxylic acid or aminomethylcyclohexanoic acid or amstat or anvitoff or cl?65336 or cl65336 or cyclocapron or cyclokapron or cyklocapron or exacyl or frenolyse or hexacapron or hexakapron or tranex or TXA):ab,ti

\#7 MeSH descriptor Aminocaproic Acids explode all trees

\#8 MeSH descriptor 6-Aminocaproic Acid explode all trees

\#9 (epsikapron or cy-116 or cy116 or epsamon or amicar or caprocid or lederle or Aminocaproic or aminohexanoic or amino caproic or amino $\mathrm{n}$ hexanoic or acikaprin or afibrin or capracid or capramol or caprogel or caprolest or caprolisine or caprolysin or capromol or cl 10304 or EACA or eaca roche or ecapron or ekaprol or epsamon or epsicapron or epsilcapramin or epsilon amino caproate or epsilon aminocaproate or epsilonaminocaproic or etha?aminocaproic or ethaaminocaproich or emocaprol or hepin or ipsilon or jd? 177 or neocaprol or nsc?26154 or tachostyptan):ab,ti

\#10 (aminocaproic or amino?caproic or aminohexanoic or amino?hexanoic or epsilon-aminocaproic or E-aminocaproic):ab,ti

Anti-fibrinolytic use for minimising perioperative allogeneic blood transfusion (Review)

Copyright (@) 20II The Cochrane Collaboration. Published by John Wiley \& Sons, Ltd. 


\section{WHAT'S NEW}

Last assessed as up-to-date: 31 January 2010.

\begin{tabular}{l|l|l}
\hline Date & Event & Description \\
\hline 31 May 2010 & New citation required and conclusions have changed & $\begin{array}{l}\text { The searches were updated to February 2010. An additional } \\
40 \text { trials have been included. The updated data show a lower } \\
\text { rate of death with the lysine analogues than aprotinin, which } \\
\text { has been withdrawn from world markets. }\end{array}$ \\
\hline
\end{tabular}

\section{H I S T O R Y}

Protocol first published: Issue 1, 1999

Review first published: Issue 1, 1999

\begin{tabular}{lll}
\hline Date & Event & Description \\
\hline 10 September 2008 & Amended & The text of'Type of surgery' under 'Aprotinin' in the 'Effects of interventions' section was amended. \\
\hline 8 September 2008 & Amended & Converted to new review format. \\
\hline
\end{tabular}

\section{CONTRIBUTIONS OFAUTHORS}

Contributors (names are listed alphabetically)

Paul Carless (University of Newcastle) obtained relevant papers, applied inclusion/ exclusion criteria to retrieved papers, quality assessed trials, extracted data from the trials, entered data into RevMan Analyses, entered study details into Review Manager 4.2.8, and co-wrote review; Dean Fergusson (ISPOT Coordinator*) co-conceived the review, performed the original literature searches, data extraction, and analyses; David Henry (University of Newcastle) obtained funding for the study, was involved in study design, screened abstracts and titles for relevant articles, and co-wrote review; Katharine Ker (London School of Hygiene \& Tropical Medicine) performed updated literature searches extracted data and co-wrote the updated review; Annette Moxey (University of Newcastle) obtained relevant papers, applied inclusion/ exclusion criteria to retrieved papers, quality assessed trials, extracted data from the trials and entered data into MetaView 3.1; Dianne O'Connell (University of Newcastle) provided statistical consultancy for the review, checked data for consistency, analysed and interpreted the results, provided methodological content, and co-wrote review, Barrie Stokes (University of Newcastle) provided statistical consultancy for the review and performed Bayesian analyses.

* ISPOT - International Study of Peri-Operative Transfusion 


\section{DECLARATIONS OF INTEREST}

None known.

\section{SOURCES OF SUPPORT}

\section{Internal sources}

- Special purpose grant, Hunter Area Pathology Service, Australia.

\section{External sources}

- Australian Health Ministers' Advisory Committee. National Health and Medical Research Council of Australia, Australia.

\section{N DEX TERMS}

\section{Medical Subject Headings (MeSH)}

6-Aminocaproic Acid [therapeutic use]; Antifibrinolytic Agents [*therapeutic use]; Aprotinin [therapeutic use]; Erythrocyte Transfusion [*utilization]; Randomized Controlled Trials as Topic; Tranexamic Acid [therapeutic use]; Transplantation, Homologous

\section{MeSH check words}

Humans 